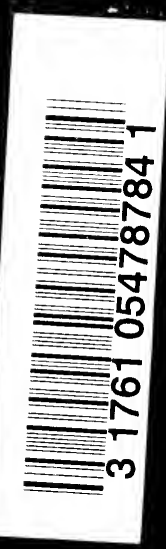




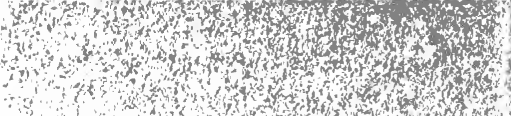

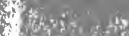

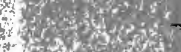

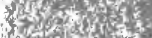

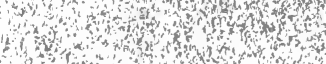

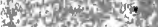

4. tow 020 int

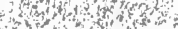

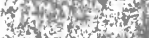
(

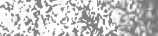
sing its and rents o s ins

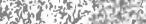

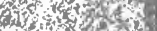

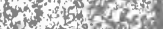
m

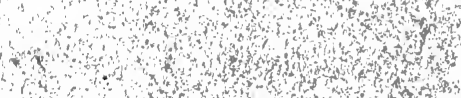

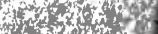
(4) 3.

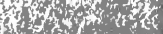
in (3) for trats.

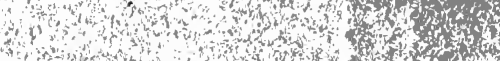

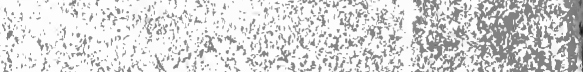
4
3

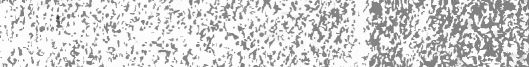
3.

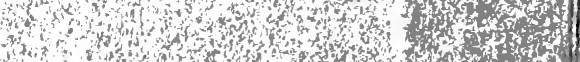
istion tet. and (1)

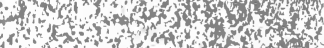





\section{AN INTRODUCTION TO THE STUDY OF SCIENCE}




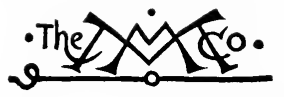

THE MACMILLAN COMPANY

NEW YORK - BOSTON - CHICAGO - DALLAS ATLANTA - SAN FRANCISCO

MACMILLAN \& CO., Limited

LONDON - BOMBAY - CALCUTTA

MELBOURNE

THE MACMILLAN CO. OF CANADA, ĹtD. TORONTO 


\section{AN INTRODUCTION}

TO THE

\section{STUDY OF SCIENCE}

A FIRST COURSE IN SCIENCE FOR

HIGH SCHOOLS

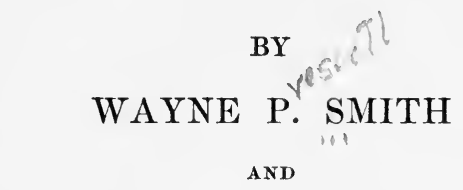

EDMUND GALE JEWETT

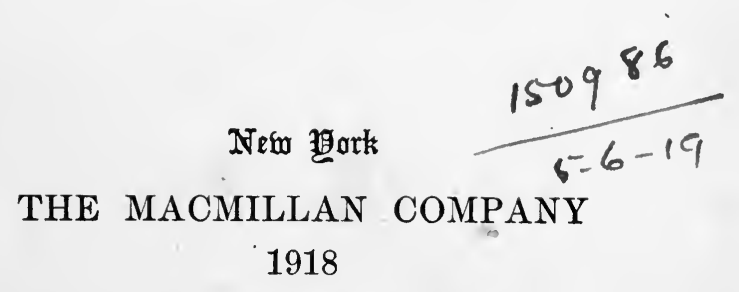

All rights reserved 
Copyright, 1918,

BY THE MACMILLAN COMPANY.

Set up and electrotyped. Published August, I918.

Norwood 呫ess

J. S. Cushing Co. - Berwick \& Smith Co.

Norwood, Mass., U.S.A. 


\section{PREFACE}

The purpose of the book is to introduce boys and girls in their first science course in high school to a scientific study of some of the principal features of their environment. Such an introductory course in the scientific study of common things is now generally recognized as an essential part of the educational opportunities afforded by our secondary schools.

The general method of the book in dealing with the topics, or projects, selected for study is broadly inductive or heuristic, the presentation following the time order of mental development of boys and girls as closely as effective teaching permits. This emphasis upon the psychological rather than the traditional logical or dogmatic method of procedure aids in realizing the purpose of the book, which is to guide students in the development of a scientific habit of mind, that is of a method of investigating and mastering their projects and problems, which is economical as well as effective.

By dealing intensively and extensively with significant and typical features of everyday experience, the student is led to observe intelligently and with a definite purpose dominating his observation, to test experimentally the observations, and in due course to form a working understanding or theory of the subject under consideration. This is vastly more educative and valuable than any fund of so-called useful information a textbook may offer. The information, therefore, which is given in the book is to be used as a tool or means of understanding and acquiring better control of the situation in hand; it is not for the sake of some possible future emergency that may never occur.

Such a course as is here outlined helps to fulfil the function of scientific instruction in our public schools. This function is conceived to be the initiation of young students into what scientific thought has accomplished for the human race. One 
effect that reasonably may be hoped for is the training of boys and girls to rise above the merely local and temporary aspects of common things through the discovery and understanding of principles that are scientifically assured, that are independent of personal prejudice or habit, and that make for a better understanding and wiser use of the forces and resources of the larger environment of which the local and temporary is a part.

The following features are mentioned as significant in relation to the method of the book.

(1) Experimentation, an integral part of the whole study, is used to test, emphasize, clarify, or illustrate ordinary observations or beliefs, and to show the why and the how much in a given situation. Experiments are described not to foreclose, but to stimulate the students' ingenuity in devising different ways and means of dealing with a project. The aim is to make the students feel the need of experimentation, - not to force a set experiment upon them as a task, which is both irksome and profitless. Experimentation is just a controlled method of observation; as such it is more than a mere manipulation of materials and apparatus. To experiment involves an ènd or aim and with this the choice of ways and means of reaching the desired end. To help young students to understand the need of controlled observation and to train them in choosing intelligently the means and materials by which ends may be realized is one of the most important aims of any introductory course in the scientific study of the things of common experience.

(2) Scientific ideas and facts which emerge in course of study are used in discovering and interpreting new significant aspects of the environment. It is the use, not the acquisition, of knowledge which is educationally worth while. This use it is the aim of the book everywhere to emphasize.

(3) No effort is made to force the adult divisions of scientific knowledge upon the beginner in scientific study. In dealing in a scientific way with familiar subjects of the household, the garden, the community, the farm, the factory, and the great 
industry of transportation, special points of view, purposes, and methods emerge, which are defined gradually and appropriately to the students' needs and interests and in such a way as to lead them to organize what they know and think about the subjects. It is recognized, to quote the words of Professor Dewey, that "it is only because we have different interests or different ends, that we sort out the material and label part of it science, part, history, part, geography, and so on." So with the differentiation of science into its conventional branches it must be an objective, and not the beginning of scientific study; and progress in making such divisions depends upon needs, interests, and ends.

(4) Emphasis is constantly placed upon the utility of a scientific understanding of things in acquiring a better control and use of them for the purposes and welfare of mankind.

(5) The study of projects suitable to a given class or place is facilitated by the abundance of material which the book affords. The book provides sufficient work for a year, with opportunities for selection to meet special requirements. For a half-year course selections may be made readily by the aid of the topics into which the chapters are divided.

In the preparation of the book, which has extended over a period of six and more years, a great indebtedness to many teachers and scientific works has been incurred. The use of the information and help thus gained is such as to make specific mention of sources almost impossible; general acknowledgment is made with gratitude and appreciation. It is a pleasure to express our obligation to Professor George I. Finlay of New York University for helpful criticisms made in the manuscript; to Ivy G. Smith for untiring help in preparing the manuscript, for many of the drawings, and for the index ; and to The Macmillan Company for generous coöperation in practically every stage of the making of the book.

New York City, May 20, 1918. 



\section{TABLE OF CONTENTS}

\section{CHAPTER I}

The Weather .

How Forecasts are Made, 1; A Study of Weather Maps, 4; Air Pressure, 10; The Barometer, 13; Winds, 19; Cyclones and Anticyclones, 25; Local Storms, 34; The Moisture of the Atmosphere, 42.

\section{CHAPTER II}

Fire and Heat . $. \quad . \quad . \quad . \quad . \quad . \quad . \quad$.

Heat - Its Uses and Control, 58; Why Fire Burns, 69; Temperature. - The Thermometer, 84; How Heat is Transferred, 93 ; Products and Materials of Combústion, 113 ; Measuring Heat, 141.

CHAPTER III

REFRIGERATION

CHAPTER IV

Artificial Lighting

Illuminating Gas, 160; Electric Lighting, 165; Colors of Objects, 179; General Requirements in Artificial Lighting, 181.

\section{CHAPTER V}

The Supply and Uses of Water.

The Water Supply, 186; The Water Supply of Cities, 188; Rural Water Supply, 197; Dams, 200; The Water Supply and Disease, 206; Water as a Carrier of Sewage, 210 ; Purification of Water, Municipal and Domestic, 218; Outline for the Study of Local Water Supply, 223; Water Pressure, 225; Water Power, 233 ; Water Wheels, 238. 
Vehicles of Transportation, 253; Roads, 264; Railroads, 266 ; Curves, Inertia and its Effects, 274 ; Bridges, 280; Steam and the Locomotive, 289; The Production and Control of Steam, 297; Flectricity as a Motive Power, 316; Gasoline as a Motive Power, 330 .

\section{CHAPTER VII}

Transportation on Water.

Buoyancy, 338 ; Stability, 344 ; Rowing a Boat, 349 ; Sailing a - Boat, 353; The Steamship, 362; Instruments of Navigation, 370 ; Harbors, 379 ; Inland Waterways, 380.

\section{CHAPTER VIII}

Transportation in Air

Balloons, 390; The Airplane, 394.

\section{CHAPTER IX}

Beilding Materials, Their Use and Origin . . . . 403

Wood, 404 ; Stone, 406 ; Groups of Rocks, 413 ; Some Manufactured Mineral Produets, 417.

\section{CHAPTER $\mathrm{X}$}

Steel and Iron

Properties of Iron, 431; Iron Ore, 432; Reduction of Iron Ores, 437 ; Steel, 441 ; Wrought Iron, 449.

\section{CHAPTER XI}

Plants in Relation to Man

The Soil and Plants, 454; Natural and Artificial Selection, 477; Pollination and Seed Production, 483; Improvement of Plants, 496; Propagation of Plants, 499; Mendel's Law of Inheritance, 505 ; Manufacture of Food by Plants, 515. 


\section{CHAPTER XII}

Insects and Human Health .

A Typical Germ Disease - Malaria, 523; The Mosquito, 535

\section{CHAPTER XIII}

Microörganisms in Relation to Man . . . . . 546

The Dust of the Atmosphere, 546; Molds, 549; Yeasts, 558; Bacteria, 570.

\section{CHAPTER XIV}

The Protection of Health . . . . . . . . 588

Infection and Immunity, 588; Modern Theories of Immunity, 599; Vaccines and Antitoxins, 604. 


\section{INTRODUCTION TO THE STUDY OF SCIENCE}

\section{CHAPTER I \\ THE WEATHER}

\section{How Forecasts are Made}

1. The atmosphere and life. - The ocean of air at the bottom of which we live is one of the most important parts of our world. We do not usually recognize its importance unless we are threatened with deprivation of it, or are subjected to air that is bad and unsafe to breathe. Without air, however, life is impossible. Air that is impure or robbed of its usual properties undermines health and causes life to become feeble and miserable.

No other part of our world exercises so great and uninterrupted influence upon all living things as does the air. The air and its changes determine largely our manner of living and working, the kind and amount of things we grow in the soil or produce in factories, the sort of food we eat, and the kind of shelter and clothing we enjoy. It may indeed be said that it determines the kind of civilization the people of any given region of the earth may have.

2. Weather and climate. - The changes in the conditions of the atmosphere from one state to another, such as hot or cold, wet or dry, clear or cloudy, calm or windy, are generally summed up in the term weather. By this term we refer to the conditions of the air at any particular time or for a given 
period, such as an hour, a day, a week, or even for a season. The total series of weather changes and conditions is generally described by the term climate. This term is sometimes considered to be equivalent to the average weather of a region. Such a use of the term is inadequate, because climate must include several factors neglected in any account of the average weather. Some of the factors which climate necessarily includes are these: the extremes, the irregular variations, and the mean of such unusual temperatures; the humidity, cloudiness, rainfall, and the amount of rainfall accompanying cyclonic and local storms; the distribution, duration, and frequency of any weather conditions. If you consider the average weather of your own locality, you will at once realize that you get from it a very inadequate idea of the climate, that is, the total series of weather conditions.

3. Kinds of climate. - Climates are roughly distinguished from one another by such descriptive terms as mild or rigorous, hot or cold, wet or dry ; or as the climate of Florida, California, New England, the Bermuda Islands, Italy, Siberia, and so on. We are familiar also with the classification of climates by zones, as the tropical, the two temperate, and the two polar zones. There are other classifications based on the conditions affecting plant life, animal life, or general geographic considerations. Perhaps the following classification is as useful as any: the oceanic climate, sea level continental climate, and mountain climate, with such subdivisions as coast climate, desert climate, and monsoon climate. This classification gives due consideration to the conditions which actually control climate, namely, sun, water, land, and altitude. It gives moreover a peculiar definiteness to the several climate zones.

4. Prediction of weather changes. - Common consideration of the weather does not stop with the mere statement of the present conditions of the atmosphere, but it leads to the effort to forecast the kind of weather changes to be expected. Men have learned that while the unfavorable features of the weather, 
such as extreme cold or heat, and violent storms, cannot be controlled or modified by human power, the discomfort and inconvenience which they cause may be avoided in large measure. To avoid them, however, it is necessary to know beforehand what sort of weather is likely to come. This men have learned to do by discovering and interpreting the conditions which are supposed to control the succession of weather changes. Those who have made a thorough study of such conditions and their significance are able to predict with a high degree of accuracy weather changes for one, two, or even three days in advance.

Any prediction of daily weather changes depends for its degree of probability upon the kind and extent of the observations. If the important conditions of the atmosphere are not observed, or are observed inaccurately, the predictions of weather changes will be untrustworthy. If, however, the conditions are observed carefully and accurately, and then interpreted properly, the weather may be forecast with a fair degree of probability. If such observations be made with the aid of instruments such as the thermometer and barometer, the prediction will have a higher degree of probability. When the local observations are supplemented with reported observations of the whole continent, a prediction based thereon will be almost certain of fulfillment. A forecast made in this manner is invaluable to men, for it makes it possible for them to prepare for unfavorable extremes of weather and thus to experience a minimum of loss or discomfort and a maximum of profit.

The conditions of the atmosphere which must be observed in order to make dependable forecasts of weather changes, are: (1) the appearance of the sky, as at sunrise and sunset, or the halo around the sun or the moon; (2) the form and movement of the clouds; (3) the direction, force, and shifting of the winds; (4) the temperature; (5) the relative humidity or moisture of the atmosphere, which may be felt in its effect 
upon the skin or the mucous membrane of the nose and throat; (6) the pressure of the air, sometimes vaguely described as the "lightness" or " heaviness" of the air.

\section{A Study of Weather Maps}

5. A typical forecast. - The weather forecast for Saturday, December 25, 1915, as published in the morning papers of New York and vicinity was as follows: "For eastern New York, rain in the south and rain or snow in northern portions to-night. Sunday clearing and eolder; strong southerly winds, shifting to westerly." The changes occurred as predicted. Who made the predietion? How was it known what weather changes would occur in this particular region?

Weather forecasts are made by expert officials of the Weather Bureau of the Department of Agriculture of the United States. Any person with practice and knowledge of the few principles involved may make fairly probable forecasts for one or perhaps two days.

6. How weather maps are made. - At government weather stations, which are distributed at advantageous points throughout our country, daily observations are made at eight o'clock morning and evening (Washington time). Observations are made of the temperature and pressure of the atmosphere, the state of the weather, whether cloudy or clear, the direction and force of the wind, and the precipitation of rain or snow for the past twelve or twenty-four hours. This information is reported by telegraph immediately to Washington, Boston, Chicago, San Francisco, New Orleans, and other centrally located cities, where it is recorded upon large outline and topographical maps of the United States. Forecasts are then made of changes to be expected within the following twentyfour to thirty-six hours. The map is printed and copies are mailed for distribution less than half an hour after receiving the reports. The predictions are communicated to the Press and to stations where weather signals are displayed. In case 
of the sudden development of a storm and its approach to localities where it may cause damage, warnings are immediately telegraphed so that signals may be shown. Such warnings are especially important to sailors and fishermen and to fruit-growers in Florida and California.

The weather map which gives the information concerning weather conditions and probable changes may present difficulties to those who have not studied its main features and their significance. The following study will help the student to recognize the essential features promptly and to interpret them accurately. It will also aid in understanding the general principles involved in.weather observation and prediction.

The weather maps, Figs. 1, 2, 3, copies of government maps, give graphical representations of the atmospheric conditions of the country for December 24, and several days following. The dotted lines, which represent red lines on the original maps, give the temperatures, with the numbers printed near the ends of the lines or on circles, expressed in degrees Fahrenheit. Only temperatures divisible by ten are given, as the map would be crowded with lines if all variations were shown. All places, for example, reporting a temperature of $20^{\circ} \mathrm{F}$. are connected by a dotted line; similarly all reporting $30^{\circ} \mathrm{F}$., and so on from minimum to maximum. It may be assumed that the temperature at any point between two lines, as those marked $20^{\circ} \mathrm{F}$. and $30^{\circ} \mathrm{F}$, is roughly indicated by the distance between the lines.

7. Isotherms. - The lines running through places having the same temperatures and showing the temperature conditions of the country for a given date, are called isothermal lines, or simply isotherms. The term is made up of two Greek words, isos meaning equal, and thermos meaning heat, or equalheat. Note that the word thermos supplies the first syllable of thermometer, which means heat-measure.

The isotherms are very irregular in comparison with the parallels of latitude. They curve northward toward a region 


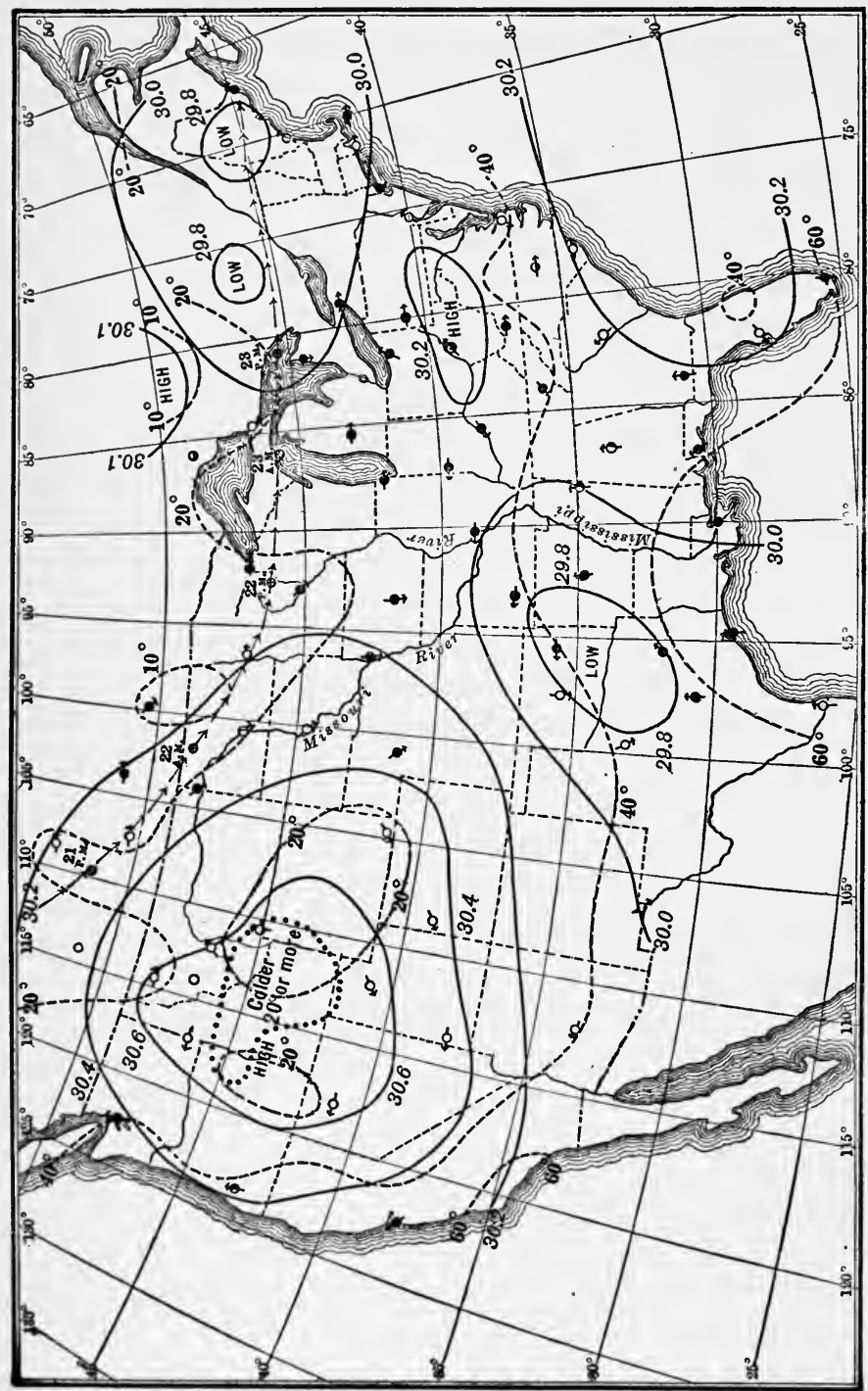

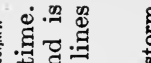

규

记

행

वृ ह

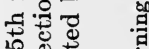

1.

일

$\infty$ क

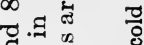

त

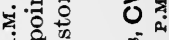

$\infty$ क

+0 क

I

पदि ठ है है

त्ञ

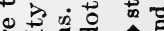

त्ड

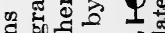

-

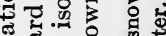

b d

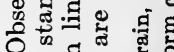

웜

1

당ㅇ 웅

-

सं一 ता

㲾

t)

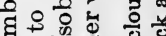

రृ 0 D

ค ల

웡 क्ष

क्ष

\& $0=3$

प टै

엉

तै

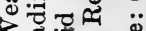

<

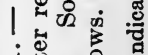

- 1 क्ष

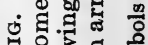

넌 해 음

लำ है 


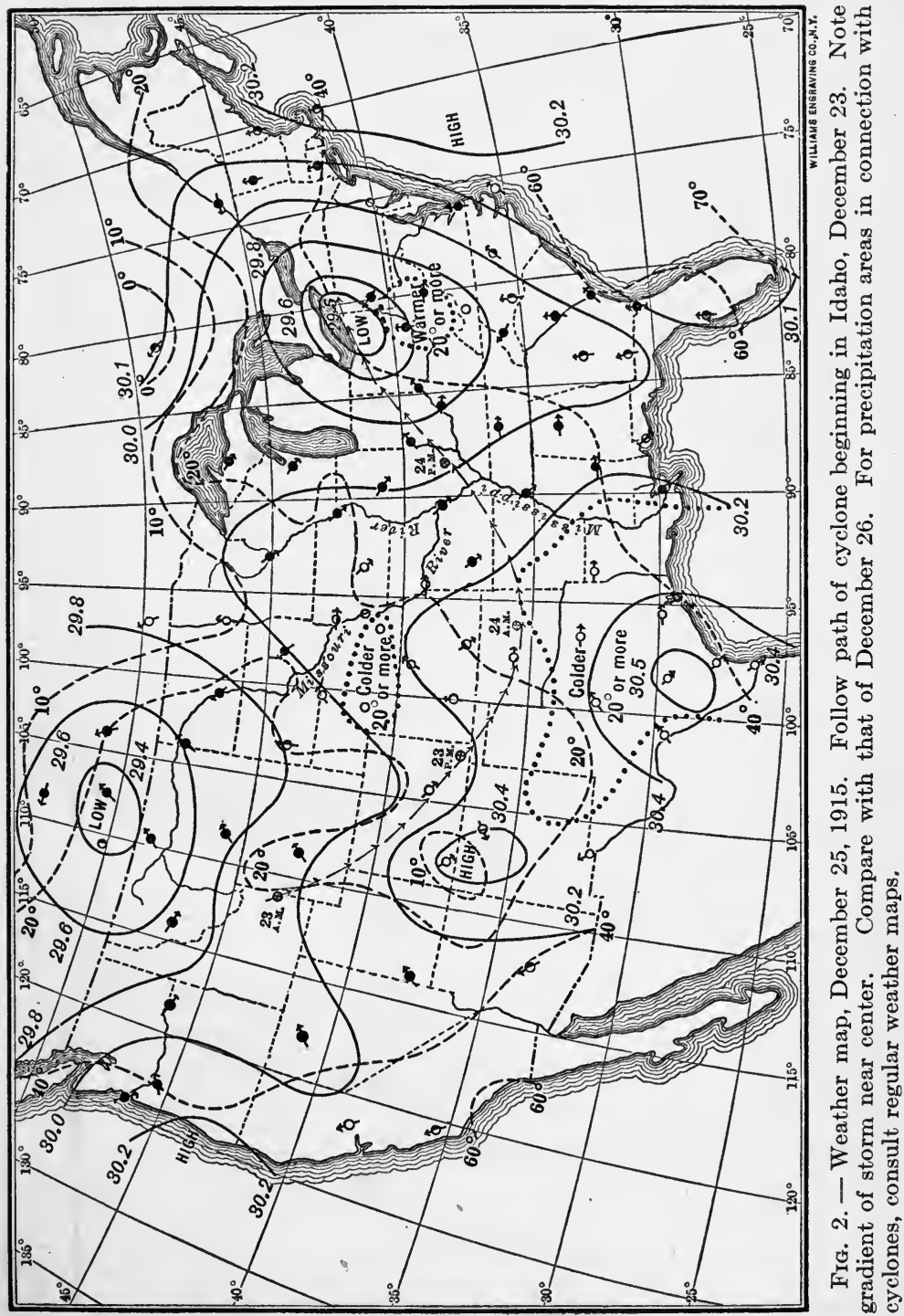




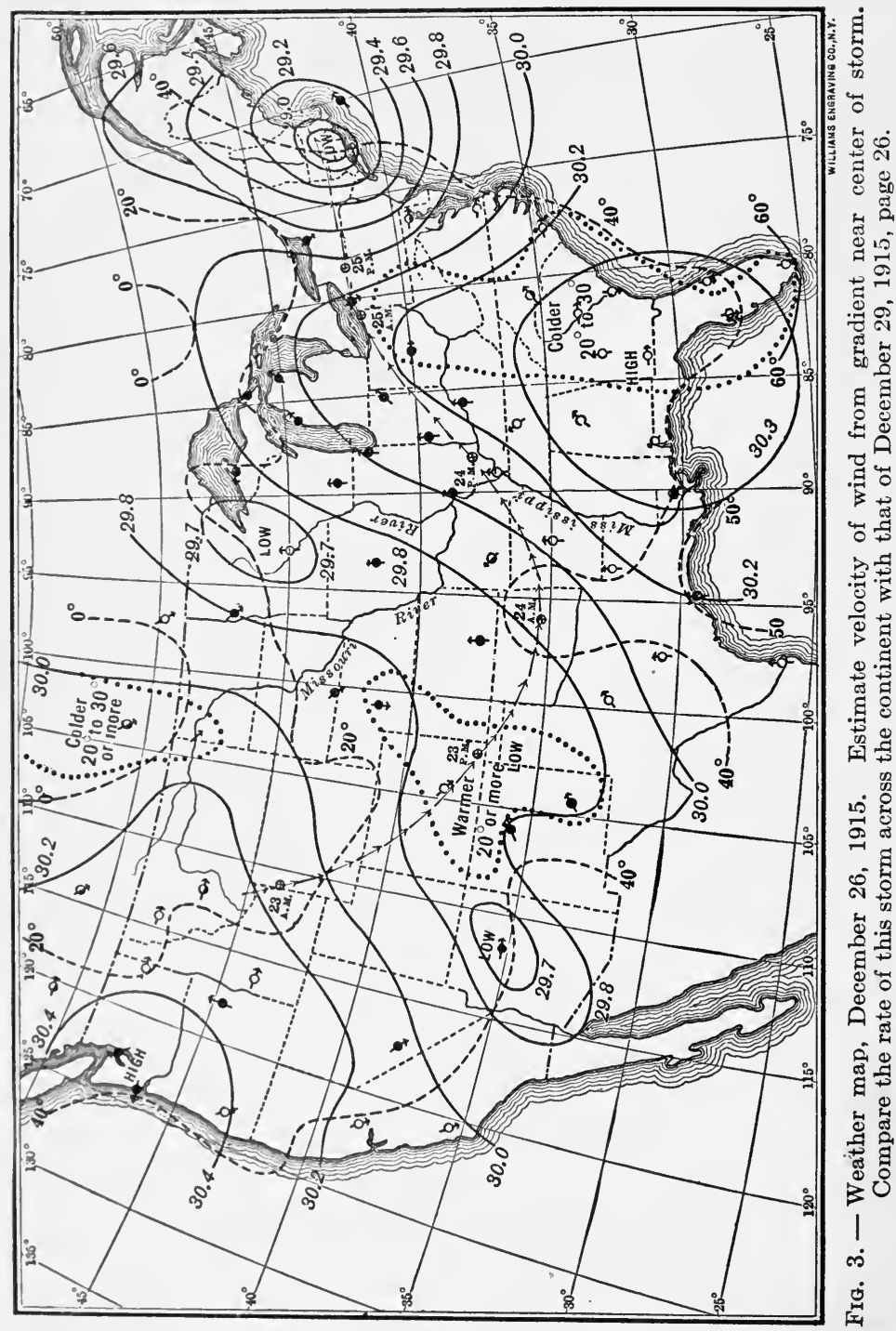


over which there is marked a "low," and southward toward a region marked " high." A relatively low temperature is registered in association with the "highs"; as, for instance, on the map of December 24, the area marked "Colder $20^{\circ}$ or more" is associated with the " high" over southern Idaho and eastern Oregon.

Consider the isotherms curving northward in the region of Maine and Canada, over which there is a "low." With this low the temperature is relatively higher than that of lower latitudes, as in the upper Mississippi valley. Is it to be expected that the isotherms follow parallels of latitude? Give reasons for your answer.

8. Isobars. - The heavy continuous lines on the maps are drawn through places which report the same atmospheric pressure. This is measured in inches of mercury (page 16) with intervals of tenths or hundredths of an inch. The numbers are printed at the ends of the lines.or near circles representing pressures, such as $30,29.9,29.65$. The lines are sometimes nearly straight, but usually curved so that they resemble the letter "U," or run in loops or circles. In each complete circle is printed "high" or "low." "High" appears in circles with a pressure above 30 , as 30.2 or 30.7 . "Low" is printed in circles with pressures below 30 .

What is the average pressure given on any of the maps? How.far is it from thirty inches? What is the greatest pressure on the map of December 24? What is the lowest pressure? Where are these areas located? How many "lows" are there on this map? How many " highs"? Compare the temperatures associated with the low-pressure areas with those associated with the high-pressure areas.

The lines connecting different places having the same pressures on a given date are called isobars. The word is composed of two Greek words, isos meaning equal, and baros meaning weight or pressure. The word baros forms the first syllable of the word barometer, which means weight-measure, the name of 
the instrument by which the pressure of the atmosphere is measured.

Isobars are drawn without regard to altitudes, as on mountains or high plateaus. The legend on the weather map is: "Barometric readings are reduced to sea level and standard gravity." Air pressure diminishes with ascent above sea level, and it increases with descent below sea level, as in a mine or shaft. This general fact is recognized by the observer at any weather station when he adds to the actual pressure of the elevated locality enough to equal the pressure which the locality would have if it were at sea level. You may correct weather map isobars for high altitudes, as explained on page 15 .

Isotherms are similarly constructed without reference to altitude. The snow line of high mountains must be at or below the freezing point, $32^{\circ} \mathrm{F}$. It would be impossible to give on weather maps the temperatures of high elevations without distorting the isothermal lines and giving facts which would be generally misinterpreted. When you desire to obtain from a weather map approximately the temperature of a high altitude, you may subtract one degree from that recorded, for each 270 feet of elevation (page 392).

\section{Air Pressure}

One may find in personal experience evidence that the air exerts pressure. In ascending a high mountain or descending into a deep shaft, or again in riding up or down in a swift elevator in a tall building, one may feel the effects of the change of air pressure upon the ear drums. In the swiftly moving elevator the discomfort experienced may be relieved by swallowing. But what does the fact observed signify? Ordinarily the pressure of the air is exerted equally upon both sides of the ear drums and is therefore not felt. A sudden change in pressure upon one side, as in an elevator ascending twenty to thirty-five stories, causes discomfort. By the act of swallowing, 
the Eustachian tubes are opened, admitting air and equalizing the pressure upon both sides.

At very high altitudes, as on mountains, the pressure is not quickly and easily equalized, and much discomfort and even illness may result. This is due to the expansion of the air in the blood in response to the decreased external pressure upon the body. But the important fact is that air in usual situations exerts pressure equally and in all directions, and because it is equal and in all directions, the pressure is not felt. The pressure is felt only when it is suddenly made unequal.

9. Pressure exerted by air. - How great is the pressure of the air upon things? You may get an idea by covering a glass full of water with a sheet of paper and then inverting the glass. The air pressure upon the paper is enough to support the water in the glass. This shows that pressure is upward as well as downward. A simple experiment will illustrate the important fact.

Exercise: Showing air pressure. - Connect a flat-sided tin can by a piece of rubber tubing with an air pump and exhaust the air from the can. The effect of the pressure of the air upon the outside surface of the can is evident. Or this experiment may be performed more easily, by putting a very small quantity of water in a can that may be tightly stoppered with a rubber stopper. Bring the water to boiling and when the steam is issuing freely from the can, cork it securely. Cool and observe the result. The air is expelled by the steam which, condensing to water, leaves a partial vacuum in the can. Another illustration of the fact of air pressure may be had in this way. An ordinary electric incandescent bulb incloses a fairly complete vacuum. If the glass near the brass part is softened by a hot flame, it cannot withstand the outer pressure of the air and is forced inwards. These simple experiments show clearly that the air exerts pressure.

That the air has weight has been shown by many scientific experiments, and the weight of a given volume of air is known definitely. A strong vessel with a capacity of one cubic foot is weighed with its air content; the air is removed by an air 
pump and the vessel weighed. The difference between the two weights is equal to the weight of a cubic foot of air, that is, one and one-fifth ounces. This accounts for the fact that the air exerts pressure upon the surface of the earth.

Pressure of air at sea level. - The depth of the ocean of air is anywhere from fifty to two hundred miles. The largest bulk, however, is within the fifty-mile boundary, and beyond this is only one twenty-thousandth of the total volume of the air. By careful experiment and calculation it has been learned that the air pressure at sea level amounts to 14.7 pounds to each square inch of surface.

Questions. - Is this more or less than a ton to a square foot? Assume that the surface area of the body is 12 square feet. What is the amount of air pressure upon the body? Why do we not feel this tremendous pressure? Why do we feel its reduction at high altitudes? Do these observed facts indicate that the pressure of the air is equal in all directions? Summarize the points which support your answer.

10. Measuring air pressure. - The pressure of the air is not shown on weather maps as "pounds per square inch," but in the height in inches of a column of mercury which balances the pressure of the air. If water were used instead of mercury for a barometer, the pressure would be stated in feet and inches; for water is about one fourteenth as heavy as mercury. Mercury is exactly 13.6 times as heavy as water, volume for volume. We shall consider a water barometer later. We have now to find out how the air pressure may be determined by the height of a column of mercury.

Exercise. - A cubic inch of mercury weighs .4908 pound. The pressure of the air at sea level upon a square inch of surface is given as 14.7 pounds. A column of mercury with a cross section of one square inch, which would balance or equal the pressure of the air at sea level, must be $14.7 \div .4908$ or 29.95 inches high, or in round numbers 30 inches high. What do you find to be the average pressure given on the weather maps? Would you infer from these facts that 30 inches is the "normal atmospheric pressure"? 
Imagine a glass tube with one end sealed, thirty-eight inches long and with a cross-sectional area of one square inch, filled with mercury, and standing with the open end in a basin containing a quantity of mercury. The tube is arranged so that the mercury may move freely in and out of the tube; but the upper part of the tube is a perfect vacuum, which means that there is no air to press upon the mercury column in the tube. The pressure of the air upon the surface of the mercury in the basin prevents the rest of the mercury from escaping from the tube, just as air pressure holds a sheet of paper under a tumbler filled with water and prevents the escape. of the water. But the mercury is free to move in or out of the tube as the pressure upon the surface in the basin varies. That is, the mercury column will change in height as the pressure upon the surface in the basin increases or decreases. Now suppose that you find the height of the mercury column to be just thirty inches. What is the pressure of the air which balances this? Multiply the weight of one cubic inch of mercury by the height of the column.

Exercise. - Will the column of mercury in the tube rise or fall as the pressure of the air increases? Why? If the pressure of the air upon the surface decreases, how will the height be changed? Explain. What condition makes it possible for the mercury to rise in the tube when the air pressure upon the surface of the mercury in the basin increases? If the upper space in the tube were filled with air, could the mercury column rise? Explain your answer.

The mercury column measures 31 inches. What is the air pressure in pounds?

If the mercury measures 28.4 inches, what is the air pressure?

The air pressure upon a square inch of surface is 12.4 pounds. What will be the height of a mercury column which balances it?

\section{The Barometer}

11. Construction of a barometer. - It will be a useful experience to make an instrument like that just described. Such an instrument is called the mercury barometer. What does this 
term mean? (See page 9.) A barometer for experimental purposes should be made whether the school has a standard barometer or not.

Exercise: Making a barometer. - You will need these materials : a strong heavy glass tube with one end sealed, about thirty-six inches long and with about a three-sixteenths inch bore; a wide-mouth glass bottle of four-ounce capacity; a small quantity of clean mercury; a

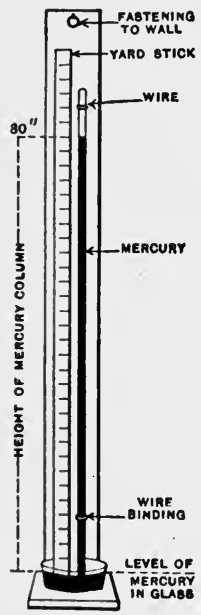

Fig. 4. - A simple barometer, showing arrangement of tube, yardstick, and support.

This simple barometer will meet all ordinary requirements, although it is not as accurate as one needed for scientific observations. You should compare it with a standard barometer, observing any differences in the readings, and learn to correct possible errors. It will give the best results if kept in a room where the temperature remains fairly constant. Record readings daily at about the same hour. Preserve the record for later use in making weather forecasts.

It should be emphasized that a comparatively small difference in barometric readings represents a considerable difference in the pressure of the atmosphere. If 30 inches of mercury represent a pressure of 14.7 pounds per square inch, what will 29 inches represent? Ex- 
press the same pressures in pounds per square foot, and per square yard. Suppose that upon the outside wall of the schoolroom there is an unbalanced pressure, represented by a reading of 30 inches outside and 29 inches inside. What would be the total difference in pressure? Do you think the wall would withstand the outside pressure? Give reasons.

The normal air pressure of your own locality may be determined very simply if you know the elevation. Air pressure decreases within the range of 10,000 feet at the rate of about one tenth of an inch in the height of the mercury column for each ninety feet, or one inch for each nine hundred feet. Thus Spokane, Washington, 1800 feet above sea, level, has a normal average atmospheric pressure of twenty-eight inches. Leadville, Colorado, 10,600 feet elevation, shows a normal barometric reading of twenty inches, or about ten pounds of air pressure to the square inch. Suppose your locality is 2800 feet above sea level, what will be the normal barometric reading? What the amount of air pressure?

12. Water barometer. - We may now consider a possible water barometer. A cubic inch of mercury is 13.6 times as heavy as a cubic inch of water. If the mercury column is thirty inches high, a water column having the same cross-sectional area would be equal to 13.6 times 30 (the height in inches of the mercury column) or 408 (the height in inches of the water column), or 34 feet. That is, the average normal air pressure would support a column of water to the height of thirty-four feet. This emphasizes one advantage of using mercury for the measurement of air pressure.

Exercise: The suction pump. - An interesting application of this fact is found in the old-style suction pump. It was learned long ago that water could not be "lifted" by such a pump as much as thirty-four feet from a well. And finally it was discovered that the pump does not "lift" the water at all ; that as the suction exhausts the air from the pipe and eylinder of such a pump, the pressure of the air upon the free surface of the water in the well forces the water upward into the pipe and cylinder. The water rises to a height at which the weight of the column of water is equal to the pressure of 
the air upon an equal area of the free surface of the water in the well. At normal atmospheric pressure this height is not more than thirty-

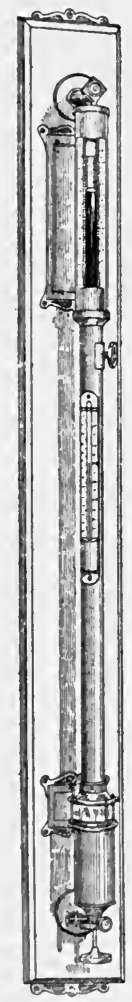

Fig. 5. - Standard barometer, of the type used by the United States Weather Bureau. four feet; but it is usually somewhat less. If a perfect vacuum were secured, how high would the column of water rise? Does this agree with what you have learned of the behavior of mercury in the barometer? Draw a simple plan and explain the operation of the suction pump. If the mercury reading is thirty-one inches, how high would a water barometer stand?

A different scale is sometimes used in our country, although its use is mostly restricted to laboratories for scientific research. This is the metric scale. On this the average normal atmospheric pressure reads 760 millimeters or 76 centimeters. A cubic centimeter of mercury weighs 13.6 grams. What is the average air pressure at sea level, expressed in grams per square centimeter?

13. Kinds of barometers in use. - The standard barometer such as that employed in the stations of the United States Weather Bureau is provided with a scale reading inches and hundredths of an inch, and with an adjustable cistern of mercury (Fig. 5). Another kind of barometer is the self-recording or barograph (Fig. 6). This is a metallic barorneter equipped with a drum and operated by clock work. The record chart, which may be for a day or a week, is attached to the drum. The barograph is sometimes combined with a temperature recorder or thermograph." The chart of such an instrument shows the pressure and the temperature changes together with the hours and days of the week. The barograph is constructed on the same principle as the metallic or aneroid barometer. 
The aneroid or notliquid barometer (Fig. 7) consists chiefly of a cylindrical metal box made of thin corrugated steel or German silver, with an elastic top, and a mechanism to show upon the dial the differences in air pressure. The box is

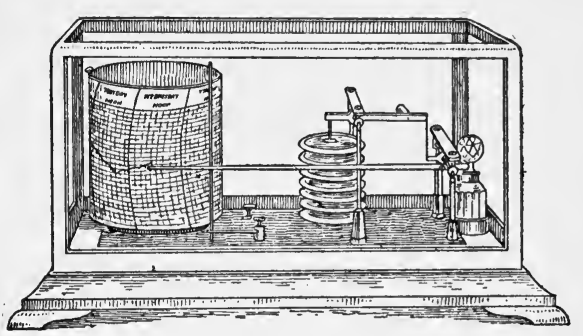

FIG. 6. - Barograph. Note differences between barograph and thermograph. Explain how each works.

a partial vacuum, so that an increase of pressure upon the top forces it inward, and a decrease allows it to curve outward. The pointer attached indirectly to the box indicates

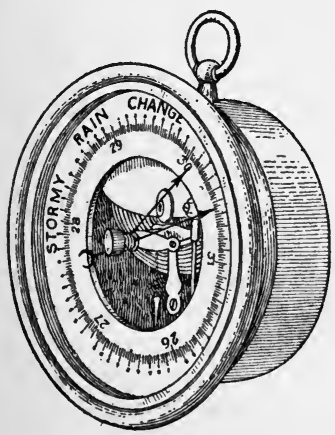

Fig. 7. - Aneroid barometer. the air pressure on the dial in " inches and fractions thereof " in correspondence with the readings of the mercury barometer. The legend usually found on the dial of the aneroid barometer, as "Stormy," "Rain," "Fair," "Change," "Very Dry," is of little or no value. The aneroid is made to meet many different needs, such as those of travelers and engineers as well as for stationary use. It is sometimes made to give the altitudes for the readings of air pressure.

\section{SUMMARY}

Men cannot control weather changes. They can predict changes and make provisions to meet them.

Weather predictions are made more reliable by use of such instruments as the barometer, thermometer, and hygrometer, and by taking into account conditions over wide areas.

Weather conditions observed for forecasting are (1) the appear- 
ance of the sky, (2) clouds, (3) winds, (4) temperature, (5) humidity, and $(6)$ pressure.

Official forecasts are made and published by the United States Weather Bureau.

Weather maps show atmospheric temperatures by isothermal lines, and pressures by isobaric lines.

Air exerts at sea level an average pressure of 14.7 pounds per square inch, called one atmosphere.

The pressure of the air is due to its weight.

A barometer is an instrument for measuring the pressure of the air.

The suction pump depends on the pressure of the air to force the water into its cylinder.

An aneroid barometer, made to record the changes in atmospheric pressure, is called a barograph.

\section{REVIEW QUESTIONS}

1. What is the importance of air to living things? 2. What are some of the special things one mentions when discussing the weather? 3 . Why are we concerned in forecasting the weather? 4. What aids do we have in making local observations? 5. What should supplement local observations to make forecasts accurate? 6. What are the conditions of the atmosphere upon which forecasts are based? 7. How are daily forecasts made in the Federal Weather Bureau? 8. How are the forecasts communicated to the people? 9. Of what practical use is their publication? 10. Explain how weather maps are prepared. 11. What are the sources of the information used by the Weather Bureau in preparing maps and forecasts?

12. How are temperatures indicated on weather maps? 13. What is an isotherm? 14. What is the relation of isotherms to lows and highs? To parallels of latitude? 15. How is the pressure of the atmosphere shown on weather maps? 16. What is an isobar? 17. What two observations are recorded on weather maps, not as actually made but as they would be at sea level? 18. In interpreting weather maps what correction must you make to obtain actual pressures and temperatures of places above sea level?

19. What experience may one have of change in pressure with rapid change of altitude? 20. Why do we not ordinarily feel the pressure of the atmosphere? 21. Why do many people have "mountain sickness" when they reach high altitudes? 22. In what ways can the fact of air pressure be demonstrated? 23. How may air be weighed? What is the approximate weight of one cubic foot of air? 
24. (a) About how deep is the atmosphere? (b) What pressure does it exert per square inch? 25. By what means can the pressure of the air be determined? 26. How do changes in air pressure make changes in the height of the mercury column?

27. Describe the process of making a simple barometer. 28. Show how important small differences in barometric readings are. 29. How may altitude be determined approximately by means of the barometer? 30. Why is mercury more convenient than water for making a barometer? 31. In what position is each of the valves of a lift pump when the plunger goes up? When it goes down? 32. Explain why the suction pump does not lift water from the well. 33. (a) In what units is pressure of the air ordinarily expressed? (b) What units are often used in the laboratory for the same purpose? 34. (a) Describe the construction and operation of an aneroid barometer. (b) What advantages does it possess? 35. What is (a) a thermograph? (b) A barograph? (c) A barothermograph?

\section{WINDS}

14. Direction of winds. - Locate on the weather map for December 26, page 6, the places showing the greatest differences in pressure. Estimate the approximate distance between a high and a low area, such as those over the Atlantic coast states. Consider the direction of the wind, as indicated by arrows, between the two centers. Do the winds move directly away or outward from the high? Do they move at right angles to the isobars, this being the shortest path between the two areas? About halfway toward the low, do the arrows indicate the wind to be going directly toward the low? Compare the diagrammatic map (Fig. 8), in which these points are emphasized more sharply. Is the direction of the winds between the two eastern high and low centers eastward? Follow the course of a wind toward the center of a low and describe its general direction. As a matter of fact, winds about high and low centers do not always complete a circular course, but they do have a general direction which may be described as circular or spiral.

With this understanding of the general circular or spiral 
course of the winds about a low and a high pressure center, we may now consider a particular instance. Take first the low area and its accompanying winds. Observe on the map that the winds are indicated as moving almost parallel with the isobars in a direction opposite to that of the hands of a watch or clock over the dial. This direction is generally described as anticlockwise. Note also that the direction is slightly inward or toward the center, even while keeping nearly

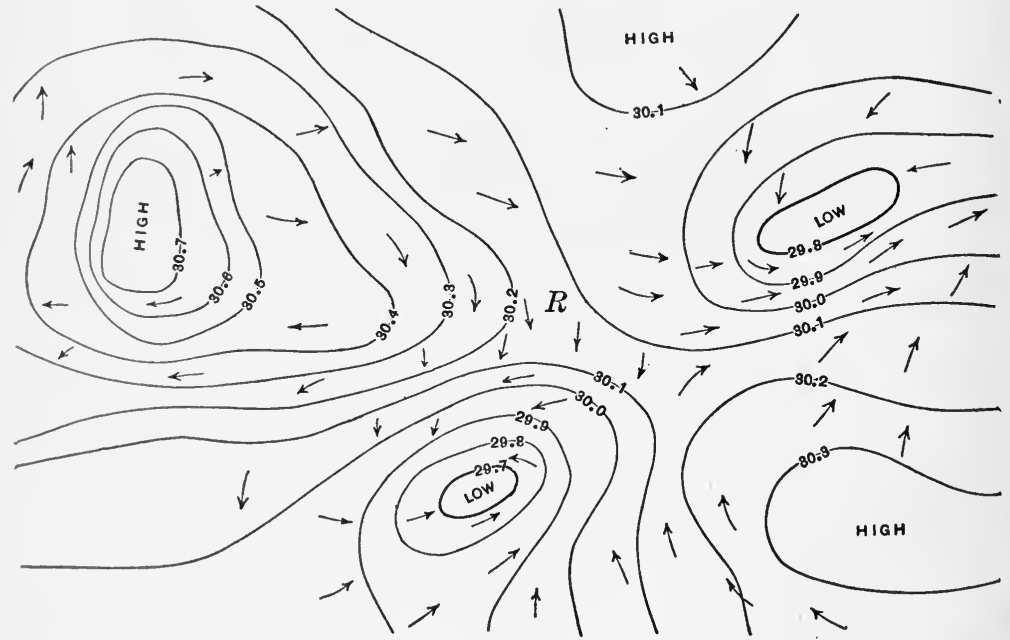

FIG. 8. - Graphic representation of areas of high and low pressure, with the lows separated by a high-pressure ridge, $R$. Direction of winds indicated by arrows. Compare with weather map of December 24, 1916.

in alignment with the isobars. This is the essential point, the generally inward and circular direction of the winds about a "low." Because of this feature the low region is called a cyclone, from the Greek word kuklos, meaning a circle.

When you consider the high-pressure areas, you find exactly the opposite condition. While moving clockwise and yet nearly parallel with the isobars, the winds generally tend outward or 
away from the center. Because of this feature the high pressure area is called an anticyclone.

Over the eastern section of the continent, winds from a highpressure area in the south move in an easterly direction rather than due north toward a low area in the north. A rule has been formulated which enables one to determine the direction winds actually have. It runs, "Stand with your left hand pointing toward the northern low and your right hand pointing toward the high, and the wind will be blowing on your back." In the southern hemisphere the wind would blow on your face. Why are winds deflected thus from the due northern or southern direction? This question must now be considered.

1. Winds starting from a high-pressure region in the south toward a low-pressure area due north are deflected toward the east, as you find indicated on the weather maps. This is due to the rotation of the earth about its axis. How this result is brought about may be suggested in this way: A hunter seeing a duck flying due east plans to shoot it when it is directly north of him. Where must he aim in order to hit the duck : directly at it, or ahead, or behind it? Or, suppose you are on a train that is running due east at a rate of sixty or more miles an hour. A post that will be passed stands several hundred yards north of the track. As you pass it, you are to aim a rifle directly at it. Will the bullet thus aimed hit the post? If not, to which side of the post will it go?

Consider now a similar case, but more clearly connected with the central problem. A fixed point on the equator is moving due east through a distance of 1000 miles an hour. Another fixed point due north on the 45th parallel is moving through a distance of about 750 miles per hour; and another fixed point due north on the 60th parallel about 500 miles an hour; while at the pole the distance traversed would be about zero. Now imagine a very powerful gun located on the equator and aimed at the fixed point due north on the 45th parallel. The bullet is supposed to keep at a uniform distance from the 
earth. Where, relative to the target aimed at, will the bullet be when it reaches the 45th parallel? Will it be to the east or west of the target? Apply this to the change in the direction of the winds when moving from a high-pressure area in the south to a low-pressure area due north. Will a moving mass of air hit the center of the low or pass to the east of it?

Exercise. - Suppose that a mass of air, a wind, starts from a highpressure area in the north towards a low-pressure area due south. In what direction will it be deflected by the rotation of the earth? Explain and illustrate.

Every weather map shows that the general direction of the wind may be modified locally by the character and temperature ofthe land surface. Mountains and valleys, proximity to the ocean or other large body of water, and the temperature differences peculiar to each, may be taken as the chief causes of such change in the wind's direction. Land surface with its marked inequalities offers greater resistance to winds than does the ocean. Consequently, on the ocean the wind tends to circulate completely about the center of a low-pressure area, while on land the circulation is more or less interrupted. Strong winds are more noticeably interrupted by land surface conditions than are gentle winds and breezes. If winds of your locality diverge from the general wind system to which they belong, find causes and explain.

15. Velocity of wind. - Common experience has hit upon certain signs as indicative of the strength or velocity of wind. When the leaves of trees and bushes are lazily swinging to and fro, the wind is described as a light breeze, and as a fresh breeze when twigs and branches are swinging gently. When branches and tops of trees are vigorously swayed to and fro, and fallen twigs and smaller branches are moved over the ground, the wind is said to be strong to high; and if branches or tops are broken and bushes bend close to the ground, it is called a gale. A hurricane with a varying velocity of eighty to one hundred 
fifty miles an hour may mark its path with ruins of unroofed or overturned buildings and fallen trees.

The Beaufort wind scale is now employed by the Weather Bureau to indicate velocities in forecasts and warnings. The numbers 0 to 12 are given in such forecasts to represent the velocity of the wind. The meaning of the numbers is given in the table.

\begin{tabular}{c|l|l}
\hline \hline Numbrrs of Scale & Kind of Wind & Miles per Hour \\
\cline { 2 - 3 } 0 & Calm & From 0 to 3 \\
1 & Light air & Over 3 to 8 \\
2 & Light breeze & Over 8 to 13 \\
3 & Gentle breeze & Over 13 to 18 \\
4 & Moderate breeze & Over 18 to 23 \\
5 & Fresh breeze & Over 23 to 28 \\
6 & Strong breeze & Over 28 to 34 \\
7 & Moderate gale & Over 34 to 40 \\
8 & Fresh gale & Over 40 to 48 \\
9 & Strong gale & Over 48 to 56 \\
10 & Whole gale & Over 56 to 65 \\
11 & Storm & Over 65 to 75 \\
12 & Hurricane & Over 75 \\
\hline \hline
\end{tabular}

Measuring Wind Velocity. - Such practical estimates are valid for many purposes. For the exact purposes of science the velocity of the wind is measured by instruments which indicate miles per hour or pounds pressure upon a unit area of surface. The instrument for measuring the strength of the wind is called the anemometer. The term is made up of two Greek words - anemos and metron - meaning wind-measure. A common form of the instrument is illustrated in Fig. 9. The hollow cups and axis or spindle are made to revolve by the wind; each revolution is registered by a system of dials so arranged that five hundred revolutions per hour indicate one mile velocity of wind. It is similar to the speedometer of a motor car.

The velocity of the winds is not shown directly on weather maps, 
though on some maps it may be given for certain localities in a marginal table. But the conditions upon which the velocity of the wind depends are represented and may be interpreted with fair accuracy. The chief factors in such interpretation are the differences in pressure between a high and a low center,

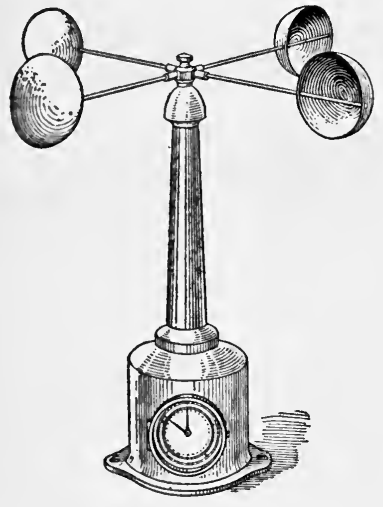

Fig. 9. - Anemometer. and the relative space between the isobars. Consider these points on the maps given.

On the map of December 24 (Fig. 1), note the pressure and the spaces between the isobars of the low area over Texas and Arkansas. The mean velocity of the wind was not quite twenty miles an hour. The low on the next day's map shows a deepening of the storm center from 29.7 to 29.56 , and the crowding together of the isobars. The mean wind velocity has increased, though at only a few places did it reach as much as thirty-six miles an hour, as, for example, at Columbus, Ohio, and Erie, Pennsylvania. The same low on the following day is over the southern part of New England. The storm center has deepened, the barometer reading is 28.9 inches, and the isobars are crowded much more closely together. The wind velocity in the region of the low was generally in excess of thirty miles an hour, while in the vicinity of New York City it reached sixty-eight miles an hour. Observe that the strongest wind of a cyclone or storm center is usually northwest of the central low and therefore immediately follows the low. What have you found to be the conditions determining the velocity of the wind, as shown on these maps? Test your conclusions by study of other weather maps.

Wind velocity and the gradient. - As a general rule the greater the difference in pressure, and the shorter the distance between the isobars, the greater is the velocity of the wind. The less 
the difference in pressure and the wider the spaces between the isobars, the less is the strength of the wind. The difference in pressure for a definite distance, as for a degree of latitude, or a given number of miles, is sometimes called the gradient. The gradient is at once seen to vary from steep to slight, and the wind accordingly from a hurricane or a gale to a very gentle breeze. A gradient of one inch of mercury for five hundred miles indicates a wind with a velocity of approximately fifty miles an hour. A gradient of seven tenths of an inch indicates a wind velocity of thirty to thirty-five miles an hour; of five tenths of an inch, about twenty miles an hour, and four-tenths, about nine or ten miles an hour. Test these figures by comparison with weather maps and reported wind velocities.

\section{Cyclones and Anticyclones}

16. Paths of Storms. - What paths do cyclones or storm centers generally follow? The path of the cyclone just studied for wind force is shown on the map of December 26 (Fig. 3). It was reported as appearing on the morning of the twentythird over southeastern Idaho. Estimate the distance it traveled in the three days. About how many miles did it move each day? An hour? Did it maintain the same rate of progress on its entire course? Compare with this path the path of the cyclone shown over Maine on December 24 (Fig. 1). A third typical path is shown (Fig. 10) for December 29. This cyclone deepened or gained in intensity during twentyfour hours while over Louisiana; on the day before it had little force. It entered by way of southern California.

The general direction of cyclonic storms is from west to east, but mountains and watercourses and the earth's rotation divert as well as distort them. Cyclones seldom pass over the Rocky Mountains. If they do, they lose in intensity and are greatly distorted, as is also the case with those passing over the Appalachian ranges. Cyclones developing in the northern part of the Rocky Mountain system or entering from the North Pacific 


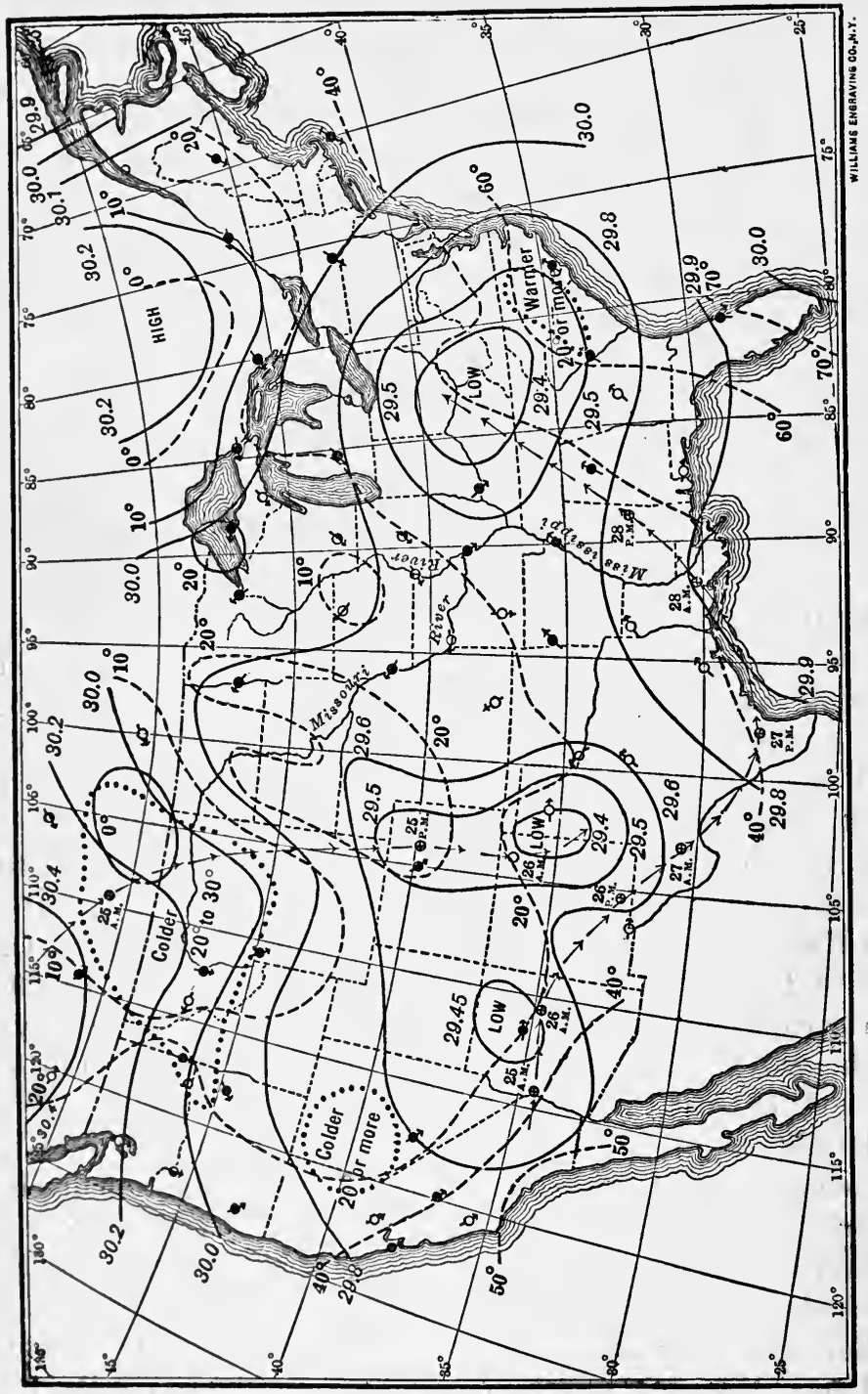

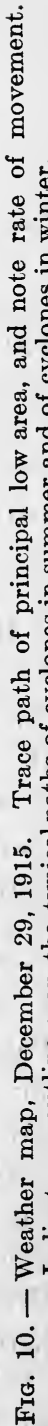


through British Columbia move southeastward as far as Montana, where some of great intensity swing east and pass through the Great Lakes and St. Lawrence region. This is one typical path of storms. Others continue south to Colorado or farther, then swinging northeastward, pass up the Ohio valley over New England, and out by way of the St. Lawrence. Another path has been indicated for the map of December 29. These are the usual paths of cyclones. The path of the West India hurricanes will be considered later.

17. Progress of cyclones. - A mean rate of progress of cyclones is estimated as approximately thirty-five miles an hour for the winter months and twenty-five miles an hour for the summer months. The rate for individual storms varies greatly. A cyclone may maintain a rate of fifteen miles an hour throughout its course and, while not severe, may be very widespread. Another may reach the tremendous speed of seventy miles an hour and be intensely severe but more limited in extent. This fact concerning the rate of progress is significant in connection with the so-called spiral movement (pages 19-21) of the winds about the central low. The center moves more rapidly than the winds, which cannot therefore complete even approximately the spiral course. On the ocean, with no obstacles to circulation, and in the tropics, where cyclones move sometimes more slowly, winds attain a very nearly complete spiral course and acquire greater rotary energy. On this point compare the typhoons of the China Sea, the cyclones in the region of the Bay of Bengal, and the West India hurricanes.

18. Diameter and form of cyclones. - The extent of a cyclonic area may be estimated roughly from the weather map. What are the approximate diameters of the cyclones shown on the maps in the text? The form tends generally to be elliptical or oval, with the narrow side usually pointed in the direction in which the cyclone is moving. In the larger number of cases the direction is northeast. The progress of the storm is slower when the broad side faces due north than 
when it faces the northwest. Test these points by the maps.

19. Rainfall. - The area over which rainfall is distributed in connection with a "low" is determined by the direction from which the supply of moisture-laden air comes. The rainfall is usually on the eastern and southern side and near the center of a "low." There is, however, little regularity in the position and area of rainfall, as is shown on maps having precipitation indicated.

20. Weather features of cyclone area. - The characteristic features of a low or cyclonic area are represented graphically in Fig. 11. The first approach of the low area is usually indicated by the appearance of the dainty, feathery clouds called cirrus. They are often described as mare's tail clouds. They are between five and seven miles high, and are more or less irregular in form, sometimes like badly frayed plumes or streaks of haze. They move in advance of the low center by many hundred or even a thousand miles. The cirrus clouds, which are made up of minute ice particles or spicules, merge gradually into a cobweb-like sheet or layer cloud called the cirro-stratus. This extends over more or less of the heavens and is often made conspicuous by the halo formed by the sun .or moon. Such cloud formation is due to the condensation of the moisture of the warm air, which, forced upward, overflows far and wide the denser masses of air.

The barometer begins to fall with the approach of the cirrostratus; the rate of its falling indicates the speed at which the storm area is moving; and the amount of its fall indicates the intensity of the storm.

To the east and south of the cyclone the winds blow. from the east or southeast, and the temperature rises. To the north and west of the center the winds blow from the northeast quarter, and the temperature falls.

Clouds denser and lower than the cirro-stratus may appear and move directly inward toward the low center. These, from 
their heaped-up form like billows of smoke or steam, are called cumulus clouds. They have various forms and sometimes combine with other cloud forms. With low area centers they

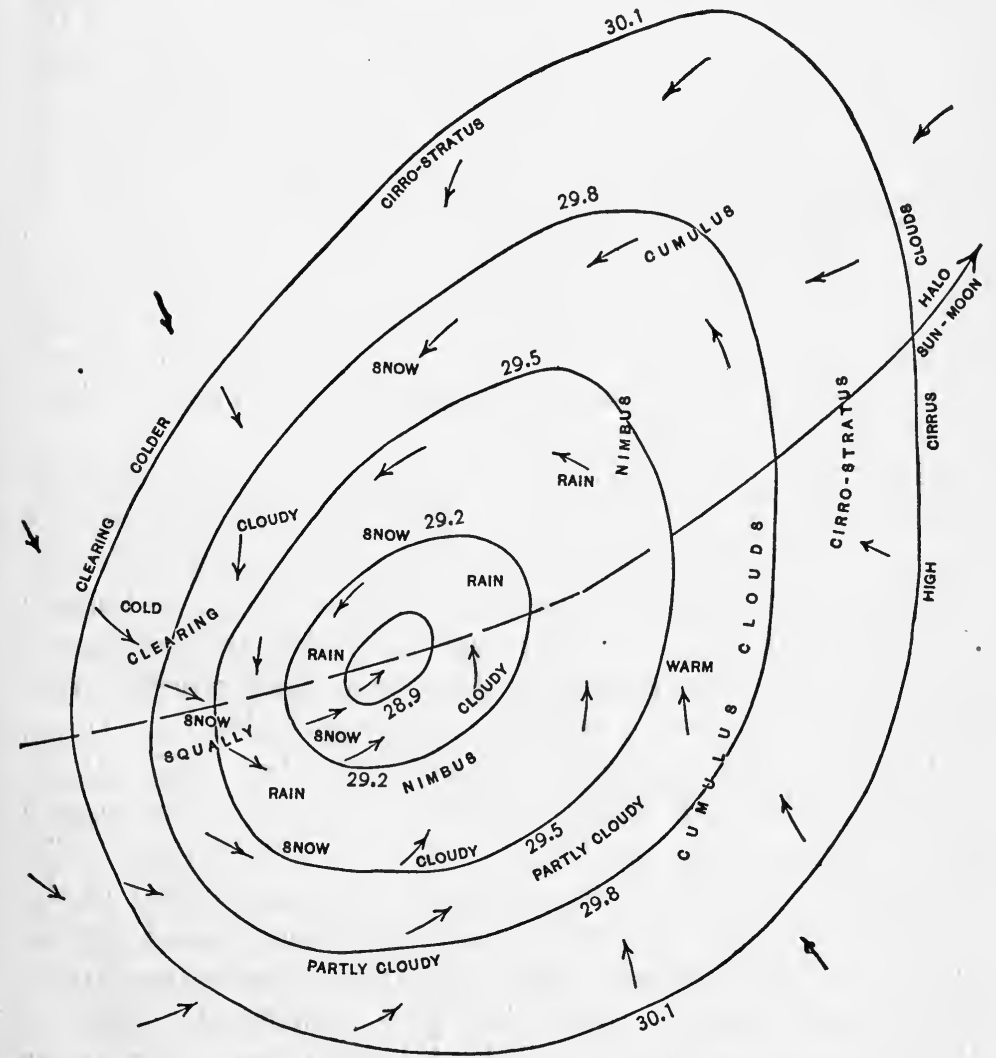

FIg. 11. - Graphic representation of the chief weather features of a cyclonic area. Based upon the weather map of December 26, 1915.

are usually in layer or stratus form, and dark in color. As the warm, moisture-laden air sweeping in from the east or southeast is lifted upward by the cold currents from the north, the cumulo- 
nimbus or nimbus clouds are formed, that is, clouds from which rain or snow falls.

As the center of the low area approaches one's locality, the wind south of the main path shifts from the east or southeast until it comes from the southwest. North of the path of the low the wind shifts around until it comes from the north and finally from the northwest. The center of the low area may be indicated by the barometer, which not only stops falling, but may for a short time show a slight rise. Sailors call this the "eye of the storm," for there is a temporary calm with perhaps a bit of clear sky. With the passing of the center the barometer rises steadily. The winds blow increasingly from the northwest quarter on the north side of the path, and from a westerly direction on the south side. The sky clears and the air becomes cool and invigorating.

21. Hurricanes. - Reference has been made to the typhoons of the China Sea, the cyclones of the Bay of Bengal, and the hurricanes, which, originating in the region of the West Indies, sweep with destructive violence over the Gulf and Eastern states of our country. The strength and violence of such storms may be studied in reports on the storm which worked great destruction of property and loss of life in Galveston, Texas, in 1900. A similar storm occurred in September, 1915, but did comparatively small damage because of the protective seawall and other measures provided after the earlier storm. The hurricane of 1915 made its way across the eastern part of the continent, passing over St. Louis, then northeast, and out by way of the St. Lawrence valley. Hurricanes sometimes strike Florida and Alabama, where they work immense damage, and then sweep over practically all the Atlantic coast. They occur usually during the summer months, from July to October, but with greatest frequency in August and September.

Hurricanes of this sort develop in the tropical region, probably along the edge of the Belt of Calm, called the doldrums, east of the Windward Islands, and in their passage over the ocean 
they acquire tremendous rotary velocity. The winds of hurricane force are usually restricted to the central area or to a diameter of two hundred fifty to three hundred miles, and they blow with a velocity ranging from eighty to more than one hundred miles an hour. The circular form of the tropical cyclone is somewhat distorted and its force is greatly diminished in passing over the land surface. The entire diameter of the storm may exceed a thousand miles. Exceptionally heavy rainfall accompanies these storms, often as much as an inch of rain falling in an hour.

One of the greatest services of the United States Weather Bureau is to forecast and issue warning to shipping and other interests concerning the approach of these destructive hurricanes. Prediction has attained a high degree of probability.

22. Anticyclones. - The anticyclonic area is quite the opposite of the cyclonic. The pressure of the anticyclone is greatest at the center and gradually diminishes with more and more widely spaced isobars toward the circumference. With the anticyclone, light winds may blow almost parallel with the outlying isobars for a considerable distance, but they become increasingly variable or shifting as the center approaches. With the very marked high-pressure areas in winter strong, cold winds are occasionally associated, which, with the precipitation due to sudden condensation of the water vapor in the air, give rise to blizzards or to driving rain. The winds of an anticyclone may reach Texas, where they produce a rapid lowering of the temperature and sometimes precipitation, either rain or snow. These are known throughout the Southwest as "Northers." There are also the cold waves, which precede the high centers and may extend over nearly all the territory east of the Rocky Mountains.

Anticyclones move more slowly than cyclones in crossing the continent, their rate of movement averaging from six to eight miles an hour less. Most of the anticyclones enter through British Columbia, moving in a southeasterly direction until 
they reach Montana, where they swing directly east or somewhat south of east and cross the country. A few develop in the central Rocky Mountain region; and a very small number, formed in the vicinity of Hudson Bay, move south through Manitoba and then eastward over the Great Lakes. Trace the paths of several anticyclones across the continent. Compare with these the paths of cyclones. Are they the same? What kinds of weather accompany anticyclones? Are they always accompanied by fair weather?

Observation of the behavior of anticyclones or high-pressure areas suggests that there are two varieties: one supplying the adjacent lows with descending and outflowing air, which is cold and dry; the other remaining practically inactive for many days over a particular locality, or moving so slowly that it may require weeks in crossing the continent. In these anticyclonic areas, winds may circulate around the outer isobars, but there is little or no evidence of descending air in the central region.

Weather observations should be made daily by each student, preferably morning and evening. The following items should be observed and recorded in convenient form, with a column for results of comparison with the United States Weather Maps. Test also the general weather features of cyclonic areas, which have been described above.

A useful form is as follows:

Name of Observer

\begin{tabular}{|c|c|c|c|c|c|c|c|}
\hline \multirow{2}{*}{ Date } & \multirow{2}{*}{ Hour } & \multirow{2}{*}{ Pressure } & \multirow{2}{*}{$\begin{array}{c}\text { TeMPer- } \\
\text { ATURE }\end{array}$} & \multicolumn{2}{|c|}{ WIND } & \multirow{2}{*}{$\begin{array}{l}\text { APPEAR- } \\
\text { ANCE OF } \\
\text { SKY }\end{array}$} & \multirow{2}{*}{$\begin{array}{l}\text { RESULTS } \\
\text { OF COM- } \\
\text { PARISON }\end{array}$} \\
\hline & & & & Direction & Force & & \\
\hline & & & & & & & \\
\hline & & & & & & . & \\
\hline
\end{tabular}

A graphical representation of the daily pressure and temperature may be made to illustrate clearly the changes. Such a diagram should extend uninterruptedly over one or more weeks. 


\section{SUMMARY}

Winds about a low-pressure area moving spirally inward in a counter-clockwise direction, in the northern hemisphere, constitute a cyclone.

Winds about a high-pressure area moving spirally outward in a clockwise direction, in the northern hemisphere, constitute an anticyclone.

Winds forced from the high-pressure areas directly toward the lowpressure areas are modified in direction by the rotation of the earth and local causes, like mountains.

The velocity of the wind depends mainly on the pressure gradient, is determined by the anemometer, and is designated in miles per hour, or by such terms as " breeze " and "gale."

The low-pressure areas follow typical paths across the continent, with an average velocity of about 30 miles an hour. They are likely to be storm centers, the greatest rainfall generally occurring to the south and east of the center.

The three chief forms of clouds are cirrus, cumulus, and stratus. Clouds from which rain is falling are nimbus clouds.

Anticyclones, or high-pressure areas, which usually follow the paths of cyclones but travel more slowly, are generally associated with clear weather. In winter they may bring cold waves and blizzards.

Typhoons of the China Sea, cyclones of the Bay of Bengal, and hurricanes of the West Indies are rotary storms of large dimensions and destructive violence. Hurricanes sometimes enter the United States from the Gulf of Mexico.

\section{REVIEW QUESTIONS}

1. How is the direction of the wind indicated? 2. (a) What is the direction of the wind between a high and a low? (b) What is its direction about a low? (c) About a high? 3. What does the word "cyclone" mean as used by the Weather Bureau? 4. What does the word " anticyclone" mean? 5. What is the rule for determining the direction of the wind when the position of a high and low are known? 6. Which way are winds deflected in the northern hemisphere? Why? 7. Mention and describe certain local conditions that affect the direction of the wind. 8. What are some of the terms used to indicate different wind velocity? 9. With regard to difference in velocity how many winds are named on the Beaufort scale? 10. (a) What is the use of an anemometer? (b) How is it constructed? 11. (a) Is the velocity of winds generally recorded on 
a weather map? (b) From what conditions can the velocity be roughly inferred?

12. What is meant by "gradient"? 13. Describe the paths followed by typical cyclones in the United States. 14. What is the average velocity of cyclones? 15. Does any mass of air moving spirally into a low commonly complete the circle? 16. What is the average diameter of lows? 17. What is the general form of a low as shown by the isobars? 18. Where, with reference to a low, is rain' most likely to occur? 19. What conditions as to clouds, rain, temperature, and direction of wind indicate to an observer the approach of a low? 20. Describe the changes in direction of the wind when a low passes to the north of an observer. To the south. 21. What are the usual conditions of sky, wind, and temperature when a low is receding?

22. What are the direction and character of the winds in a high? In regions around a high? 23. With what pressure conditions do cold waves occur? Blizzards and northers? 24. What are the typical paths of anticylones? 25. Anticyclones may be grouped into what two types? 26. (a) Where do hurricanes originate? (b) What are typical paths? (c) In what season are they most likely to occur?

27. What are storms similar to hurricanes called in the Bay of Bengal? In the China Sea? 28. What velocity may be reached by winds in a hurricane? Over what area? 29. What rainfall per hour would be considered very heavy? 30. Of what importance is the prediction of hurricanes?

\section{Local Storms}

There are two great types of local storms, the tornado and the thunderstorm. The tornado belongs to the class of whirlwinds which includes the simoom peculiar to the Sahara desert, the waterspout, and the smaller whirlwinds formed frequently in almost every part of the country.

23. Tornadoes are of local origin and must not be confused with cyclones, as they are often incorrectly called. The tornado is comparatively small and operates within a limited range of territory; but it is at the same time the most violent and destructive of storms occurring on land or sea. The area of the destructive sweep of tornadoes varies from twenty feet to a thousand feet in diameter. They may travel anywhere from 
one mile to two hundred miles; but the path of devastation seldom reaches thirty miles in length. They occur in all months of the year, but with the greatest frequency in the period from April to July. They are closely associated with hot, oppressive, sultry days, a gentle south wind, and with lofty cirrus clouds flying in the same direction with the wind. These are regarded as significant signs in certain localities. Tornadoes usually occur in the afternoon or early evening.

The central and eastern part of the United States is the general region in which tornadoes occur. They develop with the greatest frequency in the region along the Missouri and Mississippi rivers, and certain sections of the Ohio valley, but they also appear in northeastern Texas, in Alabama, Michigan, New York, and occasionally in certain inland sections of New England.

Tornadoes are developed many hundred miles south or southeast of a cyclonic storm center. The whirlwind is promoted by the meeting of several currents of air, as the colder air of the north and the moist, warm air coming from the south, and by a very rapid rise in temperature of the air near the ground. As the warm air with its burden of water vapor is lifted up more or less vertically by the cold-air currents, it expands with the decreased pressure, and, acquires in its upward course an increasingly rotary motion. The moisture, condensing as it rises into the upper region, releases heat energy, which augments the force of the rotation and at the same time causes a decrease of pressure at the center. The greater the supply of moisture, the greater will be the amount of heat energy released and consequently the stronger the suction at the center. When the moisture supply is exhausted, the tornado ceases. By the partial vacuum of the spiral, air near the ground is sucked up with almost unimaginable force. The noisy rushing of air up a heated chimney with a forced draft is similar. It is possible that the terrific roaring, which may be heard for miles, is due to this violent rotary motion., 
The tornado resembles a gigantic funnel, the small end of which dips from time to time to the earth, cutting a swath through trees, buildings, or whatever may be in its path, while the larger end is spread out with masses of black cloud. The velocity of the rotary motion of the lower end of the tornado can be estimated only from its effects. But it seems probable that it reaches a velocity of four hundred to six hundred miles an hour.

The very low pressure within the central part of the whirl or tornado is the important fact in understanding the explosion of closed houses or closed rooms within houses. The air pressure may be reduced one third of the normal. Calculate the difference between the suddenly reduced pressure on the outside of a closed house and the normal pressure retained within the house as 4.9 pounds per square inch. The tremendous difference in pressure is easily seen to be more than enough to account for the explosion. Buildings which are open may be wrecked, but not by exploding.

The fact is important in explaining the large breakage of plate glass windows in New York City and other cities within a radius of many miles of the dynamite explosion on July 31 , 1916, on Black Tom Island. The sudden decrease in pressure on one side of the glass was so great that the glass could not withstand the normal pressure on the other side. In this case the windows fell outwards, or the air in the building expanded and found escape where the resistance was least.

A heavy fall of hail accompanies tornadoes. The lightning and thunder are often terrible. Where tornadoes occur it is of practical importance that every one know the signs of the approach of such storms, and realize that safety lies to the northwest or southeast of their path, because tornadoes almost invariably move toward the northeast. The rate of progress is usually about forty miles an hour, but it may vary from fifteen to one hundred miles an hour.

Cloudbursts and waterspouts. - Tornadoes with their,swiftly 
ascending whirls of moisture-laden air and the formation of the cloud within the central area may yield a heavy fall of rain. Where the updraft is too strong, this occurs not as a rain, but as a torrent, commonly called a cloudburst. When passing over lakes or oceans or even large streams of water, such whirlwinds may suck up water and scatter it in a whirl, called a waterspout, over a considerable space; but the larger part of the water is generally due to rapid condensation.

24. Thunderstorms. - Only certain features of this variety of storm need to be mentioned. The range of occurrence of thunderstorms is wide both north and south of the equator. They occur with greatest frequency and violence in the tropical regions and diminish toward the higher latitudes. In the United States they occur most frequently and with greatest severity in the general region of the Mississippi valley and in Florida, less often in the region north and east of the Mississippi, and with still less frequency west of the Rocky Mountains. In several sections of California thunderstorms are of rare occurrence.

A thunderstorm resembles a tornado in several respects. It is of local origin, develops generally on the southeastern or southern side of a cyclone; and travels in most instances towards the northeast over a tract not more than two hundred miles long and seldom more than twenty-five miles wide. The rate of progress varies from fifteen to twenty-five miles an hour. Like the tornado, a thunderstorm depends upon a sufficient supply of hot, moisture-laden air started upward by currents of cold air or by its own movement up the sides of hills or mountains. In its upward movement (Fig. 12) it sometimes acquires the rotary or spiral whirl, as shown in clouds characteristic of the tornado. The surface air flows inward toward the central updraft area, sometimes with great velocity. When the surface air currents fail to supply moisture and heat, the energy of the storm diminishes and is finally dissipated. Thunderstorms are usually accompanied by heavy rainfall and occasionally by hail. 
The electrical display and the majestic roar of thunder are interesting features. It is possible here to mention briefly only a few of the important conditions upon which electric discharge or lightning depends; and these are given primarily to stimulate further observation and study of such phenomena.

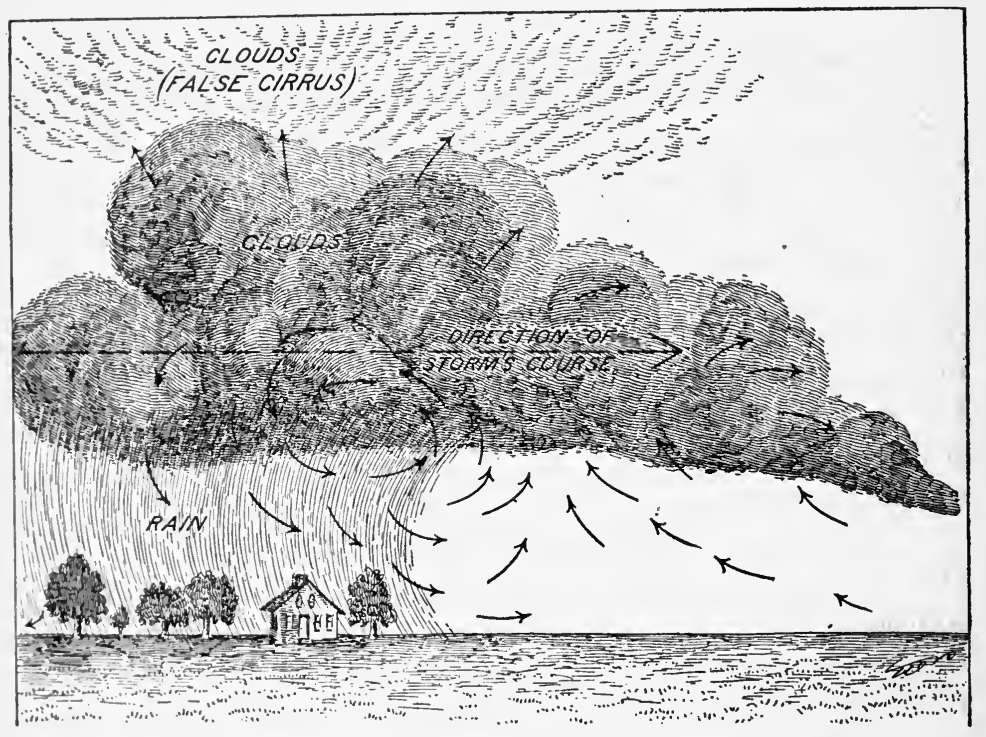

FIg. 12, - Vertical section of a thunder storm, showing the vertical whirl. A whirl parallel to the ground would be a whirlwind, tornado or waterspout. (After Arrhenius.)

Exercise: Electrical discharge. - If the school has an electrical machine, such as the Wimshurst, and condensers or Leyden jars, the essential features of electrical discharge may be illustrated. Try making sparks pass through different distances and media, as wet and dry paper. If the charge is great and the distance rather long, forked sparks may be produced. Zigzag paths are easily illustrated. The sound by the sparks may be considered as thunder.

Electrification of the air. - The air that lies nearest the earth is practically devoid of electrical energy; but the layers above 
the immediate air envelope of the earth are charged with electricity. By the use of observational balloons and other means of investigation, it has been discovered that there is a regular and rapid increase in the amount of electricity until a height of about four miles is attained. Beyond this limit the quantity of energy seems to remain constant. During fair, dry weather the electric energy is equally distributed. It is prevented from passing to the earth by the dry air near the earth surface ; for dry air is a very poor conductor of electricity. In cloudy weather the differences in electrification are much greater at different altitudes. It is only in cloudy weather that lightning occurs. But atmospheric changes, such as the winds, or the ascent to the upper regions of water vapor produced by evaporation, and especially the condensation of moisture to form cloud and rain, bring about marked disturbances with more or less concentration of electrical energy within limited areas.

The electrical discharge. - In condensation, electricity exists only on the surface of water particles that make up a cloud. With continued condensation the water particles combine again and again to form larger drops or globules. In this coalescence of globules the surface area is rapidly decreased, which means a reduced capacity for holding the electricity. Every globule has about all the electric charge that it is capable of holding. When two globules combine, the new surface area formed is only about one fourth larger than the surface area of either of the globules, while the electric charge is about doubled. The inevitable result is that a cloud in which condensation continues is having its capacity for electricity rapidly reduced and its electrical charge made more concentrated and powerful. The electric charge finally becomes so powerful that the cloud can no longer retain it or the intervening mass of air block its transfer; then it forces its way to an oppositely electrified cloud or to the earth.

Such an electrical discharge is known as lightning. It at 
first equalizes, for a time at least, the difference in electrical energy between two clouds or a cloud and the earth. In its passage through the air, much of its energy is changed into heat, the air particles being thrown into swift vibration and becoming white hot, incandescent, or luminous (page 166). The transfer of electrical energy is so rapid that only a flash is visible. The white-hot air expands suddenly and violently, and instantly contracts, or cold air rushes into the partial vacuum, giving forth a succession of vibrations that produce the noise of thunder.

The distance of a thunderstorm from an observer may be readily estimated. The flash of lightning is seen almost as soon as it occurs. The sound or report of the thunder travels more slowly, about eleven hundred feet per second at ordinary temperature. The distance is approximately the product of the rate at which sound travels and the number of seconds elapsing between the flash and the thunder.

From the account of the accumulation of electricity upon raindrops, it should be evident that a heavy downpour of rain will greatly diminish the electrical charge of the cloud. When occasion offers, observe whether the sharpest lightning precedes rainfall or not; and whether or not there is a diminution of the lightning after the beginning of rainfall.

When directed toward the earth, an electrical charge follows the path of least resistance, "striking" usually the highest points, as towers, steeples, or trees which are especially good conductors because of their water or sap. In passing down a tree to the earth, the electricity follows the moisture, which, instantly vaporized by the heat, expands and explodes, tearing off the bark or splitting and shattering the tree. Lightning striking dry sand fuses it and makes what are called "lightning tubes."

Kinds of discharge. - In certain circumstances when the air. is heavily charged with electricity, there may occur brush-like flames on high, sharp-pointed objects, as trees, flagpoles, or 
masts of ships. Occasionally there is a crackling noise accompanying the brush-like flames, similar to that of the flow of electricity from points on condensers. This kind of discharge is called St. Elmo's fire. Discharges between clouds occurring generally in brush-like forms may be what is called sheet lightning. A very powerful discharge encountering great resistance and passing over a long distance produces branches and is known as forked lightning.

Lightning rods or conductors have been extensively employed in many localities to avert the dangers from electrical discharges. When lightning conductors are of suitable metal, such as copper, of sufficient number, are thoroughly insulated from the building and connected with the earth through water or damp soil, they may be effective. They are intended to prevent violent, destructive discharges by securing the silent transfer of electrical energy from the upper air to the earth and thus equalizing the electrical difference between them.

\section{SUMMARY}

Two types of local storms are the tornado and the thunderstorm.

The tornado belongs to the group of rotary storms, which includes simooms of the Sahara desert and waterspouts.

Tornadoes develop southeast of the center of lows and travel toward the northeast. They occur usually in the summer months in the Mississippi valley.

Thunderstorms are local disturbances in cyclonic areas. They are due to down drafts of cold air and up drafts of warm, moist air. The normal electrification of the atmosphere is disturbed, and lightning produced.

\section{REVIEW QUESTIONS}

1. What disturbances similar to tornadoes occur over the sea? Over the Sahara desert? 2. Compare a tornado with a cyclone (lowpressure area) as to diameter, velocity of wind, distance traveled, time of year in which they occur, time of day, and effects. 3. On what sort of day are tornadoes most likely to occur? 4. In what part of the year are tornadoes most frequent? 5. Describe the development of a tornado, its position with reference to a low, and other favoring 
weather conditions. 6. What extreme velocity may be developed in the whirl of a tornado? 7. Explain why buildings of ten burst outward as a tornado passes. 8. What knowledge of the behavior of tornadoes may enable one to avoid them, or find protection from them? 9. Describe a waterspout.

10. In what regions are thunderstorms common? 11. Describe a thunderstorm. 12. How can a lightning flash be illustrated in the laboratory? 13. What is the normal electrical condition of the air? 14. What conditions change the normal electrification? 15. Under what conditions does an electrical discharge take place? 16. Why does the electrical discharge produce light? Sound? 17. (a) How fast does sound travel? (b) How may this fact be used in determining the distance of a thunderstorm? 18. Why is lightning less frequent in the middle of a storm than at its margins? 19. (a) Why are trees and steeples frequently struck? (b) What causes the wood to splinter and fly? (c) What are "lightning tubes"? 20. Describe the various types of electrical discharge. 21. How may lightning rods protect a building?

\section{The Moisture of the Atmosphere}

25. Relative density of moist and dry air. - The study of cyclonic and anticyclonic areas and of local storms suggests an important question which may be stated thus: Why does warm, moisture-laden air rise when it is met by cold, dry air currents? The answer may be indicated by a simple experiment and observations.

Exercise: Behavior of warm and cold air. - Insert a rubber stopper, through which is passed a glass tube six to ten inches long, into a thin glass flask or test tube. Put the end of the tubing into water, and alternately warm and cool the flask. Observe the behavior of the water in the tube. What do you infer as to the change in volume of the air when warmed and when cooled? When does its volume increase and when decrease? What does this show as to the relative weight of equal volumes of warm and of cool air?: When a given volume of warm air meets an equal volume of cold air, which will be forced upward?

Study the behavior of warm and cold air in a room heated by a stove or other appliance. What is the temperature of the air near the floor? Of that near the ceiling? Can you detect anything sug- 
gesting eirculation of the air in the room? What air moves upward and which settles toward the floor? Compare also the behavior of smoke issuing from a chimney into the open air with the behavior of warm air in a low-pressure area. The heated air from the chimney may sometimes be seen to continue upward and take a slight whirling or rotary motion before it spreads out and disappears. Does this resemble the central draft of a low-pressure area, or of a tornado as described above?

Air that contains water vapor is shown by the barometer to be lighter, volume for volume, than dry air. A cubic foot of steam, which is water vapor, is lighter than an equal volume of dry air at the same temperature. Consequently the pressure of air with any mixture of water vapor will be less than that of dry air; and the greater the amount of water vapor which air contains, the less its weight, as shown by the barometer.

It appears from this that a cubic mile of cold, dry air encountering near the surface of the earth a cubic mile of warm, moist air will invariably force the latter wherever the resistance is least, which is usually upward. In its upward progress it expands and cools, and its moisture is condensed to form cloud and perhaps precipitation as rain, snow, sleet, or hail.

26. Humidity of the atmosphere. - The atmosphere always contains moisture or water vapor whether visible or invisible, but in varying quantities. Moisture is in fact one of the most important constituents of the atmosphere. It has apparently great influence upon the development of storms (page 35). Its importance in relation to health is generally recognized, though not as yet thoroughly understood.

Water vapor is supplied to the atmosphere by evaporation, which occurs at any temperature from water, ice, or bodies containing water in any form. The only condition that checks evaporation is complete saturation of the air. Evaporation is facilitated by high temperature and by moving air, as wind, which replaces a mass of air already saturated with a mass of comparatively dry air. Compare the effect of different temperatures or moving air upon drying clothes. 
Water vapor or gas is invisible, but when condensed or made liquid, is visible as cloud, fog, mist, or rain. Upon what does its form of existence in the atmosphere depend? Consider these familiar instances: The warm, moist air of the house or kitchen is invisible, but as it escapes through an open window into the outer cold it forms a cloud. Observe the condensation on cold windows or woodwork. The air exhaled from our lungs is heavily laden with moisture, which is, however, invisible unless the outer temperature is below a certain point, or one breathes upon a cold surface, such as glass or bright metal. At what time of the day does a mist appear? Why does it disappear soon after sunrise? During what part of the day is dew deposited? What causes it to disappear? What becomes of mist and dew after the sun begins to shine upon them? The air in clear, warm weather contains a relatively large amount of invisible moisture, but lowering the temperature below a certain point makes the moisture visible as cloud, mist, or dew. Thus it appears from these observations that the form of moisture in the air depends upon the temperature. It also is seen that the amount of water vapor that air may contain depends upon the temperature; the warmer the air, the greater the amount of moisture; and the lower the temperature, the less the amount it can hold.

The amount of moisture that the atmosphere may contain can be considered in the following way. Suppose a mass of air at a definite temperature, as $32^{\circ} \mathrm{F}$., is supplied with water vapor through evaporation until it contains as much as it can hold. Evaporation proceeds rapidly at first, but gradually becomes slower until it ceases altogether. When evaporation ceases, the air is said to be saturated at the given temperature. If the temperature of the air is raised, its capacity for moisture is increased, and evaporation is resumed. Suppose the temperature is raised to $68^{\circ} \mathrm{F}$., the air can then hold approximately four times as much moisture as it could at $32^{\circ} \mathrm{F}$. (Table, Fig. 13). Evaporation will continue until the air is saturated at that 
temperature, or the water vapor exerts enough pressure upon the water to stop evaporation.

Absolute humidity. - When the total amount of water vapor in the atmosphere is considered with no reference to the temperature and the amount the air might hold, it is described as the absolute humidity. The absolute humidity of the atmosphere is measured by the hygrometer or the barometer, and is expressed in inches or fractions of an inch of mercury; or it may be expressed in ounces per cubic yard of air, as in the following table. Only temperatures of common significance are given.

\begin{tabular}{c|c|c}
\hline $\begin{array}{c}\text { TEMPERATURE } \\
\text { FAHRENHEIT }\end{array}$ & $\begin{array}{c}\text { OUNCES PER CUBIC } \\
\text { YARD }\end{array}$ & $\begin{array}{c}\text { Pressure IN INCHES } \\
\text { OF MERCURY }\end{array}$ \\
\hline $32^{\circ}$ & .13 & .18 \\
$41^{\circ}$ & .18 & .25 \\
$50^{\circ}$ & .25 & .36 \\
$59^{\circ}$ & .35 & .50 \\
$68^{\circ}$ & .47 & .70 \\
$77^{\circ}$ & .63 & .98 \\
$86^{\circ}$ & .83 & 1.24 \\
$90^{\circ}$ & .93 & 1.45 \\
$95^{\circ}$ & 1.05 & 1.63 \\
\hline \hline
\end{tabular}

FIG. 13. - Capacity of air for water vapor at different temperatures.

Exercise. - Let us assume that a cubic yard of air is saturated at $50^{\circ} \mathrm{F}$. How much water vapor or gas does it contain? If saturated at $86^{\circ} \mathrm{F}$., how much vapor does it contain? About how many times as much vapor is required for saturation of air at $77^{\circ} \mathrm{F}$. as at $50^{\circ} \mathrm{F}$.? As at $68^{\circ} \mathrm{F}$.?

Consider the influence of changes of temperature upon saturation of the atmosphere. Assume that air at $90^{\circ} \mathrm{F}$. contains .47 of an ounce of water vapor per cubic yard. To what temperature must the air be cooled to bring about saturation? Does evaporation occur when the air is saturated? If now the temperature of the air be warmed to $90^{\circ} \mathrm{F}$. and maintained there with continued evaporation until the air is saturated, 
how much water vapor will the air contain? By what two methods may saturation be produced? Do both methods depend upon temperature? The relation of saturation and temperature may be shown in the following manner. A mass of air is saturated at $50^{\circ} \mathrm{F}$., and with no change in its water-

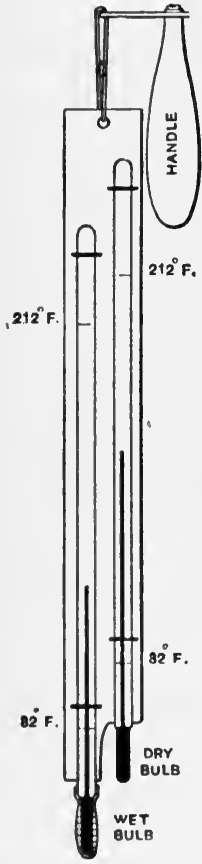

Fic. 14. - Sling hygrometer. vapor content is then warmed to $68^{\circ} \mathrm{F}$. The absolute humidity of the air is .25 of an ounce per cubic yard. At this temperature the air can contain twice as much moisture before it becomes saturated. Expressing this as a ratio, the air contains $.25 / .47$ of what it can hold, or in percentage, about 53 per cent of its total capacity. The same mass of air may be imagined as cooled to $50^{\circ} \mathrm{F}$. It now contains all the water vapor possible, without condensation, or $.25 / .25$, or 100 per cent, its maximum capacity. What would be the ratio of the amount of vapor present to the total capacity of the air, if its temperature were $86^{\circ} \mathrm{F}$.? $95^{\circ} \mathrm{F}$.?

Relative humidity. - The expression of the actual amount of water vapor in the air as the percentage of the total amount that air can contain at a given temperature is called the relative humidity. It is the relative humidity, that is, the ratio of the vapor present to what the air can contain, that determines our experience of drying air or of damp air.

The presence of water vapor in the air, although invisible, is detected and measured by an instrument called the hygrometer, from two Greek words, hugros, meaning wet, and metron, measure (Fig. 14). This instrument really consists of two thermometers, placed upon the same support. The mercury bulb of one is wrapped with a piece of porous cloth which is attached to a short length of lamp wicking. The other end of the wicking is kept in a 
small vessel filled with water. The water is transferred by the wicking to the cloth, where it is evaporated. The evaporation of the water from the cloth wrapping of the bulb cools the mercury and lowers the mercury column in the tube. The more rapid the rate of evaporation, the lower the mercury in the tube will fall, and the greater will be the difference between the readings of the two thermometers. This indicates that the air has much less water vapor than it can contain. The slower the rate of evaporation, the less will be the difference between the two thermometers, and the greater will be the amount of vapor in the atmosphere. If the two thermometers have the same readings, it is evident that the air has all the moisture it can hold, or one hundred per cent, and that evaporation cannot occur in this situation.

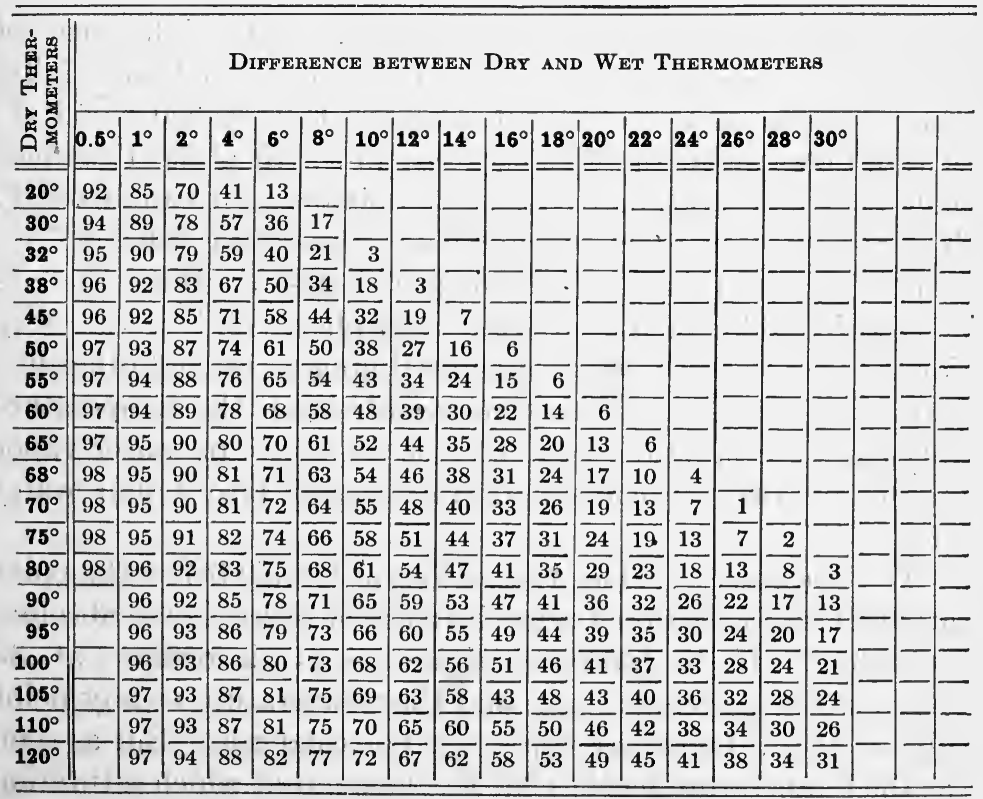

FIG. 15. - Relative humidity, per cent-Fahrenheit scale. 
The dry-bulb thermometer gives the air temperature. The wet-bulb thermometer gives what is sometimes called the sensible temperature, that is, the temperature which we experience or feel.

Exercise. - To obtain the relative humidity in percentage we take the readings of the two thermometers, find the difference between them, and then consult a table that gives the relative humidity in per cent. Suppose the dry-thermometer reading is $80^{\circ} \mathrm{F}$. and the wet $70^{\circ}$, giving a difference of $10^{\circ}$. In the table, Fig. 15, begin with the dry-thermometer temperature of $80^{\circ}$ and follow the row of figures across the table until you reach the figure in the column headed by the difference $10^{\circ}$. This figure gives the relative humidity in per cent, or 61 per cent.

1. The reading of the wet-bulb thermometer is $45^{\circ}$ and that of the dry is $69^{\circ}$. What is the relative humidity? 2. The wet thermometer is at $78^{\circ}$, and the dry at $80^{\circ}$. What is the relative humidity?

27. Temperature and condensation. - The influence of temperature upon condensation is to be considered more fully. Suppose a mass of air saturated at $68^{\circ} \mathrm{F}$. is cooled to $59^{\circ} \mathrm{F}$. without other change. What will occur? Air at $59^{\circ} \mathrm{F}$. cannot hold in the form of vapor as much moisture as it can at $68^{\circ} \mathrm{F}$. The result is that with the fall of the temperature below $68^{\circ} \mathrm{F}$., vapor begins to condense upon dust motes, of which there is an incalculable number in the atmosphere, and in this way forms cloud or fog. When deposited upon grass and other like objects, it forms dew. In other words when the temperature falls from $68^{\circ} \mathrm{F}$. to $59^{\circ} \mathrm{F}$., 0.12 of an ounce of water vapor per cubic yard is condensed or changed into liquid water (Fig. 13).

The dew point. - Does the cooling of the air below the point at which condensation begins increase or decrease the absolute humidity? If the humidity of a mass of air remains at .35 of an ounce per cubic yard, and the temperature at any point above $59^{\circ} \mathrm{F}$., the water vapor will not condense. But as soon as the temperature reaches $59^{\circ} \mathrm{F}$., the point at which saturation is 100 per cent, and continues to fall below this point, condensa- 
tion begins and cloud is formed or dew is deposited. Thus the reduction of the temperature below the point at which saturation of the air occurs causes the condensation of the invisible moisture, and of course reduces the absolute humidity. The temperature at which condensation begins, as shown by the deposited dew or cloud, is said to be the dew point. With an absolute humidity of .35 of an ounce per cubic yard, the temperature $59^{\circ} \mathrm{F}$., which is the temperature of saturation, is also the dew point - the point at which invisible vapor is converted into visible liquid or water.

Exercise. - If the dew point is $59^{\circ} \mathrm{F}$., and the temperature is $77^{\circ} \mathrm{F}$., what is the relative humidity?

When the air has a temperature above that of saturation or the dew point, will a lowering of the temperature increase or decrease the relative humidity? What will be the effect upon absolute humidity of any fall in temperature above the dew point? Below the dew point?

A simple experiment will illustrate clearly the important facts about the dew point, and its variation with the relative humidity of the atmosphere. Into a bright tin can or a porcelain pitcher about half full of water put some cracked ice or snow, a little at a time, and stir carefully with a thermometer. As soon as condensed moisture begins to appear on the outer surface of the vessel, take the thermometer reading. This is the temperature at which condensation occurs, that is, the dew point. Try the same experiment on different days, and compare the thermometer readings. What do the results show as to the dew point? What do they show as to relative humidity?

The dew point may be found approximately for all temperatures in this way. The result is most nearly correct when the temperature is about $70^{\circ} \mathrm{F}$. Multiply the difference between the readings of the wet and dry thermometers by 1.8 , and subtract the product from the temperature reading of the dry thermometer. This gives the dew point accurately enough for practical purposes.

The facts which have been considered in the study of humidity and condensation in relation to temperature are directly useful in explaining the characteristic weather changes, as cloud formation and precipitation, dew and frost (Fig. 16). 
Cloud. - The warm, moisture-laden air blowing usually from the southeast or south toward a low-pressure area is lifted or forced upward as it approaches the center by the cold air blowing from the northeast or north. The air as it rises expands and cools. When it reaches the temperature of satura-

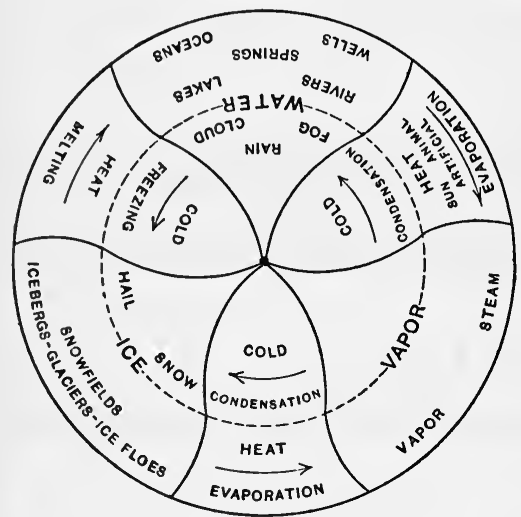

FIG. 16. - Diagram of the water cycle. tion or the dew point, the relative humidity is 100 per cent. As it passes below this point, condensation begins or water vapor is converted into visible moisture deposited upon dust motes in the air. This is cloud. Fog is formed when the moist air is cooled near the earth.

Rain.-Continued reduction of temperature below the dew point causes the condensation of an increasing quantity of water vapor. The water particles combining become large and heavy enough to fall through the cloud, and coalescing with smaller particles on the way may eventually become heavy enough to fall to the earth as rain. Sometimes it happens that raindrops formed very high in the air have to pass through a warmer and less humid layer of air, where they may be evaporated and thus fail to reach the earth.

Cyclonic storms, which come from the northwestern section of our country, distribute but little rain until they enter the region of moisture-laden winds from the Great Lakes. The lows that cross the southwestern section of the country may yield comparatively little rain over the eastern part of the country, unless they are deepened by the moist, warm winds from the Gulf and the Atlantic Ocean. Such deepened storms usually distribute heavy rainfall. Note the deepening of the 
low as shown on the weather maps for December 24, 25, 26, 1915, pp. 6-8. Tropical storms, as the West India hurricanes, are attended by exceptionally heavy rainfall. But the rain that follows the passage of a low center is generally very light. Rain sometimes falls with the approach of the high-pressure or anticyclonic area. Why?

Distribution of rainfall. - The inequalities of the land surface have much to do with the distribution and mean annual amount of rainfall in the different sections of our country. Make a comparative study of a physical and a rainfall map of the United States. The information thus gained, together with the facts concerning the paths of cyclones and local storms, will help in understanding the distribution of rain.

In the western section of the country the moisture-laden winds from the Pacific are forced upward by the Coast range to a high altitude, where the low temperature causes much of their vapor to condense and fall as rain. The rainfall amounts to as much as eighty or one hundred inches a year. East of the mountain range is a lowland region which gets only about one fourth as much rain in a year. The Cascade, Klamath, and Sierra Nevada mountains rob the winds of still more moisture, while the land to the east as far as the Rocky Mountains has a mean annual rainfall under ten inches. In the Rocky Mountain region the rainfall is again large, amounting in certain localities to about forty inches a year. Why?

Exercise. - Explain the fact that the semi-arid strip of country east of the Rocky Mountains, including the western part of Nebraska, Kansas, Oklahoma, and a narrow strip of Texas, has a very light rainfall. Why do not the moist winds from the Pacific Ocean yield moisture for this region? Explain why the Atlantic coast states have a larger rainfall than the central states. Why do they have less rainfall than the western coast? From what source do the Atlantic coast states get their moisture? Show direction of winds bringing moisture from the Gulf and the Atlantic Ocean, and their relation to the lowpressure areas. Why is the precipitation greatest in the central states during the summer season? 
Hail. - In some localities hail may fall during the summer. Examination of large hailstones shows that they are made up of alternate layers of clear and of opaque ice. This suggests the manner of their formation. Raindrops formed very high and falling through air currents with a temperature below freezing become frozen. Then falling through warm, moist air or through a rain cloud, they accumulate a covering of water, are caught and carried by a strong updraft into colder air currents, where the water is frozen into clear ice. They acquire here a coating of snow-like ice, and again falling into a rain cloud receive another coating of water. Thus they are whirled many times through alternate warm and cold air currents before they become heavy enough to fall through the updraft or are thrown beyond its control and fall to the earth.

Snow. - When water vapor condenses at a temperature below the freezing point, it forms small ice particles which combine to form snow crystals. Snow crystals frequently have six rays from a common center, as you may find by examination; but they may take any of a large number of hexagonal shapes. Snow crystals unite, forming snowflakes which, if the temperature is favorable, fall to the earth.

Snowflakes imprison in their feathery masses a great deal of air. Air and snow together make up a blanket that protects from freezing the soil and the plant and animal life below its surface. It does this by checking the escape of heat from the soil. It also keeps a fairly uniform soil temperature by retarding the escape of heat and protecting the soil from the direct rays of the sun. Among other benefits of the snow may be mentioned its influence upon the humidity of the atmosphere, upon the conservation of water in the soil, upon the stream supply, and upon the lumbering industry. Explain how these are influenced.

Dew. - Often in clear weather the temperature of the land surface and of many kinds of objects on the land is lowered at night by radiation of the heat, which was received from the sun 
during the day, to a point at which the water vapor in the air immediately surrounding them is condensed and deposited as dew. Dew may be deposited in daytime or at night; but it is formed more frequently at night when the sky is clear and the air quiet or only slightly moving. In experiments to determine the dew point, it is found that the temperature at which condensation occurs depends upon the relative humidity of the air. The more moisture the air contains, the higher relatively is the temperature at which dew is formed. The less the amount of moisture, the lower is the dew point. This accounts for the very heavy dews in regions near bodies of water, as along the Pacific coast, where in summer the moist sea breezes blow. It also explains the very limited dew deposits inland.

Land and vegetation lose heat much more rapidly than air and other gases generally. Thus vegetation, such as grass, which holds but little heat, loses it by radiation soon after sunset. When grass is thus cooled, it chills the air in immediate contact with it, and causes its moisture to condense and be deposited as dew. Stones and soil may have deposits of dew, but less frequently, because they receive heat from below as they radiate it from their surfaces, and therefore cool more slowly to the dew point. Air cools still more slowly, which explains why mists are formed later at night. Land radiates as well as absorbs heat more rapidly than water. Make use of this fact in explaining land and sea breezes; also in explaining why air over large bodies of water may not be cooled as frequently as that over land to the temperature at which dew is deposited.

Frost. - If conditions favor the formation of dew and the temperature is likely to fall to freezing or below during the night, frost is to be expected. When the temperature of the land and of the air near the land surface is at or below freezing, the condensed moisture particles are frozen to form small ice crystals or frost. If the temperature is at $28^{\circ} \mathrm{F}$. or lower when 
condensation occurs, white frost is not formed, though plants are frozen. Vegetation thus frozen turns black later, and a black frost is said to have occurred. Frost is one of the adverse climatic conditions with which farmers contend, and because of the importance of protecting growing vegetation from it, the United States Weather Bureau forecasts and issues warnings when frost is likely to occur. Possible damage by frost may be reduced or averted by smudge fires, irrigation to saturate the air with water vapor, and by coverings for more delicate plants.

Frost is favored in spring and fall by the passage of a highpressure center with its accompanying clear, calm, and cool nights. If in such a situation, the barometer is above the normal or rising ; if the temperature is falling and the dew point is below $40^{\circ} \mathrm{F}$. and falling, frost is quite certain to occur.

Frost may be predicted for particular localities with much certainty by aid of an hygrometer. If the dew point is near the freezing point, the temperature falling below $50^{\circ} \mathrm{F}$., and the sky clear with no winds, then frost is probable. Another method may be used. When the temperature of the dry thermometer is below $50^{\circ} \mathrm{F}$., multiply the difference between the readings of the two thermometers by 2.5 , and subtract the product from the temperature shown by the dry thermometer. This gives the temperature to which the air may cool during the night.

\section{SUMMARY}

Moist, warm air is less dense than dry, cold air and is forced upward by it.

Evaporation is constantly adding to the invisible water vapor of the air.

The actual amount of water vapor in the air, which may be expressed as ounces per cubic yard, is the absolute humidity of the atmosphere.

The ratio of the amount of moisture in the air to the amount it would contain if saturated is the relative humidity.

Relative humidity may be determined from readings of the wetand dry-bulb thermometer, by the use of a table. 
The temperature at which condensation takes place is the dew point. The dew point varies with the absolute humidity of the atmosphere.

When water vapor condenses on cold objects, it forms dew; when it condenses on minute particles in the atmosphere, it forms fog, cloud, or rain, depending on the position and size of the drops.

Sleet is frozen rain. Hail is generally made up of alternate layers of ice and snow.

When water vapor condenses below the freezing point, it forms frost on objects, and clouds or snow in the air.

\section{REVIEW QUESTIONS}

1. Why does moist, warm air rise? 2. How can you demonstrate that warm air is less dense than cold? 3. What effect upon the density of the air has water vapor? 4. What change in the water vapor of the air takes place when air rises to regions of less pressure? 5 . What is the source of the water vapor which is in the air? 6. What evidence have you that water exists in the air as an invisible vapor? 7. (a) For what is the hygrometer used? tion of the wet- and dry-bulb hygrometer.

(b) Describe the construcon which it works. 8. Which thermometer indicates the " sensible" temperature? 9. What is meant by saturated air? 10. About what is the maximum amount of water vapor which may exist in the air at a temperature of $95^{\circ}$ F.? At normal room temperature? 11. In what two ways can we express absolute humidity?

12. Explain the effect of temperature upon saturation. 13. Give from the table, page 45, figures not given from the text to show that you understand the meaning of relative humidity. 14. Define relative humidity. 15. Absolute humidity remaining constant, under what change of temperature does the relative humidity rise? Fall? 16. What is any temperature called at which condensation begins? 17. Given an absolute humidity of .63 ounce per cubic yard and a temperature of $86^{\circ} \mathrm{F}$., how many degrees must the temperature fall to produce saturation? If it falls to $59^{\circ} \mathrm{F}$., how much moisture per cubic yard will be condensed? 18. (a) Describe an experiment in which the dew point was found directly. (b) How may it be computed from the reading of wet- and dry-bulb thermometers?

19. Explain the formation of clouds. 20. Why does it rain? 21. Low-pressure areas in crossing the continent give conditions for precipitation usually in what region? 22. Where in the western states is the annual rainfall exceptionally heavy? Why? 23. Where in the 
western states is the annual rainfall very light? Why? 24. Compare the rainfall of the eastern slope of the Rocky Mountains with the western slope of the Appalachian Mountains. Give a reason for the difference. 25. How is hail formed? Describe the structure.

26. How is snow formed? Why may a snowflake be called crystalline? 27. What are the useful effects of a snow blanket? 28. Under what conditions is dew deposited? 29. Which loses heat by radiation more rapidly, air or solid objects? What does this explain? 30. Under what conditions does frost form? 31 . What means may be employed to prevent damage from frost? 32. Explain how a local observer may predict frost. 33. (a) Compare highlands and valleys as to their dangers from frost. (b) Why is frost more frequent in valleys than on highlands? (c) How does the cold air of the hills promote cooling of adjacent valleys? 34. What is the value of irrigation when frost is threatening? 35. Why is a frost not nearly so probable if the dew point is at or above $42^{\circ} \mathrm{F}$ ? 36 . Smudge fires with their dense smoke may keep the temperature of an orchard five or six degrees above that of the surrounding country. Would this ordinarily be high enough to prevent an orchard from freezing? Explain.

\section{SUGGESTIVE QUESTIONS}

1. "Without air life is impossible." Explain how fishes live. 2. In what ways does electricity enable us to prepare for unfavorable changes in the weather? 3. When the wind is blowing directly from the west, in what direction with reference to the observer is the low area? The high? 4. If the air is still and an automobile without a wind shield moves through it eastward at a rate of 50 miles an hour, how may the occupants describe the apparent direction and velocity of the wind?

5. Show in what ways the climate determines the particular occupations of people in your locality. The agricultural products. 6. Does frost or freezing kill a plant? Explain your answer. 7. What is meant by " black frost"? 8. If the air in a closed jar is not saturated, in what different ways can it be brought to saturation? 9. Are" clouds formed in your locality or do they always come from a distance? Give reasons for your statements. 10. Find the relative humidity by means of a wet-and a dry-bulb thermometer in the early morning, at noon, in mid-afternoon, and at night. Account for the differences. 11. Air is warmed by the furnace. What change occurs in its absolute humidity? In its relative humidity? 12. Air is warmed by a 
kerosene oil stove. What change occurs in its absolute humidity? In its relative humidity?

13. Explain why different clouds in the sky may be traveling in different directions at the same time. Why the wind may be in a still different direction. 14. Account for the different colors of clouds. 15. Account for the differences you may observe in the falling of raindrops and snowflakes. 16. Almanacs are sometimes valued for their weather predictions. Compare the actual weather conditions of a given period with the predictions of representative almanacs. What is your conclusion as to the value of such predictions? 


\section{CHAPTER II}

\section{FIRE AND HEAT \\ I. Heat, its Uses and Control}

28. Uses of fire. - What are some of the important common uses of fire? Prepare a list of its uses in the home, in industries, and in the arts and sciences. Compare present uses of heat and heating appliances with those of the age of the Pilgrims in New England.

Fire has played an important rôle in the spread and settlement of men over all the earth, as is quickly recognized from a rapid survey of a few of the chief facts. What was the custom of the ancient Greeks in respect to fire when they were sending a colony of the young away to a new land? How did the Romans show their regard for fire? Without the aid of fire could men have conquered the northern and colder regions and acquired their natural resources? How in our present world could manufacture, transportation, and travel continue without the energy produced by fire?

The production of fire and the discovery of its uses must be considered supremely important events in the history of mankind. By means of fire man has increased his control of the world and its resources. Through its use he is enabled to endure the rigors of climate, to increase wonderfully the food supply, and to improve food materials for all purposes. The. clothes we wear, our buildings, the tools, machinery, utensils, and instruments employed in the industries, are largely made possible by the magic effects of fire. Fire and the wheel have together been the means whereby man has made into thoroughfares the vast and uneven stretches of land, the restless oceans, 
and the trackless air. They are the means by which the present world of art and achievement has been created.

29. Sources of heat. - The directly available heat energy of the sun is far from being sufficient for all the needs and purposes of man. In cold and damp weather men must for the sake of health and comfort supplement the sun's heat with that produced from fuel. Hence they have had to find fuel.

Exercise. - What are the chief fuels in common use? What is the relative abundance of each? Its source of supply? What are the advantages, such as cleanliness, convenience, economy, etc., attending its use? What does the cost to the consumer indicate as to its abundance? How does the cost of transportation affect the selling price of fuel? What other reasons can you find for the use of any particular fuel, such as artificial gas, wood, oil, gasoline, alcohol, coal, and the like?

The fuels with which you are familiar may be altogether unknown to some of the earth's inhabitants. Explorers report leaving coal with entire safety among certain tribes of the Eskimos, who call it "black stone" and do not know how to burn it. You may find in books of travel accounts of other people who are unfamiliar with various kinds of fuel. You may never have seen the kind of fuel used in Ireland, and found in certain places in our country. It is called peat (page 455). The inexperienced would be puzzled to make a fire with it. What kind of fuel do the Chinese make use of in the "hand warmers"? What other unusual fuels are used in foreign lands?

Other sources of heat. - Heat may be produced in varying amounts by other means than by burning fuel. If you take a small amount of borax in your hand and wet it with water, you will feel the heat released by the action of the borax and water upon each other. Quicklime when slaked with water yields considerable heat (see page 418). If sulfuric acid is mixed with water, heat is developed in a similar way. There are other changes of the same sort by which heat is produced.

All of the ways mentioned, together with the burning of fuel, are described by scientists as chemical changes, for they are brought about by combining two or more different substances 
in such a way as to yield a new product. The burning wood, for example, yields light, heat, smoke, and ash, all of which are new and different from the wood and the air which combines with it in burning. Such changes are different, moreover, from the merely physical changes such as pouring sand from one bucket into another, sawing wood, or whipping eggs to a stiff froth (page 131).

There are still other ways of producing heat. You know that if you rub your hands together briskly, they become warm, and that by rubbing a piece of metal on cloth or wood, heat is produced. By hammering a piece of metal such as lead or iron it is made warm, and may be made too hot to hold by the continued impact of the blows. When a tire is pumped very tight, the air is compressed and yields heat. Thus by friction, impact, and compression, heat is produced.

A very small amount of heat is derived from the interior of the earth. The central mass of the earth, though solid and not molten, is supposed by scientists to have a temperature of possibly $36,000^{\circ} \mathrm{F}$. Heat from this source may be released through volcanoes, geysers, and hot springs as well as in other less conspicuous ways.

30. Kindling a fire. - The commonplace procedure of kindling a fire involves a more or less definite knowledge of an important fact which is often described as the kindling temperature of substances. This fact we understand in a general way to mean that a given substance, such as paper, wood or coal, begins to burn at a certain temperature. What this means in particular may become clear in the following exercise.

Exercise. - What are the usual means of starting a fire? How did primitive people produce fire? Try to kindle a fire with a rapidly. rotating stick on a board and some inflammable material. Can it be done quickly? Why were special efforts made by people of the ancient world to keep a fire overnight or for longer periods? Try starting a fire with a reading glass. Upon what sort of material must the sun's rays fall to kindle readily? How does a reading glass differ from a piece of ordinary window glass in respect to form and effects? 
By what means did the early settlers in America kindle fires? Explain the action of a flintlock gun or pistol in discharging the load. Gas lighters of materials similar to flint and steel are in use in certain sections of the country. Friction cigar lighters are common.

How many kinds of matches do you find in use? In what respects do they differ from one another? Examine closely two or three of the more distinctive kinds. What are the special properties claimed for each? Are some of them described as " safety, strike anywhere, and with no afterglow"? Are some of them merely "safety" and to be kindled on the box in which they come? You may find what is called the "sulfur" match, which on burning gives off a gas with a disagreeable odor. Is there any marked difference in the way the wooden splints of the several kinds burn? Of what kind of wood are the match splints generally made? What reasons can you give for this? By what process is the match kindled? Can it be kindled by impact, as with a hammer? Are there matches which are proof against this?

In making matches a wood like white pine is made into splints; these are dipped into paraffin, then into melted sulfur, and when cool into a paste of phosphorus and some such material as lead dioxid, and glue to hold the substances together. Then the heads are dipped into some substance that excludes the air and insures safety. The "safety" match, which is designed to be struck on the box, differs from the ordinary kind by containing in the match head a mixture of sulfur in some form and chlorate of potassium, but no phosphorus. It may be lighted on glass. The prepared surface on the box is made of a mixture of ground glass and red phosphorus, cemented by a glue. When the match is rubbed on this, it takes up a small amount of the phosphorus, which ignites easily, kindling the sulfur and the rest of the match.

The kindling temperatures of substances. - The order in which the several substances are ignited or kindled is important. Phosphorus is kindled at a temperature but little above that usual in a room, that is, it has a very low kindling temperature. It is ignited by even the slight friction caused by drawing it over a smooth surface. Sulfur kindles at a temperature a little higher than that of phosphorus. It is ignited by the burning phosphorus and is made to burn more vigorously by the lead dioxid, called an oxidizing material, contained in the 
match head. With the burning sulfur several kinds of wood are ignited, for they have a kindling temperature just a little higher than that of sulfur. Thus each of the substances in the match is selected because of its "kindling temperature."

Does the usual method of laying the materials for a fire in the range or fireplace indicate a knowledge of their different kindling temperatures? Explain how you would arrange the materials for a hard-coal fire in the furnace or range. You may be familiar with what is known as the "hot blast" stove. Is the fuel arranged in the same way in it as in an ordinary stove? Why? Have you tried to make a fire in the woods with only damp wood available? How did you do it? A woodsman does not start a fire with paper. Taking your list of fuels, arrange them in what you think to be the order of the kindling temperature from lowest to highest. Add to your list some substances which are not fuels, as straw, woolen and cotton cloth, iron, asbestos, and so on. From this it will appear that knowledge of the kindling temperatures of different kinds of fuel and other materials is valuable not only in making matches or in starting fire, but in selecting materials for different purposes, as clothing and buildings.

31. Draft in fireplace or stove. - As you sit comfortably before the fire in the fireplace or stove, you seldom consider, unless the stove or fireplace smokes, why it draws. When the fire is first lighted, the smoke may issue freely from the stove and fill the room, but after a while it goes up the chimney as desired. Why does it behave in this fashion? Why, in fact, does it draw at any time? A simple experiment will demonstrate draft very clearly and will aid in understanding its conditions.

Exercise. - Provide a box similar to that shown in Fig. 17 with dimensions about $6^{\prime \prime}$ by $6^{\prime \prime}$ by $15^{\prime \prime}$, with one side covered with a piece of glass and two holes in the top as indicated in the figure. By making a sliding bottom the candle can be shifted from one position to another without opening the box. Over the holes place glass chimneys. 
When the candle is burning and placed under one chimney, hold a piece of burning paper or Chinese joss stick over the other. What is the course of the smoke? What difference is there in the temperature of the air coming out of the box and of that going in through the other chimney? Determine with a thermometer. Move the candle from one chimney to the other, but do not change the burning paper. What happens to the course of the smoke? Change the burning paper to the other chimney. What is the result? Try the candle about the center of the box, between the two chimneys. Try the smoking substances over each chimney. What is the behavior of the smoke? It may be necessary to let the candle burn in this position a few minutes to get a characteristic result. Shift by degrees the candle from the middle toward one hole or the other.

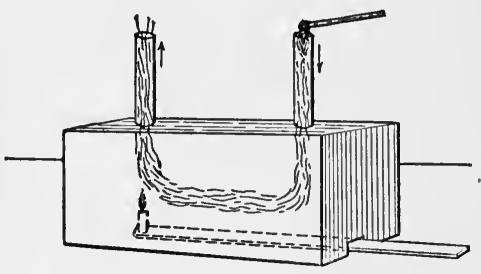

Fig. 17. - Apparatus to illustrate air currents in unequal heating.

Observe the gradual change in the course of the smoke, and the temperature of the air entering and leaving the box.

State the relation you observe between the position of the burning candle and the course of the air as shown by the smoke. Does the smoke enter the box with the warm or with the cold air? State the relation of the temperature of the ingoing and outgoing air to its course as indicated by the smoke. If the candle is not burning, will there be any air circulation through the box? Why?

Why warm air rises. - The problem now is to ascertain why the warmed air rises and issues from the chimney, and the cold air enters the box. The experiment makes it clear that as the position of the candle is changed, different parts of the air are heated and a different course of circulation results. Temperature and air movements seem to be connected. Where a given volume of air is unequally heated, currents are produced ; the warmer air ascending, perhaps being forced upward, and the colder air descending. Why changing temperature causes such circulation of the air is illustrated by the following experiment.

Exercise: Relation of volume to temperature. - Fit into a thin glass flask a one-hole rubber stopper, and into this insert a six to ten inch 
length of glass tubing of very small diameter. Put the end of the glass tubing into some water so that a drop becomes inclosed in the tube. Cool the flask by wrapping it in a cloth wrung out of cold water. In which direction does the drop of water move? Remove the cold cloth and warm the flask with the hands. Note the direction of movement of the water. Does warming increase or decrease the volume of the inclosed air? What is the effect of cooling?

A similar experiment will illustrate a change in the weight of a unit volume of air. Put the end of the tubing into water in a beaker,

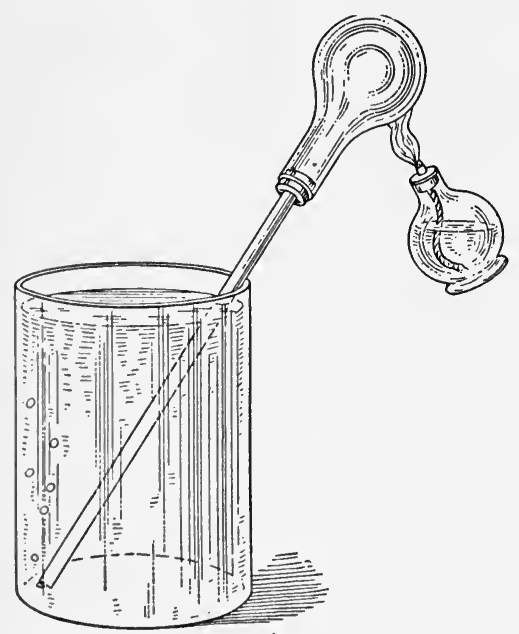

FIG. 18. - The volume of air changes with changes in temperature. Explain how this device illustrates the fact stated. as in Fig. 18, and heat the flask. What does the escape of bubbles of air mean? Does the air remaining in the flask weigh as much as the original air content? With this fact in mind, do you think that a cubic inch of cold air weighs the same as a cubic inch of warm air? Will it weigh more or less? Explain now the circulation of air in the box-and-candle experiment.

Explain why a tire tube inflated with cold air may " blow out" when heated. .To what may the heating be due? Will a basket ball, partly filled with cold air and then warmed so that it becomes tight, weigh more after heating than before? Explain your answer.

These facts are fundamental not only in the case of the problem in hand, but with the larger air currents, such as local winds, planetary winds, and zones of temperature.

Repeat the experiment of air expansion under changes of temperature with some other gas, such as illuminating gas. You will find. that it behaves exactly as air does under similar conditions.

The general law. - A long series of experiments with gases has made it possible for scientists to formulate the general law that all gases expand with the rise of temperature and contract with the fall of temperature. 
The law applied to draft. - The draft, as in the fireplace, is easily understood by the aid of the facts you have studied experimentally. The air, which is directly heated by the fire, expands and, as it expands, occupies more and more space, so that a cubic foot of the expanded air weighs much less than a cubic foot of the colder, unexpanded air. Since the pressure of the air is the result of its weight, the downward pressure of the air in the chimney cannot be so great as that of the outside air. The outside, colder air falls toward the place where the pressure is diminished through expansion due to rise in temperature. The heavier air forces its way into the fireplace where it is heated and then in turn is forced upward through the chimney by incoming volumes of colder, heavier air.

The process is the same as that observed in the course of the smoke-laden air passing through the box and its chimneys. It is due to the unequal heating of the air by the candle in the one instance, by the fireplace in the other instance. The principle is identical in both cases, in fact in all examples of draft, whether in range, furnace, or fire in the open air.

Control of draft. - Heating appliances have been equipped with certain more or less effective means of control of draft and of escape of heated air. It is through such means that all the heat produced by a fire does not follow the path above described and waste itself in the great outdoors. Dampers of different kinds are familiar. What devices of this kind are on your range at home? When a fire has burned long enough to establish a good draft and a bed of hot coals, the draft is partly cut off by closing dampers in the range or chimney. The escape of the heated air is checked so that the range may become more uniformly heated. Dampers are provided to direct the course of the heated air through the range to its several parts, as the oven, for example. In many pipes there is what is known as the " air damper," which when open admits cold air directly from the room. This cold air reduces the temperature of the air in the pipe and chimney, and checks 
the draft through the range, thus saving fuel. Means for the control of the draft are often provided at the top as well as the bottom of the fire box of the range. It may be noted that one of the chief defects of heating appliances is the waste of fuel and loss of heat, although this is controlled in some measure by dampers.

Exercise. - Make a sketch of the course of air through a range, and show how and to what parts of the range it may be deflected by dampers.

Stoves sometimes smoke disagreeably when the fire is first kindled. Making use of the facts just observed, explain the smoking stove and how it may be remedied. What must be the conditions before the draft is fully established and the smoke passes out through the chimney?

Forced draft. - Intense heat is frequently necessary in generating steam for locomotives and stationary engines. One way of securing this is by increasing the draft. Locomotives make use of the exhaust steam to increase the draft. As this means a great loss of energy and an increased cost of work performed (cf. page 295), other devices have been coming into use, such as fans and compressed air. Fans may be used either in the flues to accelerate the movement of the waste gases, or to force air under and through the grates. For the same purpose chimneys of great height are built. The height of the chimney adds to the volume of warmed and therefore lighter air which is being forced out by the colder air rushing into and through the furnace. The result is a greatly increased draft. Have you known of smoky chimneys remedied by being built higher? What other ways do you know for forcing draft? Explain the operation of the blacksmith's forge.

32. The sun's heat. - The natural and direct source of heat energy is the sun. Upon this energy we depend for light and heat and life. How great is our dependence is not always recognized. The sources and materials of artificial heat coal, oil, natural gas, and the like - are stored-up energy derived from the sun, millions of years ago. It is diff- 
cult to realize the vast amount of heat radiated by the sun. It is estimated that in the tropics the sun's heat is received at the rate of one fourth of a horse power for each square foot of surface. In general the sun radiates upon one square mile of the earth's surface an amount of energy equivalent to the several millions of potential horse power of the waters falling over Niagara. What must be the total amount of energy radiated by the sun throughout the universe! Our earth receives only a small fraction, that is, about one two-millionth of the total amount. It is upon this comparatively small fraction of the sun's energy that we are entirely dependent for life, light, heat, and power. It is absorbed by plants and animals, by the land and water of the earth, by the water vapor and dust particles in the air. It is the chief factor in causing evaporation and the promotion of air currents. It also determines the amount and distribution of the rainfall and with this, the distribution, kind, and abundance of plant and animal life upon the earth.

Men have long sought for ways and means to utilize directly the sun's energy. Progress, however, has been slow and successful in comparatively few fields. The means which we have to-day are of the simplest kind, such as windows and mirrors to reflect and concentrate the sun's energy. Many uses of the sun's heat are common, such as drying certain food materials, as fruits, vegetables, meat and fish, or in accelerating plant growth in hothouses. Appliances consisting of mirrors have been devised to gather and apply at a desired point the sun's energy for such work as pumping and heating water and operating small electric generators. A solar motor has been made which produced enough steam to pump about 1400 gallons of water a minute. Experiments with solar motors are now being made in the southwestern section of the United States. Such a motor may prove to be one of the means in reclaiming vast areas of now barren land where water is available under the surface, but not generally on it. 


\section{SUMMARY}

Fire was one of the most important discoveries in primitive times; by its aid men are steadily increasing their control of nature.

Our natural source of heat is the sun. Our main controllable source is the chemical process of combustion. The chief fuels are coal, wood, petroleum, and gas.

Fires may be kindled without matches by friction. The use of matches reduces the amount of friction needed by using substances of low kindling temperature.

Drafts are convection currents due to temperature changes and consequent changes in the density of the air.

The control of draft by dampers or its increase by blowers and exhaust fans help in controlling the temperature of fires and the rate of combustion.

The sun is the ultimate source of all forms of energy that we use. To it is due the energy of the wind and of falling water, the heat from fuel, and the electric current.

Direct use of the sun's energy is made in many ways, such as in agriculture, in heating and lighting buildings, in preserving foods, and in operating solar motors.

\section{REVIEW QUESTIONS}

1. What rôle has fire played in the history of civilization? 2. MMention several important ways in which man's use of fire enables him to gain control of the world. 3. What other inventions are to be compared with fire in importariee to man?

4. Why is heat from artificial sources necessary? 5 Name the most important common fuels used in difierent parts of the world. 6. Mention and describe other means of producing heat besides burning fuel.

7. Mention several methods of kindling a fire. 8. Describe typical ways of primitive peoples. 9. What are the essential characteristics of matches for common use? 10. Describe the making of matches, mentioning materials and purposes of each. 11. Explain the meaning of kindling temperatures. 12. What use is made of the knowledge of kindling temperatures in making matches? In starting fire? 13. What use is made of this knowledge in selecting substances for clothing and buildings?

14. Describe an experiment which illustrates the behavior of air in a chimney. 15. What are the effects of heat upon air? 16. What is the effect of rise and fall of temperature upon all gases? 17. By 
the aid of the facts illustrated experimentally, explain draft in a chimney. 18. How is draft controlled in heating appliances? Describe a modern range and show location and purpose of dampers and other devices for control of draft. 19. Why does a fire smoke sometimes when first started in a range or stove? 20. Mention several conditions in which forced draft is necessary. 21. By what means may it be secured?

22. Is there any known energy which is not derived from the sun? Explain. 23. How can the heat from coal or wood be the stored-up energy derived from the sun? 24. Give an idea of the amount of heat that the earth receives from the sun. 25. What is the importance to us of this received solar energy? 26. By what devices have men sought to utilize directly the sun's energy?

\section{SUGGESTIVE QUESTIONS}

1. How could you make a fire in the forest without paper and matches? 2. What is the most effective fuel, taking into consideration cost, cleanliness, and convenience? 3. A fireplace smokes badly whenever the fire is burning. How would you remedy it? 4. What important factor in the climate is typified by the draft in a chimney? 5. How could you make direct use of the sun's heat in warming water for the household? 6. Mention several industries in which heat from fuel is indispensable. 7. Mention several uses of the sun's heat energy made directly by us in our daily life. 8. Have you read of any people who did not know of fire or make use of it? What progress had such a people made in the arts and crafts of civilization?

\section{Why Fire Burns}

33. Early theories. - Although great advance had been made in the use of fire for heating and lighting, and in the industries and arts, there was practically no understanding of the conditions, process, and products of combustion, before the latter part of the eighteenth century. It had been generally imagined that when anything burns, such as wood or a candle, some substance escapes. This substance was supposed to resemble the air or be " ethereal," and it was named by the scientists of that age "phlogiston." No further knowledge of what makes a fire burn and what happens in burning was 
gained, however, until the age of Scheele, Priestley, and Lavoisier.

Lavoisier's experiments and discoveries. - Lavoisier, a Frenchman (1743-1794), who is now called the "father of modern chemistry," proved that combustible materials do not lose, but on the contrary gain weight, when burned in air. This completely upset the old belief. Lavoisier began a series of experiments to discover the source and nature of whatever it is that combines with substances burned in air. He had learned from Joseph Priestley (1733-1804), an English clergyman, that the air is not a simple substance but is made up of at least two different substances. Lavoisier experimented with many combustible substances, among them lead, which is interesting to work with, has a low melting point, and shows plainly its combination in combustion with some other substance.

Exercise: Burning lead in air. - It is well worth while to perform the following experiment with lead, by means of which Lavoisier made important discoveries. Weigh accurately a small amount of bright lead, the dish in which it is to be melted, and the metal seraper which you will need to remove the burned lead from the dish. Melt the lead and then heat it strongly above the melting point. As a yellowish film is formed over the surface, scrape it to one side with the metal scraper. When all of the bright lead has disappeared, weigh accurately the product, that is, the yellowish substance, and also the dish and the scraper because some of the substance may adhere to them. The result will show that the product or burned lead weighs more than the original lead, and that the dish and the scraper have not lost in weight. This indicates that the increase in weight is due to something probably derived from the air.

This experiment was followed by another in which Lavoisier sought to ascertain whether all or only a part of the air combines with the heated lead. He put a measured quantity of lead into a dish, which was then covered closely. The dish was heated. A very small amount of powder was produced, and in additional experiments it was found that this amount 
could not be increased by longer heating. A lighted splinter was thrust into the dish and the fire was extinguished at once. This proved that the remaining air does not support combustion. The experiment also proved that only a portion of the air is involved in the process of burning. It must be, so Lavoisier concluded, this portion of the air which is added to the weight of the products of combustion.

34. Air and combustion. - It has been proved by the investigations of later scientists that only a small proportion of the air combines with burning substances, and that it is this proportion which makes the products of combustion weigh more than the original combustible materials. In our study we shall seek to demonstrate this fact clearly and also to determine how much of the air is used in combustion and how much is not, or how much supports combustion and how much does not. We shall also try to learn by observations and experiments what are the essential properties of these two parts of the air, taken separately or mixed.

It will be worth while to demonstrate the fact that air does combine with substances in being burned, and that the products of combustion weigh more than the substances burned. The following experiment will present a striking illustration of the important facts.

Exercise: Weighing products of combustion. - Fit a wire gauze cone snugly inside a lamp chimney about one third of the distance from the bottom (Fig. 19). Fill the chimney above the gauze with pieces of caustic soda (sodium hydroxid), which will absorb the products of burning. Fasten a short piece of a candle on a cork large enough to fit the lower end of the chimney, and into the sides of which notches have been cut to allow the air to pass through. Put the cork with candle into the chimney and stand the whole thing on one arm of a balance. Balance it exactly with weights and very small shot or dry sand. Then light the candle and let it burn in the chimney on the scale arm. What happens? Does the result indicate that the products of combustion weigh more than the visible materials before being burned? 
Air indispensable to burning. - At this point the question may be raised whether the air is indispensable to burning. The experiment just performed seems to show that it or a part of it combines with the burning candle. The old experiment of Lavoisier with burning lead in a closely covered dish pointed

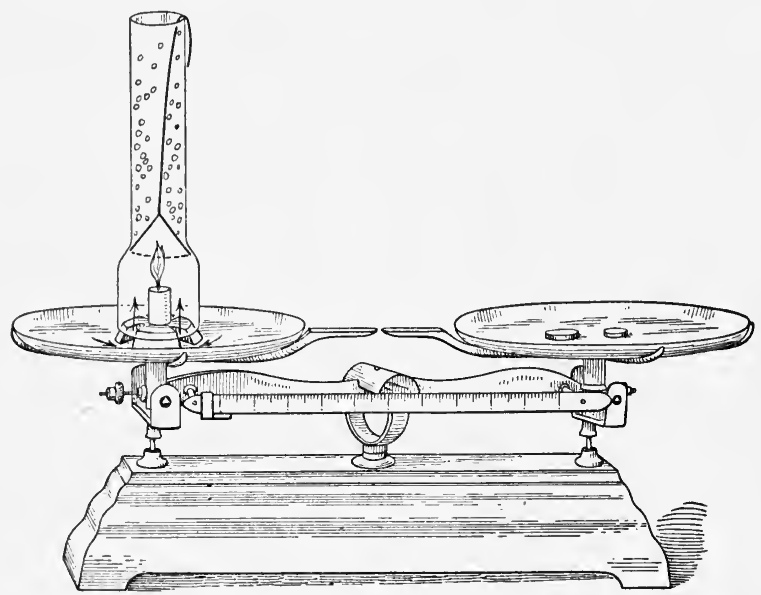

Fig. 19. - Caustic soda in the lamp chimney absorbs the products of combustion. The burning candle produces substances which are shown by this experiment to weigh more than the amount of candle consumed.

to this conclusion. But it may require a clearer demonstration before the answer can be given positively. Is air indispensable to burning? You may find out the important point in this way.

Exercise: Relation of air to combustion. - Hold a lamp chimney over a burning candle, so as to allow about one half an inch space at the bottom for air to pass through. Gradually decrease the space and observe the effects on the flame. Finally shut off the draft entirely. Does the candle continue to burn? Cover the top of the chimney with a piece of cardboard. What happens? (Compare this with the ways of controlling the draft in a range or stove.) What do the facts observed indicate as to the influence of air on combustion? Does the candle burn more brightly with a draft from below than it does when in the open air? Why? Does the brightness of the flame 
increase when the draft is increased by having a small opening at the bottom and the top of the chimney? Does the fact that the candle flame flickers uncertainly and is finally extinguished when the air is shut off completely at the bottom and the top, prove that air is important for burning? Does it prove that air is indispensable to burning?

What proportion of the air is active or unites with burning substances may be determined in several ways. The two ways which are suggested will bring out the essential points. Both experiments may be started at the same time, though one requires several days to give a satisfactory result.

Exercise: Part of air used in combustion. - You will need two quart glass jars with wide mouths; a short piece of candle

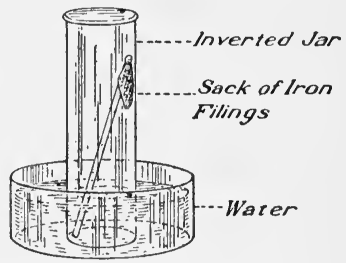

FIG. 20. - The iron filings becoming rusty or slowly oxidizing use up a portion of the air imprisoned in the jar. How much of the air is thus used? fastened to a cork large enough to support it when floating and small enough to enter the mouth of the jar; three ounces of bright iron filings tied in a bag of cheesecloth, and this fastened to one end of a glass rod about five inches long ; a piece of glass tubing bent as in Fig. 21.

Moisten the bag and iron filings and arrange over water in a granite pan, as in Fig. 20. Mark the level of the water with a rubber band or a sticker label. Let the jar stand undisturbed for several days, but observe frequently and record heights of water level. When level becomes stationary, mark it and test the residual air in the jar as directed in the next experiment.

Arrange glass tubing, which should be about one inch high inside the jar, as in Fig. 21, and fasten with rubber band. Use limewater or caustic potash solution in the granite basin.

Light the candle and then cover carefully with

FIG. 21. - How to arrange glass jar, glass tube, and candle over water. the jar, forcing this down until the water runs into the shorter end of the glass tube. Note that the water closes the tubing, thus preventing the escape of air from the jar. Observe the behavior of the candle flame. What does it show concerning the necessity of air to 
burning? Observe the rise of water in the jar. If limewater is used, explain the change of color (page 124). Let the jar stand undisturbed until its temperature is that of the room. Mark then the level of the water in the jar. Taking the original level as indicated by the shorter end of the glass tubing, how much air by volume has been used in the burning? How can you measure the volume? What is the fractional part of the original volume contained in the jar, again measuring from the end of the tube? After removing the tube, slip a piece of glass over the mouth of the jar while in the water, and holding it securely invert the jar. Slip the cover to one side and insert a burning splinter or candle. What does the result show?

When the experiment with the iron filings is finished, compare results of both experiments. Is the volume of remaining air the same in both cases? Does it have the same properties? If the products are the same, is it justifiable to infer that the process is the same, though very much slower in one case than in the other?

Of the total amount of air imprisoned in the jar at the beginning, approximately one fifth has been used in burning. This part plainly supports combustion.

35. The residual gas, nitrogen. - The residual part of the air, about four fifths of the original volume, shows by tests that it does not support combustion. It remains in contact with water with no appreciable decrease in quantity, and is thereby shown to be not soluble in water to any great extent. It remains in the jar, moreover, while you insert a burning candle or match; this indicates that it has about the same weight, volume for volume, as the air. The fact that it does not combine with the burning candle shows that, as compared with the other one fifth of the air, it is sluggish and inert.

The residual gas was perhaps first discovered by Scheele, in experimenting with iron filings. Because this gas refused to support combustion and seemed incapable of sustaining life, Scheele called it " vitiated air." It was found to be abundant in some well-known compounds, as saltpeter or niter (potassium nitrate). Lavoisier, who made chemistry a measuring or quantitative science, determined the actual importance of the gas in the atmosphere, and because of its niter-forming nature 
he named it nitrogen. This is the name by which it is now generally known.

Properties and uses. - Nitrogen constitutes about four fifths of the total volume of the atmosphere. It does not support combustion, nor does it support animal life if confined in it. But it is indispensable to animal and plant life, and forms an essential part of their substance and food. Although they live in an atmosphere containing nitrogen in such quantity, animals do not directly absorb and assimilate it. They depend for their supply upon plants, which in turn depend upon compounds of nitrogen in the soil, as nitrates (saltpeter) and ammonia. Some plants, as beans, peas, and alfalfa, known as legumes, are important for the quantities of nitrogen they derive from the air through the activities of bacteria, which are plants of microscopic size, living in the nodules of their roots (page 573). These minute plants absorb nitrogen from the air in the soil, use it as food, and throw off in waste products such compounds of nitrogen as the legumes need for food.

A summary of the points already considered will be helpful in the study of other problems in the process of combustion. First. It appears that lead, and some other metals, as iron and copper, are changed when heated in the air into substances that weigh more than the metals from which they are produced.

Second. It appears that the air or some part of it is necessary to this change in the metals; if the air supply is shut off, the change is not effected, no matter how much heat is added.

Third. The experiments with a candle show that air is necessary to combustion, for when shut off entirely, the candle ceases to burn.

Fourth. The proportion of the air concerned in combustion is found to be about one fifth. The approximate four fifths left, called nitrogen, does not support combustion, but extinguishes flame and fire.

From these facts it may be inferred that about one fifth of the air, the active part, may combine in combustion with other 
substances; and that this accounts for the increased weight of the products of combustion above the weight of the original visible substances that are burned.

36. The active part of air, oxygen. - It is necessary to study specially the active part of the atmosphere. It has been learned that, when separated from the active one fifth of the air, nitrogen not only does not support, but also promptly extinguishes fire. Hence it may be inferred that nitrogen mixed with the active part of the atmosphere dilutes it and consequently retards combustion. The importance of this may be readily imagined. If the air were entirely composed of the active substance, a fire once started would burn up everything combustible on earth. If the atmosphere were wholly nitrogen, fire and life would be impossible. But mixed as they are, the mixture supports safely both fire and life.

The active part of the atmosphere is not easily separated from it; but it may be derived without difficulty from many compounds which it helps to form. Yellow lead or litharge, for example, when heated in an open crucible to a high temperature, becomes quite red, or forms in burning a new compound known as red lead. In burning, the active part of the air combines with the yellow lead, yielding a product of combustion, just as bright lead did in the experiment just cited.

Red lead is a familiar commercial article, commonly utilized in making paints for ironwork and farming implements, and even in the manufacture of finer paints for artists. It might be used as a source of the active part of air, if it were not necessary to employ high temperatures to make it give up this constituent. If a small quantity of red lead is heated in the air to a high temperature, at which the color changes rapidly, it gives up a substance which causes a glowing match to burst into flame. This indicates that it yields under the influence of heat some of the active part of the air. The same result can be obtained by vigorously heating iron rust, copper rust, 
or any compound of similar nature. The air which combines in burning or rusting is liberated from the burned or rusted product by heat.

A considerable amount of this gas is needed for study, and it may be derived readily from several compounds which are inexpensive and easily obtained. The most common is chlorate of potash (potassium chlorate). To determine whether or not this gives up the same gas, heat a few small lumps in a hardglass test tube. When the lumps are breaking down rapidly, introduce a glowing splinter into the tube. What happens? What does the result indicate as to the gas liberated? Does it behave like the active part of air in supporting combustion?

The gas which is produced from red lead and potassium chlorate, and is supposed to be the active part of the air, was probably first discovered by Scheele. Because it sustains life, he named it "vital air," and because it supports and is indispensable to fire, he called it " fire air." Independently of Scheele, Priestley discovered and gave a very complete description of its nature and behavior. Lavoisier, however, gave it the name by which it is known to-day. He found it present in many acids, and came to think of it as an "acid former." He accordingly named it oxygen. What does the term mean etymologically?

Exercise : Laboratory production of oxygen. - To produce sufficient oxygen for study, arrange apparatus as shown in Fig. 22. Put into a large-diameter, hard-glass test tube a mixture of 10 grams of potassium chlorate and 10 grams of powdered manganese dioxid. Close the test tube with a one-hole rubber stopper, into which you have inserted a piece of glass tubing about 4 inches long. Attach to glass tubing a piece of rubber tubing 18 or more inches long ending in a short piece of glass tubing. Arrange the jar in water so that you can readily insert under its mouth the end of the glass tubing. Have ready also three or four large bottles (eight to ten ounce capacity) filled with water and covered with a square of glass. Apply heat carefully and gradually to the test tube, and when gas is escaping freely, put end of glass tubing under the jar in the basin of water. The gas bubbles through the water and, entering the jar, displaces the water. 
When the jar is full of gas, cover with glass and remove, invert, and leave covered. Fill the other bottles in the same way, covering closely with glass or wet filter paper. When all the bottles are full, remove the rubber tubing from the water and take the flame at once from the test tube. If water enters the test tube, it will cause trouble and perhaps injury.

Exercise: Oxygen and combustion. - Make the following tests with the collected gas. After every test close each bottle promptly to retain the products of combustion for later tests.

1. Char a splinter or match and then bring it to a glow, not a flame. A Chinese joss stick is better, for it glows easily. Insert in a bottle.
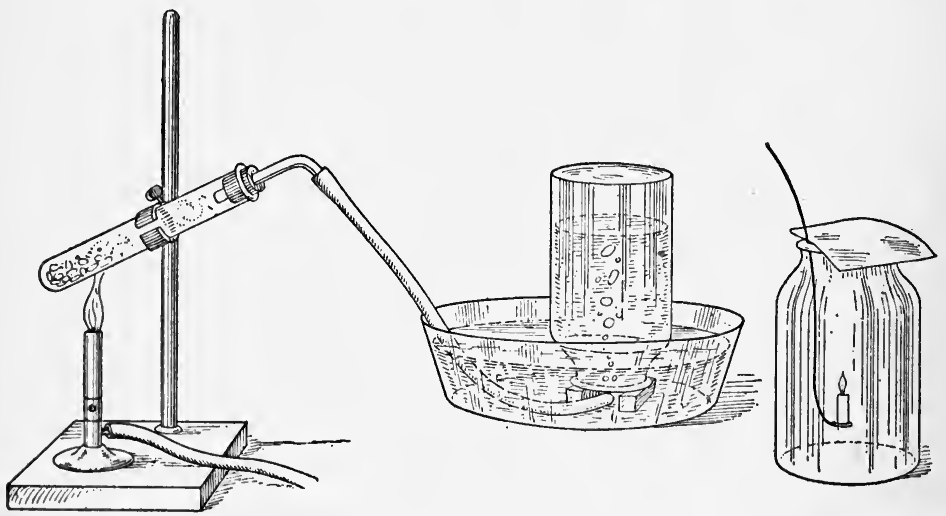

F1G. 22. - Potassium chlorate is decomposed by heat, liberating oxygen, which is captured in a bottle over water. Candle burning in jar of oxygen.

What happens? Repeat this until the reaction fails to occur. 'Does the gas make combustion take place? Does the gas burn? Does it seem to be a good supporter of combustion?

2. In a second bottle of oxygen insert a lump of charcoal by means of a deflagrating spoon or a piece of wire. Keep the bottle closed as much as possible while the charcoal is left there. What takes place? Remove and heat the charcoal to a bright red glow. Introduce it into the same bottle. What is the result? What does this indicate as to the temperature of substances igniting in oxygen? Must they be at a kindling temperature?

3. Put into a bottle enough dry sand to cover the bottom about one half inch deep. Cover bottle quickly to prevent escape of gas. 
Wind into a tangled mass, about seven inches of a twelve-inch length of very fine iron wire. Hold the mass in a flame until it becomes red hot. Insert in bottle just prepared with sand. What happens? What reason do you find for the sand? Does the iron burn? Will iron burn in the open air, even when heated red hot? Does this indicate that oxygen is a better supporter of combustion than ordinary air? What does it suggest concerning the importance of nitrogen in the atmosphere? Fasten a small lump of sulfur on a fine iron wire; light the sulfur and introduce it into a jar of oxygen. What happens to the iron wire? Why? How was it ignited? Can it be ignited by burning sulfur in the air? Close each bottle immediately after testing it.

4. Put a small lump of sulfur on a deflagrating spoon, light and insert into a bottle. Try the same with a small piece of yellow phosphorus, if available. What is the difference between its burning in air and in oxygen?

5. Try bottle No. 1, which has failed to support combustion in a glowing splinter or charcoal, with a burning candle. Does the candle burn when introduced? Does this seem to show that oxygen is especially responsible for burning?

Physical properties of oxygen. - Oxygen, which you collected in bottles, is invisible, and is found by test to have neither taste nor odor. When the bottle is left uncovered for a moment, the gas does not escape; but if it were lighter than air, it would be forced out as soon as the bottles were opened. It is heavier than nitrogen. Oxygen may be cooled sufficiently to become a liquid, just as water vapor when cooled becomes liquid water. These characteristics of oxygen are sometimes called its physical properties, - properties that may be known through our senses, as sight, hearing, touch, taste, muscular sense, smell.

Chemical properties of oxygen. - The tests show something of the behavior and nature of oxygen in combustion. The glowing splinter or charcoal bursts into brilliant flame in oxygen, but by comparison burns faintly in the open air. Iron burns very slowly in the air, or rusts as we commonly describe it; but in pure oxygen it burns with readiness. Other substances like iron behave in the same way in oxygen. Phosphorus, although it ignites slowly, burns freely in air, but burns with 
dazzling brilliance in oxygen. These are only a few typical instances, but you would find the same result with a large range of substances. Only a few, as gold and platinum, are not affected by oxygen in this way. On the whole it may be concluded that oxygen unites freely with burning or sufficiently heated substances introduced into it.

Another characteristic of oxygen is to be noted. You collected it in bottles first filled with water. The gas, making its way through the water to the surface, that is, to the bottom of the bottle, forced the water downward and out until the gas alone filled the space. This indicates that oxygen is not very soluble in water. It is, however, slightly soluble, which is especially important when considered in connection with a fact of common experience that only in the presence of moisture is oxygen active in rusting iron and certain other metals, in fermentation and decay, and in the drying of such oils as linseed. Perfectly dry iron does not rust. But there is always enough moisture in the atmosphere to enable oxygen to begin its work on iron and other metals when these are not protected as by a moisture-proof oil.

In slow combustion, or oxidation as it is commonly called, oxygen becomes more active as the temperature rises. In a haystack or pile of oil-saturated rags, the temperature may become so high as to cause spontaneous combustion. Hay that is not thoroughly dry is favorable to oxidation. Oxygen of the air uniting with the moistened substance effects chemical changes that bring about a rise in the temperature. Hay is a poor conductor of heat, which, being therefore largely retained within the stack, accelerates the process of combustion. The result may be a charred stack of hay and possibly an outburst. of flame if the charred substance is suddenly exposed to the air or to a greater supply of oxygen. Oiled waste is similarly oxidized and when the kindling temperature is reached, ignition occurs. Thus heat increases the facility of oxygen in combining with many substances. 
When oxygen combines with substances both in slow and rapid combustion, the resulting products of the process are compounds known as oxids. The yellowish powder produced by burning lead in the air or in oxygen is the compound lead oxid, familiar in commerce as litharge. Burning sulfur in the air or in oxygen produces a disagreeable gas, which is also a compound called sulfur dioxid. The prefix di means two, and when so used, means that two unit parts of oxygen are combined with one unit part of sulfur. Charcoal is practically all carbon, and so, too, is the charred stick. These burning in oxygen give as a product carbon dioxid.

Oxygen and life. - Oxygen is indispensable in the processes of life. In living organisms oxidation or slow combustion occurs in the production of energy necessary for all kinds of activity and for bodily heat. The human organism is, in fact, an engine which utilizes food as fuel; but in doing this the food must be burned or oxidized. The oxidation of food fuel or of living tissues, into which food is converted, is carried on by the aid of oxygen received from the lungs and circulated throughout the body by the blood. The blood also carries off and eliminates with the help of the lungs the waste products of oxidation. Thus our lungs perform a double service to life, receiving oxygen and throwing off oxids or waste products of combustion.

Oxygen in medicine. - Oxygen is frequently administered in medical practice to accelerate combustion in the living body. A good whiff of oxygen just at the beginning of a sprint or a long run gives additional energy and endurance. It is exhilarating, almost intoxicating, in the outburst of energy thus liberated. In medicine it is utilized for relief from suffocation, asphyxiation, and drowning, and for many other purposes. Describe the pulmotor and its use in resuscitation of individuals overcome by smoke or poisonous gas.

Oxygen in industry. - You may infer from your study of oxygen that the greater the amount of it added to a burning substance, the more rapidly the substance burns and the more 
intense the heat. It is this property which makes oxygen useful in many industries, being utilized in generating very high temperatures needed in working with metals, as in welding, cutting, and the like. It is combined in practically pure form with some highly combustible substance, such as hydrogen or acetylene gas. The flame of hydrogen and oxygen gives a temperature of approximately $4000^{\circ} \mathrm{F}$.; while the oxyacetylene flame gives about $6500^{\circ} \mathrm{F}$. at the hottest point. Students will find in current magazines accounts of the various industrial uses of oxygen and may report on such to the class.

Abundance of oxygen. - From the chemist's point of view oxygen is one of the most active substances known. It unites freely with practically all other substances. It is also one of the most abundant of known elements. It forms about one fifth of the atmosphere and eight ninths of the water; it enters largely into the composition of almost all kinds of rocks, and into all forms of living matter. It is estimated to constitute about forty-eight per cent by weight of the whole surface of the earth, of all that the earth contains, and of the air enveloping it (page 433).

\section{SUMMARY}

Our knowledge of the nature of fire dates from the time of Lavoisier, in the last quarter of the eighteenth century.

Lavoisier showed that burning is oxidation.

Only oxygen, which constitutes about one fifth of the volume of the air, is useful in burning. The remaining four fifths is mainly nitrogen, which is an indispensable element in the foods of plants and animals.

Oxygen is active in burning, nitrogen is inert and dilutes the oxygen of the air.

Oxygen may be obtained by heating various compounds, such as potassium chlorate.

Pure oxygen supports rapid combustion, even of iron. water.

Oxygen is a colorless, odorless, tasteless gas, slightly soluble in

Oxygen dissolved in water causes the rusting of iron. 
Slow oxidation of many oils and of some other substances may result in spontaneous combustion.

Oxids are products of the union of oxygen and other substances.

Oxygen is indispensable to life, is useful in medicine and industry, and is the most abundant element.

\section{REVIEW QUESTIONS}

1. How did Lavoisier prove that a combustible substance burned in air gains in weight? 2. What does lead burned in a closed dish show? 3. What proportion of air seems to be used in such burning? 4. Describe how it may be shown that a certain part of the air is necessarily concerned in combustion.

5. What do you find to be the properties of nitrogen as to solubility in water? support of combustion? support of life? weight? activity? 6. Why was nitrogen called "vitiated air"? What is its proportion in the air? 7. What is the use of nitrogen in the air?

8. What part of the air unites actively with burning substances? 9. What are the characteristics of oxygen as to visibility? taste? odor? solubility? support of combustion? support of life? 10. What is oxidation? Give instances of different rates of oxidation of substances. 11. Describe a method for the production of oxygen on a large scale. 12. What are the physical properties of a substance? 13. What are oxids? Why are they so named? 14. What is the meaning of the term "dioxygen" or dioxid? 15. What use is made of oxygen in life processes? in medicine? in manufacture? 16. Why does the chemist consider oxygen as especially active? 17. What is the relative abundance of oxygen?

\section{SUGGESTIVE QUESTIONS}

1. Why is metallic lead not found in nature? 2. If air is indispensable to combustion, why does the fire in a stove or furnace keep overnight when all drafts are closed? 3. Fires in holds of ships are sometimes extinguished by blowing in steam from the boilers. Explain. 4. Describe some experiment not in the text to show that air is necessary to burning. 5. Why would prolonged inhalation of oxygen not be altogether beneficial? 6. What does it mean to say that gold does not "rust"? 7. Explain "spontaneous combustion," and if possible give instances. 8. Suppose the proportion of oxygen in the air were decreased by one half ; what changes do you imagine would have to occur to make human life possible? 


\section{Temperature - the Thermometer}

37. Our feeling of temperature. - Have you not observed that of many individuals in a room no two may feel or judge the temperature to be exactly the same? One may find it too warm for comfort, another, too cold. The same individual, moreover, may one day experience no discomfort in a room with a given temperature, but the next day be extremely uncomfortable when the temperature is the same. Is our feeling or judgment of the temperature of any external object, such as water, air, wood, iron, stone, or cloth, reliable? Can you judge accurately how hot or cold a body is? The following experiment will demonstrate to what extent we can depend upon our sensations of temperature and its changes.

Exercise. - Hold one hand in water as hot as it can be endured, and the other hand in cold water, for two or three minutes. Then put each hand alternately into lukewarm water. What are your sensations of temperature in each ease? Can you judge accurately the temperature of lukewarm water in this way? Put the hand that was held in cold water into the hot water, and then into the lukewarm water. What are your temperature sensations at each trial?

What do these experiences indicate as to the reliability of our feelings of temperature and its changes? Do you imagine that an individual in a fever can feel or judge the temperature of a room correctly? Explain. What do you infer from this as to the relation of our bodily condition to sensations of temperature of external things?

It is interesting to note how we get sensations of heat and cold. It may be imagined that we feel heat and cold equally over all the skin. With a cold nail having a sharp point or a lead pencil touch different places on the back of your hand. Do all places give sensations of cold? Mark such as do with black ink. With the point of the nail warmed, find spots that give a feeling of warmth and mark them with red ink. Do the cold and warm spots coincide? Apply. the warmed nail point to a spot which is marked black as designating a cold spot. What is the result? Try a cold point upon a warm spot. Are the spots well distributed over the back of your hand? Do they merge into each other or are they quite distinct? The temperature spots, as they are named, are distributed more or less sparsely over the whole body. 
38. Measurement of temperature. - It is necessary in very many processes of manufacturing and in the household to secure and maintain a definite temperature. Since feeling is too variable to be taken as a guide in such matters, an instrument is necessary which will determine reliably and automatically temperature and its changes. Such an instrument is the thermometer. With its aid we can find exactly the temperature of any substance, such as the air, water, fire, earth, or any living organism.

Construction and action of the thermometer. Examine a thermometer (Fig. 23). The usual household type is simple in construction, made of a glass tube with a very small but uniform bore and with a small bulb at one end. The bulb is filled with mercury which usually reaches some distance into the tube. The tube is either graduated or fastened to a graduated scale. Measure the spaces. Are they equal? The graduated distances are determined by means of what are known as the fixed points, which are the freezing and boiling points of water. When the fixed points are determined for any thermometer, the distance between them may be easily divided into equal parts. How many spaces are there between the fixed points of the thermometer under examination?

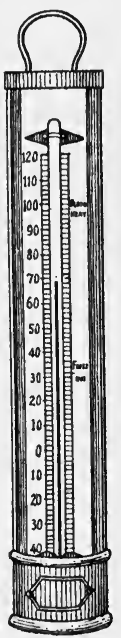

Fig. 23. A household thermometer.

You will observe that when the mercury in the bulb is warmed, as with the hand, the height of the column in the tube changes. It rises to a higher level, which may be read on the scale as degrees. When the bulb is cooled, the mercury column falls. The behavior of mercury under the influence of heat and cold is a fact of great importance in understanding how temperature is measured. Air was found to behave in similar fashion, page 64; it expanded with warmth and contracted with cold. It is known that all gases behave in the same way.

Water is not used in thermometers because it does not expand 
uniformly between the freezing point and the boiling point, and because it becomes solid at freezing temperature and vaporizes at boiling. The substances commonly utilized in thermometers of the "liquid" kind are mercury and alcohol. Alcohol is adapted to temperatures that fall below that at which mercury freezes or becomes a solid, as it does in the high latitudes and very high altitudes. Both mercury and alcohol expand and contract uniformly throughout a wide range of changes in temperature. They are sensitive to very slight

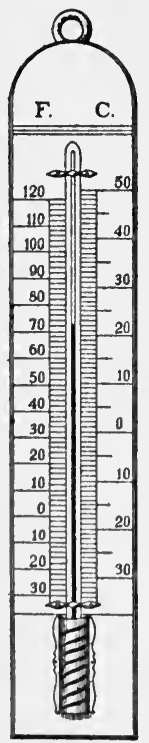

FIG. 24. - Fahrenheit and Centigrade scales. differences in temperature and show relatively large changes in volume as a result. They respond promptly to such changes and are also easily visible or can be made so by using a glass tube with small bore. They report quickly and accurately, therefore, the actual temperature of any body.

Exercise. - Other important features may be noted. Why, for instance, is glass used for the tube? How does glass respond to changes in temperature, as eompared with mercury? Why is the bore of the tube very small and uniform? Why is there a bulb at the lower end and why is it much larger than the bore of the tube? What other substances would do as well as mercury and alcohol in the liquid thermometer? Explain your answer. What must be done to make alcohol easily visible in the tube?

On the Fahrenheit scale (Fig. 24), which is commonly used, the freezing point is designated as $32^{\circ}$ and the boiling point $212^{\circ}$. This gives an interval of $180^{\circ}$ between the two fixed points. The zero, $0^{\circ}$, is $32^{\circ}$ below the freezing point.

Fahrenheit, a German scientist (1686-1736), in making the scale, took as one of the fixed points the temperature of the human body, which he called $100^{\circ}$. The other fixed point he found by a mixture of ice, sal ammoniac, and water, and this he called $0^{\circ}$, zero. He divided the interval into 100 equal 
parts. On this scale it appeared that water freezes at $32^{\circ}$ and boils at $212^{\circ}$, at normal atmospheric pressure. Later it was decided to take the melting of ice and the boiling of water as the fixed points and to make the scale accordingly. By this scale the bodily temperature is found to be about, $98.4^{\circ} \mathrm{F}$.

The fixed points of the thermometer are readily determined. The freezing point is found by packing a thermometer in pure cracked ice or snow in a glass funnel. The melting ice or snow soon causes the mercury thread to stand at a certain level, where it remains until all the ice is melted. This level, which is the point of freezing or melting, is marked on the glass tube. The bulb is then immersed in boiling water. The mercury soon registers a high stationary level, which is the boiling temperature and is so marked. What is the freezing temperature on the Fahrenheit scale? What is the boiling temperature? How many degrees are there between the freezing and the boiling temperature?

The Centigrade scale is used practically throughout Europe and South America, and is the scale employed in almost all scientific work. On this scale the freezing point is designated $0^{\circ}$ (zero), and the boiling point $100^{\circ}$. The interval between these fixed points is divided into one hundred equal parts. Centigrade is thus seen to mean centi, one hundred, and gradus, degrees, or one hundred degrees. Readings above and below the fixed points are similarly determined by the same distance for each degree. It is well to notice that when readings below zero on either scale are given, they are written with the minus or negative sign (-) as, for example, $-4^{\circ} \mathrm{C}$. or $-12^{\circ} \mathrm{F}$.

Comparison of the two scales. - The Centigrade and Fahrenheit scales (Fig. 24) are so widely used that one must be able to compute promptly from the readings on one the equivalents on the other. They may be represented as parallel stairways, the ground floor of one marked $0^{\circ} \mathrm{C}$., and of the other $32^{\circ} \mathrm{F}$. The top landing of one is marked $100^{\circ} \mathrm{C}$., and of the 
other $212^{\circ} \mathrm{F}$. There are accordingly $1 \mathrm{CO}$ steps of the one stairway, and $212-32$, or 180 steps on the other (Fig. 25).

To find the equivalent of one scale on the other is simple. On the Centigrade scale 100 steps indicate the same difference in temperature as 180 steps on the Fahrenheit scale. How many steps F. are equivalent to 10 steps C.? How many

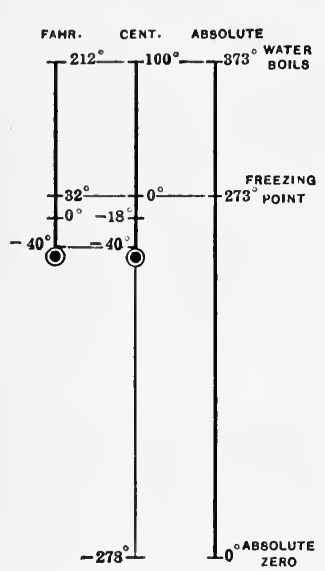
steps F. to 5 steps C.? Expressed in the form of a fraction one degree $\mathrm{C}$. is equivalent to $\frac{9}{5}$ of a degree $\mathrm{F}$. What part of one $\mathrm{C}$. degree is one $\mathrm{F}$. degree?

Exercise. - The reading of a C. scale is $50^{\circ}$. What is the equivalent on the F. scale? How many steps is this above the freezing point?

Find the equivalent on the other scale of each of the following: $14^{\circ} \mathrm{F}$; $; 70^{\circ} \mathrm{C} . ;-20^{\circ} \mathrm{C}$.; $-40^{\circ}$ F.; $37^{\circ}$ C.; $98.4^{\circ}$ F.; $68^{\circ}$ F.; $212^{\circ}$ F.; $98^{\circ} \mathrm{C} . ;-273^{\circ} \mathrm{C}$.

39. Kinds of thermometers. - Weather

Fig. 25. - Diagram a thermometer known as the "maximum showing different temper- and minimum." The instrument consists
ature scales.

of two thermometers, one containing mercury for maximum temperatures, and the other alcohol for minimum temperatures. The bore of the maximum or mercury thermometer is very much constricted just above the bulb; mereury forced by expansion through the constriction cannot return. It registers the maximum temperature during a given period, such as twelve or twenty-four hours. The minimum or alcohol thermometer contains a small index, much like a double-headed pin. The index is drawn downward by the fall of the surface of the alcohol and remains at the lowest point reached until released by the observer.

The clinical thermometer of medical practice may be mentioned. It is a maximum mercury thermometer, with Fahren- 
heit scale, reading from $92^{\circ}$ to $110^{\circ}$, but with each degree subdivided into fifths. How does a physician take one's temperature? How does he make the mercury return to the bulb after using?

A common thermometer is the aneroid or metallic, which usually consists of brass and steel strips made into a spiral coil. The thermograph (Fig. 26) is similarly constructed, but besides indicating the temperature changes, records them on a chart graduated for days and hours and moved by clockwork. The principle of the metallic thermometer is precisely the same as that of the liquid or gas thermometer. Certain metals expand and contract in definite amounts with thermal changes and

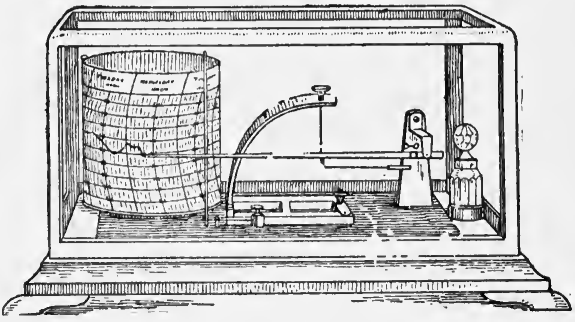

FIG. 26. - Thermograph. A seven day record sheet is fastened to the cylinder which is turned by clockwork.

are utilized to show such changes. Their changes in volume are accurately determined for given differences in temperature, just as in the case of mercury or hydrogen.

The ordinary mercury thermometer is useless for temperatures above $357^{\circ} \mathrm{C}$. $\left(680^{\circ} \mathrm{F}\right.$.), for mercury boils at this temperature. It may be made useful for temperatures as high as $500^{\circ} \mathrm{C}$. $\left(932^{\circ} \mathrm{F}\right.$.) by filling the space above the mercury with nitrogen. Higher temperatures, however, are needed in many manufacturing processes. In the electric furnace, temperatures may reach $3600^{\circ} \mathrm{C}$. to $4000^{\circ} \mathrm{C}$. In scientific investigation a temperature of $7800^{\circ} \mathrm{C}$. has been obtained. (See page 175.) Such temperatures are above the range of ordinary thermometers. They may be approximated by estimating from the color and the quantity of the light produced. Thus scientists estimate the sun's temperature to be about $6000^{\circ} \mathrm{C}$.

Instruments, called pyrometers, are used for very high tem- 
peratures. Some are made of clay in the form of pyramids or cones. A pyrometer may have two or more cones in series, each cone made of a different kind of clay so that they melt at different temperatures. The melting temperature of each is known, and that just showing the effect of heat is taken to indicate the temperature.

Thermometers containing a gas, as hydrogen, are necessary in temperature measurements of extreme accuracy and delicacy. These are usually employed in laboratory investigations. The standard thermometer for the world, however, is a hydrogen thermometer, kept at the International Bureau of Standards, near Paris.

Mercury freezes at $-39^{\circ} \mathrm{C}$. What degree on the F. scale? The mercury thermometer is therefore useless for temperatures below this, as in the polar regions and at very high altitudes. Alcohol, which freezes at $-130^{\circ} \mathrm{C}$. or $-202^{\circ} \mathrm{F}$., is used for such temperatures. When temperatures below the freezing point of alcohol are to be determined, they are measured with thermometers made of hydrogen gas or of helium, one of the rare gases of our atmosphere.

It is interesting to note some of the low temperatures obtained in scientific research. Air becomes liquid at a temperature of about $-212^{\circ} \mathrm{C}$., or $-350^{\circ} \mathrm{F}$. Hydrogen, when liquefied, produces by its rapid evaporation a temperature of about $-258^{\circ}$ C. Helium is liquefied and by rapid evaporation produces a temperature of $-272^{\circ} \mathrm{C}$. The lowest possible temperature is $-273^{\circ} \mathrm{C}$., known in science as absolute zero. The boiling point of liquid air (the boiling point being the temperature at which the liquid is changed into vapor, just as water at $100^{\circ} \mathrm{C}$. is converted into vapor or steam) is about $-191^{\circ} \mathrm{C}$. The boiling point of helium is about $-268.5^{\circ} \mathrm{C}$.

Man has learned how to produce and use extremes of temperature greater than those found in nature. The lowest temperature obtained is as low, perhaps several degrees lower, than the estimated lowest temperature of the great void among 
the stars and planets. The highest temperature obtained is about $7800^{\circ} \mathrm{C}$. or about $1800^{\circ}$ higher than the estimated temperature of the sun. Such achievements illustrate man's increasing control of the forces of the universe and his progressive adaptation of them to the needs and purposes of mankind.

40. Familiar instances of changes due to heat and cold. Common experience presents many examples of changes in mineral substances under the influence of heat and cold. Boiling water poured upon a cold heavy glass dish is likely to cause it to break. Why? How are fruit jars prepared for hot fruits or vegetables? What would happen if fruit jam at nearly boiling temperature were poured into a cold glass container? It is sometimes difficult to unscrew the metal top from a glass jar; but if the top be submerged for a moment in hot water, it may be easily unscrewed. Explain what happens. Which responds to the heat more quickly, glass or metal? You may find in the home other instances of such changes caused by heat and cold.

Exercise. - What causes a wheel, as of a wagon, to "freeze" on the axle? How does the plumber provide for changes in the length of steam and hot-water pipes?. Are such pipes fastened securely to walls and floors of buildings? Why? Are the ends of the steel rails on railroads close together at ordinary temperatures? When the weather is very cold? When it is very hot? Explain the conditions observed. Are steel bridges bolted securely to the piers? Explain. Find other examples where provision is made for changes in materials used in structures and in products of manufacturing.

\section{SUMMARY}

Thermometers are used because we need to determine temperature more accurately than we can with our unaided senses.

The expansion of liquid mercury is uniform and continuous with rise of temperature.

Alcohol is used in place of mercury when low temperatures are to be indicated.

On the Fahrenheit scale the freezing temperature of water is marked $32^{\circ}$ and the boiling temperature $212^{\circ}$. 
On the Centigrade scale the freezing point is $0^{\circ}$ and the boiling point $100^{\circ}$.

One degree Fahrenheit is equivalent to $\frac{5}{9}$ of one degree Centigrade.

Maximum and minimum thermometers register the extremes of temperature, generally for a day.

Physicians use a maximum thermometer called the clinical thermometer.

A hydrogen gas thermometer is the standard for the world.

A metallic thermometer with a recording apparatus constitutes the thermograph.

Instruments for measuring temperatures above $650^{\circ} \mathrm{F}$. are called pyrometers.

Alcohol because of its low freezing point is used for temperatures such as occur in the Polar regions.

Expansion and contraction due to changes in temperature are provided for by builders and engineers.

Extremely low temperatures have been obtained by the evaporation of liquid air, liquid hydrogen, and liquid helium.

Man has learned how to obtain and use extremes of temperature greater than those found in nature.

\section{REVIEW QUESTIONS}

1. Mention several important uses of a thermometer. 2. Explain the fact that the mercury in a thermometer rises as the temperature rises. 3. Give some reasons why mercury is more suitable than water for thermometer construction. 4. Why is a large bulb and a small bore desirable?

5. Give the reading for the freezing point and the boiling point of water on the Fahrenheit and Centigrade scales. 6. Describe the construction and working of a maximum thermometer. Of a minimum thermometer. Of a thermograph. What are the advantages of a thermograph?

7. How can furnace temperatures be estimated? 8. What is the lowest temperature obtained? How?

\section{SUGGESTIVE QUESTIONS}

1. If glass expanded more than mercury for the same rise in temperature, which way would the mercury in the thermometer move as the temperature rises? 2. Why are rivets inserted red hot in steel construction? 3. What is the estimated temperature of the sun expressed in degrees Fahrenheit? 4. Is the center of a steel arch bridge 
higher in summer or in winter? Of a suspension bridge? Explain. 5. A party is being equipped for Arctic exploration. What kind of a thermometer and barometer should be taken? Explain. 6. Mention several practical uses of our ability to obtain unusually high and low temperatures. 7. Mention several industries in which exact measurement of temperature is indispensable. Give reasons. 8. Would exact measurement and control of temperature in baking or roasting foods be advantageous? In what ways?

\section{How Heat Is Transferred}

Heat produced by burning fuel in a range is transferred to other bodies in several ways (Fig. 27). The range and cooking utensils placed upon it are heated in one way. The water in the teakettle or the hot-water tank is heated in another way, as is also the air of the room. Objects which are exposed to, but not in contact with, the range are heated in a third way, as may be seen when some article is injured by being left too near the fire. The three ways in which heat is transferred from its source to other objects are now to be studied in some detail.

41. Conduction. - First of all, heat, as you know, is transferred from the fire or range to objects in contact with it. What happens to an iron poker when it is held with one end in the fire? Will it

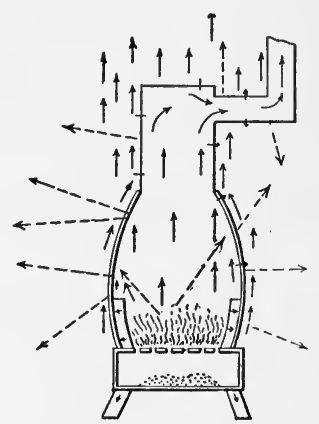

Fig. 27. - Three ways of transfer of heat. Conduction $\rightarrow$; convection $\longrightarrow$; radiation $\cdots-\cdot-\rightarrow$. Explain how each is brought about. finally become too hot to hold in the bare hand? Does the heat travel visibly from one end to the other? You know that the poker is heated throughout its length, or, as it is commonly described, the heat is conducted by the metal. So with the utensils on the top of the range. Heat is conducted from the fire by the range and by the utensils. As all substances are composed of countless minute particles, it is assumed that each particle transmits to its neighbor the heat it receives, 
which process is known as conduction. Any substance which is thus heated by being in contact with a source of heat is called a conductor.

Examples of heat conductors. - When the poker becomes too hot to hold with the bare hand, one may use a holder of such material as paper, cloth, wood, or asbestos. While we know from common experience that all sorts of substances may be heated by conduction, we know also that some are heated at different rates of speed and with different degrees of resulting temperature in various parts. We know that paper is a poor conductor, as are the other substances named above. What utensils are of ten made with wooden handles for use about the fire? Why are lid-lifters equipped with handles of different material or relatively small diameter wire of the same metal? What substances besides wood are used because they are poor conductors of heat?

Exercise: Relative conductivity. - The differences in conductivity of several common metals may be illustrated experimentally. Pro-

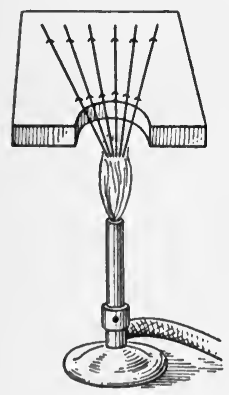

Fig. 28. - Arrangement of metal rods to determine their relative heat conductivity. cure a six-inch length of each of the following metals, all being of the same diameter as that of a telegraph wire: iron, copper, brass, aluminum, lead, or other available metals. They may be tested in either of these ways.

(1) Dip the metals in melted paraffin and when thoroughly coated, allow them to cool. Arrange them so that the ends are simultaneously heated by a flame, and the rest of each rod is shielded from the heat by a screen. Note the difference in the rate of melting on the rods. Does this indicate the rate at which each conducts heat?

(2) Arrange the rods, fan-shaped, upon an asbestos plate (Fig. 28), and supported high enough to allow an alcohol lamp or a Bunsen burner to stand with the flame touching the ends. After heating a few moments move a match head slowly along each rod toward the flame. The points at which ignition occurs indicate the comparative conductivity of the metals. Classify the metals according to their ability to conduct heat.

Exercise: Water as a heat conductor. - Fill a test tube nearly full of water and, holding the lower part of the tube in your hand, apply heat to the top of the water (Fig. 29). Does the water conduct heat rapidly? Another experiment may be substituted for the above. 
Into two test tubes nearly full of water put two equal sized lumps of ice, one weighted with lead so that it will sink to the bottom of the tube. Apply heat simultaneously to the top of the one containing the weighted ice and to the bottom of the other. In which does the ice melt first? What does this indicate as to the heat conductivity of water? If it were as good a conductor as iron, would the ice melt in both at about the same time? If heat is applied long enough, in which would the water boil first? If water were heated by conduction only, it would boil thoroughly and just as promptly no matter where heat might be applied.

\section{Conductivity of substances.-} Some substances in common use, arranged according to their conductivity of heat, are here given. Silver is the best conductor of all

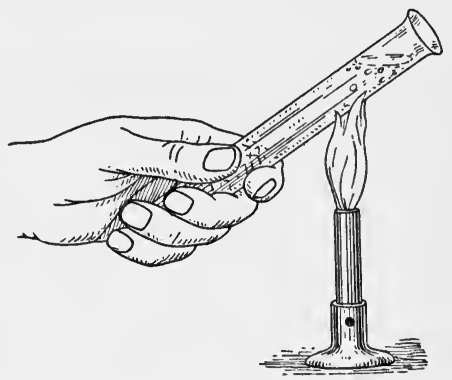

Fig. 29. - Experiment to show that water conducts heat. What does this show as to its rate of conducting heat? metals, and following it in order are copper, gold, aluminum, and iron; then glass, brick, stone, water, asbestos, cotton fiber, wood, paper, cork, woolen fiber, silk, fur, feathers, sawdust, and air. Water is one of the best conductors of ordinary liquids, an important fact in many of its uses. Air, which is typical of the gases, is the poorest conductor in the list. To summarize: metals are better heat conductors than ordinary liquids, and liquids are superior conductors to the gases.

Exercise: The materials of which clothing is made have a wide range in their conductivity of heat. This is suggestive in selecting materials for hot and cold weather. Cotton goods, for example, are not only fair conductors of heat, but are also permeable to air or allow free circulation. Are they better adapted to hot weather or cold weather use? In extreme cold, would they check sufficiently the escape of heat from the body? Materials such as woolen goods, furs, feathers, and eider down hold in their minute interstices quantities of still air. Such materials with their inclosed mass of air form thus a kind of blanket only slightly permeable to air or allowing a very slight circulation. When used as elothing or covering, they 
imprison the air which immediately envelops the body and has the same temperature as the body. They prevent the escape, except in small measure, of bodily heat and keep the body comfortable in extremely cold or windy weather. Which will be safer, clothing of cotton or of woolen goods, if soaked with water? Explain your answer. Why do explorers in the Arctic regions use tents of silk? Why do many persons place folded paper under their clothing in cold or windy weather?

The substances of which buildings are constructed are selected partly for their poor conductivity of heat. Stone, brick, cement, glass, and wood are poor conductors and make it practicable to maintain a very different temperature within a building than without. What is their use in this respect in hot weather? In cold weather?

Would a house built with double walls holding between them a blanket of " dead" air be warmer in winter and cooler in summer than if it were constructed with a single wall? Explain. Would the doublewalled house be more rapidly heated in cold weather? What is the advantage of wrapping steam or hot pipes with asbestos paper, or painting them with asbestos paint?

How are substances that are bad conductors of heat useful in refrigeration? How is ice kept in the ice house, or when being shipped? How may it be prevented from melting quickly in an ice chest? Examine a "fireless cooker." What is it that keeps the contents of the cooker at a cooking temperature for a considerable period of tıme?

Exercise: Sensations of temperature. - Touch with the hand in close succession several different kinds of substances in the same room and apparently having the same temperature, such as woolen goods, cotton, fur, wood, iron, oilcloth, tile, and marble. Are the sensations of temperature the same? Fur and woolen goods feel warmer to the touch than iron and marble. The explanation is based on their conductivity of heat. Woolen goods either take no heat from the hand, or give more heat to the hand than they receive; consequently they cause a sensation of warmth. Tile, oilcloth, marble, and the-like take up more heat than they give, with the result that one receives from them a sensation of cold. Suppose now that the iron, marble, and such substances have a temperature higher than that of the body. Will they feel cold or warm? Will they feel warmer than wood at the same temperature? Explain this fact.

42. Convection. - The comforting fact is that air, which is one of the poorest conductors of heat, can be heated by fire. The water in the teakettle boils, and the water in the tank 
becomes hot in due course. The result is not due to conduction of heat, which has just been considered, but to another method of heat transfer.

Recall the facts observed in the experiment with the box and candle, and in those experiments illustrating the changes in unit volume of air under the influence of heat and cold.

You recognize at once that the air in the box near the burning candle became warm, expanded, and was then forced away by the colder and denser parts of the air in and surrounding the box. The same thing occurs in the case of the warming of the air of a room by fireplace or stove. The air adjacent to the fire becomes warm, expands, and loses density. It is then forced upward by the denser part of the air, thus promoting a circulation that eventually equalizes the temperature of all the air of the room. The heat is thus seen to be carried away from the fire by the air currents.

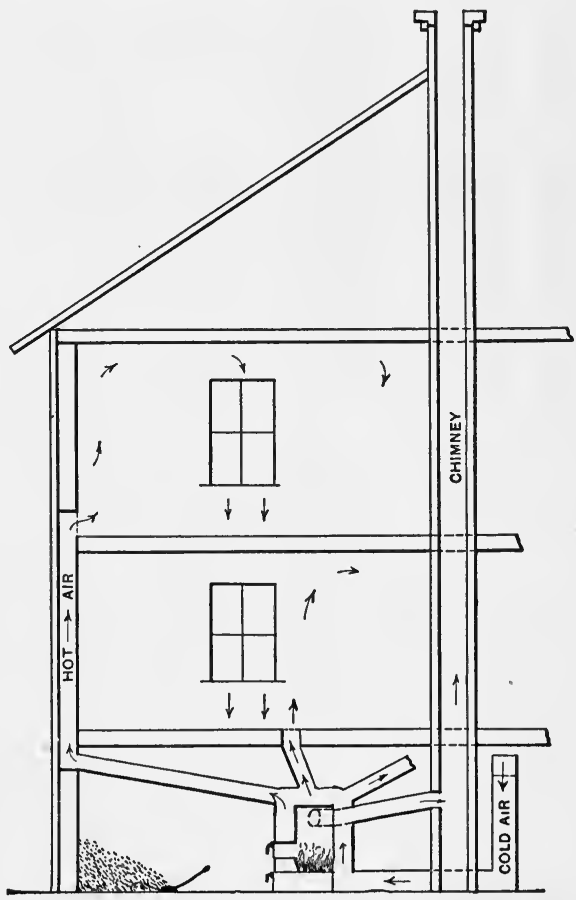

FIG. 30. - Showing convection currents in heating a house with a furnace. Trace the course of the warm air to and through each room illustrated.

Exercise: Convection currents in air. - By means of the smoke from a burning joss stick trace the course of air currents in a room heated by a stove. They will be found to follow a course similar to that of the currents produced in the box-and-candle experiment. 
Make a diagram illustrating the facts of air circulation in the room.

Heating buildings by means of " hot-air" furnaces (Fig. 30) depends upon this manner of heat transfer. Make a plan of a furnace and distributing pipes, and cold air supply. Show the course of the air from its reception by the cold-air box to its delivery in

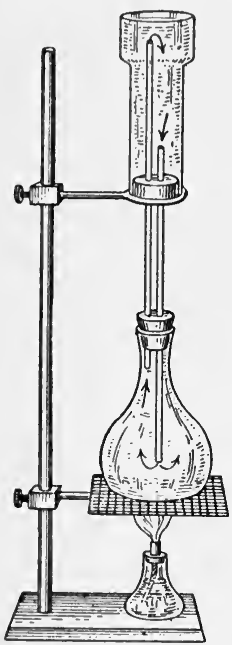

FIg. 31. - Simple representation of a hot-water system. Identify the warm and the cold water currents. Explain transfer of warm water from lower bottle to the upper. Is any of the water in the upper bottle heated by conduction? Explain your answer. different parts of the building. Explain what takes place as the air comes into contact with the hot furnace. Why does it move through the distributing pipes and not out through the cold-air box? If the hot air from the registers fails to reach all parts of the room, will the temperature in all parts of the room be the same?

Liquids, which as you know are poor conductors, distribute heat in the same way as air. Portions being heated lose density and are forced away from the heat source by the denser portions. You may readily demonstrate the fact by applying heat to the bottom only of a test tube full of cold water. Observe the way in which the heated portions of the water are transferred from bottom to top. Are there currents downward as well as upward? Try this out by applying heat to the top of water in a test tube, holding the lower part of the tube in the hand. The course of the heated parts of water will be made more conspicuous if some small particles of coloring material, as aniline dye, are put into the bottom of the tube.

Exercise: Convection currents in water. - The important fact may be illustrated clearly by either of the following demonstrations. Set up the apparatus shown in Fig. 31. Put some coloring matter in the bottle or lamp chimney to show the course of circulation. The chimney may be taken to represent the hot-water tank; the glass tubing to represent the pipes connecting the tank with the water coil in the range 
or heater, here represented by the lower bottle. Apply heat to the lower bottle. Explain results.

Meaning of convection. - The process of transfer of heat in this manner by gases, such as air, and by liquids, such as water, is known as convection. The heated portions of a given volume of a substance are moved from place to place through differences in density. Eventually the whole of a given mass, as the air of a room or the water in a boiler, becomes hot by the actual circulation of the heated portions or particles. Very ancient peoples made use of convection, as is evident from their practice of putting hot stones into water contained in vessels of bark or skin. To-day the fact is utilized in hot-water systems, in steam and hot-water heating, as well as in hot-air heating.

Exercise. - In the diagram of Fig. 32, identify the cold water supply pipe, the course of water to and from the range, and the hot-water delivery pipe leading to laundry, kitchen, and bath. Explain how the water is heated;

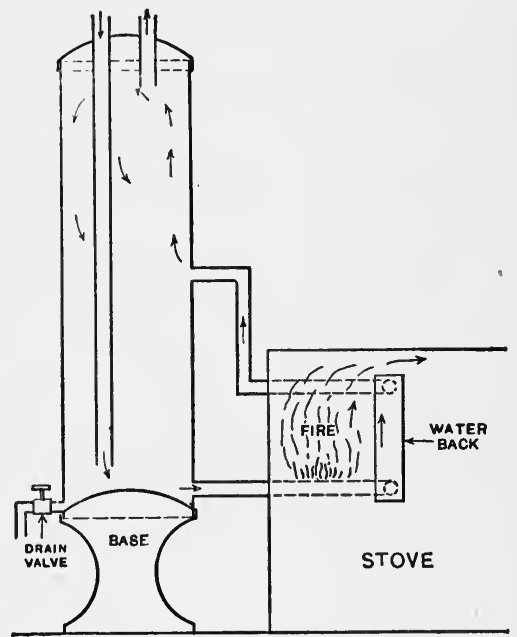

Fig. 32. - Water heater and hot-water tank. Trace the course of the hot and cold water through the tank and the water-back. how circulation is brought about and the water in the tank heated. Apply the facts that have been brought out experimentally.

Exercise. - The use of hot water for heating buildings is becoming very common. The accompanying diagram (Fig. 33), presents the chief features of such a system. Trace the course of cold and hot water in the system. Explain why the water circulates in the pipes and boiler. What is the use of the "expansion tank"? Of the overflow pipe? Why are these above the rest of the system? Mention other familiar uses of water as a medium for the transfer of heat, in the household and in manufacturing. 
Water has a property which makes it an excellent medium for heat transfer besides its differences in density due to unequal heating. Every one knows that a hot-water bag is superior to a hot flatiron as a foot warmer. Why this is so is not difficult to demonstrate.

Exercise: Relative heat capacity. - Place upon the top of a range where the heat is uniform equal weights of water, iron, and any other

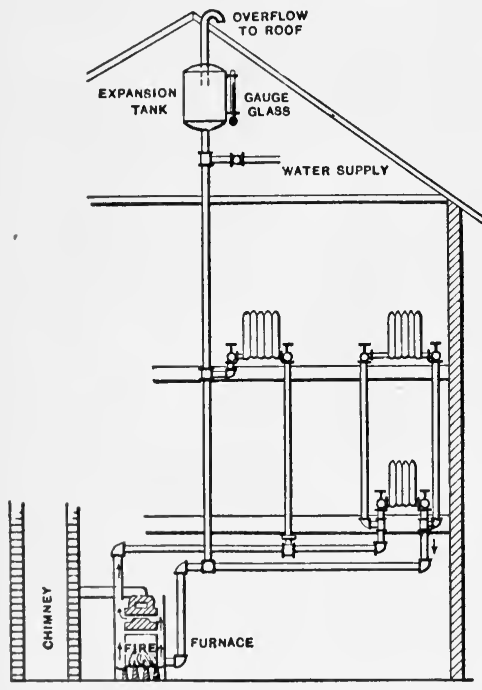

Fig. 33. - Trace the course of cold and hot water in the system. material that you may have convenient, as lead, aluminum, stone, or brick. It is soon apparent from the thermometer that the temperatures of the several substances change very unequally and at different rates of speed. The lead becomes hot very promptly, the iron more slowly, and water most slowly. The fact is that the amount of heat necessary to raise the temperature of a given weight of water (one kilogram or one pound) through one degree Centigrade or Fahrenheit will raise the temperature of an equal weight of aluminum about five degrees, of iron about ten degrees, of silver about twenty degrees, and of lead thirty degrees. Which of these substances takes and holds the largest amount of heat? Which has the lowest capacity for heat?

We must consider not only the capacity of a substance for heat, but also the way it gives up heat. A good medium for heat transfer should hold a great deal of heat and at the same time be capable of surrendering it freely to surrounding objects which are colder. Try this experiment to show the relation between heat capacity and heat delivery and the rank of water among other substances in this respect. 
Exercise. - Take equal weights, as one hundred grams, each of water, iron, lead, aluminum. Heat these in a vessel of boiling water until they have a temperature of $100^{\circ} \mathrm{C}$. Then put each into a vessel containing one hundred grams of water at zero Centigrade. Stir each mixture with a thermometer until it gives a stationary temperature for two or three readings. Assuming that the loss of heat in transfer and the heating of the vessel is negligible, the results will be approximately these: The one hundred grams of water at $100^{\circ} \mathrm{C}$. raises an equal mass of water from zero to $50^{\circ} \mathrm{C}$., that is, the quantity of heat given up by hot water is approximately equal to the quantity of heat taken up by the cold water. The results for the other substances are quite different.

The mass of lead, one hundred grams, at the temperature of $100^{\circ} \mathrm{C}$., raises one hundred grams of water from $0^{\circ} \mathrm{C}$. through about $3^{\circ}$. The heat given up by the lead in eooling from $100^{\circ} \mathrm{C}$. to $3^{\circ} \mathrm{C}$, , or about $97^{\circ}$, is just enough to raise the temperature of the water $3^{\circ}$. The same weight of aluminum raises the temperature of the water about $17.6^{\circ} \mathrm{C}$., or nearly six times as many degrees as the lead. One hundred grams of iron effects a rise of temperature in the water of about $11.3^{\circ} \mathrm{C}$, or just about one half as many degrees as effected by the aluminum. This is a point worth noting regarding aluminum cooking utensils.

From these facts it is evident that water is five times better as a receiver and deliverer of heat than aluminum, the best of the substances considered, and is many times superior to the others. Indeed scientists have proved that water, weight for weight, takes up a larger amount of heat, holds it better, and gives it up more gradually and in far greater amount, than any other of the ordinary substances. Because of these properties water is not only useful as a medium of heat transfer in warming our homes and public buildings, but is also selected as the standard for measurement of heat capacities of all known substances.

Exercise. - Compare the advantages of hot-water heating with those of the hot-air furnace and steam; as to cleanliness, ease of control, quietness of operation, fire risk, maintenance of uniform temperature, and influence upon the humidity of the air of the room or building. 
Convection currents of the air and the ocean. - The distribution of heat or cold by convection is familiar on a large scale in the local winds, in the great trades and prevailing westerlies, called the planetary winds (Fig. 34), as well as in the ocean currents. Many have experienced the hot winds that occasionally sweep across the prairie region of the central United States, or the cold winds that descend from high mountains. These are typical cases of convection currents. Then, as you know from your study of geography, the Japan

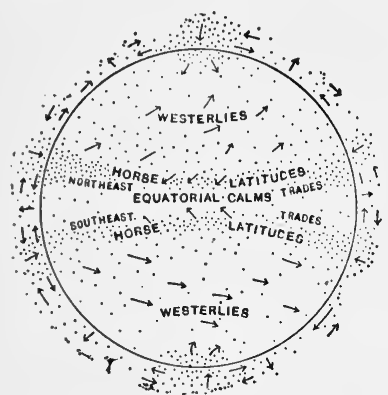

Frg. 34. - Diagram of planetary winds, showing belts of high and low pressure, and upper air currents. and the Gulf streams, the Labrador and other cold streams are examples of convection currents in water.

Ventilation. - Heating is closely related to ventilation, for both depend upon convection and diffusion. It is generally recognized that health and working efficiency depend largely upon the condition of the air which is breathed and which bathes the body continuously. Air that dries out rapidly the bodily tissues, as in nose and throat, or is excessively hot, dead, and of disagreeable odor, is not healthful, and does not permit us to make the best use of our physical and mental energies. We need air of a proper temperature, with adequate moisture, and moving sufficiently to bathe our bodies continuously but without draft. The actual amount of air supplied for each person, which is often stated to be about fifty cubic feet per minute, is not nearly so important as that the air be live or moving, and have the other qualities just given.

Exercise : Ventilating systems. - Make a study of any ventilating system. How is fresh air supplied, by force fans, windows, or other openings? Are the openings arranged with reference to the heat supply, such as radiators? Or is the air circulated at the temperature it has outdoors? Is the air of the room live, that is, actually circulating 
in the breathing space? How is this secured? Often one may find a layer of dead air, cold and ill-smelling, resting upon the floor and reaching possibly to the height of three feet or more. Hot air, when admitted through vents a few feet below the ceiling, remains at about the same level, and when the windows are open at the top, it escapes. If the windows are opened at the bottom, the cold, fresh air sinks to the floor. Warm and cold air should diffuse or mix. How can this be brought about?

The humidity of the air of a room may be determined by the aid of the wet- and dry-bulb thermometers (page 46). Ordinarily the effect upon the mucous membrane of the nose and throat is fairly indicative of the humidity. If a hot-air furnace is utilized for heating, is it equipped with a water pan for making humid the heated air? What other provisions do you know for supplying the air with sufficient moisture?

What should be the temperature of a room in which physical work is performed? Mental work? How is the temperature of the room or building regulated, that is, does it depend upon human control or a mechanical device? The instrument in common use for this purpose is the thermostat. This depends upon the expansion and contraction of metals under the influence of heat and cold, and is so adjusted that a variation of about two degrees will cause the heat supply to be turned on or shut off.

43. Radiation. - Heat from range or fireplace is transmitted to objects in a different way from the two which have been studied. As you sit in front of the fireplace your face and hands may almost blister with the heat, and your back be freezing. The air between you and the fire is decidedly cooler than your face and hands. How can you verify this? Objects below the level of the fire and sufficiently near to it are warmed and sometimes burned by the excessive heat. But a screen placed between the fire and the objects cuts off the heat supply, as you know, for you sometimes protect your face by means of a paper or book. If the air were moving from the fire toward you, it might bring heat to you by convection; but convec- 
tion currents rise, and the air may be absolutely still. The only means of conduction in this case is the air.

How the sun's energy reaches the earth. - Let us consider this matter with reference to the way in which the sun's energy is transmitted to our earth. Objects upon which the sun shines are warmed. But is the air warmed through which the sunlight passes? If the air transmits sunlight by conduction, it must be warmed to at least the same degree as are the objects upon the earth. Experience, however, affords many facts which indicate that this is not the case. On the desert when the sun -shines directly upon the sand, the reflected heat from the sand is intense. But the air is comparatively cool, as one realizes on entering a shaded place. Mountain climbers and explorers in the high latitude of the Polar regions quickly learn that the sun's rays are cruelly hot, blistering face and hands when exposed to them directly; but the air may be at a temperature very many degrees below zero.

Aëronauts ascending in balloons about seven miles find the cold unendurable. Registering thermometers and barometers sent far higher in experimental balloons furnish evidence that the temperature rapidly becomes lower with increasing altitude, and the air itself exceedingly rare. The atmosphere does not extend above the surface of the earth more than two hundred miles (see pages 12 and $391 \mathrm{ff}$. .).

These facts seem to prove that the air cannot be a conductor of the sun's energy to the earth, any more than it can be a conductor of heat from range or fireplace to adjacent objects. The air does not reach far enough toward the sun to be a conductor.

In fact air is one of the worst conductors of heat. It is only one twenty-fifth as good a conductor as water, and water is about one hundred times worse than iron. Evidently the sun's energy is not conducted by the air, but transmitted by a third method.

Glass is a better conductor than air or even water. The sun shines through glass windows and warms objects upon which 
its rays fall. Is the glass as warm as the objects in the direct sunlight? If glass conducts the sun's energy, it must be as warm or even warmer than the objects. But you find that glass is only slightly warmed by the transmitted rays. With the aid of a reading glass start a fire in some paper or burn a hole through a piece of cloth. When the substance is smoking hot, feel the glass. Is it as hot as the burning substance? Is it much hotter than the air? Plainly, glass is a good medium for the transmission of sunshine, absorbing and converting into heat only a very small amount. Observe its use in hothouse or sun parlor. Suppose the windows were made of thin sheets of copper instead of glass. Would the room be warmed by the sun when shining directly upon such "windows"? Do you think it would be possible to keep the room warm with heat from fuel on a cold day? Does it seem that the sun's energy can be transmitted by conduction through the air any more than through glass?

Exercise: Third way of heat transference. - The principal points may be brought out clearly by observations on an incandescent lamp. Observe that the hot filament of a tungsten or carbon lamp throws out some heat. This cannot be transmitted entirely by conduction, for the vacuum or inert gas within the bulb and the glass are poor conductors. Clasp the bulb with the hand and turn on the electric current. Is the heat instantly sensible? Turn off the current. Does the heat diminish gradually as it does in the case of cooling iron or water? Repeat this until the facts are clearly grasped, and you have proved whether or not convection or conduction accounts for the heat transfer.

An iron ball, three to five inches in diameter, with a hook or knob to which a wire may be attached, is heated until it is practically red hot; then hung by the wire in about the center of the room and half way from the ceiling to the floor. Now find by trials in what directions and at what distances you ean feel with your hand the heat radiating from the ball. Is the heat thrown out in all directions, downward as well as upward? Do convection eurrents move downward? Do they move upward? Interpose between your hand and the hot ball successively a sheet of paper, a piece of glass, and a sheet of asbestos paper. What happens? With a small mirror see if you 
can deflect the heat from the ball to your hand held to one side. Does the mirror feel as warm to the touch as your hand does when receiving the reflected heat or when held in the same place as the mirror?

Give reasons for the fact that the heat from the hot ball is not transferred by conduction or convection. In what direction do convection currents usually move? Is there any possible medium for conduction?

In what sort of path do the heat rays move? Can they be deflected by a mirror or bright smooth metal surface? In what kind of path ds they move after being deflected? Can you determine the angle of reflection of such deflected rays?

Do the sun's rays follow the same sort of path as that of heat from the hot ball? With a mirror reflect the sun's rays to' one side upon your hand. Do you feel the heat thus reflected as strongly as when the hand is directly exposed to the sun?

Meaning of radiation. - Evidently heat energy travels from one place to another by a method that is different from conduction and convection. It moves along straight lines and does not appreciably heat the medium through which it is transmitted. These two facts distinguish sharply this method of transfer from the other two. This third way is generally known as radiation.

Exercise. - Test your knowledge of radiation as a method of transfer of heat energy by the following questions. How could you locate without touching it, a hot but utterly black stove which has no light or other qualities to reveal it to hearing, smell, or sight? What fact enables you to define its location? Has the path of the heat rays anything to do with your locating the stove? Has the heated part of the air anything to do with it? Suppose the heat is deflected by a mirror or other reflector, can you then locate the stove without approaching it closely? If you know the angle of the reflected heat rays, can you locate the stove?

Place a thermometer eighteen inches from the center of the flame of an alcohol burner and on the level with it; and another an equal dis-॰ tance directly above the flame. Which heats up more quickly? Which records the greater temperature? How is heat energy transferred in each ease?

Radiant energy. - The facts of observation emphasized by the experiments enable one to understand the theory of radia- 
tion and radiant energy held by scientists to-day. In the first place, scientists prefer to speak not of the "heat," but of the "radiant energy" of the sun. For heat is only one effect of radiant energy; another effect is light. Every body whether stick, stone, metal, plant, or animal, which has exposed surfaces, is radiating or giving up energy. The lower the temperature of the body, the less is the amount of energy it radiates. The higher its temperature, the greater the amount of energy radiated. But radiation occurs as long as the temperature of a body is above what is known as absolute zero, $-273^{\circ} \mathrm{C}$.

In the second place, every body, according to this theory, is composed of countless exceedingly minute particles, called molecules. The molecules are unceasingly active; their activity varies, however, according to the temperature of the body. In a piece of cold iron, for example, the activity of the molecules is very slow in comparison with that of the molecules in a piece of red-hot iron; while in a mass of molten iron the molecular activity is wonderfully increased above the latter. The activity of the molecules of a body increases with rise in the temperature of the body.

Now whenever a body is warmer than any surrounding objects, or whenever its molecular vibrations are more rapid than those of the surrounding objects, this body is said to radiate or give up energy more rapidly and freely than the other objects. The radiant energy given up always moves in straight lines. This your experiments make quite clear. If the energy is deflected from its original path, its direction is then determined by the angle of the reflecting surface with the direction of the radiant energy before being deflected. This you know by your experiments in reflecting sunlight upon objects at a distance and in changing the direction of the heat from the iron ball.

The ether as the medium of transfer. - Radiant energy is transmitted by radiation and through a medium which science 
assumes as pervading the whole universe of space and matter. This medium is called the ether. The ether envelops and permeates all things; it fills the infinite space between the heavenly bodies of the universe and the most minute spaces between the molecules of all solids, liquids, and gases. It is supposed to be rigid and at the same time elastic, offering no resistance to bodies passing through it. Because of these properties it transmits radiant energy in all directions and in straight lines by radiation or in the form of waves. It is the medium of transfer of radiant energy. Through this medium the sun's energy passes with a velocity of about 187,000 miles a second, but the medium itself is not heated.

Effects of radiant energy. - The molecular vibrations of a body, such as of the sun or a hot range, are communicated to the all-pervading ether, causing it to vibrate. When the vibrations radiating in all directions along straight paths fall upon a body, they promote or accelerate its molecular activity, that is, they heat the body. As the body's molecular vibrations become more rapid and violent, its molecules are farther and farther repelled by and from one another; this appears to us as the expansion of the body. Air, as you know, expands with rising temperature. All other gases, liquids, and solids behave in a similar way.

When a body, on the contrary, is no longer exposed to the vibrations from a source of radiant energy, as the sun, it gives up or radiates its own energy more rapidly than it receives energy. Its molecular vibrations diminish, the paths of the molecules become slower and shorter, or, as we commonly express it, the body contracts. Contraction with the lowering of temperature is characteristic of all substances except water.

Heat is only one result of radiant energy. Radiant energy of the sun or any other source, such as a lamp, has different effects as determined by the nature of the body or the organ upon which it falls. The sun's energy falling upon the earth 
and the objects on the earth is absorbed and converted into heat so long as the vibrations do not exceed four hundred billion a second. When the rate is between four hundred billion and eight hundred billion vibrations a second and these fall upon the eye, the organ of sight, they are converted into light. Both heat and light must therefore be considered as effects of radiant energy.

Other effects may be mentioned. Radiant energy, when it falls upon certain substances, induces in them chemical changes and is in this respect known as chemical energy. This effect is familiar in photography, for it is this sort of energy which produces the changes on the exposed plate or film in the camera. In other substances or conditions it causes electrical changes, and is described as electrical energy. Because of the different effects which the sun's energy produces in different kinds or qualities of bodies, it can be correctly spoken of, not as heat or light, but as radiant energy.

Color and surface in absorption and radiation. - Besides the different heat capacities of familiar substances, common experience furnishes many instances where the color and the character of the surface of objects affect the capacity for absorption of heat energy. Snow sprinkled with soot or ashes melts more rapidly than snow which is clean and white. A square of black cloth and another of white cloth of the same material and size, spread over snow so as to receive equally the sun's rays, show very different results in a short time. Under the white square the snow melts very slowly, and more rapidly under the black, which seems to mean that the black absorbs and transmits more heat energy than the white. The importance of color in heat absorption and radiation or transmission may be shown in several ways.

Exercise : Radiating surfaces. - Get two bright tin cans of equal capacity, which may be closed tightly with stoppers. Fit into each stopper a thermometer. Blacken one can with lampblack by holding it over a kerosene or gas flame. Fill both eans with water of the same 
temperature as it comes from the faucet, close them with the stoppers, and hang by a cord or wire in direct sunlight. Take thermometer readings at intervals of ten minutes. In which can does the water heat more rapidly? At the end of an hour which shows the higher temperature? Which color is thus shown to be the better absorber of heat energy?

The effect of color and bright surfaces upon radiation, or the giving up of heat, may be determined by filling the cans with hot water of the same temperature and hanging them in a cool place. Which radiates heat more rapidly? At the end of a given period of time which is the cooler?

Objects that are black and rough are good absorbers of heat energy ; but they also give up or radiate their heat very rapidly. Smooth, bright, or highly polished articles reject or reflect a large proportion of heat energy and absorb it slowly; but when they are heated, they radiate heat much more slowly than objects of the other class. A bright stew pan absorbs heat more slowly than a black iron pan. But when the bright pan is hot, it retains its heat better than the iron utensil. Explain why a reflector, such as that used for a lamp, which is exposed to a large part of the heat energy of the light, does not become as hot as the lamp chimney or other objects as near.

Hence, it may be stated that ready absorbers are ready radiators, that is, they give up heat rapidly; and slow absorbers are slow radiators, that is, they retain their heat well. Explain the use of aluminum cooking utensils on the basis of these facts.

Exercise. - With these points in mind, explain whether lightcolored or dark-colored clothing is better for hot weather wear. Compare the advantages of light-colored clothing with those of dark colors for winter weather. Explain the fact that animals in the Aretic regions are usually covered with white fur. Is absorption or radiation of heat more important in this instance? May the white have other advantages?

How does knowledge of the facts studied help in understanding such features of climate and weather as the following: the difference between inland and seacoast climate; the differences in temperature of land and ocean, of land and sea breezes, and of mountain and valley breezes? If it is helpful in explaining winds, in what way does it account for the distribution of water vapor and rainfall? Upon what facts do ocean currents depend? 


\section{SUMMARY}

Three ways of transferring heat energy are conduction, convection, and radiation.

A conductor of heat is a substance which readily transmits heat from one part of itself to another.

Metals are the best conductors, liquids are poor, and gases poorest. The warmth of clothing depends mainly on the inclosed air spaces.

Convection, or the conveying of heat by the movement of the hot body, is illustrated by the rising of hot air from the stove and the movement of heated water in a hot-water system.

A given weight of water absorbs or gives out more heat for a change of one degree in temperature than any other common substance.

Water is taken as the standard for heat capacity.

The winds are convection currents.

A knowledge of convection is useful in controlling ventilation.

Radiant energy from the sun comes to us through cold space.

Good absorbers are generally good radiators.

\section{REVIEW QUESTIONS}

1. In how many ways is heat transferred? 2. What is the necessary condition of heat transfer by conduction? 3. Give the explanation of the process of conduction. 4. How would you show the different conductivities of familiar metals? 5. How would you prove that water is a poor conductor? 6. What are the best known conductors? 7. To what class of substances do they belong? 8. What is the best known conductor? 9. What is the poorest conductor named in the list? 10. Give reasons to show that liquids and gases are not heated entirely by conduction. 11. What use is made of our knowledge of the heat conductivity of materials used for clothing? 12. Explain why woolens, fur, feathers, and eiderdown are poor conductors. 13. What use is made of the knowledge of conductivity of different substances in the construction of buildings? 14. Give examples of the use of poor conducting materials in retaining heat or cold in cooking or preserving food. 15. Why do some substances, like wool, feel warm and others, like marble, feel cold to the touch?

16. Explain why air when warmed by the heat from a stove moves away from the heat source to other parts of the room. 17. How can you show such circulation of heated air? 18. What use is made of the transfer of heat by air? 19. Trace the course of air from coldair box to furnace, and thence to rooms and its circulation in a room. 
20. What substances besides gases transfer heat by convection? 21. How would you show that water transfers heat by convection? 22. What use is made of water in conveying heat from one place to another? 23. Describe how you would show water's capacity for heat in comparison with that of other materials. 24. How does the heat capacity of water make it especially useful in heating by convection? 25. Why is water taken as the standard in measuring the heat capacity of different substances? 26. Give instances of convection currents that are important in climate.

27. How is ventilation related to heating by convection? 28. What kind of air should we have in our homes, schools, churches, and other buildings to insure us good health and vigor? 29. What means are used to supply fresh air in our homes and public buildings? 30. How may the humidity of the warmed air be regulated? 31 . How is temperature regulated?

32. Give facts which prove that one sitting before a fire may be warmed by a way of heat transfer which is neither conduction nor convection. 33. Give facts showing that the sun's energy does not warm the earth by conduction or convection. 34. What does the reading glass show? 35 . What direction do heat rays follow? 36. How can you prove this? 37. In what conditions does a body radiate energy? 38. Explain how energy is radiated by a body. 39. What medium transmits radiant energy? 40. How is a body heated by radiation? 41. What effect does a rise in temperature have upon a body? 42. What happens to a body in giving off heat? 43. What other effects does radiant energy produce besides heat? 44. How would you show that the color and character of surface of a body affect its capacity for absorbing heat? 45. For retaining heat? 46. Of what use is the knowledge of this in selecting cooking utensils? In selecting fabries for clothing?

\section{SUGGESTIVE QUESTIONS}

1. What sort of material, wood, stone, or iron, would you select in building a house for comfort in a hot climate? In a cold climate? Explain. 2. What advantages and disadvantages would a heating stove made of white tile have? Why? 3. Why is the air in the gallery of a theater more uncomfortable in hot weather than that of the main floor? 4. Would a hothouse made with oiled paper instead of glass be satisfactory? Explain. 5. How could a house be safely heated in cold weather by conduction? 6. Suppose heat were not transferred by convection in air and water. What would be the chief climatic 
changes in your own locality? In the Pacific Northwest? In the Mississippi valley region? 7. What do you imagine would be the effect upon agriculture in these regions? 8. You may shape a piece of clear ice like a reading glass and the sun's rays passing through it will heat one's hand very noticeably before the ice shows much sign of melting. Explain.

\section{Products and Materials of Combustion}

44. Oxygen in all products of combustion. - It has been learned that when such metals as lead and iron or such substances as phosphorus and sulfur are burned or oxidized, new substances called compounds are produced. Because of the presence of oxygen in these compounds or products of combustion, they are called oxids, such as iron oxid, lead oxid, sulfur dioxid and so on. You will find this to be true of any product of combustion, that is, it is an oxid or contains oxygen no matter what the other elements may be. We have now to try to capture the products of burning some common substances and learn from them what they are and from what substances besides oxygen they are produced.

We may take the candle as a typical instance of burning materials, a kind of miniature gas plant. A candle is composed of cotton wick and some combustible material, such as tallow, paraffin, or the like. Heat melts the substance which then gathers in a cup-like depression around the wick and passes up through the wick in a stream. Near the top the liquid is changed by heat to a gas which burns. Flame is always a burning gas. If the material be wood or coal, it must none the less be changed into gas to produce a flame. If you watch burning wood or coals, you may see jets of gas pouring out, which finally ignite and burn as flame. When all the gas burns off, or, as we say, when the wood or coal burns to "coals," there is no more flame, just a glowing mass of coals.

Extinguish the flame of a candle and observe that the melted substance around the wick becomes solid just as it was before 
melting. But the part of the candle which has been burned does not become solid. What has become of it? It is commonly said that it has been burned. The candle as a candle ccrtainly does not exist after burning, but the substances of which it is composed are by no means lost. A burning candle produces light and heat, which may be measured exactly; but with these we are not now concerned. The products of burning are what we are interested in; and these we must seek in different ways.

45. Is water a product of combustion? - You have often observed water forming on the bottom of a pan placed over the fire, and perhaps wondered how it came there. Usually the fact is dismissed with the assumption that it must have been on the outside of the pan beforehand. But it is worth investigating to find out whether or not this assumption is correct. A simple way of testing it is this :

Exercise. - Hold a test tube which you have made perfectly dry outside, and which is nearly full of cold water, about two inches above the burning candle, or at such height as will avoid sooting. What appears on the outside of the tube? Can you identify it by touch or taste? If you should weigh the tube and its conțents before and after this experiment, you would find that the weight is increased by the amount of moisture formed on the outside. Where does it come from? Certainly not from the water in the tube. Can it come from the candle? There is no visible sign of water in the candle. May there be some substance in the eandle, which in the form of gas combines with oxygen to produce this moisture?

Moisture, which seems to be one product of the candle burning in the air, may be peculiar to the candle. Try the same experiment with any kind of flame available, as gasoline, kerosene, alcohol flame, or illuminating gas which certainly contains no water. It would hardly be possible to mix these with water and produce a useful flame. You discover by trial that each of them produces moisture which in sufficient quantity may be identified as water.

Composition of water. - It is necessary to find out of what water is composed, so that we may know what in the burning candle unites with oxygen to form water. 
The study of water may be carried on in several ways. Two are suggested here. The first is strikingly brilliant, and may be done accurately with the aid of a delicate balance. The experiment is instructive also without weighing.

Exercise. - Cut off and, if practicable, weigh accurately a small piece of magnesium ribbon. Burn it in the air, on a piece of asbestos paper, and weigh the product. Oxygen in the air unites, as you know, with burning substances and forms oxids. In this instance the product of combustion is magnesium oxid. The additional weight shows the quantity of oxygen combined with it in burning. But the principal point is that oxygen is indispensable in burning magnesium.

Fill a beaker about two thirds full of water, and cover closely with a heavy piece of asbestos paper, through which is made a slit just large enough to admit the magnesium ribbon. Boil the water until the air is expelled from the beaker by the steam, and then insert the ribbon which you have lighted by the fire of the burner. The magnesium ribbon continues to burn just as freely as it did in the air. The product is identical with the product of magnesium burned in air, that is, magnesium oxid. Where is the oxygen necessary for burning obtained? What conclusion can you draw as to one of the constituents of water?

Another interesting phenomenon may be observed if the room is darkened during the experiment. Above the asbestos and about the slit is a flame, which evidently is not altogether due to the burning magnesium. The magnesium is deep in the steam and above it is a space through which no flame is carried. But outside is this ghost of a flame. Now if one substance contained in water is oxygen, as your observation shows, another substance seems to burn when it comes into contact with the oxygen in the outer air. The union of the oxygen from water with the burning magnesium must have set free the other substance, or substances, which are apparently combustible.

Caution. - The intense brilliancy of the flame makes it necessary to wear blue glasses to protect the eyes. To see the flame burning above the slit, hold a strip of eardboard between the magnesium and the eyes.

Exercise: The decomposition of water. - We may analyze water into its constituents by passing through it an electric current, a process known as electrolysis (electro-lysis, meaning to loosen or dissolve by electricity). A comparatively simple apparatus can be made. The materials needed are a granite or porcelain dish about six inches 
in diameter and four inches deep; copper wire well insulated and heavily paraffined; two short pieces of carbon, about the size of a pencil, such as used in an electric arc lamp; three or four dry cells; about one ounce of sulfuric acid mixed with about two pints of clean water; two large test tubes.

Attach each piece of carbon to one end of the wires, by wrapping tightly, and arrange in the dish about two and one half inches apart (Fig. 35). Holding carbons upright, pour melted paraffin into the dish to a depth of at least half an inch. When the paraffin is hard, cut two or three ditches in the paraffin about one fourth of an inch deep and radiating from the carbons. These should be an inch or two long to provide free exit for water in the tubes. Connect the

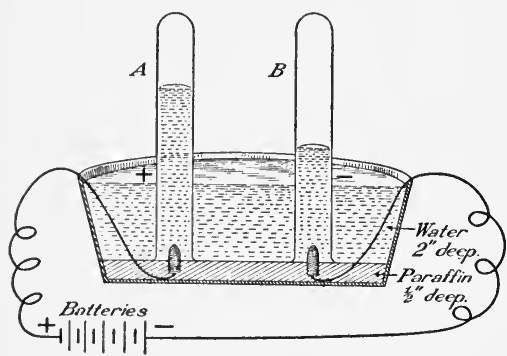

FIG. 35. - Simple apparatus for decomposing water into its elements by electricity. other ends of the wires to the batteries in series. Pour into the dish a solution of water 30 parts and sulfuric acid one part to the depth of about two inches. Fill the test tubes with the mixture and invert over the carbons. If the lighting system furnishes direct current of 110 volts, this may be used with a 16 candle power lamp in series to give resistance. This current will give results more quickly.

The effect of the electric current in the water is soon evident from the small bubbles which, passing from the surface of the carbons to the upper parts of the test tubes, force the water in them downward and out. This process may be continued indefinitely with merely the renewal of the supply of water, the acid remaining undiminished. What conclusion can you draw from this as to the source of the gases?

Observe that one tube is filled nearly twice as rapidly as the other. By means of exact and delicate apparatus it can be demonstrated that the gases combine exactly two parts by volume of one to one part of the other in forming water. In your experiment the results showing this may be only approximate because of the partiäl absorption of the gases by the water; but they are sufficiently reliable for all practical purposes.

Remove the tube which is filled first, holding it in the inverted position. Place over the carbon another tube prepared as before described. Into the tube you have taken from the dish, insert a 
glowing splinter. It seems to be like nitrogen, for it extinguishes the fire, and it is certainly not like oxygen, which would have produced a burst of flame. Now introduce a burning splinter or a candle. Do not be alarmed if a slight explosion occurs. Hold the tube securely, always inverted, and observe closely what happens. The flame of the eandle is extinguished; but unlike nitrogen, the substance itself burns with a pale bluish flame that runs upward into the tube. Note the heat produced. After the burning ceases, examine the inside of the tube. Is it moist? Does the moisture tend to form drops of water?

Oxygen, you have learned, is indispensable to combustion, and it must unite with the burning substance in the tube; their product is apparently moisture or water. Before you draw a final conclusion, examine the gas collected in the other tube. Remove, close immediately with your thumb or a stopper, and invert it to upright position. Insert a glowing splinter. What is the result? Repeat this until the reaction fails. What substance is this?

Apparently water can be decomposed into two constituents, one of which is oxygen, as shown by its support of combustion, and the other a gas which does not sustain combustion, but is itself combustible, burning with a pale bluish flame and much heat. If all these points are not clear, repeat the tests with the second tube of substance collected.

Inflammable air or hydrogen. - It is interesting to find that Henry Cavendish (1731-1810), an English investigator, discovered this combustible gas and carefully studied its behavior.

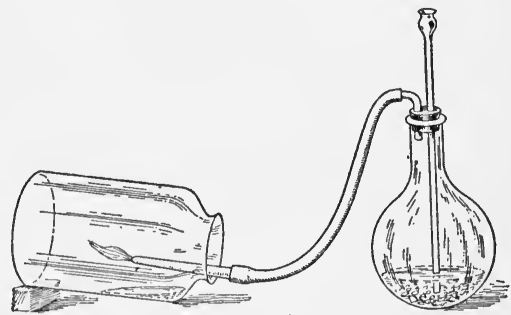

FIG. 36. - The product of hydrogen burning with air. Enough may be obtained in this way to test for its properties.

Having learned the properties which your tests make clear, he called it "inflammable air." He proved that when it burned with the oxygen of the air in a perfectly dry bottle (Fig. 36), the only product of the combustion was moisture, which condensed into small drops of water. The question " by what substances 
is water formed?" he answered thus: "water is formed by the union in combustion of oxygen and "inflammable air." "

This name for the substance is not convenient and falls short also of indicating its rôle in water and in other compounds. Because it is one of the chief constituents of water, and because when burned in oxygen either pure or as present in the air, it produces only water, it was named by Lavoisier "waterformer " or hydrogen.

We learn from our study that at least one of the products of the candle burning in air is water. The analysis of water by the electric current makes it certain that water is composed of at least two substances, oxygen and hydrogen. Thus the circle seems to be complete. Hydrogen, when burned either in pure oxygen or in the oxygen contained in the air, produces water, and water is decomposed by a certain amount of electricity, which is the equivalent of a certain amount of heat, into hydrogen and oxygen.

The production of hydrogen. - Water is clearly one of the products of the burning candle. The technical name of water is hydrogen oxid, showing that it is a compound of hydrogen and oxygen. Before we seek the source of hydrogen in the burning candle, we may advantageously study more fully the nature, behavior, and principal compounds of hydrogen.

From water. - Hydrogen is abundant in water, existing in the ratio of two volumes to one of oxygen. It can be liberated from water by electrolysis, as you have learned; but it may be set free more rapidly by certain metals, such as magnesium, calcium, sodium, iron, and zinc. Iron and zinc require high temperatures. Magnesium acts, as you have observed, in steam, separating oxygen and hydrogen and liberating the latter. Sodium reacts too violently for safety. Calcium liberates hydrogen in a safe but also rapid manner. A quantity of hydrogen may be obtained by holding a bottle filled with water over a small lump of the metal calcium placed in a vessel 
of water. The ascending gas displaces the water and occupies the space in the bottle.

Another way of getting hydrogen, and one which shows at the same time the wonderful attraction of metals for oxygen, is to pass steam over red-hot iron fragments. The steam is broken down, the oxygen uniting with the hot iron, and the hydrogen passes off. A similar reaction occurs in passing steam through white-hot coal or carbon in the manufacture of "water gas" (page 162). Water gas burns with a flame like that of hydrogen, or is almost non-luminous.

From acids. - A very common laboratory method of obtaining hydrogen with the apparatus shown in Fig. 37 is the following. Put a small quantity of granulated or moss zine into the flask and supply through the funnel tube small amounts of dilute sulfuric acid. The generated gas is conducted through water and is collected in bottles over water. If air is mixed with the gas, a more or less dangerous explosion is certain. Students should exercise care, and the instructor should supervise the experiment closely. When the hydrogen is coming freely from the end of the rubber tubing, collect four or

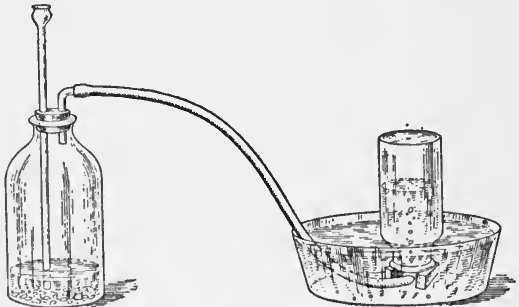

Fig. 37. - Apparatus for making and collecting hydrogen. The hydrogen of the sulfuric acid (hydrogen sulfate) is replaced by zinc.

more bottles of the gas. Slip a piece of glass under the mouth of each bottle as it is filled; lift the bottle vertically and set it away in an inverted position.

The reason for using zinc and sulfuric acid in generating hydrogen is that zinc, or any other of a whole series of metals, enters into an interesting combination or exchange with sulfuric or certain other acids. Zinc is a simple substance, or an element. Sulfuric acid is a compound produced from sulfur, oxygen, and hydrogen. When the acid is brought into contact with zinc, it begins at once to exchange hydrogen for the zinc, 
with the result that the hydrogen is set free. Hydrochloric acid makes the same sort of exchange.

Exercise: To show properties and behavior of hydrogen.- (1) Into one bottle, held inverted, thrust a glowing, but not burning candle. After you note the reaction, introduce a burning candle, which, securely attached to a wire six inches long, should reach nearly to the bottom of the bottle. The fire of the candle is extinguished, but the gas in the bottle ignites. Withdraw the candle slowly, and it is ignited by the burning gas. Does the flame of the gas show what sort of gas it is? Hydrogen when not mixed with oxygen ignites without explosion.

(2) Place a bottle upright on the table, open it for a moment, and then throw a burning match into it. What happens? What does the result indicate as to the cause of the difference in reaction from that in the former test? Why does leaving the bottle uncovered in an upright position make this difference in the contents of the bottle? Test your answer to the question by the following:

(3) Hold a perfectly dry bottle mouth downward and directly over the mouth of a bottle containing hydrogen. After a minute or so, cover both bottles and stand in upright position. Drop into each a burning match. What does the result show as to the content of the bottle previously filled with air? What as regards that filled with hydrogen?

When air and hydrogen mix in the bottles, the product is said to be a mixture. But when oxygen and hydrogen unite in combustion, the product, which is water, is said to be a compound. Are hydrogen and oxygen more nearly like the separate gases when they form a mixture or when they form a compound? You will find it helpful to remember this distinction between mixture and compound.

Does this test indicate which of the two, hydrogen or air, is the heavier? What does the method of transferring hydrogen from one bottle to another indicate as to its density in comparison with that of air?

(4) Test with burning candle to assure yourself that hydrogen ignites without explosion when unmixed with oxygen, and burns with a pale, bluish, almost invisible, but intensely hot flame. Observe especially the heat produced.

Summary of properties. - Hydrogen is invisible, and has neither taste nor odor. When you pour hydrogen from one vessel into another, you must pour it upward as in a sort of "looking-glass world." This indicates that it is lighter than 
air. It is not only lighter than air, but it has less density than any other known substance. Because of this fact it is taken as the standard of density for all gases. These are what we consider its physical properties, that is, properties which appeal to our senses.

The wonderful lightness of hydrogen is the reason for its use especially in experimental balloons which ascend to high altitudes. It is, however, expensive to produce and tends to leak through silk. Consequently illuminating gas, which is cheaper and does not escape rapidly through silk, is generally used in ordinary airships and balloons.

Hydrogen has other properties, as your experiments show. It unites very freely with oxygen. When mixed with oxygen and exposed to flame its union is sudden and explosive. Although it does not support combustion, it is itself combustible, burning in oxygen with a pale, bluish, intensely hot flame. This indicates that hydrogen is very active. The heat which it produces is so intense that in the oxy-hydrogen flame it readily cuts, as if with an extremely sharp knife, a steel bar two feet thick. A given weight of hydrogen burning in oxygen produces a greater amount of heat than an equal weight of any other two known substances. Its heat value (page 143) is more than four times that of pure carbon. The product of burning hydrogen is water. And the heat evolved by a given weight of hydrogen combining with a given weight of oxygen is sufficient to raise through one Centigrade degree of temperature about four thousand times its own weight of water. To break water down into its constituents requires exactly as much heat as is produced by them in uniting. Because of its inflammable nature one might not expect to find hydrogen free in the air ; but it does exist in traces; in your experiments you probably allowed some to escape.

Hydrogen in compounds. - The importance of hydrogen in life may be more fully appreciated when one recognizes the large number of compounds in which it is an essential 
constituent. The most universal and abundant is water. Hydrogen forms about one ninth of the weight of water, or more exactly it is one part by weight to 7.94 parts by weight of oxygen. But volume for volume, hydrogen is only about one sixteenth as heavy as oxygen and about one fourteenth as heavy as air. The volume or bulk of hydrogen is twice that of oxygen in water.

Hydrogen dioxid is a familiar compound, of which much use is made for disinfectant, aseptic, and bleaching purposes, and in the restoration of old works of art. It is known commercially as hydrogen peroxid. It is a very unstable compound, which in decomposition readily liberates about one half of its oxygen, the rest being just water. Because of its ready release of oxygen it is useful in oxidizing dead and putrefying tissue, in destroying germs or infective substances. The solution which is common in the market does not usually have more than three per cent of the full strength of the dioxid.

Hydrogen combines with nitrogen to form ammonia, a compound which is noticeable by its odor where organic matter is putrefying. Ammonia, used in refrigeration and ice-manufacturing, is produced in commercial quantities from bituminous coal as a by-product of coal gas and coke manufacture (page 163).

Hydrogen is an essential constituent in all acids, of which vinegar is a type, and in all substances which neutralize acids, of which ammonia water, caustic soda, and caustic potash or lye are examples. When to these compounds all fuels, except pure carbon, and all foods are added, in which hydrogen is an essential, the importance of the substance in the things upon which life depends is made strikingly evident.

We have now to return to the inquiry as to the source of the substances which combine in the burning candle to form water. Water is composed of oxygen and hydrogen, as the study of the composition of water revealed: Oxygen clearly is taken from the surrounding air, but hydrogen must be derived from 
another source, since it does not exist free in the air in quantities sufficient to account for the amount of water produced. We seem to be forced to conclude that hydrogen is a constituent of the substance of the candle. This does not seem improbable. The substance of the candle is melted and passing upward through the wick is changed by the heat into gases which burn. Hydrogen must be one of the gases thus produced, because water is one of the products of the burning candle.

Exercise: Testing for hydrogen. - Burn a lump of pure earbon, such as charcoal, in oxygen. Cool the test tube or bottle to condense any water vapor produced. Is moisture formed? If not, what does its absence indicate as to the substances formed? Was hydrogen present?

Test other familiar substances for hydrogen. Rendered beef suet, lard, tallow, or butter, may be made into small candles with wicks of cotton twine. If you test gas, alcohol, or kerosene as suggested on page 114, and find water as the product of their burning, you may conclude that they contain hydrogen.

46. Another product of combustion. - The flame of the candle gives considerable light. The flame of hydrogen is hot, but does not produce much light. It is in fact non-luminous. This leads us to inquire what in the substance of the candle is the cause of its light. We may find this by capturing and questioning the products of its burning. We must provide, however, a different condition from that which made the water vapor visible as moisture. We know of course that, whatever else any product of combustion may contain, it will always contain oxygen, or be an oxid compound. We may imagine also that, like water, it will not support combustion. These characteristics we may expect in any product of burning substances.

Limewater - an indicator of one product of combustion.The products of the burning candle are easily captured by holding a wide-mouth bottle directly over the flame. By cooling the bottle the water vapor is made visible. But it may be that 
other products remain invisible in spite of the cooling and may be made visible by some other condition. Now scientists have discovered that limewater renders visible another of the products of combustion. Pour a small quantity of limewater into the bottle and mix the contents by shaking. The limewater turns a milky white color. This proves that some product of combustion is present which is not made visible by cooling. That it is not the water vapor which has this effect, may be proved by mixing some clean water with limewater.

Exercise. - The problem may be simplified considerably by burning some pure carbon or charcoal in oxygen. Into a bottle full of oxygen first pour about two ounces of limewater, close the bottle with a stopper, and shake vigorously. Oxygen produces no change in the color of the water. Now introduce a lump of charcoal which has been heated to a bright glow. When the oxygen is consumed by the burning, close the bottle and shake. What change occurs in the color of the limewater? As neither oxygen nor the water vapor produced by hydrogen burning in oxygen has a similar effect, you are justified in concluding that it must be the product of the burning of carbon, which turns limewater milky white.

You have learned that pure carbon and oxygen combine in combustion to form a new compound which is an oxid compound. Now one of the best established facts of chemistry is that in ordinary conditions carbon completely burned unites in the ratio of one part to two parts of oxygen. This is what occurs in the present instance, and the product must therefore be carbon dioxid. Carbon dioxid is the only odorless, invisible gas which has this effect upon limewater, and this may, therefore, be taken as an infallible test for its presence.

Carbon in the candle. - Recall now the facts observed when you mixed limewater with the products of the burning candle. Evidently carbon must have been burned to produce the carbon dioxid, the presence of which was shown by the limewater test. The carbon can have in this case but one source, namely, the candle. It is clear, therefore, that the substance of the candle contains both hydrogen and carbon. 
The products of burning sulfur, phosphorus, iron, and whatever else you preserved in the bottles used in experiments with oxygen may be tested for carbon dioxid. What does the test show in each case? What is the name of each of the products in which oxygen occurs?

47. Ash. - A product of combustion of many kinds of fuel is the ash. Ash, as the non-combustible residue, is a mixture of clay and such mineral substances as phosphorus, lime, potash, and iron in different compounds. Ashes of certain hard woods are sometimes used as fertilizer, or are leached with water for their potassium carbonate or "lye." This is useful in making soap. A very small percentage of wood is incombustible, as experience shows. Of coal about ten per cent is usually estimated to be ash. Coal ash has no fertilizing value.

48. Incomplete combustion: smoke. - The flame of the candle, if the wick is properly trimmed and combustion is not interrupted, gives off no smoke or gases except water vapor and carbon dioxid. We know from experience, however, that burning may be checked in various ways, as by holding a cold object, such as a porcelain or glass dish, in the flame, and smoke be produced. Part of the smoke blackens the object, or leaves a deposit of soot. Imperfect combustion in a range or any fuel-burning appliance causes the production of quantities of smoke, part of which escapes by way of the chimney and part as soot clutters up the flue. The fact that smoke is produced in the candle flame only when burning is checked, suggests that it may be due generally to interrupted and therefore incomplete combustion. If this is true, it should then be possible to make combustion complete. This possibility may be tested in the following simple manner

Exercise : Smoke and soot. - Put a small quantity of powdered soft coal or a few small bits of dry wood in a hard glass test tube, and close the tube with a rubber stopper in which is inserted a piece of small diameter glass tubing with one end drawn to a fine tip. Heat to a high temperature. When smoke issues freely from the tip, apply to it a lighted match. Does the smoke ignite and burn? Heat until no more smoke appears. Remove the charred substance from 
the test tube, and when cool heat it directly to a high temperature. It will burn completely if charcoal ; or if coke, all but a bit of ash will burn, for both are practically pure carbon. How can you prove that they are carbon? Does this show that smoke consists largely of unburned substances which will burn in proper conditions?

Heat a small amount of soot directly in a flame, as you did the carbon. Does it burn when heated to a high temperature? If it does, and produces carbon dioxid, as you may determine by the limewater test, what do you infer it to be? Does it behave as charcoal and coke in similar conditions?

In combustion carbon particles of very minute size combine at any temperature which is too low to effect complete combustion. Thus are formed visible masses of unburned carbon particles which remain suspended in the invisible gases evolved in imperfect combustion. The gases are combustible as your experiments show, and burn to water vapor and carbon dioxid. They are usually made up of hydrogen and carbon monoxid, which gases form what is known as water gas (pages 162-3). These facts prove conclusively that smoke with its visible soot and invisible gases is not a useless material to be driven off and lost.

Make tests of other fuels for carbon by the same method followed in collecting smoke from the candle. You might test, among others, kerosene, alcohol, and illuminating gas. If any of these fails to show the presence of carbon by forming soot, its products of combustion may be tested for carbon dioxid.

In the use of coal or other carbon compound for the production of heat, it is possible to allow the carbon to be wasted in two ways: (1) it may be driven away in fine particles, forming a black smoke; (2) it may unite with only half the amount of oxygen necessary for complete combustion, and the incompletely burned carbon or carbon monoxid escape as an invisible.gas.

It is well established that one pound of carbon produces, if burned completely, 14,544 British Thermal Units (page 142). It has been proyen also that one pound of carbon produces about 5000 B. T. U., when it is only partially burned or changed 
merely into carbon monoxid. This represents a loss of about 10,000 B.T.U. per pound of carbon or a waste of nearly two thirds of the fuel. If the soot together with carbon monoxid escapes, the combustion is much less perfect, and the loss of fuel and therefore of money is correspondingly greater.

Smoke and soot are of practical interest to every one. - In complete combustion smoke is not driven out and lost, and soot is not accumulated in the range and chimney or distributed broadcast. In incomplete combustion, fuel is wasted. Stoves and heating appliances in general do not secure complete combustion. With proper control, however, fuel may be burned more completely and the heat more fully utilized. This is why men in charge of industries where great quantities of fuel are consumed, employ every possible means to secure complete combustion, or allow no smoke to escape. They are concerned in stopping waste and saving money. The smoke nuisance, as this is experienced in many places, is thus an economic problem as well as one of health and beauty.

Conditions of incomplete and complete combustion. - We may summarize briefly the important conditions favoring incomplete combustion and the consequent loss of fuel. These are:

1. An insufficient supply of air or oxygen.

2. Combustion somehow checked and the temperature reduced below the point at which carbon particles are completely oxidized or burned to carbon dioxid.

3. An accumulation of ashes in the grate or bed of coals, which prevents air from coming into contact with the heated carbon and liberated gases.

4. Fuel supplied in such a way as to prevent the burning of the gases liberated by the heat.

The conditions of complete combustion are these:

1. Proper regulation of the drafts.

2. Keeping coal nearly free from ashes and the grate clean to insure entrance of abundant air.

3. Proper distribution of fresh fuel over the hot coals. 
The observance of these conditions will insure better control of fire, more economical consumption of fuel, and it should stimulate to a fuller utilization of the heat produced.

49. Forms of carbon. - Soot or lampblack is produced on a large scale for commerce from natural gas, coal gas, or petroleum. It is deposited in a relatively pure form on the inside of the retorts, in which coal is distilled for illuminating gas. Its familiar uses are in making black paints, printing inks, and shoe polish. It is made into carbons used in electric arc lights, and into the carbon plates for dry batteries. Examine the carbon of a dry battery.

Diamonds and graphite. - Interesting forms of carbon are found in the diamond and graphite. The diamond when burned in a closed retort becomes like graphite; when heated in oxygen it is burned to carbon dioxid. Carbon may be changed by a very high temperature (about $3000^{\circ}$ C.) and enormous pressure into diamonds. (Consult an encyclopedia for information about Henry Moissan and his work in producing diamonds.) Graphite is used in making lead pencils, in paints which are especially protective against rust, in making crucibles and other containers to withstand very high temperatures, and as a lubricant.

Charcoal made from wood is almost pure carbon. In its modern manufacture, wood is heated in closed retorts with no access of air. Certain by-products are secured, such as wood alcohol and acetic acid, both being of great importance in chemical manufactures. Combustible gases are also collected and piped to the furnace as fuel. The process is known as wood distillation.

Coke. - Carbon constitutes a large percentage of coal, lignite, . and peat. Coke which is almost pure carbon, is obtained from coal heated in much the same manner as wood for charcoal. Coke burns at a high temperature with little flame and no smoke.

50. Carbon compounds. - Carbon is found in many compounds of great importance. Carbon and hydrogen combined 
constitute such substances as natural gas, coal gas, coal tar, and petroleum with its derivative products, such as kerosene, gasoline, naphtha, benzine, and paraffin. The compounds of this group are generally designated as hydrocarbons. These are all very combustible, and because of the large proportion of hydrogen they contain, yield an especially large quantity of heat. Some of them, such as gasoline, benzine, and naphtha, are so highly combustible as to be explosive; they contain unusually large proportions of volatile hydrocarbons.

Many useful compounds are formed of carbon, hydrogen, and oxygen, as for example, wood, lignite, peat, coal, and alcohol. The table on page 144 shows the percentage of hydrogen and carbon in these substances and also in those grouped under the hydrocarbons.

Nature often works in seemingly contradictory ways. By a change in the proportions of the elements, a very different series of substances results. Carbon combines with hydrogen and oxygen to produce such familiar compounds as the various kinds of sugar and starch. These are fuels, but of a class quite different from that given above. They make up a large part of the fuel or food supply of mankind. This group of carbon compounds is called the carbohydrates, the oxygen and hydrogen being united in the same ratio as in water. The same three elements are combined, moreover, in such common substances as vinegar, acetic acid, citric acid abundant in lemons and oranges, and tartaric acid especially abundant in grapes.

51. Carbon and hydrogen in paraffin. - The products of the burning candle have been captured and the secret of their immediate source discovered. Water and carbon dioxid have revealed the elements of which they are composed. The little ash from the burned wick may be neglected. But a new problem is presented. How can the candle be composed of carbon and hydrogen? Carbon as shown by soot or charcoal is black. Hydrogen is invisible as a gas and does not become a liquid or 
solid except at extremely low temperatures. The candle does not resemble either of its constituents.

Compare with this the composition of water. Water in the electrolysis experiment was found to be composed of two invisible gases, and it certainly does not resemble them as they exist separately or when mixed. But water is not a mixture such as one may make of sugar and sawdust, or sand and salt. Water is a compound. A compound involves change in the structure of the substances combined to produce it. Hydrogen and oxygen are changed in their structures in combustion, and so form water. Water is changed in structure when electrical energy, the equivalent of a certain amount of heat, is applied to decompose it into its elements.

Take now the case of the candle. Heat melts and vaporizes its constituents which, when they unite in burning with oxygen, form new compounds. By the same line of reasoning, one may suppose that hydrogen and carbon were changed in their structures when they were combined to form such compounds as petroleum and its derived product, paraffin. The candle, is therefore a typical compound and in no way resembles in structure its constituents as they are when existing separately.

Compound and simple.substances. - Compound substances can be analyzed into two or more substances. Such compounds as carbon dioxid, wood, coal, water, petroleum, sugar, and starch are examples. Just as the paraffin of the candle is decomposed into its constituent substances by burning, so other compounds by suitable treatment may be decomposed into their constituents. But some substances which unite to form these compound substances, or are liberated from them by decomposition, show themselves incapable of further decomposition under any kind of treatment. Such substances are called simple substances or elements to distinguish them from compound substances. Of simple substances or elements, among which are hydrogen, oxygen, nitrogen, and carbon, with which you have become familiar, there are a few more than 
eighty known at present. Compound substances are almost beyond enumeration.

Physical and chemical change. - All compounds are the products of what scientists describe as a chemical change. This is very different from a physical change. The candle may be melted into a shapeless mass of paraffin, and then restored to its original form with practically no loss of material. When, however, the candle is burned in air, its structure is forever destroyed, and by no art or trick of magician or scientist can it be restored from the products. Melting the candle is a physical change which gives nothing new ; burning is a chemical change which produces new compounds.

The distinction between the two kinds of change may be emphasized by another example. One may pour water from one vessel into another, or carry water from the Atlantic Ocean to the Pacific, but one does not change its structure or produce a new compound. Water may be changed into a solid, liquid, or gaseous state; but its structure remains unchanged. It is still water in any of these states. But when water is decomposed by electricity or other means, it ceases to exist as water; and in its place are two gases, invisible and odorless, one supporting combustion, the other highly combustible. This is a chemical change. Again when hydrogen is burned in oxygen, both are changed structurally or chemically, and produce a new compound substance. Decomposition is a chemical change. Production of new substances is a chemical change. Change that does not affect the structure of a substance or produce new substances is physical. There are many examples of both kinds of change in the world about you, for example, cooking potatoes, baking bread, mixing soap and water, sawing wood, or breaking stone.

52. Carbon dioxid. - The study of how fire burns and of the products of burning brings to light, among other important facts, that the mixture which we call air consists of nitrogen and oxygen in large proportions, and carbon dioxid in much smaller 
proportion. It appears that wherever combustion occurs, whether slow or rapid, carbon dioxid is produced. Combustion is going on everywhere in the world, so that the quantity produced must be large. The following data may be suggestive of the amount produced by just one sort of combustion, that of coal.

Coal on the average contains about eighty per cent carbon, a small percentage of hydrogen, and a small amount of incombustible substances that remain as ash. More than a thousand million tons of coal are mined and burned in a year. The burning is often incomplete as evidenced by the quantities of escaping smoke and soot; but for our present purpose it may be assumed that combustion is complete.

Carbon, when burned, combines with enough oxygen to produce more than three and one half times its own weight of carbon dioxid; that is to say, one pound of carbon unites in combustion with enough oxygen to produce three and one half pounds of carbon dioxid. How many pounds of oxygen are taken from the air for each pound of carbon burned?

Now multiply the thousand million tons of coal by eighty per cent, the average amount of carbon, to find the total carbon content; and multiply this product by three and one half to find the quantity of carbon dioxid produced. The output of carbon dioxid from this one source is seen to be stupendous.

This is only one of the many ways in which carbon dioxid is produced and liberated in the air. All fuels and all foods contain carbon in large proportions, and when consumed are oxidized or turned into large amounts of carbon dioxid. Let us consider a few typical instances from everyday life.

Exercise: Testing for carbon dioxid. - Blow through a glass tube into a beaker containing limewater. What does the reaction indieate? If you can get a bellows, fill it with air from near the floor of the room and force it through the limewater. Is the effect similar to that of air expired from the lungs?

Heat a small quantity of sugar in a test tube or iron spoon until 
the water is driven off ; then burn and capture its product in a bottle. Add limewater and shake. Does the test show the presence of carbon dioxid? What does this show as to the composition of sugar? Try the same experiment with other familiar substances, as starch, dried vegetables, and fruits. Different substances may be assigned to different members of the class for testing and report of results.

The following materials may be tested:

1. Mix a small quantity of baking powder in water, and heat gently.

Capture and test the gas evolved with limewater.

2. Mix one ounce of baking soda with one half ounce of cream of tartar; dissolve the mixture in water, heat gently, and test the gas produced.

3. Dissolve half a teaspoonful of baking soda in warm water and mix with sour milk; warm slightly if necessary; test the gas produced. Other substances, as lemon juice, eider, grape juice, may be similarly mixed with soda and the product tested.

What do these tests show as to the use of carbon dioxid in the home? Bread made with baking powder or with yeast in fermentation (page 562) owes its lightness and consequent digestibility in large part to carbon dioxid. Fermentation of fruits and fruit juices (pages 565-6) produces carbon dioxid. What use is made of this in preparing beverages?

Amount of carbon dioxid in expired air. - Fats, oils, sugars, and starches when consumed as food are changed in the body by union with oxygen into water and carbon dioxid. As long as we live, whether at work or at rest, we are oxidizing carbon in some form and eliminating carbon dioxid. This means that respiration is necessary both to supply oxygen and to eliminate carbon dioxid. Each individual sets free on the average about two pounds of carbon dioxid a day. The expired air contains approximately 16 per cent oxygen, 4.38 per cent carbon dioxid, and is saturated with water vapor.

Exercise : Laboratory production of carbon dioxid. - A quantity of the substance is needed for study of its behavior and nature. For its production you need apparatus similar to that used in producing hydrogen (page 119). Into the flask put a small quantity of well broken marble, cover with water, close with the stopper, and then pour some hydrochloric acid into the funnel. The gas is quickly gener- 
ated and by conducting it through a rubber tube, the end of which should reach the bottom of the collecting bottle, enough may be secured. Fill several bottles and cover each promptly with glass or wet filter paper until ready for use.

Exercise: Properties of carbon dioxid. - To determine when a bottle is full, have a candle fastened to a wire so that it will reach the bottom of the bottle. Light it and let down slowly. When it meets the gas, it will be extinguished. Relight and try again and when extinguished, observe the level at which this occurred. Repeat until you have the bottle full.

Experiment with the gas in these ways:

(1) If you are not certain of its influence upon combustion, introduce a burning candle into a bottle of the gas. For comparison put a burning candle into a similar bottle of air. Does the gas generated from the marble support combustion?

(2) Put some limewater into a bottle, close, and shake vigorously. What does the reaction of the limewater indicate as to the content of the bottle?

(3) Turn a bottle upside down and insert a burning candle; when extinguished, remove, light, and again introduce it. Repeat several times. The behavior of the flame and of the glowing wick of the candle at successive introductions is interesting. Toward the end of the trials is the fire completely extinguished? If not, explain why it is not. Would the gas remain in the bottle, if it were lighter than air? Compare with this the fact that you collect it in open bottles. Can you guess approximately the difference between the weights of the gas and of air?

(4) Fill a bottle containing gas one third to one half full of water. Cover with the palm of one hand and shake thoroughly. Note the sensations on the surface of the hand. What do they suggest as to the behavior of the contents of the bottle? Submerge the mouth of the bottle in water and open. What happens? What does this show as to the amount of gas absorbed by the water? Is the gas soluble in water?

(5) Mix a small amount of clean water with the gas in one bottle by shaking vigorously. Taste the mixture. Recall the taste "of " soda water." In what respects are the two tastes similär?

Exercise. - Prepare a summary of the points observed as to the behavior and nature of carbon dioxid.

1. What is its influence upon combustion? What facts support your conclusion? What use is made of it in fire extinguishers?

2. Is carbon dioxid soluble in water? What effect does it have on 
the water in which it is dissolved? Compare soda water, or any of the carbonated waters on the market. Water at ordinary temperature is capable of absorbing practically its own volume of the gas.

3. Is carbon dioxid heavier than air? Where, when unmixed with water, would you expect to find the gas? Would mines and deep wells be included in your list of places?

4. What test is used to determine the presence of carbon dioxid? Is the substance produced from marble and hydrochloric acid the same as that you collected from burning carbon in air or oxygen, or that contained in air expired from the lungs?

Carbon dioxid is more than half again as heavy as the air. That is, if a unit volume, as a cubic yard, of air has a weight of 1 , as one gram or one ounce or one pound, an equal volume of carbon dioxid has a weight of 1.5 grams, ounces, or pounds.

Distribution and abundance. - Carbon dioxid is universally distributed in the air, in water in the soil, and in rocks, as well as in all living things. In one kilogram of air carbon dioxid weighs $.5 \mathrm{gram}$. In a liter of air it makes up but .3 of a cubic centimeter. Although it is half as heavy again as air, it is generally kept well mixed by air currents, clouds, and rainfall. It forms about .01 per cent by weight of ocean water. Many underground waters-are highly charged, as their effervescence shows when they are released from earth pressure. The effervescent waters of many springs are widely known, as those of Saratoga Springs, New York. Carbon dioxid is especially abundant in such rocks as limestone, marble, and chalk, which because of this fact are known as carbonates, limestone and marble being called calcium carbonates. For commercial purposes carbon dioxid is prepared from limestone and marble by heating or by treating with acids. By volcanic action great amounts are returned to the atmosphere from the vast underground stores. In the process of decay, all organic matter, plant and animal, which contains a large proportion of carbon, liberates quantities of the gas. This is largely absorbed by water. In its union with water; carbon dioxid permeates the crust of the earth, dissolving many substances which are carried away by the water and redeposited elsewhere. Thus together 
with water, carbon dioxid has a great share in shaping the crust of the earth and bringing into circulation substances that millions of years ago were buried deep in the earth's crust. After its action upon rocks beneath the surface, it escapes to the air, ready to begin another cycle of the same kind. For carbon dioxid not only unites freely with water, but just as freely escapes from it.

The carbon cycle. - In this manner not only in life, but in death and decay, and in all the processes of nature, carbon

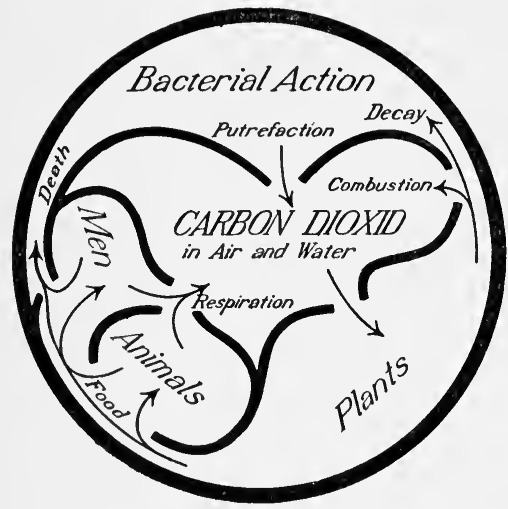

FIG. 38. - The carbon cycle. Trace its progress from air to air, and show by what agencies the cycle is made possible. dioxid is being produced and released in the air. The diagram (Fig. 38) presents graphically the chief stages in the great cycle of this important substance. Air is taken up by plants, carbon dioxid is decomposed, oxygen and other gases liberated to the atmosphere, and the carbon assimilated with other substances in forming sugars, starch, other food materials, and plant tissues. Animals eat plants or plant products and thus get the carbon compounds they need for making tissue and producing energy. Some of the carbon is oxidized and the carbon dioxid returned by the lungs to the air. Animals and many plants die and decay, a process carried out by bacteria and molds, in which carbon is oxidized and set free as carbon dioxid. Again it is absorbed by plants and so on, cycle following cycle eternally.

Formation of coal. - The importance of carbon dioxid to plants to-day (pages $515 \mathrm{ff}$.) is suggestive when we consider the luxuriant growths of vegetation that were necessary to form the immense stores of coal and the reservoirs of natural gas 
and other hydrocarbons upon which we now draw for heat and power. In ordinary conditions when a tree falls in the forest, it decays or oxidizes, giving up carbon dioxid and water. Finally little remains but mineral ash. If the wood of the tree is burned, the process is hastened, but the products of the burning are the same. If, however, the tree falls into the waters of a swamp and lies there, the process of decay or oxidation is retarded and much of the carbon is preserved. Such in substance are the conditions in which coal is formed.

In very remote periods of the earth's history, millions of years ago, all of the conditions were at times favorable for the formation of coal. Great swamp areas existed, and the climate was moist, warm, and equable with long seasons for growth. The temperature and humidity of the atmosphere may have been much like the condition found in a tropical swamp to-day. The vast forests and jungles appropriated the carbon dioxid from the air, utilized the carbon in building up plant tissue, and restored the oxygen to the air. The great stores of carbon in the luxuriant vegetation were not lost through decay, but preserved and accumulated mass upon mass in the swamps of that age. This continued for ages and ages, as the coal measures indicate by their extent and depth, until different conditions appeared and with these a new geological period of the earth's history. In succeeding periods the accumulated masses of vegetation with their carbon content were weighted down by deposits of mineral materials, subjected to pressure, heat, and other conditions, and gradually changed into the hydrocarbons and coal.

To-day the stored-up treasures of carbon, the products of ancient vegetation, are brought forth from their hiding places in the earth's crust and burned in producing heat and power. Thus the carbon dioxid taken from the air in the early history of our earth is being restored. In that remote period carbon dioxid may have been slightly more abundant in the air than it is to-day, but this does not seem probable. The balance of 
the constituents of the atmosphere is apparently not disturbed by the return of the vast quantities of carbon dioxid from coal and the hydrocarbons. The carbon dioxid may be absorbed, and the balance thus maintained, by the rapidly increasing extent and amount of vegetation which is being grown under man's control.

\section{SUMMARY}

The candle is a miniature gas plant.

Its flame consists of burning gas.

Two products of combustion are water vapor and carbon dioxid.

That water is composed of oxygen and hydrogen may be proven by burning magnesium in steam.

Water is composed of two volumes of hydrogen and one volume of oxygen as shown by electrolysis.

Hydrogen, discovered by Cavendish, burns in air, producing hydrogen oxid, or water.

Hydrogen may be liberated from water by certain metals.

Hydrogen is a colorless, odorless gas, much lighter than air. It burns with a hot, colorless flame, but does not support combustion.

Hydrogen may be liberated from sulfuric or other acids by zinc or certain other metals.

Hydrogen is an essential part of many compounds, such as water, hydrogen peroxid, ammonia, acids, foods, and fuels.

The presence of hydrogen in the composition of the candle and other substances may be proved by the production of water when they burn in the oxygen of the air.

Carbon dioxid, another product of combustion, is a colorless, odorless gas.

Its presence is indicated by limewater.

By the products of its combustion the candle is proven to contain carbon and hydrogen.

Mineral matter that does not burn is left as ash from many kinds of fuel.

Smoke, consisting of invisible gases and soot, is due to imperfect combustion and represents a direct loss of fuel.

Soot is unburned carbon. It may be commercially produced and sold as lampblack.

The invisible gases of smoke contain hydrogen and carbon monoxid, which are valuable fuels.

Charcoal and coke are impure forms of carbon produced by destructive distillation of wood and bituminous coal. 
The hydrocarbons, such as kerosene, gasoline, and paraffin are composed of hydrogen and carbon in different proportions. They are important fuels.

The carbohydrates, such as starch and sugar, are composed of hydrogen, carbon, and oxygen. They are important foods.

Carbon, hydrogen, and oxygen are elements.

Starch, paraffin, and water are compounds.

Changes in which new substances are produced are chemical changes; other changes are physical.

Immense amounts of carbon dioxid are produced by fires and living animals, and decaying organic matter.

Carbon dioxid for laboratory experiments is generally produced from marble or limestone by the action of hydrochloric acid.

Carbon dioxid neither burns nor supports combustion.

Carbon dioxid is one of the gases which make up the mixture called the air, and is dissolved in ocean and underground waters. It is combined with calcium in immense deposits of chalk, limestone, and marble.

The carbon dioxid of the atmosphere is one stage of the great cycle of carbon in nature.

Coal is an accumulation, mainly carbon, formed from plants which obtained their carbon from the carbon dioxid of the atmosphere.

\section{REVIEW QUESTIONS}

1. What are the most common products of combustion? 2. How can you demonstrate that water is a product of combustion? can you show that carbon dioxid is a product of combustion?.

3. How tion a substance which, when burned in air, produces only substance which produces only carbon dioxid.

5. What substance is always a constituent of all combustion products? What general name do chemists therefore give to all such products? 6. What does the experiment of burning magnesium in steam show as to the composition of water? 7. Describe a method of separating water into its constituent elements. 8. In what ratio of volume do the elements combine to form water? 9. Of the two constituents of water which one burns? Which supports burning?

10. What did Lavoisier contribute, about the time of the American Revolution, to our knowledge of the air? 11. What evidence do you find that hydrogen is lighter than air? 12. Mention two acids in which zinc will replace the hydrogen. 13. Mention two metals which will replace the hydrogen of water. 14. Describe the process of mak- 
ing water gas. Of what two gases is it a mixture? For what purposes is it used?

15. Mention physical changes which take place in the burning candle; mention chemical changes. 16. What two material products and what two effects do we get from a burning candle? From any fire made of common fuels as wood, coal, gas? 17. Is water obtained from the candle or from the burning of the candle? 18. Distinguish between mixture and compound substances, giving examples of each. 19. Mention four common compounds containing hydrogen.

20. What is smoke? Give at least two reasons why it should be prevented. 21. What are the conditions that favor complete combustion of fuel? 22. What uses are made of lampblack? Of graphite? 23. How can it be shown that the diamond is pure carbon? 24. Under what conditions does carbon crystallize to diamond?

25. How and from what material is charcoal obtained? Coke? 26. Mention several common foods that contain carbohydrates.

27. Give three sources of the carbon dioxid in the air. 28. What substance is purchased for the carbon dioxid it produces and gives up when wet and warm? 29. How does the air that enters the lungs differ from the air which is expelled from the lungs? How can you show this difference? 30. What use is made of the fact that carbon dioxid is soluble in water? That it will not support combustion? 31. Why does the percentage of carbon dioxid in the air not increase? 32. From what source was derived the material which makes our coal seams? By what agency were they formed?

\section{SUGGESTIVE QUESTIONS}

1. How can you show that expired air contains the same substances as are produced in the burning of a candle, of wood, and coal? 2. Describe the changes that occur in burning wood or coal, and state the conditions that bring them about. 3. Explain the difference between rusting, as of metal, and burning, as of oil, coal, or wood. What is the difference between these processes and the use and assimilation of foods in the animal organism? 4. Of what use is nitrogen in the air? In the soil? In such food substances as beans, meats, etc.? 5. What reasons can you give to support the statement that the supply of nitrogen in the air is inexhaustible? 6. Show in what ways carbon dioxid is essential to human life. 7. With the enormous consumption of carbon dioxid by plants, why is the supply in the air not greatly reduced? 8. Give a suitable name to the white powder formed by the burning of tin. 
9. What is the ratio by volume of the gases that compose hydrogen dioxid? 10. Why should a lantern be lowered into an old well before men go down into it? 11. After an explosion in mines men not injured by the explosion may be suffocated by " choke damp." What is "choke damp"? 12. An alcohol lamp does not deposit soot. Describe fully how you would prove that alcohol contains carbon in its composition. 13. In what way may the diamond be proved to be carbon? 14. In what ways did the formation of coal prepare the earth for man's existence? 15. When you see smoke issuing from your chimney, what use can you make of the knowledge of the products of combustion in deciding whether or not the furnace is being properly managed? 16. If black smoke appears, what may be wrong in the management of the furnace? 17. Why is the knowledge of what flue gases contain useful to an engineer? 18. Is too much air entering a furnace as wasteful of fuel as too little? 19. Why is burning a safe method of garbage disposal?

\section{Vi. Measuring Heat}

It is common knowledge that different kinds of coal vary greatly in the way in which they burn and in the amount of heat they produce. So it is with all kinds of fuel, such as wood, oil, gas, gasoline, and alcohol. We compare one kind of fuel with another, estimating their heating value by their effect in warming various things. Such an estimate is of course vague and of limited practical value; but it indicates the possibility of an exact or scientific method of measuring quantities of heat based upon its effect in raising the temperature of objects, such as iron, air, or water. If we have such an exact method of measurement, that is, a unit quantity or measure which will apply to different quantities of heat as a yardstick applies to different dimensions, we can readily apply it to the quantities of heat produced from different fuels and thereby determine their heating values or heat-giving powers.

53. Heat units. - It is not difficult to determine such a unit quantity of heat or unit measure. It involves chiefly the selection of some substance which responds uniformly to heat, showing a definite change in temperature for a definite quantity 
of heat. Water, which has the highest heat capacity of all ordinary substances and is also the most dependable in showing the effects of applied heat, is chosen as the standard substance in the measurement of heat.

The British Thermal Unit. - Those making use, according to the English system of measures, of the pound for a unit quantity of matter, and the degree Fahrenheit for a unit change in temperature, define their heat unit in the same terms. In this system, one pound of water is taken as the unit of weight, and the unit measure of heat is the quantity of heat required to raise one pound of water at $32^{\circ} \mathrm{F}$. through one degree. This is known as the British Thermal Unit, commonly abbreviated as B. T. U.

The Calorie. - Those making use of the metric units of measure and the Centigrade scale define their heat unit in a similar manner. One kilogram of water at $0^{\circ} \mathrm{C}$. is raised through one degree of temperature by a unit measure of heat called the Calorie. It is practically equivalent to $4 \mathrm{~B}$. T. U.

The gram calorie. - In scientific laboratories and in many textbooks of chemistry and physics another metric unit quantity is used. The amount of heat required to raise one gram of water at zero Centigrade through one degree of temperature is called the calorie or often the gram calorie. This is one one-thousandth of the Calorie.

54. Heat values of fuels. - In America fuels have heat values assigned to them in terms of the English system, as so many B. T. U. per pound. A pound of the best grade of anthracite, for example, is said to produce about 14,000 B.T. U. Practically, however, coal is seldom perfectly burned, and some of its heat is lost. But if all the heat from one pound of coal of this grade were applied to 14,000 pounds of pure water, it would. effect a rise of one Fahrenheit degree in temperature.

The practical point here is that in purchasing coal or wood one should consider the heat value together with the cost per ton or cord. For example, there are bituminous coals with a heat value as low as $10,000 \mathrm{~B}, \mathrm{~T}$. U. per pound, and others that 
may run as high as $15,500 \mathrm{~B}$. T. U. per pound. If the same price is charged for both, or if a higher price is charged for' a coal with a higher heat value than that charged for a coal with a lower heat value, the fact is worth knowing and computing in the cost of heating.

When the heat value for a unit measure of a given fuel is known, such computation is simple. Comparisons of one fuel with another are equally easy. There must be included, of course, the advantages which one fuel or source of heat may afford as compared with the advantages or disadvantages of another. Gas, for example, is very convenient, involving much less work than coal, and distributing heat much less widely, an advantage during hot seasons. Electricity may have advantages, such as comfort and convenience, which may more than compensate for its cost in excess of that of gas.

Heat values of chemical constituents of fuels. - In the table of common fuels and some typical coals the heat values given are at best only approximate. Coal from the same mine varies in heat value. The table shows that the heat value of a fuel depends upon the relative quantities of the combustible substances of which it is composed. The chief of these substances are carbon, hydrogen, and sulfur. One pound of carbon completely burned is estimated to give 14,544 B. T. U., hydrogen gives 62,028 B. T. U., and sulfur 4050 B.T. U. Hydrogen, it appears, has a heat value about four and one half times that of carbon. Thus a fuel, such as kerosene or gasoline, which has a large proportion of hydrogen, has a higher heat value than a fuel having a small amount of hydrogen and a larger proportion of carbon.

Calculating a fuel's value in B.T. U. - When the chemical composition of a fuel is known, the heating value of the fuel may be computed approximately by this formula:

Carbon $\times 14,544+62,028 \times\left(\right.$ Hydrogen $\left.-\frac{\text { Oxygen }}{8}\right)+4050 \times$ sulfur gives the heating value in $\mathrm{B}$. T. $\mathrm{U}$. 
Example. - A coal contains :

Carbon .

Hydrogen

Oxygen .

Sulfur.

Nitrogen

Ash

\begin{tabular}{r|r}
$\%$ & \\
55.94 & .5594 \\
5.25 & .0525 \\
19.04 & .1904 \\
4.35 & .0435 \\
.89 & \\
14.53 & \\
\hline 100.00 &
\end{tabular}

Substituting the proper figures in the formula we have

$$
.5594 \times 14,544+62,028 \times\left(.0525-\frac{.1904}{8}\right)+4050 \times .0435
$$

or

$$
8334.90+1,680.27+176.17=10,293,
$$

the B. T. U. This calculated value is approximate, but close enough for practical purposes. Problems may be based upon the table, which gives typical fuels, and coals from well-known fields of our country.

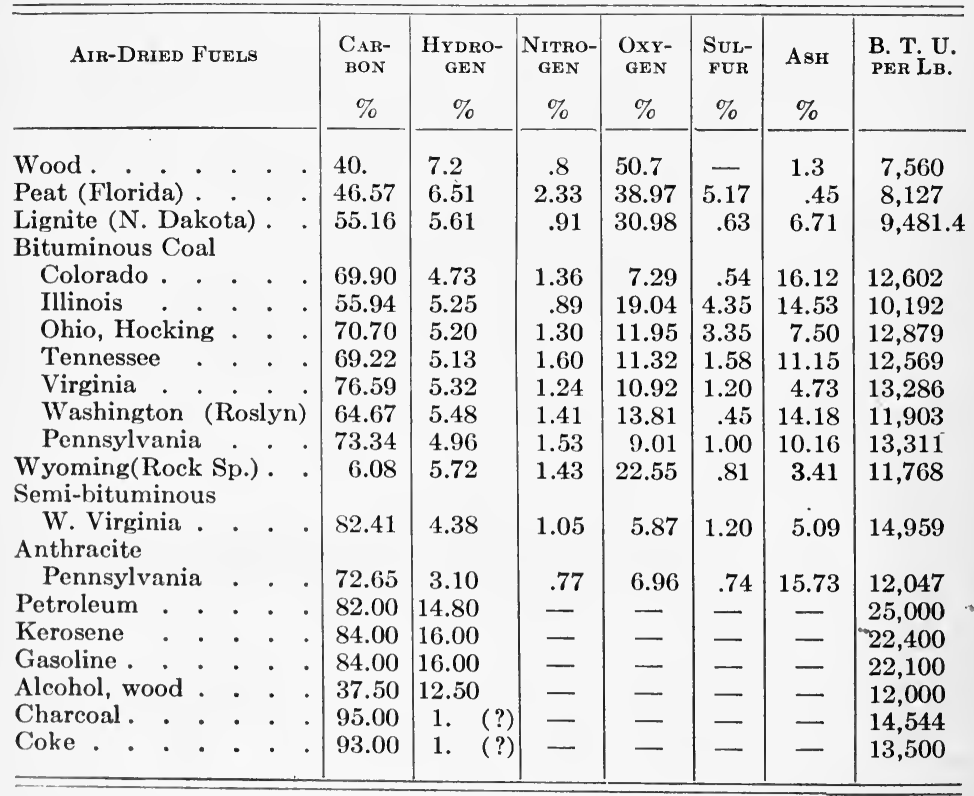

Fig. 39. - Table of fuels, their composition, and heat value in B. T. U. 
The weight of a unit measure of common fuels is as follows: coke and coal, $2000 \mathrm{lbs}$. per ton; wood, $4000 \mathrm{lbs}$. per eord; petroleum, $6.5 \mathrm{lbs}$. per gallon; kerosene, $6.1 \mathrm{lbs}$. per gallon; gasoline, $4.9 \mathrm{lbs}$. per gallon; wood alcohol, 6 lbs. per gallon; chareoal, 30 lbs. per bushel.

The heat value of artificial gas varies with the kind of gas. The range is from 150 to $700 \mathrm{~B}$. T. U. for a cubic foot. A cubic foot of coal gas has a value of about $700 \mathrm{~B}$. T. U. If it costs 80 cents per thousand cubic feet, it means that 80 cents purchases 700,000 B. T. U. of heat. You may compute the cost of an equal quantity of heat from coal, and then compare the cost of the two fuels, coal and gas.

Electricity is utilized in many sections of the country both for cooking and for heating, as well as for lighting. It is instructive to compare the cost of a given amount of heat from electricity with an equal amount of heat from any of the fuels. A kilowatt hour is estimated to furnish about $3400 \mathrm{~B}$. T. U. The cost per kilowatt hour varies greatly in different sections. If it costs two cents per kilowatt hour, it will be found to be somewhat more expensive than gas at 80 cents per thousand cubic feet; but it has advantages which are not afforded by any other source of heat supply. What are some of the advantages of gas as a fuel for cooking? For heating? Of electricity for these purposes? Compare with coal.

\section{SUMMARY}

Coal, although measured by the ton, is bought for the amount of heat it yields.

Heat is measured by the amount required to raise one pound of water one degree Fahrenheit. This is called the British Thermal Unit (B. T. U.). In the metric system the unit of heat is that required to raise one kilogram of water one degree Centigrade, called the Calorie; or one gram of water one degree Centigrade, called the gram calorie.

The heat value of a fuel depends upon its chemical composition. Fuels vary greatly in heat value.

Good coal has a heat value of about 14,000 B.T.U. per pound; coal gas 700 B. T. U. per cubic foot; and electrical energy 3400 B. T. U. per kilowatt hour. 


\section{REVIEW QUESTIONS}

1. What is the meaning of the heat value of a fuel? 2. What is the unit quantity of heat employed by British and American engineers? How is it determined? 3. What unit quantity of heat is employed in scientific laboratories and by French engineers? How is it determined? 4. Give several reasons why some kinds of coal are worth more than others. Why may one get more heat for the money. in buying a high-priced coal than in buying a low-priced coal? 5 . What constituent in gasoline gives it a high fuel value? 6. Give advantages in cooking and heating with electricity, with gas, with coal, with wood. 


\section{CHAPTER III}

\section{REFRIGERATION}

FAmiliar applications of low temperatures are made in refrigeration and in the manufacture of artificial ice. Refrigeration used in cold storage, transportation, and distribution, makes it possible for the whole world to contribute to every market its produce, such as meat, fish, eggs, butter, fruits, and vegetables, and secures a more equal distribution of foods throughout the year. Ice manufacture renders man independent of the caprices of winter and gives a product that is incomparably purer and safer for all purposes than the natural ice.

Cooling is exactly the reverse of heating. - A. stove, fireplace, or radiator, having a temperature higher than that of surrounding objects, radiates heat. In cooling or refrigeration, some substance, such as ice or liquid ammonia, with a temperature far lower than that of surrounding objects, absorbs and takes from them their heat. Ice is melted by the heat absorbed from other things. Liquid ammonia in ice making takes heat from adjacent substances and "boils," or passes into vapor. It behaves just as water does in taking up heat when it boils and vaporizes.

55. The household refrigerator. - The common household refrigerator (Fig. 40) depends for its efficiency upon several conditions. It should be of more or less non-conducting materials. If made with double walls the space between them should be filled with a blanket of " dead" air or some insulating substance, as hair felt, mineral wool, cork, or well-packed excelsior. Second, a refrigerator must secure circulation of 
air within its compartments. The air cooled by contact with the heat-absorbing ice must be allowed to fall into the food compartments, where it forces the warm air away from the food and upward into contact with the ice. Third, it should pro-

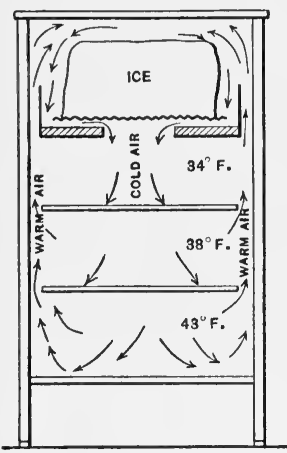

FIG. 40. - Diagram of a household refrigerator, with temperatures of compartments and circulation of the air.

vide for the melted ice or waste water to circulate over the interior surface of the refrigerator, where it absorbs still more heat from the air and food. Melted ice has a temperature of $32^{\circ} \mathrm{F}$; and if it is drained from the refrigerator with a temperature of $35^{\circ}$ to $40^{\circ} \mathrm{F}$., we know that it is reducing the temperature of the air. Finally, the efficiency of a refrigerator depends upon the amount of heat that it requires to convert ice from a solid into a liquid. This point needs consideration, since it is fundamental in all refrigeration. The freezing of water. - Water freezes or congeals only after it has given up a large amount of heat. Its temperature must reach the freezing point, and then continued loss of heat causes it to freeze without the sensible lowering of the temperature. If a pool of water is perfectly still, its temperature may fall below $32^{\circ} \mathrm{F}$., but with the slightest agitation it begins to congeal and the temperature jumps up to the freezing point. The amount of heat that water loses in changing from liquid to solid form without sensible reduction of temperature, is exactly what it takes up in changing again from solid to liquid.

Exercise: Heat used in melting ice. - Into two one-quart tin cans put equal weights of ice and of water at $32^{\circ} \mathrm{F}$., and place these in a larger vessel of boiling water. Stir contents of both cans continually with different thermometers. Remove the cans from hot water as soon as that containing ice shows its temperature rising above the freezing point. You find that melting the ice takes as much heat as is required to raise the temperature of an equal weight of water from $32^{\circ} \mathrm{F}$. to about $150^{\circ} \mathrm{F}$. or $160^{\circ} \mathrm{F}$. 
Heat of fusion. - The heat taken up by the ice merely liquefies it and is equal to the amount of heat given up by the water in changing into ice, after it reaches the freezing temperature. The amount of heat required to melt or fuse ice is called the hidden or latent heat of melting or fusion.

Assume that ice with a temperature of $32^{\circ} \mathrm{F}$., or $0^{\circ} \mathrm{C}$., is put into a refrigerator. An amount of heat which will raise the temperature of an equal weight of water from the freezing point to approximately $150^{\circ} \mathrm{F}$., must be absorbed by the ice in melting. This amount of heat is taken from the air and the food in the refrigerator. The waste water of melting ice is often about $32^{\circ} \mathrm{F}$. If made to circulate over the inner surfaces of the compartments, it will absorb more heat and drain off with a temperature as high as $38^{\circ} \mathrm{F}$. This of course means economy in ice.

Exercise. - The latent heat of melting is $142 \mathrm{~B} . \mathrm{T}$. U. per pound (for the meaning of B.T.U., page 142). Suppose a refrigerator with a capacity for 50 pounds of ice melts 20 pounds a day. The quantity of heat required is

$$
20 \times 142=2840 \text { B.T. U. }
$$

Assume also that the waste water has a temperature of $38^{\circ} \mathrm{F}$. This has taken from the air and contents of the refrigerator an amount of heat equivalent to

$$
20\left(38^{\circ}-32^{\circ}\right) \text { or } 20 \times 6=120 \text { B. T. U. }
$$

That is, the total quantity of heat which the ice in melting and the water in warming to $38^{\circ} \mathrm{F}$. have taken up is $2840+120$ or 2960 B. T. U.

The heat of fusion applied generally. - All liquids may be converted into solids, just as all gases may be changed into liquids, by lowering their temperature below a certain point. Changing any solid into a liquid involves latent heat. Mercury, for instance, solidifies at about $-39^{\circ} \mathrm{C}$. or F. It becomes liquid by absorbing exactly as much heat as it gives up in becoming solid. Now the point at which mercury or any 
solid substance becomes liquid is known as the melting or fusion point. And the quantity of heat needed to melt or fuse a solid is known as the heat of fusion or sometimes as the latent heat of fusion.

56. Production and uses of low temperatures. - Temperatures below that given by melting ice have become necessary for many purposes. Such low temperatures may be obtained by a mixture of ice and common salt (about twenty-three per cent of salt yields the lowest temperature). A still lower temperature is secured by a mixture of ice with calcium chlorid in crystals, or with magnesium chlorid. A typical use of such mixtures with low freezing point is the making of ice cream in the ordinary freezer.

Ice cream freezing. - A mixture of salt and cracked ice melts more rapidly, that is, absorbs heat more rapidly and reduces surrounding objects to a lower temperature, than does melting ice alone. The particles of the salt act upon and facilitate the separation of the particles of ice, while the particles of ice act in the same manner and to the same end upon the particles of the salt. All this requires heat energy, which must be supplied largely by the surrounding objects. Thus salt and ice

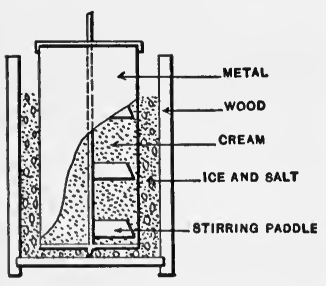

FIG. 41. - Diagram of ice cream freezer. change each other to a fluid condition, with a resulting temperature of the mixture much below the freezing point.

The ice cream freezer (Fig. 41) is usually made of material that conducts heat very poorly, such as wood or metal with a heavy paper covering. The cream container is made of good heat conducting material so that the heat from the cream mixture may pass freely into the salt and ice. As the temperature of the salt and ice mixture is many degrees below the freezing point of the cream, the heat continues to pass in the same direction, from the cream to the colder mixture, until the cream is frozen. 
57. The manufacture of ice. - This method is impracticable in refrigerating on a large scale, as in cold storage. Other methods have been devised and are now generally utilized for this purpose and for the manufacture of ice.

A study of an ice-making machine will explain also the important facts involved in current refrigerating machines. The diagram (Fig. 42) presents the essential features of one type

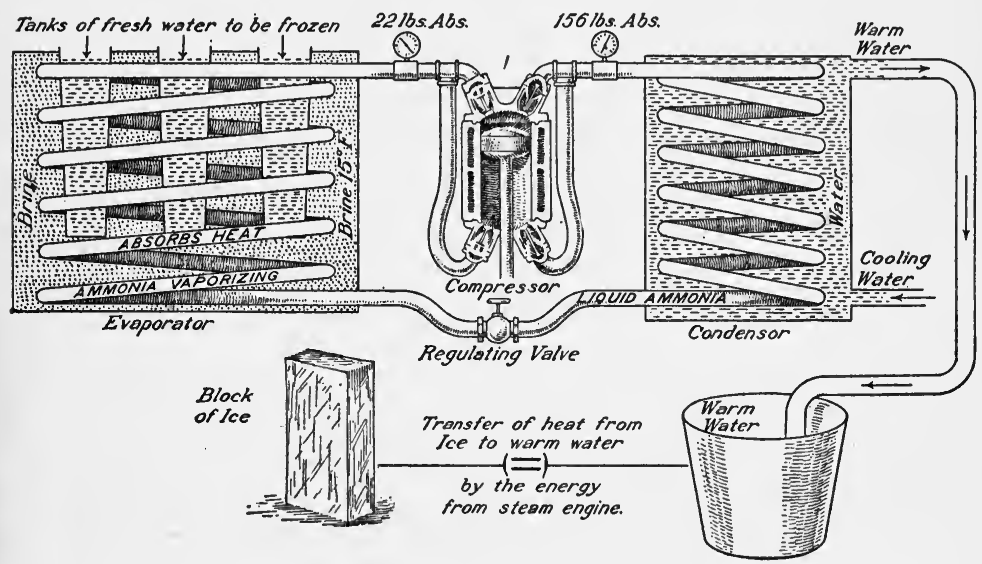

FIG. 42. - A diagrammatic representation of an ice machine. Explain each step from the compressor to the product.

of ice-making machine. The machine comprises a compressing pump, a coil of pipe immersed in running water for condensing the compressed ammonia gas, and a cooling or evaporating coil of pipe immersed in a reservoir of brine. The metal boxes or tanks of distilled water are placed in the tank of brine, where they are frozen.

The compressor, driven by an engine or dynamo, compresses the ammonia gas, and pumps it into the pipe leading to the condenser. The pressure employed in any particular plant is determined by the temperature of the water for the condenser, and by the temperature needed in the cooling or refrigerating 
compartments. As the compressed gas passes through the coil and is cooled by running water, it condenses and finally becomes liquid. The liquid ammonia collects in the lower part of the condenser coil or in a reservoir located there.

From the collecting reservoir the liquid is passed through the regulating valve into the pipe leading to the coil in the cooler or evaporating tank containing brine. The valve regulates the pressure at which the liquid is liberated in the cooler pipe. As it circulates in the coil at greatly reduced pressure, it expands, takes up heat from the brine and other contents of the tank and finally becomes ammonia gas. As-such it is drawn into the cylinder of the compressor, where it is again compressed and started on another round in the circulatory system. The heart of the whole system is the compressor, for it provides pressure and maintains circulation. Ammonia is the circulating fluid which gives up heat to the water in condensing to liquid and again takes up heat and lowers the temperature of the substances refrigerated, in expanding and becoming gas. Note the "balance," suggested in the diagram, between the temperature of the waste or cooling water and the temperature of the ice.

Refrigeration. - In refrigerating cold storage compartments, the cold brine is forced through the refrigerating pipes by a force pump. The brine circulating in the pipes takes heat from the compartments and their contents. The temperature of the brine may be regulated and thus adapted to the nature of the substances to be preserved. For example, the temperature in which eggs, apples, milk, and the like are best kept is between $0^{\circ}$ and $2^{\circ} \mathrm{C}$. $\left(32^{\circ} \mathrm{F}\right.$. to $36^{\circ} \mathrm{F}$.) ; meat and fish may be kept for long periods in a temperature of $-7^{\circ}$ to $-15^{\circ} \mathrm{C}$. (about $20^{\circ} \mathrm{F}$. to $10^{\circ} \mathrm{F}$.).

58. The production of low temperatures. - The usual system of refrigeration depends for its operation upon the behavior of ammonia gas. Some of the properties of ammonia are familiar from its use in the form of ammonia water in household 
cleansing. But we need to consider here its properties and behavior in relation to refrigeration.

Ammonia in liquid form evaporates very rapidly; and the rate of evaporation is greatly accelerated by additional heat or by currents of warm or cold air. Heat is necessary in changing a solid into a liquid, and a liquid into a vapor or gas. But in the case of many substances evaporation may be promoted by mechanical means, as release from high into low pressure, but with no supply of artificial heat. The heat, which is indispensable, must be derived from somewhere else. This fact may be strikingly illustrated in the following experiment.

Exercise: Heat absorbed in evaporation. - Pour enough water upon a board or the table top, if level, to make a small pool. Upon this pool place a thin glass dish containing a quantity of sulfuric ether. Apply a forced draft of air, as with a pair of bellows or a fan, to hasten evaporation of the ether. Evaporation requires heat. With forced or hastened evaporation the necessary heat cannot all be taken from the air. It must come from adjacent substances. In a very short time the glass is frozen fast to the board. Heat has evidently been taken from the liquid ether, from the glass, from the water in which the glass is placed, and from the board.

This illustrates exactly what occurs in an ice-making machine. The chief difference is in the mechanical appliances by which vaporizing is accelerated, and in the use of ammonia instead of ether.

Heat absorbed in expansion of gases. - Besides evaporation, which, as we have seen, may be promoted by mechanical means, there are two other facts in the behavior of a gas which must be emphasized in this connection. The first is that a gas under the influence of heat tends to expand and occupy more space. When it expands with no aid from artificial heat, it must get the necessary heat for expansion from itself and from adjacent substances. The heat it takes from itself and uses in expanding, causes directly a sensible reduction in its own temperature. This you know from common experience. Air expelled from a 
tightly inflated football or a tire tube feels cold. In the football it was probably of the same temperature as the surrounding air. When it is liberated from a high pressure into a space of lower pressure it must expand. In expanding it uses enough of its own heat to lower its temperature sensibly.

Heat liberated in condensation. - The second fact is that a gas, when compressed and cooled below its boiling temperature, condenses in giving up heat, and becomes liquid. The condensation of water vapor in the formation of clouds and rain, with the release of the heat absorbed in evaporation, is an example of the same fact on a large scale. When compression and condensation are effected by mechanical means, the heat that a gas contains is largely forced out of it. In the ice machine the expelled heat is taken up and carried away by running water.

In compensation for this loss, a liquid in expanding and vaporizing again must take up precisely as much heat as it lost in condensing and liquefying. If this heat is not provided artificially, it must be taken from the vaporizing liquid and the surrounding substances which are thereby cooled and perhaps frozen.

Application to refrigeration. - With these facts concerning the behavior of gases, the process involved in the manufacture of ice and in refrigeration is easily understood. Ammonia and certain other substances have their boiling temperatures changed in accordance with the pressure to which they are subjected. Pressure is readily controlled by mechanical means, such as the compressor pump; and the higher the pressure to which such a substance as ammonia is subjected, the higher becomes its boiling temperature (page $300 \mathrm{ff}$.). The boiling temperature of liquid ammonia at ordinary atmospheric pressure is about $-29^{\circ} \mathrm{F}$. Under an absolute pressure of 156 pounds (absolute pressure means "gage" pressure plus atmospheric pressure or 14.7 pounds per square inch), ammonia boils at a temperature of $80.8^{\circ} \mathrm{F}$. Water at a much lower temperature 
than this is easily obtained for cooling the compressed gas. With the reduction of the temperature of the gas below its boiling point, the gas condenses or liquefies, giving up its heat to the colder water surrounding the pipe.

Control of boiling point by pressure. - The boiling point of liquid ammonia at ordinary pressure is about $-29^{\circ} \mathrm{F}$. Such a low temperature is unnecessary in refrigeration. The liquid is therefore usually liberated at a somewhat higher pressure. Suppose it is released in the evaporating coil under an absolute pressure of 22 pounds. At this pressure its boiling point is about $-12.2^{\circ} \mathrm{F}$. The difference between the pressure above, and that below, the regulating valve is still very great. The temperature of the liquid in the collecting coil under 156 pounds pressure is much below its boiling point. The liquid liberated into a pressure of 22 pounds begins immediately to vaporize and to expand rapidly. It takes heat largely from itself as it vaporizes and expands, and thereby lowers its temperature to about the point at which it boils under this comparatively low pressure.

At this temperature the gas moves through the pipes, absorbing heat from them and from the brine, expanding continuously until it is drawn into the cylinder of the compressor. The brine gives up heat but does not freeze, because its freezing point is far below that of fresh water, perhaps as low as $-4^{\circ} \mathrm{F}$. The brine takes heat from the water in the molds, and by reducing its temperature to the freezing point, eventually causes it to become solid.

Ammonia is of common occurrence. - It may be detected by its odor wherever putrefying organic matter, animal and vegetable, is found, as about stables, cesspools and manure piles. It may be found by chemical tests in water where pollution has occurred from decaying organic matter or sewage. Its source for commercial purposes is the ammonia liquor produced in the destructive distillation of soft coal (page 163) in making illuminating gas. In a former age it may have been prepared 
from the horns and hoofs of deer; it was called, therefore, "spirits of hartshorn"; but it was usually obtained by the destructive distillation of the horns, hoofs, and bones of cattle.

Other substances used in refrigeration. - Ammonia is the most widely utilized substance in refrigeration, but sometimes sulfur dioxid, carbon dioxid, and ethyl chlorid are used in the same general way. Sulfur dioxid is useful in creameries where a freezing temperature is not necessary. Carbon dioxid is sometimes used on ocean liners; for, though it requires seven times the pressure necessary for ammonia, it does not escape and contaminate the foodstuffs in the cold-storage room. Ethyl chlorid is especially useful in small refrigerators for the home or market. It works under ten to twelve pounds pressure, and gives a temperature of about $28^{\circ} \mathrm{F}$. in the compartments of a household refrigerator. Household refrigeration by some such means is certain to replace the inconvenient and not wholly hygienic use of ice. There is an opportunity for some one with ingenuity to invent a system that will be economical, effective, safe, and easy to control.

\section{SUMMARY}

Refrigeration makes possible the cold storage and distant shipment of perishable foods, and gives a dependable and safe ice supply.

A household refrigerator is cooled by ice, which absorbs and removes, as it melts, 142 B.T.U. per pound.

The heat of fusion of a substance is the amount of heat required to change a pound of the substance from solid to liquid without adding to its temperature.

Ice and salt make a freezing mixture.

The ice cream freezer illustrates the use of a freezing mixture, and the relative heat conductivity of metal and wood.

The ammonia-gas ice-making plant depends upon the difference in the boiling point of the gas under different pressures. The same amount of heat which is given out in condensation is absorbed in vaporization. In the ice-making plant, heat is transferred from a colder to a hotter body by the expenditure of energy. 
Ammonia is obtained from coal, or other organic matter containing nitrogen, by destructive distillation.

Besides ammonia, sulfur dioxid, carbon dioxid, and ethyl chlorid are sometimes used in refrigeration plants.

\section{REVIEW QUESTIONS}

1. What practical uses are there for low temperatures artificially produced? 2. Describe (a) an efficient household refrigerator; (b) the circulation of air within it. 3. How are foods cooled in such? 4. (a) What is the latent heat of fusion? (b) How may it be shown in the case of fusion of ice? (c) What use is made of it? 5. (a) Of what materials is an ice cream freezer made? Why? (b) Explain why cream freezes in it. 6. Explain why water is frozen by the evaporation of ether in the experiment described on page 153.

7. What change in temperature occurs in a gas when it expands? Explain and give examples. 8. What change in temperature occurs in a gas when it is being compressed? Explain with examples. 9. Upon what facts in the behavior of ammonia does an ice-making machine depend for its operation? 10. Describe fully what occurs in the complete circulation of ammonia in the ice-machine. 11. What is the commercial source of ammonia? 12. (a) What other gases besides ammonia are used in refrigeration? (b) What are the characteristics of each?

\section{SUGGESTIVE QUESTIONS}

1. Why do we not place a hot air furnace in the attic of a house, and ice in the lowest part of the refrigerator? 2. Atmospheric pressure decreases with altitude. What happens to the temperature of a mass of rising air? 3. Water at the top of a lake is generally warmer than at the bottom; but the air at the top of a mountain is generally colder than that at the foot. Explain. 4. It is found that frost is often formed in troublesome quantities on rock drills and other machines run by compressed air. Explain why. 5. If liquid air were cheap and available, what uses could be made of it in the household? What advantages and disadvantages would its use possess as compared with ice? 6. Would a refrigerator in which the ice would not melt be useful? 7. Would a refrigerator, in which the ice melts very rapidly be efficient? 8 . Why do some people wrap ice in paper or a woolen blanket? 


\section{CHAPTER IV}

\section{ARTIFICIAL LIGHTING}

59. Early lighting. - Lighting is the most familiar of the many uses of fire. Convenient artificial lighting has become such a common necessity that we seldom stop to consider what a luxury it would have been for the savage with his flickering torch, for the Pacific Islander with the blazing kukuinut, or

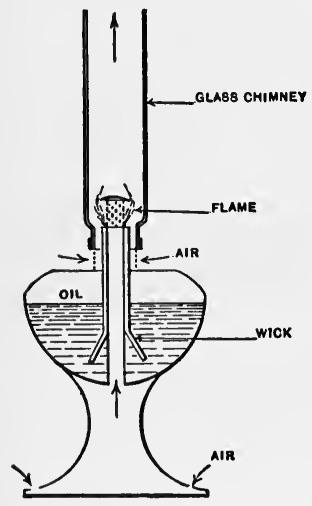

Fig. 43. - Oil lamp, with circular wick and central draft. for those peoples who made use of lamps of hollowed-out stones, shells, or gourds. The elaborate lamps of the Egyptians, the Greeks, and the Italians show a great advance over these means of lighting; but they are hardly to be compared with modern appliances, such as the electric lamp.

The oil lamp. - Not until 1783 was the familiar oil lamp of to-day invented by Argand, a Swiss physician, who devised the ring-shaped wick with the central draft (Fig. 43.). This increased the burning surface of the wick more than twice the former amount. His brother later discovered the use of the glass lamp chimney, which made the light steady and brighter.

Gas lighting. - Another important advance was made by William Murdock (1754-1839), a Scotchman. After many experiments he succeeded in producing and utilizing for lighting gas made from coal. The way had been opened for him by 
the work of Dr. John Clayton (1739) and Thomas Shirley (1759). Murdock introduced the use of gas for lighting into several factories; and by 1805 Manchester was lighting its cotton mills with coal gas. Progress in its use was fairly rapid. London shortly after 1810 made it the chief illuminant; Paris began its use in 1820 ; Baltimore in 1821; Boston in 1822 ; and New York City, which did not intro-

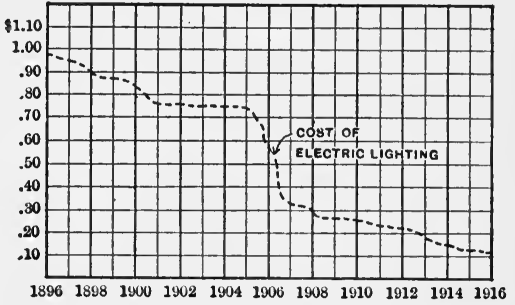

Fig. 44 A. - Graphic illustration of the decrease of cost of electric lighting in twenty years. duce it before 1823, had it in common use by 1827 .

60. Modern lighting. - The chief means of illumination to-day are kerosene oil, electricity, and artificial gas, which may

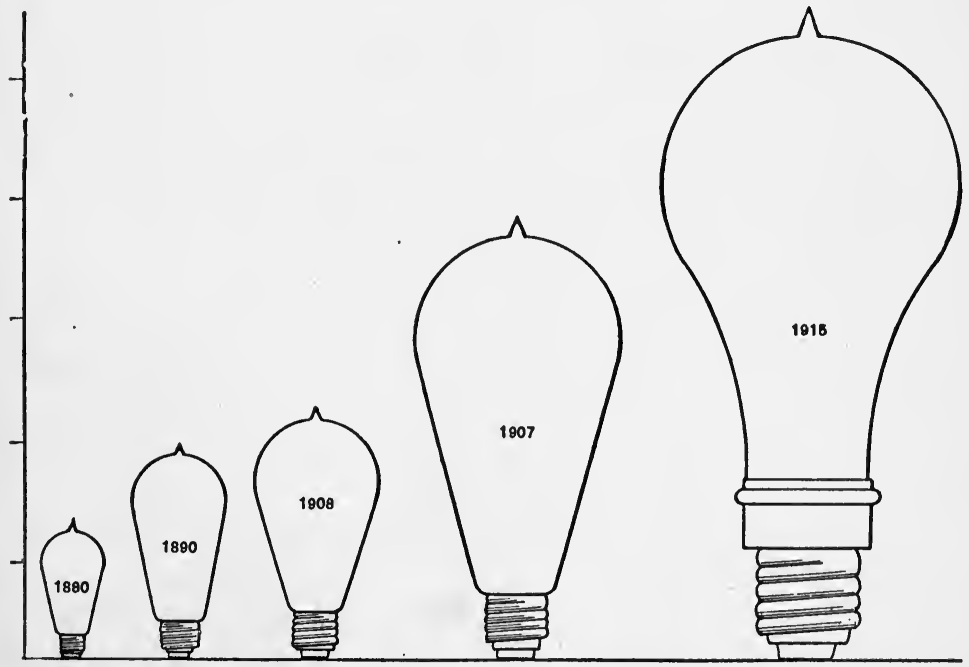

FIG. $44 B$. - The cost of electric current for lighting has decreased greatly since the year 1880 . The relative candle power purchased by the same money in given years is shown graphically. 
be enriched water gas (page 162), coal gas (page 163), acetylene or gasoline gas. The clean, convenient, and comparatively cool electric light is rapidly taking the place of all others. It has shown already (as illustrated in Fig. $44 A$ and $B$ ) an interesting decrease in cost to the consumer. Electricity bids fair to come into more general use for heating and cooking as well as for lighting, as the cost of production and transmission over long distances is reduced. Recent improvements in the incandescent lamp make electric lighting very efficient and of relatively low cost when convenience is considered. In this way it has come to pass that in contrast with the old-time means of lighting, the electric arc and the tungsten incandescent turn night into day, making possible the uninterrupted continuance of the world's work and play.

\section{Illuminating Gas}

61. The Welsbach mantle. - Certain interesting improvements have been made in the use of artificial gas for lighting. The most important of these is the Welsbach gas mantle, which gives a satisfactory incandescent light and makes illuminating gas not only less offensive, but also less expensive to the consumer. It has also made gas an active competitor with electricity in localities where the apparent cost of production or other conditions have prevented the general use of electricity for lighting. The graphic illustration (Fig. 45) shows the number of hours of twenty candle power light that each of the principal illuminants will give for ten cents. From this it is easily seen that the gas mantle occupies an important place among the modern means of illumination.

How the gas mantle is made. - The gas mantle is an interesting achievement from the point of view of science. It illustrates what may be accomplished through a knowledge of chemistry. The mantle is made of two rare metals or "rare earths," as they are generally called. One of these is the metal thorium, which is found mostly in North and South Carolina 
and in Brazil. The other is the metal cerium, which is found in the same places. These two metals are combined in the exact proportion of 99 per cent of thorium to 1 per cent of cerium. The exact one per cent of cerium multiplies the light from a thorium mantle nearly ten times, yielding a whiter, brighter light than any other proportion.

Thorium and cerium are dissolved in nitric acid. Mantles made of mercerized cotton or other suitable material sewed

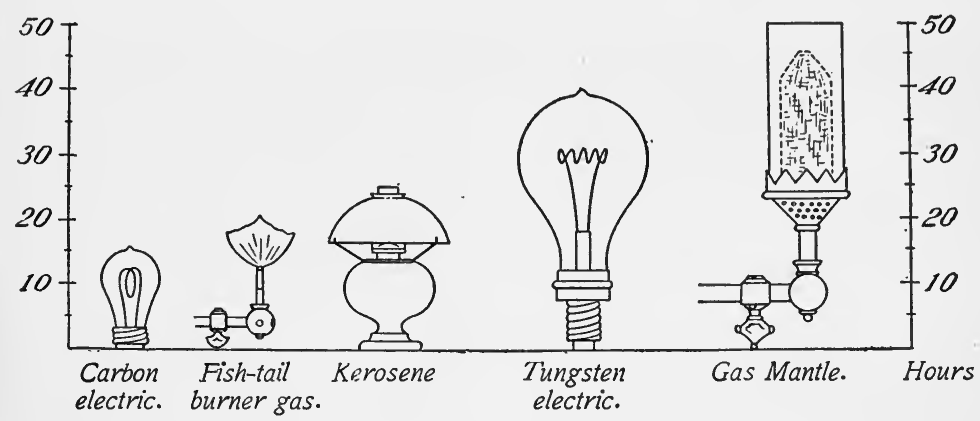

Fig. 45. - Graphical representation of hours of light of twenty candle power purchasable for ten cents. Compare carbon with tungsten incandescent, and fish-tail burner with gas mantle ; which of each set is more economical? Why?

into the desired shape with asbestos thread are dipped into and thoroughly saturated with this solution. The mantles are then burned to remove the gauze and to convert the metals into thorium oxid or thoria and cerium oxid or ceria. The mantles after burning are dipped into collodion and dried to insure their safety in distribution through the market. This dried film is burned away after the mantle is placed on a gas jet. The oxids remain as ash in the original form of the mantle. The economy of gas by the use of mantles is considerable, a good mantle consuming about one half the gas needed for the ordinary gas burner, and giving five to seven times the amount of light.

62. Artificial gas. - Artificial gas is to-day usually coal gas or what is generally called illuminating gas. The latter is an 
enriched water gas, the production of which is suggested in the experiment on page 119. Its manufacture may be briefly described.

Water gas. - The principal parts of a water gas plant are the generators, the carburetor, and the superheater. The generators are tall, vertical furnaces in which anthracite coal or coke is heated to a white heat. Dry steam is admitted through the ash pit and the grate bars and passing through the incandescent coals or carbon is decomposed into its elements, oxygen

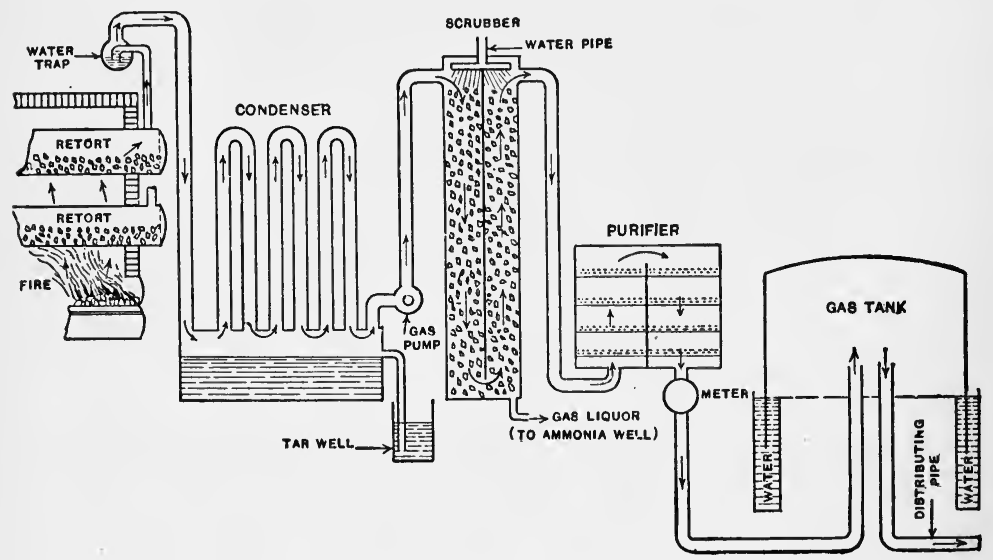

Fig. 46. - Diagram of a coal gas plant.

and hydrogen. The oxygen combines with carbon to form carbon monoxid. The carbon monoxid and hydrogen mix and form what is really water gas.

Water gas is not luminous. It is made so by mixing it with vaporized petroleum oils. The oils are sprayed into the heated carburetor and instantly vaporized. They mix with the water gas and are then conducted to the superheater which is heated to a very high temperature. The superheater breaks down or cracks the oil gases into hydrocarbon compounds which have a stable character and form the enrichment of the resulting gas. 
In some plants a scrubber is used, the work of which is explained in the description of coal gas manufacture.

Illuminating gas contains a large proportion of carbon monoxid, which is odorless and poisonous. Water gas, a mixture of hydrogen and carbon monoxid, is very dangerous because of the impossibility of detecting it by its odor when it escapes. For this reason water gas is enriched with odorous gases from oils which also make it luminous.

Coal gas. - The manufacture of coal gas may well be studied directly at a gas plant or by means of a model plant. The diagram (Fig. 46) illustrates the chief parts of the plant, and with the description of the processes may serve as a guide in studying the actual plant. The general process is known as destructive distillation. Coal, either bituminous or semibituminous, is used. It cannot be distilled and condensed as coal, but is broken down into simpler substances by the application of heat. The distilled product is gas; the by-products coke, ammonia, and coal-tar turn out, however, to be more valuable commercially than the gas.

A ton of fairly good coal yields by destructive distillation the following:

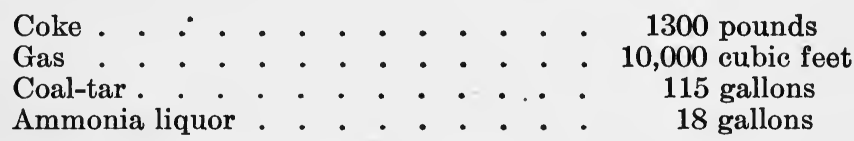

By-products. - The coke is used in producing heat for retorts, or sold for household heating. From the ammonia liquor ammonia gas or ammonia water is obtained. The usual method of making ammonia commercially is first to mix the gas liquor with slaked lime, and then to boil the mixture so as to drive off the ammonia gas. The gas is purified by being combined in solution with dilute sulfuric acid. This solution -may be evaporated to form crystals of ammonium sulfate, or it may be heated with slaked lime to drive off the purified ammonia gas. For distribution the gas is either compressed 
in tanks, or combined with water to form ammonia water (ammonium hydroxid). Ammonia water is highly concentrated, many times stronger than that sold for household use, and is employed generally for cleaning purposes, such as removing certain kinds of stains and grease spots from articles. The ammonium sulfate is one of the most valuable fertilizers, furnishing a large amount of nitrogen in a form directly available for plants. Ammonia gas is widely used in refrigeration (page $151 \mathrm{ff}$. ), and in the preparation of carbonate of soda (cf. the Solway Process in any book on industrial chemistry).

Coal-tar. - Coal-tar is the substance with which many of the modern miracles of constructive chemistry have been performed; it is a veritable treasure-trove as treated by the skillful chemist. Under proper treatment coal-tar yields a number of substances called hydrocarbons, which form the basis for many kinds of products contributing to the health, beauty, wealth, and destruction of life. The principal hydrocarbons derived from it are benzene, naphthalene, anthracene, toluene, and phenol, commonly known as carbolic acid.

From these basic substances many valuable products are derived. Phenol is converted by the action of concentrated nitric acid into picric acid used in explosives. Other products from these basic substances are aniline and many dyes derived from it ; alizarin, purpurin, and many other dyes from these; drugs such as phenacetin, acetanilid, salycilic acid, antipyrin, sulfonol, and many anæsthetics and disinfectants; some of the finest perfumes; flavoring essences, as lemon, vanilla, bitter almonds, etc.; saccharin, which is five hundred times sweeter than cane sugar but of no food value; art materials, such as printing inks, photographic developers, varnishes, etc. Indeed the old jingle is apt :

" There's hardly a thing that a man can name

Of use or beauty in life's small game, But you can extract in alembic or jar From the 'physical basis' of black coal-tar - 
Oil and ointment, and wax and wine, And the lovely colors called aniline;

You can make anything from a salve to a star, If you only know how, from black coal-tar."

\section{Electric Lighting}

63. Types of electric lamps. - Lighting by electricity is rapidly increasing in all parts of our country. This kind of lighting offers many interesting points for study, some of which may be taken up here for introductory consideration. There are three well-known and quite distinctive types, the incandescent, the arc, and the vacuum tube. We shall begin with the study of the incandescent lamp.

Exercise. - Examine a lamp elosely; if a worn-out bulb is available, it may be examined. To open it without danger to oneself and to the filament, heat it near the base with the flame of a blowpipe. When the glass becomes soft enough, it will be forced in gradually by the air. Collect the parts of the filament for examination. Heat a piece in a hot flame. Does it burn?

64. The filaments. - Filaments were formerly made of bamboo or cotton-wool ; but to-day they are mostly of metals, as tungsten, tantalum, thorium, and cerium. The bamboo or cotton-wool filament was converted into carbon and then placed in the glass bulb. Tungsten is rapidly displacing the carbon filament. The original tungsten filaments were very fragile, but with recent improvements in the preparation of the metal for this purpose the filaments are now tough and durable and the tungsten lamps may be handled as freely as the carbon incandescent lamp without undue risk.

Observe the way in which the filament is supported and arranged in such a lamp as the "Mazda" (Fig. 47). Several lateral wires are fused in each end of the glass rod which is a continuation of the glass, through which the conducting wires pass. A single continuous filament of tungsten is wound up and down on these supports. The ends of the filament are fused to the respective ends of the conducting wires. 
The conducting wires through the glass stem are of platinum. Platinum is the only metal that expands and contracts under the influence of heat at the same rate as does glass. If a metal

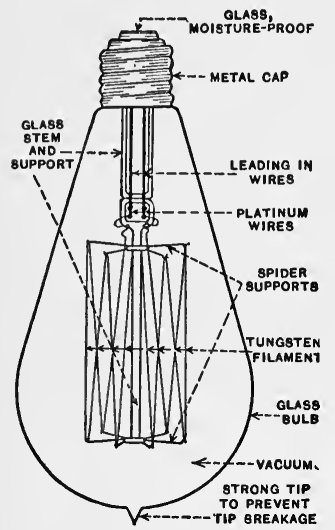

Fig. 47. - The structure of a tungsten filament incandescent lamp. with a different rate of expansion were used, it would cause leakage or breakage between the wires and the glass. Examine the way in which platinum wires are connected with the brass base, and the way in which the connection is made with the service wires through the receiving socket.

Comparison of carbon and tungsten filaments. - The diameters and lengths of carbon and tungsten filaments are very different. The carbon is thick-and relatively short, perhaps only one eighth as long as the tungsten filament. The tungsten is much finer and may be a yard or more in length. Why are there these differences?

Let us consider first a few facts about the metal tungsten. It is a comparatively rare metal, found chiefly in mines in Colorado, Montana, and California. The market quotations show that it is a valuable ore, worth about $\$ 6.50$ a pound. It is one of the heaviest and hardest of metals, being hard enough to scratch glass. What precious stone does this? Of what are glass cutters made? The melting point of tungsten, or rather the temperature at which it vaporizes, is very high; the tungsten filament vaporizes at about $3200^{\circ} \mathrm{C}$., or $5792^{\circ} \mathrm{F}$. It does not melt as ice or iron, but vaporizes directly. In this respect it is like carbon, which in the arc lamp and in the incandescent lamp vaporizes at high temperatures. The black in an incandescent bulb is due to the vapor from the filament.

The temperature of the tungsten filament may be $250^{\circ}$ higher than that of the carbon filament and not vaporize. Because of 
this higher temperature it produces more light. This accounts for the high luminous efficiency of the tungsten lamp; the more intense the heat of the filament without vaporizing, the brighter or more incandescent will be the light.

65. Conductivity of substances. - We take up now the study of the fineness and length of the tungsten filament in comparison with the carbon. In the study of heat transfer (pages $94 \mathrm{ff}$.), it appeared that different substances conduct heat with different degrees of facility. Some metals, such as silver, copper, aluminum, and iron, are regarded as good conductors; other substances are medium, and still others, such as wood, glass, paper, liquids, and gases, are so poor as to be considered heat-insulators. In the transmission of the electric current, substances show similar differences as conductors. The wide use of copper, iron, and aluminum wires suggests that these may be good conductors, and exact measurement shows them to be among the best conductors of electricity, as they were found to be of heat. Silver is the best conductor, copper next, and aluminum third.

The common practice is to speak, not of the facility with which materials conduct, but of the resistance which they offer to the electric current. It is useful to know what are good, indifferent, and bad conductors. For conductors are good or bad according to the amount of resistance they offer to the electric current. There are, moreover, no perfect conductors, that is, none that at usual temperatures would have zero resistance. And there are no substances which are perfect non-conductors or resist completely the passage of electricity. There are substances which transmit very little of the electric current and they are called insulators. Such substances include glass, rubber, mica, paraffin, dry wood, paper, etc., but they are insulators only at usual temperatures and with a certain strength of the electric current.

A list is given of common substances arranged in accordance with the resistance they offer to electricity. A perfect conducting substance would have zero $(0)$ resistance. The others 
follow in order. It must be assumed that all materials are of the same length, the same thickness or area of cross section, and at the same temperature, as $32^{\circ} \mathrm{F}$.

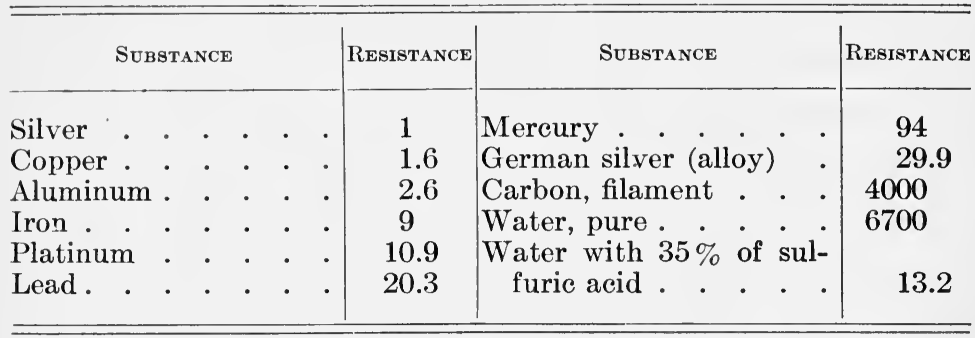

On the same scale, such materials as glass, rubber, and paraffin, which are commonly classed as insulators, would have several million times as much resistance as silver.

Resistance and temperature. - Resistance changes with temperature. Glass, for example, is an excellent insulator at ordinary temperatures, but at $200^{\circ} \mathrm{C}$. or $392^{\circ} \mathrm{F}$. it becomes a fairly good conductor. The carbon filament in the incandescent lamp has a high resistance at room temperature; but at operating temperature it loses fifty per cent of its resistance. Certain other substances have the same property. The glower of the Nernst lamp is made of certain rare earths of volcanic origin, and at usual temperature has so great resistance that it is a non-conductor. But when heated to a given high temperature, as it is in this kind of lamp by a special device, it allows the electric current to pass and is heated sufficiently to radiate light.

Certain other substances, such as tungsten, copper, aluminum, and silver, behave in the opposite way. . The tungsten filament of a lamp of candle power equivalent to that of a carbon lamp has at room temperature only one twelfth the resistance that the carbon filament has; but at the temperature of operation its resistance increases more than twelve times its original amount, so that it then has more than twice the resistance of 
the carbon filament in operation. Tungsten increases in resistance with the rise and loses with the fall of temperature. Carbon decreases with the rise and increases with the fall of temperature. Copper, aluminum, mercury, platinum, and silver behave as tungsten does, with change in temperature.

An interesting experimental result of unusually low temperatures in modifying the resistance of certain metals to the electric current may be mentioned. Metals at the temperature of liquid air show a marked decrease in electrical resistance. What is the temperature of liquid air? Recently it has been discovered that in the neighborhood of $-270^{\circ} \mathrm{C}$, $-454^{\circ} \mathrm{F}$., or $3^{\circ}$ Absolute, certain metals, as tin, lead, mercury, and platinum, show a most remarkable drop from a measurable resistance to practically no resistance to the electric current. An electric current, started in any one of these metals at this temperature or lower, continues indefinitely without the slightest sign of diminution.

Resistance and dimensions. - Length and thickness of the substance determine the amount of resistance a given material may offer to an electric current. As regards length it is generally true that the longer the wire of a given thickness, the greater is the resistance it offers. This may be demonstrated experimentally.

Exercise: Resistance and length. - Connect one "dry cell" with one hundred feet of No. 26 copper wire and this with an ammeter. What is the strength of the current shown by the ammeter? Record it. Connect cell and ammeter with fifty feet of the same wire. What does the ammeter show? Compare the readings. This strength of the current from the shorter wire will be about twice that delivered by the longer wire. Hence it generally holds that with a constant pressure the current is inversely proportional to the length of the conducting wire, the thickness remaining the same.

Exercise: Resistance and thickness. - Tests may be made with wires of different thicknesses or areas of cross section. One will find that the thicker the wire, the less is the resistance it offers, or the greater is the strength of the current which it delivers, as shown by the ammeter. The smaller the area of the cross section of a conduct- 
ing wire, the greater is its resistance to the eurrent. It resembles the effect of the size of a pipe upon the flow of water; the larger the pipe the greater the flow, and the smaller its area of cross section the less the flow. It is readily seen that this is an important factor in the selection of the size of wire for transmission of electricity. Examine a Brown and Sharp wire gauge and become familiar with several diameters of copper or other wire and their numbers according to the "B \& S " gauge.

Application of these facts to the tungsten. - What we have learned as to the relation of temperature and dimensions of the conducting material to its resistance to an electric current has to be applied to the tungsten filament. This is found to be thinner and much longer than the carbon filament of a lamp of corresponding candle power. It is at once evident that the smaller diameter and the greater length must increase the resistance to the electric current. It is plainly intended to make the electric current do the largest amount of useful work in its passage from entrance to exit. This is precisely what every electric appliance in the home is designed to do, - to make the electric current entering the house by one wire do the required amount of useful work, heating or lighting, in passing through the appliance to the wire by which it leaves the house.

The resistance of tungsten increases with rise in temperature. The higher the resistance, the greater will be the heat produced by the electric current in passing. The vaporizing temperature of tungsten is very much higher than that of carbon, as used in the vacuum of the incandescent bulb; it is, therefore, at once evident that it can be made more nearly "white hot" or incandescent, thus giving a brighter and whiter light than the carbon lamp.

To summarize: the greater the resistance of any conducting medium to an electric current, the medium remaining a conductor, the hotter the medium will become. And the longer the filament used as a radiating area, with its thickness and other conditions remaining the same, the greater will be the amount of light it will produce. 
In the usual incandescent lamp bulb there is a vacuum. But in some of the latest products the bulb is filled to about two thirds of normal atmospheric pressure with nitrogen. If air were present, the electric current would heat the filament to kindling temperature, when it would burn out. In modern lamps the aim is to reach a natural standard of 100 per cent efficiency. This standard is set by the glowworm or firefly, which produces light without heat. But at present artificial lamps fall far below this level, the carbon filament being about 3 per cent efficient; the tungsten 10 per cent; the electric arc 17 per cent; and the mercury-vapor glass tube about 6 per cent. The gas light with the Welsbach mantle is 5 per cent efficient, which is also the grade of the acetylene light.

66. Comparative life, cost, and efficiency of lamps. - It is useful to compare the life, cost of operation, and efficiency of the carbon and tungsten filament lamps. The life of the carbon filament in a 16 candle power lamp is rated at 800 hours maximum. The metal filament is rated at 1600 hours maximum. The carbon lamp consumes about 3.1 watts per candle power, or $3.1 \times 16$ or 49.6 watts per lamp. During its life of service it consumes $49.6 \times 800$ or 39,680 watts. A thousand watts for one hour make one kilowatt hour, so that the carbon lamp consumes 39.6 kilowatt hours of electric current. If electric current costs 10 cents per kilowatt hour, the cost for the operation of the lamp is $\$ 3.96$.

The metal filament lamp is rated to consume 25 watts per lamp. Multiply its life term of 1600 hours by this, and the product is 40,000 watts or 40 kilowatt hours. This at the same rate costs $\$ 4$, for the electric current. But the life of the tungsten is twice that of the carbon, which would make one tungsten equal two carbon lamps and puts the cost for current for the one, $\$ 4$, against the cost for current for the other two, $\$ 7.92$. The saving by the metal filament is worth while. 
The average candle power of the carbon filament is 13.2 , or 2.8 less than the commercial rating. The average candle power of the tungsten, sold as a 16 candle power lamp, is $\mathbf{1 6 . 5}$, or .5 more than its commercial rating. Late improvements have increased the normal life of the tungsten filament and its candle power, and have decreased the amount of electricity required per candle power.

In general, the tungsten filament lamp consumes about one third less electric energy than a carbon lamp of the same candle

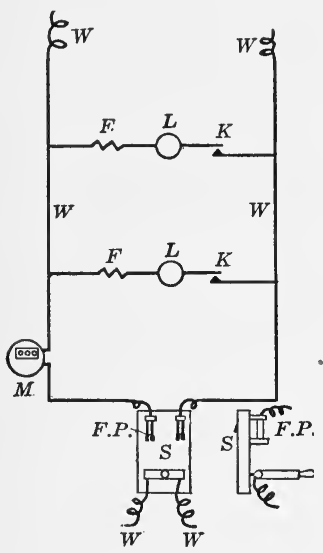

FIG. 48. - General plan for wiring house with incandescent lamp connections $(L)$, keys $(K)$, main switch (S), fuse plug $(F . P$.$) , and meter (M)$. Fuses $(F)$ to protect lamps from excess current are located conveniently. power and lives twice as long. This makes for the reduction of the cost of electric current for the consumers, and makes possible a larger use of electricity for lighting.

67. Wiring a house. - The diagram (Fig. 48) illustrates the chief features of a wiring system for electric lighting. The main switch is placed in the basement or any convenient location for connection with the main service wires and the house wires. It is so located as to enable one to cut off the electric current outside the meter. The main switch is usually a knife switch with cartridge fuses or fuse plugs. The fuses protect the house lamps and occupants from injury in case of excessive pressure of the current, and from fire in case of a short circuit in the house system. The wires are sometimes run through conduits of insulating and fireproof material. Inside switches are conveniently located in the circuit to control the lighting. The sockets in which lamps are placed are generally provided with fuses which withstand a limited pressure and thus protect the lamp. The two main wires make possible a complete circuit for the electric current (see page 328 ). 
68. Measuring electrical energy. - An electrical meter is placed in the house circuit to register the amount of electrical energy consumed. The amount is indicated by the positions of the hands over the dials. The dials indicate the energy consumed in kilowatt hours. A kilowatt hour is equal to 1000 watt hours. Suppose that there are 40 of the 25 watt tungsten lamps in the circuit. These in operation would consume electrical energy at the rate of one kilowatt (40 lamps of 25 watts each equal 1000 watts). If the lamps are kept in operation for one hour, the dials on the meter will indicate a reading of one kilowatt hour. How long must twenty of such lamps be operated to show the same amount of energy consumed? Ten lamps? One lamp?

Reading the meter. - It is a simple matter to read the meter. The first dial shows up to ten kilowatt hours, each division being equivalent to one kilowatt hour. The second dial shows ten kilowatt hours for each division or one hundred for the complete circle. The third registers 100 kilowatt hours for each division of the dial or 1000 for the circle. A fourth indicates 1000 kilowatt hours for each division.

Exercise. - Suppose, for example, the previous reading of a meter was 1362 ; and the present reading is 1379 . The difference, or 17 , indicates the amount of electric energy consumed, or 17 kilowatt hours. If the rate charged for electric energy is ten cents per kilowatt hour, the total amount of the bill for the kilowatt hours consumed would be $0.10 \times 17$, or $\$ 1.70$.

The readings for the previous state of the meter are shown in Fig. 49 by dotted lines; and the present state by the heavy lines. How many kilowatt hours have been consumed since the last reading? What would be the cost at the rate of 12 cents per kilowatt hour?

The electric current should do useful work. - The electric current which enters the house by one wire should be made to do the largest amount of useful work in any appliance before it is allowed to leave by the other wire. It is always cheaper to use the best electrical apparatus not only for lighting, but 
for heating, cooking, and power. A poorly constructed lamp, flat iron, oven, or motor consumes more current than necessary in doing the required work. For this reason it is economy to employ only the best made and most efficient appliances.

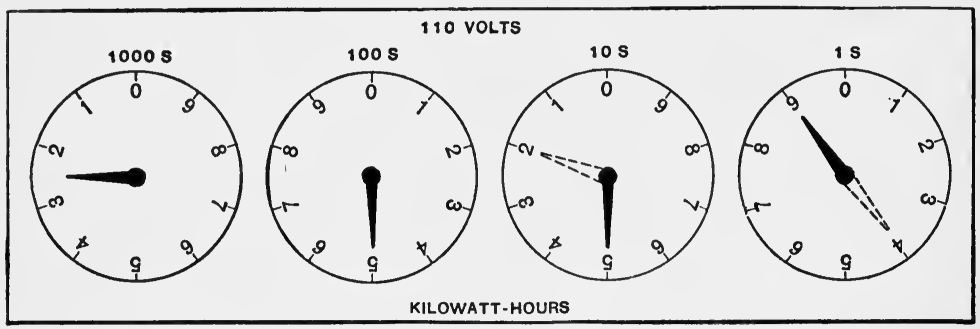

FIG. 49. - Kilowatt-hour electrical meter. Each division of the right hand dial indicates one kilowatt hour.

69. The arc light. - The mechanism controlling the carbons of the arc light, whether in the old style or in the later forms, such as the flaming arc, can be understood more readily by direct observation than from description. The "hand-con-

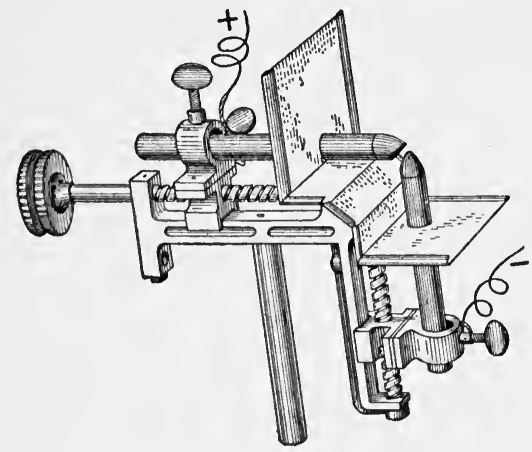

Fir. 50. - Arc lamp, controlled by hand. trolled" feed as used often in the arc light for a stereopticon (Fig. 50) is perhaps most instructive in getting the fundamental principles. In the automatic arc lamp several kinds of mechanism are employed, one or more of which you may find available for study. These are the gravity feed, clockwork, clutch, hot wire, and motor feed. A magazine arc lamp, one with six or more pairs of carbons, which are supplied as required to give a long, continuous flame, is interesting, and may be made the subject of a report by a member of the class. 
Catalogs illustrating the chief features of the mechanisms may be obtained from the local light and power plant.

In the arc lamp the carbons are arranged to meet where the light is produced. When the electric current is turned on, the carbons are drawn apart about one fourth of an inch. The current meeting the resistance of the air heats the ends of the carbons sufficiently to volatilize them. The carbon vapor streams across the break between the ends, forming the arc. The vapor is heated white hot by the electric current and makes the arc luminous.

The temperature at the crater of the arc at ordinary air pressure may reach as much as $4000^{\circ}$ to $4500^{\circ} \mathrm{C}$. This is taken as the melting or vaporizing point of carbon. It is interesting to note that in recent experiments with the carbon arc under different pressures unusually high temperatures have been obtained. Under a pressure of about 20 atmospheres (what is one atmosphere?) a brightness eighteen times that of the ordinary arc has been obtained. This indicates a temperature of about $7800^{\circ} \mathrm{C}$. (What is the equivalent on the Fahrenheit scale?) It may be possible to make use of high pressure in arc lamps. At such temperatures as are obtainable with increased pressure or even at that which is supposed to be the sun's temperature, about $6000^{\circ} \mathrm{C}$., the light produced would, within a limited area, equal sunlight in quality and intensity.

Inclosed arc. - The carbons are consumed very rapidly in the openair arc lamp. Recently the in- Frg. 51. - The inclosed arc lamp. closed arc (Fig. 51) has proved to be more economical and has driven the open-air arc practically out of the field. In this type of lamp the arc is inclosed in a glass globe which admits a very small amount of air and consequently the carbons last several times as long. 
Types of arc lamps. - The carbons are arranged in various ways. They may be vertically superposed as in the old arc lamp and in some of the latest flaming arc lamps, or they may converge (Fig. 52) as the two sides of an acute triangle; or

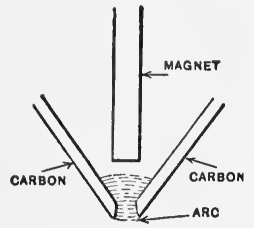

F I G. 52 . - A n arrangement of the carbons for the flaming arc light. again they may be made, as in the electric lamp of a stereopticon (Fig. 50), to meet at an angle of $45^{\circ}$. The light of the flaming arc when produced by carbons that incline and converge is thrown downward rather than horizontally. The need of horizontal distribution has led to the invention of the flaming arc with the carbons, as in the old-style arc lamp.

Composition of carbons. - The carbons used in the flaming arc light differ from those of the ordinary arc in one important respect. Certain metals are combined with the carbons for the flaming arc, which give a different quality and intensity of light. The most common of the flaming arc lights is the "yellow." This is produced by making the core of the carbon of calcium salts. The usual yellow flaming arc gives from 2500 to 3000 candle power. The so-called red light is produced by a core composed of strontium salts; and the pearl white light by barium salts. In these arcs the metals are vaporized and furnish about thirty per cent, and the vapor of the carbon about seventy per cent of the light. In one style of the arc lamp the upper rod is made of hard copper instead of carbon and lasts from 7000 to 8000 hours. The lower rod is an iron tube filled with the mineral magnetite, which burns for about 200 hours.

The recent improvements in the tungsten incandescent lamp have made it a competitor in street illumination with the arc lamp of all types. In many cities the artistic lamp stand with a group of five or more lamps is rapidly replacing the glaring arc and giving a lighting effect which the most highly improved arc cannot produce.

70. Industrial illumination. - The illumination of modern 
industries has opened a field for a very different and interesting type of electric lamp. In all industrial work where artificial light is necessary, certain definite requirements are made as to the quality, intensity, and distribution of the light. A light in any workroom must be intense enough without glare to enable the worker to see easily and clearly and not to blind or fatigue the eyes. It must be steady, unflickering, and must not cast sharp and deep shadows. The matter of safety and cost is of great importance.

The usual standard of light is sunlight or daylight. There is not at present any artificial light which equals sunlight in quality, intensity, and distribution. Sunlight is "white light," a light, that is, in which no color predominates. In artificial lights of the incandescent or arc types all the colors are combined but in a different proportion from that of daylight. The result is that the artificial lights do not give a perfect "white light."

It is known that certain colors are more fatiguing to the eye than others. Those who have experience in developing photographic negatives, know that red light has no effect upon the film or plate; while the colors at the other end of the spectrum (page 180) have a chemical effect or are photographically active. One cannot see, however, as well by red light as by yellow or green. Experiments prove that a pure green light is about eighty-three times as efficient for seeing purposes as a red light. It is also known that a one-color light is better in illumination than a many-color light. It has what is called the quality of visual acuity or sharpness. It brings details out clearly. These conditions are largely met by what are known as vapor lamps. Such lamps give light only by means of luminous vapor or gases and they are restricted to certain colors of the spectrum. The best known are the Cooper-Hewitt mercury vapor lamp and the Moore vapor lamp. The latter makes use of carbon dioxid as the gas and gives a white light. The Cooper-Hewitt lamp is widely used in industries of almost every kind, such as metal 
working plants, textile, glass, paper, and clothing manufactories, in newspaper and printing establishments, in power houses, and in photographic studios.

The mercury vapor lamp. - The Cooper-Hewitt mercury vapor lamp (Fig. 53) comprises a clear glass tube that may be twenty to fifty inches long, a reflector-holder, and an auxiliary. The reflector-holder supports the glass tube and is connected with the auxiliary. The reflector is usually polished white porcelain and flat for general light distribution or curved for

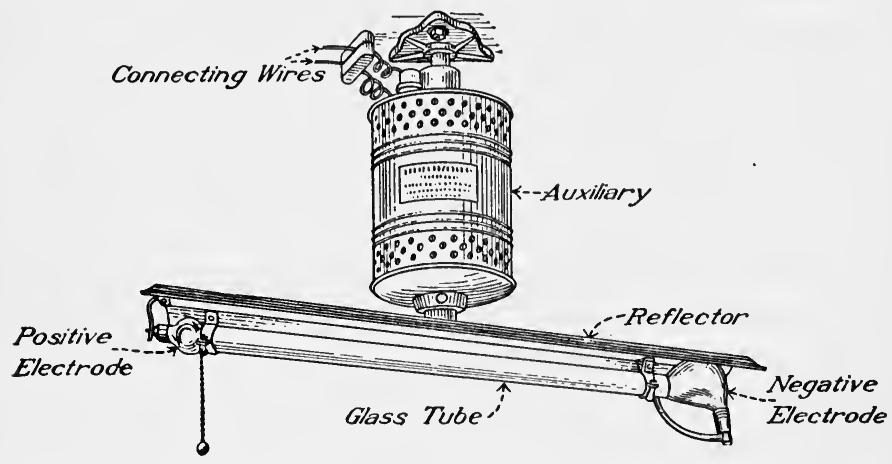

Fig. 53. - A Cooper-Hewitt Mercury Vapor Lamp.

concentrated light. The auxiliary contains the mechanism for starting the lamp and regulates the supply of electric current. One end of the tube is oval and contains a small amount of metallic mercury. Both ends contain electrodes. The end containing the mercury is called the negative electrode, which means the way by which the electric current leaves the tube. The other end is the positive electrode or the way by which the current enters the tube. In each end of the tube are sealed platinum. wires which serve as terminal connections. The air is exhausted from the tube before it is sealed.

Quality of mercury vapor light. - The quality of the mercury vapor light is peculiar as may be observed in its effect upon certain colors. Blue and green are made more intense; the 
red of one's lips appears dark or greenish black; and the white of one's skin becomes a ghastly white. White and black, which contain no admixture of colors, remain entirely normal. This is exactly the effect needed in the artificial illumination of offices and factories where there is no necessity for color discrimination.

When the rays of the mercury vapor light are analyzed by means of a glass prism, the spectrum (page 180) shows only green, blue, or violet, and faint yellow rays of light. There is no red in the light, which explains why the red color of objects is modified when seen in such light. This raises an interesting question which we shall consider in some detail.

\section{The Colors of Objects}

71. Upon what do the colors of objects depend? Do colors depend upon the nature of the objects alone? Do they depend upon the quality or kind of light falling upon them? Or do they owe their colors both to their own nature and to the quality of light which falls upon them? We can only suggest to you a way to find an answer to the question.

Common opinion may insist perhaps that the color of an object depends upon the object alone and cite the familiar fact that grass is green and the buttercup yellow, as every one knows. But is grass green and the buttercup yellow in all kinds of light? One's lips are certainly not red in the greenish light of the mercury vapor lamp.

It is easy to produce lights that do not have all the colors of the rainbow or spectrum, but are entirely lacking in one or more colors. Then we can bring within the range of such light certain objects with colors which we know in daylight and we can observe the effects. One such light may be prepared in the following way.

- Exercise. - Mix a tablespoonful of common salt with some denatured alcohol and put the mixture into a shallow dish, such as a saucer. Have some differently colored papers numbered or with labels on which the names of the colors are written. Darken the 
room and light the mixture. Try the various colors in the light. How does white appear? How do red, blue, green, and other colors appear? What change is made in the yellow? What does this experiment show as to the dependence of colors upon the nature of the objects?

The light produced by the salt-alcohol flame is shown by the glass prism to be yellow. Does this help you to understand why yellow alone of all the colors tested seems to be normal? Why does the white of one's complexion become "ghostly white"? Do you think it possible or probable that objects reflect only those colors which fall upon them? Or does it seem more probable that the color of an object depends both upon the nature of the object and upon the quality of the light falling upon it and in which it is seen?

Exercise: What the prism shows. - The following experiment will bring out somewhat more clearly the important facts. Into a dark-

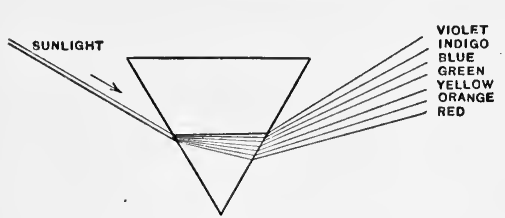

Fig. $54 A$. - Sunlight is analyzed by a prism into different colors. sible. This experiment shows also that sunlight, which we usually call white light, is really composed of many colors. With a second prism, placed as in Fig. $54 B$, this point will be emphasized. The second prism combines the colored rays into which the light is decomposed by the first prism, and throws on the screen a ray of white light.

The band of colors projected on the screen by the prism is called a spectrum. Note the arrangement of colors of the

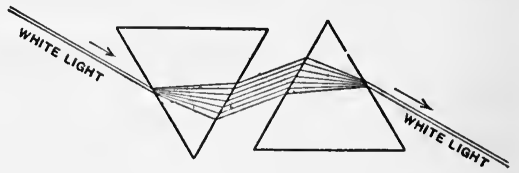

Fig. 54 B. - The different colors as analyzed by the first prism are synthesized into white light by the second prism. spectrum, passing from red at one end through orange, yellow, green, and blue to violet at the other end. Do the colors stand out clearly and distinctly separate from one another?

Pass through each color successively the pieces of colored paper which you used in the experiment with the salt-alcohol light. Observe the intensifying effect of the yellow ray upon yellow paper; but 
note that other colors are not rejected or reflected by the yellow object. Try red paper in the green and yellow of the spectrum, and note results. Does the red tend in the green light to become a greenish black as stated in connection with the color effect of the CooperHewitt light? What do you find to be the effect of the colors upon a white object? How does black appear in each color?

The facts seem to support the conclusion that the color of an object depends upon the nature of the object itself and upon the quality of the light which falls upon it. A yellow object is yellow largely because it reflects or rejects yellow light. It absorbs the lights of other colors. A pure white does not absorb any of the colors of the spectrum, that is, it rejects or reflects all of them to the eye. Black, on the contrary, rejects none of the colors, but absorbs all, and remains black, therefore, in all qualities of light.

\section{General Requirements in Artificial Lighting}

72. A good light. - The types of lamps which we have considered are sufficient to indicate the main tendencies in artificial illumination. Many other appliances for gas and electric lighting are available, but they generally depend upon the principles involved in the incandescent gas mantle, the carbon or tungsten filament, in the arc, and in the mercury vapor or vacuum lamp. They illustrate the same fundamental points as to incandescence, resistance, radiation, and vacuum, in more or less different forms.

The commercial production of lighting appliances aims to furnish an economical, efficient, and hygienic light. The initial cost of the appliance, the cost of maintenance and operation are always important in any form of lighting. The quality and quantity of light produced are considered in connection with the amount of gas or electric current consumed. The quality of light and especially its hygienic characteristics are of very great importance to the consumer. The essentials of a thoroughly hygienic, safe, and useful light may be made topics 
for special studies and reports. The following summary may serve as a guide in the examination of various kinds of lights as to the points of practical importance.

First. A thoroughly satisfactory artificial light should not injure or endanger life or property.

Second. It should be uniformly bright, steady, and so well diffused as not to cause eyestrain, or undue contraction of the pupil, both of which effects, if prolonged, will promote several kinds of eye trouble. The light radiated through a frosted bulb is diffused, that from a filament concentrated. Which light produces less eyestrain?

Third. A light should not throw out dangerous or disagreeable gases, or rob the air of its oxygen in excessive measure. Gaslight is regarded as unsatisfactory on this account. One burner consumes about five times the amount of oxygen needed by an average adult person. Carbon dioxid is produced in correspondingly large amounts. If gas is incompletely burned, its contamination of the air in a poorly ventilated room is inevitable, and special attention must be given to ventilation.

Fourth. Artificial lighting appliances should be safe and convenient for such handling as may be necessary. Movable lights should be protected and reasonably safe. How are electric lighting fixtures and appliances made safe for handling? Are special lamps made for special conditions, as in mines where gas or fire damp may occur?

Fifth. An artificial light, to be perfectly satisfactory, should not radiate heat in considerable quantity. Heat radiation means loss of light efficiency and discomfort in hot weather. It increases danger to property from fire. The latest tungsten lamp approaches the ideal of the firefly which gives light but no heat.

Sixth. A light should have such a quality as to blend completely all colors which it may contain, and allow no single color to predominate, that is, for interior illumination of homes or places where color is important, a white light is indispensable. 
For special uses, as in printing establishments, for photography, in accounting offices, where color discrimination is not required, certain colors may be absent or one color predominate, as is the case with the mercury vapor light, which has no red rays. Such a light may have hygienic advantages. For exterior illumination as in a street, where quantity of light is of first importance, a single color may strongly predominate, as in the yellow flaming arc. This gives far more light than the red or the white arc light.

Seventh. These items indicate that a thoroughly safe, useful, hygienic light must in its limited range approximate the light of the sun in steadiness, diffusion, intensity or brightness, and quality or whiteness.

The standard of lighting. - A room that is well lighted by day may be taken as the standard by which the artificial illumination of the same room may be determined. Study the way in which diffused sunlight is distributed in a room with ample windows and preferably a northern exposure. Note that the ceilings are only moderately lighted, but that the lower part of the room is abundantly and brightly lighted. Then compare with this the artificial lighting of the room, and observe especially the light distribution and concentration.

\section{SUMMARY}

Progress in artificial lighting is indicated by the use of such means as the torch, fat, oil, gas, and electricity.

Illuminating gas was first used in England about 1805.

The Welsbach mantle increases the illuminating efficiency of gas. Illuminating gas is either water gas or coal gas.

Water gas consists of hydrogen and carbon monoxid and is the product of steam and hot carbon.

Coal gas is a product of the destructive distillation of bituminous coal.

Coal-tar is the material from which are produced by synthetic chemistry, dyes, oils, perfumes, drugs, and explosives.

Two types of electric lamps are the incandescent and the arc lamp.

The filament of the incandescent lamp is of carbon or metal. 
The bulb incloses a vacuum or is filled with nitrogen.

Resistance depends upon the material, the temperature, the length, and the diameter of a conductor.

An electric current enters a house by one wire, does useful work in lamps, heaters, and motors, and leaves by another wire.

Electric arc lamps include luminous vapors, as those of carbon or mercury.

An electrode is the solid conductor by which an electric current enters or leaves a liquid or gaseous medium.

The color of an object depends on its nature and the nature of the light which illuminates it.

Sunlight is shown by a prism to be composed of all the colors of the spectrum.

An artificial light should be safe, hygienic, and in other ways resemble sunlight.

\section{REVIEW QUESTIONS}

1. What advantages do we enjoy from the use of artificial lighting? 2. What was the best method of lighting known in the palaces of the Cæsars? 3. Who invented our modern oil burning lamp? When? 4. (a) Who first made the use of gas for lighting possible? (b) What are the important facts in the development of gas lighting? 5 . What are the chief modern systems of lighting?

6. What improvement has made gaslighting more satisfactory? 7. (a) Describe the making of a Welsbach mantle. (b) What advantage has it over the ordinary burner? 8. (a) Describe the production of coal gas. (b) What important by-products result? 9. For what is ammonia used? 10. Mention an instance of the production by chemists of a substance formerly derived from plants.

11. Mention several kinds of filaments for electric lamps. 12. What are the advantages of tungsten? 13. Mention three metals in the order of their relative conductivity. 14. (a) Mention several substances which are considered insulators. (b) Are they perfect nonconductors? 15. Why is a little sulfuric acid put into the water used in electrolysis? 16. (a) What is the effect of high temperatures upon the resistance of some substances? (b) What effect has a very low temperature upon the resistance of pure metals? 17. Group together substances that have their resistance decreased by high temperature. 18. Mention several that have their resistance increased.

19. What effect is made on the current which a wire carries by an increase in length, other conditions remaining the same? 20. Which permits more current to pass, a fine wire, or a coarser wire, other con- 


\section{Artificial Lighting}

ditions being the same? 21. Why does the tungsten filament give more light than the carbon filament, when the same current is used? 22. Compare the carbon filament and metal filament lamp as to cost of current, length of service, and luminosity. 23. Describe the usual plan of wiring a house for electric lighting. 24. Show by diagrams how the electric meter is read and how the charge for current consumed is calculated. 25. Mention an instance of production of light without heat. Has man been able to duplicate this process?

26. Describe the construction and operation of a hand-controlled are lamp. 27. (a) What is the highest temperature artificially produced? Under what conditions? (b) Compare with the supposed temperature of the sun. 28. Describe the mercury vapor lamp. 29. What is the meaning of " electrode"? 30. How is light produced in the vapor lamp? 31. (a) What is the peculiarity of the vapor light? (b) How does it affect certain colors? 32. What are the important hygienic characteristics of the light? 33. Why is it especially useful in industrial plants and in printing and drafting rooms?

34. How can you show that the quality of the light affects the color of an object? 35. (a) Describe a prism. (b) Of what use is it in studying light and colors? 36. If the rainbow colors are brought together, what color is produced? 37 . What is the spectrum? 38. Name six colors visible in the spectrum. 39. What is the result if a pure red light falls upon an object which in daylight is blue? 40. What three features must a lamp have in some measure in order to be commercially successful? 41. (a) What is the standard usually. followed in providing artificial lighting? (b) Deseribe its essential features. 42. Mention seven desirable characteristics of an artificial light. 


\section{CHAPTER V}

\section{THE SUPPLY AND USES OF WATER}

\section{The Water Supply}

73. The uses of water in the household are many, such as for cooking, cleansing, carrying away waste matter from the house to the sewer or cesspool, heating by hot water or by steam, furnishing power for small motors in laundry or churn, cooling directly or by the use of ice in the refrigerator, watering garden and lawn, sprinkling dusty paths, and so on. The uses in the home have been rapidly multiplying in recent years, and this has made necessary a significant change in the sources and contral of the supply.

The average daily quantity of water used in such ways is astonishingly large. If we include with the domestic uses those of the community at large, for sewage and sanitary purposes, fires, water power, manufacturing, transportation, fountains, and general waste, the total quantity used by a community reaches an almost unbelievable amount.

A few years ago a single well or spring supplied the needs of several families; to-day such a supply would be insufficient for a single family. It has been estimated that the average quantity used for each individual was formerly between two and three gallons a day. To-day the average daily cönsumption for each individual in a community of moderate size is nearly thirty-five gallons. In manufacturing cities and towns the mean daily consumption for each person reaches approximately one hundred gallons. 
With such facts it is a simple matter to calculate the amount of water that must be provided for a city of 100,000. Assume for such population an individual allowance of twenty gallons a day; the total daily supply must be at least two million gallons. This is less than the average daily consumption per individual in most communities. The following table gives the quantities consumed in several large cities together with the percentage of water delivered through meters.

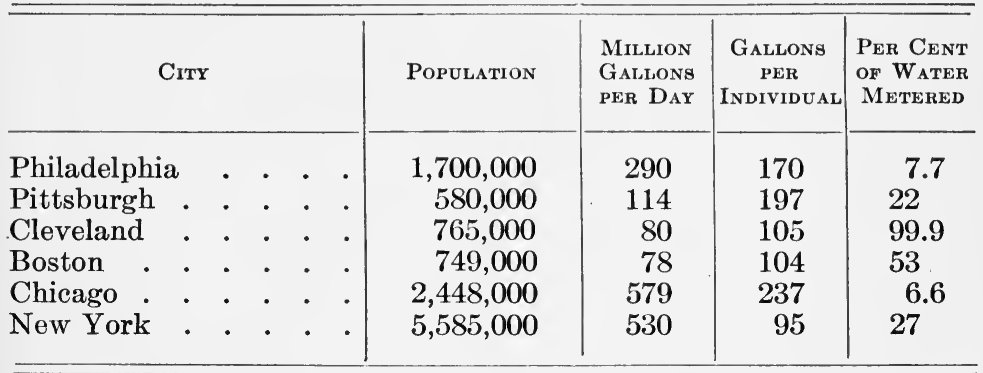

74. Public control of water supply. - It is not difficult to see why in towns and cities private control of the water supply has been replaced largely by public control. The population has increased at a remarkable rate and concentrated more and more in cities where a local and private supply is impossible. The conditions brought about by city life make local wells a menace to life and health by exposing them to contamination by filth-laden surface water and drainage. After many epidemics of typhoid fever and other diseases had been demonstrated to be due directly to polluted wells, people began not only to suspect, but also to abandon this source of supply.

Exercise: Local conditions. - There are other conditions in particular localities, which make necessary the transfer of the water supply from private to public control and supervision. These should be included in the study of the local water supply outlined on page 223 . The conditions deserving special mention include irregular rainfall; a limited watershed, making a large number of private wells with sufficient supply impossible; the multiplying uses of water in domestic, 
municipal, and industrial activities; the disposal of sewage; the necessity for economy and universal distribution throughout the community; and the belief that public interests are not always properly conserved by private interests in control of public utilities. You may find in your own locality other factors of importance.

As a result of such conditions and others of a local nature, the water supply in many places has been transferred to the control of the municipality as one of the great public utilities, in which private interests and management are undesirable. This "free gift of nature," although it is seldom really free, is now provided most conveniently for all purposes, and at a minimum cost to every member of a community. Public wisdom and enterprise are made responsible for the control of the watershed, for collecting and conserving of the rainfall on a given area, and for the distribution and economic use of water in the community. The increase in public control of this utility is shown by the fact that to-day there are more than four thousand municipal water systems in the United States, whereas at the beginning of the nineteenth century there were only seventeen.

\section{The Water Supply of Cities}

75. Conditions determining sources of supply. - The sources of the water supply of any community are determined by the rainfall and by the character of the surrounding country; the utilization of such depends upon the social and financial resources of the community. One community may look far ahead and secure possession of a desirable watershed to provide a supply that may be far in excess of current needs. Another community may suffer want and several epidemics of disease before it makes adequate provision for its present" needs. This is true of many a community to-day. Meanwhile the most available sources are being preëmpted and utilized by the more provident distant cities, although such sources may belong rightly to the near-by communities. 
Ideal supply. - Natural highland watersheds often furnish the best, safest, and most abundant supply. Mountain streams and lakes in a drainage territory free from human habitation and from possible pollution provide water requiring little or no preliminary purification. The use of such a supply depends largely upon the topography of the country and upon its distance from communities. The purchase of right of way, with the construction of reservoirs and conduits, may cost millions of dollars; but the economic profit and the benefit to the public may be far greater than the cost within a comparatively short period of time.

Mountain streams as supply. - In mountainous regions many communities secure an abundant supply of safe and useful water from near-by streams. On the score of economy such a water supply is decidedly satisfactory. It is usually clear, odorless, tasteless, and free from dangerous impurities. This is especially the case where the gathering grounds are not occupied by human beings. Still there is need of constant supervision of the source and means of supply, for through human carelessness or ignorance the water may be polluted and endanger health and life. A patrol, to prevent such accidents, is too often carried out laxly and intermittently.

The Los Angeles supply. - Los Angeles, in southern California, has gone, after careful calculation of the cost and profit, about two hundred fifty miles into the Sierra Nevada Mountains to secure an adequate water supply. On the eastern slope of the mountains, the city secured control of a watershed many hundred square miles in area, and more than four thousand feet above the level of the sea. Here rains and melting snows feed a river that formerly emptied into a dead lake called Lake Owens. Great collecting reservoirs have been constructed and are supplied by the river which has been turned into a new channel just above the ancient lake. Thence through tunnels that pierce the mountains, and through cement and steel conduits (Fig. 55) that cross the Mohave desert, the mountain 
water is brought to the city. In quantity there is more than sufficient to supply the needs of two millions and more of people. The water pressure in the city is so great as to render unnecessary any additional force, as by pumps. This, however, is

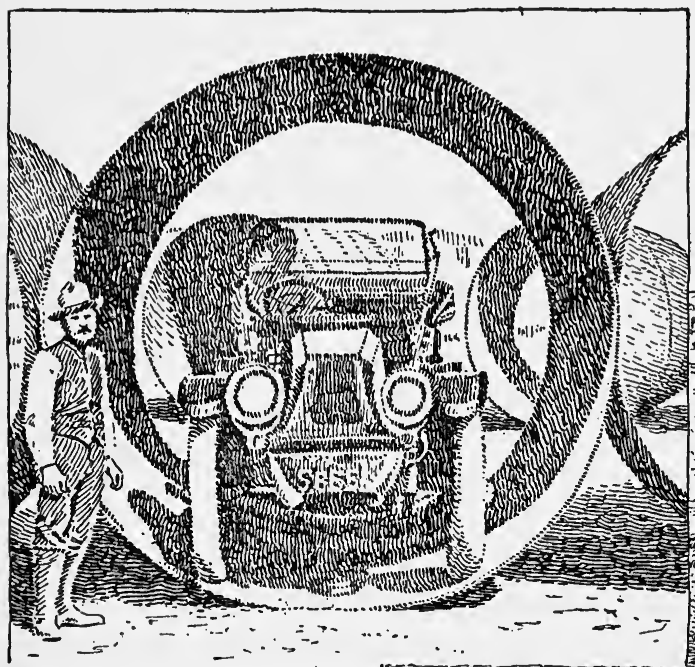

not unusual on the Pacific coast or in the Rocky Mountain region where the supply comes from great altitudes.

Fig. 55. - Section of conduit of the Los Angeles water supply. A steel siphon crossing nine-mile canyon 850 feet deep. It rises on the other side to within 30 feet of height on this side. Insert: Diameter of steel pipe is 11 feet, its length 30 feet; the steel is $1 \frac{1}{8}$ inches thick.

The New York City supply. - After many.

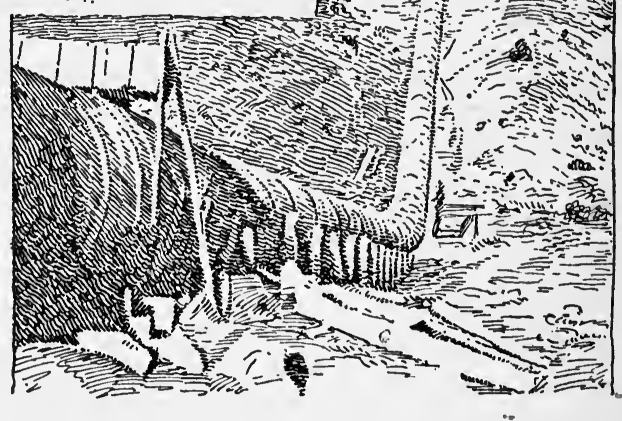
years of dependence upon the Croton watershed for its supply, New York City has gone far north into the Catskill Mountains for a source of supply that bids fair to satisfy its needs for many generations to come. It has acquired control of the 
entire area of the region which sheds its water into the eastern branch of the Delaware River. The Ashokan Reservoir (Fig. 56), the main collecting reservoir of the region, is twelve miles long, one mile wide, one hundred ninety feet deep, and holds $132,000,000,000$ gallons of water. It is five hundred ninety feet above sea level. From this reservoir the water is conveyed through an aqueduct about ninety-two miles long to the city limits at Yonkers. The conduit on the surface (Fig. $57 \quad A$ ) is what is called "cut and cover," with a base dimension of seventeen and one half feet and a height of seventeen feet. This kind of conduit extends for forty-five miles. The conduit underground (Fig. 57 $B)$ is circular, with

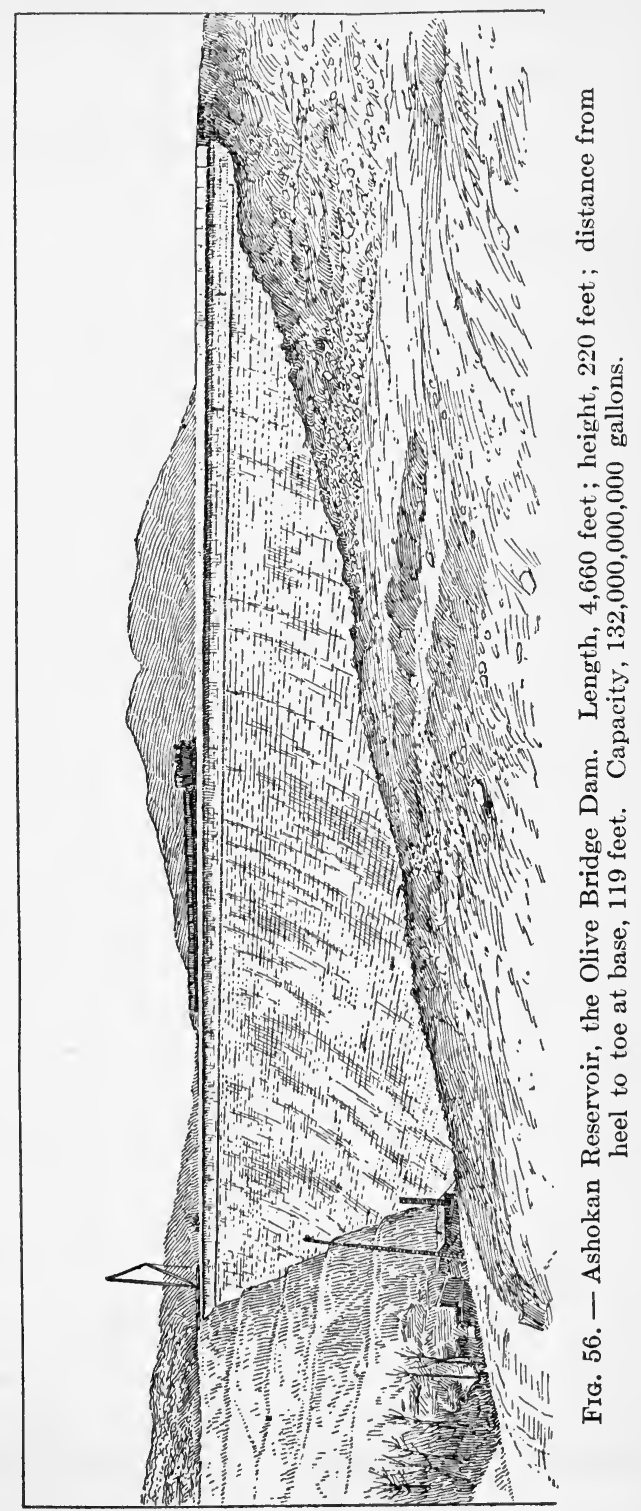


a diameter of fourteen and one half feet, and it is about thirty miles in length. Six miles of steel pipe complete this part of the system.

The water is brought from the west to the east side of the Hudson River by a tunnel like a huge inverted siphon that is

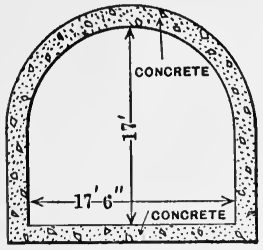

Fig. 57 A. - Cross section of the "cut and cover" conduit " of the New York City water system. 1100 feet below the surface of the river. Under Manhattan and Brooklyn, the conduit, a circular tunnel with a diameter of fifteen feet gradually reduced to eleven feet, reaches from two hundred to seven hundred fifty feet below the ground surface. It is excavated through solid rock. The water supply for Staten Island is conducted through large steel pipes laid on the floor of the bay.

The cost of this public enterprise for an adequate and safe water supply is approximately $\$ 185,000,000$. With a population of more than five and one half millions, New York City's daily consumption is estimated

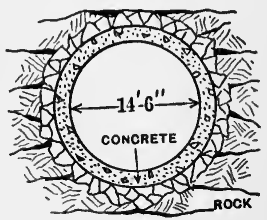

FIG. 57 B. - Cross section of the tunnel conduit excavated through solid rock. to be at the rate of one hundred gallons for each individual. The amount required will certainly become greater in the future per capita and for the inevitable increase in population.

Chicago's supply and the drainage canal. - In the great plain and prairie region of the United States the chief sources of supply are small streams, rivers, lakes, and deep wells. Cities in the vicinity of lakes get their water from them and sometimes turn their sewage into them. Chicago, for example, not only secured its water from Lake Michigan, but for many years emptied its sewage into the lake. The intakes were a mile or more from shore, which distance was supposed to be sufficient to avoid the danger of contamination by sewage. But pollution of the water seemed certain at times, and measures were taken to remove the danger. A great sanitary canal was constructed, 
running from the Chicago River to one of the tributaries of the Illinois River, along an old Indian portag? route from the Lake to the Mississippi River. Trace this on a map. Since then the health statistics of the city show that the community has been more than compensated on this score alone for the fifty and more millions of dollars which the canal cost.

London's supply. - Water secured from sources exposed to pollution, as rivers, lakes, or surface wells may be, should be purified before distribution. Many communities depending upon such a supply have adopted measures to make the water safe and to prevent accidental contamination. The common methods are by storage in still reservoirs and filtration through sand. That such means are effective may be shown by the experience of London, England.

The larger part of London's supply is from the Thames and the Lee. These rivers flow through populous districts and are made carriers of sewage above the intake of London's supply. But by employing storage reservoirs and sand filtration, London has furnished safe water for many years. It points with pride to its general health statistics, and especially to those of diseases which are supposed to be caused by germs carried by water. Storage destroys many kinds of bacteria and removes a very large per cent of the injurious matter. Filtration through sand completes the process.

The Ganges River supply. - Some rivers possess exceptional powers of sterilizing or purifying their waters. For thousands of years the waters of the Ganges and the Jumna have been regarded by the Hindus as "pure and undefiled." This does not seem possible, for into them are put all sorts of sewage and decaying organic matter, such as bodies of animals and men, among them victims of cholera. Thousands, moreover, bathe in the waters daily. But in spite of all this, the water is safe and is used for every purpose. It is interesting to find science confirming and supporting a belief that has long held sway, for bacteriological and other scientific tests have been made of these 
waters and they support entirely the old religious belief. There is some unusual potency in the waters, which destroys cholera germs within five hours or less, and other disease-causing bacteria still more quickly. It is a curious fact that water from wells in the vicinity of the Ganges River is fairly teeming with cholera germs, and must be purified before using. Boiling the water from the Jumna destroys the properties which make it safe; cholera germs and other kinds of bacteria flourish in boiled Jumna River water. What gives the waters of these rivers their germicidal properties is not known.

76. Deep wells. - Another source of supply of considerable importance and usually above suspicion of contamination is the deep well. Such a well is from several hundred to several thousand feet deep. The wells of Leipzig (5735 feet) and Berlin (4194 feet), Germany, and those at Pittsburgh, St. Louis, and Galveston, U. S. A., may be mentioned because of their depth and free flow of water.

Artesian wells. - The well passes through the local or first water table (Fig. 58) and through a layer or layers of earth

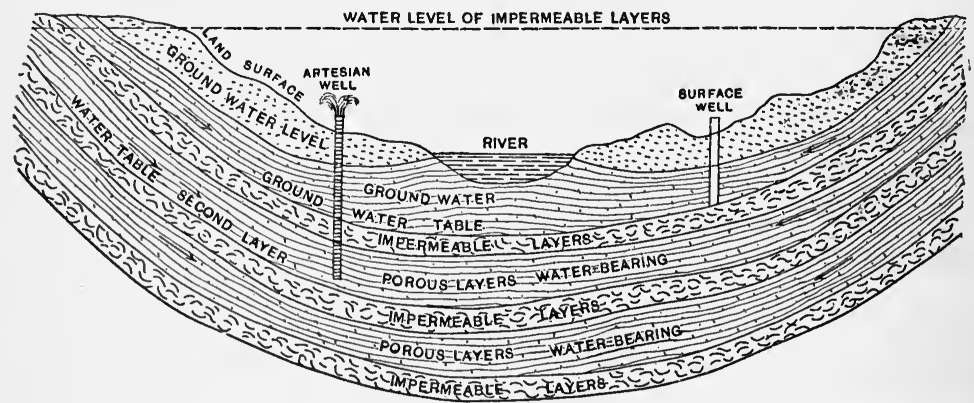

FIG. 58. - Ideal representation of conditions of artesian well; the ground water level and contour of surface; river and surface well."-

that are not permeable to water, into a layer of sandstone or other porous material which bears water. The water is thus supplied by a table that runs to a distant place of higher elevation than the well. The higher altitude, together with a large 
supply in the water-bearing layers, may give sufficient pressure to cause the water to flow freely from the mouth of the well. A well that flows thus is called artesian, from Artois, a province in France. The water sometimes spouts to a great height; in South Dakota an artesian well sent a jet more than ninetyfive feet high. If it were not for friction, the water would rise in pipes almost to the level of the source of the supply in the water-bearing layers of earth.

Artesian wells found in a plain or valley which slopes more or less gradually from high land are made possible by the character of the different layers of the earth's crust. Such conditions are found along the Atlantic, Pacific, and Gulf coasts, and on the Great Plains. Distance from the head of the water-bearing layers and the character of the intervening surface are no barrier to such a supply. Water drawn from wells at Calais, France, is carried through porous rock layers from England under the English Channel. Along the southern coast of New Jersey, as at Atlantic City, artesian wells are supplied by water that comes beneath the marshes from the mainland many miles away.

The cross section (Fig. 58) of a wide valley with a river cutting through it shows how the character of the surface and the distance from the head of the water supply cannot affect deep wells. The deep permeable strata, which supply the wells with water, secure their supply in distant highlands. The surface water is prevented from entrance into these porous layers by the first bed of impermeable material.

The water level of the ground moisture varies with the wet and dry seasons; it rises during wet weather and sinks to lower levels during dry weather. The water level is just the upper limit of water saturation of the ground, the height of water in a surface well showing the local water level. This rises or falls according to the rainfall. The general water level conforms with the elevations and depressions of the land (cf. Fig. 58), but is usually nearer the surface under depressions, such as valleys, than under hills. It often reaches the surface and forms springs 
or swamps. In regions where there is a heavy rainfall the water level will be found closer to the surface than in arid regions, where it may be very deep. The beds of stream channels are usually lower than the water level during the dry season. Where this is not the case, as in certain sections of the Great Plains, the rivers disappear largely or entirely during the dry season.

The underground course of water which may be supplied by deep wells depends upon the geological formation of the region through which it flows. In general water sinks through the soil until it reaches a water table formed by clay or rock strata impervious to water. This is sometimes called hardpan. Along the surface of this impermeable layer water slowly makes its way until it reaches crevices or porous joints; then downward it goes until it arrives at another impervious layer. It may flow on this until it emerges on the surface. It may emerge as a spring from the side of a hill; it may be captured by a deep well and flow or be pumped to the surface. Or it may enter through the floor of a river, lake, or ocean. Its rate of movement may vary from two inches to twenty feet a day, or it may be exceedingly slow. It may remain in the earth for months at a time or even for years.

Water's solvent power. - Upon all rocks with which it comes into contact, water exercises solvent power and takes a toll of mineral substances. Such deep water may therefore sometimes have a peculiar taste or color. If it comes through a limestone region, it will probably contain a great deal of lime and be " hard." If it comes from such very old rocks as the archeozoic, it will be soft, for these rocks do not contain much lime. As there are many salt deposits or rock-bound reservoirs full of saline water in the solid crust of the earth, water will sometimes be salty. It may be sulfurous; or it may contain an amount of ammonia derived from organic matter buried in ancient strata of coal or other material. Examination shows it to be entirely free from bacteria, unless it is contaminated by surface water or seepage through defective sewage pipes. 


\section{Rural Water Supply}

77. Cisterns. - A supply used in certain parts of the country is the surface water which is collected in cisterns or tanks as it flows from roofs of buildings. If this water is thoroughly filtered and stored in non-porous cisterns, it is useful for nearly all domestic purposes. It is, however, peculiarly subject to contamination, and is too often imperfectly filtered.

A cistern to be safe must be below the frost level in the ground and made of material which is absolutely impervious to surface water. Filters of charcoal and screens should be carefully supervised. To render water safe, it should be kept in a stillstorage cistern for a sufficient period of time and then be filtered into another tank for use. Why should rain water require such careful treatment? Is it not pure as it falls?

What rain water may contain. - As it rises from land and water surfaces, water vapor is entirely free from all impurities. This is nature's way of purifying water, and man follows this method in getting a really pure water for many uses. The change from a liquid to a vapor condition, called vaporization, is the first stage of distillation. In securing pure water, man controls the conditions in which condensation takes place. Nature's method of rain production is, however, different. When vapor condenses in the atmosphere, the minute water particles accumulate such impurities as dust and as they grow larger and fall through the air, they gather up more and more dust, pollen, spores, and bacteria, and absorb such gases as carbon dioxid and ammonia. Thus when rain reaches the earth, it is laden with a freight of undesirable character. Rain water with its absorbed carbon dioxid has great solvent power upon mineral substances. Besides the mass of vegetable and other organic matter collected, it dissolves and carries away mineral substances from painted roofs and similar places. Such water certainly needs to be purified. Generally only the latter part of a rainfall is turned into the cistern, the first part being allowed to waste, thus assur- 
ing some protection from dirt and other matter. Is this all that is necessary? What impurities gathered by, rain water are not dangerous? What are dangerous?

78. Springs and shallow wells. - Smaller communities and farms depend for the most part upon springs and shallow wells. Such water may in general be safe for domestic use. But certain conditions should be observed in regard to the location and protection of the supply. Springs may furnish water which is clear, tasteless, and odorless, but dangerous. If they are of surface origin, or so-called shallow springs, the danger is all the greater. Many of them are uncovered, exposed to dust, insects, and harmful bacteria. Sometimes buildings, such as stables, pigpens, and the like, are located so that they pollute the drainage supplying the springs. Such conditions must be corrected because they menace health and life.

Shallow wells are frequently found in rural districts. If a well of this kind is suitably located as regards surface drainage and subsoil, it may furnish water that is in general safe. If it receives drainage from decaying vegetation, or from house waste, cesspool, outhouse, barnyard, and the like, the water is almost certain to be contaminated and dangerous for drinking. The amount of injurious substances in the water of a shallow well depends largely upon the character of the ground through which the fallen rain must filter. If the soil is fine in texture, it will filter out much of the deleterious matter. The deeper the well and the farther it is located from the polluting sources, a desirable distance being seventy to one hundred feet or more, the better will be the filtration of the water and the safer for all uses. All surface water contains injurious substances taken from the air as well as from the soil and subsoil of the earth. When it passes through ground polluted with sewage and filth, it collects vast quantities of elements that are dangerous to health and life.

Location of a shallow well. - In view of this fact, the location of a shallow well or the use of the water of a surface spring 
should be considered with respect to these points: first, the location of the water supply with reference to the surface and the underground or subsoil drainage; and second, the disposal of waste matter such as sewage, refuse, and the like. The second point will be considered under the topic of sewage disposal (pages $210 \mathrm{ff}$.).

In regard to the first point, there are three general situations in which a well may be located. Examine the diagram of Fig. 59 II. This shows the surface drainage sloping away from the well. This is necessary in order to avoid any possibility of pollution of the water by surface waters. But the subsoil drainage is toward the well. This clearly increases the danger of the water being contaminated by surface waters that reach the subsoil in the vicinity of the well. Obviously such a location is not satisfactory.

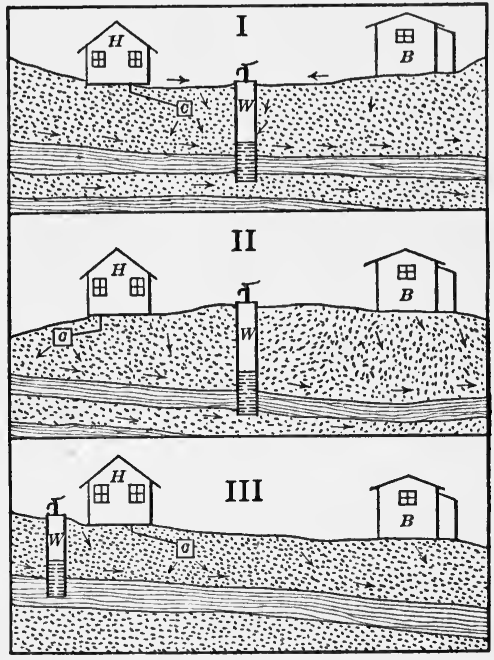

FIG. 59. - Locating a surface well. I. Surface drainage bad; subsoil drainage bad. II. Surface drainage good; subsoil drainage bad. III. Surface drainage good; subdrainage good. $H$, house; $W$, well ; $B$, barn; $C$, cesspool.

In the diagram of Fig $59 \mathrm{I}$, the well is so located as to be exposed to the drainage from the house, cesspool, barn, and other outbuildings. The subsoil drainage is bad, though it slopes away from the well in one direction. But the water is in danger of contamination by the surface waters that come from the cesspool and compost accumulations in the barnyard. Is this location better or worse than that described in Fig. 59 II?

Now examine the diagram of Fig. 59 III. In this the soil and subsoil drainage is away from the well. This represents 
a location which is altogether satisfactory and with proper care will provide a safe water.

As the diagrams show, the surface may be taken as indicating the subsoil drainage, but the subsoil slope should be definitely known before locating a shallow well. It is never economical to proceed blindly in matters which concern health and life. Protecting the water of a well. - After a well is properly located and dug, and a plentiful supply of safe water obtained, much depends upon the construction and care of the well in order to keep the water safe for all individual and domestic uses. The well should be lined with the best materials, such as specially prepared brick or stone and cement; the workmanship should be of the best. The lining should be carried above the level of the ground, and a water-tight covering provided to prevent dust, dirt, and other objectionable materials from entering. The purpose of the lining and the covering is to protect the water of the well from possible contamination by surface drainage. The ground surface around the mouth of the well should slope away on all sides so as to carry off all waste water. The ground should be kept clean also, no refuse or decaying vegetable matter being allowed to accumulate on any ground which drains toward the well.

\section{DAMS}

79. Purposes. - Dams are built for many purposes, among which may be cited the impounding and storage of water for private and municipal supply systems, for irrigation, for the generation of power, and for the purpose of diverting a stream from its usual course. Where, for example, the average annual rainfall is comparatively small and inadequate to the needs of a city, as in the case of San Francisco, immense impounding and storage dams have to be built. In the western states of our country there are extensive areas of land which with a sufficient water supply are invaluable for agricultural purposes. Where water is available during a part of the year, the United States 
Reclamation Service has made remarkable advances in constructing dams for irrigation and power purposes, which are of a size, capacity, and cost that a generation ago would have been regarded as impossible and unnecessary. Again, industrial necessity is pressing into service a small part of the total available water-power possibilities of our country. Running water that heretofore was wasted is now being restrained by dams and made to yield its power in the hydro-electric plants that are rapidly multiplying wherever conditions allow. Instances are numerous where streams have been dammed in order to divert them into a new course.

80. What a dam must do. - In the planning and construction of dams there are many indispensable conditions which must be considered if the structure is to be safe and adequate. There is the pressure to which the dam is to be subjected in all situations. It is a simple matter to ascertain the water pressure from the depth of the water and a unit area of any section of the dam. But there are extraordinary occasions of flood and storm when the incoming water may have a volume and a velocity against which a dam built for "still" water conditions would prove utterly inadequate. The great seawalls at Galveston have to resist enormous force as they meet the incoming waves of the ocean. Moving water has a velocity and energy which must be taken into account wherever such condition is possible. The dikes of Holland must meet more than still or static water pressure; they must oppose a moving force of tremendous proportions.

Diffculties in dam construction. - The design and construction of dams cannot be said to have passed beyond the experimental stage. The conditions which every dam must meet are almost impossible to predict. The strength of the materials is so variable as to defy calculation under extraordinary conditions. The foundation is always a problem, for after the construction has continued awhile it is not open to inspection, and it cannot be tested beforehand what effect 
the masonry mass may have upon it even though it be apparently a substantial rock basis. There are other conditions which emphasize the experimental stage of dam building, and indicate that present practices are no more than a suggestion of the finally approved and permanent method. The general form in use to-day is illustrated in the cross section of the Arrowrock Dam, one of the latest achievements of the

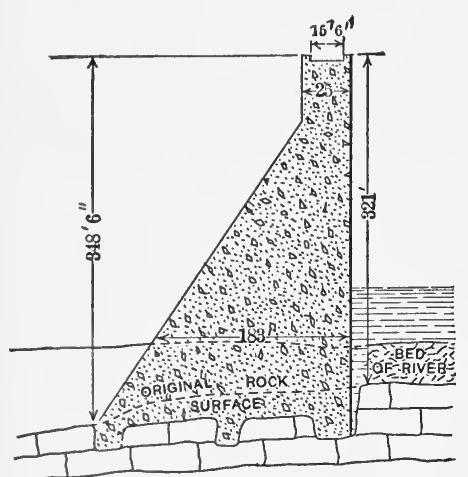

FIG. 60. - Cross section of the Arrowrock Dam, showing excavation for foundation, the general form and dimensions of the dam.

United States Reclamation Service (Fig. 60).

The Arrowrock Dam. - The difficulties met in the construction of this dam may be suggested by the fact that in order to find solid rock as a foundation for the construction, excavation had to be made through more than ninety feet of rock of volcanic origin below the bed of the river. The area at the base of the structure is more than 200 feet in width and about 1100 feet in length; from this one may easily imagine the great mass of material that had to be removed for this part of the work alone. The river at flood season has a flow of more than 18,000 cubic feet a second. The capacity of the dam is 244,300 acre feet, or about 79,800,000,000 gallons of water.

The dam is to conserve some of the available water for the generation of electrical energy which is used by the residents of the section for light, heat, and power. Its cost is very small. In some sections of reclaimed land in Idaho, electric energy is utilized not only for lighting, but for heating, cooking, and power purposes by about 50 per cent of the population. This is an item of no small value in a region where transportation facilities are limited and the cost of fuel is correspondingly high. 
The Boise River project (Fig. 61) involves an investment by the federal government of $\$ 12,000,000$, the dam alone costing about $\$ 5,500,000$. This money is to be returned to the government by the occupants of the land in twenty annual

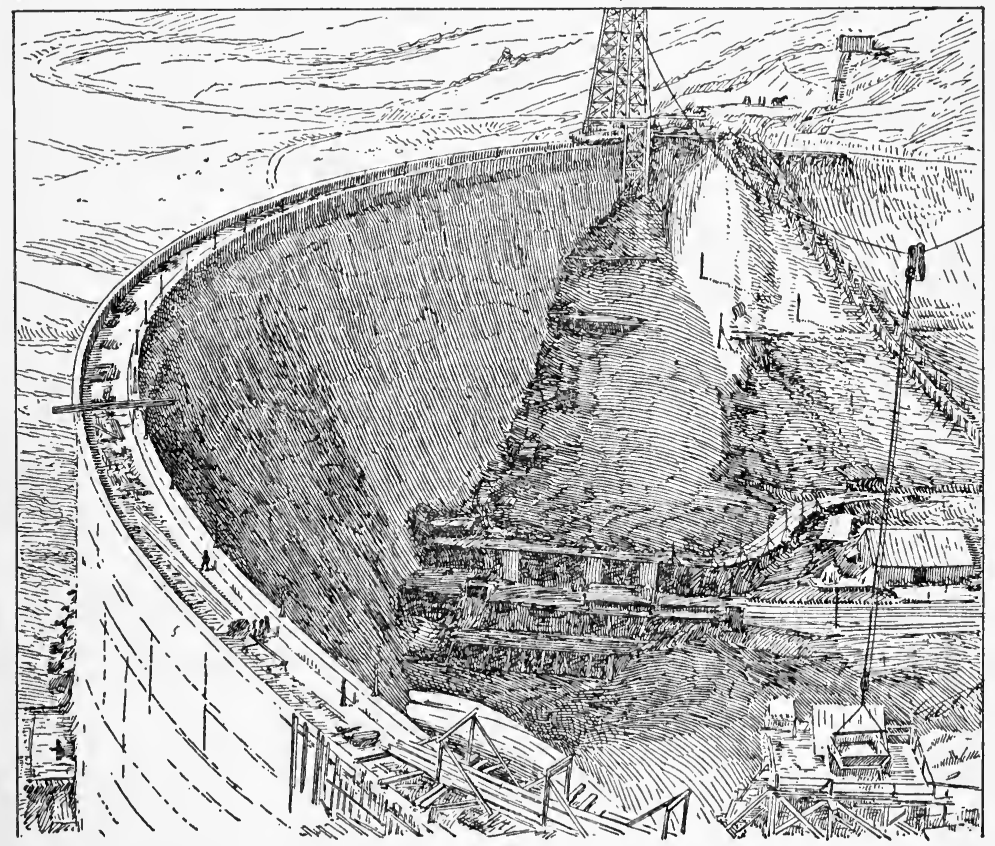

Fig. 61. - Arrowrock Dam, Boise River, Idaho, the parapet and lower side of dam. Radius of curve, 662 feet; height, 348.5 feet; length, 1,100 feet. Capacity of dam, 79,800,000,000 gallons. Cost, about $\$ 5,500,000$.

installments. A region covering 234,000 acres of desert land will be irrigated. What will be the cost per acre for water rights on the basis of the initial investment?

Exercise. - Students should obtain information regarding the work of reclamation of desert lands in the United States. Such information should include the geographical features and rainfall of the region, and the size and capacity of dams to meet the require- 
ments of the given areas. The conditions necessary in dams to insure safety in emergencies should also be studied. (Reference: Reclamation Bureau, Washington, D. C. Current periodicals, as World's Work, National Geographic Magazine, Review of Reviews, etc.)

It is estimated that the amount of land which may be reclaimed by irrigation runs from $40,000,000$ to $45,000,000$ acres. Ten million acres have been brought under cultivation and are very productive.

In what sort of places along river courses are dams usually built? Study the Shoshone Dam, Salt River project, and others. From what source does the government obtain money for reclamation projects?

What plans are suggested for the reclamation of large areas of the Sahara Desert?

\section{SUMMARY}

The average daily consumption of water per individual is 32 gallons in small communities; more than 100 gallons in manufacturing cities.

For many reasons communities have found it advisable to own and control their water supply.

An ideal water supply comes from a region high enough to give sufficient gravity pressure, where the rainfall is abundant and pollution improbable.

Typical examples of provident and safe supplies are the water systems of Los Angeles, New York, and London.

The Ganges and Jumna rivers have exceptional powers of sterilization.

Deep wells, as in Leipzig, Berlin, Pittsburgh, St. Louis, and Galveston, furnish a safe supply.

Artesian wells may be obtained where the rock formations are favorable.

Ground water found in most places is slowly moving toward springs, river courses, or the sea.

Water has great solvent power and takes up in some quantity nearly every mineral with which it comes in contact.

Cisterns for storage of rain water are necessary in some localities. They furnish soft water, but are liable to contamination.

In nature the process of evaporation gives pure water vapor. Rain formed in condensation contains impurities from the air.

Man improves nature's process by controlling the conditions and obtains pure " distilled water." 
Shallow wells should be advantageously located and carefully protected from pollution.

Dams are often needed to secure a constant water supply for cities, for agricultural purposes, for power, and for protection against floods.

The construction of dams is still experimental, as the conditions are peculiar and results cannot always be predicted.

The Arrowrock Dam is typical of the dams constructed by the United States Reclamation Service.

\section{REVIEW QUESTIONS}

1. Mention several uses of water in the household; in the community as a whole. 2. Give several reasons for the community control and supervision of water supply in towns or cities.

3 . What are the most desirable sources of water for cities? Give reasons. 4. What has Los Angeles done to provide an adequate water supply? Describe some of the important geographic conditions which had to be met. 5. Give an account of the recently completed water supply system of New York City. 6. What dangers are there in taking water for drinking from untested streams? 7. How was the water supply of Chicago affected by the construction of the drainage canal? 8. What provisions are made by London to insure a safe water supply? What seems to be the result of such precautions upon the health of the city? 9. What unusual property is possessed by the water of the Ganges River? How docs it differ from well water of the region?

10. What geological formation makes artesian wells possible? 11. Why is artesian well water safe for drinking and domestic uses? 12. What is the water level of ground moisture? Upon what does it depend? How is it related to stream channels? 13. Describe the course of water through the earth until it issues as a spring or deep well supply. 14. To what property does water owe its occasional peculiar taste, odor, and character? 15. What makes some waters hard and others soft? 16. What are the usual sources of supply in rural sections? 17. What are the sources of danger in cistern water? In shallow wells? 18. Describe how fresh water comes from salt water.

19. What is the proper location of a shallow well in relation to rock layers, buildings, and drainage? 20. What sort of construction is necessary to make the well reasonably safe? 21. How can the slope of the subsoil be determined?

22. Mention several purposes for which great dams are constructed. 23. What are some of the forces a dam must successfully resist? 
24. What difficulties are encountered in building dams? 25. Describe one of the notable dams built by the United States Reclamation Service for irrigation and power purposes. 26. What is the estimated acreage of land available for reclamation by irrigation? In what states is this land located?

\section{SUGGESTIVE QUESTIONS}

1. Which needs the more water for drinking purposes, a man or a horse? Which makes use of the larger amount? Why? 2. Give reasons for the greatly increased consumption of water in recent years. 3. If you live in the country, find out the average depth of wells in your vicinity; the character and sufficiency of the water supply throughout the year; and the safety of the water as indicated by the location of the wells relative to drainage. 4. Through what kind of ground are the wells sunk? 5. If you live where artesian wells are found, ascertain depth of wells, source, and distance of wells from source; character of water as hard or soft. 6. If you make use of cistern water, how can you make it safe for all purposes? 7 . Why do certain rivers of the central western states disappear largely or wholly during the dry season?

\section{The Water Supply and Disease}

81. Water as a carrier of disease germs. - Water is generally considered a means of distribution of several kinds of disease germs. What evidence is there to support this opinion? The chief questions to be considered in this chapter are the following. Is water a suitable means for carrying disease germs? How do such germs come into water commonly considered safe? Or is water, as we find it in nature, unsafe for drinking?

The most familiar diseases considered as water-borne are typhoid fever, dysentery, and cholera. These have become associated in the minds of many people with the water süpply. An epidemic of typhoid fever, for example, now raises specific questions as to the carrier. Is it the water polluted by sewage? Is it the milk supply, unclean food, or the house-fly? In every case investigation soon eliminates all but one or two possible 
means of transmission. We shall study the water supply in relation to this question. Let us consider typhoid fever as epidemic (meaning "among the people"), in which case the disease may be traced to a common source or means of transmission, such as the milk or water supply of a community. Typhoid fever may be widely scattered, that is, individual cases may be found in many different localities; but our concern is with the epidemic and not such individual cases. A typical instance of many epidemics which helped to establish the connection of disease with the water supply may be given in some detail.

In January and February, 1912, a city of 50,000 population in the northern part of Illinois had 10,000 cases of enteritis, similar to typhoid, and 199 cases of typhoid fever. As the epidemic occurred in midwinter, it could not have been distributed by the house-fly. It soon appeared that the only probable agencies were the milk and water supply.

The milk supply was quickly shown to be free from contamination. Fifty different dealers supplied the families in which the disease had developed, and in no instance was there a disproportionate number of cases on any one milk route. Therefore the milk supply was not the medium through which the germs were transmitted.

The water supply was investigated. Many families who had private wells escaped infection. Another interesting fact was discovered. A group of factories provided their employees with drinking water from private wells. Only six per cent of these employees developed cases of enteritis. Another group of factories provided their employees with water from the city mains. Seventy per cent of this group developed the disease.

The location of the source of the trouble was made possible by another occurrence. A destructive fire about the middle of January, just before the epidemic, had largely depleted the storage reservoir. To fill this, a well which had not been in use for a long time, was pumped. Test showed that the water 
as it came from the well was safe. But before being pumped into the reservoir, it was transferred into a pumping pit where it stood for some time. When the pumps worked at a high rate of speed, the water level in the pit was lowered and seepage through the walls of the pit was noticed. The pit was immediately emptied for examination; but overnight it was filled with "about six feet of refined sewage." The pit was found to be located where surface and other sewage drainage could reach it and seep through its porous and cracked walls. Enough did enter to cause a sudden and widespread epidemic among the people making use of the city water.

This case is but one of a large number, the investigation of which has brought about the almost universal demand for a safe water supply. The experience of such cities as Pittsburgh, Cincinnati, and Chicago, in reducing the annual death rate and the number of typhoid cases by improvement of the water supply and of the disposal of sewage, is substantially the same.

82. Koch and filtration of water. - The classical instance of disease-reduction through purification of the water is that of Altona and Hamburg, Germany. These cities secured water from the River Elbe. Altona filtered it before distribution. Hamburg turned it directly into the mains. In 1892 cholera broke out in Hamburg and before it was brought under control 17,000 cases and 8600 deaths occurred out of a population of about 600,000. Hamburg, situated above Altona, emptied part of its sewage into the river. Altona, in spite of this pollution of the water supply, had only a few scattered cases of cholera. Robert Koch, noted for his achievements in hygiene, demonstrated the value of the sand filter in this case. The cholera microbe was frequently discovered in the raw "river water; but not a single microbe was to be found after the water had been filtered.

The experience of European and American cities in purifying admittedly polluted water and the resulting improvement 
indicated by the health statistics have not been without influence upon smaller communities throughout the land. Many places have installed purifying systems, and, they claim later, the typhoid fever rate per year has been greatly reduced. It' is now one of the most popular arguments advanced to secure needed improvement of the water supply.

The following data (Fig. 62) on water supply and the typhoid fever rate in several communities in New York State are taken. from the bulletin published by the New York State Department of Health in January, 1913. Your own community may be similarly studied with the aid of data afforded by the Health Department records.

\begin{tabular}{|c|c|c|c|c|c|c|c|c|}
\hline & 1899 & $1900-5$ & 1906-7 & 1908 & 1909 & 1910 & 1911 & 1912 \\
\hline Albany . & $80^{1}$ & 24.8 & 20.1 & 10.9 & 10.8 & $14.9^{2}$ & 17.8 & 17.7 \\
\hline Auburn. & & 23.8 & 9. & $46.6^{3}$ & 17.5 & 8.6 & 7.9 & 11.2 \\
\hline Elmira ${ }^{4}$. & & 45.5 & 36.4 & 30.7 & $33.5^{5}$ & 26.9 & 13.3 & 15.9 \\
\hline New York & & 18.6 & $16.5^{6}$ & 12.8 & 12.7 & 11.6 & 11. & 9.7 \\
\hline
\end{tabular}

FIG. 62. - Typhoid fever rate per 100,000.

The data presented in this table may not prove conclusively that improvement in the water supply is followed directly by a decrease in the typhoid fever death rate. The connection, however, is close enough to support the general belief that safe water must be supplied in the interest of public health. Filtering and storage systems are necessary to prevent the distribution of water which may be accidentally contaminated by disease germs, even though the watershed may not be occupied for human habitation and the water is considered safe. Clear, odorless, and tasteless water may be fatally loaded with

1 Water filtered after 1889.

${ }^{2}$ New and improved filtration after 1910.

${ }^{3}$ Violations ordered removed 1908; note since then decrease in rate.

${ }^{4}$ Water filtered after 1896 because of epidemic.

${ }^{5}$ Improved supply and filtration since 1909 with use of chemicals.

- Strict patrol of watershed since 1907. Note low rate generally and decrease for 1910-12. 
disease germs introduced accidentally, carelessly, or ignorantly. To prevent disaster from such accidents it is necessary to have dependable protective measures in constant operation.

\section{Water as a Carrier of Sewage}

83. Water supply and sewage. - One of the gravest dangers to the gathering grounds and the source of a water supply is its possible pollution by human waste matter. Too frequently sewage in large or small quantities is turned into such areas. Communities which depend upon a water supply thus exposed to pollution must provide for its purification or risk several kinds of serious and filthy diseases. The greatest necessity is to dispose completely and effectively of all human waste matter or sewage. With this problem every one is or should be vitally concerned.

The disposal of sewage strikes at the health and life as well as the happiness of every member of society. It should not be considered as merely a municipal problem, but one that touches every individual; for only when it is made the active concern of every one can it be satisfactorily and effectively handled. The old-time, easy-going attitude toward the disposal of human waste products, both of the sick and the well, is now generally condemned as intolerable, if not criminal. To endanger human health and life through careless and imperfect disposal of sewage is morally criminal, as poisoning wells is legally criminal. For sewage, as has been proven beyond all question, may transmit the germs of at least five filth diseases, typhoid fever, enteritis, cholera, diarrhea, and dysentery. The germs of these diseases come from those who are or have been victims of the diseases. They are transmitted to healthy persons through the medium of food and water contaminated by sewage. There is no other medium of transfer, so that if this problem were intelligently met and all human waste completely destroyed or disinfected, the diseases would eventually disappear.

There is a twofold problem in the disposal of sewage and waste 
matter, - that which concerns the individual and the individual family as in rural districts, and that which concerns the entire community or city. In the city, for instance, there is the waste of the household, the factory, and all industrial and commercial institutions. We do not need to consider here the removal of such materials as garbage, ashes, paper, cans, bottles, etc., which are often collected for their commercial value. We have only to consider the methods employed for the disposal of sewage and the ways by which it may be treated to remove all danger to human life and health.

84. Some ways of sewage disposal for cities. - Sewage is frequently turned into a system of conduits and discharged from these wherever sanitary wisdom or often carelessness or false economy may decree. The conduits may be on the surface, as in certain parts of South America, Asia, and in some localities in the United States. The only approximately sanitary sewers are placed underground, and are watertight except for entrance and discharge of sewage or for temporary opening to remove obstacles clogging up the sewers. The sewers must be watertight to prevent pollution of the ground through which the conduits are laid, and also to prevent the entrance of surface drainage and materials that would tend to block the system. The provision of openings at suitable places is necessary to give ventilation and to allow chemical treatment to destroy offensive odors.

Water is the carrier of sewage in the community as well as in the individual organism. Practically all the water used in a community is turned into the sewage to carry away waste products. Where surface drainage is conducted by gutters and basins into the same system, it helps to flush and prevent the more or less permanent deposits from clogging the pipes. When rainfall is carried off in surface gutters, additional water is necessary at times thoroughly to cleanse the sewer system. This means an increase in the quantity of water consumed and of the water supply ; but it cannot be regarded as waste or extravagance. 
The particular way by which any community disposes of its sewage depends upon the geographical features of the region, as well as upon the sanitary wisdom and efficiency of the community. A town or city situated near an ocean, a lake, or running stream, probably makes use of such an easy and inexpensive means of disposal. One may find that the chief municipal problem is to avoid a nuisance or the possible pollution of its own water supply. The danger to other communities is not always considered. The connection of any recent epidemic and the contamination of the water supply by sewage should be investigated. One should observe how waste is removed and destroyed or allowed to pollute the water supply.

Need of sewage disposal plant. - Where sewage is discharged into the collecting area of a water supply, it should be thoroughly treated in a modern plant for sewage disposal. Such a plant is usually elaborate and costly in construction and operation. A coarse screen is used to remove large materials, such as gravel, pieces of wood, and so on. Septic tanks (see page 215) of large dimensions are used for the decomposition or putrefaction of the organic matter. The final product, called the effluent, may for greater safety be treated where it is discharged with some chemical substance as hypochlorite of lime. Soil filtration or stream action may be counted on to remove any possible remaining elements of danger.

The house system of drainage is an important part of a sewage system. All branches of waste pipes should be as short as possible and lead directly from fixtures to the main waste pipes. Open, easily accessible, and thoroughly ventilated pipes are now considered the most sanitary. To prevent the entrance into the house of sewer gas, which is not only unpleasant but dangerous, traps are provided. These in general are U-shaped (Fig. 63) so as to retain enough water to block the passage of gas.

A practical point in the care of such devices may be mentioned. Grease and other clogging materials accumulate in the traps 
and connected pipes and unless removed will cause trouble. The regular use of concentrated lye and boiling water will keep the pipes free from all such materials.

When waste pipes are not in use for a long period, the water in the traps is evaporated. This gives free passage to sewer gas. To prevent this it is necessary to pour a small quantity of kerosene or a similar oil upon the trapped water and to close securely the waste openings. This is especially necessary if the pipes are to be left unused for several weeks.

A large supply of water is needed to carry away all the house waste. In the larger pipes materials tend to accumulate and unless removed by a strong flow of water will stop up the passageway. To aid in the removal

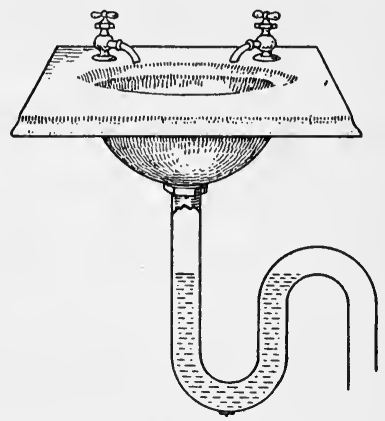

Fig. 63. - A water trap to block sewer gas. of waste matter the rainfall is often turned into the house sewer. If this is not done, it will be necessary to provide at frequent intervals extra water for the purpose.

85. Rural sanitary sewage disposal. - Modern sanitary methods and devices are becoming more and more common in many rural sections of our country. The old imperfect ways of disposing of sewage are being replaced by those which completely purify or convert it into safe and valuable fertilizing compounds. Such methods and devices are much less expensive and more effective than laying conduits to a more or less distant stream or body of water. They are the result of sound sense and scientific knowledge of the conditions, agents, and process of sewage putrefaction and purification. The wonderful discoveries of Pasteur proved that the process of decay or putrefaction as well as fermentation is due to the activities of certain kinds of exceedingly small, microscopic plants, called bacteria. These bacteria flourish in certain conditions which 
are easily provided in several simple and more or less permanent ways.

The old-time cesspool and the modern septic tank made of cement are two familiar devices which are widely used in providing the conditions in which putrefying bacteria thrive and work. In the use of the septic tank there are none of tre dangers to health which attend a carelessly kept cesspool.

Both of these devices require a sufficient water supply. This should be available in every rural home, together wi h the conveniences which such a supply makes possible in the kitchen, bathroom, laundry, and garden. There are several comparatively inexpensive and easily cared for systems which give such a supply. A tank placed in the attic, or elevated outdoors, or a tank in the cellar where pressure is supplied by a compression air-pump may meet this need. The tank may be kept full from an upland reservoir, from a well pumped by a gasoline pump or windmill, or from a stream by the aid of a hydraulic ram. All of these are familiar in rural life..

The cesspool. - With an adequate water supply the sewage problem may be settled for a time by the cesspool, the cheaper and more easily constructed of the two devices named. This is merely a hole in the ground, about ten feet deep and three or four feet in diameter, lined with uncemented stones, and carefully covered with heavy planks. It should be fifty or more feet from the house. The drainage from it should be away from the water supply. The cesspool requires care, regular cleaning, and at intervals a strong lye solution must be added to dissolve the heavy greasy scum that collects upon the surface and the stone lining. Such treatment does not favor the beneficial bacteria to any great extent, but some manage to thrive and bring about desirable changes in the contents.

The septic tank. - The septic tank, which was first tried out at Exeter, England, in 1893, has much more to commend it than the cesspool. It is made of concrete and provides permanently the conditions which favor rapid and complete putre- 
faction of sewage. It may have any external form, but should have two or, better, three divisions of the interior. The design which is given in Fig. 64 is simple, effective, and not expensive to construct. The dimensions of a tank must vary with the amount of sewage it is to contain or with the average size of the household. The dimensions given will give a tank large enough to meet the requirements of a family of six to eight.

The tank should be located at least twenty feet away from the house and where the drainage of the surface and subsoil is away from the water supply. It may be located so that the

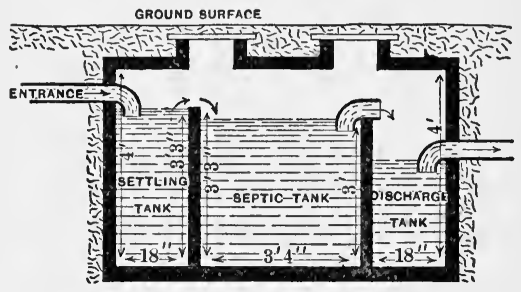

FIG. 64. - Lengthwise section of a septic tank.

drainage can be conducted to a field or to the garden. As the upper surface is at least one foot below the surface of the ground and usually covered with soil, no question of appearance is involved.

The excavation should be made large enough to allow for the framework and molds for the walls. It may be five feet wide by nine feet long by six feet deep. The molds are to be made of ordinary lumber with one side surfaced; the inside surface of the cement should be smooth. The cement floor should be five inches thick, and the walls and partitions about four inches thick. The entrance and discharge pipes are put in place while the cement work is being done so that they will be solid and of a piece with the whole tank.

86. Kinds of bacteria in sewage. - Such a tank is septic because it provides the conditions in which putrefaction occurs rapidly and thoroughly. It provides such conditions permanently and effectively. Putrefaction is brought about by the activities of certain bacteria. Bacteria of many different varieties are already in sewage in countless numbers, millions 
and millions to the cubic inch. Some of these dissolve organic matter, converting it into compounds that are not only free from dangerous or offensive elements, but also valuable as fertilizer.

There are in general three kinds of bacteria in sewage, two of which are harmless to human health and are beneficial in such conditions as the septic tank provides. The third kind is harmful, disease-causing, or pathogenic, as it is usually described.

The bacteria of one of the harmless groups flourish only where the supply of air or oxygen is abundant. They work near the surface of the soil, as in matter spread thinly over the ground or in wood such as fence posts, where one may find evidence of their work in the decay near the surface of the ground. These bacteria throw off waste products containing much more oxygen than do the substances upon which they live. For this reason as well as the fact that they depend upon oxygen for their existence, they are called oxidizing or aërobic bacteria.

The bacteria of the other harmless group which promotes decay of organic matter thrive in the absence of air or oxygen, that is, in just such conditions as are provided by the septic tank. These become firmly established in such a place, and liquefy, putrefy, and purify all its contents. Because they thrive in the apparent absence of oxygen, they are called anaërobic bacteria. The central division of the septic tank is most favorable, for there the sewage is still or at most moving very gently. They reside mostly in the sludge formed on the bottom, although the slow eddying of the liquid brings some to the surface, where they inoculate incoming matter and start its decomposition.

It is easy now to understand why at least three divisions of the septic tank are necessary. Because sedimentation and agitation of the liquid are unfavorable to these bacteria, the first compartment is provided to allow settling and prevent the disturbance of the second or middle division. In the first section the incoming matter loses its sediment and is slowly and gently transferred into the middle compartment. The 
coarse parts of the sediment are broken up and in time liquefied unless they are unusually resistant material. Such substances as paper, cotton cloth or similar vegetable fiber, wood, and certain kinds of wool fiber are dissolved in the course of a few months. The third division effects a continuous removal of the purified liquid without disturbing the main body.

Disease-causing bacteria. - The third group of bacteria found in sewage and due to human waste is the disease-causing or pathogenic kind. This group forms a real danger to the health of all members of the family and community. One variety of the pathogenic bacteria is that known as the typhoid fever bacillus or germ. This may come from two sources: first, from the discharges of patients suffering with the disease; second, from individuals who are typhoid carriers. Some of these may never have had the disease, and be immune to it, yet carry the germs and distribute them unknowingly where much harm may result. Other individuals who have suffered with typhoid fever may continue to harbor the germs after recovery and throw them off in such ways as to endanger the health of others. There are, moreover, other diseases, such as dysentery, cholera, diarrheal disorders, and hookworm, which are distributed through the careless and imperfect disposal of human waste products. The germs of such diseases may be transferred accidentally to places where food and water may be contaminated. It often happens that vegetables like lettuce and celery, which are eaten without cooking and upon which dangerous microörganisms thus escape destruction by heat, are fertilized or otherwise polluted with unpurified sewage. Water which appears to be pure may be swarming with deadly microorganisms transferred to it through carelessly distributed human waste matter. Thus such germs find their way from the ill, or those who have been ill with such diseases, to those persons who are healthy.

These facts, which have been demonstrated repeatedly, should make clear the necessity and obligation of every one to 
employ such measures as will insure the widest margin of public safety. In rural sections or where the community does not provide a sewage system, the septic tank offers the safest and most economical insurance against the spread of disease. The conditions that the tank favors are exactly those in which the beneficial bacteria live and work and bring about the utter destruction of the pathogenic bacteria or germs of all kinds. In community sewage systems, disposal plants with chemical treatment of the sewage are necessary. For the important thing is to prevent the escape of the disease-causing germs and bring about their destruction. It is, however, a factor of commercial importance that sewage completely purified by bacterial action or chemicals is a safe and valuable fertilizer.

87. Chemical treatment. - In city sewage disposal plants the effluent is generally treated with chemicals to insure absolute safety. Hypochlorite of lime (chlorid of lime) in the ratio of one pound to ten thousand gallons is added. The same precaution may be taken with the effluent of the septic tank. A small amount of chlorinated lime sprinkled at the opening of the discharge pipe will do the work. All the precautions suggested may be taken to mean that the sane, scientific treatment of all human waste and sewage in order to destroy all pathogenic germs and prevent the spread of disease is incomparably easier and more economical than any known method of curing disease and its ravages.

\section{ViI. Purification of Water-Municipal and Domestic}

88. Still storage. - Storage in still reservoirs is one of the most important and effective means in securing safe water for all ordinary purposes. It has additional advantages : it conserves and makes possible a more equal distribution throughout the year; it mixes and equalizes waters from different sources. By mixing water from a source which may have been accidentally infected, with waters from safe sources, the danger of spreading disease is greatly reduced. 
Sedimentation takes place in still water, the very fine suspended matter, both inorganic and organic, settling to the bottom. Coloring matter is generally removed, and hard water is greatly modified for the better. The most important fact is that water of most dangerous sort can be purified by still storage.

It has long been known that water becomes safe and pure if stored long enough in casks or stone jars. It does not matter how polluted water may be at the beginning. At certain stages of the process the water may be vilely odorous; but eventually it becomes odorless, tasteless, and safe. From the old so-called practice of letting water purify itself, came the suggestion of storage reservoirs. It is now known, however, that water in storage is purified through the action of bacteria. These minute plants may flourish in the water for a while, but after consuming all the food materials in the water, they perish and settle to the bottom as sediment. Careful investigations have shown that many kinds of bacteria are destroyed and other kinds greatly reduced in numbers by the storage of water for two or three weeks. The germs that cause such diseases as cholera and typhoid fever are greatly weakened, and they and other dangerous germs are destroyed when the water is stored for a longer period. This fact alone is sufficient to justify storage in still reservoirs wherever the water supply is exposed to contamination.

Another advantage of still storage which should be mentioned is that it gives opportunity to test water previous to filtration or distribution. If an outbreak of disease that may be waterborne should occur, all sources of supply may be tested at once and the suspected source of supply shut off. Storage also reduces the work of the filter and increases its effectiveness. Filtering devices may fail accidentally to remove all impurities ; storage prevents such accidents.

There is a disadvantage in the still storage of water ; for the water may be contaminated while in storage. Exposed water catches dust, pollen, spores, and minute seeds from plants, and 
encourages their rapid growth. Algæ, masses of thread-like plants commonly called "pond-scum," flourish in still water, if there is a sufficient food supply. Algæ, in themselves undesirable enough to demand instant removal, point to a still more serious condition on the watershed or in the storage reservoir. The food of these plants consists of nitrogen compounds or nitrates. Nitrates come from three sources: (1) from drainage from richly fertilized fields; (2) from decaying organic matter, such as dead animals or plants, on the watershed or in the reservoir ; and (3) from sewage or human waste products. None of these has any place on the watershed or in the reservoir. The presence of algæ may, therefore, be taken as a warning that a thorough examination should be made in orrler to find and eliminate all sources of pollution from the collecting and storage areas.

89. The sand filter. - The schematic section of a sand filter (Fig. 65) shows more clearly than a description the

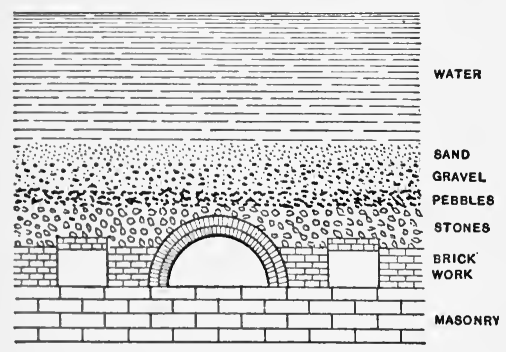

FIG. 65. - Cross section of a sand filter. essential features of its construction. There are several kinds in use, and all of them are reasonably satisfactory. Those of the type illustrated remove, after still storage, approximately 99 per cent of the remaining bacteria and other microörganisms. As the cost of the sand filtration, including all interest on original investment, operation, repairs, supplies, and depreciation, is comparatively small, estimated at about $\$ 1.00$ per 100,000 gallons of water, it does not seem difficult to justify its use. Even where water is supposed to be pure, as from a mountain stream, filtration minimizes the possibility of accidental contamination.

Such a filter as that illustrated will furnish water at a rate of about two gallons an hour for each square foot. This is 
equivalent to over $2,500,000$ gallons an acre a day, which is taken as the best rate of filtration. The operation is not satisfactory for a much higher rate, as it cannot remove many of the deleterious substances.

A sand filter depends not so much upon the materials and its mechanical parts as upon bacterial activity. The materials should primarily allow the water to filter through very slowly. Filters freshly installed or cleaned have slight effect on the water. Investigations show that bacteria in water passing through filters just cleaned amount to more than 1800 to each cubic centimeter. Before cleaning there were only forty to a cubic centimeter. On the floor of the filter is formed in time a peculiar gelatin-like substance which is the habitat of beneficial bacteria. Cleaning removes this and largely destroys the bacteria. The mechanical catching of suspended matter is only one part of the function of a filter. The invaluable part is the bacterial destruction of organic matter.

90. Purification in the home. - There are often occasions when water should be purified in the home. The market is well supplied with porous, charcoal, and other ingenious filters. These as may easily be shown are of little value except to remove suspended matter of certain kinds. If the water is suspected of carrying impurities, a simple and effective means of purifying it is by boiling. Disease-causing bacteria and other organisms are killed by boiling. Spores of certain kinds are not so affected; but these are not considered harmful. Water that is boiled should be allowed to stand before decanting into containers for storage. The peculiar flavor may be remedied by vigorous shaking to aërate it thoroughly, or by cooling.

\section{SUMMARY}

Cholera, typhoid fever, dysentery, and certain other diseases are often water-borne.

Many epidemics proven to be due to polluted water show the necessity for purifying water. 
The disposal of sewage is the most important problem in providing a safe water supply.

Sewage disposal plants are used by some cities.

In rural sections a concrete septic tank is the most effective means of disposal for a single household.

The three main groups of bacteria found in sewage are the aërobic, anaërobic, and pathogenic.

Typhoid bacilli come from human beings who are typhoid patients or typhoid carriers.

The effluent from a public or private septic tank may be treated with chemicals to insure absolute safety.

Storage in still reservoirs increases the safety of water through sedimentation, the activities of beneficial bacteria, and the weakening or destruction of pathogenic germs.

The presence of algæ in a reservoir indicates sources of danger on the watershed.

Sand filters, such as are used by municipalities, are effective in removing pathogenic germs through the action of beneficial bacteria, and are inexpensive to operate.

Water for the home may be made safe by boiling.

\section{REVIEW QUESTIONS}

1. What is the general belief as to the transmission of disease by water? 2. What evidence is there in support of this belief? 3 . What agencies are known to be involved in the transmission of typhoid fever? 4. Give in brief form an account of the method employed in tracing a typhoid epidemic to its source and in bringing the disease under control. 5. What is the lesson from the experience of such cities as Hamburg and Altona, Germany, Pittsburgh and Chicago, U. S. A., in dealing with water-borne disease? 6. What famous scientist was associated with the perfecting of the sand filter? 7. For what other discoveries is he renowned? 8. What general conclusion can be drawn from the facts presented in the table, page 209, as to the relation of water to health?

9. Is water safe for drinking merely because it is clear, odorless, tasteless, and cold? Explain your answer. 10. Mention five diseases which may become epidemic through the imperfect or careless disposal of human waste matter and sewage. 11. What are the common methods of sewage disposal in urban and rural districts? 12. Why is the septic tank better than the cesspool? 13. Describe the plan and operation of the septic tank. 14. What kind of bacteria does it 
favor and make use of in sewage purification? 15. (a) What kind of bacteria promotes decay in the presence of air or oxygen? (b) What are pathogenic bacteria? 16. What is a typhoid carrier? 17. Of what practical importance is it to every one to know that certain diseases may be distributed by food and water contaminated by human waste or sewage? 18. Why should each individual consider the health of others in disposing of waste products, refuse, etc.?

19. What are the advantages and the disadvantages in the still storage of water? 20. What especially dangerous elements are removed from water by still storage? 21. What happens to water when stored in casks for a long period of time? Explain. 22. In what ways is a sand filter useful and effective? To what is its effectiveness due? 23. Why is it sometimes necessary to purify water in the home? 24. What are the usual methods employed? Describe each. 25. Give reasons why the ordinary filter in the home is not a protection from disease germs. 26. How may such a filter endanger health?

\section{SUGGESTIVE QUESTIONS}

1. Give reasons why a community should be held responsible for typhoid fever cases due to a contaminated water supply. 2. Why should a community seek to discover the source of the disease and bring it under control, as well as the agency in its distribution? 3. How may a community effectively protect its citizens from the diseases mentioned in the text? 4. Typhoid fever is preventable by vaccination. Why is it to the interest of the community to vaccinate at public expense all its citizens, especially if they intend to travel abroad. 5. Explain why city water may generally be safer than a rural supply. 6. If an epidemic should occur and be suspected as due to contaminated water, what can be done in the household to insure safety in the use of the water?

\section{LOCAL WATER SUPPLY}

Exercise: The local water supply. $-A$. Is the local water supply under private or public control? To what extent is control exercised over its use for different purposes?

$B$. Study of the water provided. Is it soft, hard, or medium? How can you test this property? Examine the inside of a teakettle. Is there a white, hard substance on bottom and sides? What does this indicate as to the character of the water? How does the water 
react to soap? What effect has boiling upon its hardness? What means are commonly used to soften water?

Is the water clear or colored? Let a quantity stand in a glass for a while, and examine for sediment. What kind of sediment is precipitated, sand, clay, or vegetable matter? Evaporate about 100 grams of water in a glass beaker to find dissolved matter. When the water is nearly boiled away, remove flame or put beaker on a sand bath: Is there sediment?

Has the water any odor? The simplest way to test for odor is to fill a bottle which is perfectly clean and odorless about two thirds full, shake vigorously, and then smell immediately when the bottle is unstoppered. Has the water an odor when warmed? Fill a beaker nearly full, cover closely with glass cover and heat gradually. When the water is hot, remove from heat, and as you lift the cover, smell the vapor in the glass. If the water has odor, it indicates the presence of undesirable materials, living or inorganic. A rank odor like that from decaying matter is evidence of the presence of organic matter.

$C$. Has the water good pressure as it comes from the faucet? Is the pressure sufficient for fires and other emergencies? Is it due to the natural pressure, caused by the altitude of the reservoir which supplies the distributing mains? If mechanical means are employed to secure pressure, find out what they are and how regulated. If there is a water tower in the system, explain its operation. What conditions reduce the original pressure due to gravity or altitude of distributing reservoir?

D. At what distance is the source of supply from the community? What is the character of the intervening country? Describe the conduits, their course, and capacity.

What kind of water source is used? Is it surface, as stream, shallow well, or lake, or is it ground or deep water, as deep well or spring? Describe the collecting and conserving reservoir; its construction, capacity, and elevation above places of distribution. Is there any vegetation growing in the reservoir? Is there much of it? Of what kinds? Do you find algæ or other weeds growing in the water? The presence of algæ is very important (cf. page 220). Why? What animals, if any, occupy the water? What effect do you suppose they may have on its purity or safety for domestic use? If fish is abundant, is fishing in the reservoir allowed? Do you think this practice would have any influence upon the quality of the water?

$E$. What measures are taken by the community to prevent the pollution of the water and watershed? Is there a regular patrol? What power is vested in the patrol? 
F. The watershed. What is its extent? The character of the ground, sand, clay, gravel, rock, or loam? What kinds of vegetation grow on the watershed? What is the approximate amount of the weed type? About how much decaying vegetable matter is produced annually? Does much of the vegetation produce spores or pollen? Do you think spores or pollen make water less safe for drinking and domestic use? Cf. pages 197,220. Is the area inhabited by animals? What kinds and how many? Is it occupied by people? About how many? What do the people dwelling there do with refuse and sewage? Are any of the lands under cultivation? What fertilizers are used on such lands? How could this affect the water supply?

$G$. Is the water purified? If done by a storage reservoir, describe the location and other important features of the reservoir and state how long water is stored before distribution. See page 219 for proportion of bacteria killed by still water storage. If there is a filter system, describe it, giving size and capacity, arrangement of materials through which water is filtered, amount delivered in twenty-four hours, supervision and other interesting features. Compare action of a sand. filter with that of a household filter. What percentage of bacteria is removed by a sand filter? Are chemical substances used to sterilize the water? If so, what kind? Is the water tested regularly? How often? Has any epidemic such as typhoid fever been suspected as due to, or positively traced to, the contamination of the water supply? What facts support the conclusion? How was the water polluted? What was the source of the pollution? Give a summary of the chief impurities, both dangerous and harmless but undesirable, which may be found in water.

$H$. The sewage system. What is the local method of sewage disposal? What is the relation of the place where sewage is emptied to the source of water suppiy for your own and neighboring communities? If cesspools are used, are they so located as to avoid pollution of the water supply? If a sewage disposal plant is in operation, describe its general plan and operation. What disposition is made of the product of such a plant?

\section{Water Pressure}

Practical importance of water pressure.-Water supplied within the same system has different rates of flow or different pressures at different places and elevations. The feeble, slow trickle from a faucet on the third floor or higher is frequent in 
many city systems. When water is being used freely for sprinkling lawns or in laundry work on basement or first-floor levels, often little or none is available on higher floors in that locality. This is especially the case when the pressure is not increased by special means, such as the force pump, or when the reservoir is not high enough to give sufficient gravity pressure at all times throughout the system.

91. How pressure is secured. - It appears in the study of water supply that many cities, as Los Angeles, Denver, and New York, depend upon the elevation of the source or the distribution reservoir for the necessary pressure for all ordinary demands. - In the case of New York City the Catskill supply is sufficiently elevated to force the water to a height of 240 feet above sea level, equivalent to about twenty stories. In buildings exceeding this height, special means are provided by the owners to secure adequate pressure for usual service and for emergencies. The elevation of the Los Angeles supply, which is more than 4000 feet, gives a pressure which, after deducting for loss due to friction in conduits, is easily sufficient for all possible needs.

Many cities obtain their water from a source which makes the use of natural or gravity pressure impossible. Where a suitable hill is available it may be made the site of a storage and distributing reservoir, to which the water is pumped by force pumps. In many places where the supply is provided by deep wells, by a lake or a running stream, it is delivered directly into the mains and distributed at the desired pressure by pumping plants. In the country pressure may be secured by a supply tank elevated on a trestle out of doors or located in the attic of the house. Water is delivered to the tank by a force pump. operated by a windmill or a gasoline engine. Sometimes water may be supplied to the tank by a hydraulic ram. If the tank is placed in the basement, it may be made air-tight and water delivered at desired pressure by means of a compressed air machine or pump. 
92. Measuring water pressure. - A matter of practical concern to any one about to install a private water supply system, or to an engineer in charge of the installation of a municipal system, is how to determine what the pressure of the water will be for any given part or place of the supply system. There must be a minimum rate of flow at every point of delivery, and this cannot be left to chance or mere guesswork. A water supply that gives only a trickle at any place in a system is worse than none. Only the barest outline of the chief factors of this problem can be given here; but this may serve as introductory to a complete study of it such as may be found in any treatise on the mechanics of fluids, under the title hydraulics.

A cubic foot of water weighs about 62.5 pounds. A tank containing water to the depth of twelve inches sustains by its floor a pressure of 62.5 pounds for each square foot. The pressure on each square inch is $\frac{1}{144}$ of 62.5 pounds, or 0.434 of a pound. If now the height of the column of water is increased to two feet, it makes the pressure upon each square foot $2 \times 62.5$, or 125 pounds. What is the pressure upon each square inch? If the water were eight feet deep, the pressure would be $8 \times 62.5$ pounds per square foot or $8 \times 0.434$ for each square inch.

Water incompressible. - The weight, it is to be noted, does not change for a unit volume at different depths. Water is practically incompressible, and therefore at any depth or with any head or fall the pressure may be computed by multiplying its pressure at the depth of one foot by the total depth in feet. Pressure is always proportional to depth.

93. Pressure in moving water. - The conditions just stated hold for still water pressure but do not entirely apply to water flowing through pipes or conduits. Experience shows that the flow of water from pipes near the distributing reservoir is full and forceful, while, at a distance, unequal and inadequate pressure is the rule. Even though the supply reservoir is elevated above all places in the distributing system, the difference 
in pressure is conspicuous. What is the cause of the difference in the rate of flow?

Friction in pipes. - Water is retarded in its flow by the lengths, varying diameters, inner surfaces, and bends or angles of the pipes. These conditions, with possible air resistance in pipes, reduce the original pressure. All these factors are included in the word friction.

The amount of friction due to the inner surfaces of pipes depends upon the materials of which the conduits are made. It varies with wood, iron, steel, cement, and other materials. Wooden pipes, such as are generally used on the Pacific coast, give, when new, less resistance than iron or steel pipes, and become smoother and offer still less resistance after being used a few years. Iron and steel pipes, on the contrary, resist increasingly with use. Rivets in steel pipes cause additional friction. These facts indicate some of the obstructions to the flow of water, which are due to different kinds and ages of materials.

The loss of pressure due to length of pipe is considerable. A pipe 10,000 feet long and 1 foot in diameter, with a fall or head of 100 feet, delivers about 4.25 cubic feet of water a second. At this point it would throw vertically a jet of the same volume only a little higher than the upper surface of the pipe. At the reservoir a jet of water of the same volume would, if directed vertically, reach very nearly the 100 foot level. The pressure 10,000 feet away is thus perceived to be very much less than that at the reservoir. A working rule is that the loss of pressure through friction is directly proportional to the length of the pipe.

In laying long pipes it is customary to use pipe of large diameter, because the larger the diameter of the pipe, the less. will be the friction, or the greater the flow. There is loss of pressure due to the entrance of water into the pipe at the reservoir. In determining, therefore, the rate of discharge of water at any place in a system, it is necessary to take into consideration all these factors which cause friction or retard the flow. 
The standpipe. - During the daytime, water is usually drawn in largest quantities at all points in a system. The flow consequently becomes very feeble at the places farthest from the source of supply. To remedy this a water-tower or steel standpipe is often used in long pipe lines. This is a sort of supplementary reservoir, which stores up excess water when the demands are relatively small, and furnishes additional supply when the demands are greatest. In its operation it resembles the air reservoir of a force pump. The compressed air cushion in the standpipe (cf. page 231) assures a steady and sufficient flow at all points.

94. How water transmits pressure. - The way in which water in a closed container, such as a tank or a system of pipes, transmits pressure was interestingly demonstrated by Pascal (1623-1662), a French scientist. He filled a small barrel with water and fitted in the bunghole a vertical tube many feet in length. Into this tube he poured water until the pressure became so great as to burst the barrel. The weight of the water in the tube was comparatively small, yet it exerted enough pressure distributed with equal intensity in all directions or over the entire inner surface of the barrel, to cause the barrel to burst. The important point is that when pressure is applied to any unit area of a liquid in a closed container, it is transmitted in exactly the same amount against every unit area of the container in contact with the liquid. The pressure is exerted also in a direction which is perpendicular to the area in contact.

Suppose that the area of the cross section of the tube is one square inch, then the pressure of a column of water twelve inches high would be 0.434 of a pound. This pressure is exerted upon each square inch of the inner surface of the barrel and in a direction which is perpendicular to the surface at every point. If the height of the column is increased to two feet, the pressure per square inch is $2 \times .434$, or .868 pound. In short, increasing the height of the column increases at exactly the same rate the pressure upon each unit area in contact with the liquid. 
Hydraulic press. - The fact that water is practically incompressible and transmits pressure equally in all directions in a closed container is made use of in many ways in the industrial world. One of the many kinds of machines which depend upon these properties of water is the hydraulic press.

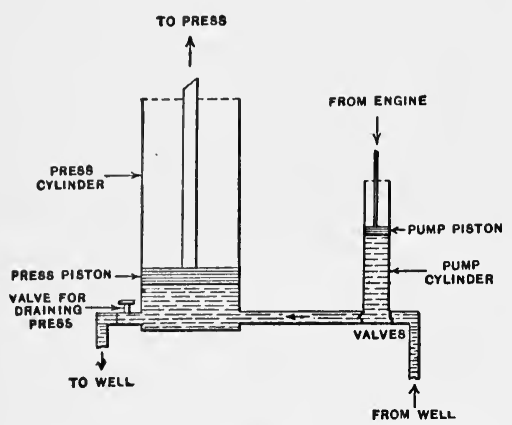

Fig. 66. - The hydraulic press. The pressure on the pistons is proportional to the square of the diameter. If a pressure of one pound is applied to the pump piston, what is the total pressure transmitted to the press cylinder? Scale one eighth inch to one foot.
This machine is used in many ways, as in baling cotton, in the manufacture of linoleum, in pressing olives, grapes, and the like. The hydraulic elevator is still to be found in many places. The chief features of the hydraulic press are illustrated in Fig. 66.

Exercise. - Suppose the area of the pump piston is $\mathbf{1 0}$ sq. in., and that of the press piston is 100 sq. in. What will a pressure of $50 \mathrm{lbs}$. upon the pump piston exert upon the press piston? The pressure is transmitted by water in a closed container equally and in all directions, so that the 5 lbs. pressure of each square inch of the pump piston gives a pressure of $5 \mathrm{lbs}$. to each square inch of the inner surface of the press in contact with the water. The total pressure exerted upon the press piston is therefore equal to 5 (pounds per sq. in.) $\times 100$ (area of press piston) or 500 pounds. How may the mechanical advantage of a press be increased? Would there be any advantage if the pistons had the same area?

95. The force pump. - All pumps that have their cylinders above the surface of the water supply depend upon air pressure (page 15). The lift pump delivers water without pressure. The force pump delivers water with pressure. The pressure may be utilized in delivering the water at a considerable distance from, and height above, the cylinder. The amount of pressure 
depends upon the amount of force applied to the piston and the strength of the pump.

A simple form of the force pump is illustrated in the diagram of Fig. 67. It is seen to be somewhat different from the suction or lift pump. The piston in this form is solid and valveless and made to fit the cylinder very closely. The discharge pipe is provided with a valve $(D)$ and with an air chamber. The upward stroke of the piston admits water into the pipe and cylinder; and the downward stroke forces the water to close the intake valve $(C)$ and to open

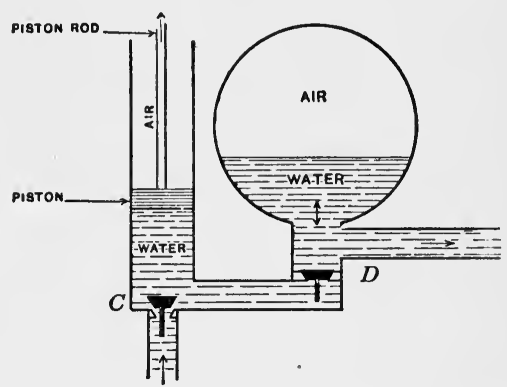

Fig. 67. - Diagram of force pump. Explain action of valves $C$ and $D$ as the piston moves up and down.

valve $(D)$. It also drives the water from the cylinder into the discharge pipe. Part of the water is forced into the air chamber, compressing the air, which, as soon as the pressure from the piston ceases, expands like a coil spring and causes the water to flow more uniformly.

There are many forms of the force pump, examples of which may be found in rural homes. They may be operated by hand, by a windmill, by a steam or gasoline engine. More complex, double action pumps are employed in the large pumping plants of cities where great pressure must be supplied. Such, too, are the pumps on fire engines. By these pumps a stream of water may be delivered with tremendous velocity and steadiness. The ammonia compressor shown in Fig. 42 is of the double acting type.

96. The centrifugal pump. - The centrifugal pump may be used where it is necessary to lift great quantities of water a short distance. The centrifugal pump may be likened to a fan such as is used to drive air through a building or to force the draft of a furnace. The wheel is inclosed in a water-tight metal casing. 
The important features are shown in Fig. 68. The water enters the wheel at the center, is caught by the blades and driven with

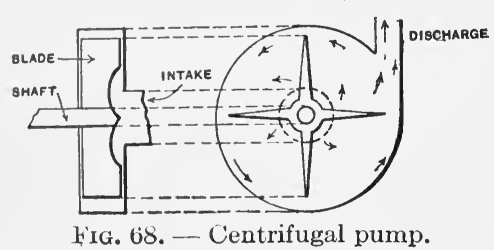
great velocity into the space surrounding the wheel and into the delivery pipe. The rotary force of the wheel is so great that it delivers the water steadily and in large volumes.

\section{SUMMARY}

Water pressure in supply pipes is due to gravity or force pumps.

Water pressure due to gravity varies with the depth, and is $\mathbf{6 2 . 5}$ pounds per square foot for each foot of depth.

Water pressure in pipes is reduced by friction.

The amount of friction in a pipe increases with the length of pipe, the roughness of its inner surface, the number of bends, and the decrease of the diameter of the pipe.

The pressure and flow at places distant from the reservoir or pumps may be equalized by the use of a standpipe.

An increase in pressure on any area of an inclosed body of liquid is accompanied by an equal increase on every like area of the liquid. This is Pascal's principle, and accounts for the action of the hydraulic press.

The force pump delivers water under pressure.

The centrifugal pump depends on rotation and is used to lift great quantities a short distance.

\section{REVIEW QUESTIONS}

1. What is the importance of water pressure in any supply system? 2. By what means is pressure secured in different systems? Give instances of each. 3. How is pressure of water in a tank measured? 4. Explain why water has a constant density at different depths. 5. What conditions reduce the pressure or retard the flow of water in a system of pipes? 6. Compare wooden conduits with steel pipes in their effect upon water pressure. 7. Explain how length and diameter of pipes modify pressure. 8. Explain how a standpipe works. 9. What is shown by Pascal's experiment? Give mechanical uses of this fact. 10. Describe the force pump and its operation. 11. Describe the operation of the rotary pump; and state its relation to air pressure. 


\section{SUGGESTIVE QUESTIONS}

1. If the pressure at a certain depth in salt water is 100 pounds per square inch what is the pressure at twice the depth? At three times the depth? 2. A householder finds that water flows with greater force from the faucet at midnight than at midday. Explain. 3. Show that there is less surface in large than in small pipes in proportion to the amount of water carried by the pipe. 4. If water were used at the same rate at all times during day and night would a standpipe be of any use? 5. What different devices do you know by which water pressure is increased in emergencies, as for a fire? 6. If there were no leaks and no water being used in a eity system of water pipes and the pressure were increased at the pump from 20 to 40 pounds per square inch how much change of pressure would there be in distant parts of the system?

7. Boilers are tested by filling them with water, then forcing into them a little more water. If by one stroke of the pump, after the boiler is filled, the pressure at the pump is increased by fifty pounds per square inch, what increase in pressure is thereby given to each square inch of the inner surface of the boiler? If the boiler were filled with air, would each stroke of an air pump of the same size increase the pressure on the inner surface at the same rate? Explain the difference. If the pressure were increased to the bursting point, which of the two substances, water or air, would cause a dangerous explosion?

\section{Water Power}

97. Importance of power. - The things required by man to satisfy his needs are produced by very complex activities, directed by men who are familiar with, and in some degree masters of, the resources of nature. The whole earth, through farm, forest, mine, river, lake, sea, and air, is made to give of its raw materials and to supply the power whereby manufacturing, transportation, and communication are made possible. Of the whole complex system of modern life and industry the power plant, without which machinery and appliances would be useless, is the central organ.

98. Sources of power. - The power plant to-day derives its energy from three main sources, fuel, water, and wind. Of all fuels, coal furnishes about 75 per cent, and oil, natural gas, 
and wood together, less than 3 per cent of the total power utilized by the industries of the United States. Falling water, the use of which is rapidly increasing for the direct production of power as in the hydro-electric plant, furnishes about 20 per cent. Wind, utilized for running pumps and small electric generators in rural districts, and all other sources make up the small remainder, or 2 per cent.

Power and the coal supply. - The industries of our country utilize annually more than thirty-five million horse power. About twenty-seven million of this is furnished by heat from fuel. Falling water contributes more than six million, which amount is being gradually increased. The current rate of consumption of bituminous coal is over $450,000,000$ tons, and of anthracite about $85,000,000$ tons a year. At this rate the known coal supply will last according to authoritative estimate for a period of seventy years for anthracite, and six to eight times seventy for bituminous. As the supply approaches the point of exhaustion and becomes increasingly difficult to mine, the cost must increase accordingly. In order to economize the known resources of coal, it is necessary to make all possible use of other available sources of power.

Available water power. - The amount of power available from falling water is, according to one estimate, about $100,000,000$ horse power. This estimate is based upon several conditions. First, it requires the construction of reservoirs to collect and conserve the flood waters and all suitable stream flow of the country. Second, it requires the conservation of the forests, and the reforestation of watersheds from which the forests have been removed. Forests are supposed not only to protect watersheds, but also to store up rainfall, effecting more equal distribution of stream flow throughout the year. Third, it seems to require a single, central, and wise control of water power and all the conditions upon which the water supply depends, together with the protection of the public interests in granting the use of water-power privileges. 
These conditions are not met at present and may not be met for a considerable period of time. There are, however, many available streams for the development of power, which, if not prevented by political and other factors, could be profitably utilized. The almost inexhaustible sources of water power in the Pacific coast states, in the Rocky Mountain region, and in many sections of the eastern part of the United States, are largely neglected or their use denied. A familiar instance of largely unused water power is the Niagara Falls together with the rapids of the Niagara River. This is one of the best known large volume and fairly abrupt fall rivers in the country, and offers immense advantages in the production of power. In its course of thirty-six miles, the river falls 326 feet, 206 feet of this fall being included by the rapids and Niagara Falls. The height of the falls is about 159 feet on the Canadian side, and 160 feet on the American side. The quantity of water flowing over the falls is estimated at 222,400 cubic feet a second. How many pounds will this equal? It is estimated that by proper conservation of the flood waters of the river $4,000,000$ to 6,500 ,000 horse power could be developed. At present a few hundred thousand horse power, a mere fraction of the total available power, is utilized by the Ontario and American hydro-electric plants.

The conditions upon which the above estimate of the possible water power of the United States is based, are at present impossible of realization. A more moderate estimate which takes account only of the average stream flow, puts the available amount at about sixty million horse power. The significance of these estimates is sharply emphasized by the fact that the total amount of water power now developed from falling water is hardly one fifth of the amount available according to the minimum estimate.

Comparative cost of power from fuel and water power. Approximately 500,000,000 tons of coal are consumed annually in producing $27,000,000$ horse power. Only 3 to 18 per cent 
of the heat developed from coal is actually utilized, the rest escaping as loss or waste. In a moderately efficient plant it costs an average of thirty dollars a year to generate one horse power from coal. This includes the cost of plant, maintenance, labor, and fuel. Most plants, however, do not keep the cost per horse power much below fifty to sixty dollars a year. Only the most efficient power plant working at full capacity every day in the year can bring the mean cost as low as fifteen dollars.

It is generally estimated that it costs on the average just about one half as much per horse power, or fifteen dollars as against thirty dollars for coal power, to generate power from falling water. And at least one half of the total amount of power now developed from coal might advantageously be derived from falling water. The total saving of coal by the substitution of water power would be not only large, but also widely distributed. The known coal supply would be conserved, thereby increasing by just so much the wealth of the nation, that is, the wealth of all its citizens. The cost of coal to the consumer would be kept at a reasonable figure. At present its cost is high and increasing - too high when considered on the basis of its efficiency in generating utilized heat; and it must increase as the supply decreases. The use of water now running to waste would thus not only postpone additional increase in the cost of coal for the consumer, but also reduce the cost of power to a remarkable degree (cf. Fig. 79).

To illustrate the economy of coal that may be effected by the use of falling water for the production of power, we may refer to the hydro-electric plant at Big Creek, California (Fig. 69). This plant will furnish 350,000 horse power to Los Angeles and vicinity. With a total head or fall of about four thousand feet, utilized in two power stations, and the immense volume of water supplied by the extensive area of the watershed, this seems to be a reasonable estimate. With such a fall one cubic yard of water is equivalent to fifteen pounds of coal in producing energy. It takes on the average six pounds of coal per hour to generate 


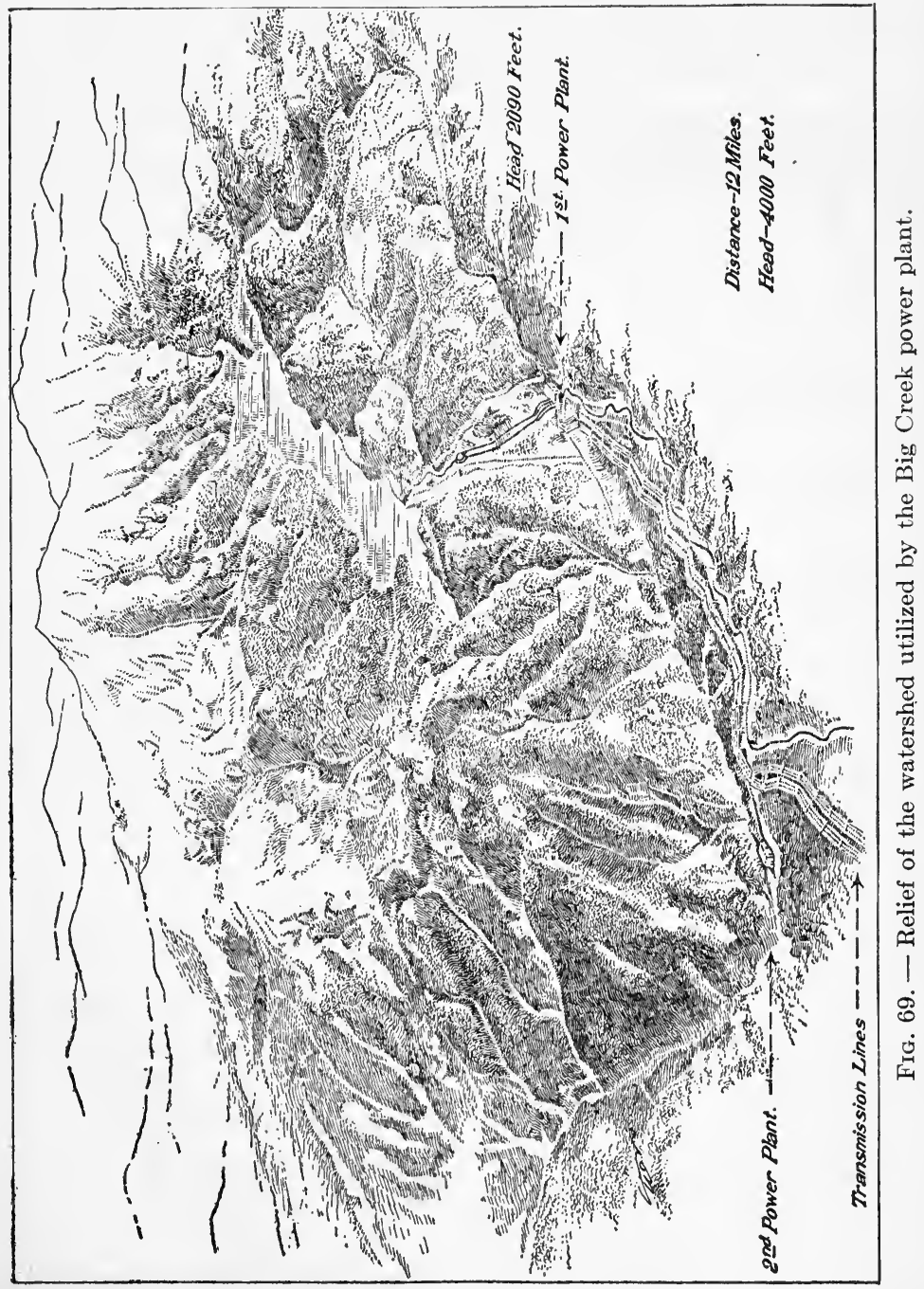


one horse power of steam. It is therefore readily computed that the amount of energy produced by the supply of water with this fall is equivalent to that delivered by approximately $7,800,000$ tons of coal a year.

\section{Water Wheels}

99. Modern water wheels. - It is just such conditions and possibilities as these which have brought about a wide-spread revival of water power. The quaint, old-fashioned water wheels, known as the overshot and undershot wheels, while chiefly of historical interest, revealed a principle that has been worked out more fully in modern wheels and turbines of the highest efficiency and adaptability to different conditions. The modern water wheels and turbines are adaptable to the volume of stream flow, to the fall or head of water, to different rates of speed and to almost unlimited demands for power. They have made it possible to harness and utilize streams that have plunged riotously to waste or those that have rolled sluggishly to the sea.

The Pelton wheel. - Falling water is made to yield its energy for useful work by two chief kinds of machines - the Pelton water wheel, designed to utilize a high fall and limited quantity of water, and the turbine, adapted to a low head and large quantities of water.

The Pelton wheel (Fig. 70) invented by a carpenter whose name the wheel bears, is made to generate from one fifth of a horse power to 20,000 horse power. At Rjukan, Norway, ten sets of Pelton wheels are installed in the first power plant, each set developing between 14,000 and 19,000 horse power with a fall of 970 feet. A second power plant, three miles distant, and 909 feet lower, uses the same water. The electric current thus generated is chiefly used in manufacturing nitrates for fertilizers.

The Pelton wheel is extensively used on the Pacific coast, where it was invented. It is especially adapted to the kind of 
water supply of that region. The streams that flow from the high watersheds of the mountains along the entire coast, not only furnish at present enormous quantities of power, but are capable of supplying approximately one third of the total possible water power of the United States. The mountain streams which were early used in hydraulic mining for gold, are now being harnessed to produce the energy required for industrial and domestic purposes.

The Pelton water wheel is impelled by water issuing from nozzles from a common supply pipe. As the speed of the wheel must be regulated or kept practically. uniform, and a change of pressure in the pipes cannot be made without endangering the pipes, the nozzles are made so that they can be deflected from or toward the buckets of the wheel. The ad-

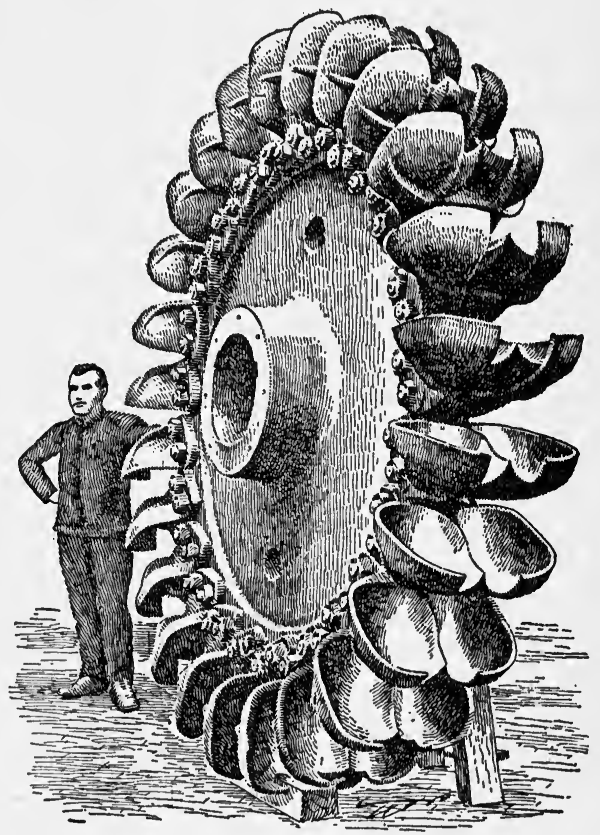

Fig. 70. - The Pelton water wheel, one of those used in the Rjukan, Norway, hydro-electric plant, developing about 7500 horse power. Estimate its diameter. justment is made automatically by a governor, which corresponds to the governor on a steam engine.

We can only with difficulty appreciate the velocity and force of water having a fall of 1000 to 2000 feet. The conduits at Rjukan, Norway, are of steel one inch thick to withstand pressure of more than 450 pounds per square inch. The actual 
pressure in the lower sections of the pipes is 420 pounds per square inch. The Big Creek power plant, California, utilizes in each of the two stations a head of nearly 2000 feet. The water, with a velocity of 350 feet a second, is forced through nozzles six inches in diameter, and as it issues from the nozzles resembles a solid rod of polished steel. The wheels, ninety-four inches in diameter, arranged in pairs, revolve as fast as the driving wheels of an express locomotive traveling one hundred miles an hour. So accurately, however, are the buckets of the wheel adjusted to the angle at which the water strikes them, that there is no shock of impact. So nicely adjusted also is the speed of the wheel to the velocity of the water issuing from the nozzles, that it brings the water practically to a standstill as it leaves the buckets. Thus the water is made to give up almost all its energy.

This point may be made clear in this manner. Suppose that the buckets on the periphery of the wheel are moving in the same direction and with the same speed as the water; they can therefore take from the water none of its velocity and consequently none of its energy. If the buckets, on the contrary, were kept stationary, they would throw the water back with about the same velocity which it has when it strikes them. If, however, the buckets move at a speed about one half that of the velocity of the water, they take from the water and utilize practically all its energy. For example, the velocity of a jet of water is one hundred feet per second, and the speed of the buckets of the wheel is fifty feet per second. The water moves forward fifty feet a second faster than the bucket which it enters. As it leaves the bucket, it must move backward with a speed of fifty feet per second with reference to the bucket. . Since the bucket is moving forward at the rate of fifty feet per second, the water is left practically at a standstill with reference to a stationary object.

Engineers compute accurately the velocity of a jet of water when they know the head or the vertical distance to the intake. 
With the velocity determined, they calculate what must be the proper velocity of the rim of the wheel. Then they plan for such a diameter of the wheel, and size of dynamo, as will give the proper speed to the buckets and secure the maximum amount of energy from the falling water.

The amount of work that a Pelton water wheel delivers for useful purposes (page 245) is unusually large in comparison with machines depending upon fuel. With a medium high fall of ten to twelve hundred feet, a wheel of ten to twelve feet in diameter will put out in useful work from eighty to eighty-five per cent of the total energy put in by the falling water. This is nearly six times the amount of useful work a steam engine performs with an equivalent amount of energy furnished in fuel. It is also more efficient than the undershot wheel, which it resembles in some respects.

The water turbine. - The water turbine is adapted to large quantities of water with a low head or fall. The head may vary from three feet to six hundred feet. In the usual style of turbine the water is admitted all around the circumference of the wheel through jets. It is deflected by guides upon the blades of the wheel, and as these lie across its pathway to the center of the wheel where it escapes, it pushes them to one side, thus making the wheel rotate. This is shown in the diagram of Fig. 71. Turbines may be horizontal with vertical shafts, or ver-

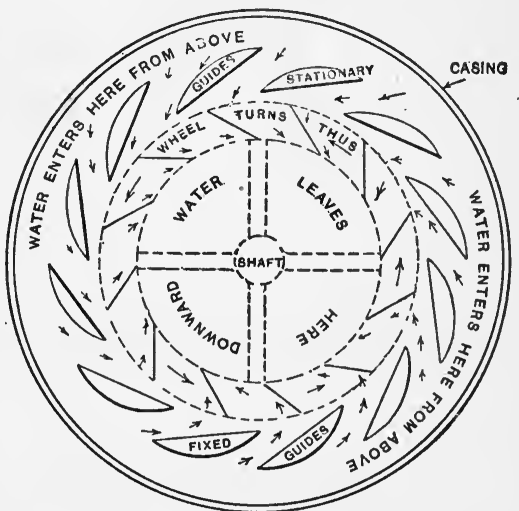

Fig. 71. - Turbine water wheel. Section through wheel and guides viewed from above.

They are made with one wheel, tical with horizontal shafts. or with two wheels fastened 
turbine is supplied with water from a common pipe and discharges it through a common pipe. The efficiency of the water turbine varies from eighty to ninety-five per cent (page 245).

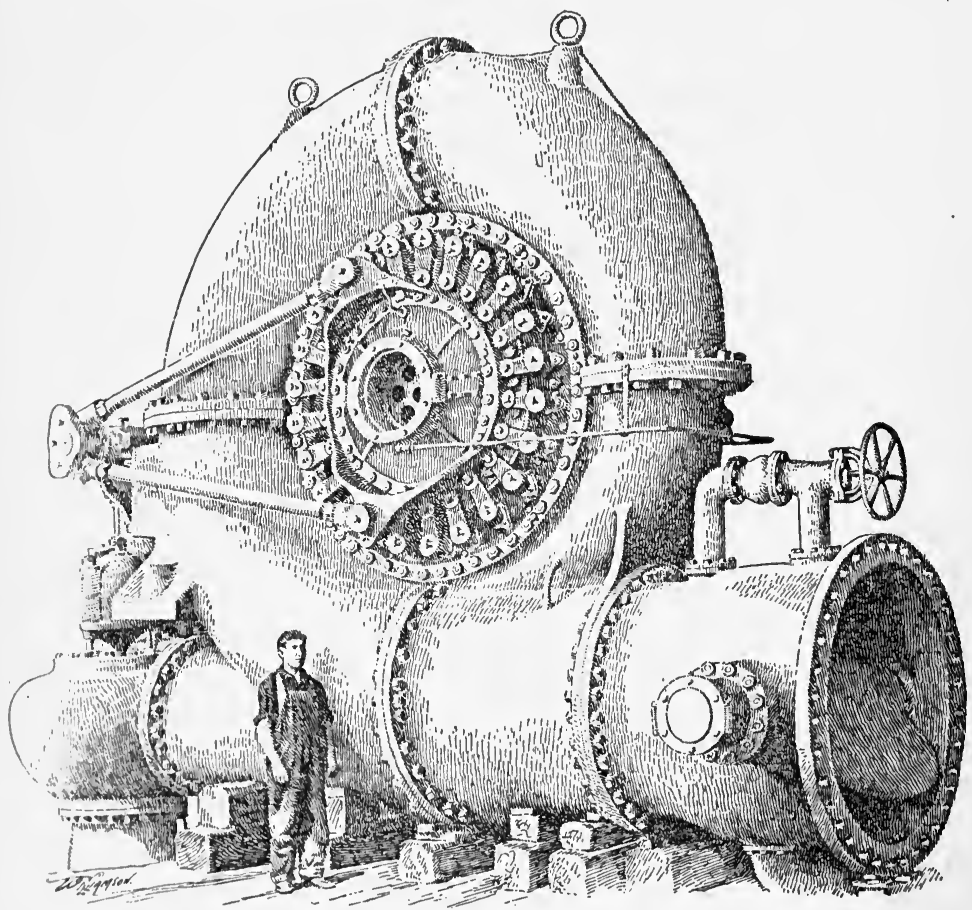

FIG. 72. - A ten thousand horse power water turbine for hydroelectric plant. Diameter of valve (right of picture) is 5.5 feet. Estimate diameter of turbine casing from height of man standing beside it. Turbine is adapted to a head of 519 feet and about 450 cubic feet per second, and will make 350 revolutions per minute.

Turbines vary in size from a few inches to fifteen or more feet in diameter, and in capacity from that of a toy pin wheel to that of a wheel producing 18,000 or more horse power (Fig. 72). A western plant has in operation four large turbines, each producing 18,000 horse power from a fall of about 500 feet. At 
Niagara Falls the. Ontario Power Company has now in operation fourteen twin-wheel turbines, each of which, from a supply by a pipe nine feet in diameter and with a fall of about 140 feet, produces 12,500 to 13,000 horse power. The plant at Keokuk, Iowa, on the Mississippi River, is equipped with turbines which generate, besides the power utilized locally, 231,000 kilowatts of electricity, equivalent to about 300,000 horse power, which is transmitted to St. Louis, 144 miles distant.

100. Horse power and efficiency of water wheels. - In the study of water power and machines for converting water power into working energy, we have mentioned frequently "horse power" and "efficiency." These terms are so commonly employed in describing power sources and machines that they need to be understood. We may consider them in the following manner.

Method of calculating energy of falling water. - Assume that the volume of a stream is two hundred cubic feet a minute, and that it has a useful fall of fifteen feet. The weight of 200 cubic feet of water is the product of $200 \times 62.5$ (pounds per cubic foot), or 12,500 pounds. Energy is measured and expressed in "foot-pounds," which means the amount required to lift vertically one pound one foot (cf. page 269). In this case the amount of energy produced by one pound of water falling a distance of one foot is one foot-pound. The total mass of water weighs 12,500 pounds, and in falling a distance of one foot gives 12,500 foot-pounds. But it falls 15 feet, and gives, therefore, 12,500 $\times$ 15 or 187,500 , the number of foot-pounds of energy. This may be expressed in a general proposition: The amount of energy is found by multiplying the unit of energy (one foot-pound) by the number of units of weight (pounds) and the number of units of distance (feet).

Calculation of horse power. - We have now to find out what horse power is represented by a body of falling water giving 187,500 foot-pounds per minute: First, what is a horse power? An interesting story connected with this term makes clear its meaning and use. 
About the year 1780, James Watt, who had manufactured and was then trying to sell steam engines to mine operators, discovered that he had to compete with the horse as a motive power. Coal and water were hoisted from the mine by a rope running over a pulley or wheel at the top of the shaft, and to one end of the rope the horse was hitched. As the horse walked away and pulled, it lifted the load. Watt quickly found it necessary to prove that his engine could do more work in the same period of time than the horse. To do this he had first to show what a horse power represents in foot-pounds per minute or other unit of time. By careful experiments he found that an average draft horse could lift a load of 150 pounds when walking 220 feet a minute ; or it could lift a load of 75 pounds when walking at the rate of 440 feet a minute, or 300 pounds when walking 110 feet a minute. In all these cases the horse is doing the same amount of work. The product of any set of two terms (weight and distance) given by each trial is 33,000 , the footpounds of work in one minute. This was therefore taken by Watt, and since then generally used, as representing one horse power, that is, the performance of work at the rate of 33,000 foot-pounds a minute.

It is a simple matter now to compute the horse power of the given fall of water. The fall, as you see, performs in one minute 187,500 foot-pounds of work. The horse power is to be found from the proportion 1 (h.p.): $x$ (h.p.) :: 33,000:187,500, or $x$ equals 5.6 horse power. That is, the horse power of a given fall of water is equal to the number of times 33,000 is contained in the number of foot-pounds of energy produced by the water per minute.

Exercise. - 1. Suppose that 650,000 tons of water pass over the Niagara Falls each minute, and the useful head is 160 feet. What will be the total horse power of the water?

2. The Keokuk power plant delivers 300,000 horse power of electrical energy, which is only 85 per cent of the total energy furnished by the water. What is the total horse power of the water? 
3. If 300 cubic feet of water fall each second a distance of 25 feet, how many foot-pounds are available? How many horse power?

4. How many horse power will a stream furnish which has a head of 10 feet and a flow of 50 cubic feet a second?

101. Efficiency of machines. - It is a common practice to-day to speak freely of the efficiency of anything. The term was originally applied to machines, and we shall here consider it in its original use. What is meant by the efficiency of a machine? Let us study this with reference to the available energy of a body of falling water and a water wheel or a turbine. A stream of falling water yields 187,500 foot-pounds of energy a minute. A water wheel utilizing this energy in doing useful work would, if perfectly efficient, deliver 187,500 foot-pounds, or exactly the amount that it receives. But a machine takes in more energy than it puts out in useful work. In every machine energy is utilized in overcoming friction and is wasted in many ways. No machine is perfectly efficient, that is, no machine puts out in useful work as much energy as it takes in.

Efficiency is expressed in percentage. A machine is described as so many per cent efficient, as for instance, 10 per cent, 25 per cent, 85 per cent, 90 per cent efficient. This depends upon accurate measurement of the output of useful work, and the input of energy. Suppose a water wheel delivers 150,000 footpounds of useful work; and the amount of energy available from the falling water is 187,500 foot-pounds. What is the efficiency of the wheel? It is shown by the ratio, 150,000 : 187,500 , or .8 , or .80 , or 80 per cent. The wheel is therefore 80 per cent efficient.

The efficiency of a machine is in general the ratio of the useful work delivered to the energy supplied, or

$$
\text { Efficiency }=\frac{\text { Useful work delivered }}{\text { Energy supplied }} \text {. }
$$

A machine is a device for transmitting or transforming energy already existing. It cannot create energy. The amount of 
work performed by a machine is never greater than the amount of energy supplied or put into it, that is, it cannot be more than 100 per cent efficient. Do these propositions state different facts or the same fact in different ways? If this is the correct view of the world of energy and of the purpose and efficiency of machines, can a plan to invent a machine that will create energy be sane? Is perpetual motion possible? Explain.

Exercise: Horse power and efficiency. - 1. A stream flow of 400 cubic feet a second has a head of 35 feet. What is the total amount of energy of the water per minute, expressed in foot-pounds? At what rate is the energy available, expressed in horse power?

2. A horse power is equal to 746 watts; and 1000 watts equal one kilowatt; or a kilowatt is equal to about one and one third horse power. How many kilowatts of electricity will the above determined horse power develop?

3. The Keokuk power plant produces 300,000 horse power. How many kilowatts will this equal?

4. Suppose the ascertained horse power of Problem 1 were utilized in water turbines of 90 per cent efficiency. What would be the amount of horse power delivered?

5. A Pelton wheel delivers 1100 horse power of useful work out of a total 1500 horse power input of energy. What is the efficiency of the wheel?

6. A machine delivers in useful work 18 per cent of a total input of 560,000 foot-pounds of energy a second. What is the horse power delivered? What is the horse power of the energy supplied?

102. Water power and transmission of energy. - The revival of the use of water power has been largely dependent upon progress in invention of machinery for the production and control of electrical energy. As long as the use of power was restricted to the immediate vicinity of its production, the use of falling water was necessarily limited to a few localities: Water for power purposes is not often available where the power is needed. Consequently other sources of energy, such as fuel for steam and later for. internal combustion engines, had to be utilized. Meanwhile men learned to produce electrical energy economically, to transmit it over long distances, and to 
make use of it not only in lighting and heating, but in transportation, manufacturing, and communication. To-day, falling water, wherever found, can be harnessed to produce electric energy, which may be transmitted a distance of five hundred miles and then converted into mechanical energy. This distance is only a beginning which the future will greatly extend.

Transmission of the electric current over long distances by means of a comparatively thin copper or aluminum wire at a relatively small expense is only one of the principal advantages of electricity. To-day electricity is transmitted over distances which a few years ago were considered impossible. The electric energy produced at the hydroelectric plant at Keokuk is transmitted to St. Louis, a distance of 144 miles. The current is carried at 110,000 volts on a five eighth inch copper cable, which is supported on steel towers eighty feet high and eight hundred feet apart. From the power plants at Niagara Falls electricity is transmitted about 160 miles in the United States, at 60,000 voltage, on aluminum wires one and one eighth inches in diameter; and in Canada at 110,000 voltage to more distant points. The Big Creek Power Company in California to-day transmits current with perhaps the highest voltage and over the longest distance. The two power stations, four miles apart, are located about seventy miles from Fresno, at Big Creek (cf. Fig. 69). The electricity is transmitted at 160,000 voltage to Los Angeles, about 240 miles distant. The six cables of aluminum are each one inch in diameter. The power is received at a sub-station, where it is transformed and distributed for domestic, municipal, transportation, and manufacturing purposes.

The transfer of electric energy over long distances at unusually high voltage is made possible by the transformer and the electric switch. At the power station the electric current generated is stepped up or transformed (see page 329) to a very high voltage for long distance transmission. At the receiving station the high voltage is transformed or stepped down to the 
low voltage required for various uses. The tremendous pressure at which electric current is transmitted would be impossible without the use of the modern switch. When a circuit is broken, the electric current tends to jump across the break, as experience teaches. The electric current meets the resistance of the air and forms an arc (page 175) of such high temperature as to melt and vaporize the metal of the ordinary switch. The modern switch is made to operate almost instantaneously by springs, and the are is controlled by powerful magnets.

The uses of the electric current have multiplied rapidly in recent years, with the invention of appliances for many purposes, in the home, the factory, on railways, and for communication. Lighting, heating, and cooking by electricity are becoming common in many localities. In manufacturing, the electric current is used in operating tools and machines of all sorts, in welding, in forge and foundry work, and in electric furnaces for making steel, aluminum, and nitrate (page 446). The improvement of motors, together with the improved means of transmission, bids fair to revolutionize transportation (pages $316 \mathrm{ff}$.). The progress achieved may be taken as prophetic of future advance in the uses of the electric current and in the utilization of falling water for the generation of electric energy for all purposes.

\section{SOMMARY}

The use of power is one of the principal characteristics of modern civilization.

Power plants derive energy from fuel, falling water, and wind.

The use of water power saves the coal supply.

Falling water yields only about one fifth of the $60,000,000$ horse. power readily available in the United States.

The average cost of energy from falling water is about $\$ 15$ per horse power for a year; from fuel about $\$ 30$.

The Big Creek-Los Angeles power plant yields power equivalent to that from $7,800,000$ tons of coal a year.

Modern water wheels are highly efficient and adaptable. The Pel- 
ton water wheel is 85 per cent efficient and adapted to a high head and small volume of water, as in Scandinavia and on the Pacific Coast.

The water turbine is 95 per cent efficient and adapted to a low head and a large volume of water, as at Niagara and Keokuk.

Energy may be measured in the units which are used in measuring work.

The amount of energy represented by a quantity of falling water is found by multiplying the unit of energy (one foot-pound) by the number of units of weight (pounds) and the number of units of distance (feet).

Power is the rate in time of doing work.

The English unit of power is defined as the horse power.

The horse power is doing work at the rate of 33,000 foot-pounds a minute.

Efficiency equals the useful work delivered divided by the energy supplied.

A machine transmits or transforms energy already existing, but does not create it.

The use of water power is increasing because of improvements in switches, transformers, and other appliances for the transmission of electric energy.

Examples of long-distance transmission lines are those between Keokuk and St. Louis, Big Creek and Los Angeles, and in the region of Niagara.

Electrical energy has a wide and increasing use for domestic and industrial purposes.

\section{REVIEW QUESTIONS}

1. Of what importance is power to man? 2. What is the relation of water pressure to the use of water for power? 3 . What are the chief sources of energy for power purposes? 4. What proportion of the total power used is derived from each source of energy? 5. What is the situation in regard to the known coal supply? How many years is it estimated to last at the present rate of consumption? 6. How would the increased use of water power influence the coal situation?

7. What are the estimated water power resources of our country? 8. What energy might be harnessed in the Niagara River? What are the chief obstacles to this? 9. Compare cost of water power with that of heat from fuel. 10. What is the estimated saving of coal per year in the California Big Creek power plant? How is this estimate made? 
11. What machines make use of the energy of falling water? 12. Describe the Pelton wheel, giving conditions to which it is adapted and recent notable installations of it.

13. Describe the water turbine; state conditions to which it is adapted; and examples of its use in well-known plants.

14. Compare the Pelton wheel and the water turbine, giving at least one point of similarity and one of difference.

15. What is a machine? Can a machine create energy? Cite examples of different kinds of machines in common use.

16. Explain the method of calculating horse power as worked out by Watt.

17. Explain: Horse power equals $\frac{\text { Foot-pounds per minute }}{33,000 \text { foot-pounds per minute }}$.

18. Explain : Efficiency equals $\frac{\text { Work delivered }}{\text { Energy supplied }}$.

19. What connection is there between the improvement of means of transmitting and controlling electric energy and the development of water power? 20. Mention instances of long distance high voltage transmission of electricity. Give important details. 21. Give at least five uses of electricity.

22. If there is a flowing stream in your vicinity, show what favorable and what unfavorable conditions it may present as a source of water power? 


\section{CHAPTER VI}

\section{TRANSPORTATION ON LAND}

103. Importance of transportation. - The importance of travel and transportation is more or less clearly recognized by all civilized peoples. Progress in the materials and arts of living is closely associated with the steamship and the railway. We may realize this by considering several groups of facts with reference to our own localities.

1. Consider what the local markets offer, that could not be had without the modern facilities of transportation. Food, clothing, tools, machinery, building materials, fuel, and many other necessities are imported in part from distant places. With the old means of carrying goods, most of the articles now considered necessities would be luxuries or would be impossible to obtain. Indispensable to transportation, moreover, is the present means of refrigeration. Modern carriers may perform wonders in speed, but without refrigeration meat, fish, fruit, and vegetables, milk and butter and other delicate foodstuffs could not be kept fresh during the long journeys.

Exercise. - Much may be learned of the geography of the productive regions of the earth and the importance of transportation by preparing a list of the goods in the local market brought from distant places; by studying the characteristics of the places where they are produced, as to soil, climate, methods of production, people, etc.; and also the carriers by which the goods have been transported to local markets.

2. The presence of people of different nationalities in practically every community is obviously a result of the increasing 
facilities for travel. This may be taken as a sign of the possibility that eventually all the nations of the earth may become one, commercially at least, through the use of natural forces in machines that triumph over space and time.

Exercise. - Prepare a list of the different nationalities represented in your local population; by what carriers they came; the living conditions and government of the country from which they eame; and the rate of increase of foreigners in your community in relation to improvement of facilities of transportation.

3. Progress in any of the factors of human welfare has always been associated with the exchange of ideas and resources among individuals or groups of people, such as tribes or nations. A group of individuals cut off from communication with other groups makes slow progress, if any, in what we call civilization, frequently becoming unprogressive and perhaps, in course of time, falling below its former stage of civilization.

104. Transportation and topography. - The character of the land surface, or its topography, has much to do with the development of transportation and the progress of its inhabitants. In a region where travel is difficult and transportation impossible, except of the smallest amount of goods, the inhabitants generally will make little advance in commerce and its associated arts and industries. Exchange between two individuals or groups of people is incomplete until the goods are transported to the place of the owner. Where transportation is impossible or too costly of time and labor, exchange is practically unknown. If, on the contrary, a region, by its level land and navigable waterways, favors travel and transportation, the groups of people dwelling therein are brought into contact; they develop commerce and with this the industries, arts, and refinements of civilization. Many examples of both these conditions are recorded in history and are also found to-day in many parts of the world.

From this it is evident that one of the most important conditions of transportation is the topography of a country. 
Topography means the inequalities of the land surface, which form natural barriers to free and easy traffic. Such obstacles include mountains, deserts and plains, rivers and lakes, canyons and rocky gorges, swamps, etc. With the improvement of vehicles and the use of motive forces other than those inherent in man and animals, roadways must be constructed or the inequalities be leveled so that vehicles may move over them with speed and safety. Thus mountains are cut through or tunneled, gorges are made to provide roadways of light grade, canyons, rivers, and swamps are spanned with structures of steel and concrete, while even lakes and parts of the sea are crossed by massive viaducts over which vehicles of tremendous power and weight travel. All work of making straight and level the ways regardless of surface inequalities is done with the purpose of securing the most economical, safe, and rapid travel and transportation.

\section{Vehicles of Transportation}

105. Primitive vehicles. - It was early discovered that a long, heavy stick or a small tree could be moved with less effort by dragging than by carrying. This suggested to primitive peoples a better way of transporting game or household goods than by carrying them on the head or shoulders. A small tree, or two slim poles fastened together, much like the thills of a buggy, could be utilized as a vehicle for transporting goods, being dragged by man-power, or later by animals. One end of this simple vehicle rested upon the ground; near this end the load was fastened, and the other end, like the handles of a wheelbarrow, was lifted and pulled. By this means a heavier load could be transported with less effort than by carrying. The vehicle resembled a wheelbarrow without a wheel, to be pulled instead of pushed.

A vehicle 'of this kind was used by certain tribes of Indians in North America for transporting their possessions from one hunting ground to another. In recent years one might have seen 
in the far western states certain tribes of Indians making use of these primitive vehicles pulled by ponies.

106. The vehicle as a machine. - This simple machine, for a vehicle is a machine, involves an important principle which is applied to-day in the most varied and intricate ways. By its use a limited force may be so directed as to overcome a large resistance. The principle is fundamental, but it can be presented here only in the simplest form.

In order to understand the advantage of such a vehicle or machine, let us consider the following example. Suppose a plank ten feet long represents the vehicle. As it lies on the ground, a load of 200 pounds is placed so that its center of weight is one foot from one end. If the weight of the plank is neglected, it will be found by trial that the force required to lift the other end of the plank will be twenty pounds. By shifting the load nearer to the end which is to remain on the ground, the force required to lift the other end is decreased. By shifting the load toward the end to be lifted, the lifting force must be increased. Suppose that the load of 200 pounds is located five feet from the end that is to rest on the ground, the lifting force at the other end must be just one half the weight of the load, or 100 pounds. That is, the product of the weight and its distance from the place or edge where the plank rests on the ground is found to be equal to the product of the lifting force and its distance from the end that remains on the ground. Or in this case, $200 \times 5=$ Force (100 pounds) $\times 10$.

The advantage of such a machine as a vehicle is evident. It requires much less force to lift and drag a load over the ground with such a machine than it does to carry it, if it can be carried. Give examples which verify this.

The lever. - The crude vehicle embodies an idea which has had remarkable development and varied application in different machines. To-day we call such a machine a lever. Its chief features are the point whereon the lever rests, called the ful- 
crum; the distance from the fulcrum to the apparent center of the weight, which is one arm, and the distance from the fulcrum to the point of application of force, which is the other arm. The arms are distinguished as the weight or resistance arm and the force arm. In the case of the primitive vehicle, the fulcrum, the weight arm, and the force arm are easily identified. Imagine the crude conveyance fitted with a wheel, the axle of which is the fulcrum, and you have a sort of wheelbarrow. What are the weight arm and the force arm of a wheelbarrow? Imagine now that two such simple levers are joined by their fulcrums on a common axis, and you at once recognize that the resulting machine resembles the nutcracker, one kind of lemon squeezer, and many other familiar tools. All of these are applications of the principle of the lever.

The fulcrum, the weight, and the force do not always occupy the relative positions shown in the drag and the wheelbarrow. The weight may be located at one end of the lever, and the fulcrum at any point between the weight and the force. Thus the crowbar may be used as such a lever or pry in moving heavy bodies. The principle involved in this use of the lever is exactly the same as in its use as a lifting machine (Fig. 75).

An example will make this clear. A stone weighing 450 pounds is to be moved by a pry. If the length of the weight arm is six inches and the length of the force arm 4.5 feet, what amount of force will be required? The relation of force and weight is exactly that stated above. The product of the weight and the weight arm is equal to the product of the force and the force arm; or $450 \times .5=$ Force $\times 4.5$, or 50 pounds, the amount of force required.

If the fulcrum is exactly midway between the centers of force and of weight, the weight and force must be equal. This is illustrated in the trip scales, or the teeter-board with equal weights on the ends. Both of these are equal-arm levers. With unequal weights the arms of the teeter-board are of unequal length; as shown in Fig. 73. 
Exercise. - Assuming that the weight is not given, how would you find it? How would you find one unknown length? Explain the relation between "pull of earth" and "push of stump," Fig. 73.

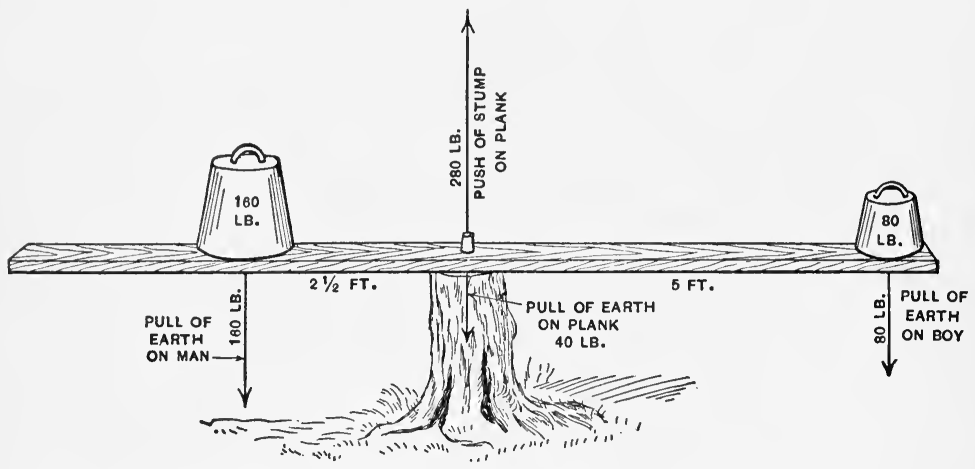

FIG. 73. - Showing the relation of weights and arms of the lever; and the relation of the downward pull and the upward push. The weights may represent a man and a boy.

To get a clear and definite idea of the principle of the lever, plan and carry out a series of experiments with a meter or yardstick and different weights. Make problems involving the relation of weight

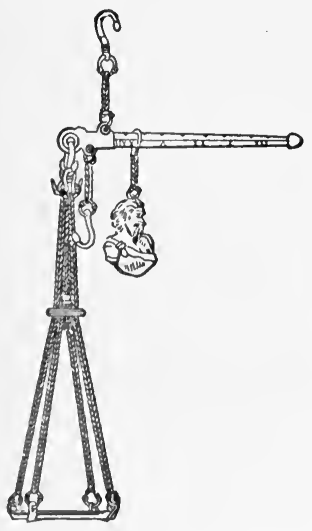

FIG. 74. - A Roman steelyard. Identify the fulcrum and the arms of the lever. and force and the length of the weight and force arms. Study the trip balance with its movable weight on the arm. Note that the weights are made to apply at definite centers. Study also a chemist's balance. What is the importance of having the centers exceedingly fine and hard? (Fig. 74.)

Adaptations of the lever. - The simple form of the lever used as a lift or a pry (Fig. 75) has been adapted to many different uses and converted into important parts of many tools or machines. A small crowbar bent somewhat at the fulcrum resembles a tack puller, and by a little more bending may be converted into a claw hammer, a nail puller, or other similar tools. Examine some common 
utensils such as a can opener, a spade, a doubletree of a wagon, a spoon, knife and fork, and so on, to see whether they make use of the principle of the lever, and if so, how.

Exercise. - At first sight a crank, such as that of an automobile, a windlass, a pedal crank of a bicycle, or a driving wheel of a locomotive, may not be associated with the principle of the lever as it appears in the crowbar. But imagine a crowbar bent into the form of a crank, and the resemblance is unmistakable. Note that the crank has its fulcrum, its resistance arm and force arm, just as the simple lever has. Identify each of these in a windlass, a bicycle pedal crank, a Dover egg-beater, or any other machine of this character.

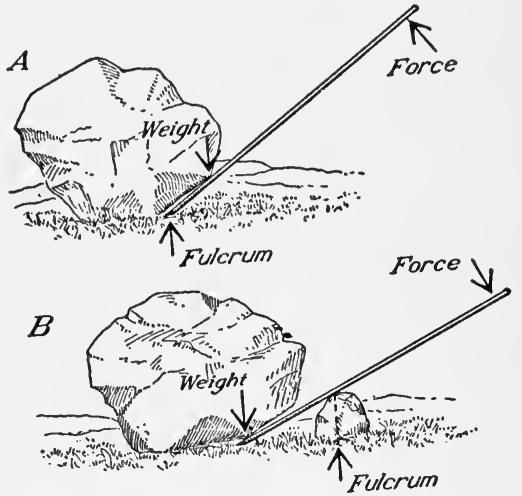

Fig. 75. - The lever. $A$, its use as a lift ; $B$, its use as a pry. In what other way may the lever be used? Give illustrations.

What is the effect of increasing the length of the force arm? Of shortening it? Such tools, moreover. as scissors, pliers, forceps, and the like, are obviously based on the principle of the lever, for they simply combine two levers on a common fulcrum or axis of movement (Fig. 76).

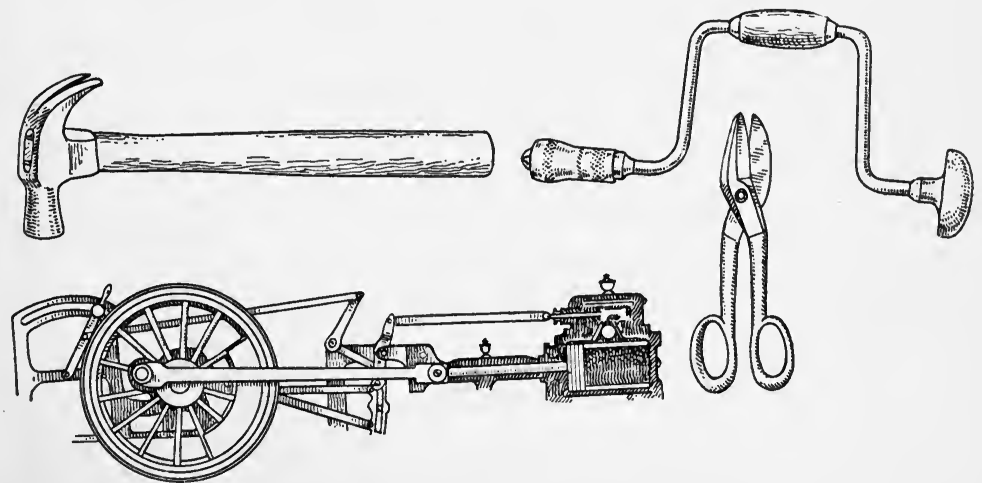

FIG. 76. - Identify in each of the tools or machines the fulcrum and arms of the lever; show how the lever is used in each case. 
These few applications of the principle of the lever may suffice to indicate its importance to man. The mechanical advantages were probably first studied and described by the ancient Greek mathematician and scientist, Archimedes (about 287-212 B.c.), who in his enthusiasm over its importance declared that if he had "a place whereon to stand" and apply his lever, he could move the world. From this it is evident that inventors in primitive ages devised in the lever a most useful machine. Its utility, in whatever form it may be embodied, lies in the fact that it gives man with his limited power a great advantage in overcoming resistance or weight. This is true whether it is employed in moving heavy bodies or in doing other kinds of work. Through his ingenuity man has turned the principle of this simple machine to all sorts of complex and intricate uses in machines ranging from the ordinary pry to the windlass, the watch, the steam shovel, and the locomotive.

107. Friction. - The use of the drag as a vehicle leads directly to the discovery that a heavy load can be moved more easily over a smooth surface than over a rough. Dragging may be made easier by means of sled runners; but with the use of runners there must be some improvement of the trails or roads over which heavy loads are frequently dragged or hauled. It is a clearly recognized advantage to reduce as much as possible the resistance encountered in moving heavy loads over roads. To-day such resistance is generally known as sliding friction. Thus progress in road making is intimately associated with improvements in vehicles; both being designed to reduce sliding friction to the minimum.

The wheel and axle. - One of the most important discoveries,. ranking with that of fire and of the bow and arrow, was the wheel which turns on a shaft or axle. To make the ends of poles or the runners of a sled or drag slide over rough or soft earth, was far from easy, and it required great strength for heavy loads and allowed only a slow rate of progress in favor- 
able conditions. But with a pair of wheels rolling under the load, transportation became a source of pleasure as well as profit. The one-wheeled vehicle, like the wheelbarrow, and the two-wheeled, like the cart and the chariot, revolutionized travel and the transportation of goods over the land.

Rollers. - It is not known when or by what people the wheel was first used. The ancient Egyptians and the dwellers in the valleys of the Tigris and Euphrates made use of it on chariots. Perhaps it was suggested by the roller and the lever, which were used in transporting heavy stones for the Egyptian pyramids, statues, and temples. Rollers of thîs kind are still used for moving buildings and other heavy masses, and they are adapted to many other purposes, as in sawmills or in portaging boats around rapids. What advantage does the roller give over sliding? Is there more or less friction with the roller? Explain your answer.

Exercise: Means of reducing friction. - One or two experiments will help in understanding the value of the roller and the wheel in reducing friction. Get a block of wood, load it to weigh about 200 grams, and fasten a serew eye into one end. Insert the hook of a spring balance into the screw eye and weigh the block. Then with the balance in a horizontal position and in line with the screw eye, draw the block slowly and steadily along on the table. Take several readings of the balance as the block moves. If the readings vary, take the mean or average. Which now is the greater, the force required to lift the block, or the force to pull it on the table? Does it generally take more strength to carry an object than to drag it?

Place the block on a pair of rollers and draw it along on the table. Take readings of the balance as the block moves. If the readings vary, take the average. Which now requires the greater force, dragging or pulling the block on rollers? Repeat with wheels.

Another point should be noted. With the block at rest on the table pull slowly and gradually on the balance and take the reading just when it is about to start and again when it is moving steadily. Compare the two readings. What do you think the difference in the required force indicates?

If in walking one feels a foot slip, does one instantly check the slipping foot or throw the weight on the other to maintain or regain bal- 
ance? Explain. The same fact is illustrated in the locomotive. An engineer in starting a train may sometimes let too much steam into the cylinder, so that the driving wheels slip and spin on the rails. If he supplies more steam while the wheels are slipping, will the engine start the train? What must be done to give the wheels a chance to take hold? Does this agree with the facts observed in the case of the sliding block? Why and how is the track sanded?

Advantages of friction. - The facts presented in the experiments show both the advantages and disadvantages of sliding and rolling friction. They emphasize especially the necessity for a certain amount of friction between the wheels and the track in order that the train may move. A frictionless contact of wheels and track would make a start on a level track impossible. Imagine, for instance, a locomotive trying to start on greased rails. Furthermore, locomotives and many other moving bodies must stop. What is the necessary condition of stopping a train, a wagon, an automobile, or a boat on the water? Explain each particular case.

There is friction also between the axle and its bearings. This is much less than that between the wheels and the track. Is this friction of the axle and its bearings advantageous or not? Suppose that instead of greasing the axle, the track were greased. What would be the result? Consider this in another way. The diameter of a wheel is two feet. What is its circumference? The diameter of the axle is four inches. What is its circumference? On the basis of these dimensions, how many times as far as the axle slips in its bearings will the wheel roll on the track? Which will be the more advantageous, reducing the friction between the wheel and the track, or that of the axle in its bearings? Explain your answer.

Friction is commonly reduced in axle bearings by the use of lubricants such as oil and graphite. Other means of overcoming such friction are by the use of what are called frictionreducing metal alloys. One of the most familiar anti-friction alloys is Babbitt metal. If we examine the bearing of a small 
engine, a windmill, a railroad car, or any similar bearing, we find that it comprises two parts, one the cast steel frame, and the other the brass or gun metal bush, as it is called, which provides an exceptionally smooth surface. The brass bush is often grooved inside in the direction of the axle. The grooves are filled with a white metal alloy, which, while fitting closely about the axle, yields sufficiently to weight or pressure and causes less friction than cast steel bearings. When worn out, the alloy may be renewed without difficulty.

Exercise. - If convenient, a student may be assigned to visit a factory or mill where babbitting is done, and report to the class on the process. He may report also on other means employed in the factory in reducing friction in machinery.

As an instance of means employed to overcome useless friction, the bearings of a modern bicycle may be examined. Students may be expected to explain their construction, operation, and mechanical advantages. Evidently the bicycle needs an adjustable bearing which, as may be seen, is secured by the use of one or more rings of case-hardened steel balls running in a groove. Both ball and roller bearings are utilized in automobiles, since they cause less friction than any machine which slides; and, when speed and power are necessary, means which reduce sliding friction are utilized.

Exercise. - What are the chief applications of the roller in modern machinery? Compare these applications with its use by the ancients, determining in what particular ways progress has been made.

Rolling friction. - The friction between the wheels and the road is peculiarly interesting, although not quite so obvious on steel rails as on dirt roads. On a highway a little hill of dirt (Fig. 77) is formed in front of the rolling wheels of a loaded vehicle. This is crushed, but it requires energy to do it. As the friction thus encountered is continuous, the expenditure of energy in overcoming it must also be continuous. The same kind of friction is met on the steel rails over which a train passes. 
The rails are depressed ever so slightly by the wheels, and each wheel must be pulled continuously from the bottom of the

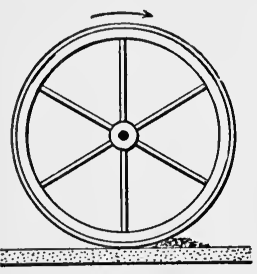

Fig. 77.-Diagram showing rolling friction on road (greatly exaggerated). depression. This adds to the labor of the locomotive. Resistance of this kind is known as rolling friction.

The rolling friction of wagons and automobiles is reduced by the use of wheels with broad tires, which prevent their sinking deeply into the road. In order to eliminate in large part the rolling friction of railroads, roadbeds are built solidly of crushed, closely packed rock, heavy ties or sleepers being used ; the size, weight, and hardness of the steel rails are increased; and smooth, properly tempered surfaces are provided on the wheels of engines and cars. Examination of a track will show to what extent these means are utilized.

\section{SUMMARY}

By means of transportation the whole earth may be made to contribute to the needs of any community. By travel individual and social progress is accelerated.

The topography and climate of a country largely control and modify the means of transportation.

A primitive vehicle of transportation is the pole drag. It is based on the principle of the lever.

The forces in a lever are inversely as the length of the arms.

The use of the lever is illustrated in such appliances as (1) the teeter-board and equal-arm balance, (2) the crowbar, (3) the clawhammer, (4) pliers, (5) the nutcracker, (6) the windlass, (7) the locomotive driving wheel.

The use of the roller substitutes rolling friction for sliding friction.

Friction of a wheel on the ground, or rolling friction, is reduced by the use of a hard roadbed. Friction of a wheel on its axle is reduced by means of oil, by babbitting, and by roller or ball bearings.

Sliding friction decreases when an object begins to slip.

\section{REVIEW QUESTIONS}

1. Mention several facts that show the importance to your community of transportation. 2. Mention several food products of com- 
mon use in your community, that have been transported at least five hundred miles. 3. How have modern facilities for travel changed the population of your community? 4. What are the greatest geographical obstacles to travel and transportation? 5. What effects have geographical conditions on the civilization of a people?

6. How may the transporting capacity of a man or a horse be increased by the use of two poles? 7. If a plank is of uniform dimensions and weighs 40 pounds, what force will be required to raise one end? 8. If the plank is 16 feet long and a weight of 300 pounds is placed 2 feet from one end, what force must be used to lift the other? 9. Where are the fulcrum, the force arm, and the resistance arm of a nutcracker? Of a pair of pliers? Of a wheelbarrow? Of the drive wheel of a locomotive? 10. If the force is applied ten times as far away from the fulcrum as the resistance, how many times the magnitude of the force is the magnitude of the resistance? 11. Mention several appliances which are modified forms of the simple lever. 12. What advantage did Archimedes claim for the lever? Who was he and for what was he noted?

13. Mention early uses of the roller and the wheel. 14. What is sliding friction? 15. Mention several instances where friction is necessary. 16. Does sliding friction increase or decrease when slipping begins? 17. What practical knowledge of friction does the locomotive engineer or the automobile driver need? 18. Why do ear wheels turn and not ordinarily slide on the track? 19. How is friction reduced in a wagon, bicycle, automobile, windmill, and railroad car?

\section{SUGGESTIVE QUESTIONS}

1. Is starting friction greater than sliding friction? 2. What does the experiment with the block and spring balance show as to this? 3. Does sliding friction decrease with increase of rate of movement? Does the spring balance show greater or less force when pulling the block slowly or when pulling it rapidly? 4 . If the sliding surfaces of block and track were lubricated, would the friction decrease? Why? What other means might be employed to reduce friction? 5. Do you know why a motorman or engineer on a steam locomotive always reduces the air pressure on brakes when the car or train is just about stopping? 6. Name some of the uses of friction as shown in the common utilities of the home. Of the shop. Why do nails that are well driven hold securely? How should tent stakes slant, toward or away from the tent? Why? Why are engines able to start on an apparently smooth track? 7. What is the principal advantage of rollers, balls, or wheels, over sliding? 
8. In any machine you find convenient, as a sewing machine, grindstone, lathe, or bicycle, show what combinations of the simple lever, the roller, and the wheel and axle are made. 9. Show that the roller, wheel and axle, and the crank are modified forms of the lever.

\section{ROADS}

108. Roads and vehicles. - As the vehicles used in transportation are improved and multiplied, and the motive forces employed become increasingly those of steam, electricity, and gas, the roads over which carriers must travel are necessarily improved. In mountainous regions where trails are difficult and narrow, pack animals, such as the horse, the mule, the llama, or the birro, serve until traffic becomes too heavy. Then wagons are introduced and trails must be changed into roads or new roadways built over which wagons can move. Such improvement is often slow, as may be observed to-day in many rural districts where the farmers suffer yearly loss because of the disproportionate expense of getting their crops to market. The roads are unfit for loaded wagons and impossible for motor trucks. Public interest in good roads is rapidly developing, however, not only supporting, but even demanding, more and better roadways; for it is realized that the expense of transportation is borne not only by the farmers, but by the consumers as well.

With the rapidly increasing use of the automobile the construction of roads has become more and more of a professional engineering problem. The work is now regarded as too important to be left to unskilled supervision, as it was a few years ago. To-day the most durable materials must be utilized and the surface form must be such as to insure perfect drainage and to facilitate transportation. But with the present tendencies in road construction we may compare the ancient Roman roads, which are the most solid and durable ever constructed, the Claudian Way being still in use after two thousand years of service. Does your observation of recently constructed 
roads lead you to suppose that they will last as long as the Roman roads? Explain, giving examples of different kinds of roads.

109. Kinds of roads. - In cities, where traffic is exceptionally heavy, streets are macadamized, are paved with bricks or wooden blocks, or have a concrete base covered with a layer of asphaltum and sand. Asphaltum, like petroleum, is a natural product, obtained mainly from the asphaltum lakes in Trinidad, South America. In rural districts roads are often dirt-covered, more or less stone being mixed with the dirt. Sometimes country roads are made by covering a layer of coarse, well-packed rock with finer rock, which is bound together by tar, crude petroleum, or asphaltum. Dirt roads may be merely oiled with petroleum, or they may be plowed up and mixed with crude oil and then packed until they become solid and smooth. What kinds of roads are found in your vicinity?

A good road is higher in the center, sloping gradually towards the sides. What is the advantage of this surface form? Culverts under the road take care of temporary streams, while bridges are built over permanent waterways. Hollows or ravines may be filled in or bridged. Hills are graded down or cut through to secure relatively light grades.

110. Roads and motive force. - The use of steam, electricity, and gasoline as motive forces has made necessary radical changes and improvements in the kind and nature of the roadway. Solid dirt or macadamized roads are fairly useful for slow transportation of small tonnage; but with the present industrial and commercial activities such roadways are entirely inadequate except for a limited tonnage over short distances, as from farm to station or elevator. The chief conditions in road making to-day are determined by the available motive forces, that is, the pulling power and the speed. The importance of the roadway to motive force may be illustrated in the following example. 
Let us assume, for the sake of keeping the number within easy comprehension, that a horse exerts a pulling or tractive effort of 100 pounds, although a horse usually has greater pulling force than this. On a soft, dirt road of the usual country type, such a tractive effort will haul a load of about 400 pounds. On a macadamized road, smooth and level, it will haul a load of about 3000 pounds. On steel rails and a level track it will haul a load of approximately 20,000 pounds. This illustrates clearly why the heaviest hauling is done on rails. Speed of movement may be considered in the same connection; but it will not essentially modify the numbers given for the load and the pulling force.

\section{RaILroads}

111. Conditions determining location of railroads. - Several features of railroad construction are worthy of careful study because of the mechanical principles which they illustrate. A railroad is located in a new country where it will secure the greatest natural advantages in construction and hauling, and also obtain whatever traffic the region may afford through its mining, agriculture, and other industries. Formerly railroads were run in a very winding way through a hilly country to avoid many heavy grades, tunneling, and expensive bridgework. To-day speed, hauling capacity, and economy of power are necessary, and not merely ease and economy of construction; consequently roads are built directly through mountains. by tunnels and across rivers and canyons by bridges and viaducts. As instances of such road building we may refer to the Chicago, Milwaukee, and St. Paul, and the Union Pacific railroads, which save several hundred miles of trackage, reducing by several hours the running time between Chicago and the Pacific Coast, and the expense of operation which is almost enough to balance the additional expense of construction.

Railroads, moreover, are expending millions of dollars to shorten the routes and reduce the grades and curves of old 


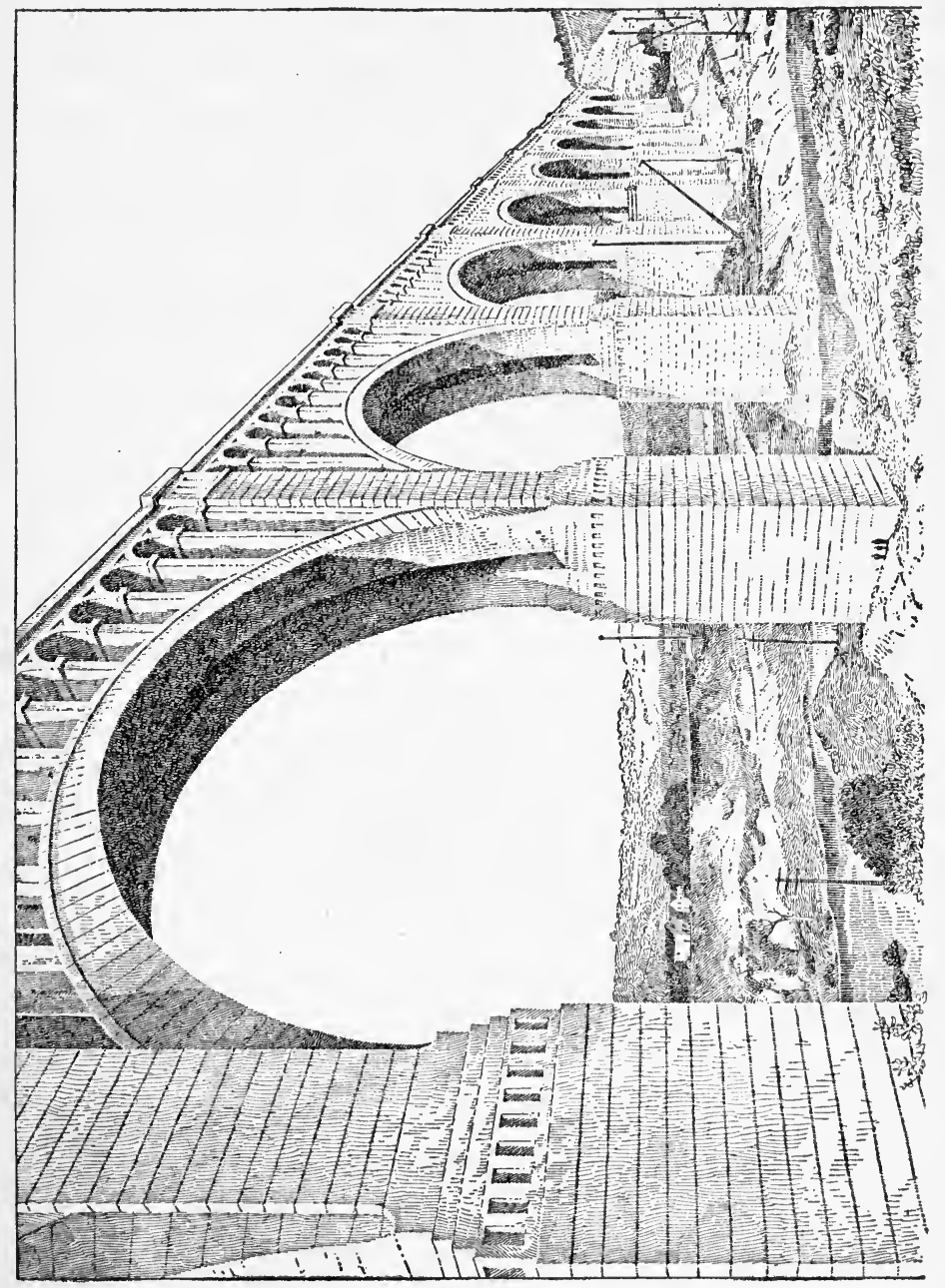

总怘军

๙

$\because 0$

동

귱

:

들

.

융

$\underset{4}{+}$

원.

경

. 에 ᄚ

$\stackrel{\square}{\circ}$

.

유요

䨔

舲

สี की

N

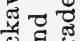

गै

$\checkmark$ \&

๑

$\leftarrow+$

$+4$

잉

త్ర

洸茨

동 욜

을 미

ㄴ 0

త్తె

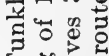

ㅂ. 요

1 氛苍

$\infty$ 党

1 8.5

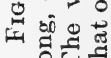


construction, in order to increase the speed and effect economy in hauling freight and passenger trains. A recently completed cut-off on a section of the Lackawanna Railroad (Fig. 78) is an example of such engineering economy. At a cost of about $\$ 12,000,000$, a saving of less than four miles in route is effected; but the new grade is such that two locomotives now haul the load which required five over the old grade; in passenger traffic twenty minutes is saved, and in freight about one hour over the old schedule.

Tunnels are necessary on many railroads, and they may be used on highways through hilly country or for subway systems in large cities. The subways of Boston and New York City, the tubes under the Hudson and the East rivers of New York, and the mountain tunnels of the Great Northern Railway in Washington may be mentioned as examples. The advantages of tunnels are many. Prepare a list of advantages for class discussion. Compare them with the disadvantages.

112. Grades. - The inclined plane and work. - Aside from commercial benefits to be obtained by locating a road through productive country, there is the necessity of avoiding long and steep grades. Every one knows that it takes less force to haul a load on the level than on a grade; and that it takes less force to lift it up an incline than to lift it vertically, that is, against the direct force of gravity. The steeper the grade, the greater must be the force to haul the load. If the grade is light, the distance to be covered is greater; but the force required at any moment is less. Another way of expressing it is to say that the amount of work done in lifting a load by the aid of an inclined plane to any given height is about the same, perhaps if anything more, than is necessary in lifting it vertically; but it takes less force at any given moment.

Suppose a box weighing 400 pounds is to be loaded on a wagon that is four feet above the ground on which the box rests. One man cannot lift the box directly; but with aid of a skid or plank 16 feet long he can load it quite easily. The inclined 
plane enables him to do what would require two or more men to do by lifting vertically. But how much less force is required with the incline than in direct lifting of a given weight? Common experience vaguely gives the ratio as less; but it is necessary in any important situation to know exactly how much less. We can determine the ratio by a simple experiment.

Exercise: Work and the inclined plane. - Let a four pound weight, a block or small box loaded with sand, represent the four hundred pound box. Attach a spring balance to it by strings so that it can be lifted vertically. Obviously it takes four pounds to lift it. If it is lifted upwards a distance of one foot, the work done is four footpounds, that is, the number of units force multiplied by the number of units distance gives as a product the number of foot-pounds. If it is lifted vertically a distance of four feet, it takes sixteen foot-pounds of work. Whenever it is required to find the amount of work done the terms are made to read " work is equivalent to force times distance."

What now is the work done in lifting the four pounds to a height of four feet by means of an inclined plane? Suppose you have a smooth plane sixteen feet long. Pull the box from bottom to top of the plane by the spring balance. What is the average reading of the balance? Suppose this takes just two pounds of force. Two (number of pounds force) times sixteen (distance in feet) equals thirty-two foot-pounds. This shows that more work is done in lifting the load by means of the inclined plane than by lifting it vertically. But less force is required to do the work.

The four hundred pound box may accordingly be lifted to the wagon by means of the inclined plane with about one fourth of the force required to lift it vertically. This of course implies that friction is reduced to a minimum.

The experiment may be carried out with wheels, which give rolling instead of sliding friction. Assume that the load of four pounds is lifted to the required height by means of the incline and wheels, and minimum friction, with a force of 1.4 pounds. Making the necessary substitutions in the formula, then 1.4 (number of pounds) times 16 (number of feet) equals 22.4 , the number of foot-pounds, or the amount of work done. Remember that the total amount of work necessary to lift the 
box vertically four feet is only 16 foot-pounds. If now it takes 22.4 foot-pounds to lift the load the given height with the aid of incline and wheels, it evidently takes considerably more work than is performed in lifting the same load vertically. But the work is so distributed as to make possible its performance by a relatively small force.

To what is this extra work due? It is found to be due in part to the resistance or friction encountered by the rolling wheels on the track and on their axles (page $259 \mathrm{ff}$.). Some of the friction is eliminated by lubricating the axles, but the remainder is enough to account for the additional work.

Grades and distances. - In building roads engineers plan, wherever a change in level is necessary, as in crossing mountains, to keep the grade as light as possible in order to save motive force, and to keep the distance as short as possible in order to reduce to a minimum the friction in hauling. Highways are not kept as strictly within a limited range of grade as are railroads. But heavy grades on highways are usually short. For railroads, a rise of more than two and one half feet in a hundred feet, or more than a two and one half per cent grade, reduces greatly the hauling efficiency of the road. Light grades and long distances are preferable to heavy grades and short distances.

Exercise. - Is the total amount of work done in lifting a given load up a long incline greater or less than that done in lifting it to the same elevation up a short incline? In which case is the greater amount of force required?

Grades and tractive effort. - The motive force of railroads is limited by certain almost unchangeable conditions. Increased motive force in a steam engine necessitates increased size and weight of engine, which two factors are restricted by the nature of the roadbed, the size and strength of the rails, the gauge of the track, the headway clearance of bridges and tunnels, and many other conditions which are practically unchangeable. 


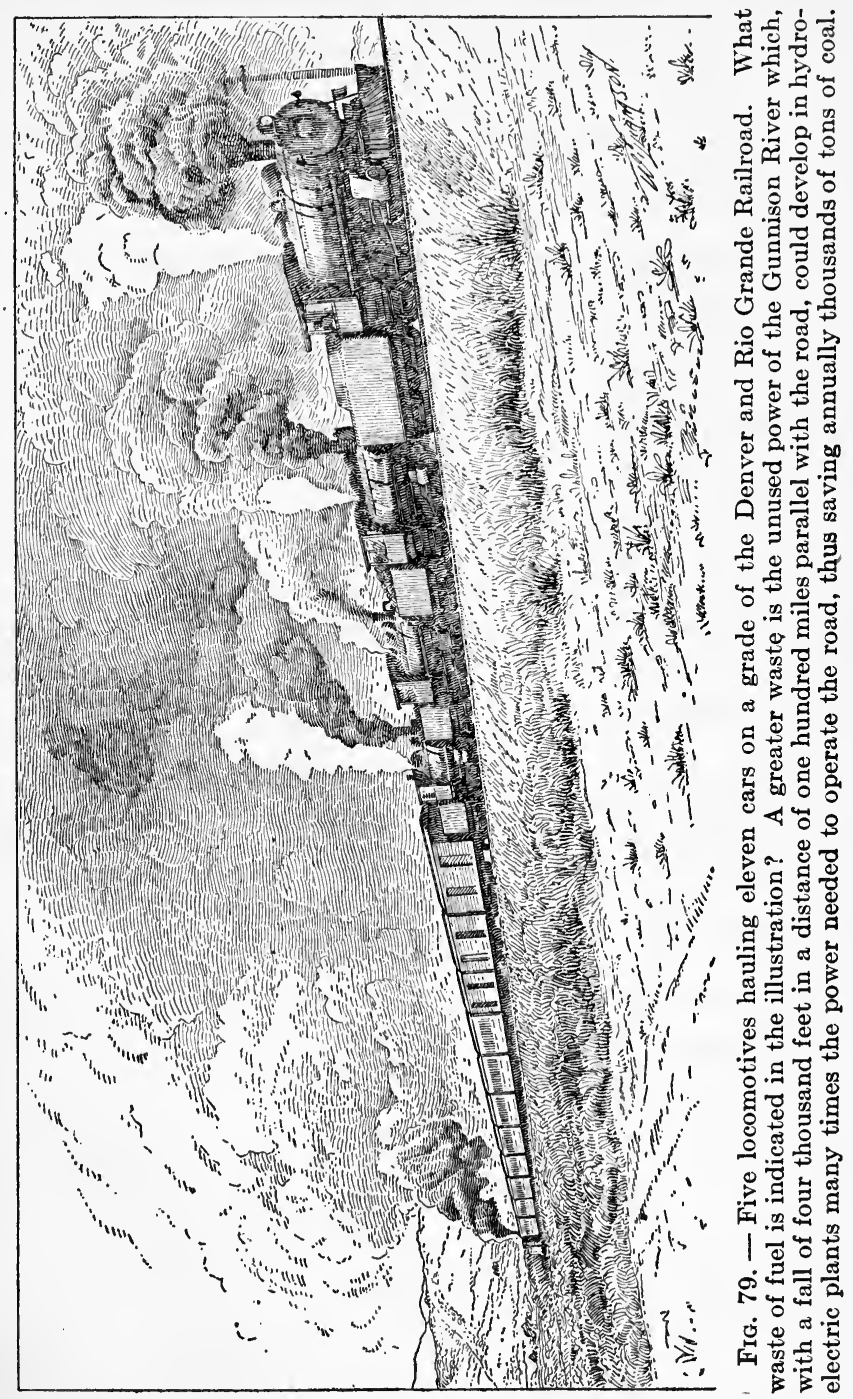


Steam engines are well known to be inefficient - the most modern type yielding hardly 18 per cent output of work for the 100 per cent input of fuel or heat energy. The pulling force, or tractive effort, as it is called by engineers, of an engine is estimated at about one pound to five pounds of weight. Suppose an engine, including the tender and its load, weighs one hundred tons; it has an estimated tractive effort or pull upon the drawbar of twenty tons. It can haul on a level road about four thousand tons. An engine of this size is a pygmy in comparison with the locomotives of to-day; but it illustrates the ratio of weight to tractive effort.

Assume that a trainload of one thousand tons is hauled on a perfectly level track by a pull of five tons. On a road with a rise of fifty-two feet in a mile, or a one per cent grade, the same load will require a tractive effort of fifteen tons. On a two per cent grade, the amount of tractive effort needed is about twenty-five tons, or five times that required for a level track. If the grade is two and one half per cent, beyond which engineers prefer not to go, in building railroads, the tractive effort for the same load is about forty tons. This shows why light grades are necessary in order to secure the highest hauling efficiency of the road, and to avoid excessive wear and tear on rolling stock and track. The illustration (Fig. 79) shows the combined tractive force of five engines doing on a heavy grade what one engine does easily on a level or even a light grade.

Grades on transcontinental lines. - The data in the accompanying table show clearly what modern engineering has achieved in building the great transcontinental railroads. Calculate the per cent of grade of each. 
TABLE OF ELEVATIONS AND GRADIENTS

\begin{tabular}{|c|c|c|c|c|c|c|c|c|c|c|c|}
\hline \multirow{2}{*}{\multicolumn{8}{|c|}{ RAILWAY }} & & \multirow{2}{*}{$\begin{array}{c}\begin{array}{c}\text { Highest } \\
\text { Summits }\end{array} \\
\text { Feet }\end{array}$} & \multicolumn{2}{|c|}{$\begin{array}{c}\text { Maximum Gradient } \\
\text { Per MiLE }\end{array}$} \\
\hline & & & & & & & & & & Eastbound & Westbound \\
\hline $\begin{array}{c}\text { Grand Trunk Pac } \\
\text { One summit }\end{array}$ & ific & • & · & - & • & - & - & & 3712 & 21 & 26 \\
\hline $\begin{array}{c}\text { Canadian Pacific } \\
\text { Three summits }\end{array}$ & $\cdot$ & $\cdot$ & $\cdot$ & . & . & - & . & • & 4146 & 237 & 116 \\
\hline Great Northern . & $\dot{.}$ & $\dot{.}$ & $\dot{0}$ & $\dot{0}$ & $\dot{0}$ & . & : & : & $\begin{array}{l}3370 \\
5569\end{array}$ & & \\
\hline Three summits & . & . & . & . & . & . & . & • & $\begin{array}{l}5532 \\
2849\end{array}$ & 116 & 116 \\
\hline Northern Pacific & . & . & . & ${ }^{\circ}$ & $\cdot$ & - & . & . & 5569 & & \\
\hline Three summits & $\cdot$ & . & $\cdot$ & . & - & . & . & & $\begin{array}{l}5532 \\
2849\end{array}$ & 116 & 116 \\
\hline Santa Fe System & - & . & - & . & - & - & - & & 7510 & & \\
\hline Six summits & . & - & - & . & . & & . & . & $\begin{array}{l}7453 \\
6987 \\
7132 \\
2575 \\
3819\end{array}$ & 175 & 185 \\
\hline Chicago, Milwauk & zee, & and & d S & t. & Par & & . & & 5788 & & \\
\hline Four summits & . & $\cdot$ & $\cdot$ & - & - & & - & - & $\begin{array}{l}6322 \\
4163 \\
3010\end{array}$ & 87.6 & 105 \\
\hline Union Pacific & & . & & & & & & & 8247 & & \\
\hline $\begin{array}{l}\text { Omaha to San } \\
\text { Three summi }\end{array}$ & $\begin{array}{l}\text { Frar } \\
\text { its }\end{array}$ & ncis & sco & . & . & - & . & & $\begin{array}{l}7017 \\
5631\end{array}$ & 116 & 105 \\
\hline Western Pacific & • & . & . & - & . & . & - & . & 5712 & & \\
\hline Two summits & - & - & . & . & . & . & . & . & 5018 & 52.8 & 52.8 \\
\hline
\end{tabular}

Exercise. - Which of the railroads in this list reaches the highest elevation? Which has the highest maximum grade? Locate on railroad maps the summits of the transcontinental lines. What are some of the chief objections to high elevation for freight traffic? For passenger traffic? What advantages for passenger traffic are offered by high elevation in the hot season of the year, as via the Santa Fe? What advantages are afforded by the elevation, as via the Northern railroads? What disadvantages in the winter season?

The highest elevation reached by any railroad in the Western Hemisphere is that of the "Trans-Andean Railway" of South America. What is its altitude? 


\section{Curves, Inertia and its Effects}

113. Curves. - Few roads are straight for a long distance, usually having many curves, some of which are very sharp. Country roads are not built at present for high speed, especially around turns, although on certain mountain roads the sharpest curves are constructed to secure the safety of descending vehicles. Accidents occur because of the attempt to take improperly constructed curves at too great a speed. On railroads, however, curves are designed and constructed to secure both speed and safety. Trains make nearly the same speed on ordinary curves as on straight track; but if the track on such curves were as level as that of a street railway, or the usual highway curves, disaster would certainly result if attempted at high speed.

114. Effect of change of direction. - We may realize the essential conditions that determine the construction of tracks on curves for high speed, by referring to several common experiences. If you are running along a straight path at top speed and try suddenly to turn sharply to the right or left without checking speed, you find that the path followed goes far beyond the path intended and in the direction of the straight course you are running. The momentum carries you beyond the planned course. Bicycle riders or motor cyclists know well how to maintain their balance in turning a corner or a sharp curve in the road. They may reduce their speed somcwhat, but frequently by leaning toward the inside of the curre they take it safely at a fairly high speed. When throwing the hammer, the thrower feels its pull outward, while he pulls it inward; when released the hammer flies off on what is called a tangent. Many of you have swung a pail of water through an inverted position without spilling a drop. The water tends to go on a tangent, but is prevented by the pail and the inward pull of the arm. 
Exercise. - On a track of narrow cardboard or sheet tin bent in the shape of a trough, and curved to form a vertical loop as in Fig. 80, a marble will loop the loop just as a skillful bicyclist, automobilist, or aviator can do in his machine.

Other instances may be cited, but all point to the same principle, that is, a moving body tends to continue to move in the same direction in which it was started, until compelled by some other force to stop or change its direction. Complementary to this is the familiar fact that a body stays where it is put, until moved by some external force. There is a question involved here which must be answered before considering how railroad curves are built

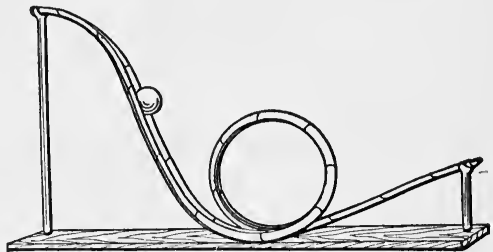

Fig. 80. - A trough made of cardboard, tin, or wires bound together, and shaped as in figure will guide a marble around the loop. What is the principle involved?

to permit speed. When a body is moving in a circle about a center, in what direction does it actually tend to move? Does it move directly away from the center? Or does it tend to move in a straight line continuous with its direction at any given moment? The answer to this question may be found experimentally in the following way.

Exercise. - Attach a string eight or more feet in length to a wooden ball. Take hold of the string two or three feet from the ball and swing the ball in a circle, the plane of which is parallel to the floor and just a little above it. Mark the center of the circle described by the moving ball. When the ball is moving steadily, release the string. Observe the course of the ball. Does it move away from the center of the circle? Does it move in a straight line continuous with its direction at the moment of its release?

115. Inertia. - Consider now the behavior of bodies at rest, which is complementary to the behavior of moving bodies. Place on the table a visiting card or other small card with part of it extending beyond the table's edge; and place on the card 
a penny or any similar body. Snap the card directly on the edge; it is driven away, leaving the penny in practically the same location. Passengers in a car which starts suddenly tend to stay where they are or fall in a direction opposite to that of the moving car. Passengers on a car which suddenly stops do not cease instantly and entirely their motion in the direction they were traveling. Cite other examples from your experience and observation. Do they not all point to the fact that whether in motion or at rest things tend to continue in their given state? This characteristic of things is generally called inertia.

Law of inertia. - Sir Isaac Newton (1642-1727) formulated what he called the Law of Inertia, which is one of his Three Laws of Motion. He stated the law of inertia in these words: " Every body continues in its state of rest or of uniform motion in a straight line, unless it is compelled by other forces to change this state." This is exactly what your observations lead you to conclude. Since we are concerned with motion, we may limit the statement as follows: "A body in motion tends to move in the same direction unless it is compelled by external forces to change that direction."

116. Elevation of the outside rail. - In the construction of curves or bends on railroads where speed, safety, and comfort

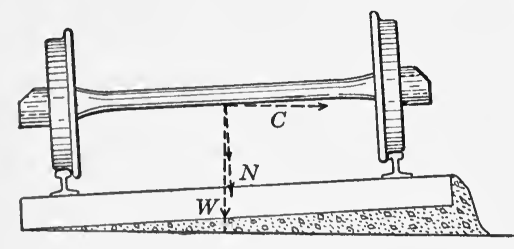

Fig. 81. - Track on curve, showing lines of forces which keep the car in a position normal to the track. are necessary, the inertia of moving bodies is accurately computed and provided for. The track is elevated on the outside of the curve exactly enough to compel the train easily and gradually to change its direction, to allow the safe maintenance of a given rate of speed, and to secure the equal distribution of the load upon both sets of wheels and rails. To have the load bear too heavily on the outside wheels, their flanges, and the 
outside rail would be dangerous. The result of the elevation of the outside rail is that the inertia of the moving train which is acting toward the outside of the track, shown by the dotted line $C$ (Fig. 81), and the weight of the train acting toward the inside of the track, $W$, balance each other and keep the train in a position which is normal to the track, $N$.

The cross section of the curve of a typical course for motor car racing (Fig. 82) illustrates the point clearly. This has an outside elevation of 25.6 feet over the

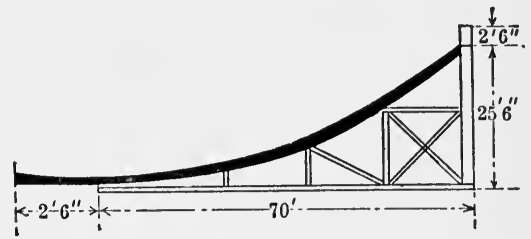

FIG. 82. - Cross section of motor car racing track. A car moving at a rate of 110 miles per hour has a position normal to the track on the outside elevation. inside level of the curve. It is designed to allow a motor car, moving at the rate of about 100 miles per hour, to maintain a position normal to the track surface at the outside of the curve. The normal position is the resultant of two factors: the one, the tangential force of the rapidly moving car, which is acting horizontally, and the other the weight of the car, which is

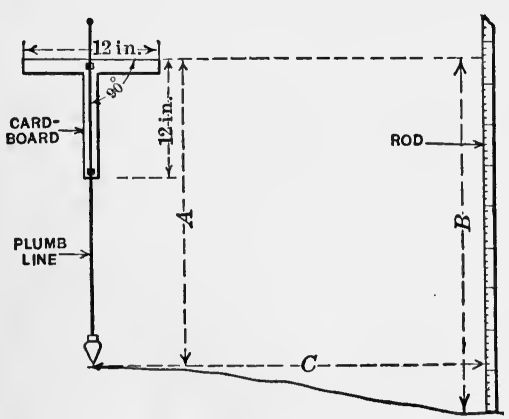

FIG. 83. - A simple level for surveying. acting vertically. Make a diagram and explain.

117. Exercise: Measuring grades. - Grades may be measured with a fair degree of accuracy by means of the simplified form of the surveyor's level shown in Fig. 83. Cut the " $T$ " from heavy cardboard, according to the dimensions given in the diagram. Fasten the plumb line to the " $T$ " so that the angle designated in the diagram shall be exactly $90^{\circ}$. If greater accuracy is desired, make two readings, with the instrument reversed for the second reading. The upper end of the plumb line may be fastened to a tripod or other steady support. The plumb bob should just clear the ground. 
The rod may be made of a square piece of wood six or eight feet long and marked off in feet and tenths of a foot. The rodman may use a white card as an indicator, which is to be moved up and down on the rod until the top of it is at the right level, as is indicated by the signals of the one who is sighting across the top of the "T." The rodman takes and records the reading. After each reading is taken, the instrument is moved to the position of the rod, which is advanced to another suitable position.

The distance $A$ is always known; the distance $B$ is shown by the rodman's reading and record. The distance $C$ is to be measured with tape (a steel tape is best). The difference between the distance $A$ and the distance $B$ gives the elevation for the distance $C$. The per cent grade is found by determining the number of feet rise in a distance of 100 feet.

\section{SUMMARY}

The improvement of roads must accompany the improvement of vehicles of travel and transportation.

Roads are built of various materials, such as dirt, stone, asphaltum, concrete, and brick.

The harder and smoother and more nearly level the road, the heavier the load a horse or other motive power can pull.

Formerly railroads and highways were built with many curves and high grades to save cost of construction. To-day they are built with few curves and low grades to save cost of operation.

A long grade to reach a certain elevation reduces the tractive force needed, but increases by extra friction the amount of work done.

Work is expressed in foot-pounds. A force of one pound acting through a distance of one foot does one foot-pound of work.

In spite of the added friction, light grades are to be preferred.

The resistance of the rail or road to the passing of the wheel is called rolling friction.

According to the principle of inertia a body in motion tends to move in a straight line. This tendency is the tangential force of the body.

As a train rounds a curve, the tangential force tends to tip the train outward. This tendency is counterbalanced by gravity, which is brought into action by the elevation of the outside rail.

\section{REVIEW QUESTIONS}

1. In what ways do transportation and travel depend on roads? 2. Mention examples from ancient times of good roads. 3. Describe the form, construction, and materials used in city streets. In a good 
road. 4. (a) What motive forces have been employed in land transportation? (b) In what way are they dependent on roads? 5. Compare the loads that can be pulled by a horse on soft roads, hard roads, and steel rails.

6. Give instances of the modern tendency of railroads to shorten routes. 7. Why are tunnels necessary? Mention several wellknown tunnels. 8. (a) Why are there grades on highways? (b) What determines the steepness of the grade? 9. (a) Compare the force required to move a weight up an incline and to lift it directly. (b) Compare the total work done in each case. (c) What is the advantage of the incline? 10. What necessary relation between grades and distances is considered in building a railroad to cross a certain elevation?

11. (a) What conditions determine the size and power of locomotives? (b) Can these conditions be easily modified? 12. A train may be how many times the weight of a locomotive which is capable of pulling it? 13. (a) The tractive effort of an engine is what part of its weight? (b) What is rolling friction? (c) How is it reduced on railroads? $(d)$ on highways?

14. Why are not all roads straight? 15. Give examples from experience of the tendency of moving bodies to go in a straight line. 16. Explain how an automobilist may "loop the loop." 17. (a) Does a body tend to move straight away from the center of a curve or tangent to it? (b) How can this be demonstrated? 18. (a) What property of a body is referred to by the term inertia? (b) What instances illustrating this property of bodies have you observed? 19. State Newton's Law of Inertia. 20. Why are running courses in a gymnasium usually elevated on the outside? 21. What force is used to balance the inertia in a train which is taking a curve? How?

\section{SUGGESTIVE QUESTIONS}

1. Why are grades necessary in roads? What means are sometimes used to keep a low per cent grade? 2. What is the relation of grades to motive power? 3 How high would you have to raise one end of a level plank which is 10 feet long in order to give a grade of 2 per cent? 10 per cent?

4. What is the grade of some portion of the street or road near the school, as determined by means of the instrument described on page 277 ?

5. Determine the height of a hill by the same instrument, and the grade of a road leading to its summit.

6. Cogwheel railroads are sometimes used on very steep mountains, 
as Pikes Peak, Colorado. Explain how this makes possible a high tractive effort in proportion to the weight of the locomotive. Would such locomotives be geared to move slowly or rapidly? Why?

7. What evidence is there that rails are depressed as the wheels of the train pass over them?

8. Mention several good illustrations of friction taken from a baseball game; a football game.

9. How has the use of the automobile influenced road building in your vicinity? In the country at large?

10. The United States Government gave the Union Pacific Railroad Company a large amount of land to encourage the construction of the road. Justify this use of the public lands.

11. Why are steel rails not made in the form of solid, rectangular bars?

\section{BRIDGes}

118. Primitive and modern bridges. - There are few roads that do not need bridges. Highways and railways are making increasing use of them in order to economize time and cost of trackage and hauling. Not very many years ago a stream or an abyss that could not be spanned by a felled tree or by a rope used as a bridge, had to be forded, or crossed by a long, difficult route. The log bridge and the swinging suspension bridge of primitive construction shortened many roads, and also suggested how man might make further use of his ingenuity in overcoming such obstacles to rapid and easy travel. The primitive bridge involved the principles which underlie modern bridge engineering. To-day these principles are realized more fully in such designs as give the greatest possible strength and utility, and in construction with such materials as stone, concrete, and steel. The modern bridge is one of the finest examples of scientific and artistic engineering. It is a thing of beauty as well as of utility. It is also an instance of the success which men have achieved with the aid of scientific knowledge in conquering some of the greatest obstacles to transportation and travel.

119. The principle of the girder. - You may find in your vicinity examples of the simple bridge with log or heavy timber 
girders covered with planks. The principle upon which the structure depends may be illustrated in a very simple way. Lay a stick of wood upon two end supports, resembling the end piers of a bridge. Press with the hand upon the middle of the stick, gradually bending and finally breaking it. Observe closely the ways in which the force is applied and the resistance encountered. The wood fibers in the upper layers of the stick are crushed together, and those in the lower layers are pulled apart. Engineers speak of the force applied above as the crushing force, and of the resistance of the material to this force as the crushing strength. The pull or force applied to the lower layers is called the tensile force, which is met by the tensile strength or resistance of the material.

These are the essential facts in all bridge construction, of the simplest as well as of the most complex form of structure. A study of the familiar types of bridges will make these facts clearer, and it will show also how the crushing and tensile strength is distributed among different parts in order to secure greater strength and at the same time to avoid increasing greatly the weight of the bridge.

120. The truss bridge. - Simple girders of wood or steel may meet all ordinary requirements over short spaces or spans. But longer spans are very often necessary, over which simple girders cannot be placed without increasing excessively the weight, and decreasing the supporting strength of the structure. Evidence of this you may find in bridges in your neighborhood. For long spans a type of bridge known as the truss is often constructed; this you will perceive is but a modified form of the girder type.

The chief features of the truss are illustrated in diagram $B$, Fig. 84. Note that the crushing and tension layers of the girders are now actually divided into two sets of members, which are called the compression and the tension members. Compare diagrams $B$ and $A$. In the triangle type the girder may be considered as twice as long as that in $A$, but divided 
into two equal parts by the support at the center. The working lengths are thus from the center $b$ to the ends $A$ and $C$. Suppose a load is resting upon the center of the bridge. 'Where

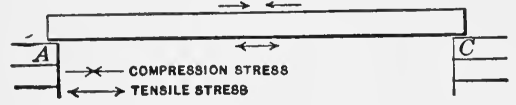

A. Single girder.

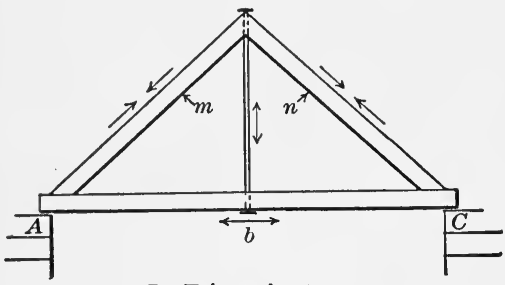

B. Triangular truss.

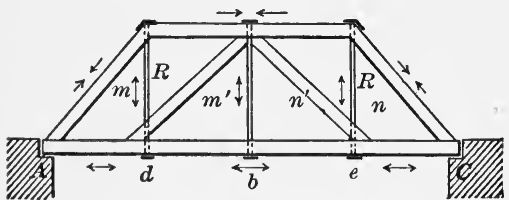

C. Howe truss.

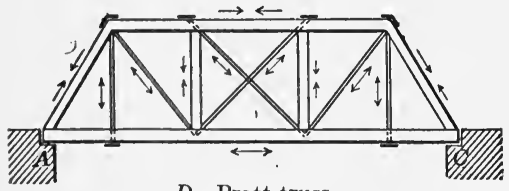

D. Pratt truss.

Fig. 84. - Typical forms of the truss bridge. The simple girder and triangle make possible bridges of great length and strength, and relatively small weight. does the weight actually rest? How is it transmitted to the supports? Trace out the members which transmit it to the supports. Which are the crushing or com. pression members? Which are the pulling or tension members?

Howe truss. - It is evident from this that the triangle type not only gives greater strength than a simple girder, but also makes possible much longer spans without intermediate piers or supports. By a modifi. cation of the triangle, still longer spans are possible and with an increase rather than a decrease in strength for sustaining maximum loads. A modified triangle is shown in diagram $C$. This is known as the Howe Truss. Can you find the complete triangle? By imagining it traced out in continuous form, you realize that it would reach, if it were not thus divided, an undesirable height for long spans. Hence it is cut into two parts, and the top $m^{\prime}, n^{\prime}$, is lowered to rest at points $d$ and $e$. The apex reaches the top bar which connects the sides of the triangle. 
Exercise. - Suppose a load is at the center of this bridge. By what parts is the load supported? How is it distributed to the supporting parts? What is the work of the steel rods, $R R$, running from the girder at $d$ and $e$ to the sides of the triangle? What is the function of the upper part of the triangle? Is this function the same as it would be if the triangle were not divided?

Pratt truss. - Study the distribution and transmission of the weight in the truss bridge called the Pratt Truss, diagram $D$. Suppose a load is at the center of the bridge, what members support it? Which are compression and which tension members? Which of the forms of the truss is best adapted to long spans between supports or piers?

Exercise: Strength and the form of bridge members. - The materials needed are several strips of stiff cardboard three inches wide and twelve inches long; a twelve-inch piece of telegraph wire or similar wire; and a spring balance of several pounds' capacity, or a series of weights.

Lay a piece of cardboard flat upon two supports representing the end piers. Supports should be high enough to allow use of the balance or weights in exerting stress upon the span. Make record of results in each case. Test by pulling with balance at the center of the cardboard strip. Test a similar piece placed on edge. How are floor joists placed? Bend a strip into the form of a semi-circle or arch, support ends to prevent their spreading, and test its strength at center. Make a form with the two sides of a triangle, support so as to prevent spreading, and test its strength at apex. Try the wire in similar ways.

Compare the load supporting capacities of the different forms tried. How much more strength has the triangular form than the semi-circular? than the piece placed on edge? than that placed flat? In which form does the wire show greatest strength in supporting the load? What different forms of the supporting members do you find in the bridges in your vicinity? What three kinds of bridges do the forms tested represent? Which form shows the greatest number of advantages?

Cantilever bridges. - The cantilever is a special form of the truss type of bridge. The most familiar form of cantilever construction is the springs of a wagon or automobile (Fig. 85), 
where the axle supports the center in much the same way as a pier supports the central part of a cantilever bridge. Exam-

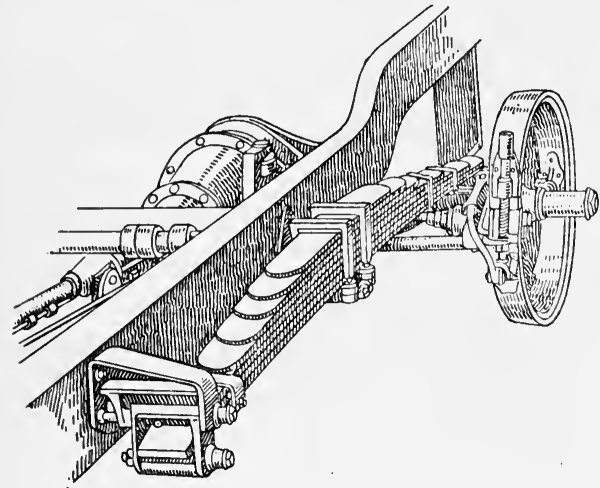

Fig. 85. - The principle of the cantilever applied to an automobile spring. Compare with its use in the cantilever bridge, Fig. 87 . ine Fig. 86 for the distribution of forces and members. One of the most noted creations of this type is the railway bridge across the Firth of Forth, near Edinburgh, Scotland. This has two main spans (Fig. 87) each 1710 feet in length, and supports two railway tracks. Its rigidity is such that express trains may cross it at the rate of sixty or more miles an hour. The large tubes, eight to twelve feet in diameter, and usually of circular section, are the compression members. The lattice work parts form the tension members. The illustration shows that tubular framework is combined with the cantilever design. The new Quebec bridge across the St. Lawrence River is of the cantilever design, with the principal span 1800 feet long, which is probably the longest cantilever span ever built.

121. Tubular frame. Several kinds of bridges have been worked out on the basis

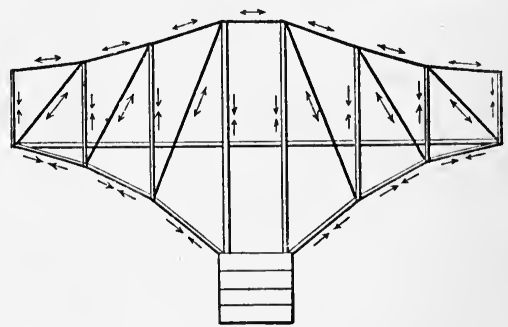

Fig. 86. - Diagram of a cantilever bridge, showing typical truss förms in arrangement of tension and compression members. Compression members $\rightarrow \leftarrow$ Tension members $\leftarrow \rightarrow$ of these facts. One kind is the tubular. It resembles the frame of a bicycle which is made of tubes, instead of solid 
steel. The tubular form makes the machine lighter and, with the triangular arrangement of its tubes, stronger than a solid steel frame of the same weight. The bicycle frame is really a bridge, with the two axles as its supports. The same sort of form is sometimes used in bridges. The tubular form gives

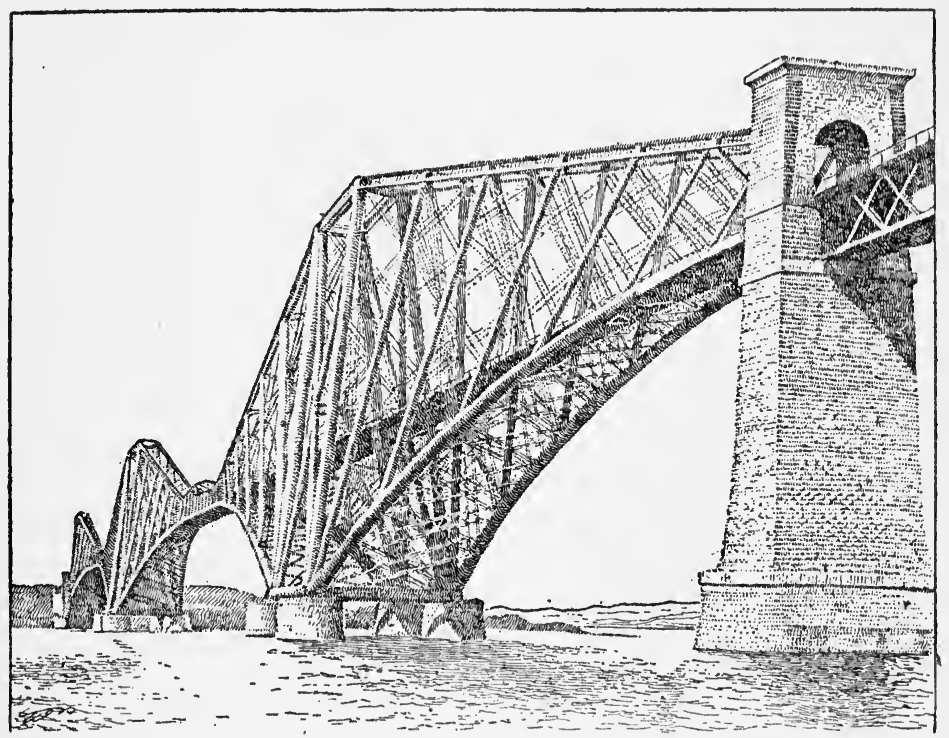

FIg. 87. - Cantilever bridge. Tubular members: Length of two main spans is $\mathbf{1 7 1 0}$ feet each. Identify the compression members; the tension members.

strength and lightness, an important factor where the spans are long. An example of such is the bridge across the Menai Straits for the London and Northwestern Railway. Tubular framework is combined with different types of bridge construction, such as the cantilever (Fig. 87) and the arch.

122. The suspension bridge. - Another type of bridge is the suspension. It is suspended very much as the hammock from two supports. Tall, massive towers furnish support for heavy wire cables. The ends of the cables are held fast 


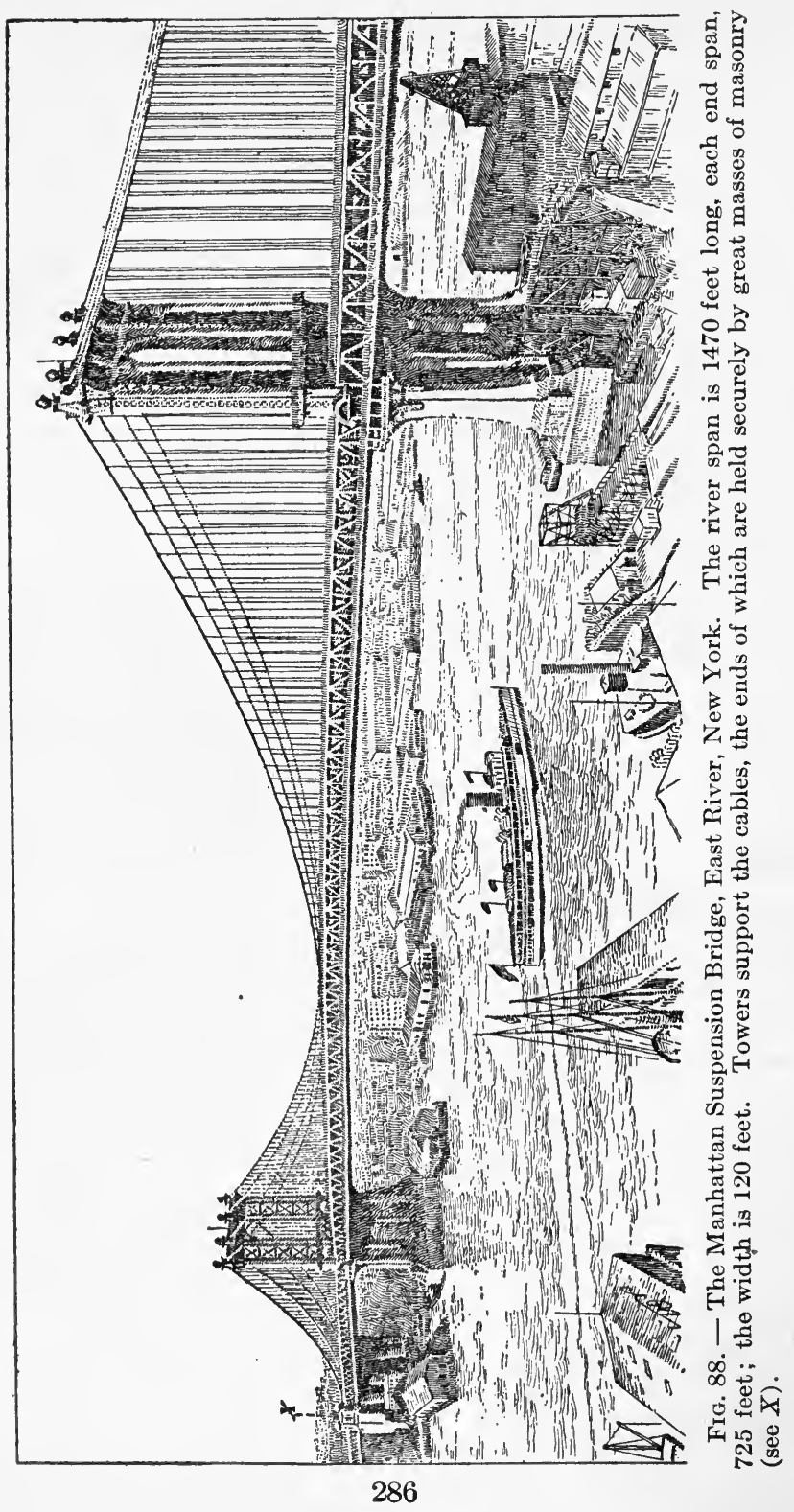


by great masses of masonry. The cables are of steel fifteen or more inches in diameter, selected and formed for tension strength. The suspension bridge is peculiarly adapted to long spans. The famous Brooklyn Bridge over the East River, New York, graceful in its lines, and, when built, the largest and strongest in the world, comprises two shore spans of 900 feet each, and a central or river span of 1595 feet. Since its completion in 1883, many bridges of the same type have been constructed, exceeding it in size and capacity. The Manhattan Bridge (Fig. 88), a short distance from the Brooklyn Bridge, has a main span of 1470 feet, a width of 120 feet, with a capacity of four electric railways, a roadway, and two footways.

123. The arch. - Quite the reverse of the suspension bridge is the arch. The materials of which the arch is constructed are selected and formed to withstand crushing force, while the suspension materials have to withstand tension. The arch must have very heavy piers against which it may push without spreading. Stone and steel are the usual materials employed. The arch bridge across the East River at Hell Gate, New York (Fig. 89) has a main span between piers of 1017 feet and a rise from the hinges to the crown of the main arch of 220 feet. The capacity of the bridge is for more than four trains of heaviest possible load, on four tracks. Some idea of the weight of the whole structure may be got from the fact that the average weight for each lineal foot is 26 tons.

\section{SUMMARY}

A simple girder supported at the ends sustains a crushing force in its upper portions and a tensile force in its lower portion.

The truss is like a girder, having the upper and lower portions separated into distinct members. This makes possible the use of longer spans and gives greater strength than a simple girder. The truss consists of a series of rigid triangles.

Tubular frame bridge construction is illustrated by the bicycle.

The suspension bridge, built of materials having great tensile strength, is adapted to very long spans. Notable instances are found in the Brooklyn and Manhattan bridges, New York City. 


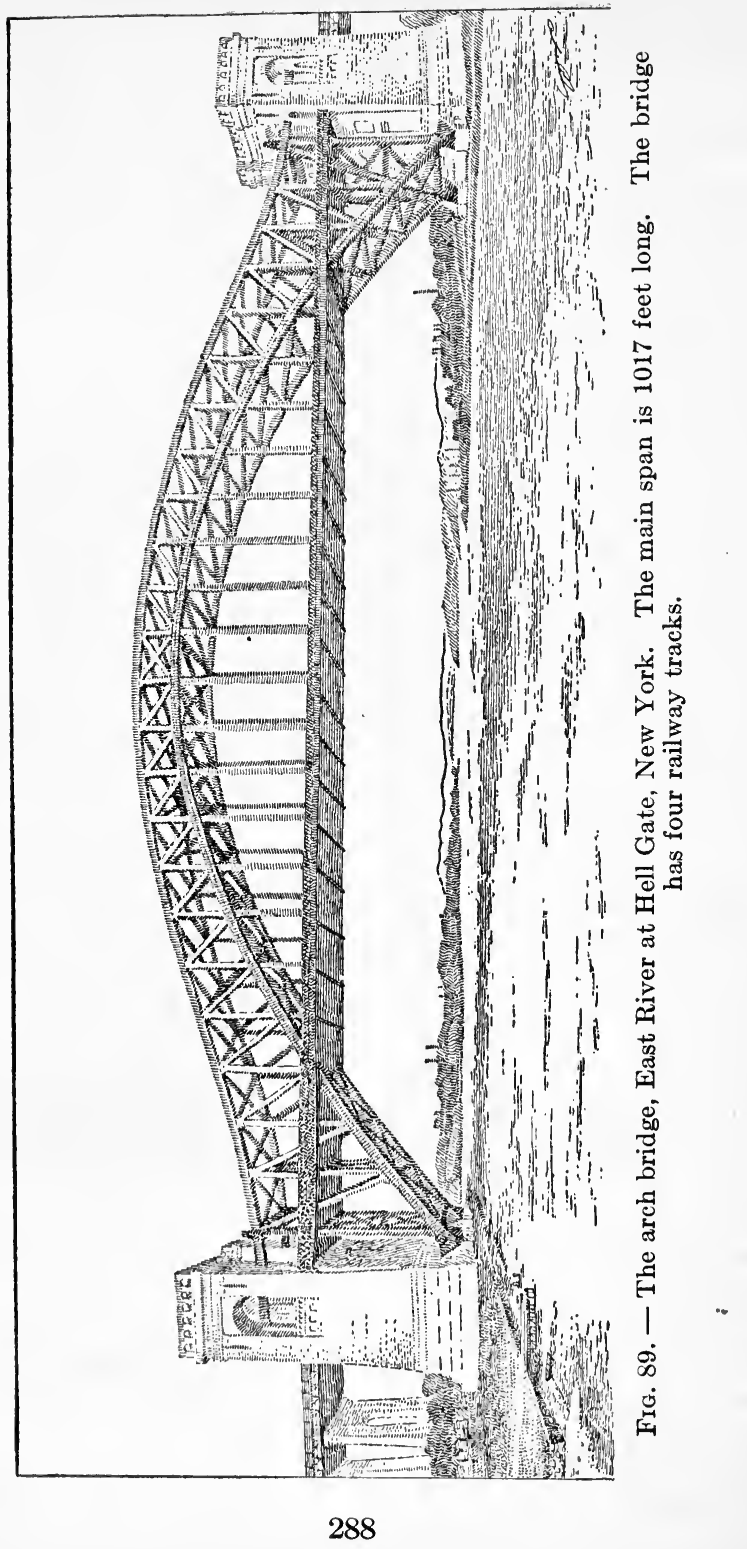


The arch bridge, represented by the steel arch over the East River at Hell Gate, New York, is built of materials that resist crushing forces.

The cantilever is like the truss, supported not at the ends but at the middle.

\section{REVIEW QUESTIONS}

1. Why do we need bridges? 2. Describe primitive types of bridges. 3. What is meant by (a) crushing force? (b) tensile force? (c) crushing strength? (d) tensile strength? 4. Show how a simple girder supports a load. 5. What advantage has the truss over the girder? 6. Why is the frame of a bicycle made of steel tubes rather than of steel rods? 7. (a) Mention an instance of the use of a suspension bridge. (b) How is the weight supported? 8. (a) What materials are usually employed in such construction? (b) Describe the railway bridge across the Menai Straits.

9. (a) How does the principle of the arch bridge differ from that of the suspension bridge? (b) What is the chief feature of the arch bridge? (c) Of what materials is it usually built? 10. (a) What is the chief feature of a cantilever bridge? (b) In what other uses is the same principle employed? 11. Indicate the compression and tension members of the arch bridge, Fig. 89.

\section{SUGGESTIVE QUESTIONS}

1. When you try to split wood across the grain, what sort of strength or resistance do you encounter? 2. Why have some kinds of wood better erushing resistance than others? Which kind is better for bridge structures? 3. (a) What types of bridges depend almost entirely upon the crushing strength of materials? (b) What types depend mostly upon the tensile strength of materials? (c) Will a bridge that depends upon both kinds of strength be better than one that depends chiefly upon one kind? Explain.

4. Indicate the compression and tension members of $(a)$ a simple girder bridge, $(b)$ a truss bridge, $(c)$ a suspension bridge, $(d)$ an arch bridge, $(e)$ a cantilever bridge.

5. Give instances where the same principles of compression and tension members are used, as in the frame of a bicycle, of an automobile, and of a house.

\section{Steam and the Locomotive}

124. Early locomotives. - The story of the application of steam to locomotives for land transportation is full of romance. 
It is impossible to give here even a bare outline of the story; but students may find in histories of invention and encyclopedias interesting accounts of the lives and achievements of the men who contributed to the development of the steam engine. Here we may merely mention the names of James Watt, Thomas Newcomen, Richard Trevithick, and George Stephenson. Richard Trevithick built in 1802 a road steam

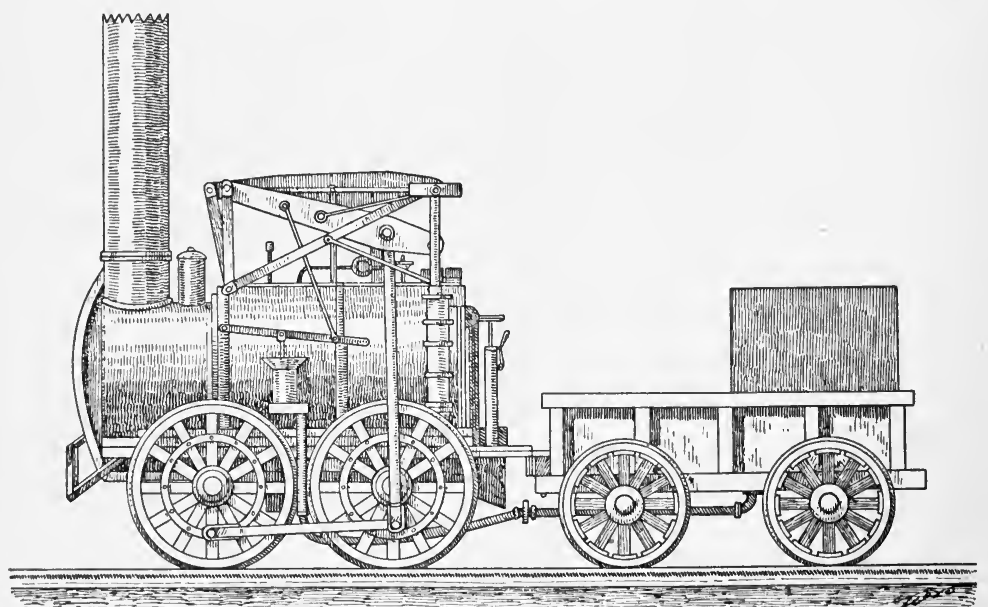

Fig. 90. - The "Stourbridge Lion," the first locomotive brought from England to America (1829). How is the power applied to the driving wheels?

engine, and in 1804 a track steam engine, both of which startled the world by their speed and power. George Stephenson's pygmy engine of 1815 and his record-making "Rocket" of 1829 are instructive forerunners of the modern locomotive. Mention must be made also of the Stourbridge Lion (Fig. 90), the first locomotive to be brought from England to America, in 1829. Among American inventors we may name "John L. Stevens, who in 1826 designed and built the "first locomotive made in America, driven by steam, upon a track" ; and Peter Cooper, who, in addition to his other noteworthy achievements, constructed the historic "Tom Thumb" steam engine. 
These early engines are the beginnings of the development of a kind of locomotion which has completely revolutionized travel and transportation. The engines produced by George and Robert Stephenson, especially the type in which the power of the piston is directly transmitted to the driving wheels (Fig. 91), anticipated the most recent and powerful creations.

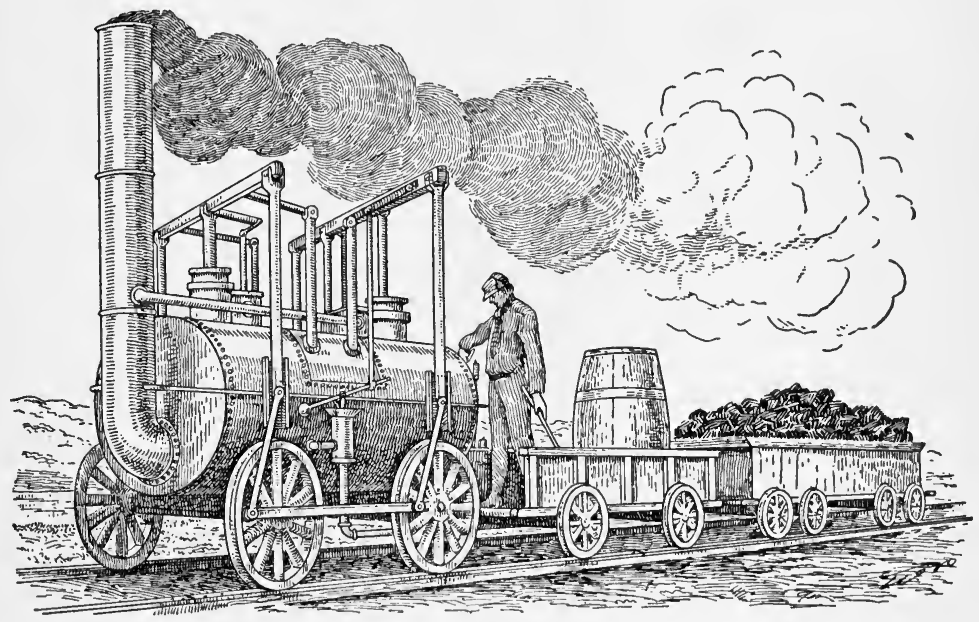

Frg. 91. - Stephenson's engine "Billy No. 1" patented 1815. Direct transmission of power to the driving wheels. The engine weighed 16,600 pounds, and the tender 8600 . It could haul 50 tons at five miles per hour, or 70 tons at lower rate of speed. Compare with power transmission of the "Stourbridge Lion," showing advantages.

The modern locomotives easily surpass the earlier types in power, speed, facility of control, and efficiency; but they are constructed in accordance with the principles of steam locomotion which were worked out a hundred years ago.

125. The modern locomotive. - As an example of what modern locomotive engineering is producing, we may refer to the present most powerful and largest engine in the world, a three-engine or triplex type, built in the Baldwin locomotive works for the Erie Railroad (Fig. 92). 


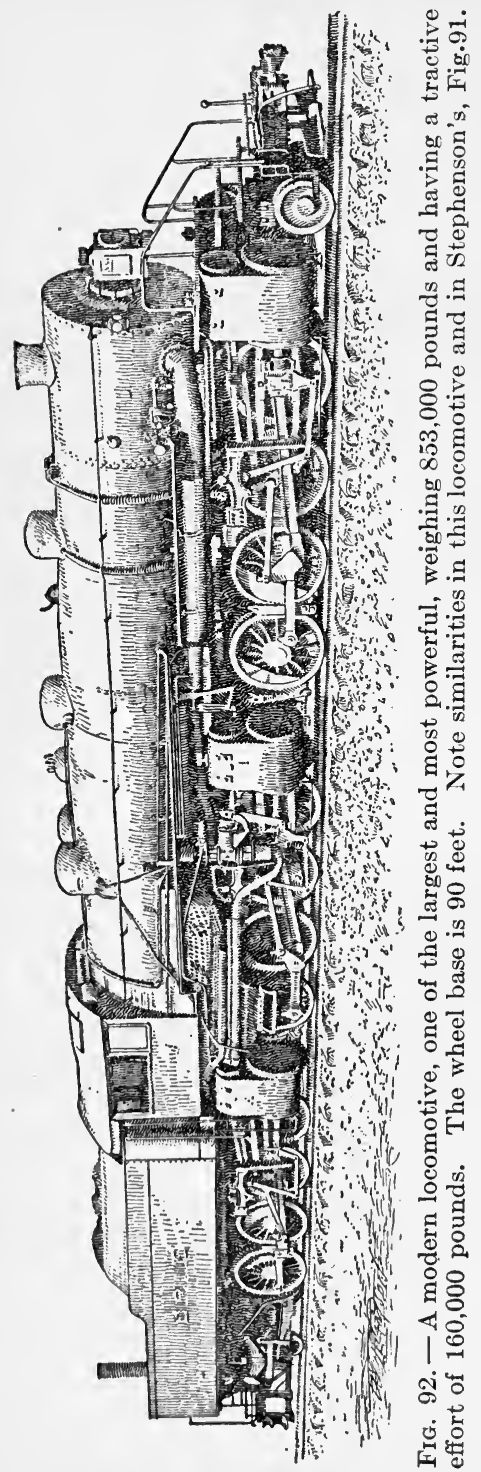

The Erie locomotive works as a "pusher" on a long, heavy grade. Its weight is 853,000 pounds, with a wheel base of 90 feet and a total length of 105 feet. Its direct pull on the drawbar, or tractive effort, is 160,000 pounds. It has moved a train of 250 loaded cars of fifty tons' capacity each, making a total load of about 18,000 tons, at a speed of 15 miles an hour. It is capable of moving a train of 640 loaded cars with a total weight of 45,000 tons, and nearly five miles long. The most powerful engine prior to this has about two thirds the tractive effort, or 115,000 pounds, and can push a train of about 30,000 tons.

The Erie locomotive is of the Virginian Mallet type, compound, and made up of three sets of engines with three pairs of cylinders, each pair driving a group of eight wheels coupled, each wheel being sixty-three inches in diameter. Fuel is supplied by a mechanical stoker at the rate of at least one hundred pounds per minute. The cylinders are thirty-six inches in 
diameter and have a piston stroke of thirty-two inches. Steam is supplied at a gauge pressure of 210 pounds, and utilized in expansion at the ratio of 1 to 2 (compare pages 294,309 on pressure and expansion in separate cylinders). The engine performs the work of three engines of the large freight service type.

Comparison with Stephenson's locomotive. - Compare these data with what we know of the first locomotive (Fig. 91), in which Stephenson, about the year 1815, worked out successfully the direct transmission of power to the driving wheels. The engine with its tender weighed 36,000 pounds; it hauled a train of ten cars or wagons, weighing with their load about 50 tons, at a speed of five miles an hour, or a load of 70 tons at a much less speed. It had four drive wheels, each two feet in diameter.

126. The operation of the steam engine. - The principal parts of the steam engine cannot be illustrated perfectly in a diagram; they should be studied with the aid of a model and by observation of an actual engine. The important parts, besides the boiler, are the steam chest, the slide valve, the exhaust port, the cylinder, and the piston, which is connected by a rod to the crank shaft of a stationary engine or to the driving wheels of a locomotive.

The operation of the engine is suggested in the diagrams of Fig. 93. Steam is admitted, as in diagram 1, through pipes by a valve which is under control of the engineer, into the steam chest $C$, and then into the cylinder through the right end port $B$. The high pressure steam drives the piston toward the left, and at the same time forces the used steam out through port $A$ and the exhaust port $E$.

In diagram 2 , the slide valve is closed when the piston stroke is about one half complete, to allow the steam by its continued expansion to complete the stroke. Just before the stroke is completed, the valve $A$ is opened to admit steam to form a cushion, which prevents pounding and also furnishes enough live steam to begin the return stroke. 
In diagram 3 , the valve at the left is open, admitting steam for the return stroke. The used steam is expelled through port $B$ into the exhaust chamber. In diagram 4 , the valve cuts off the supply of live steam before the stroke is more than half

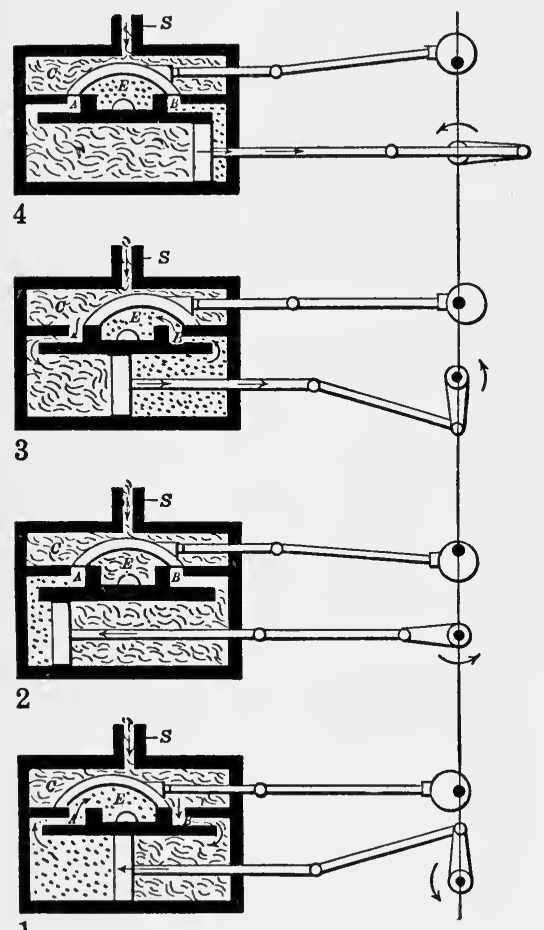

1

Frg. 93. - Diagram to show the operation of the steam cylinder. completed. The valve may be regulated to cut off the live steam when the piston stroke is one third, one fourth, or one fifth complete. This is done to obtain the expansion of the steam to three, four, or five times its original volume in the cylinder. The expansion utilizes heat energy which, aside from the loss in other ways, is equivalent to the work performed on the piston and through it on the driving crank or wheels of a locomotive.

The slide valve in the steam chest is operated by an eccentric. The eccentric is either a wheel or a crank attached to the shaft of the driving wheels of a locomotive. The wheel eccentric may be easily observed on a stationary engine. On late types of the compound locomotive what is known as the Walschaert's valve gear is generally used. This makes use of a crank and because of its action is popularly called the "grasshopper gear." The external features may be noted in Fig. 92. 
127. The condenser. - It was formerly the practice to exhaust the used steam into the open air or, in the case of locomotives, into the smokestack to increase the draft through the fire box. In many modern engines, the stationary, marine, and locomotive, the used steam is saved by being discharged into condensers.

The condenser promotes two kinds of economy and thus increases the efficiency of the engine and the saving of fuel. In the first place, the condenser chamber receives the exhaust steam into a pressure which is somewhat less than that of the atmosphere, or a partial vacuum (page $301 \mathrm{ff}$.). When exhaust steam is expelled into the air, against atmospheric pressure, it uses up energy which might well be given to the piston stroke; for the live steam has to drive out the used steam as well as drive the piston. With the low pressure condenser the used steam is taken from the cylinder with practically no help from the live steam. Consequently the live steam expends its energy upon the piston or in doing useful work. This is economical of energy and in turn means a saving of fuel.

The condenser effects another important saving. Steam in condensing gives up a large amount of heat energy (page 307), but the water of condensed steam retains also a large quantity of heat, its temperature being just a little below that of boiling water. It must therefore be evident that to collect and condense steam and to return the water with its heat content to the boilers is a saving of fuel that is quite worth while. One may calculate what such a system of saving means in terms of money from the actual cost of fuel and labor necessary to bring a given quantity of water to the boiling temperature.

Condensers have been used on marine engines for years, and recently have come into wide use on stationary engines. On locomotives the adoption has been slower because of almost unchangeable conditions, such as the size and weight of the engine, and the limited quantity of cold water available for 
condensing the steam. Locomotives must also have some means of forcing draft, which is readily secured by the exhaust through the smokestack.

The fundamental fact in the operation of the condenser is illustrated simply in the steam-heating system. In this the steam is cooled in the pipes and radiators and the water or the condensed steam is returned to the boiler by gravity, or by a pump where the return is from pipes lower than the boiler. In condensers on engines the steam is cooled and condensed more quickly by being conducted through pipes that are immersed in cold water.

\section{SUMMARY}

The modern steam locomotive represents the work of many inventors. Locomotives of to-day are powerful and heavy and may be made up of two or more engines.

The essential parts of a steam engine are the steam chest, slide valve, cylinder, and piston with connecting rod to crank or driving wheel.

By the connecting rod, the to-and-fro motion of the piston is transformed into the rotary motion of the wheel.

The condenser saves heat energy by exhausting the steam at less than atmospheric pressure, and returning to the boiler the hot water condensed from the steam.

\section{REVIEW QUESTIONS}

1. During what period was the steam engine developed? 2. What men in England and America are associated with its development? 3. What distinctive advance was made by the Stephensons? 4. Describe the big locomotives of the Erie Railroad. 5. Describe any big locomotive you have seen. 6. Compare the hauling power of early and recent locomotives. 7. What other motive power is a competitor with steam in transportation? 8. What are the working parts of a steam engine? 9. How does the steam perform work in the cylinder? 10. How is the slide valve moved and controlled? 11. What use is ordinarily made of exhaust steam in a locomotive? 12. What two economies are effected by a condenser? 13. In the condenser how is the steam condensed? 


\section{SUGGESTIVE QUESTIONS}

1. Compare advantages and disadvantages of running long trains with heavy locomotives and short trains with medium weight locomotives.

2. What practical conditions seem to limit the size of locomotives in length, height, and weight?

\section{The Production and Control of Steam}

128. Uses of steam. - Every one knows something about steam and its behavior in certain conditions. All are familiar with its uses in heating buildings, in cooking, in cleaning, in providing heat for drying such articles as clothes, lumber, fruits, and vegetables, in manufacturing water gas or producer gas (see page 162), and as energy for locomotives and stationary engines. This common knowledge may be taken as the basis for a study of some of the more important facts regarding the nature of steam, its production and control in performing useful work for us.

129. How steam is generated. - We shall begin by studying experimentally the principal conditions in which steam is produced.

Exercise. - Into a boiling-flask or beaker put about four ounces of water of freezing temperature if available and cover partly with a piece of asbestos board. Place the vessel over a flame and observe closely the behavior of the water throughout the experiment.1

When the water is boiling, hold the thermometer in the vapor just

${ }^{1}$ It will be an aid to careful observation and to the interpretation of the observed facts, if you keep a record of the following points: (1) the weight of the water with which you begin the experiment; (2) its temperature; (3) the time required to raise it from its initial temperature to boiling temperature; (4) the temperature of the water when boiling; (5) the temperature of the water vapor or steam while it remains in contact with the boiling water; (6) the effect of additional heat upon boiling water, as its temperature, behavior, and generation of steam; (7) the behavior of the steam bubbles from the moment and place of their formation to their escape at the surface; and (8) the difference in their size or volume at different depths and different temperatures of the water. 
above the water. Does it register a lower or a higher temperature than boiling water? Continue the boiling long enough to determine accurately the temperature of steam.

Observe the bubbles formed in the water at practically all places in contact with the vessel soon after heat is applied. How can you prove that they are not steam bubbles? How can you prove that they are air bubbles? What happens when water is allowed to stand in a glass for a while? If you cannot answer these questions fully at once, keep them in mind for later consideration.

Does continued application of heat to the water cause a rise in the temperature of the water or the steam above $212^{\circ} \mathrm{F}$.? Apply heat in larger or more intense amount to bring out the result unmistakably. It may appear that more intense heat causes more violent boiling and an increased rate of evaporation, but no change in temperature of water or steam.

Is the steam visible when it is imprisoned in the bubbles? Is it visible as it escapes from the water? Or does it remain invisible until it has left the surface of the water, as when it enters or leaves the neck of the flask? Have you observed steam rushing from the spout of a teakettle or the "blow-out" of a safety valve on a boiler? Is it visible directly when it makes its escape?

Insert a thermometer into the space above the water surface and try to determine whether steam remains invisible as long as it retains the temperature of boiling water. Do the observed facts support the view that steam remains invisible as long as it retains the temperature of the boiling water; and that it becomes visible when its temperature is reduced below that point?

The behavior of the water where the heat is directly applied may be made easily visible by putting some solid coloring matter, as aniline dye, in the flask. The water in contact with the heated surface is warmed and loses density. Steam bubbles are produced there, and they too are less dense than the water from which they are generated. Both the heated water and the steam bubbles are forced upward by the cooler, denser water. descending from the upper layers. As the steam bubbles strike the layers of colder water, they are cooled and collapse. The slight noise associated with their collapse may help in understanding the "singing" of water in the teakettle or boiler. As the temperature of the upper layers of water be- 
comes more nearly equal to that of the lower layers, the steam bubbles expand as they approach the surface. The fact is explained thus: the heated water and the steam bubbles at that part of the flask where heat is applied, are under the pressure of the water above as well as the pressure of the atmosphere exerted upon the surface. As the bubbles rise to the surface, they meet with less resistance or pressure than that exerted upon them at the bottom, and they expand. Their rate of movement increases at the same time, because by their expansion they displace more water which in turn forces them more rapidly toward the surface. At the surface the bubbles break the film of water enveloping them and are what we generally call steam.

130. Boiling temperature and high pressure. - In your experiment water is made to boil in the open air, at ordinary atmospheric pressure. Steam is generated, however, in boilers at a pressure much greater than that of normal atmosphere. The steam gage may show a pressure from one pound to two hundred fifty pounds per square inch. What happens as regards the boiling temperature of water when the pressure is increased? Does it remain the same as at normal air pressure, or, if not, what change is effected? The answer to the question may be obtained by the aid of an experiment.

Exercise. - The apparatus illustrated in Fig. 94 is designed to allow increase in pressure to be made at will and thus to indicate immediately what change occurs in the boiling temperature of water under more than atmospheric pressure. The experiment is safe, if performed with reasonable caution.

A laboratory boiler is the best apparatus for the demonstration. But if such is not available, set up the apparatus shown in Fig. 94. The flask should be of large capacity, round bottom, and very strong, tough glass. Make a pressure gage of a twelve-inch piece of glass tubing, $\frac{3}{16}$ of an inch bore, bent into the form of a double U, but with one arm at least six inches long. Insert tubing in stopper as in diagram. Put in mercury as in diagram. Put a thermometer in the middle hole so that the bulb will be well covered with water. Into the third hole insert a piece of glass tubing to which is attached a piece 
of rubber tubing; this will serve as an escape valve. A pinchcock on the rubber tubing will aid in regulating the escape of steam. Place the boiler thus equipped and about two thirds full of water on a stand and secure it with a clamp. Apply heat.

Observe the thermometer and pressure gage as the water is being brought to boiling. When the water boils vigorously and steam

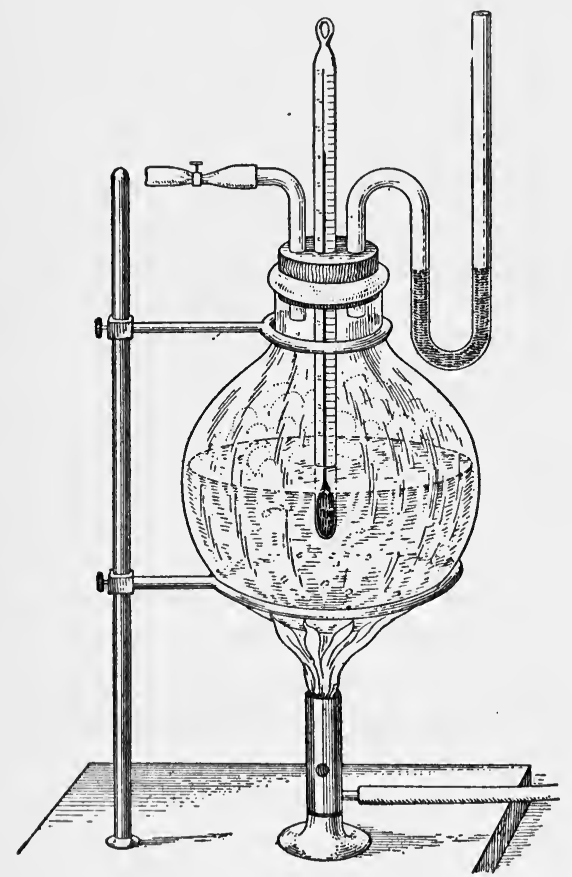

Fig. 94. - Apparatus for demonstrating changes in boiling point due to changes in pressure. issues freely from the escape valve, close the valve partly to prevent the free escape of steam. The pressure is increased, as the mercury in the gage shows, and the thermometer indicates a change in the temperature of the water. Apply carefully different degrees of pressure by closing the valve more and more, and note the corresponding changes in boiling temperature.

Boiling temperature raised with increase in pressure. - The temperature of boiling water (page 87) is not changed with any amount of added heat so long as the pressure remains unchanged; the water boils more violently or is converted more rapidly into steam. In this experiment an increase in pressure is followed immediately by a rise in the temperature, which shows that the boiling temperature of water is raised. The table (page 312 ) gives the boiling temperatures for certain pressures above and below atmospheric pressure, and may be consulted for further emphasis of the fact brought out in your experiment. 
High pressure for cooking. - The application of high pressure to raise the boiling temperature is familiar to those who make use of vessels for cooking at high altitudes. Such vessels are equipped with covers to be secured with clamps, with a pressure gage and a safety valve. They are tested to withstand a pressure of one hundred pounds per square inch. This will give a boiling temperature of $338^{\circ} \mathrm{F}$. (See Table of Pressures and Temperatures, page 312.)

131. Boiling temperature and low pressure. - What change is effected in the boiling temperature of a liquid by the reduction of the pressure below that of ordinary atmospheric pressure? There is an ancient puzzle which has to do with the difficulty of cooking eggs in boiling water at an altitude of ten thousand feet or more. The problem has to be met by those who dwell upon high mountains or plateaus, anywhere from five thousand to fifteen thousand feet above sea level. Does the boiling temperature of water at such reduced pressure fall in a manner corresponding to its rise under increased pressure?

A very interesting way to demonstrate the influence upon the boiling point of water of pressure below that of the atmosphere is the following. The demonstration should be started at the beginning of a class period, for it will continue, with a little attention, for an hour or more. It is much more striking than the usual experiments for this purpose.

Exercise. - Arrange the apparatus as shown in Fig. 95. Pour into beaker $A$ about a pint of water at $70^{\circ} \mathrm{C}$. or $158^{\circ} \mathrm{F}$., and an equal amount of cold water into beaker $B$. Put into each beaker a thermometer. The $U$ tube should be eight or more inches long, three fourths of an inch in diameter, and of heavy, strong glass. After closing one end of the tube securely with a rubber stopper, pour into it about three inches of water. Let the water run to the closed end of the tube. Holding it carefully over a flame, bring the water to boiling. When the steam escapes freely, close the end loosely with a rubber stopper and when steam fills the entire space, close it tightly. The steam expels almost all the air from the tube. Put the tube, with the water all in one end, into the beakers as in Fig. 95. 'The cold 
water in $B$ causes the steam to condense rapidly, leaving a partial vacuum, thus reducing considerably the pressure upon the free surface of the water. The water begins to boil, large bubbles of steam passing over to the other end. If the boiling is too violent, withdraw the tube partly from the beakers. The boiling will moderate as the temperature of the water in the tube falls. When all the water has

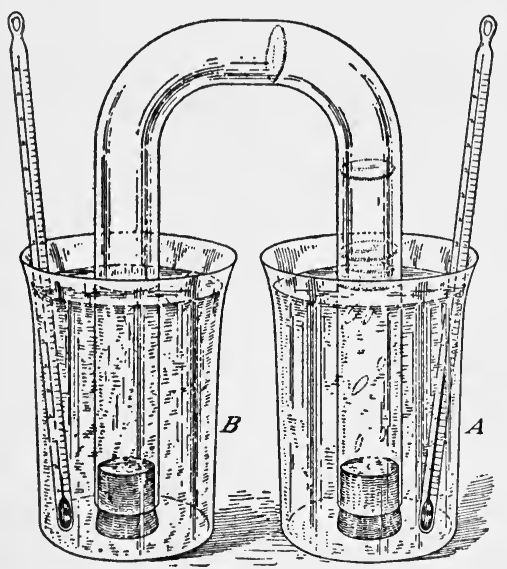

Fig. 95. - At lower than atmospheric pressure water boils at low temperatures. This simple apparatus demonstrates the fact. passed over into the other end of the U, the tube may be reversed to continue the process.

The water in beaker $B$ will soon be too warm to condense the vapor. Replace it with colder water, and continue to cool gradually until you have it as cold as a mixture of salt and ice will make it. The temperature of the water in the tube may fall to $68^{\circ} \mathrm{F} .\left(20^{\circ} \mathrm{C}\right.$. $)$ or lower during the class period, and the water will continue to boil vigorously. To maintain ebullition longer, it is necessary only to have the water in $B$ cold enough to cause condensation.

The behavior of the bubbles indicates that there is very little, if any, pressure upon the free surface of the water. The steam volumes rising from the stopper continue unbroken until they reach the surface where they are as large as the diameter of the tube. If the pressure were uniform at all depths of the water, the size of the bubbles would of necessity remain unchanged.

Boiling temperature lowered by reduction of pressure.-By reducing pressure or resistance to the expansion and escape of the vapor or steam, the boiling temperature of a liquid is.caused to fall below that at ordinary pressure. A piece of ice in a vacuum as complete as can be made would produce steam; or water at a normal freezing temperature would boil under the same conditions. This may be demonstrated with suitable apparatus. But a fact of fundamental importance is that 
heat is present in any substance which has a temperature above absolute zero or $-273^{\circ} \mathrm{C}$. If pressure is reduced low enough, any volatile substance will boil at a correspondingly low temperature (see page 312 ), and its steam will have the same temperature as that of the boiling liquid under that pressure.

Some industrial uses of low boiling temperature. - Many industries make use of the fact that the boiling temperature of a liquid can be lowered by the reduction of the pressure upon its free surface. If such substances as milk, eggs, sugar sap, and cider were boiled at ordinary pressure, they might be easily scorched or otherwise damaged. They are usually evaporated under low pressure or in vacuum pans at relatively low boiling temperatures. The fact is turned to advantage also in economizing fuel. In the manufacture of glycerine, for example, from the waste lye solution from soap making, the evaporation is carried on at different pressures and temperatures. The steam produced from the first evaporator is conducted to and boils the liquid in a second evaporator which is kept at a sufficiently lower pressure. The steam from this is likewise made to boil a third, and so on possibly to a fourth evaporator. Thus the latent heat of steam is utilized three or four times, saving almost two thirds or three fourths of the fuel required for evaporation at normal atmospheric pressure.

The chief points presented in these experiments and observations may be summarized briefly.

First. The boiling temperature of water remains the same under the same general conditions.

Second. The temperature of a boiling liquid remains the same, if pressure is constant, until all of the liquid is vaporized.

Third. The boiling temperature of a liquid varies with pressure; rising or falling as pressure increases or decreases. Pressure may be controlled mechanically, and thereby the boiling point as well.

Fourth. The boiling temperature of a liquid is that at which the pressure of its saturated vapor, produced at the place of 
application of heat, is equal to the pressure upon its free surface. When equal, the water vapor as bubbles will rise to, and escape from, the surface of the liquid. This is " boiling."

Fifth. The temperature of steam remains the same as that of boiling water as long as it is in contact with it.

132. Amount of heat required to produce steam. - After water has been brought to the boiling point by heat, it requires a great deal more heat to change it into steam. You can make a rough judgment of the additional heat needed by experimenting with a given quantity of water, as fifty grams by weight, and comparing the time required to raise its temperature from $32^{\circ} \mathrm{F}$. to $212^{\circ} \mathrm{F}$. with the time required to convert a certain fraction of the water into steam. If, for instance, ten grams of water were evaporated in a given time, the total quantity or fifty grams would be vaporized probably in five times the time required for the ten grams. The result is of course only approximate, but good enough for practical purposes.

The quantity of heat absorbed by water in being raised from freezing to boiling temperature is usually referred to as the heat of water, or as applied to all liquids as the heat of the liquid.

The heat of steam is the other factor of importance. The additional heat necessary to change water into steam does not register sensibly or by the thermometer. The general belief is; however, that somehow the extra heat is not lost or wasted if the steam generated is utilized for heating or for power. This implies that steam may contain the heat of the liquid and also heat supplied in changing the liquid into steam. The facts may be made clear by experiments.

Exercise: Heat of steam. - Balance a beaker containing a thermometer on a pair of trip scales, and pour into it enough water at $32^{\circ} \mathrm{F}$. to balance a hundred gram weight. Arrange a boiling-flask with about fifty grams by weight of water (the apparatus used in showing relation of high pressure and boiling temperature will be excellent); connect the escape valve by rubber tubing, tipped with 
a short piece of glass tubing, with the water on the balances. See that the tubing reaches nearly to the bottom of the water in the beaker, but does not touch the beaker. Balance exactly. Bring the water to boiling and continue heating until a ten gram weight on the scale pan is balanced by the condensed steam in the beaker on the opposite pan. This means that ten grams of water have been transferred by steam from the boiling flask. What is the resulting temperature of the water in the beaker? Your result is satisfactory if it is about $58^{\circ} \mathrm{C}$. or $136^{\circ} \mathrm{F}$.

Determine the heating effect of boiling water upon water at freezing temperature. Balance a beaker containing 100 grams of cold water on the scales. Put on the weight pan a ten gram weight, then pour into the beaker exactly ten grams of boiling water. Take the resulting temperature. You may find a rise in temperature to about $9.1^{\circ} \mathrm{C}$, or $37^{\circ} \mathrm{F}$. If your result is anywhere near these figures, you may consider the experiment successful. Some of the heat is lost in different ways, as in transferring steam through the tubing, in the air, the glass, and otherwise. But you find that approximately six times as much heat is transferred by the steam as by boiling water.

Latent heat of steam. - Steam in pipes, radiators, engine cylinders, and so on, condenses, and in so doing, gives up the same quantity of heat that is required to convert water into steam. This fact has been proved experimentally. After water is heated to boiling temperature, the heat required to change it into steam is utilized in driving asunder the molecules of water from each other and setting them free in space as steam. It is also needed in making the pressure of steam equal to the pressure exerted upon the surface of the water. As there is no change in the temperature of steam, as long as it is in contact with water, from the temperature of the boiling liquid, this extra amount of heat is called the hidden or latent heat of steam.

133. Evaporation and condensation. - Water is vaporized by heat under men's control for definite purposes. In nature water is always and everywhere being evaporated or vaporized when exposed to the air and as long as the air's capacity for moisture at a given temperature has not reached its limit 
(page $44 \mathrm{ff}$.$) . When the limit of the air's capacity is reached,$ the air is said to be saturated. If the temperature of the air falls below that at which saturation occurs, water vapor condenses. If the air is warmed above the temperature of saturation, it increases its capacity for water vapor, and evaporation is renewed. As you have seen (page $48 \mathrm{ff}$.), these facts are fundamental in understanding the humidity of the atmosphere and the conditions of condensation. The chief point at present is that evaporation requires heat, the water vapor absorbing it in the same way as steam does in being produced from water by heat from artificial sources. This difference, however, must be noted : evaporation takes place only at the surface, while steam is generated within the body of water. Evaporation occurs at any temperature at which water may exist as liquid or solid; but steam is generated by boiling at what is known as the boiling temperature.

Effect of condensation. - Water vapor produced in evaporation gives up in condensation its hidden or latent heat in the same way and to the same extent as does steam in condensing. The same quantity of heat is liberated in condensation as is absorbed in evaporation. This fact is important in the study of climate and weather. Evaporation utilizes heat, which is absorbed in the water vapor, and just so much heat is taken from the total amount with the moderation or cooling of the air where it occurs, as in the tropics. When the vapor condenses in a colder region, it liberates heat energy, which warms the air of the colder region. The corresponding fact in the behavior of steam is seen in its use for heating buildings. The steam in the radiator in condensing releases heat which it absorbed in being produced from water by the heat in the. furnace.

134. Measuring heat of steam. - Heat is measured, according to the English system, by the unit quantity required to raise the temperature of one pound of water through one degree Fahrenheit (page 142). The quantity of heat necessary to raise 
the temperature of one pound of water from $32^{\circ} \mathrm{F}$. to $212^{\circ} \mathrm{F}$., or through 180 degrees is, therefore, 180 British Thermal Units. In your experiment with steam and boiling water (page 304), you find that steam gives up about six times as much heat as boiling water does. By very carefully conducted experiments it has been found that the quantity of heat required to change boiling water into steam is 972 B.T.U., or a little more than five times the quantity utilized in raising the temperature of water from the freezing to the boiling point. The facts are shown graphically in Fig. 96.

The practical significance of this difference in the heat capacities of steam and boiling water may be made clear by

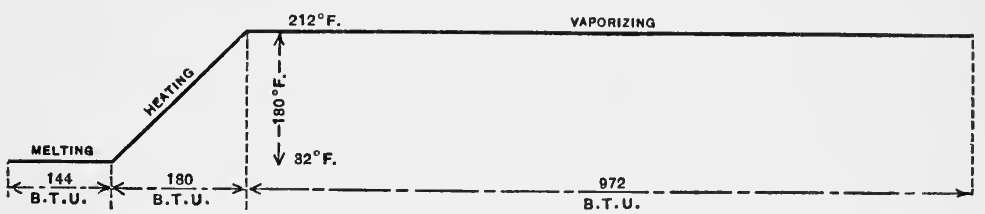

FIG. 96. - Graphic representation of heat units in converting one pound of ice into one pound of steam.

expressing the facts in the following manner. Water in cooling from the boiling temperature of $212^{\circ} \mathrm{F}$. to $32^{\circ} \mathrm{F}$. gives up heat energy to the amount of 180 B.T.U. per pound. Steam in merely changing from vapor at $212^{\circ} \mathrm{F}$. to water at the same temperature gives up heat energy to the relatively enormous amount of 972 B.T.U. per pound. Thus it is evident that the heat of steam, which is not shown by the thermometer, is a store of energy readily utilized in work of great value to the whole world.

135. What steam bubbles suggest. - In your observation of the behavior of the steam bubbles or volumes in boiling water, attention was directed to the fact that the bubbles, small at the start, expanded rapidly on their way to the surface. This behavior of the steam bubbles suggests two important points. First, the volume of steam produced from a given 
weight of water is many times greater than the volume of that weight of water. Second, the expansion of steam under decreasing pressure suggests the compressibility of steam or its elasticity. These two points we shall consider briefly with reference to their practical application in machines.

Volume of steam. - The relative volumes of equal weights of water and of steam at atmospheric pressure may be presented in this way. One pound of water occupies .016 of a cubic foot of space (62.5 pounds occupying one cubic foot of space). The steam produced from this weight of water occupies at this pressure about 26.7 cubic feet of space, or more than 1600 times the space occupied by a pound of water. You will find it helpful to make a graphic representation of the relative volumes of steam and water for a given unit of weight.

This great difference between the volume of steam generated from a given amount of water and the volume of the water becomes a serious danger in certain conditions. The water in a boiler may run too low, as the common expression has it, and the heat remain constant, possibly heating red-hot the parts of the boiler not covered by water. The intense heat concentrated upon a small amount of water causes it to boil with increasing violence, throwing it against the superheated parts. The water vaporizes with startling rapidity. The volume of steam generated from the small quantity of water is too great to escape through the safety valve or to be retained by the boiler. The result is an explosion, the tremendous energy generated in a flash, as it were, bursting through the weakest part of the boiler.

When the energy of steam is kept under control, it may be made to perform useful work, or, as it is usually stated, it may be converted into mechanical energy. This is exactly the reverse of the conversion of mechanical energy or motion into heat energy, as by friction or by compression of gas in a bicycle pump. If admitted into a cylinder in which there is a movable piston connected with a crank or driving wheel, the steam by its expansion forces the piston to one end and then to the other, 
as determined by the slide valve and its controlling mechanism. This involves the second point stated above.

The compressibility of steam together with its volume as compared with the volume of the water from which it is produced, makes it wonderfully useful for power purposes. Pressure may be exerted to compress steam by mechanical means. Like air as in the case of the bicycle pump, it may be reduced in volume and when released resume its former volume with much force. The greater the pressure, the more the volume of a unit weight of steam is decreased. And as the pressure decreases, the more the volume of a unit weight of steam increases. In the boiler, steam is produced at increasing pressure and is consequently not able to expand to its full capacity. When it passes from the steam chest, where it is under the high pressure of the boiler, into the cylinder, a space of low pressure, it expands, rapidly occupying all available space. It drives the piston forward or backward as it enters from one port or the other (pages 293-4). In expanding it uses its own heat energy and becomes cool. The same fact may be observed when air is escaping from an inflated tire or basketball. In using up its heat energy by expanding, the steam may be made to do useful work, that is, to drive the piston back and forth, its heat energy being converted into mechanical energy. Thus the steam engine is really a heat engine.

136. Superheated steam. - For the purpose of power to do work, steam is generated in boilers under high pressure and has thus a correspondingly high temperature. In many types of the modern engine steam is utilized only after it has been removed from the boilers and subjected to additional heat for the purpose of raising its temperature and thereby increasing its energy. Steam thus treated is known as superheated. A superheater is shown in Fig. 97. The superheating of steam has another advantage in the fact that it effects an economy of fuel, a matter of serious consideration to-day in view of the rapidly inereasing cost of fuel. 
137. Waste in simple engine. - In the case of a simple steam or heat engine the steam is conducted from the steam chest on the boiler to the cylinder, where it expands, utilizing heat

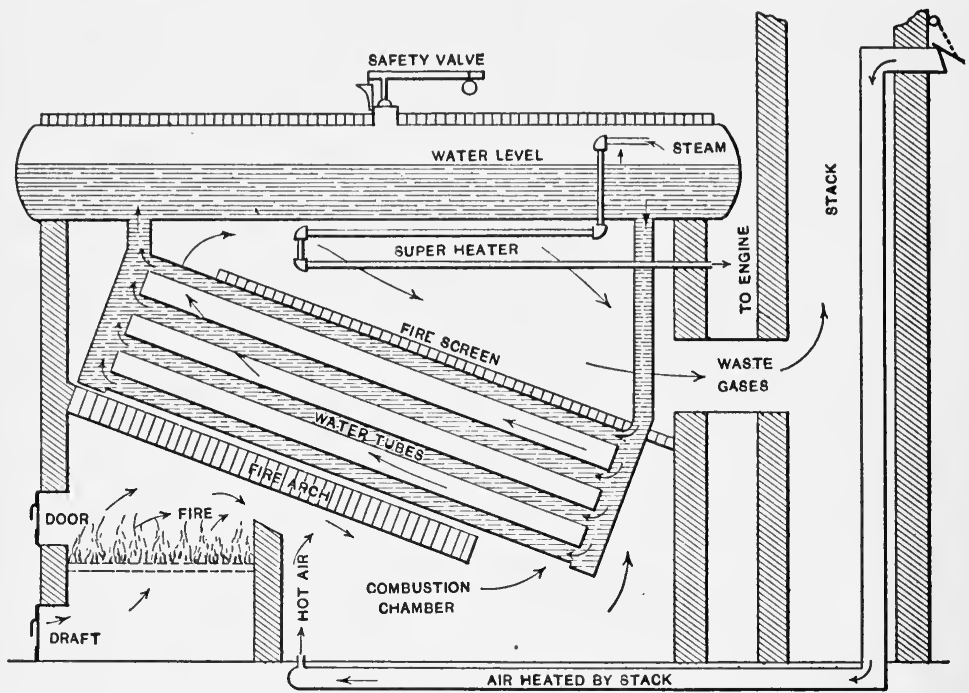

FIG. 97. - A boiler setting to secure complete combustion, with a superheater. Trace out the course of gases formed in combustion and explain possible use of them.

and driving the piston from one end to the other. The used steam is forced through the exhaust port and allowed to escape.

138. Multiple engines and compound engines. - Such an engine fails to make use of anything like the total quantity of heat energy of steam. To overcome this waste, many improvements have been made, among which are the compound, triple and quadruple expansion types of engines, and steam turbines for ocean steamships and stationary power plants. The steam turbine, for instance, is adapted to a range of pressure from two hundred fifty or more pounds absolute pressure (which is gage pressure plus ordinary atmospheric pressure) to one pound absolute, or a partial vacuum. Consult the table 
on page 312 for the expansive capacity of steam below atmospheric pressure.

The compound engine. - The compound engine is becoming familiar in locomotives. The triple and quadruple expansion types are necessarily restricted to marine and stationary use. The compound engine makes use of the steam in a series of two cylinders. The used steam from the first cylinder is conducted to, and made to perform work by greater expansion in, the second cylinder. This is generally made possible by having the second cylinder adapted to a lower temperature and pressure than the first. So perfectly is this adjusted that each cylinder gets its fraction of steam's expansion and so of its heat energy. Compound and multiple expansion engines are designed so as to divide the total energy of steam at a given pressure and temperature into two, three, four, or five parts. The steam is allowed to perform just so much work in each cylinder. When superheated, it has of course greater energy, which it gives up in its course through successive cylinders, or sections of turbines.

Table of steam pressures (for reference). - The table on page 312 gives a few typical cases of the relation of absolute pressure to (a) volume of one pound of steam in cubic feet; $(b)$ latent heat of steam in B.T.U.; $(c)$ the heat of water in B.T.U.; and $(d)$ the boiling temperature of water in Fahrenheit scale. The heat of water is the amount of heat added from $32^{\circ} \mathrm{F}$. to $212^{\circ} \mathrm{F}$., and not the total quantity of heat which water may have above absolute zero or $-273^{\circ} \mathrm{C}$. Gage pressures are given for cases below atmospheric pressure. For all above subtract 14.7 pounds from absolute pressure to find gage pressure.

Study of the data presented in the table enables one to see more clearly the enormous quantity of heat energy which a given mass of steam at a given pressure contains. It emphasizes the advantages in making use of steam as a medium for the transfer of heat. It should make clear also why steam is utilized at very high pressures in the modern engine. An absolute pressure of 240 pounds per. square inch is not unusual to-day. This 


\section{Table of Steam Pressures and Temperatures}

\begin{tabular}{|c|c|c|c|c|c|}
\hline $\begin{array}{l}\text { Absolute " } \\
\text { Pressure } \\
\text { Pounds to } \\
\text { Soutili: } \\
\text { INCH }\end{array}$ & $\begin{array}{c}\text { Gage Pressure } \\
\text { IN Pounds } \\
\text { PER SQUARE } \\
\text { INCH }\end{array}$ & $\begin{array}{l}\text { VOLUME OF } \\
\text { ONE POUND } \\
\text { IN CUBIC } \\
\text { FEeT }\end{array}$ & $\begin{array}{l}\text { LATENT HEAT } \\
\text { OF STEAM IN } \\
\text { B.T.U. }\end{array}$ & $\begin{array}{l}\text { HEAT OF } \\
\text { WATER IN } \\
\text { B.T.U. } \\
\text { ABOVE } \\
32^{\circ} \mathrm{F} \text {. }\end{array}$ & $\begin{array}{l}\text { TEMPERATURE, } \\
\text { FAHRENHEIT, } \\
\text { BOILING } \\
\text { WATER AND } \\
\text { STEAM }\end{array}$ \\
\hline 0.089 & -14.611 & 3333.3 & 1091.7 & 0.0 & $32 .^{\circ}$ \\
\hline .5 & -14.2 & 636.9 & 1058.4 & 47. & $79.9^{\circ}$ \\
\hline 2. & -12.7 & 172.4 & 1026. & 94.4 & $126.3^{\circ}$ \\
\hline 3. & -11.7 & 118.5 & 1012. & 109. & $141: 6^{\circ}$ \\
\hline 6. & -8.7 & 61.3 & 995. & 138.6 & $1700^{\circ}$ \\
\hline 10. & -4.7 & 38.4 & 982. & 161. & $193^{\circ}$ \\
\hline 14.7 & 0.0 & 26.7 & 972. & 180. & $212^{\circ}$ \\
\hline 18. & 3.3 & 21.8 & 958. & 191.3 & $222.4^{\circ}$ \\
\hline 20. & 5.3 & 20. & 960. & 196. & $228 .^{\circ}$ \\
\hline 30. & & 13.5 & 938. & 219.5 & $2500^{\circ}$ \\
\hline 50. & & 8.5 & 923. & 250 . & $281 .^{\circ}$ \\
\hline 65. & & 6.5 & 905. & 267. & $297.8^{\circ}$ \\
\hline 100. & & 4.4 & 888. & 293. & $327 .^{\circ}$ \\
\hline 125. & & 3.5 & 875. & 315. & $344 .^{\circ}$ \\
\hline 150. & & 3. & 863. & 330. & $358 .^{\circ}$ \\
\hline 175. & & 2.6 & 853. & 343. & $371 .^{\circ}$ \\
\hline 200. & & 2.2 & 843. & 355. & $382 .^{\circ}$ \\
\hline 225. & & 2. & 834. & 365. & $392 .^{\circ}$ \\
\hline 350. & & 1.8 & 826. & 375. & 401. \\
\hline 300. & & 1.5 & 811. & 393. & $417 .^{\circ}$ \\
\hline
\end{tabular}

means that steam has a temperature of $397^{\circ} \mathrm{F}$. which is the boiling temperature of water at this pressure. Its temperature may be increased by superheating. The volume of steam at this pressure, 1.9 cubic feet, shows what tremendous capacity a unit of steam of this temperature has and gives up in doing work in reaching the volume which it has at atmospheric pressure. Even this volume is increased by the low pressure turbine which gets energy out of steam at a pressure far below that of the atmosphere. Again we have an illustration of the way in which man controls natural forces for his own needs and purposes.

139. Industrial uses of different boiling temperatures. Knowledge of the different boiling or vaporizing temperatures of 
substances is of practical importance in many industries. One familiar use is illustrated in distillation, a process which comprises boiling the liquid, and removing therefrom its vapor, which is condensed to liquid by cooling. For example, pure water for drinking and cooking is now provided on ocean liners by distillation of salt water from the ocean.

In distillation either the vapor or the residue or both may be saved. Sugar sap, salt water, and the like may be vaporized for the sake of the residue alone, that is, the sugar, salt, and so on. In certain kinds of gold mining, mercury is employed to gather the grains of gold; then the amalgam of mercury and gold is broken up by distillation in which the mercury is vaporized, condensed, and saved, and the gold is secured as the residue. Water which is thought to be polluted, but must be utilized for drinking, may be vaporized, the vapor being condensed and cooled as pure water, and the residue thrown away.

Table of Some Common Substances with Their Boiling Temperatures at Atmospheric Pressure

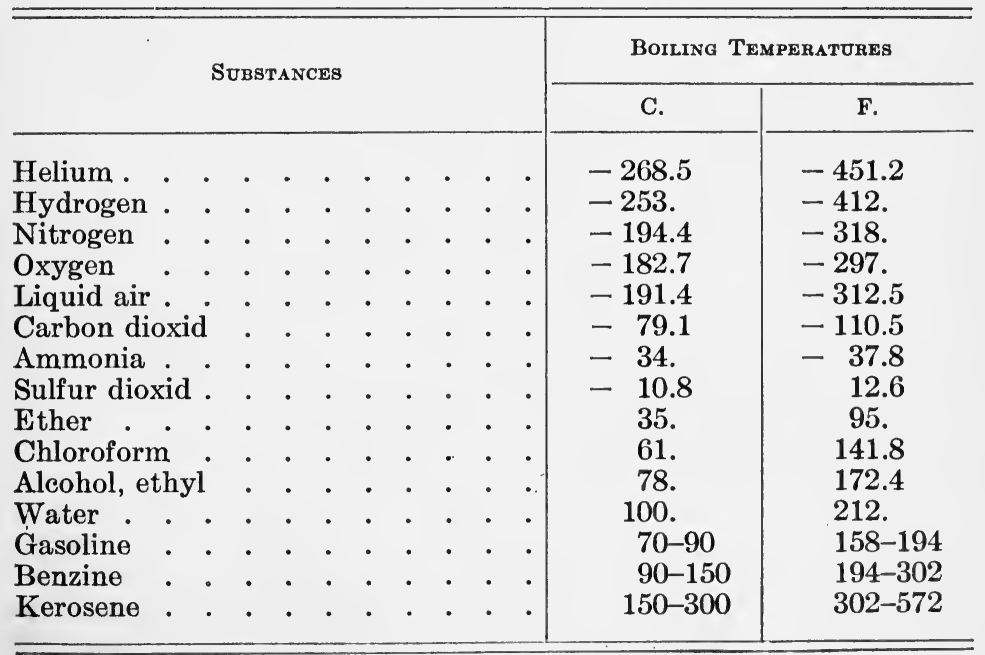


Fractional distillation. - Fermented mixtures of alcohol and water and other substances produced from grains, such as barley, rye, and corn, or from fruits, such as berries and grapes, are distilled in fractions, as alcohol and water, the alcohol being separated from other substances. The distillation of crude petroleum illustrates clearly the process of fractional distillation. Petroleum contains many different substances which have different boiling temperatures. The most volatile substances, such as gasoline, naphtha and benzine, are among the first to boil or vaporize, and separate from the rest. Gasoline boils at from $70^{\circ}$ to $90^{\circ} \mathrm{C}$., naphtha at $90^{\circ}$ to $120^{\circ}$, and benzine at $120^{\circ}$ to $150^{\circ} \mathrm{C}$. The residue after distillation yields among other substances lubricating oil, vaseline, mineral oil, paraffin, and a carbon which is used in making electric carbons. From liquid air oxygen and nitrogen are readily separated from each other, because nitrogen vaporizes at a much lower temperature than oxygen, and it is easily removed, leaving practically pure oxygen as the residue.

Boiling point of mixtures. - Mixtures of two or more substances have a boiling point somewhere between the two extremes, as determined by the proportions of the individual substances mixed. Sugar or salt or other solids of a similar nature, when dissolved in water, raise the boiling point in proportion to the amount of the solid substance in solution. Pure water boils at $100^{\circ} \mathrm{C}$; ; but all water except distilled water has more or less of impurities in solution and requires therefore a higher temperature for boiling. The boiling temperature of liquid air, as may be seen in the table, is between the boiling points of nitrogen and oxygen.

\section{SUMMARY}

Steam is produced from water by heat.

Its temperature at ordinary pressure is the same as that of boiling water, or $212^{\circ} \mathrm{F}$.

Steam becomes visible or condenses when its temperature is reduced below the temperature of the boiling water. 
Boiling temperature varies with the pressure.

The heat (972 B.T.U. per lb.) which disappears when water is converted into steam is the latent heat of steam. It reappears when the steam condenses.

The same disappearance and reappearance of heat occur in evaporation and condensation in nature.

The steam engine depends on the relative volumes of equal weights of steam and water, and on the ability of steam to expand.

The heat energy of steam is used in part in the simple steam engine, but more fully in multiple expansion engines.

Distillation depends on the difference in the boiling temperatures of substances.

\section{REVIEW QUESTIONS}

1. Mention several uses of steam. 2. Explain the singing of a teakettle.. 3. What important facts are to be observed in the boiling of water? 4. Is steam visible? Why? 5. How does the temperature of steam compare with that of boiling water? 6 . Does the application of a hotter flame to the boiling water increase its temperature or its rate of boiling? 7. How ean you demonstrate that the boiling temperature of water rises with increase in pressure? 8. How can you demonstrate that the boiling temperature of water falls with decrease in pressure? 9. What are some of the practical uses made of the change in boiling temperature due to change in pressure?

10. Which has the greater heating effect, steam or water, at the same temperature? How could you prove your answer? 11. What is meant by heat of liquid? 12. Heat energy that enters a body and produces no rise in temperature is called by what name? 13. The heat which disappears when water boils is used in performing what tasks among the water particles? 14. Distinguish between evaporation and boiling by mentioning two points of similarity and two of difference. 15. Explain the influence upon the weather of condensation and evaporation.

16. Steam heating of buildings depends upon evaporation and condensation. Explain. 17. How much heat is required to warm a pound of water from the freezing to the boiling point? 18. How much to vaporize it? 19. How much heat is given out by the condensation of the pound of steam? 20. How much is given out in the cooling of the pound of water to freezing point?

21. Compare the volume of a pound of water with the volume of the same weight of steam. 22. Why does the pressure increase in a 
closed boiler when the water boils? 23. What changes take place in the volume of steam when the pressure is increased? 24. What use is made of this fact in explaining a boiler explosion? 25. What changes take place in the volume of steam when the pressure is decreased? 26. What change takes place in the temperature of steam in expanding? 27. How may the heat energy of expanding steam be utilized? 28. A steam engine is called a heat engine. Explain. 29. What is superheated steam? How is it superheated (Fig. $23 \mathrm{H}$ )? 30. Why does a simple steam engine not make use of all the heat energy of steam? 31. What is the advantage of compound and multiple expansion cylinders used in steam engines? 32. How high are the temperatures and pressures used in modern steam engineering?

33. Mention two liquids which may be separated from a mixture because of their differences in boiling points. 34. Mention four substances that have their boiling points below the freezing point of water. 35. Mention four substances having their boiling points between the freezing and boiling points of water. 36. Mention some having their boiling points above that of water. 37. How is sugar obtained from its solution? salt? 38. What may be inferred about the strength of a salt solution from its boiling point? 39 . What is fractional distillation? Illustrate in the making of petroleum products. 40. Mention a case where distillation is carried on for the sake of the residue; for the sake of the distillate; for both.

\section{SUGGESTIVE QUESTIONS}

1. Is a thunderstorm the cause or result of " cooling the air "?

2. Establish the volume of a pound of water from the weight of one cubic foot of water.

3. Is air like steam in changing its volume with changes in pressure?

4. What changes in temperature take place when the volume of air is changed by compression or expansion?

5. What changes in air take place as it blows from an inflated tire?

6. Explain why a cloud sometimes forms in the neck of a soda water bottle when the cork is removed.

\section{Electricity as a Motive Power}

140. Electrification of steam railroads. - Wonderful as has been the increase in the use of electricity as motive force in transportation in cities and suburban districts, its installation for rail- 
roads that were previously operated by steam is most interesting and significant. The rate at which steam roads are being converted into electrically operated roads indicates that electricity
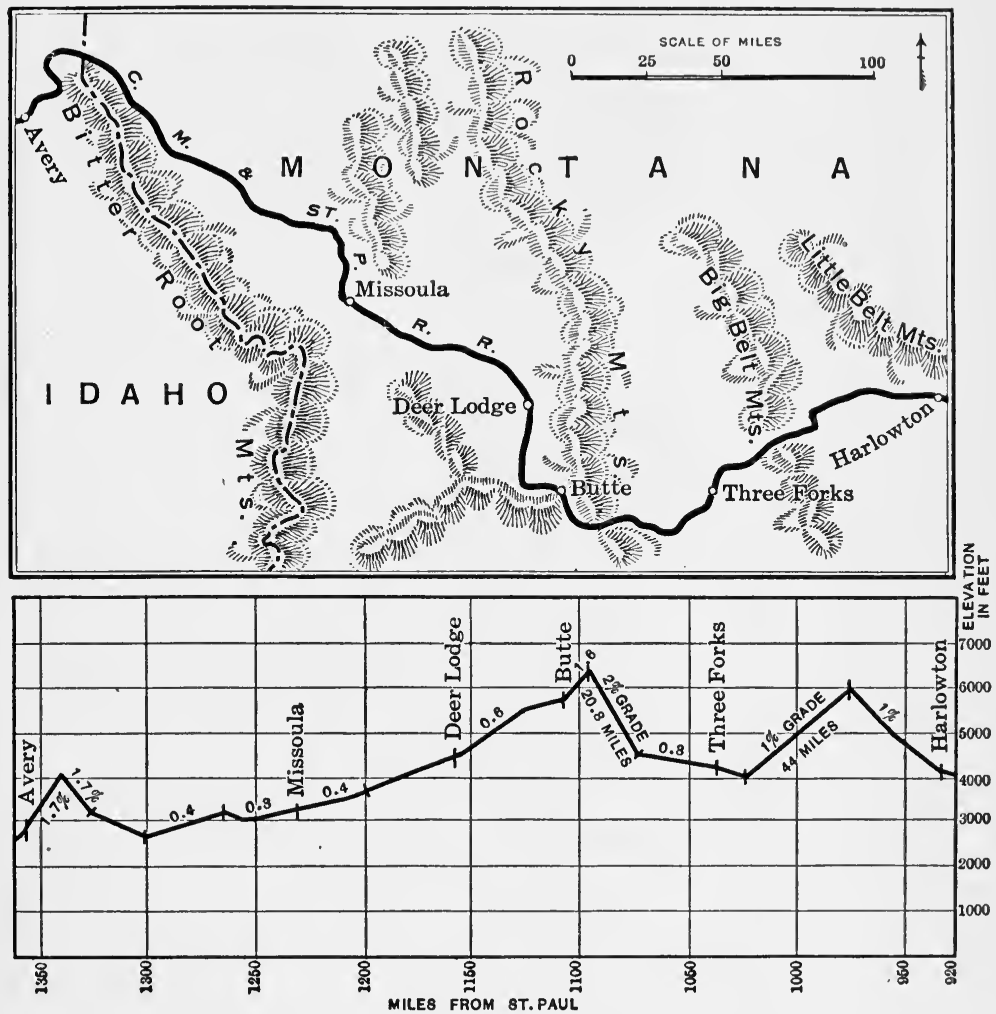

FIG. 98. - Map and profile of the section of the Chicago, Milwaukee, and St. Paul Railroad operated by electricity. Note distances and grades and compare with table of elevations, page 273 .

will be made at no very distant day the chief motive force in a large part of transportation. Already more than one thousand miles of road, comprising over two thousand miles of track, including sidings, spurs, yards, and the rest, have been electrified, 
and steam power practically discontinued on these sections. Hundreds of miles additional are well along in construction, and still more are planned.

An example of electrification of steam railroads is that of the Chicago, Milwaukee, and St. Paul. Four engine divisions comprising 440 miles of road and 160 miles of spurs, sidings, and yards are electrically operated. The stretch of road (see table, page 273) crosses three mountain ranges (Fig. 98) : in Big Belt Mountains at an elevation of 5788 feet; the Rockies at a maximum elevation of 6322 feet, this relatively low elevation for the Continental Divide being due to a tunnel half a mile long; and the Bitter Root Mountains at an altitude of 4163 feet through a tunnel nearly 9000 feet long. The east approach to the Rockies (Fig. 98) has 20 miles of road with 2 per cent grade. The west approach to the Big Belt Mountains has 40 miles of 1 per cent grade, a long, heavy pull for steam locomotives.

It is probable that the rest of the road to the Pacific coast will be electrified, aggregating a total distance of 850 miles. The Cascade range of mountains is crossed at an altitude of 3010 feet by a grade that is very light.

141. The electric locomotive. - The electric locomotive (Fig. 99) is 112 feet and 8 inches in length and weighs about 284 tons. The estimated weight upon the driving wheels is 200 tons. The locomotive is rated at 3000 horse power for continuous operation, and 3440 for one hour. The motors are flexible or like a good horse which under stress may exert additional power to haul a limit load over a steeper grade. The tractive effort or pull available for starting a load is about 120,000 pounds, but for continuous pulling is about 85,000 pounds. A freight locomotive can haul a 2500-ton train on all gradients up to and including one per cent, at a speed of 16 miles per hour; or a load of 1250 tons on gradients up to and including two per cent, the maximum gradient on the road, at the same speed. For example, one locomotive hauls a train of the larger load until it arrives at a grade steeper than one per 
cent. Here a pusher helps over the steep grade, and at the summit is transferred to the head of the trains and aids in retarding the speed down grade.

Regenerative braking. - Instead of depending upon brakes, involving wear and tear of wheels, brake shoes, and track, the motors of both locomotives when going down grade are changed into electric generators which not only brake the train effectually, but also send into the trolley 25 to 50 per cent of the total electric energy consumed. The air brakes are used only in stopping and in emergencies. The important point is that the motors are reversible in function (page 324). When going up grade they take in electrical energy and give out mechanical energy for hauling. When going down grade they take in mechanical energy and give out electrical energy. This method of braking is known as the regenerative.

The passenger locomotives are like the freight locomotive except that they are geared

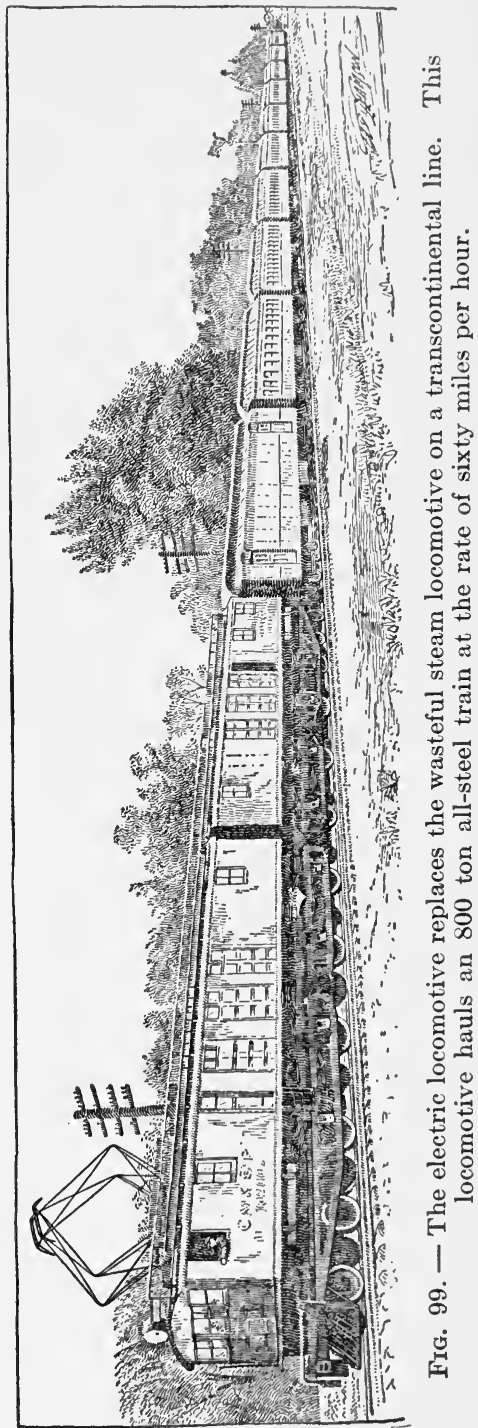


to higher speed and for a less load. They may haul a trailing load of 800 tons on the grades at a speed of 20 to 25 miles an hour, and on level stretches at a speed of 60 miles per hour.

Comparison with steam locomotive. - The steam locomotives replaced by the electric locomotives are of the large Mallet duplex or two-engines-in-one type. They weigh 6 per cent more than the electric, and have a tractive effort of only 76,200 pounds, or nearly 9000 less than that of the electric locomotives. They are rated below the electric in speed and flexibility for emergencies. Compare the weight and tractive effort of the Erie engine (page 292).

Electric current used. - The motors make use of direct current electricity at approximately 3000 volts. Alternating current at 100,000 voltage is transmitted from the hydro-electric plants of the Montana Power Company, the principal plant being located at Great Falls, north of Helena, Montana, to the railway's sub-stations, where it is stepped down by transformers to 2300 volts and converted into direct current. It is distributed to the trolley wires and collected by the pantagraph for the motors. In a complete locomotive there are eight motors which are twin-geared to each of eight pairs of fiftytwo inch driving wheels (Fig. 99).

Future use of electricity. - The change of steam railroads into electrically operated railroads is largely for purposes of economy. It promises much as to the future use of electric power in transcontinental traffic. It means the utilization of falling water now running to waste and the economy of the coal resources of the country. Twenty years will witness extensive transformations of the present means of transportation with the probability of electric energy taking precedence over steam. Even in those cases where steam railroads have been electrified with energy derived from heat from fuel, the railroads seem to yield financial profit as well as other kinds. Such returns make it probable that many similar installations will be made.

Progress in the use of electric energy in transportation may 
not be unlike that of the automobile within the past twenty years. Less than twenty years ago there were fewer than fifty automobiles in the country, and these were fantastic, almost impossible contrivances. To-day the automobile is everywhere in rapidly increasing numbers. In a similar way we may consider the present and the future of electricity in long distance transportation. To-day the alternating current is generated for transmission over long distances and then converted into the direct current for most effective use. To-morrow generators will be made which will generate direct current at high tension and potential for long distance transmission, and do away with the present expensive process and machinery for rectifying or changing the alternating into the direct or continuous current (pages 327-8).

142. The electric motor. - The electric motor changes electric energy, transmitted by wires from a battery or a generator, into power to do work or mechanical energy. The electric generator (page 325) takes in mechanical energy derived from steam or falling water and gives out electrical energy. The simplest form of the motor is illustrated in Fig. 100. When an electric current passes along the wire that lies between the poles of a magnet, the wire is made to move in the direction of the dotted line. If

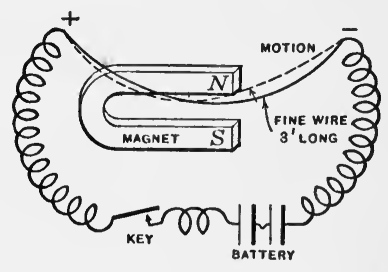

Fig. 100. - Diagram showing how a motor works. the direction of the current is reversed, the wire will move in another direction. This does not resemble a motor, but it acts exactly like one. Suppose you have a coil of wire, as in Fig. 101, and the electric current causes the coil to move from dotted line to present position. At just this point where the coil would stop, the current is reversed, with the result that the coil continues to move through another half turn. Reversing the current at this point causes the coil to be carried beyond the next stopping point. With the continued reversal 
of the direction of the electric current at the critical points, the coil known as the armature will continue to rotate. The chief

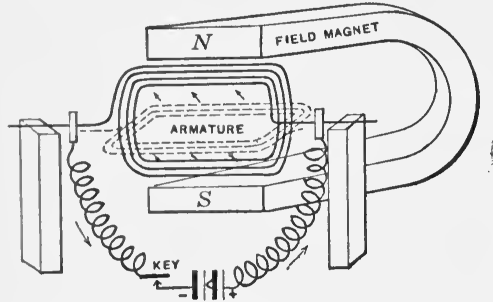

FIG. 101. - A simple form of the electric motor, showing armature, magnetic field, and source of electric energy. difference between this simple form and an actual motor is in the strength of the magnet and the number of coils of wire the actual motor has to form the armature.

The essential facts in the structure and operation of the electric motor may be learned by examining a small model motor, or better still by making a motor, for which the following plan may serve. Careful study of the motor will prepare the way for an understanding of the generator.

Design for a motor. - The framework (Fig. 102) is made of two iron bars $(I, B)$ about $\frac{1{ }^{\prime \prime}}{4}$ by $1 \frac{1}{2}$ ' by $12^{\prime \prime}$ long, and two iron bolts about $\frac{1}{2}{ }^{\prime \prime}$ diameter by $10^{\prime \prime}$ long, around each of which should be an iron sleeve $8^{\prime \prime}$ long (a piece of iron pipe of $\frac{6}{8} "$ inside diameter). The iron bolts with iron sleeves serve as the cores for what is called the field coil. Wrap the pipe with insulating tape, and then with three layers of insulated copper wire, No. 22, Brown and Sharp gauge, insulating with tape between each layer.

The rotating part, which is called the armature $(A)$, is made of a wooden wheel about $6^{\prime \prime}$ in diameter. Make a coil of soft iron wire (stovepipe wire) in the form of a circle about seven inches in diameter and as thick as a half inch rod. Wrap the coil with insulating tape. Mark it off into four equal segments and wrap each with a single layer of No. 22 insulated eopper wire. The wire may be kept unbroken between the segments as in the diagram. Fasten the wrapped coil to the wooden wheel by nailing and wedging.

Cut a three inch disk of sheet copper into quarters and fasten them $(C, C, C, C)$ to the armature with small nails. The wood is sufficient insulation between the segments. Connect the ends of the armature coils of wire, making clean connections $(A, C)$, to the segments of copper. The copper segments form what is known as the commutator.

Support the wheel upon a nail of suitable length. Provide a high 
point of support by fitting a spool into the hole of the wooden disk. Close the upper opening of the spool with a piece of dented tin (see diagram of cross section).

Support the brushes $(B, B)$ which conduct the electric current to the commutator $(C)$ on two spools $(S p)$ held in place by long nails.

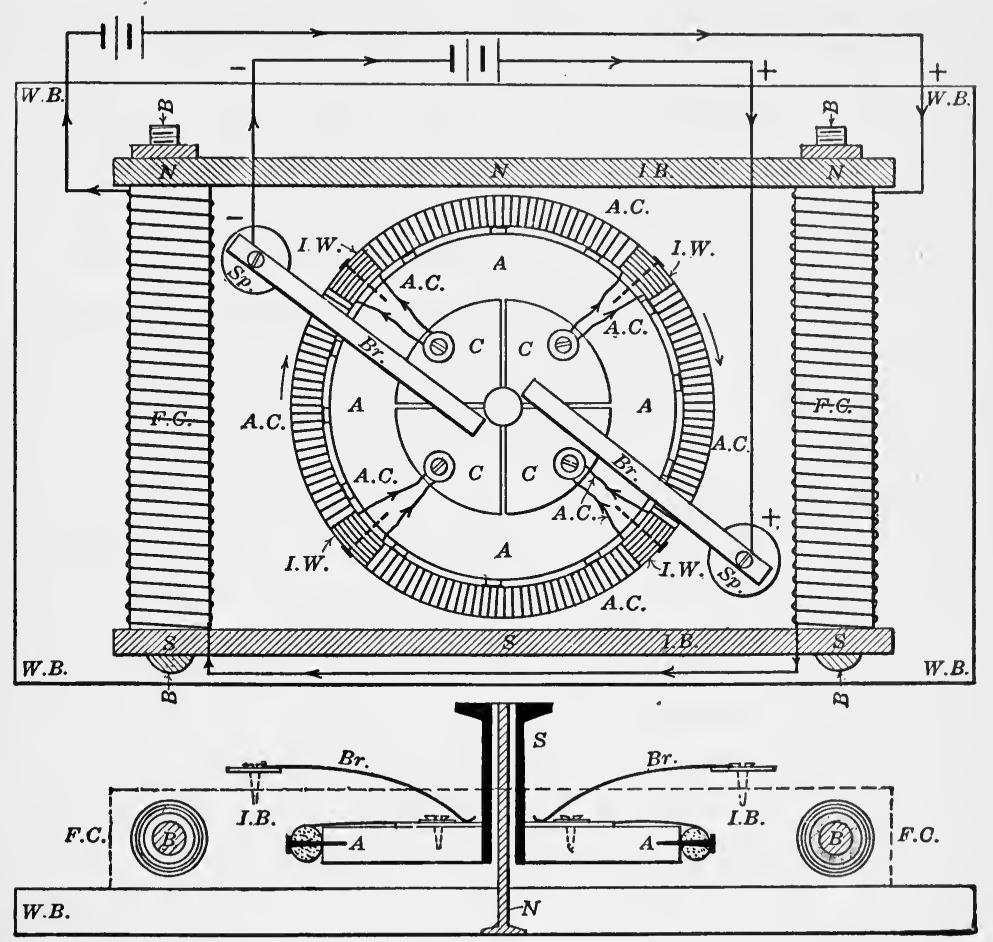

Fig. 102. - A plan for the construction of an electric motor.

See that the brushes, strips of copper sheeting, make good contact with the wires from the battery.

When you have completed the motor, set it up as shown in the diagram with two batteries (page 333) of two cells each. The current from a single battery of several cells may be passed first through the armature, and then through the field coils which form what is ealled the electromagnet. 
The direction of the armature may be reversed by reversing the direction of the electric current either in the field coils or in the armature.

If the armature were connected as a motor is, by a belt or gear to some machine, it would be harnessed to do work or to change electric energy into mechanical energy. This motor is not strong enough to operate any except the lightest machine.

The electromagnet. - The field coils form what is called the electromagnet. The electromagnet differs from the permanent magnet in this respect: its magnetic power is due to the electric current passing along the wire wound around the soft iron cores. The permanent magnet is made of a certain kind of steel which retains indefinitely its magnetic force. The electromagnet is a temporary magnet, for the soft iron loses its magnetism as soon as the electric circuit is broken. It may be pointed out that there are two complete circuits in the electromagnet: one the electric circuit by way of the wire wound around the cores, and the other the magnetic circuit produced in the soft iron cores and the bars connecting them.

The electromagnet is useful beeause it is a temporary magnet. It is used in such familiar appliances as the electric bell, the electric clock, in powerful magnets employed in metal works for lifting large loads of metal, in the Morse telegraph sounder, the automatic telephone, the galvanometer, and in generators and motors. (Examine the magnets of an electric bell or buzzer, and compare them with the magnets making up the field coils of the motor.)

The motor used as a generator. - The motor when supplied with electrical energy may perform mechanical work; or it may be reversed in function and when supplied with mechanical energy produce electrical energy. This is the case with the motors of the Milwaukee electric locomotive (page 319). If the armature of the motor you have built were made to revolve at a high rate of speed, it would generate an electric current just as a generator does, that is, the motor would become a generator. 
143. The principle of the generator. - We may illustrate the principle of the generator very simply, as we did in the case of the motor. When the end of a permanent magnet is moved across a wire as in Fig. 103, there is generated in the wire an electric current. The current produced by a magnet moving across a single wire is necessarily very weak; but it shows exactly what is done in the actual generator.

If a very long, fine, insulated wire is wound into a coil like that in Fig. 104, and made to whirl rapidly between the ends of a $U$ magnet so that the wire is near both ends simultaneously, there will be produced an electric current of considerable pressure. The current may be taken off the collectors $(C, C)$, which in the generator, as in the

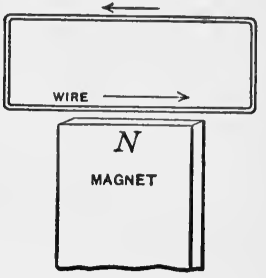

Fig. 103. - A magnet moving across the single wire toward the observer induces in the wire an electric current. This is the simplest form of the electric generator. motor, form the commutator, by means of brushes $(B, B)$, and measured by a galvanometer connected in the circuit or made to produce a spark. The coil of wire represents the armature, and the magnet represents the field magnet.

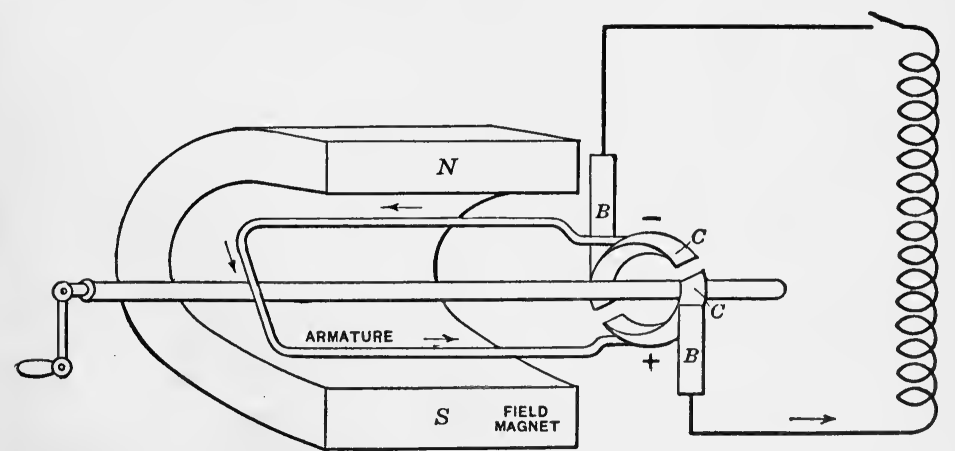

Fig. 104. - A simple generator of electric current; a single wire loop (armature) turning in a magnetic field $(\mathrm{N}, \mathrm{S})$, direction of the electric current indicated by arrows, split ring commutator $(\mathrm{C}, \mathrm{C})$ and brushes $(\mathrm{B}, \mathrm{B})$. 
Another step will make clearer the application of the principle in the actual generator. The armature is usually made of a core of sheet iron disks insulated from each other by thin paper or other substance and placed together to form a drum, and this drum wound with several turns of continuous insulated wire, the ends of which are connected with the collectors, as in Fig. 104. The field magnet is made of a certain kind of steel when it is to be a permanent magnet, or of soft iron wound with copper wire when it is to be an electromagnet. When the armature revolves rapidly in the magnetic field, electric pressure or electromotive force is generated. Although this may appear to be more complicated, it is identical in principle with the simple form first presented.

The magneto. - On automobiles or in certain telephone systems there is a small generator called the magneto. This comprises an armature with a soft iron core wound with many turns of fine wire, and a permanent $U$ magnet, between the ends of which the armature is made to revolve.

Summary. - It is necessary to keep clearly in mind that when a conducting wire is moved sidewise within a magnetic field (the field, that is, of a magnet), there is generated in the wire an electric current. This is the principle of the electric generator. When a conducting wire carrying an electric current comes within a magnetic field, it is made to move in a direction which is the reverse of that which would generate an electric current in it. This reverse motion may be continued and be made to do work as a motor (pages 321-2). A motor is made with a large number of turns of wire and with a powerful magnet so that a great twist or torque is given to the armature. By making the armature with a sufficient number of different coils of wire, the twist or torque is made continuous.

The motor and generator connected. - The electric motor may be operated by energy derived from a battery of dry or other cells. It is, however, usually operated by electric energy derived 
from a generator some distance from the motor. In either case the connections are as indicated in Fig. 105. The electromotive force produced by the generator is transmitted by a conducting wire to the motor. The pressure of the current is shown by the galvanometer $G$ placed in the circuit. The electric current is made to operate the motor and then it returns to the generator by way of the ground wire. This is exactly what one finds in the operation of motors of street cars

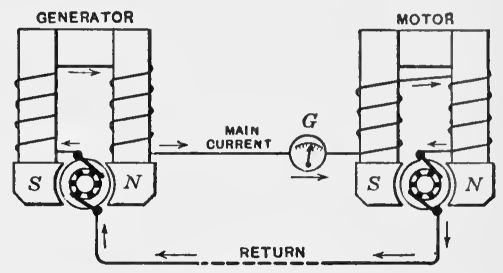

Fig. 105. - Diagram to show generator and motor connected; the electric current is measured by galvanometer (G). or electric locomotives. The current is transmitted by a trolley wire or third rail to the motor and thence to the generator by way of the rails and the ground (Fig. 106). How is the circuit completed in the case of the telegraph and telephone?

144. Direct and alternating current generators. - Electric generators are of many different kinds, but they may be grouped conveniently into two classes according to the kind of current they produce. These are the direct or continuous current (des-

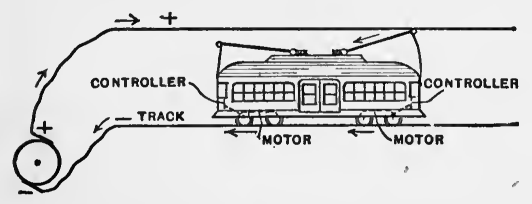

Fig. 106. - Diagram of course of electric current from generator along trolley or third rail to motor of a street car and from this through the rails to the power station. ignated as D. C.) and the alternating current generators (designated as A. C.). The direct current generator has a commutator that takes off the current generated in the revolving coils in only one direction, as illustrated in Fig. 107. The commutator is divided (Fig. 104) into two parts or segments which are insulated from each other on the actual generator, and not separated as shown in the diagram. The brushes are placed so exactly in contact with the commutator that each passes from one segment to the other at the moment the cur- 
rent in the coils of the armature is reversed in direction. You recall that as one part of the coil of wire moves across one pole of the magnet, the current is pushed in one direction, and as it moves across the opposite pole the current is pushed in the opposite direction. The commutator is the important item in this kind of generator.

The alternating current generator has no commutator. The ends of the wires of the outside circuit are directly connected to rings on the shaft of the armature. With this generator the

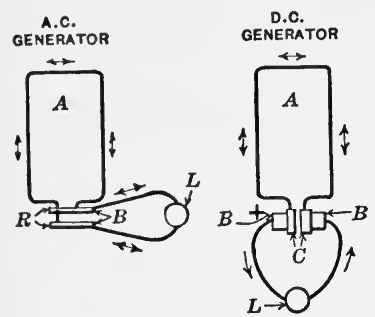

FIG. 107. - Diagrams illustrating the production of the direct and the alternating currents. $A$, the armature; $B$, brushes; $C$, commutator; $R$, rings; arrows showing the direction of the generated current. electric current in the conducting wires follows first one wire and then the other, or it has first one direction and then the other. This is different from the D. C. generator, where the current leaves by one wire and returns by the other throughout. In the A. C. generator the current is taken off just as it is generated in the coil of the armature in moving across the opposite.poles of the magnet (Fig. 107). The alternating current is generally used for ordinary lighting and heating. It may be used in specially constructed motors, but is not generally in the motors of street cars or electric locomotives. These make use of the direct current. In this case if the alternating current is generated, it must be rectified, or converted into the direct current (page 320). The alternating current cannot be used for charging secondary cells (page 333); the current for this purpose must be in one direction only.

145. The transformer. - The alternating current is best adapted for transmission over long distance, because it is easily changed from a moderate electrical pressure at which it is generated, say 2500 volts, to a very high potential, as 150,000 volts, at which it can be transmitted; and then back to a low 
pressure at 2500 or even 110 volts, at which it can be used with safety. This change in pressure is made by means of the transformer.

The principle of the transformer may be suggested, though details will have to be sought in treatises on electricity. The principle is simple and may be illustrated in the diagram of Fig. 108. A core of soft iron is made to form a circle so as give a complete circuit for the flow of the magnetic force. A small insulated wire has forty turns around the core, forming the first or primary coil. A large insulated wire has two turns around the core, and is known as the secondary coil. The feed wires coming from the generator are connected with the ends of the small wire or primary coil. The service wires of a house, for example, are connected with the ends of the large wire or secondary coil. An alternating current of about 2300 volts

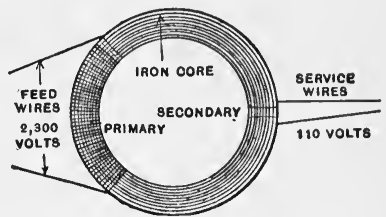

FIg. 108. - The principle of the transformer; forty coils of the primary or supply wire to two coils of the secondary or service wire upon a common core transforms the high voltage to about one-twentieth of the current.

passes along the wire of the primary coil, and there is produced in the iron core a magnetic current which moves first in one direction and then in the other (page 325). As the magnetic current passes along the iron core through the secondary coil, there is induced in this an alternating electric current of about $\frac{1}{20}$ of the voltage received, or 110 volts. The pressure or voltage is thus transformed or stepped down to that which may be used safely in the house for lighting and heating. The loss of energy in the transformer is very small.

The principle may be expressed as follows: "The volts of an alternating current received in the primary coil are to the volts delivered by the secondary as the number of turns of wire of the primary is to the number of turns of wire of the secondary." If there were no loss in the transformer, the electrical energy delivered would be equal to that received. 
From this it should be evident that the voltage of an alternating current may be stepped up as well as stepped down. Explain how this can be done.

\section{Gasoline as a Motive Power}

146. The gasoline engine. - The remarkable increase in travel and transportation on streets and highways in recent years is due not only to the application of electricity, but also to the development of the gasoline motor for use in vehicles of all sorts.

The gasoline motor is often described as an internal combustion engine. This is because the fuel, a mixture of gasoline vapor and air, is supplied directly to the cylinder where it is ignited and by its expansion in combustion furnishes the working power. The steam engine, for instance, receives through pipes steam from a boiler under which a fuel has been burned. Both types of engine are heat engines, for they convert heat energy into working or mechanical energy. The chief difference between them is the place where the fuel is burned.

Its operation. - A gasoline motor may consist of a single cylinder, or of two, four, six, eight, or twelve cylinders. But the operation is the same for twelve as for one cylinder. If possible, examine a gas engine and find out the important parts and the work each does in operation. The main points of the common motor may be illustrated in the diagrams of Fig. 109. In A, the first stroke of the piston, the intake valve is open, the piston is moving forward and drawing into the cylinder the explosive mixture of gas and air. In $\mathrm{B}$, the second or return stroke, the piston is moving backward and compressing the mixture. An electric spark, which is timed, ignites the mixture, which, by exploding and expanding, drives forward the piston. This, C, is the working stroke of the engine. The fourth step, $\mathrm{D}$, shows the piston moving backward, expelling the burned gas through the exhaust valve, which is now opened. The working energy, as illustrated in the diagrams, is applied only to alter- 
nate forward strokes of the piston. The other three strokes must be made with energy transmitted from the working stroke through the flywheel, or from additional cylinders. Because of the fact that one stroke only of four is the working stroke, this type is called the four-cycle motor. The crank shaft makes two complete cycles or revolutions with energy applied to one half cycle. In a motor consisting of four cylinders the working energy is delivered upon the crank shaft for each half revolution. With six, eight, or twelve cylinders the impulses are made more and more nearly continuous.

The two-cycle motor is much lighter per horse power, without poppet valves, but less economic of fuel than the four-cycle. Its principle of operation is identical. The chief difference is that every
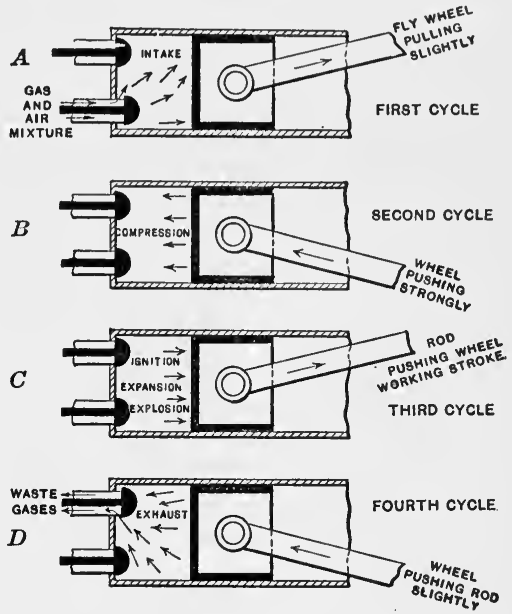

Fig. 109. - The operation of the oneworking-stroke-in-four or four cycle gas engine.

alternate stroke is a working stroke, or energy is delivered at each revolution of the crank shaft.

147. Dependence upon other mechanisms. - The development of any machine seems generally to be dependent upon the perfecting of something else. The gasoline motor depends for its usefulness upon several other mechanisms. One of these is the carburetor, in which gasoline or other hydrocarbon is vaporized and mixed with the right proportion of air. Another is the means by which the gas mixture in the cylinder is ignited. This may be effected in the stationary engine by a torch; but it is to-day generally effected by an electric spark, the electric current for which is derived from a battery of dry or other cells, 
or from a magneto. As the so-called dry cell is used for many other purposes than ignition, it may be studied first of all in some detail.

The dry cell. - The construction of a dry cell is illustrated in Fig. 110. You should break apart a worn-out cell and examine its parts. The material surrounding the carbon rod is

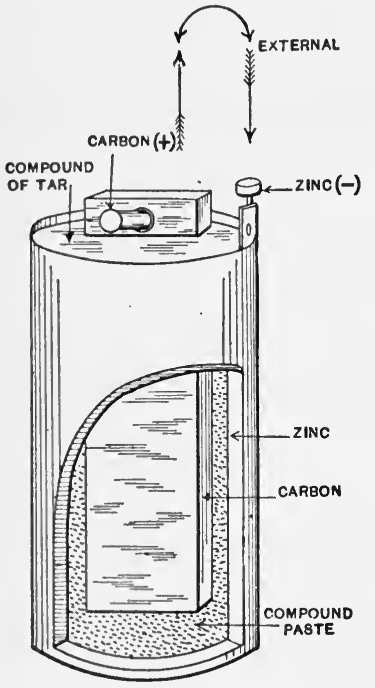

Fig. 110. - "Dry" cell. perhaps the only difficulty to be encountered. This varies in composition in different makes of cells. A very common formula calls for manganese dioxid, plaster of Paris, flour, and sawdust, mixed with a solution of sal ammoniac to form a paste. The zinc container may be protected inside with a few layers of absorbent paper, like blotting paper. When the terminals of carbon and zinc are connected in circuit, there is started a chemical process by the zinc and sal ammoniac, which in uniting generate an electric current. The electricity passes from the carbon along the wire circuit and back through the zinc. Breaking the circuit stops the chemical action and the generation of electricity. The sal ammoniac and zinc combine and form a new compound. When all the available zinc or sal ammoniac has been thus used up, chemical action ceases, or we say the cell is worn out. Sometimes the paste may be moistened by introducing a little water through a hole bored into the top covering, and the cell made active for a little longer period. This one fact suggests the inaccuracy of calling it a dry cell, for moisture is indispensable to its activity.

A battery. - When two or more cells of any sort are connected, they make up a battery. The usual way of connecting cells is 
in series. This gives a total electric pressure equal to the sum of the electric pressures of the separate cells. Such an arrangement is shown in Fig. $111(A)$. In $(B)$ the same grouping of cells is shown by symbols that are commonly used, the zinc being indicated by a heavy line and the carbon by a light line.

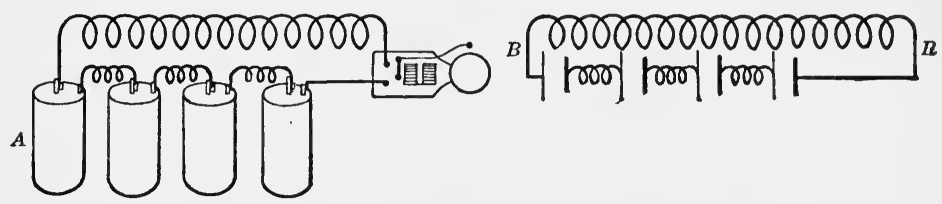

Fig. 111. $-A$, a battery of cells arranged in series. $B$, the symbols commonly used to show such connections.

The secondary cell, or storage cell as it is generally called, is utilized in all sorts of motor vehicles and in many other ways. For some vehicles it provides the motive power, for others it is used for ignition, lighting, starting, and so on. Through recent improvements its use has been greatly extended in many new fields. Trucks and delivery wagons, electric motor cars, street cars, light and power plants for the purpose of maintaining uninterrupted service, telephone and telegraph, private lighting plants for homes, and a thousand other appliances make use of secondary cells. The essential facts in its construction and operation should be studied experimentally.

Exercise. - For class study and demonstration a secondary cell of the lead type is readily made. Fasten two pieces of lead plate to a piece of dry wood about $2^{\prime \prime} \times \frac{3^{\prime \prime}}{4} \times 8^{\prime \prime}$, and connect the leads with copper wires. Place the lead plates in a glass jar of about two quart capacity and fill jar nearly full of a 15 per cent solution of sulfuric acid. Connect wires with three or more dry batteries or a direct current generator of six to ten volts capacity, as shown in diagram Fig. 112. Connect up a small electric bell as in the diagram with a switch or merely broken wires that may be closed when needed.

When the apparatus is set up, close switch No. 1, to charge the cell. Observe the changes that occur. The electric current passing along the wire and plate marked $(+)$ combines with the sulfuric acid solution and the lead to produce a visible change on the surface of the 
lead plate. This substance is brownish in color. It is a compound of oxygen and lead, and is called lead peroxid. By way of the plate, marked (-), hydrogen is thrown off. (See electrolysis of water, page 115.)

After ten to fifteen minutes open switch No. 1, to cut out the dry cell, and close switch No. 2 to connect the electric bell with the storage

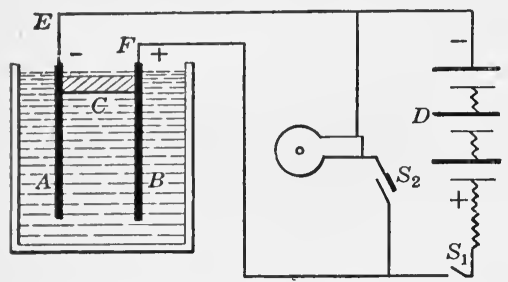

Fig. 112. - Experimental storage cell. Fasten two pieces of sheet lead, $A$ and $B$, to a piece of wood, $C$, and connect each with copper wires, $E$ and $F$, completing the circuit with dry cells, $D$, and a switch, $S_{1}$. Make a second circuit with an electric bell and a switch, $S_{2}$, as shown in diagram. To charge the cell close $S_{1}$. To discharge the secondary cell, open $S_{1}$ and close $S_{2}$. cell. In the discharge the substances are changing and generating electric current. The lead peroxid is converted into metallic lead, which is now soft, spongy, and more easily changed at the next charging. As the cell is small, it has a very limited capacity; but with repeated charging and discharging it increases in capacity. That is, the chemical substances are more easily and abundantly developed in charging, and they in turn produce a larger amount of electric energy in active circuit.

Principle of the secondary cell. - Electricity is not stored in the secondary cell, for just as much escapes in charging through the negative plate as is transmitted through the positive plate. The electric current in interaction with the lead and sulfuric acid solution forms chemical substances which, when the battery is connected in circuit, produce electrical energy.

\section{SUMMARY}

Increase in electrification of steam railways is due to greater use of water power, improvement of electrical generators, transmission lines, and motors.

When a wire is moved through a magnetic field, a current is generated. This illustrates the principle of an electric generator. A generator is a machine for transforming mechanical energy into electrical energy.

When a current is passed through a wire in a magnetic field, the wire moves in a definite direction. This illustrates the principle 
of the motor. A motor transforms electrical energy into mechanical energy.

The generator consists of coils of wire and a shaft in the field of an electromagnet.

A magneto comprises coils of wire which move through the field of a permanent magnet.

An alternating current (A. C.) generator delivers into the external circuit a frequently reversing current, just as it is produced in the coils of the armature.

A direct current (D. C.) generator, by means of the commutator, delivers into the external circuit a current in one direction only.

In general, a motor may be used as a generator, and a generator as a motor.

The electromagnet consists of a soft iron çore wound with insulated wire.

The transformer has two coils, a primary and a secondary, upon a common core. It is used to step up or step down the voltage of an alternating current.

To transmit a small current at high voltage is more economical than to transmit a large current at low voltage.

The successful use of gasoline motors in vehicles depends upon electric ignition.

Gasoline engines are of two types, the one-in-four working stroke, or four-cycle, and the one-in-two working stroke, or two-cycle.

Electricity for ignition purposes is derived from a magneto or from a battery.

A battery consists of several cells connected with wires.

The two types of cells most used for ignition are the dry cell, and the secondary (storage) cell.

The current for the dry cell (a primary cell) is due to the chemical action of the zinc and sal ammoniac.

The electric current of a secondary cell is due to chemical changes which are reversed when a current passes in the opposite direction.

\section{REVIEW QUESTIONS}

1. What progress has been made in recent years in electrifying steam railways? 2. Why has the Milwaukee railroad electrified its lines in the mountains of Montana? 3. How does one electric locomotive help another in the mountains? 4. How is the train going down grade made to help in furnishing energy for trains going up grade? 5. What natural resources are used for developing the 
power? 6. Compare advantages of steam and electricity as motive power of transportation.

7. Describe a simple electric generator and name the parts. 8. What is the difference in construction between the D.C. and A. C. generator? What parts are alike? 9. What is the purpose of the commutator? Of the brushes? 10. Mention an important difference between gasoline engines and steam engines. A fundamental likeness. 11. Upon what has the development of the automobile depended? 12. What use is made of the electric current by the gasoline engine?

13. Describe how a four-eycle gas engine works; a two-cycle. 14. Describe the dry cell. Which is the positive binding post? What is a battery? 15. Describe a lead storage cell. Does it store chemical energy or electricity? 


\section{CHAPTER VII}

\section{TRANSPORTATION ON WATER}

148. Primitive boats. - Men living near the water in olden times made boats of many kinds and of different materials (Fig. 113). Although they may not have reasoned it out, they

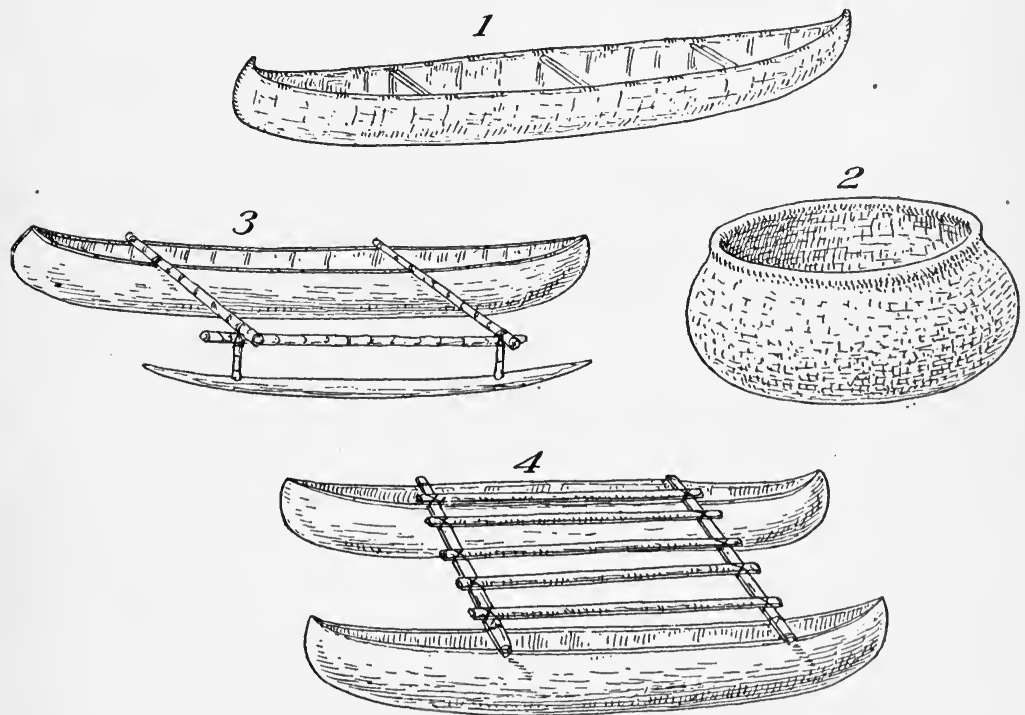

Fig. 113. - Types of boats: 1. birchbark canoe; 2. Turkish goofah; 3. canoe with outrigger; 4 . catamaran.

knew that the water displaced by a log caused it to float. Hence to decrease its weight they burned or dug out the insides of suitable logs, making the so-called dugout boats. In regions 
where large timber was scarce or unknown, rafts were made of small logs, such as bamboo stalks, tied together; or boats were made of tough bark or skins fastened over a frame (Fig. 114). Such were the birchbark canoes of American Indians, the skin

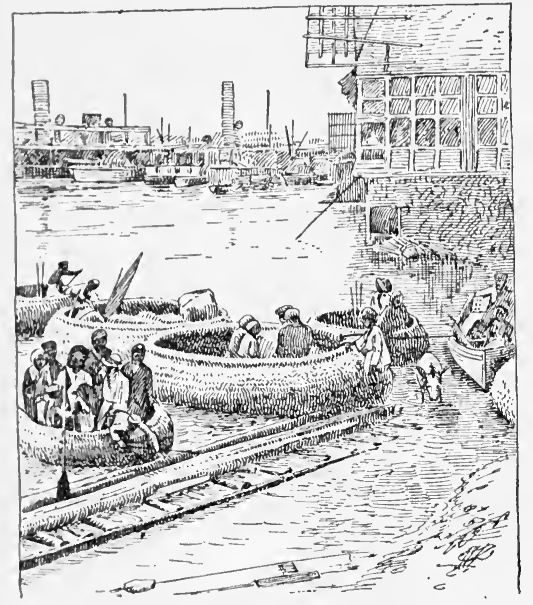

FIg. 114. - Primitive circular boats and modern steamships on the Tigris River. - (From London Illustrated News.) canoes or kyacks of the Eskimos, and the round skin boats used on the Euphrates River.

When in later ages men began to build boats of hewn planks, they opened the way for new achievements not only in the construction, but also in the propelling of boats. With the discovery of the sail as a means of making use of wind as a motive power, a new era of water travel and transportation was begun.

Youth to-day frequently beginsits experience in water navigation with craft similar to those used by men in primitive times. The rude raft or float, the flat-bottomed boat, the rowboat, and the canoe, and eventually the motor boat follow in the experience of youth in somewhat the same order as they did in the course of human history. The motor boat and the sailboat usually come late in the series.

\section{BUOYANCY}

149. Why a boat floats. - In all kinds of floating and sailing craft there is one problem to which attention is required. We are generally familiar with the various kinds of motive power employed, but we may not always understand why a boat or any other object floats. A boy weighing seventy-five pounds 
floats as well as, but no better than, a man weighing one hundred seventy-five pounds. If the boy carried a lump of lead weighing twelve and one half pounds, he would weigh one half as much as the man. But would he float? What more than weight must be considered in finding the answer to the question "Why does a boat float?"

It is a common saying that some materials float and others sink. Certain kinds of wood sink, as do lead, iron, and steel. But steel and lead are utilized in building ships, while modern dreadnaughts are armored with steel plates that are many times heavier than the same bulk of wood. What are the conditions, then, which determine whether or not a body will float in water? We shall find these conditions readily by the aid of experiment.

Exercise: Buoyancy. - Notch a can, as shown in Fig. 115, to allow water to overflow. Fill the can with water until it overflows. Weigh a suitable block of wood or any kind of material small enough to float in the can. Carefully putting it into the can, let it float naturally, catching in a large pan the water that overflows. Weigh this water and then calculate the relation of the weight of the water displaced by the floating body to the weight of the body. Does the buoyancy depend upon the whole body or only on that part submerged? When trying to float, does a man keep head and shoulders out of water, or lie so as to keep the back of his head under water? Have you tried to lift a large stone when it is in water and again when it is in the air? What is the difference in the effort required? Why? The important facts will be illustrated in the following experiment.

Weigh a small stone or other heavy object on a spring balance. The object should weigh 250 grams

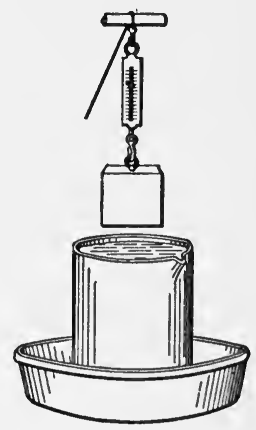

FI G. $115 .-\mathrm{Ar}$ rangement of apparatus to show buoyant force of water. at least. Lower the stone, while supported by the spring balance, into the water. Take the reading of the balance. Has the actual weight of the object, or, strictly speaking, the pull of the earth upon it, changed? Apparently this is not the case. Another force is now supporting part of the weight which, when in the air, was supported 
by the spring balance. The force which the water exerts in sustaining the weight, whether the object sinks or floats, is called the buoyant force of water.

Put the stone into the overflow can just as you did the block in the first experiment. Catch and weigh the overflowing water. What relation do you find between the weight of the water displaced and the buoyant force of the water as shown in the last experiment?

The principle of buoyancy. - The object has clearly lost in the water the weight of its own volume of water. A general statement of the fact is given: "The buoyant force of water (and you may find it holds true for any liquid) is equal to the weight of the water displaced." For a body that floats evidently displaces water equal in weight to itself, - that is, the entire weight of a floating body is apparently lost in water.

Archimedes' discovery. - There is a tradition that Archimedes, living in Syracuse, Sicily, was requested by King Hiero to ascertain whether or not silver had been substituted by a jeweler for gold in his crown. As the king would not permit Archimedes to drill into the gold crown, and as the crown was too irregular in shape to measure for volume, another method had to be devised. The scientist gave much thought to the problem. One day when taking a bath he perceived that his body was buoyed up by the water; in fact it seemed to lose weight when submerged in water. The significance of this discovery in relation to his great problem was recognized at once. He saw clearly how he could determine the weight of the gold in the crown and whether or not any substitution of a lighter metal had been made. He was so delighted with his discovery that he rushed home from the bath, shouting "Eureka ! Eureka!" a Greek word meaning "I have found it."

He weighed the crown in air, then in water. The difference between the two weights was equal to the weight of the water displaced. From the weight of the displaced water he computed its volume, which he knew to be equal to the volume of the crown. With these data he determined the weight per 
unit volume, or as we say, weight per cubic inch. As gold was the densest metal the ancient Greeks knew, it was easy to detect the presence of any lighter metal in the crown. The king, satisfied with the proof, conferred great honor upon Archimedes for his discovery. Archimedes' method may be employed to find out the weight of a unit volume, or the density, of a piece of iron, lead, or rock. For large pieces the spring balance is fairly accurate; but the beam balance is better for weighing in air and in water.

Exercise: Buoyancy and pressure. - It is now evident that the water exerts a buoyant force. Why is this the case? Punch a number of holes at different levels in a tin can. Then press the can into the water. Does it appear that the water presses in all directions? Is the pressure on bottom and sides equal, so far as you can judge from the force of the flow through the holes? When the can is full of water, lift it and observe how the pressure is exerted. Is it in all directions? If there is a difference in pressure, where is it? What seems to cause this difference? Is there more water above or below the places of greater pressure? Where does a swimmer when diving experience the greater pressure on the ear drums, near the surface or at a depth below?

Put the can into the water at any definite depth. When does the water cease to flow through the holes? When the water is completely at rest, what is indicated in regard to pressure? Is the pressure in the can equal to that outside it? Suppose the pressures were unequal, would the water remain at rest or continue in motion? What is the relation of the levels of the water inside and outside the ean, so that the pressure at any one of the holes shall be equal in both directions? What difference would you expect to find if you had pressed the can into a large body of water as a river or lake? Does pressure depend upon the bulk of water above the hole, or upon the distance of the hole below the water's surface?

Pressure and depth. - As shown in the study on water pressure under the topic of water supply (page 227) pressure varies with the depth (Fig. 116). This indicates that it is the weight of a column of water above a given place, which determines the pressure. It is well known that in deep parts of the ocean, fish and plants are peculiarly adapted to withstand enormous pressure and 
when brought to the surface explode. Experiments have been made in deep places of letting down with a sinker an empty, flat bottle tightly corked. The bottle is crushed by the enormous pressure.

Application to boat building. - Builders of ships have a practical knowledge of the facts which the experiments have illustrated. They know the density of materials of which ships are made, and can compute beforehand the weight of a vessel and its equipment; how much water it will displace; and to what depth it will sink in displacing its own weight. They also determine what load it will carry and to what depth it will sink when loaded.

Exercise. - Suppose you are making a plan for a boat of the common flat-bottom sort, with the dimensions 14 feet long, 3 feet wide, and 16 inches deep. If the materials of which the boat is made weigh 200 pounds, how deep will the boat sink? We know that one cubic foot of water weighing about 62.5 pounds exerts a pressure at a depth of one foot of 62.5 pounds per square foot. The area of the boat bottom is 14 feet $\times 3$ feet or 42 square feet. The total pressure necessary to cause the boat to sink one foot is $62.5 \times 42$ or 2625 pounds. But the weight of the boat is 200 pounds. Therefore it will sink into the water only $\frac{200}{2625}$ or .076 of a foot. Is this more or less than one inch? How much? What must be the weight of a load to cause the boat to sink to the depth of six inches? Ten inches?

150. Density and relative density. - The density of water is approximately 62.5 pounds per cubic foot. The important items in this are the weight and the unit volume. The density of any other substance is determined in the same manner, that is, its weight per unit volume. This may be expressed in so. many pounds per cubic foot, or grams per cubic centimeter. The standard of density in all scientific measurements is pure water with a temperature of $4^{\circ} \mathrm{C}$, , or $39^{\circ} \mathrm{F}$. Its density is 1.00 . The density of other substances is stated in relation to the density of water. Pine wood, for instance, has a relative density of $\mathbf{0 . 5}$, that is, it is just one half as dense as water. 
Exercise. - What is the weight of a pine timber 12 inches in each dimension and 10 feet long? How deep would it sink in water when resting on one side? Suppose it were kept standing in a vertical position in water (Fig. 116), how deep would it sink before the pressure from below would be sufficient to support it? What fractional part of the timber is out of water?

Consult the table of relative densities and that of weight per cubic foot of substances in order to answer these questions. Which of the woods, pine, spruce, oak, cedar, and lignum vitæ, would you select for lightness in making a boat? Why is cork well adapted for use in life preservers? As a float for fishing nets and lines? Would a piece of iron float on mercury? on molten glass? Explain your answers. If you had no other information

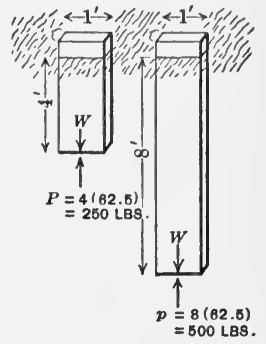

Fig. 116. - Pressure varies with the depth (diagram). than is presented in this table, would you judge that materials for building boats must be limited to woods with densities less than that of water?

\section{Weight of Common Substances per Cubic Foot}

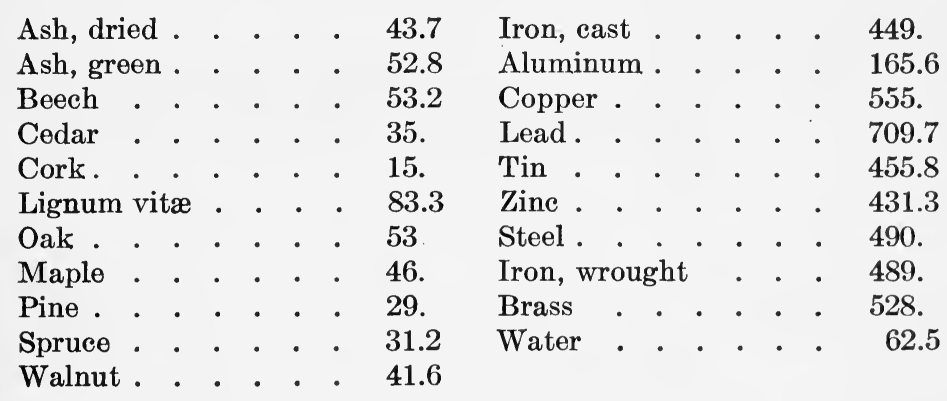

Table of Relative Densities

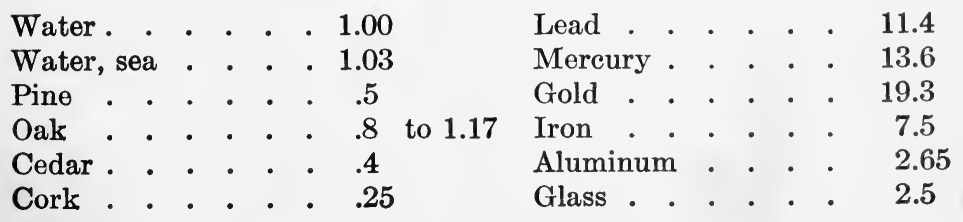




\section{Stability}

151. Stability. - Besides floating in water a boat must have stability of a kind to prevent it from tipping dangerously with ordinary shifting of its load. Disaster frequently results if the cargo shifts too much to one side, or if some one "rocks the boat." The problem for the boat builder is to design and construct a boat which will not tip over in usual situations.

You have seen and probably experimented with tumbler dolls, or possibly the bottle imp. The bottle imp is a round bottom

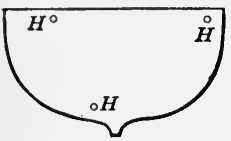

bottle weighted with some lead shot held in place by paraffin. No matter how the imp is placed in water, it will rise of its own accord to a vertical position. So it is with the tumbler doll and other similar devices, such as oil cans weighted to prevent tipping

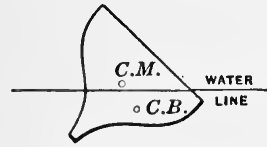

Fig. 117.- I. Form of board with position of holes for its suspension to find center of weight. II. Relation of center of weight (C.M.) and center of buoyancy (C.B.). and consequent waste of oil. It is exactly the same principle which is followed in securing stability or equilibrium for boats.

The following experiments will illustrate the essential facts :

Exercise: To find center of weight. - Saw out a piece of board at least one foot across and ton inches deep so as to represent the cross section of a boat. Bore three or more holes not far from the edge, somewhat as shown in Fig. 117. Hang the board on a nail upon which it may swing freely and come to rest without interference. Let a plumb line hang from the same nail. Draw a line upon the board to represent the position of the plumb line. Now hang the board by another hole and designate the plumb line in the same manner. Try then the third hole. Where does the plumb line pass with reference to the other lines? Is the weight of the board apparently centered at the point of intersection of the three lines? How can you prove that it is?

Attach a weight of several ounces to the part representing the keel of the boat. Proceed as formerly to determine the point at which the weight is centered. The pull of the earth upon an object is not 
actually centered at any point, but is represented as the resultant of the many. pulls or the weight of the object acting at a point called the center of gravity. Draw or imagine a water line crossing the board which is taken as the shell or hollow hull of a vessel; you will find that the center of weight is not in the solid hull, but in the inclosed space. This is not puzzling, if you consider the boat with its air space as a solid substance. Find the center of weight or gravity of a wooden or iron ring, as a barrel hoop. Where does the center lie, in the solid material or not?

Exercise: To find center of buoyancy. - Cut out of heavy cardboard a form to represent a cross section of a yacht with a deep keel. Determine its center of weight. Tip the form to an angle which would be dangerous for a boat, and draw a line to indicate where the water line is supposed to come. See diagram II, Fig. 117. Cut off the part above this line. Now find the center of buoyancy of the lower part, just as you found the center of weight. When you have done this, reattach the cut-off part by means of gummed labels; and find out whether or not the boat would tip over at the position which you find it has when at the center of buoyancy. What is the relative position of the center of weight to the center of buoyancy? Is it above, below, or by the side of, the latter? Suppose the keel weighted with lead as a ship is with ballast. Where now is the center of gravity? What is its position relative to the center of buoyancy?

152. Relation of centers of weight and buoyancy. - The experiments have brought to your attention the important facts of weight and buoyancy. It is necessary now to inquire what must be the relative positions of the two centers of weight and buoyancy to give the boat the greatest degree of stability. Study of the diagrams of Fig. 118 will help you to answer the question.

Exercise: Stability of boats. - Diagram I represents the center of gravity or weight as vertically above the center of buoyancy. Will the boat tip readily to either side? Why? In diagram II, the center of buoyancy is located to the right of that of the weight, and it is also represented as at the center of the submerged part of the boat. What will be the tendency of the boat as determined by the two forces acting thus upon it? Remember that weight is the downward pull of the earth upon a body; that buoyancy is the upward, vertical push of the water; and that both of these forces, the pull and the push, may be 
represented as located at certain points, as at their respective centers (page 256).

Diagram III shows the center of weight located to the right of that of buoyancy, and both near the edge of the boat. How will the boat behave? Will it tend to right itself or to tip farther?

Compare diagrams IV and V with I and II. In which type of hull, the flat-bottomed or the round, does the center of buoyancy shift more rapidly when the boat is slightly tipped?

What will happen with the forces located as in diagram VI? Why? Comparing diagrams VII and VIII with I and II and IV and V, which
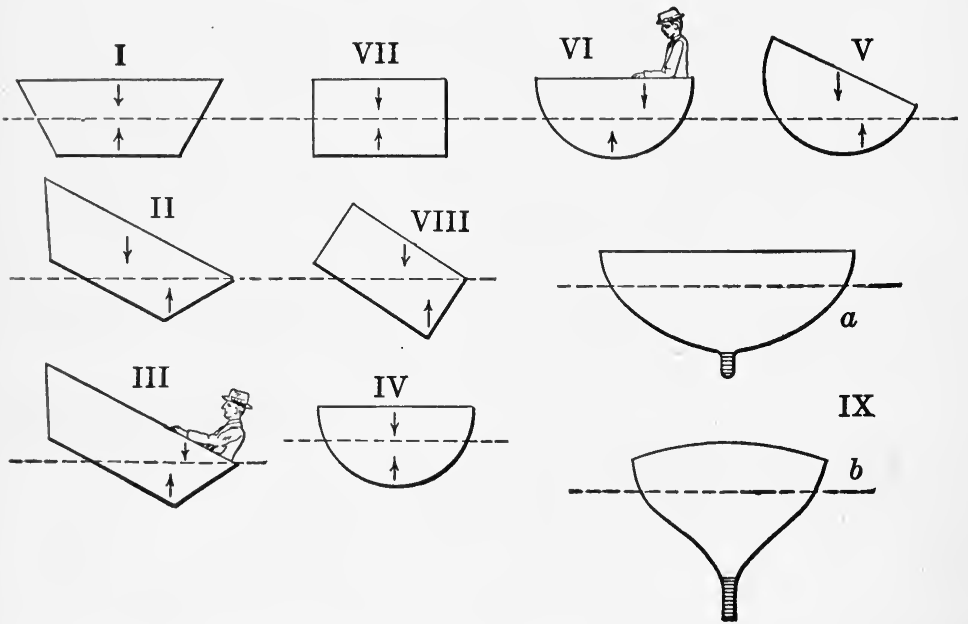

FIG. 118. - Centers of weight and buoyancy. Arrow pointing downward represents center of weight; arrow pointing upward, center of buoyancy.

type of hull would give the greatest stability? Give reasons for your answer. Suppose you have a hull with a small keel, as in diagram IX $(a)$, how would its stability compare with that of the shapes already considered? What will be the effect on stability of a long, deep keel, as in diagram IX $(b)$ ?

153. Means to secure stability. - Builders aim to secure stability of boats by design and construction, but they often make use of ballast and other devices to make boats more stable. Many inventors have devised boats and rafts that have stability 
and buoyancy, together with all necessary strength. In the submarine boat ballast and air tanks are employed. The air tanks are subdivided into such small compartments that injury to one or several does not render the boat unseaworthy. Lifeboats and rafts have been built which have shown remarkable stability in difficult conditions. A life raft, for instance, has been made with many air compartments, and under test has upheld a load of 5000 pounds placed on one side without tipping dangerously. The raft has been cut into two parts and each part has carried safely a full load of passengers.

The problem is especially interesting in connection with the sailboat (Fig. 119). A

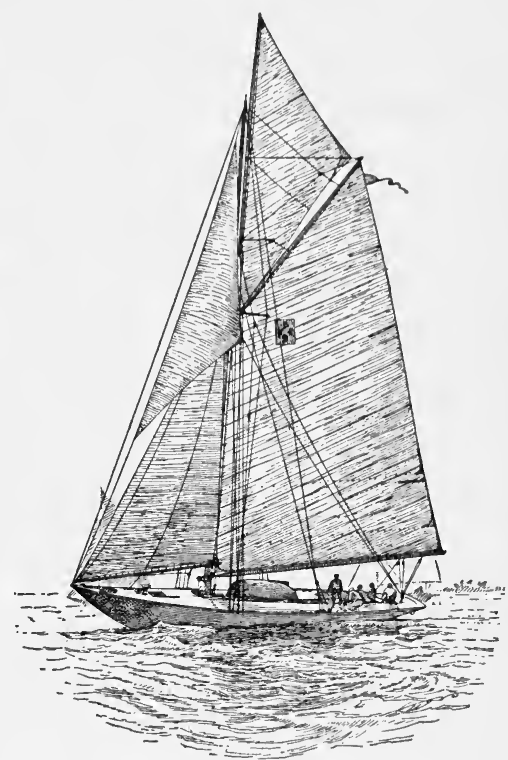

FIG. 119. - The sailboat. sailboat (Fig. 120) must have enough stability to resist the heeling force of the wind upon the sail. Ballast or a keel weighted with iron or lead are among the means employed to
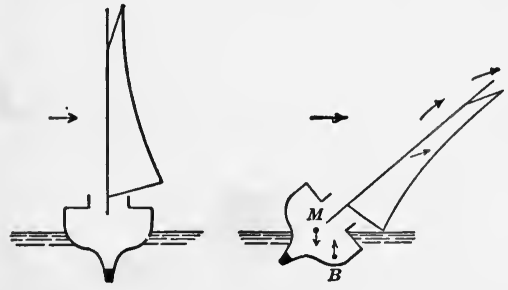

Fig. 120. - Stability of a sailboat. $M=$ the center of mass; $B=$ the center of buoyancy. make such a boat stable. The mast of a sailboat acts like a lever when the wind tips the boat. The longer the force arm of the lever (pages 254-5), the less the force of the wind required to tip the boat. But as the boat heels over, the wind spills over the top of the sail and 
exerts less tipping force. If the boat is constructed according to a good plan and has plenty of weight on its keel, it may careen with safety even when the sail is nearly touching the water. A flat-bottomed boat has great stability when tipped slightly, but becomes increasingly unstable when the tipping passes a certain point (Fig. 118). Boats that have a weighted keel or carry ballast should have inclosed air chambers to prevent sinking if accidentally overturned. The mast is made as light as possible, but it must be strong enough to carry the sail and whatever force may ordinarily be exerted upon it.

154. Stability in other objects. - Stability is not limited merely to boats or bodies intended to float in water. Vehicles of all kinds, such as carts, bicycles, automobiles, cars and locomotives, aëroplanes and balloons, are designed so as to have the highest degree of stability in usual situations. The racing automobile, for example, has its center of weight as low as possible.

Chairs, tables, furniture of all kinds, dishes, vases, tools, and utensils must have the property of stability. If chairs tumbled over when placed in their right position, they would be discarded promptly. Buildings, however tall, must be stable; and even the materials of which buildings are constructed, such as brick, stone, and steel, must have in their own shape maximum stability. Four-footed animals are more stable than bipeds; but bipeds have acquired a remarkable skill in maintaining a certain kind of equilibrium which meets most conditions of their environment. The equilibrium maintained by these is mobile, adaptable to different conditions. It differs greatly from the unstable equilibrium of such objects as the tumbler doll or the bottle imp when placed so as to raise the center of gravity, or of a pyramid when stood upon its apex. It differs moreover from the stable equilibrium of the pyramid when standing upon its base, of the tumbler doll in a vertical position or of a plank lying on its broad surface. Finally it differs from the indifferent or neutral equilibrium of a baseball, 
billiard ball, or other perfect sphere; for the center of gravity of such objects as these does not change its position however much they are rolled.

\section{Rowing A BoAt}

155. Oars and paddles. - The first motive power employed by men in propelling boats was their own strength, aided by the current, poles being used in shallow water, and then paddles or oars. Later animals walking along the banks of a stream or waterway dragged the boat; and eventually the wind was used for voyages far from sight of land.

The oar in action is much like the paddle. A paddle is held near the middle with one hand and near the end of the handle with the other. The oar is held by an oarlock which allows both hands to work one oar or two. It brings into play a fuller use of additional muscles, as those of the legs. How are racing shells arranged to utilize the leg muscles of the oarsmen?

Mechanical advantage of oars. - Oars may be pushed or pulled. Fishermen and rowers of the Venetian gondolas frequently push upon the oars. How did the ancient Greeks, Phœnicians, and Romans propel their boats? The oar or paddle is a lever. What other uses of the lever have been studied? One end of the oar is broad to secure greater resistance in the water. The oarlock is the fulcrum. The distance from the oarlock to the center of the hand is one arm of the lever, and from the fulcrum to the center of the blade the other arm. It was shown on page 258 that the lever, used in the ways there discussed, gives the mechanical advantage of greater force in overcoming resistance. The lever used as an oar gives the advantage of speed because of the greater length of the resistance arm relative to the length of the force arm.

Why are long oars used on racing shells? What is the advantage of short oars?

Action and reaction. - Why does the backward pressure of the blade against the water cause the boat to move forward? 
Let us neglect the boat and consider merely the blade and the water. From common experience it may be inferred that the pressure of the blade upon the water is equal and opposite to the pressure of the water upon the blade. For example, when you jump from a boat, you push the boat in the opposite direction. When you push against a wharf, the wharf pushes equally against you and your boat. If the wharf should yield to your pushing, it would never aid you in getting your boat away from it. You count upon the return push of the wharf. A motor car may run perfectly and yet make no headway over a stretch of sandy road, or ice. The road is not pushing against the wheels. The road must react to the action of the wheels if the car is to move backward or forward.

The reaction force may be used in performing work, as is well illustrated in the self-loading or automatic gun. The recoil or kick of the gun depends generally upon its weight and the force of the exploded cartridge. This force, which would appear as the gun recoil in the ordinary gun, is made to act upon certain springs which operate to eject the empty shell, to throw into the chamber a fresh cartridge, and to cock the hammer. The two factors, the explosion reaction and the strength of the springs, together with the weight of the gun, are so nicely balanced that one experiences practically no recoil.

This is the relation which holds between the pressure of the oar blade and the pressure of the water. The two forces are equal and opposite. The action of the oar is equaled by the reaction of the water upon it. The result is that the boat moves forward when the oar blade exerts backward pressure against the water, the pressure of which is forward. In this the oar is taken as a part of the whole boat, which it practically is.

The third law of motion. - The idea of action and reaction as being equal and opposite has a wide application in our work with forces and motions. It is useful in understanding facts in many fields of experience which cannot be discussed here. In the study of physical science, for instance, you will find 
that it is this principle which is used in explaining the relation of the sun and the earth, of the moon and the earth, and of every body falling toward the earth or of every force exerted upon the earth. Its significance is so far reaching that it has been accepted as one of the great laws of motion. Sir Isaac Newton, one of the greatest scientists, made this his third law of motion and expressed it in this general form: "To every action (or force) there is an equal and opposite action (or force)."

\section{SUMMARY}

A floating body is one that displaces its own weight of water; a sinking body is one that displaces less than its weight.

The amount of buoyant force exerted by any liquid on any body is equal to the weight of the liquid displaced by the body. This is Archimedes' principle.

Buoyant force is due to the pressure of the liquid.

The density of a substance is its weight per unit of volume.

Relative densities are often given in which water is taken as the standard.

The stability of a boat depends upon the relative positions of its center of weight and its center of buoyancy. It is secured by the form of the boat and the proper placing of ballast.

Stability is especially necessary in a sailboat, because of the force of the wind on the sail.

Small boats are generally propelled by paddles or oars, which are forms of the lever and illustrate its principle.

The propulsion of a boat illustrates Newton's third law of motion: Action and reaction are equal and in opposite directions.

\section{REVIEW QUESTIONS}

1. What are the relative advantages and disadvantages of travel by sailing boat and steamship? 2. Describe some primitive boats. 3. Under what conditions does an object float in the water? Under what conditions does it sink? 4. State the principle of buoyancy. 5. (a) Tell the story of Archimedes and the golden crown. (b) What was the new method employed in determining the purity of the gold? (c) Of what use is this method to us? 6. What facts regarding pressure can be demonstrated by means of a tin can full of holes? 7 . What is the relation of pressure to depth? 8 . What is the relation of pres- 
sure to the volume of water? 9. What would happen to a flat-sided bottle which is stoppered and sunk in the deep sea? 10. What facts should builders of boats know?

11. What is the meaning of density? Of relative density? 12. Will iron float or sink in melted zinc? In melted lead? 13. How much of a cork is under water? Of a block of pine? Of a block of oak? 14. Compare the volumes of a pound of cork and a pound of pine.

15. What is the importance of stability in boats? 16. Describe how the center of weight of a piece of board may be found. 17. What is meant by the center of buoyancy? 18. What are the relative positions of center of weight and center of buoyancy when a boat tends to right itself? When it tends to overturn? 19. In designing a boat what is planned to give great stability? What can be done in loading the boat? 20. How is the idea of stability applied to other things than boats?

21. Mention some simple means of propelling boats. 22. Compare the oar with the paddle. 23. Show that the oar is a form of lever. 24. Give an illustrative problem to show the forces that come into play when the oar is used. 25. Explain why the backward push of the blade against the water drives the boat forward. 26. State the law of reaction.

\section{SUGGESTIVE QUESTIONS}

1. What do you imagine would be the result of trying to swim in a tank of mercury? (Density =13.6.) 2. What if one should fall into a tank of gasoline? (Density 0.7.) 3. Boats before launching are often painted one color above the water line and another below. What must be known to determine the position of the water line? 4. Some governments require that a licensed ship have marked on its side the water line as it would be when the ship is loaded with the total weight of eargo allowed for safety. How does the government inspector know where the line should be? 5. In rivers near the ocean fresh water may be on top and salt water below. Explain.

6. What sort of stability has ( $a$ ) a boat? (b) a table? (c) an orange? $(d)$ a football player? (e) a tree trunk? ( $f)$ a cube? ( $g)$ a 'dog? 7. Why does a person lean forward when carrying a heavy load on back or shoulders? To one side when carrying a suitcase with one hand? 8. Can you stand with both heels against a wall and pick up a hat in front of you without falling? Why? 9. Why is a racing automobile made to run close to the ground? 10. Why do racing yachts have a very deep keel? 11. Why will a boat tip more easily when its occupant is standing than when sitting down? 12. When 
running at top speed, what is the position of the trunk relative to the feet and legs? Why? 13. Why is the shifting of a cargo dangerous? 14. What do sailors mean by a "list to port"?

\section{Sailing a Boat}

156. Early method of sailing. - Sailing a boat into the face of the wind is now so common as to provoke little or no curiosity. In the early centuries of sailing, however, this was considered impossible. The seamen of Phœnicia, Greece, and Rome waited for favorable winds, to carry them, if possible, directly to the desired port. Even triremes might be equipped with sails and delay sailing in order to take advantage of favoring winds. To-day, whether the direction of winds is favorable or not, they are utilized in sailing by skillful adjustment of hull, rudder, and sails, in accordance with certain well-established laws or principles. Winds cannot be changed by man to suit his wishes; man must adapt himself and his means to suit the winds and make what use he may of them for his purposes and advantages.

157. Sailing into the wind. - How can a boat sail at an angle of forty-five degrees or more into the face of the wind? This is, in spite of its familiarity, an interesting problem, and its method of solution has a wide application to other situations. For this reason it is worth special study. It may be well, however, to begin with a commonplace example, that of pushing a wagon with a wagon pole, or a stick, as children often do with toy

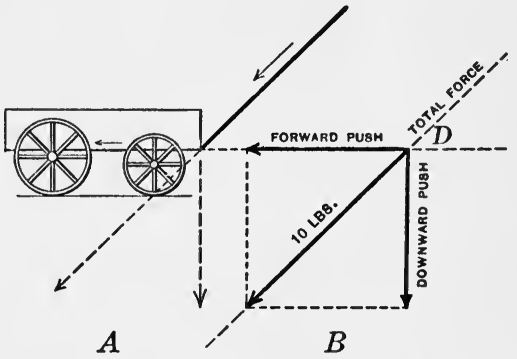

Fig. 121. - Showing how a single force may have two effects acting in different directions.

express wagons. Although the conditions are different, the fundamental facts are the same in pushing a wagon and in sailing a boat into the wind. 
Exercise: Relation of forces as in pushing a wagon. - Assume that the angle in the illustration, Fig. $121(A)$, is $45^{\circ} .^{1}$ A pushing force of

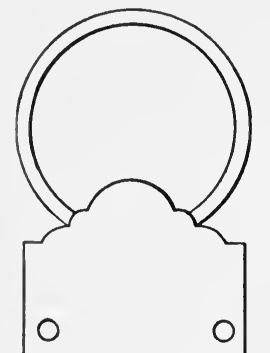
ten pounds ${ }^{2}$ is exerted on the stick. As is evident from the direction of the stick, part of the pushing force is expended upon the ground, or downwards, and may be regarded as wasted or ineffective effort. Only a part of the effort serves in moving the wagon forward. The problem now is to find how much of the total effort, ten pounds, is wasted in the downward push, and how much is applied to the wagon effectively.

Graphic solution. - The problem may be solved graphically, ${ }^{3}$ that is, by representing the forces by lines drawn to scale. As in Fig. $121(B)$, draw a line parallel to the surface of the ground as a base

${ }^{1}$ Angles may be measured with a protractor. Or take a square piece of paper, fold in half diagonally; the acute angle formed is $45^{\circ}$. To find an angle of $15^{\circ}$, cut the paper on the crease, then fold the $45^{\circ}$ angle into three equal parts and cut. Each will be $15^{\circ}$. How can you find an angle of $60^{\circ}$ ?

2 Measuring force. - Force is measured by its pressure or pull, and the quantities may be stated in ounces and pounds (or in grams and kilograms in the metric system). A sack of flour placed on the platform of a balance is weighed. The flour is removed, but the weights are left unchanged. One may grasp the platform with the hands and exert a pressule sufficient to balance the weights that measured the weight of the flour. Plainly the force exerted in the pressure is exactly as many pounds as the flour weighed. Consequently we speak of a pressure of fifty pounds just as properly as of a weight of fifty pounds of any commodity. So with the pull exerted by a force. A spring balance (Fig. 122) registers the pull which an applied force or a given weight of iron or sugar may exert. Weight and force are then equivalent. And it is a common practice to speak of a pull or a push of ten, twenty, or a thousand pounds whether the force be exerted by man, animal, or anything else, such as falling water, wind, steam, or electricity.

${ }^{3}$ Graphic representation of force. - Both force and weight may be represented graphically. A given length of line may be taken to represent a given amount or measure of force or weight. A line one fourth of an inch long may represent a force of ten pounds; a line four times as long will represent by the same scale forty pounds of force. The length of a line may thus show the magnitude of a force. The direction in which the force is act-

FIG. 122. - Spring balance. What is the equivalent of 64 ounces in grams? ing may be shown by arrows. It is equally simple to show the point at which a given force is applied. Thus the magnitude, direction, and point of application of a force may be represented by a line drawn to scale. 
line. Put a dot $D$ on this line to represent the point at which the stick is applied to the wagon. Assume that the stick forms with the base line an angle of $45^{\circ}$. Extend the line representing the stick indefinitely. Suppose the scale is a line one fourth inch long for each pound. Measure off the required distance according to the scale on the line representing the stick from the point $D$. This length is two and one half inches. Take the line just measured as the diagonal of a rectangle and complete the rectangle. Measure a horizontal and a vertical line. Are they equal? (The values are only approximate in the case of an angle of $45^{\circ}$.) Applying the scale to your measurements, how many pounds of force do you find exerted on the vertical line? How many pounds of force are exerted in the downward or wasted effort? How many pounds are effective? What is the total force exerted?

Put the stick at a smaller angle with the base line, as for instance at an angle of $15^{\circ}$. Extend the line representing the stick in the direction the stick is pushing. Measure off the length to represent the force applied (say ten pounds). Complete the parallelogram. What is the length of the perpendicular to the base? What is the length of the base line of the parallelogram? What amount of force is wasted in the downward push? What amount of force is effective? Why is more force effective in this trial than in the former $(B)$ ?

Let the angle formed by the stick with the base line be $60^{\circ}$. What will be the amount of wasted or ineffective force? What will be the effective force? Make a drawing to scale.

In what position must the pole be to push the wagon with the least effort or force? Will it form an angle with the ground surface? Will the angle be acute or obtuse? Explain and illustrate graphically your answers.

Experimental solution. - The important facts may be demonstrated by means of an experiment. In this we shall consider that the pulling forces shown on the spring balances are equivalent to the pushing forces represented in the case of the wagon.

Arrange, as illustrated in Fig. 123, three spring balances just in front of a blackboard or upon a table. Draw a line of indefinite length in continuation of that formed by the string of balance $A$. Take this as the diagonal of the parallelogram which is to be completed. Draw lines also to represent the strings of balances $C$ and $B$.

Now exert upon balance $A$ a pulling force of ten ounces. Record the readings of balances $B$ and $C$. Measure on the diagonal a length, as $P$ to $D$, which according to your seale represents the magnitude of the force exerted. Complete the parallelogram by drawing from 
point $D$ lines parallel to the lines representing the strings of $B$ and $C$ balances. Measure two adjacent sides and compute their numerical value by your scale. Do they agree with the recorded readings of the balances? Do they correspond with the results you obtained in the case of $B$, Fig. 121 ?

Change the direction in which balance $A$ is pulled in order to produce results similar to those brought out in the study of the wagon.

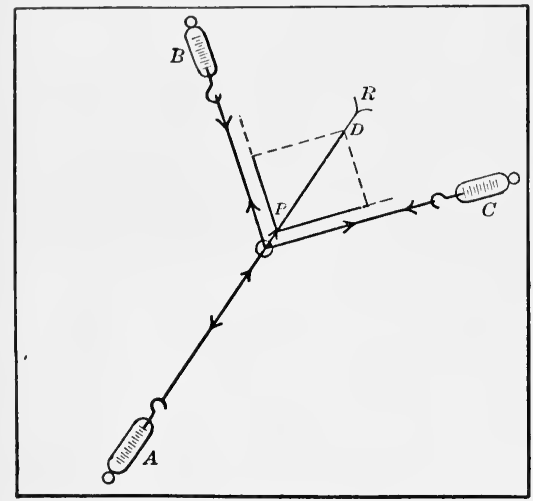

FIG. 123. - Arrangement of spring balances to show the relation of a resultant to its component forces, or of a single force to its resolved forces.

they be expressed? How may they be represented? What characters of force may be represented graphically?

In order to test the value of the method and its application to cases where a given force is broken up or resolved into two forces, the one effective and the other wasted, we may take a problem similar to those studied, namely the towing of a ship through a canal, Fig. 124. A ship is towed by an electric tractor. The tractor exerts a direct pull upon the towline of 1000 pounds. The towline pulls the ship not only ahead, but also toward the bank of the canal. The pull toward the bank is counteracted by the shape and position of the ship's hull and by the rudder. What, as represented in Fig. 125, is the amount of each of the resolved forces? Let the towline be twice as long, as shown by the dotted lines. What do you find to be the magnitudes of the resolved forces?

Suppose the tractive or pulling force is 800 pounds, and the angle formed by the towline with the line representing the path of the 


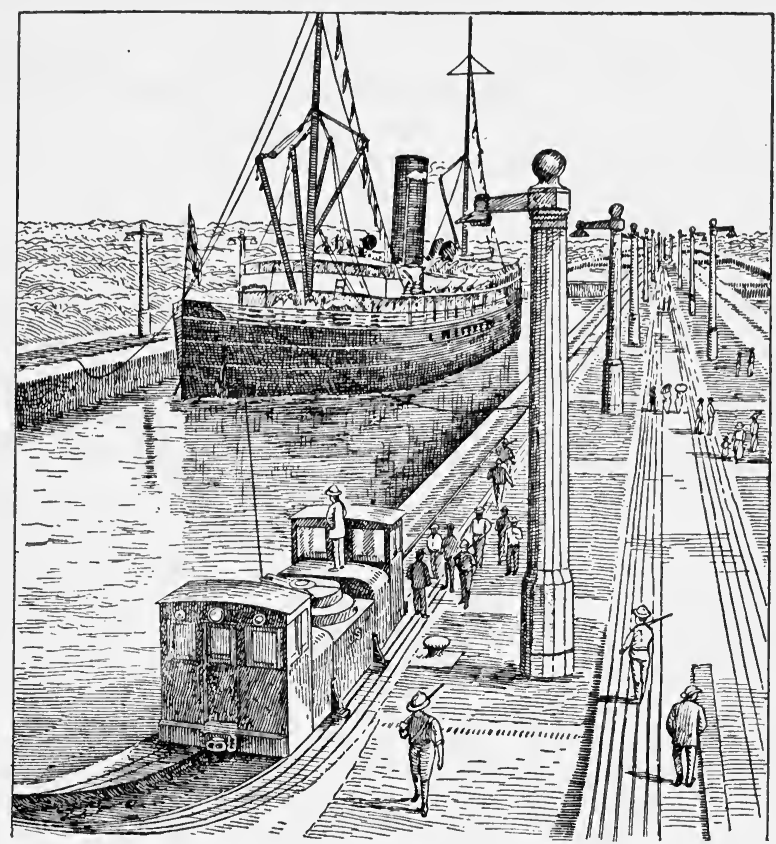

Fig. 124. - The "Alliance" passing through Gatun Locks, Panama Canal. This was the first ocean steamship to pass through the canal.

ship is $15^{\circ}$. Show graphically the magnitude and direction of the resolved forces. Try different lengths of the towline, for example, three times the first length, and find the magnitudes of the effective and wasted forces. Will the effective force be greater or less with increase in length of the towline? How does the effective force depend upon the angle made by the towline with the direction of the ship?

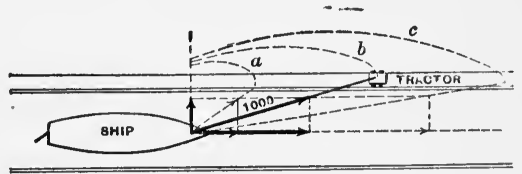

FIg. 125. - Towing a ship through a canal.

Application to sailing. - To return to the sailing boat and the problem of its making headway when the direction of the wind forms an angle of $45^{\circ}$ with the direction of the boat, we 
have but to apply the facts developed in the example of the wagon, and illustrated in towing a ship in a canal.

Exercise: Relation of forces in sailing. - In diagram $A$ of Fig. 126 , is shown the relation of the forces when only the sail and the wind are considered. The wind, let us say, is from the north and the boat is headed westward. The force of the wind, $A F$, is equivalent to 300 pounds. This is resolved into two forces, the one, $A P$, which is perpendicular to the plane of the sail, and represents the pressure on the sail, and the other, $A W$, which is parallel with the sail and represents the waste force called the "spill" of the sail. The figure is

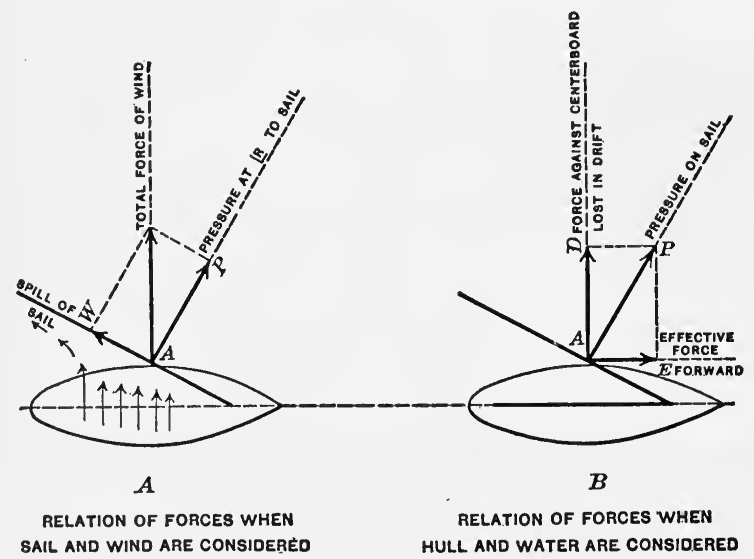

Fig. 126. - Boat sailing with the wind abeam at $45^{\circ}$ angle. Scale $\frac{1}{16} "$ to 30 pounds.

drawn to the scale of one eighth of an inch to 30 pounds. What is the length of the diagonal? Of the perpendicular and parallel lines respectively? What is the effective force? What is the waste force shown by the spill of the sail?

The resistance of the hull and centerboard of the boat together with that of the water must be considered. This requires that the sail pressure $(A P)$ or 240 pounds must be further resolved. The step is illustrated in diagram $B$ of Fig. 126. The pressure on the sail $(A P)$ exerts an effective force, $A E$, parallel to the centerboard, and also a drift force, $A D$, perpendicular to the centerboard. How much force is exerted against the hull and centerboard and wasted in drift to leeward? How much force is effective in moving the boat forward? 
Sailboats may sail more directly into the wind, or the angle formed by the sail with the direction of the wind may be much larger than $45^{\circ}$. The principle involved is exactly the same. Suppose the force of the wind is 300 pounds, and the sail is set as shown in A, Fig. 127. What is the amount of the effective force? What amount is wasted in the spill of the sail?

With the results obtained, you may compute the pressure of the sail force utilized in making headway, and that exerted upon the hull and centerboard, which is lost in leeway. Cf. B, Fig. 127 .

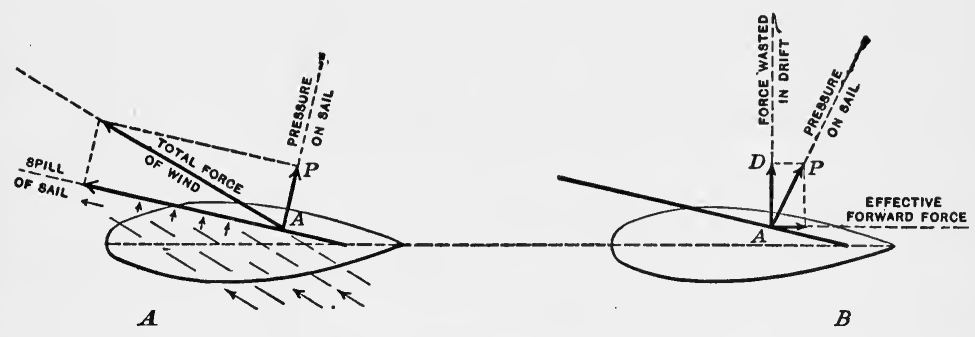

Fig. 127. - Boat sailing into the wind. $A$, relation of forces when the sail and the wind are considered. $B$, relation of forces when the hull and the water are considered.

Suppose the wind is southwestward and a sailboat has to make a point almost directly northeast of its present position. In what direction will it head on its first tack? What direction on the next? Show graphically the course of the boat in making its destination.

If the wind exerts a force of 240 pounds, and the angle formed by the plane of the sail and the direction of the wind on each tack is $45^{\circ}$, what are the resolved forces? Resolve then the sail force into the force which drives the boat ahead and that which is wasted. Solve the problem graphically as well as numerically.

158. Principle involved in resolved forces. - The facts developed in the study of the problem of sailing a boat have a wide application. They are promptly recognized in such familiar devices as wall brackets, street lamp brackets, bracket supports of trolley wire, bridges of the truss type, and roof trusses, and in such feats as flying a kite or piloting an airplane. Because of the wide range of application, it has been found necessary to try to state them in a convenient general proposi- 
tion. Such a statement is the following, though it should be considered as merely suggestive and you should try to express in your own terms the essential facts.

Any force may be resolved into two or more forces. When the effects of a single force act in a direction different from that of the original force, the effects may be determined by taking the line representing the direction and amount of the given force as the diagonal of a parallelogram, whose sides are drawn from the point of application of the force in the directions which the resolved forces must follow. If, moreover, the diagonal is drawn to a convenient scale, the numerical values of the resolved forces may be found by measuring the sides and multiplying their lengths by the scale number.

This general statement of the facts is known in physical science as the resolution of forces.

Parallelogram of forces. - The resolution of forces is but a half truth; for two or more forces may combine at a common point to produce a single effect, just as readily as a single force may be resolved into several effects. That is, the resolution of forces must be supplemented by the composition of forces. The chief difference involved is that in composition the forces uniting at a common point are described as the component forces and their effect as the resultant. The method of determining the magnitude and direction of a resultant of component forces is similar to that of determining the magnitudes and directions of the effects of a resolved force. The resolution and the composition of forces are included in a single general principle known as the parallelogram of forces.

Exercise: On the parallelogram of forces. - Because of the importance of the principle, it will be helpful, if time allows, to demonstrate experimentally by such an arrangement of spring balances as in Fig. 123 , the following :

1. The direction of the resultant of two components is always more nearly in alignment with the direction of the greater force.

2. The magnitude of the resultant decreases as the angle formed by the components increases; or 
(a) With an angle of $180^{\circ}$ the resultant is equal to the difference of the components.

(b) With an angle of zero the resultant is equal to the sum of the components.

3. No resultant can be greater than the sum, or less than the difference, of two components.

\section{SUMMARY}

Sailing at an angle into the wind was unknown to the ancients.

A force may be measured in pounds and represented by a line which shows its point of application, direction and magnitude.

Two or more forces may be found which, if substituted for one given force, would produce the same effect. This is called the Resolution of Forces.

A single force may be found which, if substituted for two or more forces acting at a common point, would produce the same effect. This is called the Composition of Forces.

In either case what is not known about the directions or magnitudes of the forces can be determined by means of a suitable parallelogram.

The resolution of forces is illustrated in a multitude of devices, such as the wall bracket, the truss bridge, the pushcart, the canal boat, the sailboat, the kite, and the aëroplane.

Sailing a boat into the wind may be explained by the principle of the parallelogram of forces.

\section{REVIEW QUESTIONS}

1. By what means can apparently adverse conditions be utilized by a sailing vessel? 2. (a) What is illustrated by the pusheart and stick? (b) In what two directions is the force applied by the stick working? (c) How may the forces involved be measured? (d) How may they be represented graphically? 3. (a) Show graphically the direction and magnitude of the forces when a foree of 25 pounds is applied to the stick which is at an angle of $45^{\circ}$ with the horizontal line. (b) When the angle is $15^{\circ}$. (c) When the angle is $0^{\circ}$. 4: Which is the useful force and which the wasted? 5. Show graphically that a long towline on a canal boat is more advantageous than a short one.

6. (a) Explain from a diagram how a boat can sail across the wind. (b) Into the wind. 7. Mention several instances when the effects of one force are seen in two different directions. 8. State the principle of the resolution of forces. 9. What are component forces? What is the resultant? 10. How is the composition of forces related to the resolution of forces? 11. If a clothesline will break with a pull of 100 
pounds, can it be made to support a weight of 100 pounds if it is hung very slack between two posts? If stretched taut before the weight is attached?

\section{SUGGESTIVE QUESTIONS}

1. A writer in the first century of the Christian era mentions a delay of several days in which a ship waited for a fair wind, that is, one in the direction he wished to go. Would a modern sailing vessel have waited? Explain. 2. Can a sailing vessel go directly into the wind? Why? 3. A big bellows was fastened to the deck of a ship and made to blow on the sails in a calm. The ship moved slowly backward. Explain. 4. If a windmill were placed on a boat and geared to a screw propeller, would it make the boat go? Would it force it directly into the wind? (The answer to the latter question depends upon the slant of the windmill blades on the wind, and the pitch of the propeller blades.)

\section{The Steamship}

159. The modern ocean liner. - Within the past hundred years the sailing ship has been rapidly replaced by the steam vessel both for passenger and for freight traffic. The demand for speed, safety, or seaworthiness, and comfort in travel has affected water transportation as well as that on land. The study of the behavior of water and winds, the invention of means to overcome their adverse influence, and to reduce to a minimum friction and waste, have brought about the building of ships which are marvels of grace, utility, and speed. Compare the Clermont of Robert Fulton with a modern Atlantic liner and with a destroyer or torpedo boat. In speed, in safety, in size and economy of operation and maintenance, the differences are most pronounced. A transatlantic liner to-day (Fig. 128) makes twenty-five or more knots, and a destroyer does its forty knots, equivalent to forty-five or more land miles per hour. A modern liner carries a crew of more than 1000, and a passenger list of from 3000 to 4000 , besides thousands of tons of freight in its holds. Equipped with all possible conveniences and the luxuries of the best hotels, the modern liner is a veritable 
floating palace. With its wireless communication, it publishes a daily paper giving the news of the world to its passengers a thousand miles from land.

In size the steamship now measures as much as 950 feet in length and 100 feet in width, with a displacement of 60,000 to

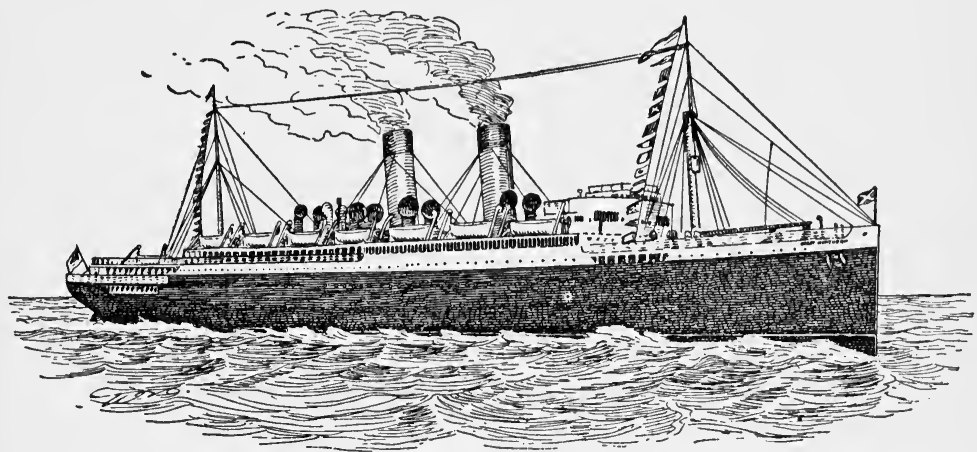

FIG. 128. - A modern steamship; steel hull, oil burning, Parsons' turbines, and triple screw propellers.

65,000 tons. It is altogether probable that the 1000 -foot liner with 100,000 tons displacement will be an early achievement in boat building.

160. Use of steam in navigation. - The application of steam power to ocean navigation is as full of romance as is that of steam and water power to the generation of electricity in present industrial life. Many methods have been tried. An old boat had paddles fixed on frames which moved them after the fashion of oars in the hands of oarsmen. The paddle wheels gradually took on the form of those used to-day on side-wheelers and stern-wheelers for river and ferry traffic. The connection of the power developed by the engine was made with a walking beam from the cylinder to the crank on the wheel. To-day where a light draft is necessary paddle boats are employed. For deep water another means of propulsion has been developed. The screw propeller is employed exclusively on ocean steamers 
and on most types of motor boats. The propeller is much like a ventilating fan, the blades sloping forward so that as they are turned they cut the water in front and force it backward, away from them. This causes the ship to move in a direction opposite to that in which the water is forced. In the wake of a screw there is a stream of water opposite to the direction of the ship. If a ship reverses its propellers and moves backward, which way is the water forced? A ship may be equipped with two propellers, "twin screws," or four, "quadruple screws," as are several great liners. The reason for the increase in number of screws will appear later.

The steam engine. - The engines of the great ocean liners are marvels of mechanical perfection. For more than thirty years triple and quadruple expansion engines have been in use and have been improved in many important ways. The steam is superheated and utilized at high pressures, and much of the energy is taken from it in its course from and to the boilers. Economies in fuel are most important. Liners of an older type consume about 3000 tons of coal every twenty-four hours; while one of the latest and largest ships afloat gets greater power and a higher rate of speed from the consumption of only 1000 tons in the same period of time. This indicates the progress achieved in increasing the efficiency of engines hitherto considered standards of perfection.

The steam turbine. - In the age of Archimedes, Hero invented a steam engine, in which a principle different from that of the reciprocating engine was involved. It was made in the form of a turbine and driven by the reaction of the escaping steam (Fig. 129), much as a certain kind of lawn sprinkler is driven by the escaping water. The steam turbine designed by . Sir Charles Parsons and demonstrated publicly in 1897 is a great advance beyond the simple turbine of Hero. Parsons' Turbinia, a ship built to test his turbines, developed a speed or 34 or 35 knots, equivalent to 40 miles per hour. The highest speed previously attained was about 24 knots. Recently 
ocean liners have been equipped with highly developed turbines and reciprocating engines, a combination yielding greater power without increase in consumption of fuel.

Advantages of the steam turbine. - The reciprocating engine, with back and forth stroke of the piston, stopping momentarily at every stroke, produces a continual jarring when operating at high speed. The vibration of the express ocean liners became more and more intolerable as their triple or quadruple expansion engines were driven hard to reduce the time required for crossing the ocean. The jarring was reduced somewhat by certain improvements. Meanwhile the steam turbine proved its value and either supplemented or replaced the reciprocating engine. The turbine is noiseless, free from vibration, steady, of comparatively small size and weight, and of tremendous power production with consequently greatly increased possibilities of speed. As to weight and power production, an old low-pressure engine produces about two horse power for each ton of machinery; the turbine produces more than six horse power for
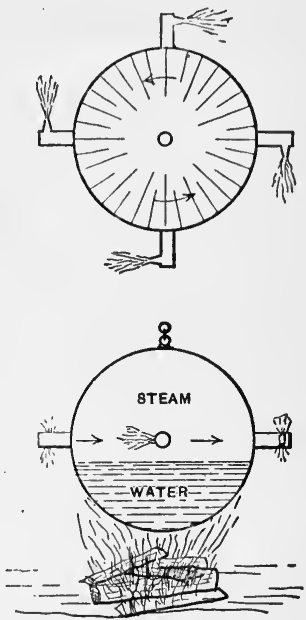

Frg. 129. - Principle of the steam turbine, as illustrated in Hero's steam engine (about 120 B.C.).

each ton. The turbine is also more efficient than the best type of reciprocating engine; and it is more flexible in construction, adaptable to high and low pressure, thus taking from steam all its energy.

One great steamship with quadruple screw has six turbines adapted to high and low pressure of steam. Steam is admitted to the first series at a pressure of 210 pounds to the square inch (see table, page 312). It is conducted from these to the low pressure turbines and then into condensers. The turbines develop about 70,000 horse power and make possible a speed of 
more than twenty-six knots, equivalent to about thirty miles an hour. Another liner may be taken as a type of the combination of reciprocating engines and turbines of low pressure (Fig. 130). The engines are of the quadruple expansion type, and utilize the steam at an initial pressure of 215 pounds per square inch. The steam performs work in the reciprocating engines

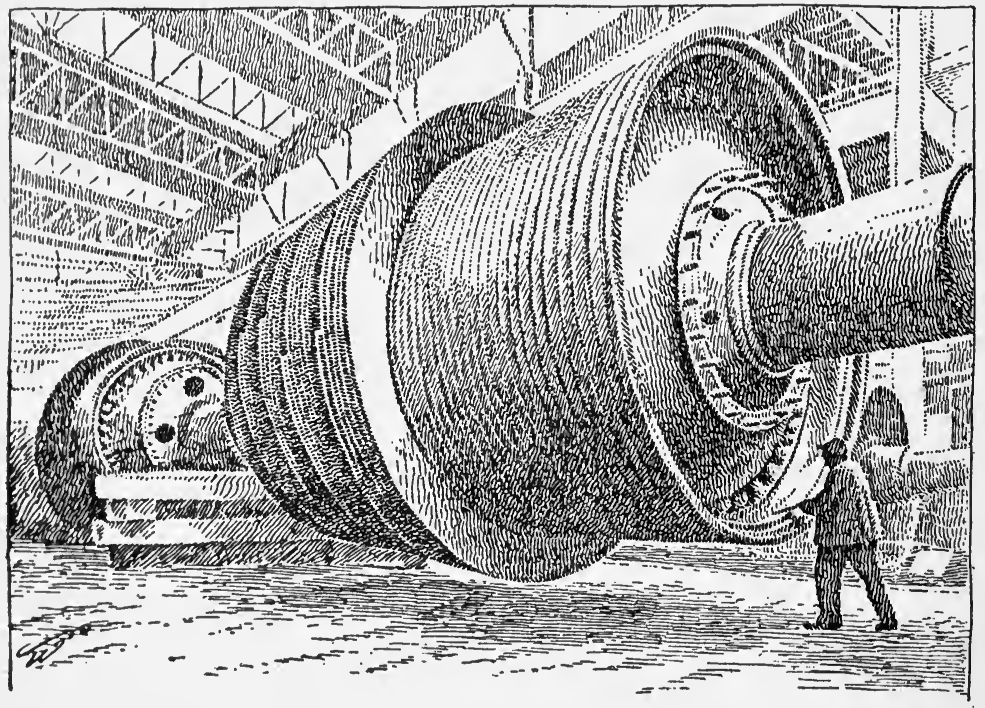

Fig. 130. - A rotor of a low pressure turbine of a great ocean liner. The diameter is 18 feet.

until it has about nine pounds pressure per square inch. Then it is conducted to the turbines, where it expands until it has a pressure of one pound per square inch (Fig. 131). The turbines develop 16,000 horse power, which with the 30,000 horse. power produced by the reciprocating engines gives a speed of approximately twenty-two knots.

Turbines are employed to supplement reciprocating engines not only on steamships but also in stationary power plants, such as the subway power plant of New York City. The 
turbines receive the used steam from the exhaust of the engines, and furnish about as much power as do the reciprocating engines before discharging it into the condensers. It involves no increase in fuel consumption. This, like the use of water power, illustrates the advance in power development in the last few years.

Defects of the turbine. - In the use of the steam turbine there are at present serious difficulties, one of which is the impossibility of reversing it. A modern steamship equipped only with turbines has four turbines for driving the ship ahead, and two for

Nozzles

Moving Blades

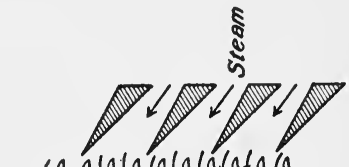

Stationary Blades

Moving Blades

Stationary Blades

Moving Blodes
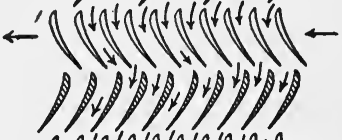

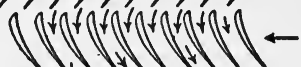

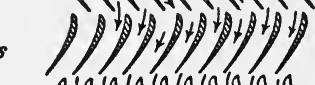

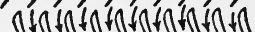

driving it astern. When reversal is necessary, the steam is shut off from the four turbines and supplied to the two propelling the ship backwards.

Several methods of solving the difficulty have been attempted. One is to equip the steel shafts of the turbines with electric generators and to utilize the electricity in driving the propellers. The high speed, the invariable output of power, the dependability, and efficiency of the steam turbine make it ideal for driving electric generators. Reversal of electric motors does not interrupt the work of the turbines. Another method is to have the steam turbines operate a water pump which in turn operates water turbines that drive the propellers. The water is used repeatedly until it becomes hot through friction, when it is turned into the boilers, and an equivalent amount of cold water supplied automatically. This method is highly efficient, yielding about 90 per cent, or a loss of about 10 per cent, of the power developed by the steam turbine. 
161. The submarine. - The submarine has been rapidly developed within a period of five years. It is constructed so that its total weight is just a little less than the weight of its own volume of water; hence by admitting water to air-tight compartments or tanks, it is made to sink to any depth desired. When under way the boat may be made to dive, like a whale or porpoise, by a horizontal rudder. Tilting the rudder causes the boat to rise or descend. When the water is expelled from the tanks by compressed air, the boat is lifted to the surface by the buoyant force of the water below it.

The Diesel oil motor or engine is generally employed on submarines. When the boat is lying idle, this operates dynamos which charge storage or secondary cells. When the boat is under water, the motive power is the electricity generated by these cells. On the surface the Diesel engine furnishes the power directly for the propellers. The storage batteries furnish electricity for lighting.

Submarines are made to resist the pressure at depths of 100 to 200 feet, in very rare cases to withstand the pressure at 1000 to 5000 feet. The air necessary for the men is supplied by tanks of compressed air or oxygen, and the exhaled carbon dioxid is absorbed by sodium hydroxid.

When the submarine is below the surface of the water, objects like ships may be seen by means of the periscope. This is a tube twenty or more feet long, with an arrangement of lenses and mirrors, that transmit light rays to the observer in the conning tower of the boat. The submarine illustrates the principle discovered by Archimedes as to water's buoyant force and displacement.

162. General importance of travel and transportation. Travel and transportation have become indispensable in modern life, and they have been made easy, speedy, safe, and economical by man's conquest of natural obstacles, by his discovery and control of motive forces other than those inherent in men and animals, and by his construction of vehicles to move 
on land, in water, and in air. Thus a new world is being created. All parts of the earth, even the remotest, are being brought into close relationship with one another through travel and commerce. The old provincial ideas and beliefs of isolated groups of people are being gradually modified by knowledge of the world gained through modern facilities of travel and communication. Through such knowledge and respect for the customs and beliefs of strange peoples, gained largely by way of travel and commerce, the whole world is being bound together in closer international relationships that may lead eventually to a federation of all nations.

\section{SUMMARY}

The demand for speed, safety, and comfort has brought about the substitution of the steamship for the sailing vessel.

Steam power is applied to navigation generally by the use of the screw propeller.

The modern marine engine is a marvel of perfection in efficiency, power, and dependability.

The first steam engine mentioned in history was the reaction steam turbine of Hero.

Sir Charles Parsons was one of the first to apply the steam turbine to navigation.

The steam turbine produces less vibration, occupies less space, and has less weight than a reciprocating engine of equal power.

The steam turbine as applied to navigation has one great defect, its inability to reverse.

The submarine, by changes of its density, can be made to travel on or below the surface of the waters.

In submarines, internal combustion engines, usually the Diesel, are generally employed when on the surface, and storage batteries when submerged.

\section{REVIEW QUESTIONS}

1. Why has steam largely replaced wind as a motive power for boats? 2. Give a short historical account of the use of steam as a motive power for boats. 3. Give the important facts as to the size, . capacity, speed, safety and comfort of a modern ocean liner.

4. What were some of the early devices for propelling steamships? 5. Describe the modern engines of ocean liners and compare their efficiency with other types. 6. (a) Describe the screw propeller. 
(b) Show how it operates. 7. (a) Who made the first steam turbine? (b) Describe and explain its operation. 8. Compare the steam turbine and the reciprocating engine as to the advantages and disadvantages for navigation. 9. (a) Mention a modern steamship which is equipped exclusively with steam turbines. (b) One which combines reciprocating and turbine engines. 10. Describe a steam turbine of the Parsons type. 11. In what way does the Curtis turbine resemble the Pelton water wheel? How does it differ from it? 12. Why are steam turbines employed to utilize the energy of the exhaust steam from reciprocating engines? 13. How does a steamship with turbines provide for reversing? 14. What devices have been made to reverse the direction of the screw propellers without reversing the steam turbines?

15. Explain how the submarine submerges or emerges. 16. (a) What motive power is used by submarines when at the surface? (b) When submerged? (c) What type of engine is employed? 17. What forces must a submarine withstand? 18. Describe a periscope and explain its use.

19. In what ways has the application of steam power to navigation affected human life?

\section{Instruments of Navigation}

The routes over which vessels travel are unmarked except on the charts of the ship's officers. To determine the location of the vessel accurately and to steer the course required, it is necessary for the mariner to have certain instruments as well as charts showing sailing routes. The indispensable instruments are the compass, the sextant, and the chronometer. To-day a steamer or sailing vessel has also a log, a barometer, and other instruments in its equipment.

163. Invention of the compass. - The invention and use of the compass made possible the ocean voyages. of exploration and discovery in the fifteenth and succeeding centuries. When or by whom the compass was invented, or when and how it was. introduced into Europe, are not known. Tradition has it that the Chinese more than two thousand years ago discovered the "magnetic needle" and made use of it in traversing the great plateaus of the empire. Certainly the compass was used in travel on land before its use in navigation was discovered. 
164. The lodestone. - The earliest compass was probably made of lodestone, which is widely distributed in nature. The ancient Greeks were familiar with it and some of their scientists described its property of attracting and repelling iron particles. The ore is a compound of iron and oxygen, known as magnetite (page 434). Lodestone has the capacity of imparting to iron or steel its magnetic properties with no loss to itself, and also the property directly associated with the compass, of assuming, if left free to move as when floating on a bit of cork, a definite direction with respect to the earth, that is, pointing nearly north and south.

165. A simple compass. - It was early learned that a bar or iron or steel, when magnetized, behaved like the lodestone, pointing nearly north and south. A steel needle was therefore substituted for lodestone in the compass, an improvement greatly increasing its usefulness. The needle was then, as now, balanced in a horizontal position on a pivot (Fig. 132). The important fact is not the way in which it is pivoted, but the magnetic property of the needle itself, because it may be suspended by a long hair or a Fig. 132. - A simple compass. single fiber of silk, or floated on a bit

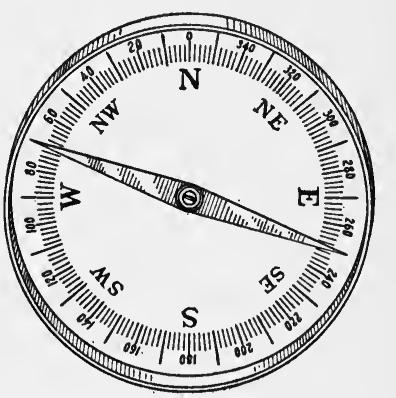
of cork on water. Such a simple compass is useful for experimental study of the behavior of the needle.

The ordinary compass is made of a magnetized bar of steel supported horizontally on a steel or jewel pivot above a graduated disk marked N., S., E., W., and inclosed in a brass case covered with glass. The mariner's compass (Fig. 133) is much more complicated, but exactly the same in principle. The circle on the disk is divided into degrees and then into thirty-two parts, each $11 \frac{1}{4}^{\circ}$. The instrument is mounted so as to maintain a horizontal position whatever may be the position of the vessel. 
Brass and glass do not interfere with the magnetic influence of the earth or other magnetized body. An iron box would

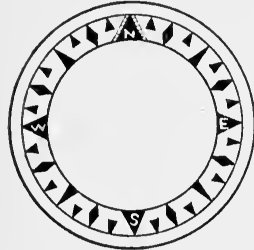

Fig. 133. - Dial of mariner's compass. quickly take from the needle its magnetic properties. This fact as to the behavior of iron is utilized in making watch cases for those who frequently work about powerful magnets, as electric generating plants. Watches with iron cases are protected from the undesirable magnetic effects.

Variation of the needle. - Early in the thirteenth century the magnetic needle had ceased to be merely a curiosity to the educated men of Europe. But little is known of its use in navigation before the age of Columbus. One fact had been pointed out, namely, that the needle pointed slightly west of what was supposed to be true north; but this was attributed to the faulty construction of the instrument. The true north was determined then, as now, by observations on the sun and the north star. Columbus noted this variation of the needle and by his observations showed that the instrument was not wrongly constructed. His observations led to an interesting discovery.

When leaving Lisbon Columbus noticed that the needle pointed west of the true north. Later when just a little west of the Azores he observed to his astonishment that the variation had disappeared. The needle pointed to what was determined to be true north. As he continued farther westward, he found the needle vary more and more toward the east of north. Later observations verified his report of the facts. The magnetic pole does not coincide with the geographic pole.

The line of no declination. - Columbus found that the line on which the needle pointed true north or showed no declination, as it is now called, a short distance west of the Azores. It is now known that the line of no declination moves gradually westward at the rate of about one degree in fifteen years. At one time it passed through Paris ; to-day it passes along a line 
that runs through South Carolina and Michigan to the north magnetic pole of the earth, which is located northwest of Hudson Bay, about longitude $97^{\circ}$ west. The declination or deflection of the needle at all points east of this line is thus constantly increasing, and that of all places west of the line decreasing. The declination of the needle at New York is about ten degrees west. That at San Francisco is about sixteen degrees east, and that at Seattle about twenty-two degrees east. Local declination may be determined by the following method.

Exercise: Finding local declination. - Determine first of all the correct north and south line of your locality. This you may do by observations on the north star. The orbit of the north star is about the size of the circumference of the moon and is completed by the star every twenty-four hours. Twice in each twenty-four hour period the star is directly north. This occurs when it is directly above or below the star in the crook of the handle of the Great Dipper. Observations should be made at such times, although the error of observations at any other times is very slight. The observations may be made more accurately by using illuminated plumb lines. When you have determined the true north and south line, the angle which is formed by the needle of the compass with that line gives the local declination.

Steel ships and the compass. - The construction of ships with iron and steel gives greatly increased strength, size, and safety, but it also makes uncertain and difficult the use of the ordinary compass. The great mass of iron and steel not only influences the magnetic needle, but itself becomes magnetized through the influence of the magnetism of the earth. This condition of the ship interferes directly with the needle and deflects it from the direction it would take under the influence of the earth alone. Compasses of the usual kind have to be tested by astronomical observation so that the error may be known and corrected.

The influence of the magnetism of the earth upon iron and steel may be observed experimentally. The experiment which we shall describe was made by William Gilbert, physician to 
Queen Elizabeth, and illustrated by an interesting woodcut in a book published by him in the year 1600 . The quaint picture shows a blacksmith holding a bar of iron in alignment with the meridian, north and south, and hammering it vigorously. The iron bar is thereby magnetized. It was known also at that time that a bar of cooling iron would become magnetized without hammering, but that the process could be hastened by hammering. The same experiment may be performed to-day to illustrate the important facts.

Exercise: Magnetizing by induction from earth. - You will need a compass, a hammer, and a piece of iron pipe or rod about three feet long. If the iron has been lying about for some time, it may be magnetized. It should be tested for magnetism by holding each end successively near the north-seeking pole of the compass. Observe the behavior of the needle. If the iron attracts the north needle at each trial, it is not magnetized.

Now hold the iron in the meridian, that is, north and south, with the north end pointing a little below the horizon. Hammer it vigorously for a moment. Test the ends of the iron by holding each successively near the north-seeking pole of the compass. Note the effect of each end of the iron on the needle. Does the end of the iron which was pointed north attract or repel the north-seeking pole of the compass? How does the needle behave in relation to the south end of the iron?

Reverse the iron, and holding it in the meridian as before, strike it several sharp blows. Test each end again, as above. Compare the results with those of the former series of tests. Is the magnetic influence of the magnet changed? How can you account for the facts observed?

The earth a magnet. - In 1600 William Gilbert explained the facts observed in the experiment by the theory that the earth is a huge magnet. He mentions the now familiar fact that an iron rod suspended horizontally and with an inclination like that of the magnetic needle, pointing, that is, toward the magnetic north pole, becomes magnetized in course of time by the earth. This is done in the same way in which a horseshoe or a bar magnet magnetizes a needle or a nail. If we wish to magnetize quickly a piece of steel, such as a needle, we stroke 
it from the center to one end with one pole of the magnet, and then from the center to the other end with the other pole. It may be done without direct contact. Try it both ways. When done by contact the slight jarring hastens the molecular changes in the steel. The action of the earth is much slower, because its field is comparatively weaker than that of the magnet.

In magnetizing the iron bar or pipe, hammering accelerates the change of the molecules into alignment with the magnetic field of the earth. The suspended bar responds much more slowly. It is just the same with steel and iron ships, and steel structures on land. They all become magnetized in time by the earth.

The theory illustrated. - The way in which the molecules of an iron bar arrange themselves in response to the earth's magnetic influence may be imagined by representing the molecules as little magnets which are parallel and have their north-seeking poles at one end and the south-seeking poles at the other. In contrast with this arrangement of the molecules in a magnetized bar, we may represent their arrangement in a non-magnetized bar as so irregular that they have no combined influence so far as this can be detected by the magnetic needle.

166. The gyro-compass. - As stated above, ships of steel and iron interfere seriously with the use of the magnetic compass in navigation. A new instrument has been invented and perfected in late years, which has proved to be so dependable and satisfactory that it is now in use in practically all the navies of the world as well as on the modern ocean liners. This instrument is the gyro-compass, and is so named because it makes use of the gyroscope as the principal mechanism.

The gyroscope, which should become familiar to every one, is one of the most interesting mechanical devices. It is virtually a top. The heavy wheel with its axle in one piece with itself is mounted in a ring on pivots (Fig. 134). When the wheel is made to rotate at high speed by means of a string or otherwise, it resists very strongly any external force that would change 
its plane of rotation. This property of the gyroscope gives it an indefinite number of uses and makes it most interesting for experiments.

The gyro-compass in the form now used comprises three wheels with spindles, each wheel with its spindle being made from a single piece of flawless nickel steel and mounted

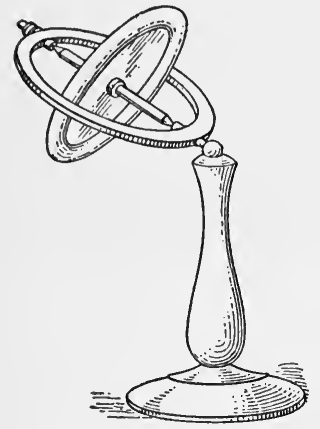

Fig. 134. - A simple gyroscope. on ball bearings. Each wheel revolves at the rate of 20,000 revolutions a minute. The motive force is supplied by a small electric motor. When the gyro-compass is properly set, it retains its direction as north and south in spite of the magnetic field of the earth or magnetized bodies, and in spite of every possible motion and position of the ship. The readings are transmitted to any place on the ship and are shown on dials in the same way as are those of the magnetic compass.

Other applications of the gyroscope. - The gyroscope has many suggested and attempted applications besides the gyro-compass. Cars have been devised to run on a single rail, retaining their balance by means of the gyroscope. Monotrack automobiles have been made in a similar way. The gyroscope has also been utilized in the construction of stabilizers for airplanes (page 399). All such applications are still in the experimental stage.

167. The chronometer. - The chronometer, a time-measurer, is just an accurate clock, the error of which is known and taken into account in making the daily reading. A chronometer runs uniformly, which is more important than that it should be regulated perfectly. Why? The time kept is usually that of the prime meridian. This enables the navigator to calculate exactly the longitude of his vessel east or west of the prime meridian. For example, a ship is at a point where the sun crosses its meridian at two o'clock by the chronometer. Is 
the vessel east or west of London? How many degrees? On the trip to Japan from San Francisco, at what longitude does the international date line occur? How many degrees west of the prime meridian? What is the hour by the chronometer when the sun crosses its meridian? How can one adjust dates without changing the chronometer?

168. Determination of latitude. - Daily observations of the sun or other heavenly bodies are made on all ships at sea to determine their location unless clouds prevent. The sextant is used to make these observations accurate. The sextant measures the angular distance of the sun above the horizon at noon. Suppose that on March 21, at longitude $30^{\circ}$ west, the sun is found to be $70^{\circ}$ above the horizon or $20^{\circ}$ from the observer's zenith. Obviously the ship is north or south of the equator just $20^{\circ}$, for on this day the sun is directly over the equator. How can one determine whether the ship is north or south of the equator? With the latitude and longitude determined, it is a simple matter to find the exact location of the ship by means of the chart.

169. The log. - The log resembles in certain respects a cyclometer or speedometer. Its mechanism is operated by a cord attached to a small propeller, which like a spoon hook is made to whirl or spin by being dragged through the water. The mechanism is connected with a hand that indicates upon a small dial the rate or speed and the distance traversed. For example, a log shows that a ship is moving at twenty knots. (A knot means nautical miles per hour.) If this rate were maintained for a day of twenty-four hours, it would be equivalent to a distance of about 540 miles. Modern invention has greatly improved the $\log$ of former times. The first kind of log used was a trailer, from the speed of which the experienced sailor could judge the rate of progress. To-day the log of some of the great liners is electric and operated by the revolving screw or attached to the propellers, every revolution of which is known to mean a certain distance. 
Dead reckoning. - In prolonged cloudy weather navigators depend upon what they call " dead reckoning." They know the general directions by means of the compass. They have the rate of speed as shown by the log. With the data thus furnished they can compute the distance traveled, and conjecture the position of the ship as to latitude and longitude. They allow roughly for variable winds, the influence of ocean currents, and possible error of the compass; and are able to locate the vessel closely enough for practical purposes.

\section{SUMMARY}

The compass depends upon the action of a magnetic needle in the magnetic field of the earth.

The angle that a magnetic needle makes with the meridian is the declination or variation of the needle. It varies slightly from year to year.

The magnetic compass is unreliable when used on steel ships, which are themselves magnetized by the earth. This has led to the use of the gyro-compass.

The chronometer is an accurate clock, the daily error of which is known. It keeps London time and is used in determining the longitude of the ship's position.

Latitude is determined by observation of the sun, or other heavenly body, made by means of a sextant.

The $\log$ is an instrument dragged through the water for the purpose of determining the distance the ship travels and its speed.

Determination of the ship's position from a former position, by means of the log and compass, is called dead reckoning.

\section{REVIEW QUESTIONS}

1. (a) What are the instruments of navigation necessary to a sailing vessel? (b) Which of these were used by Columbus? 2. What was the relation of the compass to the geographical discoveries of the fifteenth century? 3. What peoples made early use of the compass and for what purpose? 4. (a) What is a lodestone? (b) Mention some of its peculiar properties. 5. (a) How may a simple compass be made? (b) Describe the mariner's compass. (c) What sort of box is unsuitable for it?

6. Does the compass in most parts of the earth point to the true 
north? 7. What discovery did Columbus make in regard to the variation of the needle? 8 . Where now is the line of no variation? In what direction is it moving? 9. Describe how you can determine a north and south line by astronomic observations. 10. How can you determine the variation of the needle for your locality?

11. What difficulty appears in the use of the magnetic compass on steel ships? 12. (a) How can the earth's magnetism be used to magnetize an iron or steel bar? (b) When was this method known? (c) What was William Gilbert's explanation of the action of the compass or other magnetized bodies?

13. What modern device has taken the place of the magnetic compass on many steel ships? Describe. 14. Of what use is the chronometer? On what time is it run? 15. How is the ship's latitude determined? 16. (a) What is the log? (b) How is it operated? (c) What does it show? 17. What is meant by "dead reckoning "?

\section{HARBors}

170. Improvement of waterways. - On the sea man must adapt himself and his appliances to the conditions of wind and water as he finds them. He charts his ways to avoid obstacles such as icebergs, reefs, and shoals, and to suit the prevailing direction of current and wind. With inland waters, however, man works changes that facilitate commerce; he builds harbors, dredges river channels, and constructs canals by which natural waterways are joined; and he builds boats that are specially adapted to the conditions and needs.

171. Importance of harbor improvement. - It is certain that inadequate harbor facilities have retarded to a considerable extent the construction of large vessels and the development of shipping commensurate with the demands. By enlarging and improving harbors shipping is stimulated and greatly increased; and so important is this that harbor cities and the Federal Government unite in the work. It is recognized that with channels sufficiently wide and deep, and with piers sufficiently capacious and strong, and equipped with modern machinery for loading and unloading, the largest ships, now only contemplated, will become realities. 
In even the best natural American harbors channels must be widened and deepened for the largest ships. The entrance to the New York harbor through what is called the Ambrose Channel has been dredged to give a depth of forty feet at low tides and 1000 feet width at its narrowest part. The dangerous rocks at Hell Gate on the East River were removed years ago by blasting. Where natural facilities are inadequate for harbors, breakwaters of enormous size are built. One breakwater at San Pedro, the harbor for Los Angeles, California, is 9000 feet long and cost approximately $\$ 4,000,000$. Canals are cut from the sea into fresh-water bodies, where harbors are readily constructed to supplement limited ocean frontage and facilities.

Docks and piers. - Piers must be built to accommodate the largest ships, and equipped with mechanical appliances to facilitate the handling of freight. The harbors along the Great Lakes are perhaps the most completely equipped. Large freight steamers, as many as thirty in a line, may be loaded simultaneously with from 10,000 to 15,000 tons of iron ore each in less than two hours, and unloaded in about four hours. The docks project a quarter of a mile into the lakes. The machinery for handling grain and flour, ores, coal, and lumber is the most modern and efficient. In ocean harbors piers are being rapidly improved and new piers being constructed. The eight 1000 -foot piers being built on the North River at New York will accommodate the largest transatlantic express steamers contemplated. At Boston, New Orleans, San Francisco, and Seattle, harbors and increased dockage are being steadily provided to meet the growing demands of modern shipping.

\section{INLAND Waterways}

172. River improvement. - The improvement of rivers may be illustrated by the work done on the Mississippi. Like many others running through low land, this river overflows at certain seasons. The destruction of property and the hazard 
to all land cultivation in the vicinity made necessary protective and preventive measures. The construction of dikes, called levees, helped to some extent in keeping the river within its banks; but also gave rise to new difficulties. A river restrained by dikes is usually sluggish, and if its load of suspended material is large, it deposits this in considerable quantities, thus gradually raising the channel of the river above the level of the surrounding country. A break in the levees is then worse than a gentle flooding of the land.

The Mississippi carries along on the floor of its channel enough material to cover a square mile nineteen feet deep every year. It carries enough suspended matter to increase the depth of the square mile by twenty-four feet. It would

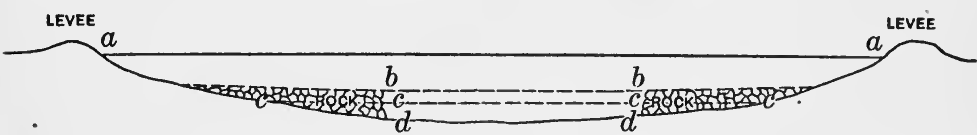

FIG. 135. - Jetties to force the Mississippi River to keep its channel clean and prevent dangerous floods.

be a gigantic, perhaps impossible, task to remove this by dredging. The engineers associated with the Inland Waterways Commission attempted to solve the problem by making the river clear its own channel. The diagram (Fig. 135) shows how this is done. The section is transverse, and the river is supposed to flow away from the observer. Jetties, large dams of loose rock, are built out from both sides of the river at intervals of about thirteen hundred feet. At low water the river flows at the level, $b, b$, and not at $c$, where it would be too widespread and sluggish. By confining the stream to the narrower channel, its usual low water depth is avoided and its flow increased and accelerated enough to clean the floor, $d, d$. At high water the whole channel $a-a$, is occupied. For the guidance of navigators the location of the jetties is shown on charts.

Future of river commerce. - The inland waterways of the 
United States, other than the Great Lakes, have now more than 25,000 miles of water used for navigation, and 50,000 miles of water that may be so utilized. In some regions competition by railroads has reduced not only the volume of river freight traffic, but also the efficiency of the service. The present situation in regard to the proper use of such waterways raises a serious problem for the whole country. Much of the produce of the land can be hauled most economically and advantageously on inland waterways; but the coöperation of all those concerned seems to be necessary to bring about the needed improvement and utilization of such natural resources for transportation.

173. Panama canal. - The remarkable work of the French engineer, De Lesseps, in the construction of the Suez Canal (a project which had been begun and partly executed by the ancients about 2000 B.c.) led to the organization of a French company for the construction of a canal across the Isthmus of Panama. This plan also had its historical anticipations in the time of Balboa and again in the plan of the Spanish Government in 1524, which, however, was opposed by the Governor of Panama as being " in opposition to the will of the Almighty who had placed this barrier in the way of navigation between the two oceans." Work on the canal was begun under De Lesseps in 1861, but failed because of many obstacles, some of which were of a kind that had not been met in the Suez construction. Not the least of these obstacles were yellow fever and malaria.

The United States purchased the rights and equipment of the French Company, and acquired with these a twenty-mile wide strip of territory across the isthmus from the Republic of Panama. The conquest of the tropical conditions and of the formerly insuperable barrier to interocean traffic was begun in 1904. Malaria and yellow fever, the dread foes of the work, were conquered through knowledge and skill (pages 523-532). The gigantic labor of construction continued with 
practically no interruption until the completion of the canal. The official opening occurred in March, 1915.

Value to trade and commerce. - The trade and commercial importance of the Panama Canal may be indicated by a few facts briefly stated. The cost is estimated to be about $\$ 375,000,000$. The length of the canal is fifty miles. The highest elevation to which ships must be raised is eighty-five feet above sea level, through the Gatun Lake and the Culebra Cut. The locks are constructed to accommodate the largest ships, being 110 feet wide and 1000 feet long.

Exercise. - The distance from New York to San Francisco via Cape Horn or the Straits of Magellan is about 13,244 miles; via the Panama Canal, 5299 miles. The canal route is shorter by nearly 8000 miles. Consider the difference in cost to shippers, if the average daily expense per shipload is $\$ 100$. The canal tolls must be included in the total expense. Find what these are for general merchandise.

The canal route distance from Liverpool to San Francisco is about 8038 miles; via the southern route, 14,084 miles, and via Suez Canal, 13,844 miles. What distance is saved via the Panama Canal over both the other routes? Estimate the saving in shipping cost. European ships clearing from Eastern Asiatic ports, as Yokohama, may save time and money by returning via the Canal, stopping en route at ports on the Pacific Coast of America for freight for European destination. By aid of distances given on the map (Fig. 136), ealculate other advantages gained by the use of the Panama Canal in oceanic transportation.

174. Other canals. - The Panama Canal is important to the United States because of its commercial, engineering, and defensive significance. It also serves as an example of the way in which man changes the waterways of the earth to suit his needs. Cities which were geographically inland have been changed into seaports and have thus acquired large maritime interests. Manchester, England, is now a seaport by means of a canal 32 miles long, which connects the city with the Mersey River and the ocean. Amsterdam, by a canal 16 miles long and 32 feet deep, brings to her docks all but the largest transatlantic liners. Holland has almost completely transformed 


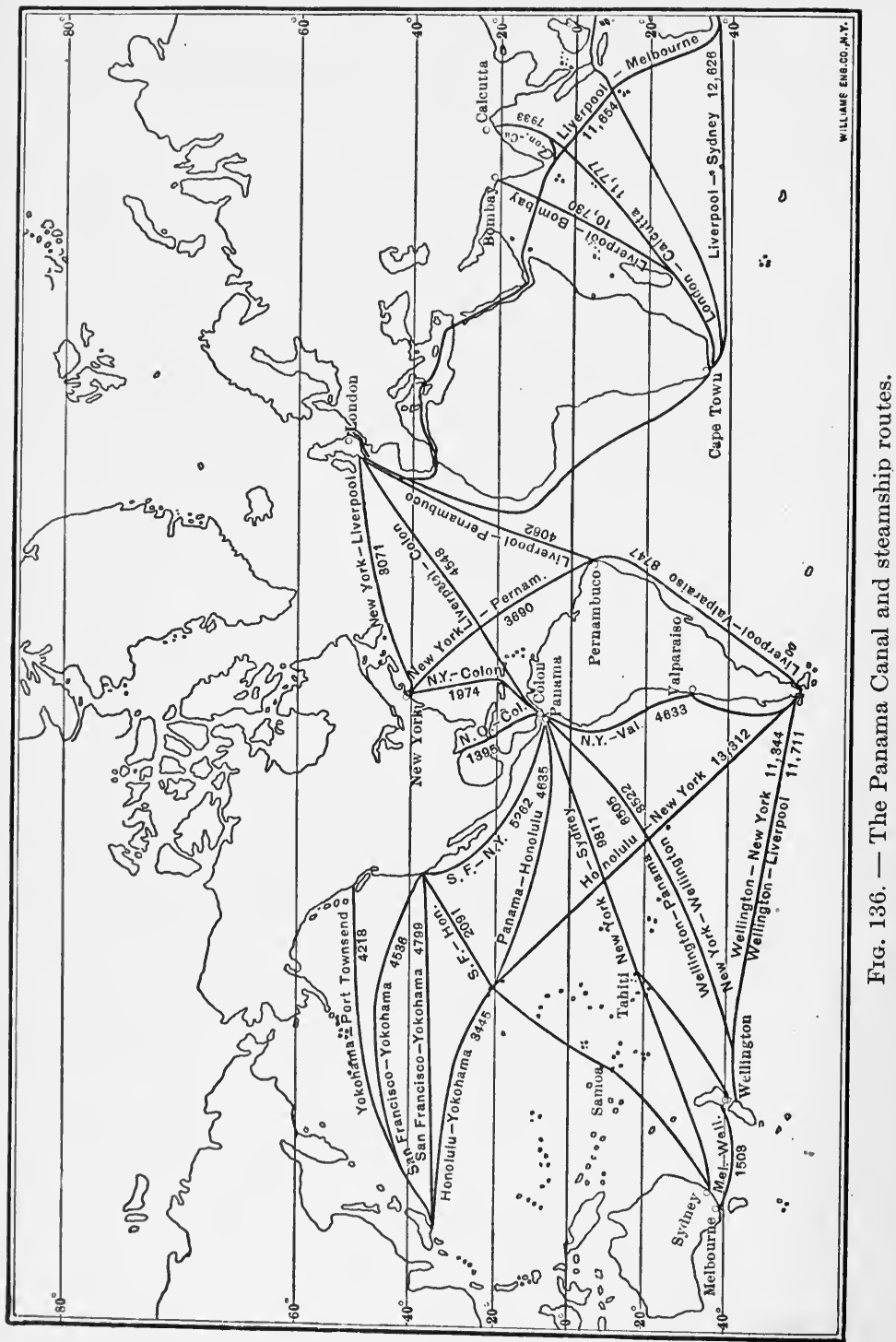


her commercial and trade life by means of canals, in the mileage of which she now leads the world. Other instances are to be found where such important constructions have changed the commercial relations and standing of cities.

The recently completed Marseilles-Rhone canal has a worldwide interest. Marseilles has a river traffic of about 40,000,000 tons a year. By means of the canal this tonnage will probably be doubled. The canal connects Marseilles and its Mediterranean traffic with Arles, a distance of fifty miles, and thus with the entire system of navigable streams and canals of the interior of France. The canal follows mostly the valley of the River Rhone, which has been little used because of its swift current and the sandbars and shoals at its mouth. A unique feature of the canal is the tunnel or subterranean waterway piercing the range of Nerthe, which has hitherto cut Marseilles off from the Rhone valley. This tunnel is 4.5 miles long and is 47.5 feet high by 77.5 feet wide. This is one of the most remarkable achievements of the French engineers in the twentieth century.

In the United States many canals of great utility have been constructed or are under way. The old Erie Canal, perhaps the first built in this country, about 1825, stimulated by its success the construction of many others. By the end of 1850 over 4500 miles of canals, at a cost of more than $\$ 200,000,000$, had been completed. A recent notable construction is the Sanitary Canal of Chicago, connecting the Mississippi River with Lake Michigan (page 192).

Canals which contribute to the immense traffic of the Great Lakes are the Soo Canal joining the waters of Lakes Superior and Huron, through which more than 50,000,000 tons of freight pass annually; and the Welland Canal around the Niagara Falls, which, however, is soon to be replaced by one to accommodate ocean-going vessels of large size.

175. Inland shipping of the United States. - Shipping on inland waters in the United States is of great importance. 
The volume of freight handled annually on the Great Lakes, for example, is enormous. Comparison with other waterways is impossible, for there is no similar amount of shipping on inland waters in any other part of the world. The Suez Canal is one of the busiest waterways; but the volume of freight handled by the Soo Canal is more than seven times that of the Suez Canal. The tonnage is far more than 50,000,000 a year, and a year there means a season of about twenty-five or twenty-six weeks only. Through the river at Detroit more than $70,000,000$ tons of freight are transported each six months of the shipping season. Approximately 80,000,000 tons of freight are carried annually over the waters of the Great Lakes.

This enormous amount of shipping is made necessary on the western side by (1) the extensive and rich ore deposits of Minnesota, Wisconsin, and Michigan; (2) the far-reaching grain-growing lands of the famous Red River Valley in Minnesota, the Dakotas, and Western Canada; and (3) the timber of the general western region sent mainly through the port at Duluth. On the eastward side, the shipping draws from the coal and manufacturing regions cargoes which are carried across the lakes and distributed at many ports for railroad transportation to inland points. At Duluth, for instance, the grain elevators have a capacity of about $40,000,000$ bushels, and the ore docks hold more than 600,000 tons in the bins. Other ports are equipped as their region of supply demands. One giant corporation alone has a fleet of more than two hundred vessels engaged in transporting ore to the amount of several million tons a year.

These few facts indicate the magnitude of the commerce of the Great Lakes, and its importance to the many cities located along the shores. Duluth, Superior, Two Harbors, Escanaba, and Marquette are among the ports of supply, while Chicago, Gary, Cleveland, Buffalo, and Toledo are well-known ports of discharge. By this great waterway the products of the East and Northwest are exchanged ; ores are brought to regions 
where fuel is obtained, foodstuffs are put into the markets, building materials supplied where needed, and coal distributed in regions where there is no natural supply. All this is done at a cost that is among the lowest in the whole system of transportation.

Exercise: How locks are operated. - The operation of a canal lock may be made clear by a diagram illustrating a longitudinal section of a lock (Fig. 137). Note these facts in your study of the diagram. A boat enters through gate No. 1, while gate No. 2 is closed. At what

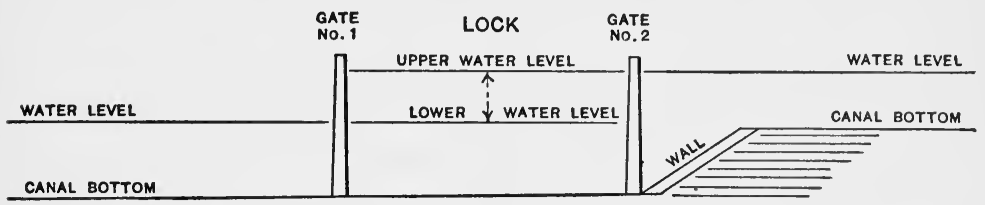

Frg. 137. - Diagram of canal lock. How is a boat lifted from lower water level to upper water level? How are gates operated to let a boat down to the lower water level?

level is the water in the lock? After the boat is in the lock, gate No. 1 is closed and No. 2 is opened. What change is thus made in the water level in the lock?

Show by diagram how a boat is lowered from a higher to a lower level of the canal. How are the gates operated for this? Describe the passage of a ship from the third level (see illustration of Gatun locks, Fig. 124) into the lake. Show how a ship passes from the lake to the lower level of the canal.

\section{SUMMARY}

The improvement of channels, harbors, docks, and facilities for handling freight makes possible the use of larger ships, insures their safety, and necessitates only a brief stay in harbor.

Navigable portions of the Mississippi and other rivers have been improved by dredging, the building of levees and jetties, and the marking of channels. They furnish a relatively cheap method of transportation.

The Panama Canal, large enough for our naval vessels, is of great defensive and commercial importance. It was begun by a French company in 1861, and was completed by the United States in 1915, at a cost of about $\$ 375,000,000$.

The completion of the canal was made possible in part by the dis- 
covery of the cause and means of prevention of yellow fever and malaria.

Other notable canals are (1) the Suez Canal, (2) the MarseillesRhone Canal, (3) the Manchester Ship Canal, (4) the Erie Canal, and (5) the Soo Canal.

Locks use the energy of falling water to lift the ships.

Our Great Lakes are a highway for a vast tonnage of ore, coal, and grain.

\section{REVIEW QUESTIONS}

1. Why is the improvement of harbors important? 2. What has been done to improve the harbor of New York? Of San Pedro? 3. What facilities for loading and unloading are found in the harbors of the Great Lakes? 4. Give an account of the amount of material carried by the Mississippi River per year. 5. (a) What has been done to prevent floods? (b) What to prevent the building up of the river bed? 6. What has caused a reduction of traffic on inland waterways and hindered their development?

7. Give a brief account of the construction of the Panama Canal. 8. (a) What are the chief advantages of the canal to the United States? (b) What are its advantages to the world? 9. What European inland cities are made seaports by ship canals? 10. (a) Explain how the locks are operated to pass a vessel from higher to lower level. (b) From lower to higher level.

11. Give an account of the importance of the Great Lakes as a waterway. 12. What materials make up the bulk of the freight on the lakes? Why?

\section{SUGGESTIVE QUESTIONS}

1. What was the effect of the construction of the Erie Canal upon the settlement of the Middle West? 2. How has inland transportation by water determined the development and location of cities? 3. What canal competes with the Panama Canal in European and Asiatic commerce? 4. Which is the shortest route between Liverpool and Yokohama? Liverpool and Valparaiso? 5. In what direction does a vessel pass from the Gulf of Mexico to the Pacific Ocean through the Panama Canal? 6. The water of Gatun Lake has become salty since the canal has been in use. As it is 85 feet above sea level, how do you account for it? 7. If the rainfall of the Isthmus should cease, could the canal be operated? 8. Will the canal be useful to sailing vessels going East? 9. How is coal transported to (a) Cincinnati; (b) St. Louis; (c) New Orleans? 10. Why did the United States Steel Company locate its plant at Gary, Indiana? 


\section{CHAPTER VIII}

\section{TRANSPORTATION IN THE AIR}

176. Early dreams of flying. - "The way of a bird in the air" was even for Solomon an object of envious contemplation. The bold endeavor of Dædalus, though quickly disastrous, the flight and speed of Pegasus and the Magic Carpet, the flying machine of Darius Green, and the far-reaching fancies of Jules Verne were anticipations of what has to-day become a reality that is still rich in unfulfilled possibilities. It is to-day comparatively simple to rival in speed the flight of a bird; and transportation through the air is now fully within the range of the practicable.

177. Types of aircraft. - Men have made the air a medium of travel and transportation by two different types of aircraft. One type is represented by the balloon, which is lighter than air, volume for volume. It is lifted by the buoyant force of the air in the same fashion as water buoys up bodies. The other type is the heavier than air machine, the air-

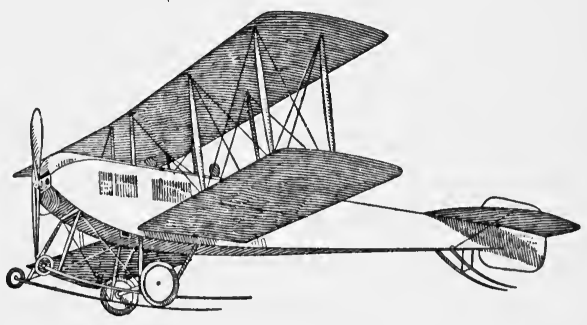

Frg. 138. - A biplane. plane (Fig. 138), which is sustained as it moves by the pressure of the air on the lower surfaces of its planes. The airplane is lifted as the bow of a boat when moving rapidly, and as the hydroplane rises when it is driven forward. 


\section{BaLloons}

178. Early balloons. - The first type of balloon with which experiments were made was the hot-air balloon. The familiar toy balloon is usually of this type. The air inside the balloon is expanded by heat and is therefore less dense than the outside air. Hence the balloon is forced upward and will remain in the air as long as the temperature difference is maintained. Such a balloon was made to carry one or two passengers, but failed to render any valuable service.

In the latter part of the eighteenth century hydrogen was discovered (page 117) and the means of producing it in quantities was devised. This made ballooning more practicable. Then the discovery was made that illuminating gas, which contains a large percentage of hydrogen and carbon monoxid, could be substituted for hydrogen; but because its average density is greater than that of hydrogen, it had only a limited use.

Balloons were not under control when in the air, save in ascending and descending. They drifted with the wind. By throwing out ballast the balloon could be made to ascend; and by opening a valve to let hydrogen escape, it could be made to descend. Balloon ascensions and drifting races furnished some excitement for the spectators and adventure for the aëronaut. Such balloons are not of great utility, however, except in the investigation of the atmosphere, its movements, density, temperature, and amount, at different high altitudes.

179. Use of balloons in study of atmosphere. - A few important facts about the atmosphere which were discovered by means of the balloon may be mentioned in this connection. Two English aëronauts in a gas balloon attained the"record height of seven miles. They found the temperature at this altitude about $-60^{\circ} \mathrm{F}$., and the pressure only one fourth that at sea level. They nearly perished in their adventure. With their limbs paralyzed by cold and weakness, they owed their 
rescue to the ingenuity of one of them, who pulled the valve rope with his teeth. A French aëronaut later reached the same altitude, but he carried with him a tank of oxygen to supply air. These discoveries of the character of the atmosphere at high altitudes led to further investigations by the aid of exploring balloons carrying no human passengers.

The exploring balloon is equipped with delicate recording instruments which respond to differences in temperature, and pressure or amount of atmosphere. The quantity of hydrogen in such balloons is such that its expansion will burst the bag at a given altitude. Why does the gas expand as the balloon ascends? The instruments are attached to a parachute which saves them from destruction. As they may be carried by air currents miles away from the starting place, they are tagged with the address and directions for their return; when the finder returns them he is rewarded for his trouble.

The upper air - its pressure and amount. - The Italians have led in such researches, one of their exploring balloons ascending to the height of 23 miles. The record of the barometer shows the pressure of the air at different altitudes. Mountain climbers had reported that there is hardly enough air at the height of 20,000 to 24,500 feet to sustain life. At about 19,000 feet practically only one half of the total amount of air is available. The barometric record obtained by the Italians' balloon verified these observations. At the height of 7 miles, $\frac{3}{4}$ of the air is left below the balloon; at 23 miles' height, $\frac{299}{300}$ is left below, only $\frac{1}{300}$ remaining above. Since the thinning out of air from sea level to the highest investigated altitude is very gradual, there is a high degree of probability that this $\frac{1}{300}$ part is so expanded and diffused that it exists in traces a hundred miles higher. Certain observations support this belief. Meteors begin to glow a hundred miles distant from the earth's surface; the heat causing the glow being perhaps due to friction with the air through which they move. The after-glow of sunset and the aurora indicate the presence of air at such altitude; indeed the 
aurora has been found by exact measurement to reach the height of one hundred miles.

The temperature of the upper atmosphere shows a similar change. It gradually falls until at the height of seven miles the thermometer registers about $60^{\circ}$ below zero Centigrade. At greater altitudes the fall is such that it appears to be altogether certain that the space beyond the earth's atmosphere has a temperature very nearly as low as what is known as "absolute zero," or $-273^{\circ} \mathrm{C}$.

180. Why balloons float. - In studying the conditions that cause objects to float in water, it was found that a floating body displaces its own weight of water. A gigantic log raft, containing millions of feet of lumber, floats because it has apparently less density than water. But a ship may be built of steel, which has a greater density than water. The reason is this: the steel structure incloses a vast body of air from which water is excluded, so that the total volume of steel and air has an average density that is very much less than that of water. Consequently a steel ship heavily loaded floats (page $344 \mathrm{ff}$.).

Average density. - The principle involved in floating a ship on water is exactly the same as that in floating a balloon in air. The average density of the balloon and its load must be less than that of the air. It is impossible to construct a steel shell for a balloon and exhaust from it the air in order to reduce its average density enough to allow it to float. A steel shell light enough to float would be crushed before the air it contains could be exhausted. What would be the pressure per square foot? If a shell inclosing a vacuum could resist the pressure, its average density would be far greater than that of the air. Hence, a light strong material such as silk or rubber is selected for the construction of the bag or envelope. This is filled with hydrogen or other suitable gas, which by its pressure against the inner surface of the envelope displaces the air, and the balloon rises.

Example. - Suppose a balloon, including envelope, basket, ropes, and other equipment, weighs 150 kilograms, and the envelope holds 
400 cubic meters of gas. If hydrogen is used, how much of an additional load can it carry at sea level or where the air is of normal average density. It is necessary to know the densities of hydrogen and of air. (What density was required in solving problems of objects floating on water?)

Air weighs 1.3 kilograms per cubic meter; hydrogen, 0.09 kilogram per cubic meter. Thus we have the following data:

Weight of solid materials of balloon . . . . . . 150 kilograms Weight of hydrogen $0.09 \mathrm{k} . \times 400$ (cubic meters) . . 36 kilograms

Total weight . . . . . . . . . . . . $\overline{186 \text { kilograms }}$ Weight of air displaced, 400 cubic meters by gas

1 cubic meter by solids

$401 \times 1.3\left(\mathrm{k}\right.$. per $\left.\mathrm{m}^{3}{ }^{3}\right)$. . . 521 kilograms

Buoyant force of air displaced . . . . $521 \mathrm{k}$.

Weight of balloon, etc. . . . . $186 \mathrm{k}$.

Unbalanced force upwards . . . . . . . . 335 kilograms

How heavy a load can the balloon carry and still float? If you desire to change these numbers to English units, you may take one kilogram as equivalent to 2.2 pounds; and one cubic meter equivalent to about 1.3 cubic yards.

181. Dirigible balloon. - Although the gas balloon has not proved to be commercially important, it has made possible a better knowledge of the world in which we live. But a new step has been taken in the construction of airships, which utilizes the internal combustion engine, electric ignition, and self-starting devices. Drifting with capricious air currents at high altitudes is not especially profitable; steering and propulsion are indispensable. Steering in the upper air is not, however, so simple as steering on land or sea with a motor vehicle; starting from and returning safely to the earth, both being hazardous to men and machinery, must be mastered. The airship, moreover, must have stability; that is, it must keep right side up, and be at all times under the control of the aëronaut.

It was early found practicable to combine without much risk of explosion the gas engine with the hydrogen balloon. The achievements of Santos Dumont in the years 1901-02 proved the utility of the petrol or gasoline engine for this purpose. The 
combination of steering and propelling by a motor made possible the present airship of the Zeppelin type. Such an airship, combining motor and balloon, is called a dirigible. The characteristic shape of the Zeppelin dirigible is determined by the conditions which it must meet. What are these conditions? How does the form of the balloon help in meeting them?

\section{The Airplane}

182. Early experiments. - The belief had long been held by a few individuals that a "heavier-than-air " machine, lifted by its own power and sustained by the pressure of the air against its surfaces, after the fashion of a kite, could be made to work successfully. In short, a machine, resembling somewhat a bird in form, seemed altogether possible. Dr. Lilienthal, a German, experimented with wings having an area of about 160 square feet, or about one square foot for each pound of his weight. By running down a hill, up which the wind was blowing, he found the current sufficiently strong to lift and float him. Unfortunately the wings collapsed when he was in mid-air and the fall killed him. Others tried the same plan, with fatal results in most cases. These experiments, however, brought knowledge of important conditions of equilibrium in air, upon which any flying machine depends. As a child must learn to maintain balance and walk before he can run, so man must master the conditions of maintaining equilibrium in the air before he can fly.

Two remarkable series of experiments were being carried on while Dr. Lilienthal and others were trying the wing-sails. Sir Hiram Maxim took up the problem and tried first to find the best possible fabric for the planes, testing silk, cotton and woolen fabrics, crêpe, linen, and other materials. His tests proved that lifting power of linen is much greater than its tendency to drift horizontally in the direction with air currents. He then worked to construct a steam engine of maximum power and minimum weight. Finally he built a machine, weighing about 7500 
pounds, that began its flight and returned suddenly but safely to land because of a broken propeller blade.

Langley's experiments. - The other series of experiments was carried on by Professor S. P. Langley of the Smithsonian Institution, Washington, D. C. All of Professor Langley's work was most carefully calculated and tested in detail. His "aërodrome," equipped with a steam engine, made a trial trip of more than 900 yards at the rate of 25 miles per hour. But this demonstration won from the newspapers and the public little more than ridicule and discouragement. Langley labored on faithfully and by his discoveries made possible the success of the present aircraft. The great obstacle to unqualified success in his machine was the motor. Late experiments with his "aërodrome" when equipped with a suitable gas engine prove that his belief in its lifting, flying, and landing properties was justified. He had determined the essential conditions of flying; starting the machine on its course, lifting, balance, rigidity, and landing.

183. Recent progress. - Undaunted by the accidents that befell other experimenters in aërial navigation, the Wright brothers, Wilbur and Orville, attacked the problem. They made themselves masters of what others had learned, especially the discoveries of Langley and Chanute. In 1901 they could glide about 600 feet downhill against the wind. By gliding they learned to control the direction and the balance of the wings or planes. In 1903 they combined with the planes a gasoline motor and made flights of about one minute's duration. Thus they created a machine that lifted and sustained itself and its load, the pilot, in the air by its own power. Gradually they perfected the machine, planes, and motor, until they could fly farther and for a longer period of time. In 1908 Wilbur Wright took his machine to France, where he made flights with passengers. He remained in the air about two hours and rose to the height of 400 feet.

Progress has been since made at a fairly rapid rate. In 1909 
Glenn H. Curtiss, whose name and work are widely known, won the race for speed at Rheims, France, attaining a speed of 47 miles an hour. To-day machines are made to carry several passengers, to move at an almost incredible rate of speed, to remain in the air for many hours, - tests have now reached more than twenty-four hours' continuous flight, - and to attain altitudes formerly supposed possible only for gas balloons. The control of the machine in the air is remarkable, as shown in the marvelous feats by expert airmen, such as looping the loop, flying upside down, and apparently leaping from place to place. The uses of the airplane and the hydro-airplane are familiar to all, but the extent of their services to man can only with difficulty be realized.

184. Airplanes and kites. - Some of the chief difficulties met in the development of flying machines have been mentioned. The principle on which the flight of the airplane depends may be comparatively easy to understand, but its realization in machinery and planes was not easily or quickly made. A flat stone when properly thrown sails a long distance, and illustrates some of the points of the flying machine. But the simplest device by which the principle of flight may be made clear is the kite, familiar in scientific investigations as well as in the play of children. The kite depends for its flight upon the moving air or wind against which its lower surface is presented at a suitable angle ; but the airplane makes its own wind by moving forward, its planes being presented against this wind. This difference may be neglected for the present.

Exercise: Why kites fly. - In the diagram (Fig. 139) the entire force of the wind is represented as located at the center of the kite. A line representing the wind force or pressure is drawn at right angles to the surface of the kite. The action of the string is shown similarly. We have to find what the lifting force is when the wind pressure and the position of the kite are known. The procedure is this: from the center of the kite, which may be represented by a point, draw a line of indefinite length and at right angles to the kite. Select a scale to be employed throughout, as 10 grams to one centimeter, in order to 
indicate the magnitude of a given wind pressure. Measure on the wind pressure line the right distance, marking the end with the point of an arrow. What is the length of a line to represent 50 grams? 40 grams? 10 grams?

From the point you have taken as representing the center of the kite, draw a vertical line to show the direction of the "lift." To find

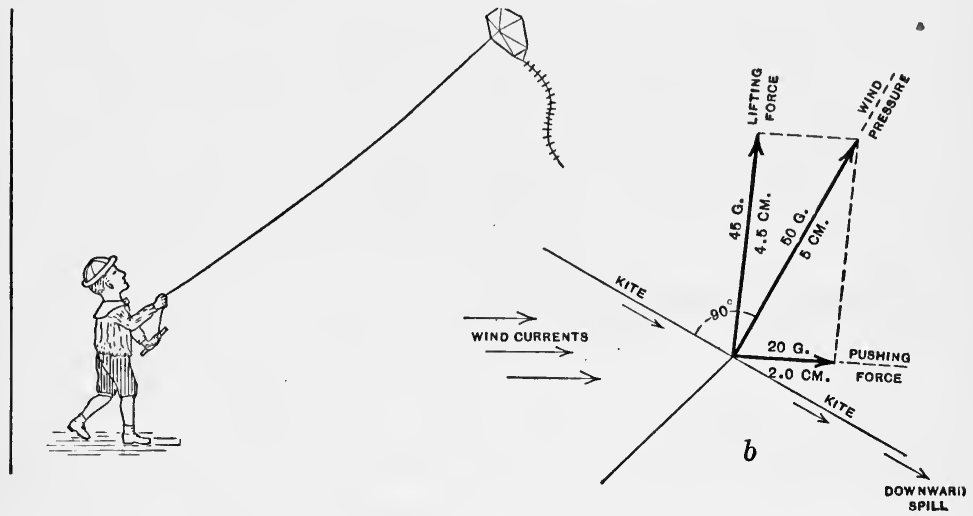

Fig. 139. - Diagram to show why a kite flies. Make your diagram on a scale of $1 \mathrm{~cm}$. to 10 grams.

the magnitude of the lifting force, draw from the end of the wind pressure line a line perpendicular to the vertical line. Measure the length of the vertical line to the point of intersection, and compute the magnitude by use of the seale number. What force would be represented by a line 4 centimeters long? 12 centimeters? 15 centimeters?

Reference to the problem of sailing a boat into the wind and the parallelogram of forces will be helpful to you in the following problems. In what position will the kite be when the lifting force is most nearly equal to the wind pressure? Show the magnitude of the drift or pushing force as you have that of the lifting force.

What must be the angle of the kite to the wind, in order that the lift and drift be equal? If this is not at once evident, it may be determined by experimental drawing with the kite placed at different angles.

Can a kite be made to fly with the string exactly vertical? If the string breaks, and the kite is allowed to move with the wind, what happens to the kite? If there is no wind, can a kite be made to fly? 
How? Can a kite be made to fly from a moving steamer's deck during a calm? If a steamer were moving at the rate of ten miles an hour, and the wind were blowing at the same velocity in the same direction, would a kite fly?

Skin friction. - Besides the drift due to the fact that some of the kite surface is presented horizontally to the air, there is also a drift due to the friction of the air moving over its surfaces. This is called "skin friction." Whether this be little or much depends upon the form and roughness of the surfaces. This factor must be taken into account in the selection of material for the planes of an airplane.

Forms of vanes of airplanes. - In the building of airplanes much careful experimental work has been done to determine

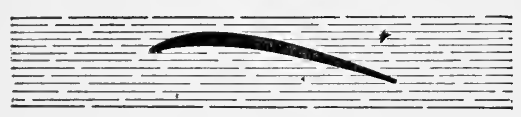

Fig. 140. - Cross section of plane and horizontal air current. Compare with corresponding cross section of a bird's wing when in flight. what form of vanes will give the greatest lift with the least drift. The drift in the airplane represents the pressure to be maintained in the forward direction by the thrust of the propellers. The

lift is the power of the machine to sustain itself and its load. Curiously enough the supporting vanes are now shaped somewhat like the curve shown in Fig. 140. The resemblance to the form of the bird's wings is apparent. Experiment has shown that this form is best adapted to flying, a fact already established by natural selection in the evolution of flying creatures.

Stability. - Besides the form of the planes there are other problems of even greater importance in the airplane. The machine must have stability, equilibrium, or balance in all directions. Otherwise it might tip to its destruction in any. one of four ways. The tipping forward and backward "can be controlled by horizontal rudders, fastened some distance astern. If the plane starts to dive forward, it is automatically righted by the increased air pressure of the upper surface of the horizontal tail piece. 
Tilting to one side or the other may be controlled by several methods. It may be partly met by the instinctive leaning of the pilot to the side opposite the tilt; but his weight is not sufficient to balance the machine. The back of his seat is often made movable, and to its top are attached wires connecting with the rear tips of the planes. If the machine tips toward the right, the aviator leans instinctively and automatically to the left. This movement pulls the rear edge of the right planes down so that they meet with more air resistance. The extra lifting force thus secured under the right plane restores the balance of the machine. Of course if the pilot wishes to turn to the right, he leans a little in that direction just as a bicycle rider does in turning a curve.

Some machines are equipped with automatic stabilizers of different sorts. These utilize the gyroscope, the pendulum, or an apparatus controlled by the pendulum. Other machines are designed and built in such a way as to secure what is known as inherent stability, or "autostability." This is secured in some measure by the arrangement of the central surfaces of the machine, a deep vertical keel, and a low center of gravity.

It is interesting to note that Professor Langley, the pioneer in America in planning and constructing flying machines, thought out carefully and described in his notes substantially every method or system, which has since been tried, to secure stability. He worked out the principles of the proportions of parts of a machine, suggested and tried apparatus for control, and succeeded in laying the foundation for the attainment of inherent stability, although this has only lately been recognized.

185. The motor. - The engine by which motive power is generated for the airplane has from the beginning been of utmost importance. A satisfactory engine must combine dependability and great power with speed and the least possible weight. A steam engine on one of the modern transatlantic liners will weigh, including the boilers, about 100 pounds for each horse power produced. A gasoline engine such as is generally used 
on the automobile has about ten pounds weight for each horse power generated.

Inasmuch as it is necessary to provide for airplanes engines that will produce one to three hundred horse power, it is evident that weight becomes a very important factor. Then there must be included the weight of fuel necessary for an engine of 100 to 300 horse power to make an unbroken trip of five or more hours. For an engine developing 100 horse power, about fifteen gallons of gasoline per hour may be used, and with this must be included several gallons of lubricating oil. Counting seven pounds to the gallon, a load of 100 gallons is seen to be no small item. Should a transatlantic flight be attempted, its success will depend in a very large measure upon the motor and the fuel load that must be carried.

Recent improvements in motor construction have brought about a significant reduction in bulk and weight together with a great increase in power and reliability. The " $V$ " form of the "twin-six" gives exceptionally great power with less weight and less fuel. A motor of recent production, water-cooled, weighs seven hundred pounds, and, with a normal speed of 1200 revolutions a minute, generates 150 horse power. Another of the two-cycle type, of the same form, and with twelve cylinders, weighs only 600 pounds and gives with a speed of 1150 to 1400 revolutions a minute, 150 to 180 horse power. These facts indicate the tendencies in motor design and construction for airplanes. A weight of two and one half or three pounds per horse power is now entirely possible. Such an achievement will do much to make the airplane a machine of practical importance in transportation and travel.

186. Commercial utility of aircraft. - Thus far the airplane, in spite of its many forms, has not had great commercial utility. But the improvements which are being made in its construction will undoubtedly be applied to machines for commercial purposes. The perfecting of the motor will eventually increase the carrying capacity of the airplane. The produc- 
tion of a machine that will be perfectly self-balancing, or " autostable" as it is described, and which will insure safety to life and to the machine, will contribute greatly to its adaptation to modern transportation.

\section{SUMMARY}

The two chief types of aircraft are the heavier than air type, and the lighter than air.

Drifting balloons are of use in determining the conditions of the upper regions of the atmosphere.

A balloon rises because it displaces a weight of air greater than its own weight.

The need of more complete control of the balloon in flight led to the invention of the dirigible.

The development of the heavier than air machines is the result of the labors of many experimenters. The pioneers include Lilienthal, Maxim, Langley, the Wright brothers, and Curtiss.

The principle of the airplane is the same as that of the kite.

Stability is secured by the design and construction of the machine, by the action of the pilot, and sometimes by automatic devices.

The success of the airplane depended chiefly upon the development of a light and efficient gasoline motor.

\section{REVIEW QUESTIONS}

1. (a) What are the two chief types of aircraft? (b) How is each sustained? 2. (a) What kind of balloon first carried passengers? (b) For what are hot-air balloons now used? 3. Compare hydrogen and illuminating gas for filling balloons. 4. How high above the earth's surface have men gone? What conditions of temperature and pressure did they find? 5. What difficulties attend ballooning? 6. (a) With what instruments are exploring. balloons equipped? (b) How is their safe return provided for? (c) To what height have they gone? (d) What has been learned by their use about the atmosphere, as to its pressure and temperature? 7. What phenomena are due to the presence of air at an elevation of fifty miles or more? 8. (a) Compare the construction of a balloon with that of a ship. (b) Why do they both float? 9. What is meant by average density? 10. What is the difference between a drifting balloon and a dirigible balloon? When did the successful use of dirigible balloons begin? 11. What advance beyond the use of the balloon was made in air $2 \mathrm{D}$ 
navigation by Lilienthal? 12. Describe the experiments of Hiram Maxim. 13. Why is it not practicable for men to attempt flight with flapping wings? 14. (a) Give an account of the work of Professor Langley. (b) What principles of aircraft did he discover? 15. Give an account of the progress in aërial navigation made by the Wright brothers. 16. What are some of the records made by airmen? What unusual feats? What do these show as to the control of aircraft?

17. In what respect does a flying kite resemble an airplane in action? In what respects does it differ? 18. (a) Why does a kite fly? (b) What is meant by the lifting force and the drifting force? $(c)$ From what are the two forces derived? (d) How is drifting prevented? 19. What is the importance of surface friction in kite and airplane? 20. What is the form of a vane? In the airplane how is the form important? 21. (a) Explain how equilibrium is maintained in an airplane. (b) What automatic stabilizers are used? 22. What difficult conditions does the pilot find in the atmosphere? 23. In what way is the airplane dependent upon its motor? 24. (a) Why are size and weight of motor important? (b) What improvements in motors have recently been made?

\section{SUGGESTIVE QUESTIONS}

1. What aircraft have you seen? Of what type was each? What speed did it make? What length of flight? 2. What would have to be done to make possible a safe and comfortable ascent to altitudes above seven miles? 3. (a) Would it be possible to make a voyage to the moon in an airship? (b) What conditions of pressure, density, and temperature would be encountered? 4. What attempts have been made to cross the Atlantic in airships? What difficulties would be encountered in such a trip? 5. What advantages do you imagine the use of aircraft would give in Arctic exploration? 6. In what ways could air navigation be of use in our country? What advantages would it have between New York and Chicago? Los Angeles and New Orleans? 


\section{CHAPTER IX}

\section{BUILDING MATERIALS, THEIR USE AND ORIGIN}

All animals need some protection from the unfavorable features of the weather and from dangerous enemies. Man has even less ability to endure unprotected the extremes of climate or to resist by his muscular strength alone the attacks of his foes. Through his intelligence, however, he has made his life more secure than that of any other animal species.

187. Primitive shelter. - In the very early ages, man found shelter from storms and from extreme heat or cold under trees or in fireless caves. He avoided dangerous animals by climbing trees. Later he learned to construct rude habitations of branches, bark and leaves, grasses, and stone. These dwellings afforded him many advantages not to be had in caves or other natural shelter. For purposes of better defense against enemies these dwellings were often built close together; and by living in villages primitive men learned the value of working together or coöperation in many pursuits.

188. Permanent buildings. - After a while men ceased to be nomads and settled down to the cultivation of the soil and to tending their flocks and herds. Then they began to build with an idea of permanence and greater protection against foreign enemies. They made use of minerals and hewn timbers for building materials. In the fertile valleys of the Tigris, the Euphrates and the Nile where the population multiplied rapidly, such a change in mode of living and building came comparatively early.

Dwellers along the Nile found stone useful for their purposes. Their engineers made use of blocks of stone of tre403 
mendous size and weight, which they transported on some sort of a sledge to the river and then on barges to their destination. The two giant statues of the Pharaohs at Thebes were each carved from a single block of stone weighing as much as one thousand tons. Probably the common people of the valley dwelt in adobe houses similar to those in the southwestern section of the United States. The Chaldeans and Babylonians made use of clay brick in most of their buildings. Are any of their structures standing to-day? Of what materials were the Greek and Roman public buildings, bridges, and homes constructed? Why were such materials used? What materials utilized by ancient builders proved most durable?

Properties of building materials. - A hasty survey of the building materials utilized in the past suggests that a perfectly satisfactory material for building must be abundant, easily obtained, readily shaped or adapted to the use or design, durable, fire-resistant, a good non-conductor of heat, practically impervious to water, and not liable to crack and crumble with changes from wet to dry or from cold to hot weather. Is there any material which meets all these requirements?

Exercise. - What qualities do trees and lumber possess? What properties are found in brick or other clay products for building purposes? What are the properties of stone, iron and steel, and concrete?

What are the chief differences between the requirements of building materials for large cities and those for villages or rural buildings? What are the principal reasons for the differences? Consider in your answers the factors of fire, collapse, and decay.

\section{WooD}

189. Properties and use. - In the United States more wood is used at present for building than any other material." What are the reasons for this? What change in regard to the use of wood for building is being made in cities? What are the reasons for such a change? Make a list of the building materials used in your community, noting especially the following points: 
those used because they are readily accessible; those because they are easily worked; those because of durability, strength, fire-resistance; those brought from other places; those used for beauty or ornamentation.

Exercise. - Study the different kinds of wood used in the construction of a modern building as a house, school, or church, and give reasons for their selection for each of these uses: structural parts as supporting timbers, rafters, and floor joists; doors, windows, and other parts of.interior finish; roof. How much of the work of preparing the materials for their present use was done on the ground? How much in a mill or factory? How much was done by hand? by machine?

190. Lumbering. - In olden times buildings were mostly constructed by slaves and with little regard to the labor involved. Materials were prepared largely on the ground and by hand. But in our day the economy of materials and construction must be considered. Consequently a great deal of the material is prepared in factories or mills by machinery and made practically ready for the builder to put into place.

The changes in transforming tree trunks into suitable materials for buildings include four groups of operations: first, the logging, which comprises felling the tree, preparing the log, and transporting it to the sawmill; second, the lumbering, which includes sawing the log into commercial dimensions and generally the drying and surfacing of the sawed lumber; third, the mill work in which much of the finishing is done of doors, sashes, windows, and the like; fourth, the assembling and fitting together of the parts by carpenters.

191. Preserving wood. - The chief objections to the use of wood in building are its combustibility and liability to early decay. Neither of these can be wholly prevented by any known means. For the preservation of wood from decay such substances as paint, wood tar, coal tar, and creosote are generally used. Paint is a mixture of oil, such as linseed oil, and 
some mineral such as white lead, zinc white, and litharge. Creosote is a product derived from coal tar. Where appearance is considered in dwellings and other buildings, paint is used. Tar and creosote are used to preserve piles, telegraph . poles, railroad ties, and shingles.

\section{Stone}

192. Properties of building stone. - Among the materials commonly used in building and construction are the various kinds of rock or stone. About $\$ 80,000,000$ worth of stone is used in this country in a year. Of the many kinds of rock used in building there are several distinct classes which have different qualities. Some are comparatively easy to cut and shape, but do not take a high polish. Of these, sandstone and slate are common examples. Other kinds, such as marble, are very heavy or hard, capable of taking on a high polish, and are used largely for ornamentation in buildings. Still others, as granite, are very hard, with gleaming crystals of beautiful colorings in their texture.

Durability is an important factor in stone used for building purposes. The climate of the northeastern part of the country is especially destructive to stone. Cleopatra's Needle, which had stood in the dry climate of the Nile Valley for more than eighteen centuries without showing greatly the effect of weather, quickly began to disintegrate after it was placed in Central Park, New York. It is now protected with a coating of paraffin. The following table shows the length of time stones can remain exposed to the weather in New York City and vicinity before their surfaces begin to crumble or flake off.

Brownstone (fine and compact) . . . . . . 100 to 200 years Sandstone (best quality) . . . . 100 years to many centuries Granite

Limestone

Marble (fine)

Gneiss 
193. Sandstone. - Sandstone is easily quarried and cut into shape for building purposes. The irregular blocks which come from the quarries are dressed either by hand or by machinery. The stone mason is then in about the position of the carpenter who has had most of his sawing and planing done at the mill. Sandrock has various colors depending upon the presence of minute quantities of iron and other mineral matter in it. It is often used in thin slabs to form facings of rough walls, or carved into various shapes for interior decorations. A certain kind of sandstone found in Ohio hardens on exposure to the air. This property gives it a wide market.

Formation of sandrock. - Notice the resemblance of a piece of sandstone to sand. It was sand before it was made rock. Sand with traces of lime was deposited as sediment in water in a region which was gradually sinking. Rocks formed in this manner are said to be sedimentary. Other deposits of clay, sand, mud, or volcanic lava buried the sand layer or stratum (strata, plural) and with their increasing depth subjected the sand to ever greater pressure. Under a blanket which may have been thousands of feet thick, the sand stratum was changed in the course of untold ages by the pressure, and was welded and cemented into sandrock. Then it was lifted above the waters by titanic forces involved in the shaping of the earth's surface and was left in the positions we find it to-day.

194. Slate. - Familiar uses of slate are for roofing and for blackboards. For both of these uses it is unsurpassed. The peculiar advantage of this rock is that it can be split along a certain plane about as easily and as smoothly as a piece of wood. This is the result of the heavy pressure to which it has been subjected in the course of its formation, and not to the fact that the original clay was deposited by the water in layers or strata.

Slate quarries may be from 200 to 300 feet deep. The blocks after being taken out are sawed into the desired dimensions 
much as blocks of wood are, although the saws move much more slowly. The splitting of slabs is the most interesting part of their preparation for market. A block about four inches thick and measuring on its face two and one half feet by five feet is placed on end and split into four slabs, each one inch thick. The slabs are put upon revolving platforms and ground to the desired thickness and smoothness of surface.

Slaty rock, which is not strong and readily crumbles under comparatively light pressure, is called shale. Slate was once shale; shale was once mud; and the mud beds were deposited by water as sediment derived from the disintegration of other rocks.

195. Granite. - The story of granite is quite different from that of slate and sandstone. Examination of a piece of granite shows that it is made up of several different kinds of minerals which are usually in crystalline form. The glassy granular particles which do not have the appearance of crystals in granite are quartz. The white, gray, or salmon-colored crystals, usually the most abundant of the constituents, are felspar. And the black glistening crystals are either mica or hornblende. Granite in which hornblende occurs instead of mica is called hornblende-granite. When felspar is in the form of large crystals embedded in a finer crystalline mass, the rock is described as porphyritic granite. Granite is variously colored and takes on a high polish.

Because of its structure and hardness granite is difficult to work and is correspondingly expensive. Its crushing strength is so great that it is often used in foundations for the heaviest structures.

Granite is very widely distributed. It occurs extensively not only at or near the surface, but perhaps as the great mass of rock structure of the deeper part of the crust. In mountain systems as the Sierra Nevada or the Himalayas, granite forms the core and appears in such magnificent "dome" structures as those in the Yosemite Valley. The giant monoliths that break 
up the level prospect of the desert lands of the West are often granite. Granite also cuts across other rock formations and is then found as veins. In the New England region granite, generally of the gray color, occurs over a large area. The abundance, durability, and beauty of this rock account for its wide use.

Granite is very durable; but it eventually decays, although the weathering process is exceedingly slow. The felspar may be broken up into very minute particles which form clay. The rest of the rock may form sand, though the mica and hornblende may finally be dissolved away by water. Or the felspar, mica, and quartz may be broken up into exceedingly fine particles to form mud. Granite is thus seen to be the source of the materials which often make up sandrock and shale.

Formation of granite. - When you first examine a piece of granite you may suppose that, like sandstone, it is made up of small particles. But this is not the case. The particles are crystals, as examination will show, and were formed in place. They also show the effects of the heat to which they were subjected and of the manner in which they were cooled. Granite is formed from the molten mass that cooled gradually and consolidated beneath the earth's surface. In the lavas which came molten to the surface are found crystals which were formed before the lavas were poured forth upon the earth. In rock of this kind the felspar crystals are conspicuous; but the rest of the rock is fine-grained, and the crystals that have had time to form under the conditions of quick cooling at the surface are very small. This kind of rock is described as porphyritic (page 408). In the rock which formed beneath the surface of the earth, the crystals are complete individuals and conspicuous, and they give to it its characteristic features. This condition of the rock structure indicates that it cooled gradually and slowly enough to allow crystallization to continue without disturbance. This is the rock commonly called granite. Because such rock is formed from molten materials, it is gen- 
erally described as igneous (from the Latin term ignis, meaning fire).

196. Limestone. - Forty per cent of all stone quarried in the United States for building and construction purposes is limestone. The annual output is valued at about $\$ 40,000,000$. But limerock is quarried for other purposes, still larger quantities being utilized in the preparation of building materials, as lime and cement, or lime for fertilizer, and in the smelting of iron and certain other metals from their ores. Great quantities are moreover used as ballast on railroads and in making highways.

Limerock is widely distributed in the United States. It is generally more accessible in regions east of the Rocky Mountains. In quality, and therefore in adaptability for different purposes, it varies greatly. The best limerock for building is found in Indiana; it is excellent on account of its good color, evenness of grain, freedom from cracks, and the ease with which it can be cut and carved with tools. The vast bulk of rock quarried elsewhere is used for making lime and cement and for reducing ores (pages 438-9).

Limerock, like sandstone, appears in layers or strata. The stratified character of the rock is significant in at least two ways. First, it indicates with certainty the relative age of the different strata where these have not been greatly disturbed by upheaval, crumpling, erosion, or other geological processes. The lower strata are necessarily older than those higher or nearer the present surface of the earth. Second, the stratification shows clearly that the materials of which such rock is composed were deposited by sedimentation.

Sedimentary deposits are made almost always under water. Well-stratified deposits occur only in bodies of still water, such as lakes or seas where the materials deposited are not regularly and continually supplied, but only intermittently as when streams swollen by rain or flood carry large quantities of suspended material. This also suggests that land surfaces and 
sea and lake bottoms have changed their relative positions, the land surfaces of to-day having been the bottoms of lakes or seas in former ages of the earth's history.

Stratification in the structure of rocks, such as limerock, sandrock, and slate, is advantageous in quarrying. The strata are separated slightly by what are called planes of cleavage and they are also broken crosswise by cracks called joints. These features are decidedly useful in quarrying and preparing the rock for use.

Limerock is soluble in practically all natural water which contains carbon dioxid, but not so freely in pure water. Parts of many kinds of rocks are easily soluble, but the relatively insoluble parts, making up by far the greater bulk, remain as soil. Limestone is almost wholly soluble. This helps in understanding the rapid weathering of limestone when exposed as in buildings, and also the formation of caves in limestone regions. Water charged with carbon dioxid makes its way through cracks or fissures of limerock, dissolving the limerock and widening the fissures into caverns. The Mammoth Caves in Kentucky and the Luray Cavern in Virginia are familiar examples of the solvent power of water in limerock. In such caverns, moreover, the water, saturated with lime carbonate, dripping through the roofs and falling upon the floors, is evaporated, leaving deposits of limestone. These deposits when suspended from the roof are called stalactites, and when built upon the floor, stalagmites; when these join, as they sometimes do, they form limestone pillars.

Many carbonated springs deposit quantities of limestone as they issue at the surface of the earth. Water, when subjected to pressure, has a greater capacity for carbon dioxid, thus increasing its solvent power and its capacity for holding dissolved limerock. This occurs when water goes down through the decayed vegetable matter in the soil and the vegetable matter and other sources of carbon dioxid in the deeper parts of the earth's crust. As water thus charged permeates limestone 
strata, it dissolves and carries away greater amounts of calcium carbonate (page 419). At the surface of the earth the carbon dioxid escapes rapidly, causing bubbling or effervescence, and the limestone is deposited in great abundance.

What is the origin of the limestone? Limestones comprise limerock, marble, and chalk. They are composed largely of carbonate of lime, though they contain many other substances which give them different textures and degrees of hardness and color. Some lime formations have already been found to be chemical in origin, i.e. the stalactites and deposits of carbonated springs.

The great bulk of limestone is, however, of organic origin. The carbonate of lime is made up of the mineral shell substance of myriads of generations of organisms that lived and died in the ocean. The shells of these animals sank to the bottom and there formed in course of time vast quantities of shell substance and fine material called ooze. The accumulated masses of both were later covered, because of tremendous earth changes, with deposits of other materials. The organic remains, lime carbonate and silica (page 424), were cemented together, subjected to pressure, and in time formed into rock.

Near the coasts of bodies of water the lime and silica were mixed with other substances, as clay and sand. Some of the rock resulting from this mixture is intermediate between sandrock and limerock, and is classed as impure limerock.

Chalk. - The lime carbonate deposits in fairly deep seas formed chalk, such as the chalk beds in England, and those in Texas, which are very similar to the ooze deposits in the oceans to-day.

Reef limestone. - Another kind of limestone formation is known as reef limestone. This is composed of the mineral remains of coral and of shellfish that lived on the coral reefs. The shell limestone, called "coquina," familiar along the Florida coast, is made up of shells of mollusks inhabiting the shallow waters of the ocean. 
Marble. - Sedimentary rocks, limerock, sandrock, and shale have in some localities been subjected to conditions that worked a complete change in their character. The conditions which produce such changes are pressure, heat, and moisture. Rock produced in this way from other rock is generally known as metamorphic (transformed). Examples of rock strata have been found which show the gradual transition from sedimentary or other rock into metamorphic rock. These show both the kinds of rock metamorphosed and the causes of the metamorphosis.

In laboratory experiments limestone has been converted into marble. Marble has been found in nature, which showed clearly by the fossils with it its origin from limerock. Besides the metamorphic change of limerock into marble, other sedimentary rocks, as sandrock and shale, are known to have been similarly metamorphosed, a sandrock composed mostly of quartz grains into quartzite, and shale into slate.

Marble is ordinarily hard, crystalline, and granular, as may be seen by means of a microscope, and it takes a beautiful polish. When produced from pure limerock, it is white; from impure, it is veined and variously colored, as blue, gray, blue-gray, yellow, and reddish. Colored marbles are generally used for ornamentation. The marble quarries of New England are well known. The Greek marble called Parian, from the island where it was quarried, is famous for its beauty and durability.

\section{Groups of Rocks}

197. Three groups. - The rocks which have been considered with special reference to building and construction have a wider significance to which attention may be directed. The kinds named are representative forms of the three great groups or classes of rocks, called sedimentary, metamorphic, and igneous.

The sedimentary rocks include, as already stated, sandstone, shale, and a familiar conglomerate rock made up of gravel 
cemented and compressed. These rocks and the materials composing them are all of mechanical origin, derived from igneous or other preëxisting rocks or minerals by weathering, or dissolved by water and transported and deposited in bodies of water. The minerals are chiefly silica or quartz forming sand beds, clay forming clay beds, and shells eventually forming carbonate of lime beds.

The limestone deposited by the water of effervescent springs, and in caves as stalactites and stalagmites, is of chemical origin. Limestone formed from the mineral remains of marine organisms, as shells and other secretions, the chalk formed from deep sea.ooze, and the coquina and reef limestone are all of organic origin.

Metamorphic rocks are formed from sedimentary and other rocks by pressure, heat, and moisture. This group includes marbles from limestone, slate from shale and quartzite from sandstone that was made up principally of quartz granules. Other rocks of this class are gneiss, which is often metamorphosed granite; bituminous coal formed from lignite and peat; anthracite coal, metamorphosed from bituminous coal; and graphite.

The igneous rocks are not stratified and contain no fossil remains. They are evidently the product of the molten mass of minerals that issued from volcanic craters or fissures, or cooled in the depths of the earth. Such rock is often thoroughly crystalline in structure, though it may be a glass rock due to a rate of cooling and hardening too rapid to allow the formation of crystals. The best known igneous rock is granite. The glass rock known as obsidian contains a large amount of silica. Lava, pumice, and basalt are familiar. Notable examples of igneous rock often mentioned are the columns of the Giant's Causeway on the western coast of Ireland (basalt rock), and the Palisades of the Hudson River. 
The Typical Stages of Rock Formation

\begin{tabular}{|c|c|c|c|c|}
\hline \multirow{2}{*}{ MineraLs } & \multirow{2}{*}{ Origin } & \multicolumn{3}{|c|}{ Stages of Consolidation } \\
\hline & & Loose as Soil & Solidified & Metamorphosed \\
\hline Quartz or Silica & Mechanical & $\begin{array}{l}\text { Sand, } \\
\text { Gravel }\end{array}$ & $\begin{array}{l}\text { Sandrock } \\
\text { Conglomerate }\end{array}$ & Quartzite \\
\hline $\begin{array}{l}\text { Quartz, Felspar, } \\
\text { and Mica }\end{array}$ & Mechanical & Mud & Shale & $\begin{array}{l}\text { Slates and } \\
\text { Schists }\end{array}$ \\
\hline Felspar & Mechanical & Clay, Kaolin & Clay Shales & Schists \\
\hline $\begin{array}{l}\text { Lime Carbonate } \\
\text { Some Magnesia } \\
\text { or Silica }\end{array}$ & $\begin{array}{l}\text { Chemical } \\
\text { Organic }\end{array}$ & Marls & $\begin{array}{l}\text { Stalactites, etc. } \\
\text { Limerock }\end{array}$ & Marbles \\
\hline Plant Remains & Organic & Peat & $\begin{array}{l}\text { Lignite } \\
\text { Bituminou }\end{array}$ & $\begin{array}{l}\text { Anthracite } \\
\text { Coal } \\
\text { (Graphite) }\end{array}$ \\
\hline
\end{tabular}

\section{SOMMARY}

Man needs protection from the weather and enemies, and a storehouse for food.

Primitive protection was early replaced by buildings of stone and brick among the Chaldeans and Egyptians.

No building material meets all desired requirements.

Wood, the most common building material, decays and is liable to burn.

Stone is preferred for fireproof structures.

Sandrock is easily quarried and worked. It was formed by the consolidation of grains of sand generally deposited in water.

Slate is split easily along one plane.

Granite is composed of particles of quartz, felspar, mica, and hornblende. It is difficult to work, but durable. It was formed by the cooling and crystallizing of molten masses.

Limestone is easily quarried because of planes of cleavage and joints. It is easily worked and fairly durable. Caves found in limerock are due to its solubility in water. Limestone is of organic origin. 
Limerock is metamorphosed into marble, sandrock into quartzite, and shale into slate.

\section{REVIEW QUESTIONS}

1. Compare man and other animals as to natural ability to endure extremes of weather and to avoid dangers. 2. How did primitive man protect himself? 3 . Give reasons why men in ancient times built permanent homes. 4. What were the chief characteristics of the buildings of the early Egyptians? the Babylonians?

5. What characteristics should an ideal building material possess? 6. Give reasons why more wood is used in building than any other material. 7. Mention four steps in the transformation of the growing tree to the finished dwelling. 8. What are the chief defects in wood as a building material? How may these defects be partially overcome?

9. Mention three classes of stone used for building and an example of each. 10. Show how the durability of stone depends on climate. 11. Why is sandstone easily quarried and prepared for buildings? 12. Why is Ohio sandstone in demand? 13. Give an account of the formation of sandstone. 14. Mention several common uses of slate. 15. Why is slate easily worked? 16. From what was slate formed? 17. Of what is granite composed? 18. What are its characteristics as a building material? 19. In what regions is granite found? 20 . What relation had granite to the formation of shale and sandrock? 21. How was granite formed? 22. What is the commercial importance of limerock? 23. In what regions is limerock found? 24. How does it appear in the quarries? 25. What does its stratified character suggest as to its age and manner of formation? 26 . In what conditions does sedimentation occur? 27. Why is sedimentary rock stratified? 28. Of what practical importance is stratification in rocks? 29. Explain how the Mammoth Cave was formed. 30. What does this show as to the solubility of limerock? 31 . How are stalactites formed? 32. What other limestone deposits aro made in this way? 33. Give an account of the origin of limestone. 34. Under what conditions is limestone metamorphosed? What is formed? 35. Mention some qualities which make marble a desirable. building material.

36. Mention the three main groups of rocks. 37. What was the origin of sedimentary rocks? 38. What was the origin of metamorphic rocks? 39. Give examples of metamorphic rocks. 40. What are the characteristics of igneous rock? 41. Mention two examples of igneous rocks. 


\section{Some Manufactured Mineral Products}

198. Lime. - Lime, whether used for plastering, or with brick and stone, is indispensable in building. Lime manufacture is an important industry, the annual production amounting in value to more than fifteen million dollars. The lime of commerce is made from limestone, fragments of marble, and sometimes from shells, all of which are grouped in chemistry under the name of calcium carbonate.

The manufacture of lime is carried on by means of the lime kiln, the essential features of which are illustrated (Fig. 141). Limestone is quarried and broken into pieces of suitable size, and then put into the kiln, where they are heated with free access of air (how does this differ from charcoal making?) so that the carbon dioxid is driven off. The product is in the form of hard, dry lumps, called quicklime or calcium oxid.

Quicklime is prepared for use in two ways. It may be air-slaked or water-slaked. In air-slaking it absorbs moisture and carbon dioxid from the air, becoming lime carbonate, a fine white powder which is useful for fertilizing. In water-slaking, it combines

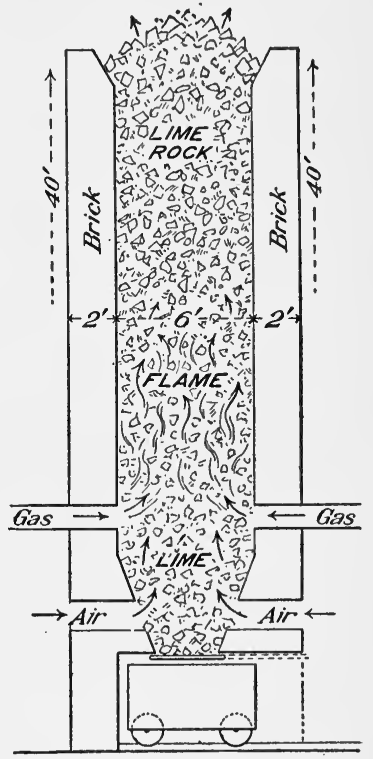

FIG. 141. - Cross section of modern lime kiln for continuous production. with water more rapidly, forming a product generally used for mortar, plastering, and other building purposes.

Exercise: Slaking lime. - The process will be more clearly understood by slaking a small quantity of quicklime and observing the changes which occur. The following is only suggestive in order to enable you to make exact observations. Put 12 ounces by weight of 
quicklime into a quart can that is standing on the bottom of an inverted pan to protect the table. Pour over the quicklime four ounces of water. Cover the can with a glass plate except a small opening through which steam may escape. Observe the temperature, the boiling, the expansion of lime lumps, and their crumbling into powder. The water, except that escaping as steam, unites with the quicklime to form a new compound.

The compound is a fine, white, dry powder. In chemistry it is named calcium hydroxid because it is calcium oxid plus water. It is a chemical compound, the product of a chemical change (page 131), in which the properties of the original substances do not appear because of their complete change in structure. The product is dry. A substance is wet only when it contains free water. Starch and sugar contain water, which, however, is not free. Weigh the product. Does the weight suggest that the product is equal to the sum of the weights of the lime and water, except for that lost in steam?

How lime compounds change in air. - Lime is often made into whitewash or milk of lime, a thin paste about the consistency of cream. When applied to walls, whitewash begins at once to change slowly its chemical nature by absorbing and combining with the carbon dioxid from the air. The product of this change, calcium carbonate, is already familiar. The same change occurs in lime plaster and mortar, but more slowly because of the larger proportion of lime contained in such mixtures. What change occurs when expired air is passed through lime water? In the hardening of whitewash, plaster, and mortar, what actually takes place is an exchange of partners. The carbon dioxid from the air, as absorbed by the lime, replaces and sets free the water, which is in turn taken up by the air as water vapor.

Chemistry and industry. - The study of the changes that occur in the manufacture of lime and in lime in its various uses may suggest the importance of the study of chemistry. The knowledge and control of chemical facts and processes are in- 
volved in the manufacture of most common necessities of modern life, such as steel, glass, copper, lead, aluminum, illuminating gas; hydrocarbons, cotton, wool and silk textiles, paper, soap, and glycerin, in preparing and cooking food products as bread, meat, and vegetables, or preparing sugar, starch, etc., and also in many operations concerned with building and construction. It is important in the industry of the household as well as in the industries of the world. Chemistry has contributed greatly to human comfort and welfare in the past; but this is only an earnest of what it will contribute in the future. One can hardly overestimate the importance of the study of chemistry in any field in which one is likely to labor.

199. Cement. - The use of cement as a building material is rapidly increasing. In the United States more than $80,000,000$ barrels of cement are manufactured every year, valued approximately at $\$ 70,000,000$. Cement is widely utilized in all kinds of construction, such as buildings, sidewalks, bridges, aqueducts, dams, and so on. When other materials are used for buildings, cement is used generally in the construction of the foundation.

Cement sets quickly and hardens in a few days after being wet, and it must therefore be kept dry in shipment and handling. The setting does not depend upon the presence of carbon dioxid as is the case with slaked lime; and it takes place also under water. In this process cement absorbs a definite amount of water and forms minute crystals, thus making an artificial rock. The rock becomes harder with the passing of years. Cement is usually mixed with sand, or with sand and gravel or crushed rock, to form concrete. Concrete may be made into any shape by being poured into molds made of wood, which are removed after the concrete has set. To secure greater strength, concrete work is often reinforced with steel rods arranged in the molds before the concrete is put in.

200. Brick. - Some sedimentary deposits have in the course of geological history been converted into shale, and some shales 
later into slate, by a very slow process of nature. (From what mineral and by what process was clay formed?) By making use of the conditions nature employed, that is, moisture, pressure, and heat, men convert clay into brick which may be harder than slate. To-day brick may be made from the raw materials in a few days, whereas in nature's course similar products may have required thousands of years.

Vast deposits of clay are found in almost every land. Some of these deposits were utilized for brick and pottery in very early history. The ancient inhabitants of the valleys of the Tigris, Euphrates, and the Nile made brick for use in buildings. To-day bricks of very many kinds are produced in great quantities, the average value of the annual output being more than $\$ 140,000,000$.

The first step in the manufacture of brick and other clay products is mixing the clay. This was originally done by hand, later by a crude machine operated by man or animal power, and to-day by machines operated by steam, gas, or electricity. The mixed clay was molded into the desired forms at first by hand, later by wooden molds, and is now molded by machines operating at a high rate of speed.

The second step of the process is baking the brick. This was formerly accomplished by slow drying in the sun; but to-day it is done rapidly by the aid of a kiln in which the clay products are heated to a temperature just below the fusion point of clay. This high temperature causes the particles to cohere closely. As the clay shrinks or contracts with the process, the bricks are generally porous. This character may be observed by placing a dry brick in a shallow dish of water; and the quantity of water absorbed may be measured accurately.

Exercise: Brick and tile making. - What different kinds of brick and tile do you find used in your community? If a brick and tile factory is located nearby, it will be profitable for you to visit it and observe the successive steps in the manufacture of clay products. Examine the clay; and ascertain whether it is the ordinary red clay, 
fire clay, or pottery clay; what materials, if any, are mixed with the clay, and for what purposes. Find out the temperature at which clay products are baked in the kiln. Note also the machines and motive power employed in the different stages of mixing, molding, transferring of molded products to kiln, and so on. Is the clay bed near the factory, or is clay transported from a distance? If the latter, find out what conditions led to the location of the factory away from the clay beds.

201. Other clay products. - By mixing different materials with ordinary clay, and by making use of clays of different kinds and grades, all sorts of brick and tile and the like are produced. Ordinary clay mixed with sand makes the glazed brick; a clay with a very high point of fusion is made into fire brick and fire proofing tile; and by a variation of the materials and processes, waterproof brick and tile, vitrified, paving, clinker, and fancy colored bricks, drain pipes and tile, architectural terracotta, and very many other varieties of clay products are now available for all sorts of building and construction purposes.

With necessary modification of process and variation of materials, earthenware, pottery, and chinaware are made in large quantities. Porcelain articles often used in the kitchen, and for parts of electric appliances are made from a mixture of pure white clay and powdered felspar, which is heated to such a high temperature that the felspar is melted and made to fill the minute pores that occur in the clay products. The value of the output of the finer clay products is about $\$ 30,000,000$ a year. The industry employs more than two thousand individuals, and with its allied branches of decorating and mining, employs several thousand more.

202. Glass. - Glass is indispensable to-day. For windows there is no satisfactory substitute for glass, although oiled paper, mica, and other substances have been utilized. For lenses and laboratory apparatus of many kinds glass has no competitor, while for the commonest articles such as bottles and dishes glass is the cheapest and most suitable known material. Glass is readily manufactured and molded into any 
form. It is also tough, hard, and strong, as may be seen from the fact that it is not easily scratched and marred and that it sustains hard usage and heavy weights.

Exercise: Uses of glass. - For what purposes other than those mentioned have you seen glass utilized? Have you noticed glass reinforced with wire net? What is the purpose of this? Modern factory buildings are largely made of glass. What kinds of glass have you seen used in this way? How is glass strengthened when it is used for practically the entire sides of buildings? What are some of the popular devices for this purpose? Consult advertising pages of periodicals, and note qualities claimed for the devices advertised.

To what extent do you think the world could get along without glass? In what ways do you think the increased use of glass would be advantageous? Can too much light be admitted into a building?

Glass manufacturing - past and present. - Glass making is a very old industry. The earliest known examples are some blue glass beads brought to England between 3000 and 2000 B.c. by traders who distributed their wares throughout Europe and told tales of ships plying the waters of the Nile and the adjacent seas. The art of glass manufacture was developed in Egypt, learned later by the Babylonians and by some of the Etruscan tribes in the northern part of Italy. In Greek and Roman art and during the Middle Ages in Europe glass making was highly developed for decorative and useful purposes.

To-day some of the most beautiful glass is made in Bohemia, where quartz sand is plentiful and well adapted to the purpose. The glass is colored by adding small quantities of minerals found in the mines of the country. Italy is also noted for its fine glass.

In the United States, Pittsburgh is the center of plate glass manufacturing, largely because deposits of sand especially suitable for this glass are found near the city. Pennsylvania, Indiana, Ohio, Illinois, and West Virginia are the chief manufacturing states, partly because of the supply of cheap fuel, and partly because of the abundance of sand of good glassmaking quality. 
Materials and process. - Of what is glass made? A visit to a glass factory reveals that sand, more or less white, is the chief constituent, and mixed with sand one or more of these substances: limestone, or chalk, sal soda (sodium carbonate), glauber's salt (sodium sulfate), potassium carbonate called potash, red lead (lead tetroxid), carbon, and arsenic. The mixture is put into large melting pots of fire-clay, and heated to a temperature at which it melts or fuses. It is boiled long enough to secure thorough mixing and the elimination of all gases. The molten mass unlike water has no definite freezing or melting point, and hardens very slowly as it cools. As it cools, it hardens sufficiently to become a plastic, pasty mass, which may be molded or blown into any desired form. It may be kept pasty for an indefinite time in a furnace, as is necessary when it is being made into articles by blowing.

Bottles, tumblers, lamp chimneys, vases, and many other useful objects are made by' blowing and molding combined. The glass-blower takes up on the tip of his blowpipe enough of the plastic mass, blows it into a bubble which is then put into a mold and blown until it fills all parts of the mold. It is trimmed and finished and transferred to the annealing furnace. Glass which cools too rapidly is very brittle; when it cools too slowly, it crystallizes much in the fashion of sugar sirup. It is therefore annealed, or gradually cooled in an annealing furnace in which the temperature is lowered as required by the product.

Window glass is made by blowing the molten glass into a large globe which by skillful manipulation is gradually lengthened into a cylinder. The cylinder is then cut open, heated, and spread out flat. Or window glass is made by machinery which gives it any desired thickness and size. Many common and cheap glass articles are pressed into shape by dies.

For plate glass the molten mass is poured upon molding tables and rolled smooth by hot rollers operated usually by hand. The form is about one inch thick. After being an- 
nealed the glass is ground down to one half or one fourth of an inch thickness and polished. The grinding is done entirely, and the polishing partly, by machinery.

Cut-glass articles are made from the lustrous flint glass. After they are molded, they are decorated with designs cut by means of emery wheels, and then polished by machinery and by hand. Imitation diamonds are similarly made from the same kind of glass. Other jewels are imitated by suitably coloring flint glass.

Sand is the principal material used in making glass. In the study of sedimentary rocks (pages 407,415 ) we found that sand is mostly quartz, which is derived from the decomposition of granite, an igneous rock. This leads to an inquiry into the nature of quartz.

Exercise. - Examine a piece of quartz, testing it for the following properties. It is crystalline, forming six-sided crystals, transparent, and very hard, as it scratches glass or is as " hard as flint," flint being a different form of the same substance as quartz or sand. It is fusible at the very high temperature produced in the electric furnace. It is often found in large crystals which may be cut into lenses for spectacles or other optical instruments, or to imitate diamonds. Its use in the Cooper-Hewitt lamp may be cited.

203. Silica. - Quartz as considered by chemists is a compound made up of two elements, silicon and oxygen. Silicon, a mineral which does not exist separately or free in nature, unites freely in the ratio of one part to two parts of oxygen to form silicon dioxid, or silica. Quartz is thus seen to be silica, and sand of the pure white variety is practically pure silica. Other sands are mixtures of silica and various other minerals.

Abundance and distribution. - Silica is very widely distributed and abundant in the earth's crust. Wherever sand is found, in whatever condition and state, whether loose or compact as sandrock or quartzite, there is silica. In the desert, on the shore and bed of stream, lake, and ocean, on mountain and 
plain, there abounds silica. Plant life contains silica, as is shown by analysis of the ash. The glistening and stiffness of many grasses, such as bamboo, are due to silica. Animal life is not without it, and certain microscopic forms contain a large percentage of silica in their composition.

Quartz in granite is pure silica; the other constituents of granite, felspar, mica, and hornblende, contain more or less silica. Much felspar is a compound of silica, alumina, and potassium. Mica and hornblende are similarly made up, though they contain in addition lime, magnesia, and iron oxid. Felspar and mica are dissolved in water, and the silica and alumina are carried away and deposited to form clay.

Clay is thus seen to contain silica. One form of clay, called kaolin, contains a very large proportion of alumina; it is the purest variety of clay and most useful in making the finest pottery and chinaware. But the other varieties contain a large amount of silica.

Table of Common Substances in Rocks and Shale

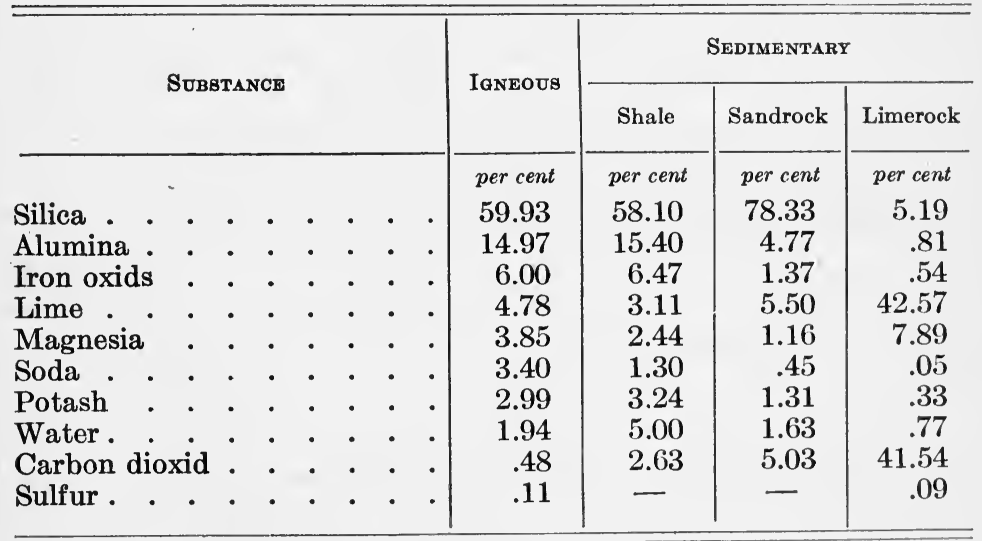

This indicates that silica is not only the most widely distributed, but perhaps the most abundant mineral in the crust of the earth. Silicon, the chemical element, constitutes about 
twenty-eight per cent of the total composition of the earth's crust or lithosphere. It is second only to oxygen, which composes forty-seven per cent of the earth's rocky crust. Next to silicon is aluminium, 7.84 per cent, and then follows iron, 4.44 per cent of the total crust (see page 433 ).

The table, given for reference, indicates the percentage of silica and other common substances in the different kinds of rocks and shales, and shows the relative abundance of certain useful minerals in nature. It is adapted from the complete table in Bulletin 491, United States Geological Survey, pages $27-32$.

204. Carborundum. - Silica is an essential material in the manufacture of what is commercially known as carborundum. This is a compound made from silica and carbon. A mixture of sand, coke, common salt, and sawdust is arranged in an electric furnace. The electric current must pass through this mass. After about eight hours of heating at the high temperature produced by the resistance of the coke to the electric current, the product is seen to be a mass of black, brilliant, and often iridescent crystals which have a degree of hardness next in the scale to that of the diamond. Hard steel is readily cut by a wheel covered with the crystals of carborundum. What are some of its uses in your neighborhood?

\section{SUMMARY}

$\$ 15,000,000$ worth of lime is produced annually in the United States.

Quicklime, or calcium oxid, is slaked with water, forming calcium hydroxid.

Slaked lime is used to make whitewash and mortar, which harden by the absorption of carbon dioxid from the air and the liberation of water.

A knowledge of chemistry is necessary to understand many.of the important household and manufacturing processes upon which our material welfare depends.

Cement to the value of $\$ 70,000,000$ is produced annually in the United States.

Cement sets by combining with enough water to crystallize. 
Concrete, or artificial stone, is made of a wet mixture of cement, sand, and gravel, or crushed rock, and may be reinforced with steel rods.

Brick, tile, and other clay products produced annually in the United States have a value of about $\$ 140,000,000$. Their production is an example of the use of heat in manufacturing.

From finer clays and related materials, with a better control of temperature, are made earthenware, chinaware, and porcelain.

The wide use of glass makes it practically indispensable.

The art of glass making was known to the ancient Egyptians and others.

Bohemia, Italy, and Belgium are notable for their glass manufacture. Pennsylvania leads in the production of glass in the United States

The chief varieties of glass are crown, flint, and hard glass.

Crown or soda glass is used for making window glass, tumblers, and bottles.

Flint glass, a lead glass, is used in cut glassware and lenses.

Bohemian or hard glass, one variety of which is Jena glass, is used for scientific apparatus.

Glass is made by melting a mixture of sand and other materials, and is shaped by blowing, molding, rolling, and grinding.

The colors of glass are due to small traces of certain minerals.

In general the larger the proportion of silica in glass, the harder the glass is and the more resistant to the effect of chemicals and high temperatures.

Sand is mostly quartz, which is a compound of silicon and oxygen, and called silicon dioxid or silica.

Silica is very widely distributed in nature and is the most abundant mineral.

Many precious stones such as the amethyst, opal, and agate are silica with traces of coloring matter.

Carborundum, made from carbon and silica fused at the temperature of the electric furnace, is next to the diamond in hardness and is used for grinding tools.

\section{REVIEW QUESTIONS}

1. Recall in detail the observations made when lime was slaking. 2. Of what advantage is the knowledge of chemistry in lime manufacture and use? 3. What is the value of the cement manufactured annually in the United States? 4. Mention some of its uses. 5. Explain the setting of cement. 6. What is reinforced concrete? 
7. From what are bricks made? 8. What is the origin of clay? 9. Why are clay beds so abundant and frequent? 10. What is the commercial importance of bricks? 11. Describe how bricks are made. 12. What other products besides bricks are made of clay? 13. Of what materials is porcelain ware made? How?

14. What are the most important uses of glass? 15. What evidences are there that the ancients made glass? 16. What countries are notable for glass making? 17. What are the chief glass making centers in the United States? Why? 18. Of what materials is glass made? 19. Describe process of making (a) lamp chimneys, (b) window glass, $(c)$ plate glass, $(d)$ cut glass. 20. Why has cheap glass often a greenish tint? Red?

21. What is sand? 22. Mention several uses of quartz. Upon what characteristics do these uses depend? 23. Give the chemical name of quartz or silica. What does the name suggest as to its composition? 24. Give an account of the distribution of silica in nature. 25 . What is the relative abundance in the earth's solid crust of silicon? Oxygen? Aluminum? Iron? 26. Of what materials is carborundum made? For what is it used? 


\section{CHAPTER $\mathrm{X}$}

\section{STEEL AND IRON}

205. The industrial and commercial importance of steel and iron in the world's work is second only to that of food production and distribution, and easily first of all the metals produced. The production, manufacture, and distribution of iron and steel and their many products involve a very large investment of capital, include hundreds of thousands of workers, and touch intimately the life and activities of the smallest community and in fact of every individual in our country. In almost every city and many of the larger towns some kind of manufacturing plant is to be found which transforms cast iron into finished products; and in many places there are factories that convert steel into almost countless articles for all known purposes.

In every phasse of modern life and its boundless activities iron in some form is indispensable. Continents are spanned by iron and steel rails, and space and time are annihilated by the iron monsters traveling over them. Every remotest part of the world is connected with every other part by a veritable network of iron wires. Rivers, lakes, and deep and wide abysses are bridged and safely crossed by means of iron; and oceans are made into highways by steel ships, propelled by iron and steel engines. The trackless air is also rapidly becoming a highway for swiftly moving ships of steel. In all fields of production, as mining, agriculture, lumbering, and fishing, in manufacturing, building, and construction, in short, in all the world's work, iron is the most useful and necessary of all the metals.

Exercise: Use of steel and iron. - Prepare a list and classify into convenient groups the common uses of iron and steel as you may observe them in the things of daily life, in the home and the com- 
munity. Explain the ground upon which you base your classification of the utilities listed.

What kinds of furniture are made of iron or steel? What advantages are gained in using such material for furniture? What disadvantages? What interior finishings of office or public buildings are made of steel and iron? What advantages are there in having window and dior frames and the doors and finishings of the rooms made of steel or steel covered? Why are steel passenger cars better than the common wooden cars? Why are steel or iron rods and rails used in concrete structures as viaducts, bridges, and buildings?

Visit a blacksmith shop and learn what different kinds of iron are used. For what purposes are the different kinds? What properties of iron make it especially useful? Can it be readily shaped as desired? Can it be welded?

What iron industries are there in your community? What sort of raw material do they use? What are their chief products?

Cast iron, which is converted into useful objects in very many localities, is easily worked and has a comparatively low fusing temperature. Visit, if possible, an iron foundry. Study the kinds of molds or patterns. What are the chief products of the foundry? What sort of iron is used for castings? How is it prepared in the foundry? How long a time is required to melt the cast iron? In what kind of furnace is this done? What fuel is used? Why?

World production. - The relative tonnage of iron and other metals for a year shows the importance of iron in the world's production. We may take pig iron as equivalent to the total output of metallic iron. The production of pig iron in 1910 amounted to more than 96 per cent of the world's total output in tonnage of metals. The value of the pig iron was about 46 per cent of the total value of the metals produced.

The United States' production. - The United States, which is the largest producer of iron, had in 1910 a total output of minerals worth considerably more than two thousand.. million dollars. This includes all minerals used in construction, as clay and stone with their products, lime, and cement, and also all fuels, as coal, petroleum, and natural gas. But if we exclude minerals and fuels and consider only the metals, such as lead, copper, aluminium, zinc, silver, gold, and iron, we have a 
total value of the metal production of $\$ 823,483,390$. The value of the iron alone amounted to about $\$ 425,115,390$, or more than 50 per cent of the total. In the year 1913, the United States' production of iron was approximately $61,924,000$ tons.

\section{Properties of Iron}

206. Why iron is useful. - What are the properties of iron which make it the most useful of metals? The commonly recognized properties are : (1) tensile strength ; (2) crushing or compression strength; (3) bending resistance; (4) hardness; (5) relatively light weight; (6) rigidity; and (7) fusing or melting point.

These properties may be considered here in the most general manner. Examples of each property in some steel or iron structure should be found to make the subject definite and concrete.

The tensile strength of wrought iron, which is almost pure iron, is, on the average, such that a bar with a cross section area of one square inch will support a weight of about 50,000 pounds.

The crushing strength of cast iron, as used in the pillars of a building, is estimated at about 40 tons to the square inch, or about five times the tensile strength of cast iron. What is meant by the tensile strength of cast iron?

Bending resistance may be suggested by a girder or beam supported at both ends, as in a bridge or a floor, and the weight applied at the middle. Iron or steel supports twice the load of any other metal in common use.

Hardness is necessary in almost every use of iron or steel. It must be hard enough to withstand a certain amount of wear through friction, and resist being scratched or marred excessively by other hard objects. Hardness is tested by the amount of pressure exerted upon a diamond point, and the depth of the scratch it makes in the steel.

Iron is one of the lightest of the common metals. Volume for volume (page 343), it is only four fifths as heavy as copper, three 
fourths as heavy as silver, and a little more than two thirds as heavy as lead; but about two and one half times as heavy as aluminum. Its relatively light weight for a unit volume makes it invaluable in large, tall structures where strength and light weight are necessary.

As is well known, iron and steel are rigid. A steel frame for a bridge, a high building, a machine, or the hull of a ship possesses a rigidity which no other material can give. A steel frame for an aeroplane is more rigid than one of wood; and rigidity of such a structure is indispensable, as the slightest yielding might lead to disaster.

Many familiar appliances show the advantage of the relatively high melting point of iron. Consider its importance in such appliances as ranges, stoves, furnaces, boilers, or anything to which intense heat may be directly applied. Its use in fireproofing buildings is also due to this property.

One property of iron, not mentioned above, needs special consideration. This is its capacity for magnetism, which is the basis of all progress in the generation and transformation of electricity (page 324). Without iron the electric generator and the transformer would be impossible: This magnetic property of iron was recognized ages ago by such peoples as the Chinese and the Greeks, who made use of it in various ways (pages $370 \mathrm{f}$.). Some ores are naturally magnetic, as those in Arkansas. These possess a strong attractive force for suitable objects that come within their range.

\section{IRON ORE}

207. Distribution of iron. - Iron is found abundantly in different compounds in almost every part of the earth. In smaller amounts it occurs in sand, clays, and rock. It is contained in solution in water, and is an important constituent of plant and animal organisms. It is present in the hemoglobin of human blood, making it possible for the blood to unite with oxygen and to transport it to the bodily tissues. 
The following table adapted from tables prepared by Professor F. W. Clark, published in Bulletin 491, United States Geological Survey, pages $32-34$, shows the relative amounts by weight in per cent of some of the more important elements of the average composition of the earth's rock crust. The table may be profitably studied, and made to show by a little ingenuity the relative abundance of the important metals; for example, taking the natural occurrence of gold as 1 , silver would be 20 , lead 4000, and iron 8,800,000. Compare with table on page 425 .

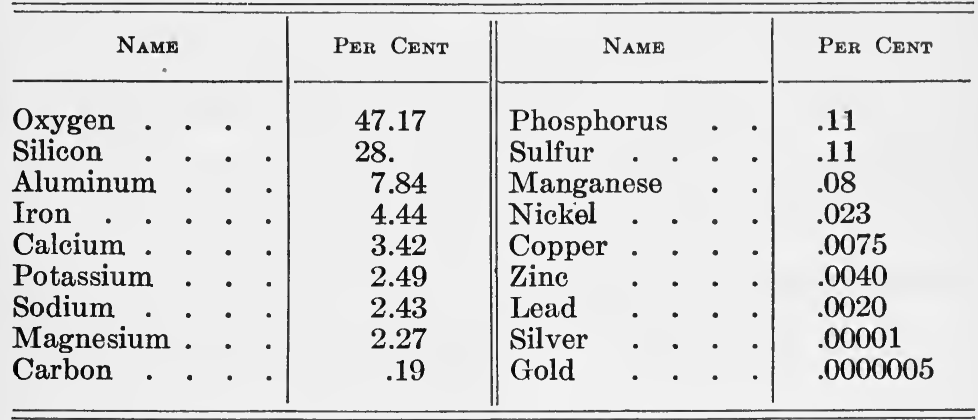

Pure metallic iron is rarely found in nature, unless it comes to earth from interplanetary space, as meteorites, some of which are practically pure iron. In the American Museum of Natural History at New York City there is a collection of meteorites of almost pure iron, brought from Greenland. One weighing $36 \frac{1}{2}$ tons is composed of about 91.5 per cent iron, 8 per cent nickel, and .5 per cent cobalt.

208. Kinds of ores. - Iron in plentiful and workable quantities is usually combined with oxygen, carbon, or sulfur. With oxygen it forms an iron oxid ore; with carbon and oxygen, an iron carbonate ore; and with sulfur, an iron sulfid ore, or iron pyrites. The iron sulfids are useful to-day not for their iron content, but for their sulfur, which is used in manufacturing sulfuric acid. The iron carbonates are important ores in England, but they form a very small part of the iron industry 
in the United States. The output in 1912 was little more than 10,000 long tons. The important iron ores to-day are those of the oxid group. These are usually classified in three groups.

The first of these groups is called the hematite. This is the most valuable of the iron ores, the production of 1912 being more than $51,000,000$ long tons. It is composed of 70 per cent iron and 30 per cent oxygen.

The second group is the magnetite. It is made up of $\mathbf{7 2 . 4}$ per cent of metallic iron and 27.6 per cent of oxygen. Although the richest in iron of the used ores, it is a very poor second in output and industrial importance. The 1912 production was less than 3,000,000 long tons.

The third group of iron ores is called the brown hematite, or simply the brown iron ores. They are distinguished from the other groups chiefly by the fact that they contain water. Because of their water content and the fact that they are found in places that either are or had been swamp land, they are often described as bog-iron ores.

There are five distinct kinds of the brown ores, which form a remarkable series on the basis of their regular increase in water content. The first of the series contains about 5 per cent of water, the second 10 per cent, and so on at regular intervals to the fifth, which contains about 25 per cent of water. If now we include the ordinary hematite, which contains no water, as the first, we have a complete series from 0 to 25 per cent water content. This is an interesting example of the way in which certain substances in nature combine in definite and multiple proportions. The industrial importance of the brown iron ores is shown by the 1912 production, which was about one half the tonnage of the magnetite ores.

209. Production by states. - Iron ores have been found and sometimes worked in every one of the states of our country; but they are to-day produced in considerable quantities in only about thirty states. These are included in four regions or districts: first, the Lake Superior region, which includes Minne- 
sota, Michigan - the first and second in order of production and Wisconsin; second, the Southern region with its center in Alabama, the third state in order of production, and including among other states Tennessee, Virginia, and Georgia ; third, the North Atlantic region in which New York, Pennsylvania, and New Jersey may be mentioned; fourth, the Western region or the Rocky Mountain and Pacific coast states, of which California, Washington, New Mexico, and Wyoming are the chief producers.

The mean annual production of these regions for a decade indicates their relative importance in this industry. The Lake Superior region produced approximately eighty-one per cent of the total output; the Southern region twelve and one half per cent; the North Atlantic four and three fourths per cent; and the Western region one and three fourths per cent.

The kinds of ores produced are hematite and brown ores in the Lake Superior region; red hematite and brown ores in the Southern region; magnetite in New York, Pennsylvania, and New Jersey of the North Atlantic region; hematite, brown ore, and magnetite in the Western region ; and iron carbonate in Ohio.

210. Mining. - Mining operations vary with the geological position and the kind and quantity of the ore bodies. Ore bodies which are horizontal or dip slightly, and lie near the surface of the

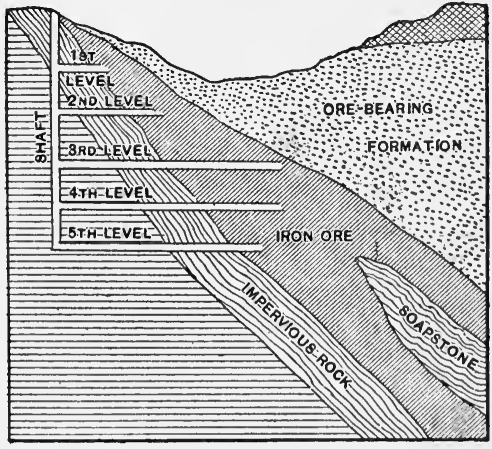

Fí. 142. - Cross section showing position of iron ore and the method of mining.

earth, are usually stripped, the ore removed and loaded upon cars by gigantic power shovels. Where the ore masses are deep in the earth, or where thin ore bodies are steeply sloping or vertical and extend to great depths, they must be reached by 
a drift mine or a shaft. A diagrammatic sketch of a shaft, with several levels or drifts which are worked at the same time, is shown in Fig. 142. On the whole, the underground method of mining iron ore is similar to that in mining coal, lead, and other minerals.

211. Formation of ore bodies. - The position in which iron ores are found suggests the question as to how they were formed. Iron-bearing formations generally are, like limerock and sandrock, of sedimentary origin. Some iron deposits, as those of the Lake Superior region, were probably of marine formation and originally composed of iron silicate and iron carbonate. The marine beds were later covered by other deposits and metamorphosed. The physical and chemical character of ironbearing formations was gradually modified. Permeating waters dissolved away the silica and carbon dioxid, leaving the iron oxids. In some large iron-bearing bodies of low grade, which were steeply inclined or tilted, the permeating waters effected a transfer of the iron from the upper parts and its concentration in the lower parts of the body. This is the probable formation (Fig. 142) of many ore bodies, as those in the Lake Superior region.

In some localities the ore bodies were formed directly by igneous action (pages 409,414), the iron being deposited by water that was originally a part of the lava. It is supposed that magnetite ore was produced in this way.

In the Birmingham, Alabama, district as many as five layers of iron ore are found separated from one another by strata of shale and sandrock. The largest seam of ore is at places thirty feet thick; and a seam which is very rich in iron, and between four and six feet thick, lies several feet below it, separated by shale and sandrock. Where ore bodies occur as strata separated by layers of shale and sandrock, there was once the sandy floor of the sea. River waters in which much dissolved iron was present flowed into the sea and deposited their iron content. Other sands covered the first deposit of iron, and these 
conditions alternated, producing stratified deposits of the above sort. At last the sea floor was upraised to form part of the land.

Some ore bodies are found which show evidences of having been formed in underground cavities. In some brown ore deposits there have been discovered iron stalactites (page 411), which plainly indicate this manner of formation.

One other kind of ore formation may be mentioned. Where limestone strata are steeply inclined, and are accessible to water that has become a carrier of iron and carbon dioxid, one or more layers of the limestone may be dissolved and replaced with iron oxids. Such formations may be extensive and may be profitably worked. In the southern iron ore regions sandstone has been similarly replaced by iron oxid. Ores thus formed are generally brown ores.

\section{Reduction of Iron Ores}

212. Manufacturing centers. - Mention is made in the section on Inland Waterways, page 386, of current methods of transportation and handling of iron ore. There are many interesting problems connected with the transportation and manufacture of iron, which may be briefly suggested here as subjects for individual study. Why are iron ores not reduced, and pig iron converted into finished iron and steel products, where the ores are mined? What conditions determine chiefly the location of iron manufacturing centers? Why are Pittsburgh, Chicago, and Birmingham the leaders in iron manufacturing?

Exercise. - Birmingham produces pig iron at less cost per ton than Pittsburgh or Chicago, although it pays more for coke and labor than either of these. A ton of iron is estimated to cost at Birmingham, $\$ 9.88$; at Pittsburgh, $\$ 13.03$; at Chicago, $\$ 13.19$. What reasons do you find to account for the difference?

213. The blast furnace. - The manufacture of pig iron or the reduction of ores consists in removing the oxygen and other 
impurities from the iron. This is done in blast furnaces (Fig. 143) which, constructed of steel lined with fire-brick, are ninety to one hundred feet high and as much as twenty-four feet in diameter. The blast furnace is kept in continuous operation.

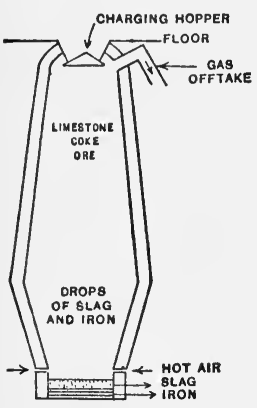

Fig. 143. - Diagram of blast furnace. The raw materials are supplied at the top, and the molten iron removed at the bottom. A modern furnace requires in the course of every twenty-four hours about eight hundred tons of iron ore, four hundred tons of coke, one hundred tons of limestone, and twentyfive hundred tons of air which, heated to $1200^{\circ} \mathrm{F}$. or $1400^{\circ} \mathrm{F}$., is delivered under a gage pressure of eight to sixteen 'pounds. The iron produced from this charge is usually four hundred tons. How many tons of ore will be reduced in a week? How many tons of coke will be required? How many tons of iron produced?

The present method of reducing iron ores is another illustration of the importance of chemistry in modern industry. Until within recent years, ore, coke, and limestone were mixed in accordance with a general recipe which made no account of ore variations or impurities. The iron produced varied in quality, but the recipe remained unchanged. But to-day there is no one recipe for preparing the charges for the blast furnace. The raw materials are subjected to an accurate chemical analysis, and on the basis of the chemical findings proportions of the materials in the mixture are computed. Then all raw materials are weighed and mixed as the analysis requires. The supply to the furnace is exactly controlled. The product is iron of the quality desired.

The process of reduction. - It is the work of the blast furnace to separate the iron from oxygen and from the impurities which the ores may contain. Moisture, organic substances, and carbon dioxid are quickly expelled in the high temperature of the fur- 
nace. The oxygen at the high furnace temperature combines freely in combustion with the coke or carbon. The heat of this combustion is increased greatly by the blast of heated air (cf. Fig. 144), which economizes fuel and facilitates the reduction of the ore. The mineral impurities or ingredients, as silica, alumina, magnesia, phosphorus, sulfur, and lime, combine in part to form a slag which is made readily fusible at the available temperature by the additional limerock. . The iron and slag melt near the hearth of the furnace and collect in it. The molten slag, which is less dense than the molten iron, floats on top. Through two taps at different levels the molten iron and slag are drawn off when necessary. Meanwhile the whole mass in the furnace settles downward, and fresh materials are supplied continually.

The molten iron when drawn from the hearth is run into great receiving ladles. In some places it is conducted directly into sand or iron molds, but more commonly the molten iron is put into great ladles where it is thoroughly mixed and equalized before being cast in iron molds. The product is the pig iron of commerce.

The slag (Fig. 144) contains many valuable substances. It may be conducted into a slag-receiving pit or into a large tank of water, called the granulating pit. Slag may be crushed and powdered for cement, prepared for fertilizer, or merely crushed for use as ballast on railroads.

The gases containing more or less dust are expelled through the top of the furnace and conducted down through flues to cleansers, which remove the dust, and perhaps some of the volatilized compounds, and cyanide and alkalies. The dust is a good fertilizer. The gas when cleaned is conducted to the furnaces, where it is burned to heat air for the hot blast, or to boilers, where it is used in generating steam.

214. The character and uses of pig iron. - Pig iron is an intermediate, not a finished, product in the iron industry. In appearance it is gray, mottled, or whitish in color, and crystalline 
峁

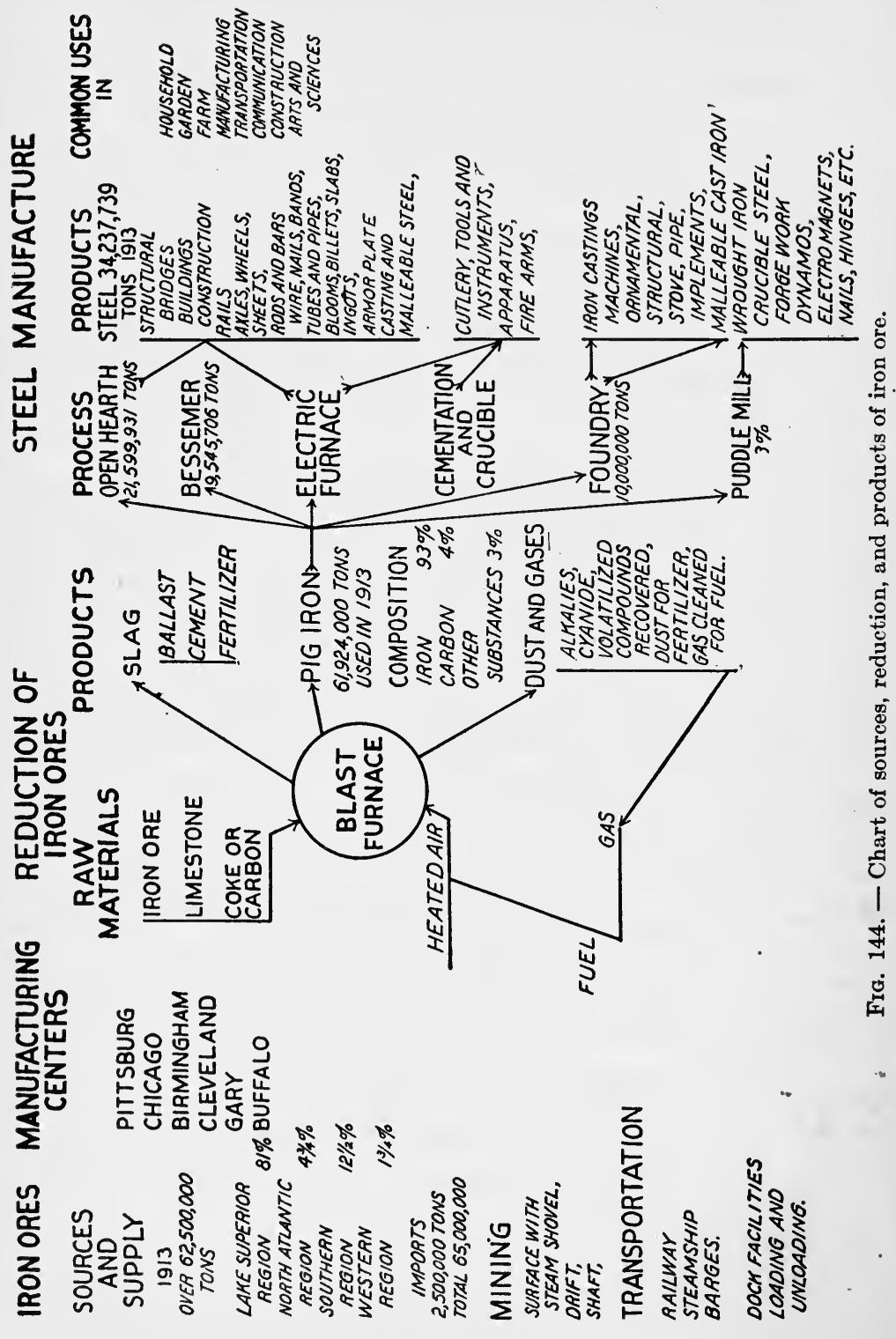


in structure. It is not malleable, but hard and brittle, easily broken by a sharp blow. It contains on the average about 93 per cent iron, 4 per cent carbon, and 3 per cent of other substances, chiefly silicon, manganese, phosphorus, and sulfur. It melts at about $1200^{\circ} \mathrm{F}$., and expands as it cools. Because of these properties, pig iron (Fig. 144) is generally utilized in three ways : (1) for iron castings; (2) for making steel; and (3) for making wrought iron. These three uses of pig iron will be considered in order.

- Iron castings. - About one sixth of the pig iron produced is used in foundries. In the foundry the pig iron is remelted and converted into such castings as are desired for machinery, agricultural implements, for car, locomotive, and railroad castings, for stove, range, and furnace castings, for soil and water pipe, pipe fittings, and valves, for structural purposes, for ornamental and many other purposes which cannot be enumerated here. But all such castings are hard, brittle, and comparatively easily broken, chiefly because of the impurities in the iron. The phosphorus is especially objectionable as it renders cold iron in any form very brittle.

To remove some of these impurities pig iron may be remelted and some of the impurities burned out. It is thus converted into malleable cast iron, a semi-purified product, containing less silicon, manganese, phosphorus and sulfur than pig iron. It is used in similar ways and for purposes similar to those of pig iron, but the castings are hard, stronger, and less brittle. They have three to four times the tensile strength, about 35,000 to 50,000 pounds to the square inch, and about the same transverse strength, as the ordinary cast iron castings.

\section{Steel}

The use of cast iron and malleable cast iron is rapidly decreasing in manufacturing and construction. These products of iron contain impurities that make them useless for very many purposes to which they formerly seemed well adapted. They 
are consequently being replaced by several kinds of steel and especially by casting steel. The result in the industry is indicated by the fact that to-day five sixths of the total output of pig iron is used in making steel.

215. The manufacture of steel. - Pig iron, either the molten or the molded solid pigs, is converted into steel in several ways. These are known as the Bessemer converter, the openhearth, the electric furnace, the cementation, and the crucible processes. In general the process of steel making is that in which pig iron is freed from its impurities, such as silicon, carbon, phosphorus, sulfur, and so on, through rapid oxidation. The Bessemer converter process, which is of great importance in the development of the steel industry, will make clear the essential features of steel making. The other processes will be easily understood from this.

The Bessemer converter is a large cylindrical vessel made of steel plates and lined usually with slabs of silica rock or firebrick. It is provided with cylindrical fire-clay bottoms; in these are many small openings through which heated air is forced under a pressure of from ten to thirty pounds. Molten pig iron is put into the converter. The hot-air blast is turned on and, blowing in a fine but powerful spray through the molten mass, produces by oxidation of the silicon and manganese enough heat to ignite the carbon. The temperature which is thus produced by the combustion of the carbon is such as burns out or converts into molten slag most of the impurities, leaving in the iron not more than one tenth of one per cent each of carbon, silicon, and manganese. As steel to be steel requires a certain percentage of carbon, this may be added by putting into the converter a quantity of molten pig iron, or some iron rich in. carbon. In this way, from molten pig iron, steel is produced "without fuel," as Sir Henry Bessemer announced in 1856 when he first described his invention.

Impurities in steel. - The Bessemer converter does not produce a steel which is free from the impurities. Sulfur and 
phosphorus which remain in steel are especially undesirable. Sulfur makes it very brittle when hot, and phosphorus makes it very brittle when cold. The character of the steel rails produced by the Bessemer process became a very serious matter when, under increasingly heavy traffic, the rails broke with startling frequency. Breakages have been greatly reduced in number by improvements in the manufacture of steel rails, but they still occur frequently enough to be a serious problem. In 1915 more than 3300 railroad accidents were attributed to broken rails, involving a property loss of nearly $\$ 4,000,000$, with more than 7000 persons injured and over 200 lives lost. It is one of the urgent problems of to-day to produce steel rails that are flawless and free from the impurities that render them unsafe.

The Bessemer converter effectively handles pig iron from ores which contain a very limited percentage of sulfur and phosphorus. As such ores have been rapidly mined and are becoming scarce, the output of Bessemer steel has decreased and may eventually cease. Improvements in the converter have been made, such as lining it with magnesia to suit ores containing a larger percentage of phosphorus, but the cost is almost prohibitive. The slag which is produced from this lining and the substances introduced with the molten iron is a valuable fertilizer, because of its lime and phosphorus content. But the greatest advance has been in the development of the openhearth process.

The open-hearth process converts into steel, satisfactorily and profitably, ores or pig iron having a wide range of impurities, and is especially successful in eliminating sulfur and phosphorus. These impurities enter into the slag which, floating on top, is drawn off at the right moment. The process is under control at every stage, which is not the case with the Bessemer process. Samples are tested frequently, thus insuring a uniform and dependable product of the desired character. In making the various kinds of steel demanded in modern construction, precise quantities of the necessary substance, such as carbon, silicon, 
nickel, chromium, and aluminum, may be introduced when needed. It is also a point of economic significance that from a given charge of iron the open-hearth yields a larger return than the Bessemer converter. It is therefore not remarkable that the annual output of open-hearth steel is rapidly increasing, the 1913 production being about $21,600,000$ tons, or more than twice the tonnage of Bessemer steel.

The chief features of the open-hearth furnace are shown in Fig. 145. The basin into which the charge is put is rectangular

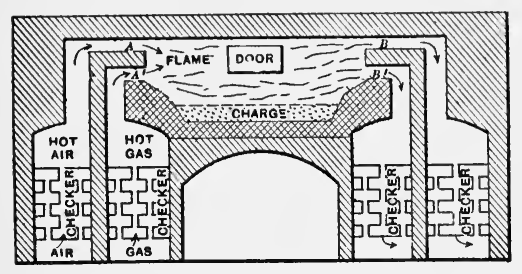

Fig. 145. - Open-hearth furnace (Diagram). The charge is introduced through the door. The direction of the air and fuel gas is frequently reversed, entering now through ports $A$ and $A^{\prime}$, and leaving through ports $B$ and $B^{\prime}$, and again passing in the opposite direction; this is the regenerative system of heating. in form and shallow in depth. The dimensions of the sixtyton type, which is the usual one for standard grades of steel, are fifteen feet wide, thirty to thirty-five feet long, and twenty inches or less in depth. The hearth bottom of the furnace, which is used to remove phosphorus and sulfur especially, is made with what is called magnesite. Magnesite is magnesium oxid prepared from magnesium carbonate by heating it to drive off the carbon dioxid, just as quicklime is made from limestone. (See page 417.) The ends, sides, and ceiling of the furnace are lined with silica bricks, which are especially resistant to high temperatures. The temperature of the furnace may reach $3000^{\circ} \mathrm{F}$. in the course of operation. The ports and chambers $\mathrm{A} \mathrm{A}^{\prime}$ and $\mathrm{B} \mathrm{B}^{\prime}$ are made with silica brick. The bricks in the chambers are arranged in such a way as to suggest the appearance of a checker board. The chambers are therefore called checker chambers or simply checkers. This arrangement is made to provide a large surface over which the air and gas must pass in entering, and the burned gases in leaving, the furnace.

The fuels generally used are natural gas, which is the best for 
the purpose, fuel oil, which is vaporized by a blast of air or by steam, and producer gas made from bituminous coal.

The furnace is kept in operation constantly. A charge which is put into the basin consists of molten iron, scrap steel, limestone, ferromanganese, and iron ore to furnish oxidizing and other necessary materials. The checker chambers, which remain hot from the previous operation, pre-heat the air and gas passing through them. After leaving the ports $\mathrm{A} \mathrm{A}^{\prime}$, the gas and air combine in combustion. The flame produced is like that of a blowpipe. It passes over the surface of the charge, oxidizing it. The burned gases pass out through the opposite ports and chambers $\mathrm{B} \mathrm{B}^{\prime}$, which they heat, and from which they are conducted to the stack. After fifteen to twenty minutes the air and gas currents, which are controlled by valves, are reversed, entering now through ports $\mathrm{B} \mathrm{B}^{\prime}$ and leaving through $\mathrm{A}^{\prime}$. The temperature of the checkers rises with each reversal so that the charge is subjected to a constantly rising temperature. Reversals are made throughout the heat which continues until tests show the desired composition of the product. The charge may be kept molten at a given temperature as long as needed. The control of the process is complete at every stage.

Chemical changes in the process. - The process is similar to that of the Bessemer converter. The flame and oxidized ore bring about the change of phosphorus into phosphoric acid, which unites with lime to form lime phosphate. This is drawn off in the slag. The sulfur combines, under the influence of the heat and oxidizing materials, with manganese to form manganese sulfid, and with lime to form lime sulfate, both of which are removed in the slag. Silicon and carbon are oxidized and pass off as gases or enter the slag.

Duplex process. - Where factories had already installed Bessemer converters, it was found that both processes could be advantageously employed. Molten metal may be first "blown" in the Bessemer converter, then transferred to the open-hearth furnace; or the two processes may be operated 
with different grades of iron. Such a combination is known as the duplex process.

Cementation and crucible processes. - For the production of the special quality of steel used in making fine tools and instruments, the cementation process is sometimes used. The general practice is to melt an almost carbonless wrought iron with enough fine charcoal to give the desired grade of carbon steel. Such steel contains from one to one and three fourths per cent of carbon. The product is sometimes called crucible steel. Strictly crucible steel, however, is made by heating molten iron in graphite crucibles, from which the iron absorbs as much carbon as is desired.

The electric furnace. - The use of the electric furnace in manufacturing steel has gradually increased since its first introduction into this country about 1908 . The annual output, which was for the first year only fifty-five tons, now exceeds sixty thousand tons. During this period more than twenty furnaces have been constructed, some of which have as large a productive capacity as the largest open-hearth furnace. By the electric furnace such impurities as sulfur and phosphorus are thoroughly removed and a steel of the highest quality is produced. Although because of its quality and the cost of production, electric steel was at first expensive, it is now possible to make it as cheaply as any steel can be made.

216. Kinds of steel. - Steel is thus seen to be a semi-purified form of iron. It retains or is supplied with a certain percentage of silicon when it is to be used for steel castings. To secure the right degree of hardness and strength it is supplied with or retains a certain percentage of carbon from that supplied in the reduction of the ore. Carbon determines the degree of hardness, or the kind of steel produced. The smaller the percentage of carbon, the softer is the steel; and the greater the carbon content within a limited range, the harder is the steel. Thus steels are classified as soft to hard, or low-carbon to highcarbon steels. They are often designated, for example, as .27, 
$.60, .71,1$, or 1.48 ; this means that a steel has .27 per cent carbon, and so on. A very soft steel, similar to wrought iron, may have one tenth of one per cent carbon. High carbon steel varies from one half to one and one half per cent of carbon.

217. Tempering steel. - One important property of steel must be considered. How this property is utilized may best be observed in a blacksmith shop where such tools as drills and picks are sharpened. Steel that has been heated red hot and then cooled slowly is very soft and useless for working hard rock or similar materials. If cooled very quickly, as by immersion in water, it becomes very hard, brittle, and likewise useless for such work. An intermediate degree of hardness or temper is necessary. Practical tool sharpeners know how to produce the right temper without the aid of thermometer or other apparatus. They have learned to interpret the colors which appear in the heated steel. For example, a sharpened edge of a drill or pick is immersed in water until it becomes black. One side of it is brightened by rubbing it on a stone or brick at the forge, when the play of colors is observed until the right color appears. Then the tool is plunged into water to cool and prevent further change. Thus a pick or drill is tempered or made with the right degree of hardness.

The colors observed by the tool sharpener indicate fairly welldefined ranges of temperature. (See page 89.) Heat passes from the main body or shank of the tool to the point, and the rise in temperature is shown by the successive colors and shades. The process is stopped by sudden cooling. The first color to appear is lemon, light at first but gradually becoming darker. It indicates a temperature of $392^{\circ} \mathrm{F}$. to $435^{\circ} \mathrm{F}$. or $440^{\circ} \mathrm{F}$. If the steel was cooled when this color reaches the very edge, it would be brittle and hard, not tempered suitably for rock cutting. An orange color follows, then a pink or " pigeon-wing," which indicates the temperature suited for rock and stone-cutting tools. A blue gives a temper suitable for mining bituminous 
coal, a tough steel point hard enough to wear well. Tempering by this method of observing temperature is limited to the range between $392^{\circ} \mathrm{F}$. and $572^{\circ} \mathrm{F}$. The table gives the important facts.

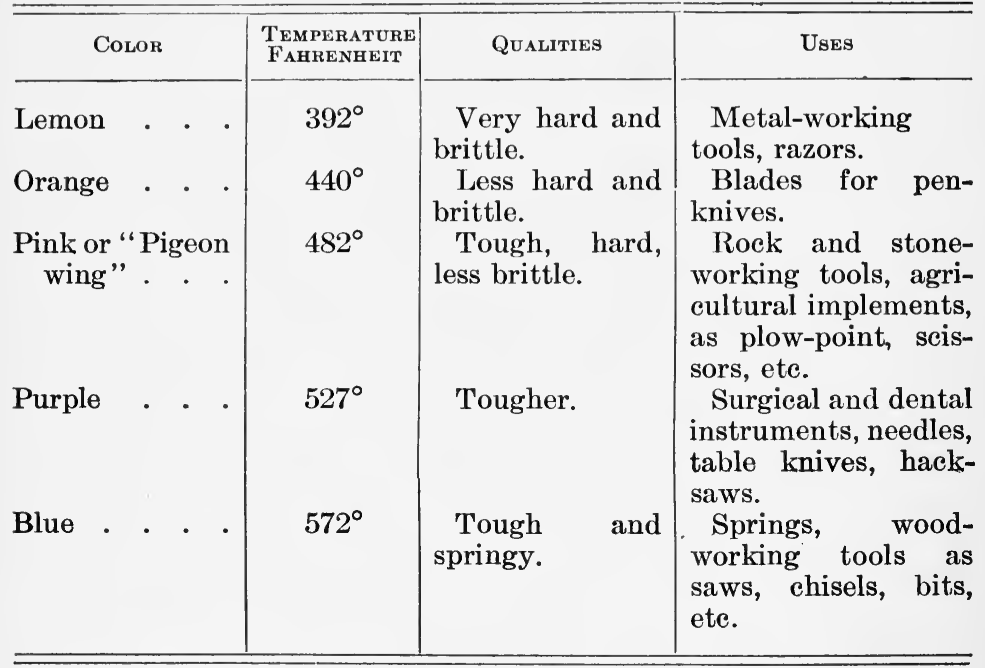

The color remaining upon steel after being tempered may be removed by polishing. Tempered tools of all sorts are usually polished enough to destroy all signs of the "temper colors." Occasionally a tool may be found which has blue markings, though these may be artificially produced.

218. Uses of steel ingots. - The general outline, page 440, shows the chief uses of steel ingots, the product of steel making. The rolling mills, steel foundries and forges, wire, rod, and nail mills, the structural shape, rail, pipe, and tube manufactures, to name only a few of the many auxiliaries which convert steel into finished products, indicate generally the extent and magnitude of the iron and steel industry of our country, and at the same time the main uses to which steel is put. 


\section{Wrought Iron}

The third way in which a small fraction, less than three per cent, of the total output of pig iron is utilized, is by its conversion in the puddling mill into wrought iron. Wrought iron is useful in blacksmith work. A small amount of it is used in the manufacture of crucible or cast steel (page 446). Because of its electrical conductivity and magnetic capacity, it is valuable as wire for such electrical communication as the telegraph, and as material for the cores of electromagnets and for the armatures of dynamos.

Wrought iron differs from low-carbon or soft steel in that it contains at least one per cent of slag. It has been described as a bundle of fibers with each fiber enveloped in slag. The slag and the purity of the iron are supposed to give wrought iron its chief value. It is nearly pure iron, never containing more than one tenth per cent each of carbon and phosphorus. But, steel of the best quality contains less phosphorus.

Wrought iron melts at about $1700^{\circ} \mathrm{F}$., but when heated red hot, becomes so plastic that it may be converted into any desired form. When it is cooled suddenly, it does not, like steel, become hard and brittle. It is generally tough, strong, and elastic. When red hot it is easily welded.

Wrought iron is produced from pig iron in a puddling furnace. The pig iron is melted, then while maintained at a somewhat lower temperature, is constantly agitated. Nearly all the impurities are thus oxidized and removed. As the iron becomes pure, it grows stiff and pasty. This is because the melting point of pure iron is higher than that of iron with its usual impurities. The pasty mass of iron is called "iron that has come to nature." The mass is divided into several parts, each of which is then rolled into a somewhat spherical form. Each mass is squeezed by a special machine into a smaller and somewhat cylindrical shape, weighing perhaps one hundred seventy-five pounds. By the squeezing, some of the slag or 
cinder is removed from the iron. The cylindrical lump of iron is called the bloom.

The bloom is rolled into what is called the puddle bar. By the rolling more of the cinder is eliminated, but the remainder is drawn out into string-like shapes which force a lengthening of the fibers of the iron grains enveloped in the cinder. The puddle bar is cut into strips usually three and one half feet long, which are piled together to equal the width of the bar or plate that is to be the final product. The bundle of strips is tied together with wire and placed in a furnace, where it is heated to a welding temperature. The heated bundle is then rolled to a plate or bar of the required dimensions. The usual length of the bars produced is seventy feet.

The finished bar is called in commerce the merchant bar. Its structure as revealed by the aid of a microscope is interesting. By the cutting, welding, and rolling the iron fibers have been lengthened greatly and now form a closely compact bundle of fibers, each of which is enveloped in its slag or cinder. The additional rolling has removed a small amount of the slag from the iron as it appeared in the puddle bar. But the final product retains from three fourths to one and one half per cent of slag or cinder.

\section{SUMMARY}

Iron is the most useful metal. It makes up 95 per cent of the total tonnage of metals annually produced. The United States is the greatest producer, with a yearly output of more than $60,000,000$ tons.

Iron is useful largely because of its strength, hardness, rigidity, density, high melting temperature, and its capacity for magnetism.

Iron in various compounds is distributed universally, as in the earth, water, plants, and animals. In abundance it is fourth among the elements.

Many meteorites are composed almost entirely of iron.

Iron ores include the iron oxids, as hematite, magnetite, and the brown iron ores, iron carbonate and iron sulfid. 
Iron ores are mined chiefly in the Lake Superior region, as in Minnesota, and in the Southern region, as in Alabama.

Methods of mining depend upon the character and position of the deposits.

Iron ores with few exceptions are sedimentary in origin.

- Iron manufacturing centers are located where iron ore, coal, and limerock can be most cheaply brought together and the output conveniently shipped to market.

Iron ores are reduced in blast furnaces. The oxygen combines with carbon and goes off as gas; other impurities melt with limerock, forming a slag. The product is pig iron.

A small part of pig iron is made into wrought iron, about one sixth is used for castings, and the rest for steel.

Steel is produced from pig iron in several ways.

The Bessemer process makes rapidly and cheaply a low-grade steel.

The open-hearth process, slower and better controlled, makes a high-grade steel, from which sulfur and phosphorus are eliminated.

The duplex process combines the Bessemer and the open-hearth processes.

The cementation, or crucible, process produces a fine tool steel.

The electric process produces the highest grades of steel.

Steel may be tempered, or made to have various degrees of hardness, by different rates of cooling.

Wrought iron, made in puddling furnaces, contains little impurity other than slag. It is made fibrous by rolling. It can be welded but not tempered.

\section{REVIEW QUESTIONS}

1. Compare the value of the annual production of steel and iron in the United States with the value of all other mineral products. With the value of the metal products. 2 . What is the approximate tonnage of iron produced annually in the United States? 3. For what uses is iron indispensable? 4. Mention some of the uses to which iron is adapted because of each of the following properties: strength, hardness, comparative lightness, rigidity, high melting point, magnetism. 5. Mention facts to show the wide distribution of iron. 6. Of what are meteorites of ten composed?

7. With what three different elements is iron generally combined? 8. Mention the chief ores of iron. Which has the highest percentage of oxygen? Which group contains water in its composition? Which is commercially most important? 9. Mention three states in order of their importance in the production of iron ore. 10. Which ore- 
producing region ranks first in importance? What kind of ores does it produce?

11. Describe how iron ores are mined. 12. Compare the formation of iron ore with that of sandrock and limerock. 13. What was the origin of the iron ores of the Lake Superior region? 14. Describe the bodies in the Birmingham region. 15. How have the brown ores been formed?

16. Describe a blast furnace. 17. Why do iron-producing firms employ chemists? 18. How is the oxygen removed from the ore in the blast furnace? 19. How are impurities like silica removed? 20. How is the molten iron from the blast prepared for commerce? What is it called? 21. What uses are made of slag? 22. Why is the dust from the smelter saved? The gas? 23. What are the characteristics of pig iron? 24. What direct use is made of pig iron? 25. How does malleable cast iron differ from ordinary castings?

26. What change takes place in the composition of pig iron when it is converted into steel? 27. What proportion of pig iron is converted into steel? 28. Name the processes by which steel is made from pig iron. 29. Describe the construction and operation of a Bessemer converter. 30. (a) What two objectionable elements does the Bessemer converter fail to remove entirely from the pig iron? (b) What effect has each of these impurities on the character of the steel? 31. Why does the open-hearth process yield a higher grade of steel than the Bessemer process? 32. (a) What is the duplex process? (b) What are its advantages? 33. What process is used for tool steel? 34. What is the source of carbon in the cementation and crucible process? 35. (a) What is the most recent process of making steel? (b) What quality of steel does it produce? (c) What are its advantages in comparison with other processes? 36 . What is steel? 37. How is its hardness dependent on the proportion of carbon it contains?

38. (a) What is the purpose of tempering steel? (b) Describe the process. 39. What does a blacksmith use as an indicator of temperature? 40. Why are steel tools and instruments polished? 41. Mention several uses of steel.

42. What percentage of pig iron is converted into wrought iron?. For what purpose is it used? 43. Compare wrought iron and soft steel. 44. What are the useful properties of wrought iron? 45. (a) How is wrought iron made? (b) What is the "bloom"? The "puddle bar"? The "merchant bar"? (c) What is the characteristic structure of the "merchant bar"? (d) How is the structure produced? 


\section{SUGGESTIVE QUESTIONS}

1. Why are meteors the only iron found in nature not in compounds? 2. Which would you compare to a rod of wood, a wrought iron bar or a steel bar? Explain. 3. How does a blacksmith weld pieces of iron? 4. If gold were as abundant as iron, and iron as rare as gold, what would be the effect on our civilization? 5. Why was copper used before iron? 


\section{CHAPTER XI}

\section{PLANTS IN RELATION TO MAN}

\section{The Soll and Plants}

219. Importance of the soil. - An old fable represents a dying father telling his sons of a wonderful fortune hidden within a foot of the surface of the garden. They, supposing that his words referred to a chest of gold and jewels, began to dig up the garden. When they had dug over the whole garden and still found no treasure, the eldest brother suggested that they should not lose all the profit of their labor, but plant the garden thus tilled. In due course they gathered a wonderful yield and began shrewdly to suspect that it was this to which their father referred as their treasure. Then they labored to put all their land under cultivation and thereby won wealth beyond expectation and greater in value than a chest of gold and precious stones.

This fable is more significant to-day than ever before. It points clearly to the ultimate source not only of a large part of human wealth, but also of all the things that supply our daily needs. The surface of the earth with its thin skin of soil only a few feet in depth produces the plants upon which we depend for food and for materials to provide clothing and shelter. The soil and its use provide not only the foundation, but the support of human life and society.

A study of soil. - What is the soil? We may learn much about it through formal descriptions in books, but we shall learn a great deal more by studying the soil directly and finding out for ourselves its essential characteristics and composition. 
A study may be carried on in the following manner and should include individual reports to the class of the results of observation.

Exercise. - Select a small area of ground about two square feet, uncultivated if possible. Dig a hole three to five feet deep. Cut one side straight and sharp. Note and record the differences in the size and character of the particles of the several layers from top to bottom. Note the changes in color, in solidity, and in compactness. What explanation can you give of the color, size, and character of the particles? How far down does what is called the soil reach? What is the general appearance of the subsoil? How does it differ from the soil? If you cannot answer this question now, keep it in mind for later consideration. What reasons can you give for the difference in compactness of the various layers?

Note the moisture at different levels. Collect carefully samples of each of the various layers of earth through which you dig, putting each in a glass jar with closely fitting cover. If you have a thermometer, you might measure the temperature of the surface soil and that of the lowest layer.

You may find some decaying and decayed organic matter, such as roots, leaves, and stalks of plants, etc. What do you find as regards the color, moisture, and compactness of this material? What is the character of the soil surrounding it? Do you find decayed vegetable matter at the lower levels of the hole? Why? Does this material seem to hold more moisture than the other kinds of soil? Such vegetable matter when saturated with water, as that accumulated in a marsh or a filled-in lake, is changed into what is commonly called muck, and this when partly dried out is known as peat. In the prairies, however, where roots of grasses have accumulated for centuries, or in woodlands, one may find great quantities of vegetable matter which decayed in the ground, became black like tar, but different from muck, and is known as humus.

The quantity of humus in the soil examined may be measured roughly. Weigh out a small quantity of thoroughly dried soil, put it into an iron pan that can be placed on the stove over a hot fire and left there for about an hour. Do not let the fire reach the soil directly. When thoroughly burned, remove and weigh it. What you have left is mineral matter. The difference between the two weights indi- . cates the amount of humus contained in the soil.

The depth of the soil or that part of the earth's surface containing humus is not on the average more than fourteen inches. In cultivated 
fields the plow seldom goes as deep as that. The layers beneath the soil are known as the subsoil. This is much more compact, harder, and contains no humus; and, if plowed up, would be destructive to profitable crops.

220. Classification of soils : On the basis of texture. - Soils are classified in several ways. It may be observed that all soils are largely composed of rock fragments of different sizes. Classification on the basis of size of particles is common. Test different soils by rubbing between the hands or fingers. If a soil feels smooth, its particles being very fine, it is clay. This, if wet and worked, will retain any shape given it. If a soil feels gritty when rubbed, it is silt. The particles of silt are so fine, however; that they can be seen only with the aid of a magnifying glass. Soil which is mostly made up of particles about the size of a period (.) is sandy, and is called sand. When it is composed of rock fragments about the size of the letter o, it is gravelly. If the soil is of particles many times the size of $o$, it is gravel. Perhaps at the bottom of the test hole is found rock, such as sandstone or limestone, or just a bowlder of rugged shape.

Soils are more than mixtures of rock fragments. They contain humus, and this adds to the above classification. Soil containing humus usually varies from a dark shade to black, according to the amount. If the humus is mixed with sand, it is known as sandy loam. If the mixture is of clay and humus, it is a clay loam.

On basis of origin. - Soils are classified also with respect to their origin. For example, the soil of the great flood plains of the Mississippi, the Nile, the Sacramento in California, and other rivers, is alluvial. The soil was transported by the rivers. from the upstream country and deposited on the flood "plains. Its fertility seems to be almost inexhaustible.

- The soil of a large part of North America is glacial in origin. Thousands of years ago great ice sheets covered an extensive area, as far south as the Mason and Dixon line in the eastern 
section of the United States. Masses of rock and earth were taken up by the glaciers and while being pushed along were crushed into fragments of different size, and deposited as moraine. The drift formed by glaciation differs greatly in character and value. On Cape Cod the soil is sandy. Over a large part of the eastern glacial region the soils are coarse, a mixture of sand, gravel, and clay, with a liberal supply of bowlders often curiously placed. In the western area, as in Illinois, Iowa, and Nebraska, the texture of the soil is finer and is exceedingly productive.

There is a third kind of soil known as the lacustrian. This is the sediment deposited on the bottoms of lakes that have since disappeared. Of the many lake plains in North America that of the rich wheat-growing valley of the Red River of the North is the most interesting. This area, which is greater in extent than the combined area of the Great Lakes, comprises what are now large parts of Minnesota, the Dakotas, and Manitoba. While the glacier was melting and slowly receding northward, it blocked the outlet of this river and caused the formation of a lake called Lake Agassiz. When the river was opened, it drained the lake. The land is very level, and the soil is of the right texture and richness to produce wheat most profitably.

221. The soil's moisture. - Soil must, however, be more than a mixture of rock fragments and decayed organic matter. You find that it is moist at different depths ; but the moisture is not like free water (page 466). This is generally called soil water or soil moisture, as it is an indispensable part of a productive soil. Large areas of the United States do not receive enough rainfall to produce more than a scanty vegetation. Such soil is arid. Soils that receive and retain enough moisture for plant production are humid. Plants that grow on arid soil are either specially adapted to prolonged drought or provided with long roots that reach deeply into the earth to find necessary moisture and food. 
Different soils from coarse to fine have different capacities for soil water. This is important in agriculture. A simple test will illustrate the principal differences in the moisture capacity of soils.

Exercise. - Weigh out from the sample soils, before they have lost their moisture by evaporation, about four ounces of each. Dry each portion thoroughly, but without burning the humus, over a flame in the laboratory or in the oven at home. After each is dried, weigh and compare the weight with that of the fresh soil. This shows the amount of soil water each contained.

Next put the dried soils into glass tumblers and pour over each just enough water to reach the surface. Drain off the free water. Weigh the soil. This gives the total capacity of the soil for moisture. Arrange the samples of soil on the basis of their respective capacities for moisture. What does this show as to the texture of the soil?

A fine clay soil holds more moisture than a coarse, sandy soil or a gravelly soil. The size of the soil particles seems to determine the soil's capacity for water. You will find by examination that the particles hold moisture only on their surface. Consequently the smaller the particles, the greater must be the moisture capacity of the soil, for the greater is their combined surface area.

Exercise. - This fact may be clearly illustrated in a simple way. A stone measuring one cubic inch has a surface area of six square inches. Break it into two equal parts; its surface area is increased by two square inches. Break it again and again until it is made into particles the size of coarse silt. The total surface area of these particles is about twenty-two square feet. Now if moisture is held only by the surface area, obviously more of it is held by the minute particles than by the original cube.

Does water readily penetrate to the interior of gravel or of larger rock fragments? Put a pebble into water and let it soak for a while. After taking it out, wipe with absorbent material to remove the thin film of water that envelops it. Break it and examine the inner surfaces for moisture. Has the moisture penetrated? Test by breaking some of the large gravel which you may have found in digging where moisture is plentiful. Select a handful of pebbles, dry thoroughly by wiping, weigh accurately, and put into an oven to dry. When removed, weigh and compare with former weight. 
Amount of soil water. - The quantity of water contained in any soil is greater than one may imagine. The surface soil may be very dry; but a few inches below there is much moisture. If you reach clay, you will find it actually wet. This is not free water, but what adheres to the particles of soil and subsoil. It is estimated that a cubic foot of clay, which when dry weighs about eighty pounds, has a moisture capacity of forty per cent or about thirty-two pounds. The moisture capacity of soils varies approximately from forty per cent to as low as five per cent of their dry weight. A hundred pounds of sandy loam may hold twenty per cent or twenty pounds of water. Humus or decayed organic material has still greater water capacity and greater retaining power.

Character of soil water. - Soil water remains after the soil has been drained of free or rain water and is quite different in character from this. Water, one of the most active natural solvents, begins to dissolve soil particles immediately when it comes into contact with them. It takes up something of practically all substances in the soil and subsoil. The finer the texture of the soil the greater is the total surface area exposed to the action of water, and the greater, therefore, is the yield of substances to its solvent power. The longer water remains in contact with soil materials, the more concentrated will be the resulting solution. Even ordinary free or well water shows under chemical examination that, although it has moved rapidly through soil and rock strata, it has dissolved away some substances, such as lime. But the quantity of materials contained in free water is very small in comparison with the amount held in solution by soil water.

Substances in soil water. - Because it is in contact with the soil particles for a longer time, soil water dissolves away and retains larger quantities of more substances than does free water. Among the substances which it thus obtains are compounds of nitrogen, potassium, calcium, magnesium, iron, and silicon. All of these are necessary as food materials for plants 
in their growth and maturity. Other substances contained in soil water and sometimes found in the ash of plants are sodium, chlorine, fluorine, and sulfur. Besides these soil water provides plants with hydrogen, oxygen, and some carbon. What amounts of these substances plants derive from soil water may be seen in the table, page 464 . How indispensable they are to plants and to all living organisms may be inferred from the fact that they are all constituents of the protoplasm, the living substance, of the cells of which an organism is composed.

222. How plants obtain soil water. - Plants utilize enormous quantities of soil water, and more than half of the total weight

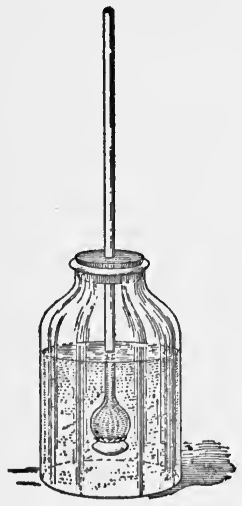

Frg. 146. - Arrangement of apparatus to show osmosis. of a living plant is due to its water content. Water is absorbed through the delicate root hairs and possibly through the tender areas of the roots adjacent to the root hairs. The sap of plants is a concentrated solution of many different substances, such as sugars and salts. This solution is denser than soil water, which, as before stated, is a weak solution of the substances dissolved from the soil. In the following exercise the plant sap may be represented by a sugar sirup or molasses, and the soil water by ordinary water. To represent the membrane covering the root hairs a piece of bladder or of sheet rubber may be used. This membrane has no openings, but is permeable by water. The apparatus may be arranged as in Fig. 146.

Exercise. - Cover the large end of a long-stemmed thistle tube, a funnel, or a tall, narrow lamp chimney with a piece of bladder or sheet rubber to be stretched tight and tied securely with a waxed string. Fill the bulb and one to two inches of the stem with the molasses or sugar sirup and arrange, as in Fig. 146, in a wide-mouth bottle or pan 
filled with water reaching to the level of the solution in the tube. Let this stand undisturbed for several hours.

A more rapid reaction can be obtained by using olive oil instead of molasses, and sulfuric ether instead of water.

Arrange a similar apparatus, but fill both bulb and bottle with clear water. Compare the results of the two. Does the water pass through the membrane in both cases? What facts support your answer to this question?

In the first experiment does the molasses pass through the membrane into the water in the bottle? Test this point. In what direction does the larger amount of the liquid move, toward the denser solution in the tube or toward the water in the bottle? Does the fact that the liquids in the second experiment are equal in density account for the facts observed? Explain.

Osmosis. - Water passes from the bottle through the membrane into the bulb and tube, and may eventually cause the solution in the tube to overflow. A small percentage of the sugar sirup passes through the membrane into the bottle, as your tests may show. The same fact may be observed when dried fruits, such as raisins and prunes with intact skins, or dried vegetables, like beans and peas, are soaked in water. This passing of solutions through permeable, but not porous membranes is known as osmosis.

A similar interchange of solutions is taking place through the membrane of root hairs and the adjacent parts of roots of plants. Soil water containing in solution food substances needed by plants is taken by osmotic absorption through the root hairs and then passed in circulation through the plant. At the same time certain substances in solution, such as carbon dioxid, are excreted through the root hairs and attack and dissolve certain materials, as limestone, in the soil, rendering them capable of absorption. That this is done may be verified by putting several small, smooth pieces of marble into soil where the roots of plants will reach them. After a lapse of several months examine them.

The root hairs are exceedingly well adapted to their work. They are so fine that they can penetrate the smallest inter- 
stices of the soil. Their tender, pliant character enables them practically to envelop (cf. Fig. 147) the soil particles with which they come into contact, and to absorb the film of soil water which covers these particles. It is nearly impossible to free root hairs from the soil without tearing them from the roots, unless the greatest care is exercised.

Exercise: Root hairs. - A study of root hairs, their short life, their position relative to the tip of the root and to that part of the root which increases in length, and other important points may be made by growing such plants as wheat, radish, bean, or corn in moistened blotting paper. When the roots are long enough, mark off with India ink equal lengths, as one sixteenth of an inch, from the tip of the root

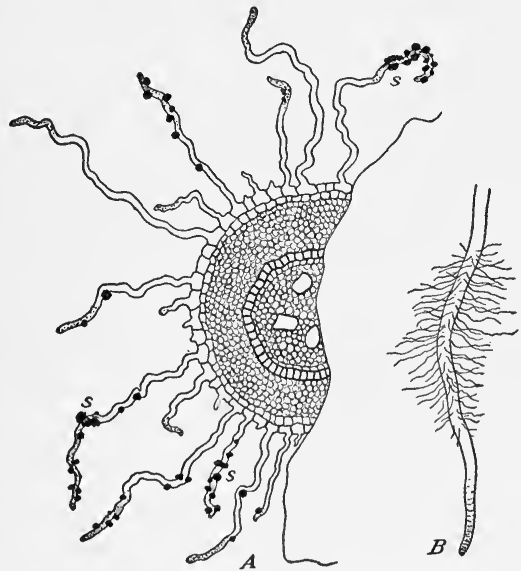

Fig. 147. - A. Diagrammatic sketch of part of a transverse section of root, showing surface cells and their extension as root hairs. Soil particles held by root hairs, $s$. $B$. Typical root with root hairs.

stages of development from the surface cells is represented diagrammatically. The root hairs are really tube-like extensions of the epidermal cells and contain in solution the organic substances (called root-sap), which make possible the osmotic absorption of soil water with its food materials. measure every day to determine how rapidly and where the increase in length occurs. Mark the extent of the root covered by root hairs and their position relative to the elongating part of the root. Note their relative number at different times, their length, sof tness and delicacy, and the short life of individual hairs. Note where new hairs are being constantly developed, and where the older ones disappear. Examine with a microseope both transverse and longitudinal sections of roots with root hairs.

In Fig. $147(A)$, a part of a transverse section of a root with root hairs at different.

to the stalk. Examine and 
The importance of root hairs is strikingly shown by a plant which has just been transplanted. For several days such a plant wilts, regardless of the nature of the soil and the care with which soil is packed about the roots. What does this indicate as to the condition of the root hairs and their importance to the plant? Can plants get the water and food materials they need from the soil without root hairs? Can they get enough of these materials if the root hairs are injured to any considerable extent?

This suggests the proper time for transplanting. If the plants are in full leaf and transpiring their usual quantity of water, can they be transplanted advantageously? Would it appear to be better to transplant them when they are not in leaf, as in early spring or late autumn? What facts have you observed which support your answer to this question? If the plants to be transplanted are in leaf, would pruning or trimming them be advisable? Why?

223. Transpiration. - That plants absorb and release through transpiration and evaporation large quantities of water as vapor may be illustrated experimentally in many ways. The several methods described in the following experiments should be considered primarily as suggestive; and you should devise ways and means of your own to demonstrate it. You may find by observation many signs of the process in plants, especially on hot days.

Exercise. - Take a potted plant which requires a considerable amount of moisture (certain lilies, such as the Chinese lily, will do); give it a full supply, and then wrap the pot carefully in a piece of sheet rubber (or moisture-proof oiled paper), closing it well around the plant stalk just above the soil or water surface. Weigh the whole accurately. Weigh again at intervals of four to six hours for two or three days. Be careful to prevent evaporation of the water except through the plant. The following data will be instructive.

Weight of pot wrapped,

Weight after 6 hours

Weight after 12 hours

Weight after 24 hours
Weight after 30 hours Weight after 36 hours Weight after 48 hours 
What do the results show? How much water is lost in a day? Does it seem to depend upon the temperature of the room where the

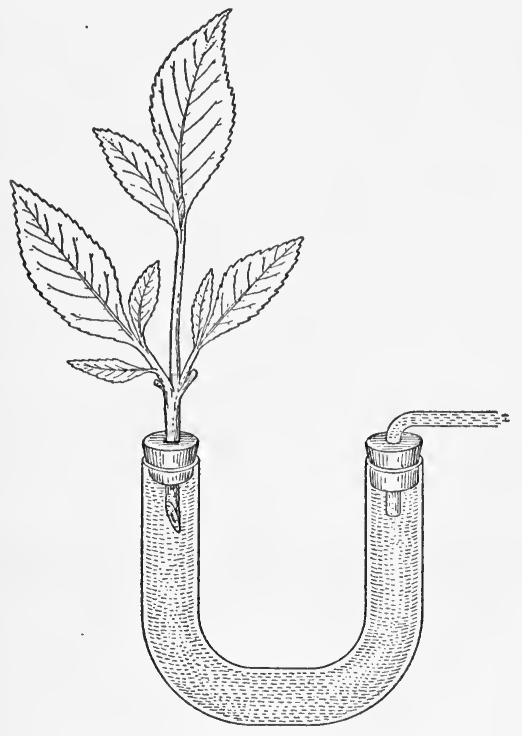

Fig. 148. - Arrangement of apparatus to show transpiration. plant is kept? Does it vary by day and by night?

The same fact may be shown with the apparatus illustrated in Fig. 148. Select a stem or shoot that is fresh and plentifully supplied with leaves. See that the corks fit close to prevent escape of water except through the branch. Mark the place the water reaches in the tubing. Set the apparatus in a good clear light.

Table of materials used by plants. - The quantities of materials that plants remove from the soil, the amount of soil water utilized and released through transpiration and evaporation, and its equivalent in inches of rainfall, are presented in the following table. The crops are expressed in bushels or pounds per acre.

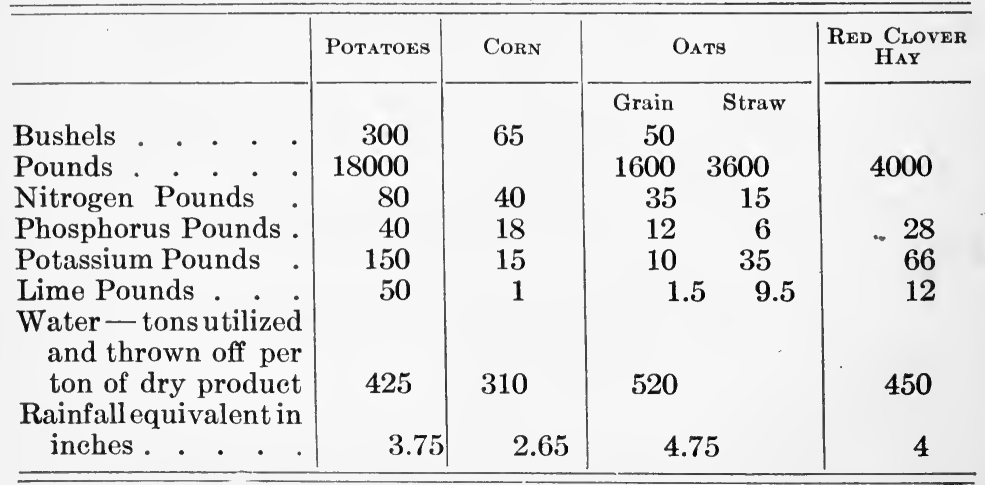


Water retained in plants. - These facts show that enormous quantities of soil water are utilized and thrown off in transpiration and evaporation by plants. But considerable quantities are retained also by plants and their products. Apples, for example, are about eighty-five per cent water; potatoes about seventy-eight per cent, tomatoes ninety-four per cent, and lettuce ninety-five per cent water. Fresh corn is seventyfive per cent, string beans eighty-nine, and dried beans fourteen per cent water. Oats fall to a lower point in water content, having only twelve per cent; and wheat has about ten per cent. A ton of dried clover hay is about fifteen per cent water ; but in its growth and maturity it has made use of more than three hundred eighty-five tons of water. In general, plants in the manufacture of one pound of dry substance utilize in transpiration from two hundred to five hundred pounds of water.

Plants and the soil's capacity for moisture. - Knowledge of the capacity of different soils for moisture is necessary in order to judge the suitability of soil for different kinds of plants. One kind of plant needs a comparatively moist soil, while another thrives best in a dry soil. Potatoes do not require nearly as much moisture as red clover. Other kinds of plants show similar variation in their need of soil water.

Crops and soil water. - When the amount of soil water required for any given crop is known, together with the mean annual rainfall of the region and the loss by drainage and evaporation, it is a comparatively simple matter to select the crops best adapted to the conditions. Where sufficient water is not provided by rainfall, or is supplied in the wrong season of the year, it may be supplemented by irrigation or conserved by dry farming (page 468). Where the soil has an excessive amount of water, it may be drained, as is done with marsh or swamp lands.

Exercise : Leaves. - Plants show interesting adaptations of size of leaves to the environmental conditions, such as soil and soil water, and the heat and humidity of the atmosphere. One may compare the 
leaves of tropical and subtropical plants with those of plants native to the temperate and colder regions. Do large leaves transpire more than small leaves? Are small leaves better adapted to a hot, dry climate than large leaves? In what ways does the size of the leaf affect transpiration?

Compare leaves of typical common plants and seek to ascertain what relation, if any, there may be between the size of the leaves and the external conditions. Examine pine, spruce, tamarack, hemlock, maple, willow, locust, oak, poplar, eucalyptus, rubber plant, grasses, and weeds.

In studying the relation of size of leaves to external conditions, consider also the relation of size and arrangement of leaves on twigs and branches to sunlight. Examine from different standpoints the exposure of leaves of a tree to light, as looking upward into the tree, and conversely looking upon a tree from some higher position. Note the comparatively small amount of sunlight that escapes through the tree to the ground. Examine the size and arrangement of leaves on such plants as the hickory, maple, horse-chestnut, willow, and oak; and then on vines such as the grape, ivy, and melon or eucumber. The significance of this will appear more fully later; at present it may be considered incidentally while dealing with other features.

224. Behavior of water in the earth. - After it has fallen upon the soil; water tends to find its way to the lowest permeable layers of the earth's crust. Many soils are superposed upon what is called "hard pan," an almost impenetrable kind of material. Water sinking to this level rests or flows slowly in the direction of least resistance, or downhill. All water which flows away in this manner and is in excess of what the soil can absorb and retain, is called free water.

Soil water, however, behaves in a very different way. It does not move actively in the soil, but, film-like, temporarily envelops the. soil particles. As the evaporation at the surface increases, the soil water moves upward slowly to the surface. This upward movement of soil water is important for plants, and its control is often of the greatest advantage to the agriculturist.

Capillary action. - This peculiar behavior of water in the soil may be illustrated by many facts of common observation. 
When the end of a strip of cloth is dipped into water, one may observe the water steadily creeping above the surface level on the cloth. A dry lamp wick inserted into the oil reservoir of a lamp carries the oil to the top of the wick, where it may evaporate or be consumed by burning. If one corner of a cube of sugar is dipped into tea or coffee, the moisture gradually extends throughout the cube. Blotting paper, wood, and many other substances illustrate the same fact. This may also be the way by which water absorbed by the roots of plants is forced to the tips of twigs and leaves, where part of it is lost through evaporation and the rest is used as a medium of circulation in the plant.

Exercise: Capillarity. - Holding closely together two pieces of glass, dip one end into water. Note the gradual rise of water between the glasses. If practicable, vary the space between the glasses, and note difference in rate at which water rises as the space becomes smaller. Arrange three or four pieces of glass tubing of different diameters, ranging from one eighth of an inch to the smallest size obtainable, in a pan containing a small amount of water. Observe the behavior of the water in the tubes of different bore. Does the result indicate that the smaller the bore, the higher and more rapidly water rises?

This behavior of water in very small spaces is usually described as capillarity. The term capillary means strictly "hair" from the Latin word capillus; capillary tubes are tubes with hair-like openings.

Capillarity in the soil. - The soil is literally shot through and through with such hair-like openings or pores. Water retained after natural or artificial drainage rises gradually toward the surface, where it is evaporated or absorbed by plants. The water is said to be "attracted" or lifted by capillarity to the surface. It is exactly the same as in the case of the lamp wick, which carries oil from the reservoir to the burning top. The rate at which capillary action of water occurs may be shown experimentally to depend upon the character or texture of the soil. 
Exercise. - Have several different kinds of soil such as clay, clay loam, sand, sandy loam, and gravelly soil ; the same number of pieces of glass tubing, each about one inch in diameter and six inches long, or tall lamp chimneys. Cover one end of each piece of tubing or chimney with a piece of cheesecloth and tie securely. Place the covered ends in a large pan and put into each one of the sample soils. Pour about one inch of water into the pan. Observe the rise of water in each, and estimate the rate of rising.

The amount of water each kind of soil will take up may be determined quite accurately by putting each tube into a separate pan and supplying measured quantities of water until the surface of the soil shows moisture. One should test the rate of capillarity with a soft mulch surface and with a hard smooth surface. These will show the effect of mulching the surface to conserve moisture, and of rolling to increase capillary rise of moisture to the surface.

Why farmers roll soil. - Many farmers, after sowing a field with grain, such as wheat or oats, roll it. As the experiment shows, this causes the water to rise rapidly to the surface. The rolling makes the capillaries or pores in the soil more continuous, with the result that the rapidly rising moisture reaches the grain and insures its germination. After seed begins to grow, the smooth, hard surface may be broken up to form a mulch, which largely prevents the escape of soil water.

Dry farming. - A large area of land in the United States does not receive sufficient rainfall during the season to raise a profitable crop. The rainfall of two seasons may be enough for one crop, or that of three years may suffice for two crops. The rainfall is conserved by dry farming. First the ground is plowed as deeply as is thought necessary to provide a deep reservoir. After a rain which is immediately absorbed by the loose, soft soil, the surface is worked into a mulch three or four inches deep. The mulch is retained through the year by frequent shallow cultivation. Thus the continuity of the capillary spaces is destroyed and water is prevented from rising by capillary action to the surface. Evaporation is also greatly reduced or prevented by the fine mulch. At the beginning of 
the second season, seed is planted and the surface soil cultivated, if necessary, to conserve moisture.

This practice is common also in many fruit-growing districts, as in California. During the dry season, when fruits need water for full development and irrigation is not available, the surface soil is thoroughly and frequently tilled, with the result that the soil moisture is conserved for the trees and is not lost by direct evaporation.

225. Soil air. - Soil air is an essential constituent of productive soil. The farmer and gardener recognize this and seek by cultivation to ventilate or aërate the soil sufficiently for growing plants. The relative quantity of air in the soil is, like that of water, dependent upon the size of the soil particles. The finer the texture of the soil, the larger the number of interstices and consequently the more air contained. The coarser the particles, the less the number of interstices and consequently the less air contained. How can you test this? But the amount of air in a soil is not the only important factor. It is the circulation of the air in the soil which is important, and which likewise depends upon the size of the interstices. The larger the interstices, the better aerated or ventilated the soil is likely to be. Thus a coarse silt or loam is better aërated although it contains less air than a very fine silt or clay.

Measuring the soil's capacity for air. - It is estimated that an average grade of soil holds about one half its own volume of air. This estimate may be tested with different kinds of soil of normal moisture and condition.

Exercise. - Put fifty cubic centimeters of each sample soil into glass jars and shake down well to get about the same compactness as in nature. Pour from a graduated beaker containing fifty cubic centimeters of water just enough to reach the surfaces of the soil. How much water is required? This represents in each its air capacity while holding its usual amount of soil water.

Now dry out each sample thoroughly, and again saturate with water. The amount of water now taken is practically equivalent to 
the total air capacity. From these data may be computed the percentage of air capacity of each.

Relation of soil air to plants. - Water may occupy all the air space in the soil. But plants which are thus deprived of soil air quickly fail. To exclude the soil air from a potted plant, cover the surface of the soil with paraffin, or wrap the pot in air-tight material, closing carefully about the stalk of the plant. Observe the result. Other conditions may be kept most favorable for plant life, but exclusion of soil air is fatal.

Many instances of the effect of water in shutting off the soil air supply of plants may be found in nature. Such plants are said to be drowned; but they are in fact suffocated. Trees whose roots may be supplied with nutrient soil, which is covered with air-tight material, as concrete or a hard, impervious clay, do not thrive, and eventually perish from lack of soil air. Many plants, however, as rice, taro, marsh grasses, water lilies, kelp and other seaweeds, grow luxuriantly in water-soaked and water-covered soil; but they are specially adapted to get the necessary air from water.

Agents of soil aëration. - How is the soil aërated? Men cultivate it, loosen it about the roots of trees and around certain farm and garden crops. But nature works to the same end in different ways. The activities of earthworms, ground moles, field mice, and ground squirrels are well known. Grasses and large plants loosen the ground with their roots and allow the air to permeate the spaces. Frost is an important factor. The expansion of freezing moisture in the ground widens the interstices between particles of soil; then, with the thawing and escape of the water, the enlarged spaces are occupied by air. Note the condition of ground which has just thawed out, especially where it was hard packed before freezing began.

Importance of soil air. - Soil air is indispensable to the existence of bacteria and other forms of microscopic plant and animal life, which are necessary to insure a fertile or productive soil. Some plants of economic value, such as the clovers, 
beans, peas, vetches, alfalfa, and the like, known as legumes, depend upon bacteria for nitrogen which they must have in order to live. It is sometimes stated, but incorrectly, that legumes obtain the necessary nitrogen directly from the air; but the fact is that all such plants are unable to assimilate nitrogen except in certain compounds called nitrates (page 572). The bacteria which live in the nodules of the roots of the legumes take nitrogen from the air and, after utilizing it for their purposes, throw it off in waste compounds or nitrates which may be directly absorbed and assimilated by the plants. The roots of such plants, when left in the soil, add to its nitrogen content, insuring thus profitable growth of other plants that are not favored similarly by bacteria.

Organic matter, such as plant stalks and roots and manure, is converted into plant food by the action of bacteria different from the kind just mentioned. These bacteria, together with molds, yeasts, and other minute organisms (page 552), bring about the decay or putrefaction of such matter. They are dependent, however, upon the proper aëration of the soil; they also aid in this by causing the decay of roots and other vegetable matter, which leave spaces where the air circulates.

Of the larger organisms living in the soil and depending upon soil air, we may mention the familiar earthworms, moles, and mice among other underground animals. These, by burrowing through the soil and by other means, contribute to its aeration and fertility.

If air is expelled and kept out of the soil, as it may be, for instance, by water, its absence is soon shown by plants. It is perhaps most easily observed in the case of the leguminous plants. The bacteria supplying these plants with nitrogen compounds are seriously affected or destroyed; and the plants become sickly, turning yellowish in color, as every gardener has observed. This unhealthy condition is due directly to the absence of the nitrogen products of the bacteria, and indirectly to the absence of soil air. 
This condition of the soil, preventing the activities of the nitrifying bacteria, is, on the contrary, favorable to the activities of a different kind of bacteria called the denitrifying. The bacteria of this group attack immediately and disintegrate the nitrogen compounds already formed, setting the nitrogen free in the air. That is, they deprive plants of the food materials provided by the other bacteria.

These facts show that the productive soil is not dead, but alive, very complex, interesting, and throbbing with energy in every particle. To regulate and adapt it to the growth of plants of economic value requires knowledge of its complex nature and properties. This knowledge must not be limited to the elementary facts that soil is composed of rock fragments of different kinds and sizes and requires humus and certain fertilizers for replenishing the substances which vegetation removes. It must include also such facts as the soil's dependence for fertility upon a sufficient amount of soil water, soil air, and aëration, and the activities of beneficial species of living organisms, both plant and animal. Such knowledge will aid, moreover, in determining with what kind of bacteria a soil should be inoculated for a given crop, such as alfalfa, as is considered necessary in modern agriculture.

226. Soil temperature. - The study of soil temperature involves many conditions, some of which have been considered in other connections. In the study of heat (page 109) it is shown that colors, kinds of surfaces, as rough or smooth, and the position of objects relative to the sun or other heat sources have much to do with the absorption and reflection of heat. Some colors absorb heat rapidly, others slowly. Some retain heat long or yield it up slowly, and others behave still differently. The kind of surface, rough or smooth, and the position of the body, as directly or obliquely exposed to the heat source, determine the amount of heat absorbed. A hill with a north exposure is not as "warm" as one with a southern exposure. In dealing with the temperature of the soil, these 
conditions must be considered, as they are vital to agricultural practice.

Exercise. - The temperature of a soil may be found by a thermometer left for a while in a hole made by driving a sharp-pointed stick into the ground. The reading shows very closely the temperature at given depths. Select different kinds of soil, and soils in different positions with reference to the sun. Tabulate carefully the results of your observations, and make use of them in consideration of the conditions of moisture, topography, surface color, surface character, and kind of soil, which determine the temperature.

Soil temperature and moisture. - Moisture and soil temperature are closely related, as may be inferred from the common observation of gardeners that a soil is cold or warm. By this it is meant that a cold soil has a large capacity for moisture and holds it long; such a soil is usually of fine texture, as clay or clay loam. A warm soil has less moisture capacity, better air-circulation and air-capacity, and is usually of a sandy character.

Water is an important factor in a soil's temperature for two reasons. First, water has a high heat capacity (page 142). It is warmed slowly, absorbing a large amount of heat without becoming sensibly warmer; and it gives up the heat it may contain just as slowly. Second, water is being constantly evaporated, and evaporation utilizes heat (page 306), thus holding down or reducing the temperature of the body giving up moisture. The heat utilized thus is taken away as insensible or hidden heat (page 305). The body giving moisture is kept cooler.

Exercise. - Apply these facts to any given soil temperature. Suppose an area of soil receives directly the sun's rays, but gives up freely a large amount of soil water through evaporation. What will be its temperature character? Suppose there is a long, hot, dry spell. What will be the result as regards the moisture retained? How will this affect the soil temperature? Does soil itself retain heat well or long? Does the observed fact indicate that the uniformity of a soil's temperature depends upon its moisture capacity and retention? 
Find experimentally the capacity of a fine clay loam for moisture, for air, and for heat; of a coarse sandy loam for each of these. Compare the two. Why is one better in all these respects than the other? In which is the rate of evaporation higher? Which in normal circumstances will have the better soil temperature for germinating seeds?

Suppose in preparing a soil for a house plant, you find its temperature is too low for the best growth of the plant. How can you correct it? How can you correct it if you find the soil too warm? How can you keep the moisture and temperature of a soil fairly stable? What is the effect of a closely packed surface upon moisture and temperature? What is the effect of a fine mulch surface? Give instances where it is important to know how to regulate the moisture and temperature of a soil.

\section{SUMMARY}

The human race is dependent for food mainly on the soil and its productivity.

Humus is decaying vegetable matter in soil. Accumulations of humus in swamps are called muck or peat.

Soils may be classified on the basis of the size of the rock particles they contain, as clay, silt, sand, and gravel. Soils containing an abundance of humus are called loams.

Soils are often named from their origin, as alluvial, glacial, or lacustrine.

On the basis of water content soils are classified as humid or arid.

The amount of soil water that any soil will hold depends mainly on the size of the rock particles and the amount of humus contained.

Water held by capillary action in the soil is called soil water. It contains something of all substances with which it comes into contact and so supplies the plants with many indispensable elements.

Soil water enters plants through the walls of the root hairs by osmosis, due to a difference in density of the sap and the soil water.

Water escapes from leaves by evaporation and passes as a vapor through the leaf pores. The process is called transpiration.

Commercial crops need different amounts of water to bring them to maturity.

A large percentage of the weight of plants and their products is due to water.

Dry farming is practiced in many localities where the annual rainfall is insufficient to mature an ordinary crop. It consists in packing the subsoil to insure its capillary action, and stirring the surface soil to decrease its capillary action and prevent evaporation. 
Cultivation of the soil kills weeds, conserves the moisture, improves the soil's texture, and increases the circulation of soil air.

Soil air, which may constitute half the volume of the soil, is essential to the growth of most kinds of plants. It is increased by the action of animals, the decay of roots, the action of frost, and by cultivation.

The soil is not dead but full of minute living things, such as protozoa and bacteria.

Nitrifying bacteria build the nitrogen of the soil air into nitrates, which the plants can assimilate. An important group uses the legumes as their hosts.

Other bacteria break down the useful nitrogen compounds and render the soil less fertile.

The temperature of the soil depends on its exposure to the sun, its color, character of surface, moisture content, and aëration.

\section{REVIEW QUESTIONS}

1. How does the old fable illustrate the value of the soil? 2. Give a summary of important observations made from the digging of a hole in the ground. 3. What is muck or peat? 4. How can humus be removed from soil? 5. What is the average depth of good soil? 6. Classify soils on the basis of size of rock particles. 7. (a) What is sandy loam? (b) Clay loam? 8. Classify soils in respect to their origin. 9. Mention regions of alluvial soils. 10. What had glaciers to do with soil formation in the northern states? 11. (a) How was lacustrine soil formed? Give examples. (b) To what are they best adapted?

12. Distinguish between arid and humid soils. 13. Describe how the percentage of soil water ean be determined. 14. (a) Which has the greater capacity for water, sand or clay? Why? (b) How do the soil particles hold water? 15. About how much water will soil hold, expressed as a percentage of its dry weight? 16. How can the moisture capacity of soil be changed? 17. What action is going on between the soil and the water in it? 18. Compare well water and soil water as to amount of dissolved material. 19. (a) What substances are dissolved in soil water? (b) Of what importance are they to plants?

20. Mention the process by which the soil water enters plants. 21. Besides the food it brings, of what use is water in the life of a plant? 22. Describe an experiment in which the process of osmosis is illustrated. 23. How may we show that substances secreted by 
root hairs aid in dissolving the soil? 24. (a) Describe root hairs. (b) Why is dirt generally found elinging to roots? 25. Give some practical suggestions for transplanting. Tell in each case enough of the structure or function of the parts of a plant to make your suggestion seem reasonable.

26. How can you demonstrate the fact of transpiration from leaves? 27. About how many pounds of water are used in the production by the plant of one pound of dry substance? 28. (a) State some facts to show the high percentage of water in garden and farm products. (b) Which of the crops cited in the table, page 464, requires the largest amount of water? Which the least? 29. In what way is the size of leaves and their abundance related to the amount of water transpired? 30. What relations do you note between leaves and sunlight?

31. Where there is too much water in the soil, what method is used to dispose of it? 32 . Where there is too little annual rainfall, what two methods are used to bring crops to maturity? 33. (a) Under what conditions is there a downward movement of water in the soil? (b) An upward movement? 34. Distinguish between free water and soil water. 35. Mention several illustrations of capillary action. 36. What is the general relation between the size of the bore of a tube and the height to which capillary water will rise in it? 37 . In what kind of soil, fine or coarse, is the capillary action most marked? 38. Explain why a newly sown field is sometimes rolled and later cultivated. 39. How may a farmer conserve water in the soil for a year or more? 40. Why does cultivation of the surface conserve moisture?

41. Compare coarse and fine soils as to the amount of air contained and the amount of circulation permitted. 42. About what part by volume of the soil is air? 43. How can the amount of air in a sample of soil be determined? 44. (a) How is a plant affected when its roots are deprived of air? (b) How do you explain the apparent exceptions? 45. Show how animals, plants, and frost may have a part in soil aëration. 46. What is the value of the air to microscopic organisms living in the soil? 47. In what way do legumes depend on bacteria? 48. How is the dead plant and animal matter in the soil changed into plant food? 49. (a) Under what conditions do nitrifying bacteria become inactive and denitrifying become active? (b) How does the appearance of the plants indicate the change?

50. What facts should one know in order to make the best use of soil in growing crops? 51. Compare the general temperature of the soil with that of the air above it. 52. Mention some conditions that control or determine soil temperature. 53. In what simple way can 
soil temperatures be taken? 54. If the experiment with the temperature of soil in prepared pots has been performed, summarize your conclusions. 55. What does a gardener mean by a warm soil? What is the usual character of such a soil? 56. Show the relation of water to the temperature of the soil.

\section{SUGGESTIVE QUESTIONS}

1. Compare the soils of the South and those of the North as to origin. 2. What is the prevailing type of soil in the vicinity of the school building? 3. (a) Are there any peat swamps in your vicinity? (b) Of what use are they? 4. Sometimes rocks used about a camp fire explode. Why? 5. What does this suggest as to the effect of temperature changes in making soil from rocks? 6. If clay retains soil water to the amount of 40 per cent of its dry weight, what per cent of the wet clay is water? 7. (a) Mention some plants that require a great deal of water; some that are drought resisting. (b) What are some of the differences in structure? 8. (a) Which is the better for plants, chemically pure water or soil water? Why? (b) Should carbon dioxid be considered the food of plants or the material out of which they make food? 9. About what part of the average rain falling on cultivated fields in your locality is returned to the air through the leaves of plants? 10. In what way do farmers of your vicinity recognize differences in soil on their farms? 11. What kind of land and what exposure is selected for the following: wheat or oats, corn, potatoes, hay, garden vegetables, orchard trees? 12. (a) Why do orchards thrive better when the soil is cultivated? (b) What conditions of growth are lacking when the soil is not cultivated?

\section{Natural and Artificial Selection}

227. Natural selection. - In only a few regions of the earth can even a small population find sufficient food materials in the free course of nature to sustain life in ease and comfort. Human population rapidly outruns natural productiveness and must therefore in some way supplement or improve nature's production, or face want and famine. In all the wealth of plant nature, man finds a comparatively small number of plants adapted to his needs, but these he selects and encourages by cultivation. Plants which interfere, he destroys or restricts in their range of growth. In the large areas set aside for grain, 
vegetables, and fruit trees, only a few stray plants of a different kind, called weeds, will be found, for these are kept out by the plow and cultivator.

In nature which at first seems to be fair and peaceful in all its ways, there is always a life-and-death struggle among all plants for light, food materials, and water; and in this struggle many are crowded out and perish. The supply of light, food materials, and the space for growth are insufficient for all plants that start growth; as a result comparatively few survive.

Exercise. - Select a small plot of ground, which may be left undisturbed all summer, and protect it by a light frame of lath about fourteen inches square, pressed firmly into the ground. Do this early in the spring just when plants are emerging from the ground. Every plant appearing within the frame and for a distance of six inches on all sides should be allowed to grow as it will. From time to time count and record the number of individuals and kinds of plants. Study the characteristies of those that seem to have any advantage over others in the struggle.

Observe and keep a record of the chief conditions to which the plants must be adapted at different times during the summer. It may be found that those survive which are most resistant to climatic extremes, such as prolonged dryness, intense heat, strong wind, driving rain, and to disease, insect and other enemies, and are also best fitted to secure necessary light, food, and water. Whether large and symmetrically developed, or small, stunted, and unshapely, the survivors possess the qualities necessary to win out in the struggle for existence.

Note, also, how plants that survive and mature are fitted to secure the propagation of their kind; that they are very prolific, producing seeds enough to plant a small field, perhaps a hundred for one that will survive at the end of the following season.

In any field or forest, along roadside, river, and fences, on mountain ranges and plateaus, on the prairies and the deserts, there are plants peculiarly adapted to the places where they grow and able to win out in the natural struggle for existence. Trees struggle for light and food materials in the forest, and those growing rapidly survive, while the slow-growing starve and eventually perish. Beneath the trees many different 
kinds of plants are usually found, suited to the conditions, and maintaining their kinds in the unceasing struggle. When climbing vines begin growth and continue to obtain sufficient nourishment, they may not only overgrow trees, but finally deprive them of light and cause their death by starvation. The survivors are necessarily few in number, but they have the qualities that insure their continuance and the propagation of their kinds. Thus it appears that nature carries on a thinningout process as unceasingly and relentlessly as the expert gardener who destroys weeds in protecting and encouraging his selected plants. The process as conducted in nature is called natural selection, and as carried on by man is called artificial selection.

In thinning out young vegetables, the smallest are usually removed and the largest, healthiest, and best located are left to mature. Natural selection, however, does not always result in the survival of the largest or best, according to man's view. In forests the largest trees may survive in the struggle for light, forcing the small and unsuccessful to perish in the shade, but in drought the small, unshapely plant with long roots and few or small leaves may survive; or it may be that one which best endures frost. On an individual tree the branches and twigs that grow most rapidly and vigorously crowd out of the race those that seem to be dormant. It is the fitness of individuals in a given set of conditions that insures survival and reproduction of their kinds.

Conditions of natural selection: prodigality of nature.-Observation of the struggle for existence among plants reveals the conditions upon which natural selection depends. The first of the conditions is the prodigality of nature, evidenced in the production of more individuals and species than can find adequate room and food for full growth and reproduction. This makes inevitable a competitive struggle for existence. Any plant, wild or cultivated, produces hundreds of seeds, only one of which will perhaps take root, grow, and mature. If a dandelion produced fifty seeds, each of which in turn produced 
fifty more, and this continued without interference, dandelions would soon possess the earth. But other plants, birds, insects, and plant diseases destroy enormous numbers of seeds and plants, so that the number remains on the whole about the same.

Variations. - The second of the conditions is that among the large numbers of offspring there are practically countless variations from the general kind. No two individuals are alike in all respects. This may be verified by examination of any number of plants grown in uniform conditions from seed selected of apparently equal quality, size, and weight, and from ancestors of the same degree of excellence.

Adaptation. - In the third place, it is altogether probable that among the numerous variations some will be well adapted to the environmental conditions, and will therefore be able to survive in the struggle and propagate their kind. In your studies of plants, look for such growing wild and in cultivated lands.

Heredity. - The fourth condition is that the successful in the struggle transmit by heredity to their offspring the qualities that made them the fittest of their kind and insured their survival. In this manner through slight variations of individuals from their kind, transmitted and accumulated from generation to generation, the new varieties may eventually form what the botanist would call new species or races. These species, like the individual ancestors, are the fittest to survive because they are adapted to their environment or are able successfully to resist adverse conditions.

228. Artificial selection. - By natural selection new species of plants have been evolved slowly. This is the method of nature, operative long before the appearance of man. By the study of plants and animals man has acquired a knowledge of nature's ways and has applied this knowledge to the development of new varieties or species at a more rapid rate than seemed possible with the undirected forces of nature. 
229. Luther Burbank. - Perhaps no better illustration is now available of the way in which this has been done than the work of Luther Burbank. He may be considered the representative of the modern wonder-workers in the plant world. A close student of plants, Burbank has learned their habits, ways of mating, and the results of cross-fertilization, and has put his knowledge into successful practice. As one result of his efforts it is estimated that, through his products, he has contributed to the wealth of the United States not less than seventeen million dollars.

Every one may have observed, as Burbank and others did, that individual plants and animals of the same species or kind vary greatly in their characters. In a melon patch, for instance, one melon vine may produce more large sweet melons than the other vines. While natural conditions, such as soil and climate, contribute to the result, the character of the seed plants is known to be much more important. Practical gardeners select the seeds of a superior plant, knowing that such are more likely to yield good melons, but they first select the plant itself because of its qualities. Men like Burbank are expert in making such selection of plants and seeds, and continue the selection of the same kind of plant through many generations. They find that artificial selection is an improvement upon and greatly hastens the process of natural selection.

Some years ago, when asked the color of a garden poppy, the answer was certain to be "red." Now blue poppies and silver-lined poppies are grown, all bred from the same original stock. Burbank one day noticed a smoky cast on the red poppies of one plant.in a bed. He marked the plant, gathered the seed, and in time planted them. Of the offspring those that showed most variation toward the bluish tint were selected.

Other plants of value and of beauty have been contributed by Burbank to the world. He experimented with the wild daisy common in the east, and with a variety procured from Europe. By cross-pollination of these two he produced many 2 I 
new varieties. He discarded all of the hybrids except a very few that had extra large fine flowers. These, however, had in his judgment one defect : they were not pure white as he desired them to be. He learned that in Japan is grown a rather small, but pure white daisy. Obtaining some of the seed of this plant, he combined it by cross-fertilization with the new large variety which he had originated. He desired to produce in a single plant the two sets of characters, that is, the large size and the pure white. He succeeded, and named the product the Shasta daisy.

Many new fruits and vegetables have been similarly produced by his labors and genius. The Burbank potato is known everywhere. The plumcot is now found in the general market, a product obtained by combining the apricot and the plum. He improved the peach and the plum as to size and quality. One of his late creations is the spineless cactus, which is expected to prove of great value, because it is excellent food for cattle and it produces a fruit said to be of pleasing flavor and of use as food for man.

\section{SUMMARY}

Comparatively few seeds produced grow to mature plants and reproduce their kind.

Selection in nature depends upon the following: prodigality of nature, individual variation from the general kind, relation of variations to environmental conditions or fitness, and heredity.

By natural selection new species are slowly evolved. By artificial selection man controls and hastens the production of new varieties and species.

The work of Luther Burbank is an example of the value of artificial selection. Some of his achievements are the blue poppy, the fragrant calla lily, the Shasta daisy, the Burbank potato, the plumcot, and the spineless cactus.

\section{REVIEW QUESTIONS}

1. Why is it necessary for man to cultivate plants? 2. What are the conditions that make it impossible for all plants that start growth to reach maturity? 3 . What are the conditions that a plant must 
survive in order to mature? 4. Do plants under cultivation have to meet the same difficulties? 5. Show that wild plants when adapted to their surroundings survive, and when not adapted, perish. 6. Give a descriptive term to the thinning-out process found in nature; to that performed by man.

7. What is meant by the fitness of individual plants to survive? 8. What are the four conditions upon which natural selection depends? 9. Distinguish between natural and artificial selection. 10. Upon what condition does artificial selection depend? 11. Will all the offspring of any parent plant be equally well adapted to their environment? Why? 12. Give some notable instances of artificial selection. 13. Give a brief account of Luther Burbank's work in improving plants. 14. How was the blue poppy originated?

\section{Pollination and Seed Production}

230. How man promotes variation. - We have considered so far only the chance variations which plants may show and which, when discovered, may be selected and encouraged to propagate individuals that repeat and accentuate their peculiar characteristics. There are, however, plants developed independently of chance variations. These are the products of man's purposeful interference with nature's course. How can this be done? The answer to this question may be found by a study of nature's method of fertilizing plants, or what is known as pollination. It is by man's control of pollination that new varieties and species of plants are produced.

How can man control the pollination or fertilization of plants? To understand this process it is necessary first of all to examine the structure of the flower, and to learn the use or function of its various parts and of the flower as a whole.

231. The flower: the floral envelope.-The brightly colored parts of the flower, which are generally the most striking, are called the petals (Fig. 149). The petals in their complete formation constitute the corolla. The principal function of the corolla seems to casual observation to be the attraction of insects.

Examine the circle of green leaves, called sepals, which com- 
pletely contain the flower when it is in the bud stage of development. The sepals thus arranged form the calyx, or cup-like part of the flower. The sepals, or the calyx, and the petals of the corolla are included in what is known as the floral envelope.

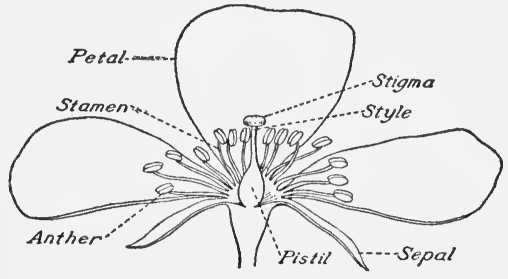

FIG. 149. - Diagram of a typical flower. The buds show that this phrase is appropriate. The floral envelope contains and protects the essential or reproductive organs of the flower or blossom.

The essential parts of the blossom comprise two sets of organs. The first to be observed are the small, stem-like parts ranged around inside the corolla. These are the stamens. Each stamen has a swollen or knob-like top, called the anther, which in the fully developed blossom is covered with a fine flour or powder. The powdery material, called the pollen, is very easily detached from the anther, as one's nose or finger may show after smelling or examining the blossom.

Remove carefully one by one the sepals and petals. To what are the stamens attached? Inside the cycle formed by them is a single stem or stalk which like a pestle is slightly enlarged at the top and much more so at the bottom. The whole part or organ is the pistil. The enlarged top is called the stigma, or when divided into two or more parts, each of the parts is called a stigma.

The swollen part at the bottom of the pistil is the ovary or egg-container. Cut an ovary of a blossom, such as an apple, peach, or plum, into sections and examine with a magnifying glass. The inner structure reveals a number of thin-walled compartments each containing a very minute body called the ovule or egg-cell. The ovules of a well-matured ovary are, if fertilized, immature seeds.

These two sets of parts, the stamens, with their pollen-covered 
anthers, and the pistil, with the receptive stigma and the ovary containing the ovules or egg-cells, are the essential or reproductive organs of the blossom. The pollen must be transferred to the stigma and thence to the ovules, if these are to be fertilized and become seeds.

Exercise. - Study the several parts and their arrangement in any of the typical flowers, such as the apple, peach, plum, tulip, or trillium. Then select other flowers for comparison, and for the sake of bringing out clearly the important features.

Fertilization. - The process of fertilizing the ovules in the pistil can be outlined here only in a general way. It may be studied more thoroughly with the aid of a microscope. The chief features of the process are illustrated diagrammatically in Fig. 150. The pollen is somehow transferred from the anther to the stigma; but it does not matter for the present by what agency this transfer is made, or whether the pollen and ovules are produced by the same individual plant or by different plants. The latter is frequently the case, as we shall find in later study.

The stigma, which you find is rough and often coated with a mucilaginous and sweetish substance, holds securely any grain of pollen that may touch it. As soon as the pollen grain is captured, it begins to grow, sending down through the stigma and the style into the ovary a very fine, hair-like tube, containing

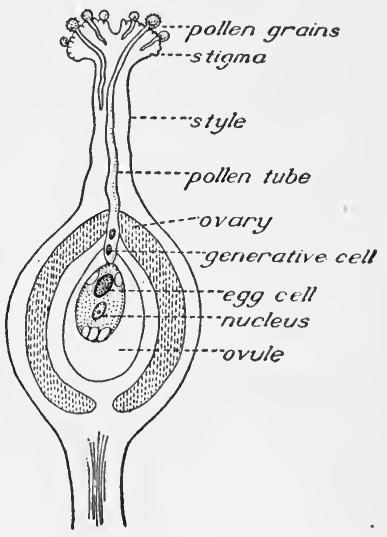

Frg. 150. - A diagrammatic illustration of a simple pistil and ovary, cut vertically, to show fertilization. Note failure of all pollen grains except one to reach the ovary; the successful one discharges the generative cell which unites with the egg cell, producing the embryo. (Strasburger.) at the lower end two protoplasmic bodies, which are the essential male fertilizing elements. The pollen tube dissolves and utilizes as food the substance of stigma and style. Within 
the ovary the tip of the tube grows flat against the side of the ovule (Fig. 151). One of the bodies, the generative cell, enters the ovule and comes into contact with the ovum or eggcell. These gradually unite, becoming a single body within a
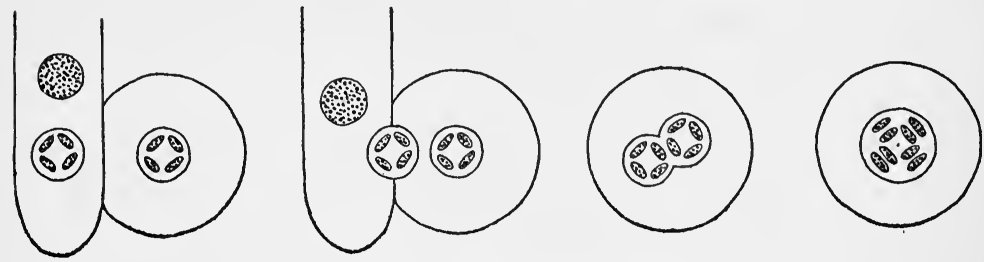

FIG. 151. - How the ovule is fertilized by the generative cell from the pollen tube (diagrammatic).

single cell-wall in the ovule. This single body, or the fertilized egg-cell, comprises the two principal original bodies derived from the parent plants, and becomes what is known as the embryo. The other body in the tube unites with another part of the cell substances in the ovule, and this combined body develops to form food which is consumed by the growing embryonic plant.

232. The seed. - The growth of the fertilized ovule is by division and subdivision (Fig. 152), until there is completed
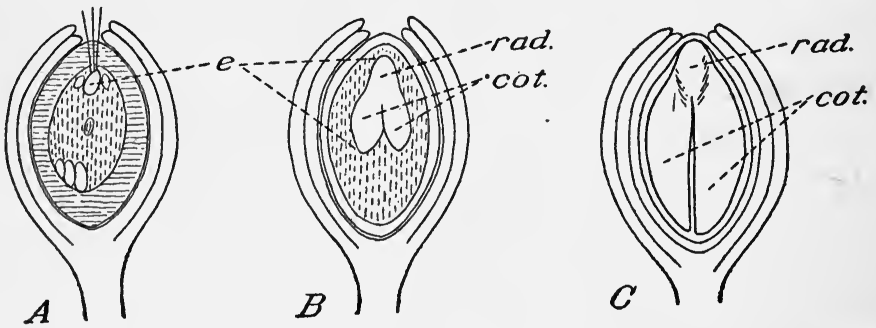

FIG. 152. - Diagram of development of fertilized egg cell. $A$, fertilized. ovule; $e$, the ovum in $A$ becomes the embryo in $B ; C$, the developed seed with cotyledons (cot.) and radicle (rad.).

the development of the embryo or miniature plant, the food supply, and the seed covering, all of which form what we know as the seed. 
Exercise. - The seed of corn, peas, beans, chestnuts, and other common plants should be examined for the purpose of identifying these parts. The food supply enveloped in the seed covering is necessary for the young plant in its early growth apart from the parent plants, and before it can assimilate from the air and the soil all the materials it needs.

The value of the food supply in seeds may be demonstrated with peas, beans, or corn. Cover loosely with mosquito netting a glass tumbler, and fill with water to touch the netting. Prepare several corn grains or other seeds by soaking in water for twenty-four hours. Leave two or three grains whole, but from the others cut away all the food material possible without injuring the miniature plant. Place the prepared grains on the mosquito netting. Keep the netting moist by adding water as needed. Observe stages in germination, course of root growth and that of stems. The rate and size of growth show clearly the value of the food supply in seed.

233. Incomplete flowers. - Some plants produce flowers which do not have all the parts or organs named. There are flowers which have both stamens and pistils but lack petals or sepals or both. The flowers of the poinsettia are without petals, but have a circle of brilliant red leaves which serve the same purpose. Some flowers have stamens, but no pistils, and others have pistils, but no stamens. The flowers which have stamens but not pistils are called staminate, and those which have pistils but not stamens, are called pistillate. These two kinds of flowers may be produced by the same individual plant, as in the corn and the squash, or by different plants of the same species, as
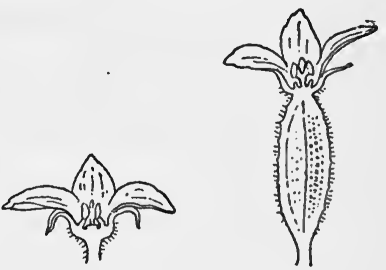

FIG. 153. - The staminate and pistillate flowers of the cucumber. (After Baillon.) in the fig, mulberry, willow, sassafras, and perhaps alders and birch.

Corn flower. - The squash or cucumber plant (Fig. 153) should be studied to determine which of its flowers are pistillate and which staminate. One of the most interesting examples of 
such division of labor by the production of staminate and pistillate flowers upon the same individual plant is the maize or Indian corn (Fig. 154). The tassel of the corn plant, at the top of the stalk, is a heavy cluster of blossoms in which are the stamens with their abundant mass of pollen grains. The

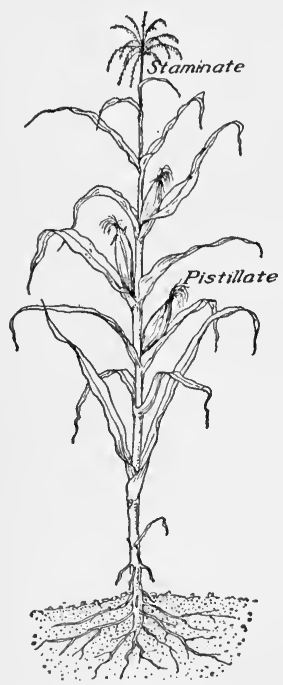

Fig. 154. - The corn plants, showing flowers. embryonic ear is the pistil, containing a large number of ovules with their silken threads, which serve as styles and stigmas. The pollen must be transferred from the tassel to the stigmas and ovules, if the cob is to produce seed or kernels of corn. Each silky thread must catch its grain of pollen, and when the pollen grain has grown through the style to the egg-cell, the silk withers or dries up at the outer end. When the chances of pollen grains finding their way to the silken stigma of the ear are considered, the comparatively small number of unfertilized ovules or missing grains is astonishing. This is even more striking when one finds only a few scattered grains on the ears of a single isolated corn plant. Why were the ovules not fertilized in such a case? Did the tassel fail to provide sufficient pollen? The remarkable fact is that the tassel provides enough pollen to fertilize a thousand embryonic ears of corn. Then why did the pollen fail to reach the silk of the ear?

234. Close- and cross-pollination. - No plant can produce seed unless the pollen from the anther is transferred to the stigma. By what means or agency is this transfer effected? In contrast to the corn, there are a few plants, such as the pea, in the flowers of which the stamens and anthers are so arranged that the pollen when mature is directly transferred to the stigmas. Such flowers are said to be close-pollinating or self- 
pollinating. On certain plants, as the wild violet, wood sorrel, and pansy, one may sometimes find small flowers which have no petals and never open; but as they produce seed they nuust be self-pollinated. In the culinary pea pollination occurs before the blossoms open. The anthers burst and provide the stigmas with pollen grains while the first petal to appear is still green. But most plants depend upon cross-pollination, and have adaptive structures to prevent close-pollination and to make use of such agencies as water, wind, or insects to secure cross-pollination.

235. Wind-pollinated plants. - The corn plant, several kinds of grasses, many trees, including spruce, pine, and conebearing trees generally, maple, elm, willow, birch, elder, and certain shrubs, depend for pollination upon the most universal agency, the wind. Many of these plants produce staminate and pistillate blossoms on the same individual, as in the corn; while others produce these blossoms on different individual plants. The staminate blossoms in both classes are many times more numerous than the pistillate. All plants dependent upon the wind for cross-pollination produce a superabundance of pollen, for naturally an immense amount must be wasted by the wind. Corn, willow, and hazel are familiar examples of this prodigality.

Adaptive features. - The corn shows a remarkable adaptation to wind-pollination. The tassel is at the top of the stalk, where it is most exposed to the wind, and the pistillate flower is lower and quite inconspicuous. The light, feathery pollen is easily detached and scattered by the slightest breeze over neighboring plants, the silken stigmas of which are so brushy and sticky that pollen once touching is held.

All wind-pollinated plants have peculiar adaptive features. Some produce pollen that is powdery or feathery, or equipped with wings, or with bladders, as the pine. The stigma on many is an interesting mechanism. Certain grasses, such as timothy, raise feathery heads high above the rest of the plant. On some 
cone-bearing trees the stigma is replaced by a peculiar arrangement of smooth, scale-like bodies that guide the captured pollen to the ovules. Trees put forth blossoms before leaves, or have their blossoms conveniently located for the wind-borne pollen.

Exercise. - Examine the blossoms of some of the wind-pollinated plants in your vicinity. What do you find the characteristic features as to size, form, color, odor, and position of blossoms, staminate and pistillate? Are color and odor of value to wind-pollinated plants? Do such plants blossom earlier in the spring than those depending upon some other agency for cross-pollination? Are the pistillate blossoms produced by one plant and the staminate by another? Are these blossoms so located that the wind carrying pollen from the staminate flower is likely to deposit it upon the pistillate blossoms?

Waste through wind-pollination. - The great uncertainty and waste involved in pollination by wind are obvious. Only where plants grow in groups, as is the case with corn and certain trees and shrubs, is there any certainty of pollination. The isolated corn plant has a very slight chance of producing seed. The pumpkin and squash would probably fail, if they were dependent upon the wind for transfer of pollen from one flower to another. These and many other plants depend upon another agency than the wind for pollination.

236. Insect-pollination. - The plant uses a great deal of energy in producing pollen, much of which is wasted.when pollination depends upon the wind. Many plants have a more economical method of securing the transfer of the pollen. By their color and perfume they attract insects, such as the bee, moth, and certain flies, to visit them. In the search for nectar the insect brushes against the anthers and transfers the pollen from them to the stigma, or to the next flower it visits. Some flowers have peculiar devices to prevent close- or. windpollination.

The wonderful adaptive structures of flowers to secure crosspollination by insects can best be studied in nature. The important points may be suggested briefly; but they should be verified in concrete illustrations of typical flowers and their 
adaptation to visiting insects. Not only flowers are provided with such adaptive structures, but certain insects also have organs that are peculiarly suited to the kinds of flowers they frequent. Such mutual adaptation of insect and flower may be noted in the case of the red clover and the bumblebee; certain long-tube flowers and the butterfly; or night-blossoming flowers and the moths. ${ }^{1}$

Colors of flowers. - Colors and odors of flowers play an important rôle in securing cross-pollination by insects. Different colors attract insects of one kind or another; the odors entice the visiting insects to search within the chalice for the minute drop of nectar which both color and odor advertise and which the insects need for food. Observe how red clover attracts the bumblebee by which alone it secures cross-pollination; how white clover and fruit tree blossoms generally attract the honeybee; many small, wide-open, yellow flowers, certain flies; red pinks and some flowers with long tubes, the butterfly; and how flowers blooming in the evening and night, which are usually white and conspicuous, attract the moths which are then flying. Colors are appropriate not only to the time of day, but also to the season of the year. One may find many instances of this and should note the visiting insects and other conditions that seem to make such color adaptation necessary.

The odor of flowers. - Odor not only supplements, but sometimes takes precedence over or entirely replaces color as a means of guiding insects to flowers. Petunias have the habit of sending forth their fragrance only in the evening, when certain moths, by which they are cross-pollinated, are flying. Those familiar with the woods will recall the heavy permeating fragrance of certain wild flowers which without their odor would escape notice, especially the small retiring kinds, the colors of which would be inconspicuous in the shade of the trees. Then,

${ }^{1}$ Students will find most interesting accounts of cross-pollination and the adaptive structure of flowers and insects in W. H. Gibson's "Blossom Hosts and Insect Guests," and Darwin's "The Various Contrivances by which Orchids are Fertilized by Insects." 
too, certain willows and maples have blossoms which possess much odor, but no petals.

Exercise. - The function of color and odor may be made quite evident by disguising the color, the odor, and again both in several flowers in the midst of the kind. Observe whether or not visiting insects neglect the disguised flowers. Observe also whether insects of a given kind, as the honeybee, visit all kinds of open flowers indiscriminately, or follow only the flowers of a certain kind of plant. This may be observed best in a garden or a field where there is a variety of plants in blossom. If insects distributed pollen among different kinds of flowers where it is useless, they would waste a large amount of pollen, the most expensive of plant products. In the study of this subject, consider such flowers as the buttercup, sweet pea, columbine, petunia, pansy, skunk cabbage, wild orchids, and fruit tree blossoms, such as the apple, the peach, or the orange, and also some nightblooming flowers:

It must be obvious that the primary function of the colors and odors of flowers is not to please man's senses of sight and smell, but to attract and secure the continued visits of insects. That many flowers do give delight through their exquisite form, color, and odor, is merely a happy coincidence.

How plants select guests. - The structures of flowers, which are cross-pollinated through the agency of insects or other

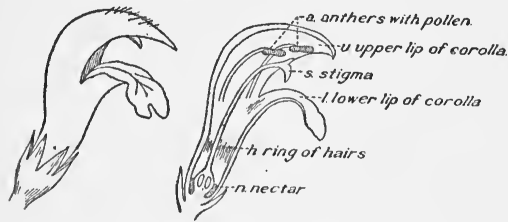

FIg. 155. - Type of flower adapted to cross-pollination by insects. Large bees alight on $l$, brushing against $s$ and $a$, thus transferring pollen from one flower to another. Small insects are excluded from nectar by $h$.

is thus powdered with pollen, with which it pollinates many stigmas as it continues its search for nectar. Red clover has ming bird and perhaps other birds, are well adapted to the purpose (Fig. 155). The sweet pea and red clover may be taken as types of such adaptation. The nectar of the sweet pea is so located in the base of the blossom that a bee is certain to come in contact with the stamens and animals, such as the hum- 
a long, narrow tube containing the honey, which can be reached only by the long proboscis of the bumblebee. Orchids of certain kinds yield their nectar only to moths with unusually long proboscides. The snapdragon opens its nectary tube only to an insect of such weight as the bee, thus excluding smaller insects and especially ants, which merely consume the nectar. The throats or tubes of many flowers are provided with hairs or bristles to exclude unbidden guests.

237. Cross-pollination and variation. - A plant which is the product of the pollen from one plant and of the ovule from another of the same species, or from another plant of a different variety of the same species, can never be a "chip of the old block." It may repeat certain characteristics of the parent plants, but it is a new variety, with striking differences from, however much it in general resembles, its kind. As is well known, plants combining the excellent characters of parent plants grow more vigorously and rapidly, have greater strength, health, and productiveness than the ancestral plants. From man's point of view such varieties are valuable; and to produce them is one of the chief aims in plant improvement.

Exercise. - That individual varieties occur in any kind of plant may be interestingly demonstrated. In soil of the kind most suitable for the selected plant, and supplied with the best degree of moisture, warmth, and light, plant at the same depth and with the same soil allotment fifteen to twenty seeds of the same crop or plant, selected for their equality in all essential respects. Such seeds as corn, pumpkin, peas, and beans will do well. The result is instructive. No two grow at the same rate, with the same vigor, strength, and health, and if the plants were observed until they matured, corresponding differences would be found. These points are fundamental in the selection of seed.

Exercise: Relation of floral structures to insect-pollination. - Observe the position of the nectar relative to the stamens with their anthers, and to the stigmas. The nectar is found in different places in different kinds of flowers, in some on the base of the stamens, in others on certain parts of the petals, and still elsewhere in others. But it is so located that insects getting it are brought into contact with the anthers and stigmas. 
Observe landing places when present in flowers; note where these are placed in relation to stigmas and anthers; whether they have any other uses than merely as landing places for insects.

(a) Many blossoms, as those of the pear and apple, have a different way of securing cross-pollination. The stigmas mature and are ready for the pollen before the pollen is ripe. This necessitates transfer of pollen from other blossoms.

(b) In blossoms of certain plants, such as the bean, daisy, and sunflower, the pollen grains ripen and are dispersed before the stigmas are receptive. These points are important in the production of new varieties by hand-pollination.

Observe the structure of .such flowers as the cowslip, primrose,. and blue flag, which have an arrangement of stamens and anthers with relation to the stigmas, that makes cross-pollination necessary, and close-pollination almost impossible.

Study the ways in which different insects, bees, moths, and butterflies, obtain the nectar and collect pollen. Examine typical insects, their bodies, legs, wings, heads, and mouth parts.

\section{SUMMARY}

The parts of a complete flower are:

Floral envelope,

Sepals, constituting the calyx,

Petals, constituting the corolla.

Essential organs,

Stamens, producing pollen,

Pistils, producing ovules.

Pollination is the transfer of pollen grains from the anthers of the stamens to the stigma of the pistil.

Fertilization consists of the union of the nucleus of the pollen grain with the nucleus of the ovum.

The fertilized ovum, using nutriment from the sap of the parent, develops into a seed, which consists of an embryo plant, a supply of nutritive material, and a protective covering.

Flowers that have stamens but not pistils are staminate. Flowers that have pistils but not stamens are pistillate. Those possessing both sets of organs are perfect flowers.

Cucumber vines and plants of corn have both staminate and pistillate flowers. 
Many plants produce perfect flowers, which because of various devices do not close-pollinate.

Cross-pollination is effected by various agencies, such as wind and insects.

Wind-pollination is wasteful of pollen, as shown by corn. A study of pollination by insects shows many mutually adaptive structures of flowers and insects.

Flowers by their colors and odors attract insects to the nectar and thereby secure cross-pollination.

\section{REVIEW QUESTIONS}

1. Of what practical importance is the control of pollination? 2. What must we know about plants to control pollination? 3. (a) What parts of the flower make up the floral envelope? (b) What advantage is it to a plant to have brightly colored flowers? 4. (a) What are the essential or reproductive organs of a flower? (b) Where is the pollen produced? (c) Where are the ovules formed? 5. What do fertilized ovules become? 6. How is the stigma adapted to hold the pollen grains that may touch it? 7. Describe the growth of the pollen tube. 8. What becomes of the two protoplasmic bodies in the pollen tube? 9. What two parts of the seed develop from the two parts of the generative cell? 10. How can you demonstrate the value of the food supply to the growing embryo?

11. What is the significance of the term staminate flower? Pistillate flower? 12. (a) Mention a species in which staminate flowers and pistillate flowers occur on the same individual plant. (b) In what species do staminate and pistillate flowers occur on different individual plants? 13. Describe the structure of the reproductive organs, and the reproductive process of corn. 14. Mention a garden plant that is self-or close-pollinated; a wild flower. 15 . Which is the prevailing type, cross-pollinated or self-pollinated plants? 16. Mention several plants that depend upon wind for the transfer of their pollen. 17. How are the following plants adapted to windpollination: (a) corn, (b) willow, (c) timothy? 18. What are some of the disadvantages of dependence on wind for pollination?

19. Mention an instance of mutual adaptation of insect and plant. 20. Why do insects visit flowers? 21. By what means do flowers advertise their presence to insects? 22. By what insect is red clover pollinated? 23. (a) What insects are adapted to reach the nectar in long tubes? (b) In night-blooming flowers? 24. What flowers depend mostly on their odor for attracting insects? 25. Why is the 
nectar of many flowers located at the base of the stamens? 26. What is the significance of the structure of the snapdragon?

27. (a) How is close-pollination prevented in such blossoms as the apple and pear? (b) As the bean, daisy, and sunflower? (c) As the primrose and cowslip? 28. How may offspring differ from their parent plants? 29. What use does man make of the normal variation of plants? 30 . How may the normal variation of any species be demonstrated?

\section{The Improvement of Plants}

238. Three ways of improving plants. - The facts concerning cross-pollination and the character of the varieties grown from selected parents have been carefully studied and applied in the improvement of all plants of economic importance. Improvements are secured in these ways: (1) by the selection of variations that show desired characters (discussed pages $481 \mathrm{ff}$.) ; (2) by controlled cross-pollination; (3) by the selection and propagation of sports.

239. Controlled cross-pollination. - Cross-pollination of selected plants presents no serious difficulties. There are two practical steps to be taken: (1) the protection of the stigma of the plant to be pollenized from possible pollination by any plant other than that selected; (2) the transfer of the pollen from the anther of one plant to the stigma of the other when this is mature and ready to receive it.

In the first, if the flower is one in which close-pollination may occur, the stamens are carefully removed before their pollen is ripe. The flower is covered with a paper bag so tied about the stem that no insects can enter, and the pistil will be kept from injury. Since the petals have no function to perform, they may be carefully cut away.

The second step in pollinating plants may be effected in several ways. One is to shake the blossom with the ripe pollen against the receptive stigma. Another is to gather the pollen in a dish and apply it to the stigma with a camel's-hair brush. The pollinated flower must be protected with a bag as previously. 
As soon as the ovules are fertilized and the fruit set, the bag should be removed. The stigma, which frequently withers like corn silk, cannot then be affected by any pollen that may fall upon it. The seed produced by controlled cross-fertilization is kept separate from other seed, and is planted in isolation from other plants. The progeny also must be hand-pollinated, and their seed selected; and this process continued through several generations until the desired variety has become relatively permanent, reproducing true to kind.

Limits of cross-pollination. - Cross-pollination is usually practiced with: (1) selected plants of the same variety; (2) selected plants of different varieties of the same species; (3) selected plants of different, but related species.

Improving a variety. - The first kind of crosszpollination is usually employed in maintaining and improving the standard of any given variety. In a plot of peas of the same variety, certain plants are selected because of their desirable characters and cross-pollenized. From the seed of these a selection of the best is made for the next planting. The plants grown from such cross-fertilized seed combine in various ways the desirable characters and are therefore generally superior to the parent plants. From the offspring, selection is made and cross-pollination effected, insuring improved or new varieties.

Related varieties. - The pollen may be transferred from a plant of one variety to the stigmas of a plant of another variety of the same kind or species. One may cross-pollinate tall peas with dwarf peas, wrinkled peas with smooth round peas. This is a specially interesting field for experiment, the one in which Mendel (pages $505 \mathrm{ff}$.) made his famous discoveries in inheritance. The method is known as hybridization, or producing hybrids, from which selection is made for propagation. Such hybrids reproduce with remarkable certainty.

Related species. - A third possible combination is to transfer the pollen from a plant of one species to the stigmas of a plant of 
another, but allied species. This has been done with remarkable success in a few instances, such as crossing blackberry and raspberry, one product of which is the loganberry. It may succeed with certain flowering plants, as geranium, narcissus, gladiolus, and certain lilies. The result is generally a failure; but if successful, such a variety must be propagated by bulbs or buds. What may be done in this field no one knows and only trials can reveal.

240. Selection and propagation of sports. - The seeds of many of the common plants grow in general true to kind, though in order to secure the best results seed must be selected from plants having the most desirable characteristics. The plants grown from seed include, among others, wheat, rye, barley, corn, onion, turnip, radish, beet, pea, bean, and many annual flowering plants. But there is a group of plants which do not as a general rule grow true to seed and are therefore seldom propagated from seed. Of these may be mentioned the potato, strawberry, raspberry, gooseberry, currant, grape, and most fruit trees. These have other ways of reproduction, as by shoots or suckers, root stalks, runners, and so on.

Seedlings. - Seeds of plants of the latter group are sometimes planted for the sake of possible variations of value, "sports" they are often called. The usual seedlings of such plants are inferior to the parent plant, e.g. seedlings from the Bartlett pear reverting to an ancestral type with fruit that is small and flavorless. But what may be produced is quite uncertain, as the following instance shows. Peter M. Gideon of Minnesota desired to grow an apple tree that would be adapted to the climate and soil of the region and bear in abundance fruit of the finest quality. He planted hundreds of apple seeds and rejected hundreds of seedlings before he found one that gave promise of value. It proved to be what he desired. The seedling yielded stems or scions for grafting and was thus propagated until it has been widely distributed over the state. The Wealthy apple has, it is estimated, increased the wealth of the 
state by more than a million dollars. Thus seedlings may have value if properly selected.

Bud varieties. - As there are new varieties possible from the seeds of the plants of this group, so there are new varieties possible from buds on the plants. In fact sports from seeds and buds are the source of many of the improvements that have been made in plant culture. Bud sports may be illustrated by the navel orange. A single bud on an orange tree grew into a branch bearing oranges without seeds, but containing in the blossom end of the fruit a small " navel " orange. The seedless orange branch was propagated by grafting on other trees, and the buds thus produced used in "budding" a large number of orange trees. In a similar way nectarines were produced as a bud variety on a peach tree. There are many cases of such chance variations continued by careful propagation among the cultivated flowering plants, such as the roses, chrysanthemums, carnations, tulips, and geraniums.

\section{Propagation of Plants}

241. Division. - Many, plants which are not ordinarily grown from seed may be propagated by buds. The method employed in this is known as division. It is the artificial separation from the parent plant of a bud, or a part having several buds, which will develop into a new and complete plant. The general method of budding is applied in several ways, i.e. layering, cutting, budding, and grafting.

In this work man has been guided by what he has observed in nature. The potato, which is a good example of propagation by buds, produces tubers on the tips of underground branching root stalks. The tubers remain after the death of the parent plant and the next season produce new plants. Men increase the number of plants as well as secure better products by selecting and cutting the tubers into sections, each of which has two or more buds, and planting the sections. Sweet potatoes, which are enlarged roots and produce shoots, are planted whole 
and the growing sprouts pulled and transplanted; or they may be grown from cuttings of the vines, or from sections of the potatoes, as in the case of the common or Irish potatoes.

Layering. - Certain plants, like the strawberry, send out runners or stolons which take root in the ground and eventually develop into complete and

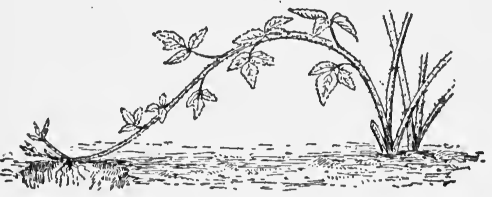

Fig. 156. - The blackeap raspberry is propagated by layering and by seeds. independent plants. The result may be gained more quickly by covering the tips of the runners with soil, and later separating them from the parent and transplanting the new plants. The blackcap raspberry (Fig. 156) propagates itself in similar fashion, and also by its seeds. The tips of its branches may be bent over and covered with soil, and when rooted, cut from the parent plant.

The same method may be used in propagating the grape, currant, blackberry, and other plants of like nature, also certain trees, as the apple, plum, and elm. When a plant develops roots with difficulty, the stem chosen is nearly severed from the parent plant by an oblique cut, a V-notch, or it has a ring of bark removed, where it is to be covered with soil. This prevents the return to the tree of the sap, which is thus made to accumulate at the wound, aiding in the development of adventitious roots. The stem may be inclosed in a box filled with soil. When it has well-grown roots, it is separated from the parent and planted in good, moist soil.

Cutting. - Buds or twigs with buds of many plants may be separated from the parent plant and used for propagation. This method, known as cutting, is not so certain as läyering, because the cuttings often perish before developing roots sufficient for growth. It is commonly known that willow branches broken from a tree and stuck into the ground will develop roots. Many other plants respond in a similar way, 
among which may be mentioned the rose, geranium, currant, quince, grape, and sugar cane. In fact some plants, like the sugar cane, have been propagated in this way for so long that they seem to be quite unable to produce seed that will grow. Cuttings from willow or grapevines, for example, grow promptly if planted in moist, warm, well-aërated soil. The willow develops roots from either end, but the grape does best from the end that was nearest the root of the parent plant.

Exercise. - Plant a grape cutting, eight or more inches long, with at least two buds in the soil and one or more above the surface. If this is done in a germinating box, the differences in the development of the buds may be observed.

Budding. - The most common method of propagating choice varieties of fruit trees, as cherry, plum, apricot, orange, and such plants as roses, is by budding. This is a method most economical of buds, when the supply is rare and limited, and requires less time for growth of plants to productiveness. Budding is generally done in late summer when the bark is readily separated
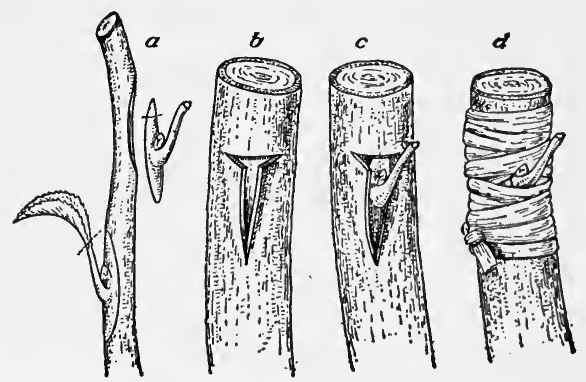

FIg. 157. - Diagram to show steps in budding. from the wood. Seedlings about one year old are usually selected for budding. They are selected from a stock which combines the characters necessary for a vigorous, healthy, productive plant. Buds are cut, as shown in Fig. 157 (a), from young stems or scions which have first been separated from the plant. The small amount of wood removed from the stem with the bud must be picked from the bark carefully so as not to injure the bud. The bud is inserted, as in (c), into a T-cut in the bark (b) which is about four or five inches above the ground. The bark is then firmly pressed and bound around 
the bud with natural raffia $(d)$. In three or four weeks the bud has grown to the stalk, and the bandage should be removed entirely or loosened and the stalk cut off close above the bud. It is worth while to bud several hardy rose stalks with choice varieties.

Grafting. - A part of a plant with several buds, but without a root system, may be cut from the parent plant and grafted into a root stalk, large branch, or top of a tree. This method of division is employed with more or less mature, woody plants like the apple, pear, peach, pecan, walnut, cherry, or plum. The same root stalk may have almost any number of grafts of different varieties or kinds, as it is not the root and stalk of the tree, but the twig with the buds that determines the character of the fruit. Burbank had more than five hundred different varieties of apples upon one stalk, all growing and bearing fruit after their respective character.

Grafting is valuable in the case of trees, the roots and stalks of which are not sufficiently resistant to soil, climate, and pests. A sturdy, hardy, vigorously growing root stalk of the proper species is grafted with the more delicate varieties. A root stalk, such as the almond, may be grafted with scions of peach, plum, or prune, or other suitable varieties. Wild root stalks are sometimes selected, as these have a hardy, vigorous constitution, supply abundant nourishment to tender, choice varieties, and contribute to the production of more abundant, larger, better flavored, and better keeping fruit.

For details of the methods of grafting, any treatise on plant culture or agricultural botany may be consulted.

242. Seed selection. - In the propagation of those plants which grow true to seed or type, it is necessary to make careful selection of the seed for every planting. In the first placè, the parent plants should be selected and their seed gathered and tested for germination qualities before being planted. The testing may be done in a germinating box or between pieces of moist blotting paper. In testing corn, for example, several 
kernels from different places on each of the selected ears should be taken, and those ears, the kernels from which do not all germinate or germinate slowly, should be rejected. In the second place, seed illustrate clearly the fundamental laws of heredity, which are just as effective in plants as in men and other animals, but more rapid. "Like tends to produce like," and seed selection should be guided therefore by the known facts of heredity, if the best and most abundant products are to be secured. The important facts are considered in the following section in connection with the study of the work of Mendel, the pioneer in the investigation of the conditions that determine the hereditary transmission of characters from parent to offspring.

\section{SUMMARY}

Men secure improvements in plants by the following methods: (1) by selection of variations that show the desired characters; (2) by cross-pollination; (3) by propagation of " sports."

Cross-pollination is usually successful only between plants of the same species.

Cross-pollination between selected plants of a given variety is used to maintain and improve the standard of the variety.

Cross-pollination between plants of different varieties but of the same species gives hybrids which may combine desirable characteristics of each of the parent varieties.

Cross-pollination between related species is rare but has produced such valuable plants as the loganberry and plumcot.

In most plant species the seeds come true to kind, as in wheat, corn, and beans. In many, as the potato, strawberry, apple, and plum, the seeds produce numerous "sports" widely different from the parent plants.

The navel orange and the nectarine originated as bud "sports."

Plants which do not come true to seed are propagated by dividing the parent plant, so as to use its buds for the origin of new individuals. Examples are found in the growth of potatoes from the eyes of the tubers, strawberries from runners, blackcaps and raspberries from tips, grapes from cuttings, oranges by budding, and apples by grafting.

Seed selection should begin with selection of the parent plants. 


\section{REVIEW QUESTIONS}

1. By what three methods may plants be improved? 2. Describe fully the steps necessary in cross-pollination. 3. Within what groups may cross-pollination be effected? 4. What may be the results of cross-pollination of selected individuals of any variety? 5 . What are hybrids? 6. (a) What is the usual result of trying to cross allied kinds? (b) Mention some instances of successful crossing. 7. (a) Mention several plants that come true to seed; several that do not. (b) How are plants of the second group propagated?

8. What is a "sport"? 9. Describe the method by which the Wealthy apple was originated. 10. (a) What was the origin of the navel orange? (b) How is it propagated? 11. Can bud variation be produced at will? 12. What two kinds of " sports" occur among plants?

13. (a) What is the general method of propagating by buds? (b) Describe the particular methods for potato, strawberry, blackcap raspberry, grape, currant, apple. 14. (a) Mention several plants that are often propagated from cuttings. (b) Describe the method.

15. (a) Describe the process of budding. (b) Mention several commercial plants propagated by budding. 16. Mention some commercial plants which are propagated by grafting. 17. What are the advantages of grafting? 18. Can grafting be successful if the stock on which the scion is placed is of a widely different species? If it is of a nearly related species? 19. (a) What is the advantage of selecting seed? (b) When should it be done?

\section{SUGGESTIVE QUESTIONS}

1. If bud sports were common, what methods of propagation would be uncertain? 2. (a) Have any new varieties of agricultural plants been originated in your vicinity? (b) Give the story of the origin and propagation. 3. Of the two kinds of flowers in the cucumber vine, which sets fruit? What is the use of the other? 4. Are the pistillate and staminate flowers of the cucumber the same color? Of the corn? Explain. 5. Compare wind-pollinated and insect-pollinated flowers as to odor and color. 6. (a) What evidence have you that some insects have a sense of smell? (b) Is their choice of odors always the same as ours? Give an illustration. 7. Mention some fruit the seeds of which are eaten by birds but not digested. What purpose does this serve in the propagation of the fruit? 8. Upon what agencies do plants depend for propagation of their kind? 9. What qualities do you find in weeds fitting them to survive but not to serve man's needs? 10. In your observation of the plants 
grown in a limited space does it appear that the conditions favor the fit or work for the elimination of the unfit? 11. Give cases of plants that show fitness in one region and unfitness in another. 12. How does knowledge of the conditions upon which natural selection depends aid men in controlling and improving plants?

\section{Mendel's Law of Inheritance}

243. Knowledge of heredity important. - It is evident from our studies of plant improvement that all methods of propagation, whether by seed or by selection and propagation of sports, are dependent upon what may be called the laws of inheritance. The whole problem of heredity, however, is far from being understood or solved; but certain facts in the propagation of plants and animals have been definitely established by experiment with a few forms of life, and it is believed further investigation will demonstrate their truth in all forms. The new knowledge of heredity has been acquired as a result of discoveries made by Gregor Mendel. It is to-day being applied in the study of human relations under the name of "Eugenics," a subject which is undoubtedly vital to all, and, so far as practicable, should be applied intelligently to the improvement of the human race.

244. Story of Mendel. - Gregor Mendel (1822-1884), to whom credit is due for the modern knowledge of heredity, was an abbot in the monastery of Brün in Austria. He devoted his leisure to experimenting with peas and other familiar plants in the garden of the monastery. In 1865 he published an account of the results of his experiments in crossing different varieties of peas; but this account passed unnoticed until the year 1900, when it was discovered almost simultaneously by several students of plant life. Since then a new period began in the study and application of the forces and processes involved in heredity. New views have been made available that are of great practical value to the gardener and to the breeder of animals, and to all who desire to understand the facts of inheritance. 
Experiments with peas. - Mendel selected for his experiments the culinary pea, because it is normally self-pollinating and therefore free from interference by other agencies. He found that the several varieties of the pea have distinctively different characters, and by testing he proved that these characters reproduce true to type. He therefore cross-pollinated different varieties of pea plants, such as tall with short or dwarf plants; those having pink or red blossoms with those having white; those producing yellow seed with those producing green; those having hard pods with those having soft; those producing wrinkled seed with those producing smooth, round seed. This he did until he learned exactly how the different varieties, when cross-fertilized, behave in successive generations. $\mathrm{He}$ discovered that in the line of descendants of hybridized peas there is never a real blending of opposite characters; but that a character persists practically unmodified however it may be combined with others.

Unit characters. - Mendel found also that particular characters, such as tallness of the plant or color of the blossoms, may be separated from a mass of other characters. Thus a plant grower may single out one character, as the stringless bean pod, from those combined in a bean pod, and by proper fertilization secure its separate continuance in all descendants.

Such a character, as tallness, color of blossoms, or color or form of the seed, is called a unit character, which means that it persists without blending in cross-fertilized descendants. This may be illustrated roughly by a mosaic made up of many different stones, each of which is intact and may be separated from the others and recombined to form other patterns. Or it resembles a chemical reaction, as when oxygen and hydrogen (page 130) combine in a definite ratio to form water. When water is decomposed, as in electrolysis, it gives up its constituents as they originally were.

How to produce hybrids. - New varieties may be produced by combining desirable characters in cross-fertilization. A 
pea bearing purple blossoms is hybridized with one bearing white blossoms. It does not matter which plant is selected for the pollen, and which for the ovule parent. The plants produced from the cross-fertilized seed inherit the characters of both parents. They are therefore called hybrids. All the plants of this generation bear purple blossoms. The plants of the succeeding generations will repeat both of the original characters in a definite mathematical ratio. This ratio will continue through all succeeding generations. It is just because of

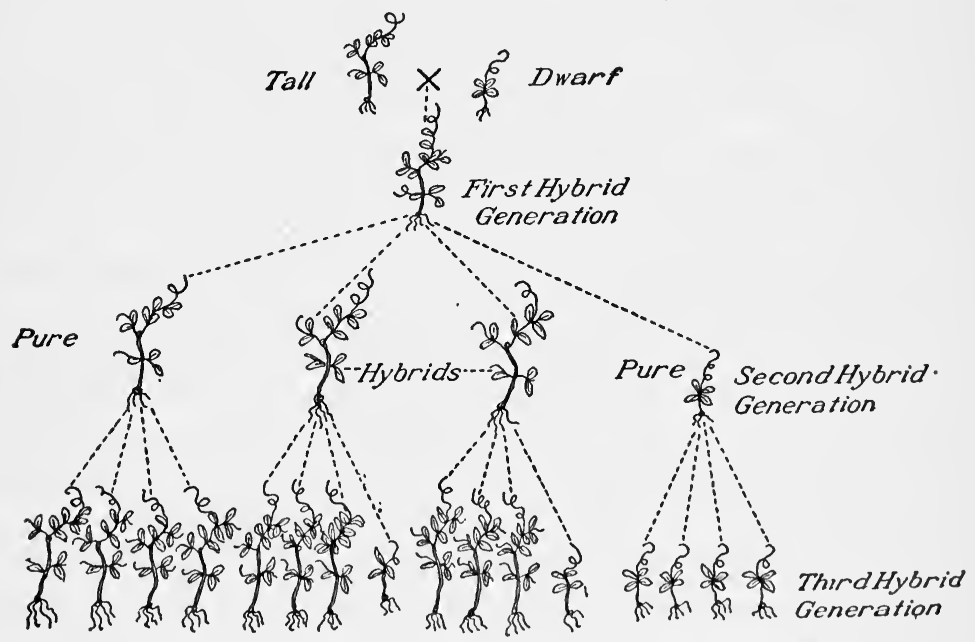

FIg. 158. - Diagrammatic representation of the principle of Mendelian inheritance.

this reappearance of unit characters in the third and succeeding generations that desirable characters may be selected and by cross-fertilization be made a permanent variety. In the same way a new variety or an old pure character may be ob- ' tained from hybrids.

Dominant and recessive. - One of Mendel's experiments in crossing different varieties of peas may be taken to illustrate the important facts and suggest the principle derived from these 
facts. Mendel, by testing through several generations, found a dwarf pea which bred true to type and did not exceed eighteen inches in height, and a tall pea that had a length of six feet. These he hybridized. The plants of the first generation of the cross-fertilized seed (Fig. 158) were all tall; in fact they were taller than the tall parent plant, growing to a height of about seven and one half feet. This is the usual result of crossing two varieties - the strong character is considerably reinforced. Because the unit character, tallness, appears exclusively in all plants of the first generation, Mendel described this character as dominant. Because the other unit character, shortness or dwarfishness in this case, disappears entirely in the plants of the first generation, it is described as a recessive character. As any one may prove by hybridizing plants having opposite and distinctive characters, the dominant character will always appear exclusively in the offspring of the cross-fertilized parents. But unit characters are never blended in the hybrids, and, however combined with one another, they retain their integrity and are transmitted unmodified to the descendants.

Succeeding generations. - The seeds of the tall hybrids were collected and planted. The plants when grown proved to be of two varieties, some tall and others short. There were no intermediate lengths of plants, as would be the case if the two unit characters had blended to form an average. The seed of this generation was planted; the seed of the short peas grew true to type ; but the seed of the tall peas produced two varieties, in the ratio of three tall to one short. So it occurred in all succeeding generations. The seed of the dwarf peas reproduced true to parent, while the seed of the tall plants split in the same ratio of three tall to one short.

245. The formal law. - Let us assume that the total number of seeds produced by the first cross-fertilized plant was twenty. These when planted grew into tall plants only. The number of seeds produced, it may also be assumed, was 256 . These when planted produced 190 tall plants and 66 dwarf plants. The 
mathematical ratio is approximately three to one $(3: 1)$. The 66 seeds of the dwarf variety produced plants true to parent type. The 190 seeds from the tall variety produced both varieties of plants in the ratio of three tall to one short plant. The seeds of these short plants produced true to type and so on through all succeeding generations. The seeds of the tall plants produced again about three tall to one short plant. This ratio is always approximately true. The recessives, as every trial shows, come true to type ; the dominants always produce three dominant to one recessive unit character.

246. Tests of the law. - Similar results have been obtained with other kinds of plants and with several kinds of animals. It holds true of wheat and corn. It has also been demonstrated in the cross-mating of white mice with gray mice, of different varieties of rabbits, poultry, cattle, and horses. What may be accomplished in further selection and hybridizing of plants and animals remains for experiment to reveal.

Application to man. - The principle in a general way should hold true of mankind. As a result of Mendel's discoveries much has been achieved in the study of human inheritance; but this study has really brought to light the difficulties and complexities which must be understood and controlled before great progress can be expected in the application of the principle to specific cases. In one case, however, we know how it works, namely, in regard to the color of the human eye.

The colors of eyes may for the present purpose be roughly classified in two large groups, the one containing all dark or brown eyes, and the other all light or blue eyes. Variations between the clear, blue eye color and the deep, dark brown eye color are neglected, as they are generally due to less or more brown pigment in front of the iris of the eye. Dark or brown eye color is known to be a dominant unit character, and light or blue eye color is a recessive. An individual plant or animal may have a character, as tallness, shortness, which is said to be pure-bred or briefly pure, when this character is derived from 
parents which had similar unit characters. So the color of the eyes of an individual is pure when it is derived from a line of unmixed unit characters, either brown or blue. An individual is, on the other hand, said to be cross-bred or hybrid in respect to a certain character, when this character is inherited from parents having different unit characters of the kind and is capable of being transmitted to offspring. Thus the eye color of an individual is hybrid when it is derived from parents one of whom possesses the unit character brown while the other possesses the unit character blue. The offspring of parents, one of whom has pure brown eyes and the other pure blue eyes, will all have hybrid brown eyes, as brown color in eyes is dominant. But they are capable of transmitting both characters to their progeny. Compare this with the scheme showing the first and succeeding generations of cross-fertilized tall and short peas.

There are several possible combinations with fairly wellknown results, of which two may be suggested. The offspring having hybrid brown eyes may mate with individuals having pure blue eyes; their children will be about equally divided into two groups, the one having hybrid brown eyes and the other blue eyes. Or the offspring having hybrid brown eyes may mate with individuals having hybrid brown eyes. In this case the distribution of eye color of their offspring would correspond with the distribution of the third generation of the crossed tall and short peas, that is, there would be a ratio of about three having brown eyes to one having blue eyes. Of the three having brown eyes, one will have pure brown eyes, and two will have hybrid brown eyes. The pure brown eyes mated with pure brown eyes will transmit true to type, just as the pure tall peas repeat in reproduction. The hybrid brown eyes will follow the rule for combinations with pure or hybrid brown, or with pure or hybrid blue. When opportunity offers, observe this in the case of parents, children, and grandchildren. It must be remembered, however, that human beings are very 
complex, combining many characters in all sorts of ways. The analysis of the physical and mental characters of man is so difficult, and human generations are so long, that comparatively little positive knowledge has been acquired, and what has been won is far from being complete. It is therefore impossible at present to apply with any considerable degree of certainty the Mendelian law, however vital this may be, to human heredity except in respect to a few relatively simple characters.

247. The Mendelian principle. - According to the Mendelian belief there is no blending of the unit characters to form a new variety in the first group of descendants of crossed parent plants, if both are of pure strain. All the offspring exhibit the dominant character, which is usually greatly accentuated. The recessive character does not appear in this generation, but will be repeated in the offspring of the hybrids.

In the next generation produced from hybrids there are usually new varieties, from which selection of desirable characters may be made for propagation under control. But on the whole the hybrids' offspring fall roughly into two groups which express the ratio of three to one; three out of four repeating the dominant character, and the fourth the recessive character of the original parents.

Practical value of the Mendelian principle. - The important fact is that hybrids are especially valuable in revealing new variations which, when selected and properly fertilized, may be productive of individual plants embodying desirable characters. Usually, however, it is only one in thousands that combines the desired characters. This one must be carefully preserved and propagated by bud and by seed. Unfortunately it requires long and patient attention to fix or make permanent a new variety so that it will reproduce true to seed. It can be done only by continuous selection and controlled pollination through many generations.

It is also possible, through the intelligent application of Mendel's law, to eliminate undesirable characters, such as 
poor resistance to disease, frost, or prolonged dryness, or a poor quality of fruit, and to replace the defective with a desirable character. This is one of the most valuable ways of plant improvement.

Suppose that only one plant in a group of others of its kind, which are infected with a fatal disease, remains immune. This plant, if selected and properly crossed with plants which are susceptible to the disease, may be made to transmit to the descendants this character of immunity to the disease. If, moreover, healthy hybrids are selected for propagation, this method is after all the most successful in conquering and eradicating plant diseases.

Improving wheat. - An interesting case may be used to illustrate this. Cultivated wheat is usually close-pollinated, showing therefore very little variation in its progeny; and it has been improved chiefly by selection of chance or sport variations. It is generally susceptible to rust and other diseases, and is unable to withstand prolonged drought, especially when ready for pollination. These characters restrict its cultivation to fairly definite regions and even to districts within these regions.

About twelve years ago a wild wheat was discovered in certain arid, stony regions of Palestine. This wheat may be a wild original of the cultivated wheat. It has several characters not possessed by modern varieties of wheat. These are, first, its cross-pollination by the aid of wind, and close-pollination in certain unusual conditions; second, its ability to grow in a hot, dry climate, owing to its special adaptive structure for checking rapid transpiration and evaporation; and third, its immunity to rust, and apparently to other common diseases of the cultivated wheat. These characters have been combined with the best characters of cultivated wheat, and the new varieties, by careful selection and control, have been made permanent and adapted to the climate of certain regions that are now, or are to become, wheat-growing. The success of 
this experiment in combining the characters of the wild with certain qualities of modern cultivated wheat will transform sections of the country too dry for domestic wheat into wheatproducing regions. Cross-pollination, moreover, always means wide variation, and this makes possible more rapid improvement in the varieties of a plant.

248. Many unit characters in parent plants. - In all efforts to improve plants it must be recognized that in every parent plant there may be many unit characters of different kinds. This complicates the problem, but the same law applies as well as when only two characters are involved. Each unit character is somehow independent, never blended with others, and may by proper control be isolated from them.

Desirable characters. - In cultivated plants such characters as vigor in germination and growth, strength, health, resistance to cold and drought, productiveness, together with a certain size and quality of fruit, are generally desired. It may be that not all these characters are combined in any individual plant or variety. But by intelligently applying the principles of the inheritance of unit characters, one may by selection and controlled cross-pollination successfully combine them in a new variety of great economic value. This involves testing, selecting, and controlled fertilization. It calls for patience at every step. Selected plants must be accurately marked, and a complete record kept, in which are given the characters and results in each generation. Although the rejection of thousands of good individuals may be necessary, if the final result is the evolution of the desired variety, this will more than compensate for the cost.

\section{SUMMARY}

A knowledge of the laws of heredity is important for all who are interested in human welfare.

To Gregor Mendel, an Austrian monk, is due much of the modern rcientific knowledge about heredity.

Mendel discovered that unit characters, such as dwarfishness and $2 \mathrm{~L}$ 
tallness of peas, are not blended in hybrids. In the first generation the dominant character alone appears. In the second generation the recessive character appears in one out of four individuals.

By selections made in accordance with Mendel's law desirable characters can be fixed and undesirable characters eliminated. An example is the production of a new variety of wheat having the desirable characters of domestic and wild wheat.

\section{REVIEW QUESTIONS}

1. In what field of knowledge was Mendel an investigator? In what way are his methods and results important? 2. Give a brief statement of the life and work of Gregor Mendel. 3. What plant did Mendel choose for his experiments? Why? 4. Mention some characters of the pea. 5. Did Mendel find that these characters in hybrids blended or mixed? 6. (a) Mention several "unit characters." (b) Define the term. 7. How does a plant resemble a mosaic? 8. (a) What result is seen in the hybrids of purple and white peas? (b) In the succeeding generations what colors appear? (c) What use may be made of the facts? 9. (a) Describe Mendel's experiment with hybridizing dwarf and tall peas. (b) Which of the two characters, tallness and dwarfishness, is the dominant? (c) What is the other called? (d) Explain each term. 10. (a) What results appeared in the offspring from hybrids? (b) Which of the two varieties produced true to type? (c) What results were obtained by planting seeds of tall hybrids? 11. Summarize the results of planting seeds of the recessives and seeds of the dominants.

12. (a) With what other kinds of plants has Mendel's law been demonstrated? (b) What kinds of animals? 13. How is Mendel's law illustrated in the color of human eyes? 14. Why is it so difficult to apply the Mendelian law to human heredity? 15. What is the ratio in the offspring of a hybrid between those showing the dominant and those showing the recessive character? 16. What advantages may be gained by applying Mendel's law to plant and animal breeding?

17. What use could be made of a plant that is found to be immune to a prevailing disease? 18. (a) What are the important characters of the wild wheat of Palestine? (b) What use is being made of these characters in the improvement of domestic wheat? 19. Mention some characters desirable in cultivated plants. What has to be done in order to combine them in one individual? In one variety? 


\section{The Manufacture of Food by Plants}

One of the most important differences between plants and animals is that plants manufacture their own food from inorganic substances, while animals do not. It is therefore upon this manufacturing ability of plants that we depend for food, directly when we live upon a vegetable diet and indirectly when we live on meat and animal products.

249. Sources of materials. - The materials that plants need for food manufacture are derived from the soil and from the atmosphere. What is derived from the soil water has already been considered. The minerals constituting the ash remains of plants which have been burned are some of the substances which have been absorbed through the roots and assimilated into the plant tissue. But more than half the dry weight of plants is made up of carbon. A small quantity of carbon is absorbed by the root hairs from the decaying organic matter in the soil, but the greater part of the supply is derived from the carbon dioxid of the air.

250. The work of the leaves: Carbohydrates. - The organs which take carbon dioxid from the air are the leaves, which are green in all food-producing plants. The color is due to small green bodies called chloroplasts present in the cells of the leaves. The chloroplasts contain a green pigment called chlorophyll, and green plants are often described as chlorophyll-bearing plants. The chloroplasts are the manufacturing centers of the leaves; they absorb the carbon dioxid, which is taken from the air through very minute openings in the leaves, and decompose it into its elements, carbon and oxygen. Oxygen is released through the openings of the leaves to the air; and the carbon is combined by the chloroplasts with hydrogen and oxygen, obtained from the water of the sap, to form new compounds called carbohydrates. It is supposed that sugars are produced first and when a certain amount of sugar is accumulated in the leaf cells, it is changed by the chloroplasts into 
starch grains. If this is true, the presence of starch in the leaves may be detected by the following test.

Exercise: Test for starch. - Prepare a thin starch paste by boiling a little starch in about one hundred times its volume of water. When this is cool add a few drops of iodine solution. ${ }^{1}$ What is the result? Boil a thin slice of potato in a small quantity of water for five or more minutes. Cool the water and add a few drops of iodine solution. Is the result similar to that with starch? Would you infer that the potato water contains starch? Make the same test with bread and any other food which you think may contain starch. The tests show that iodine solution turns starch a deep violet-blue or blue-black color.

In the late afternoon get leaves from such plants as geranium, bean, or clover; immerse them in boiling water for a few minutes, and then transfer to warm alcohol in which they should remain for several hours. Alcohol dissolves and removes the green pigment and makes the leaves nearly white. After the color is removed, wash the leaves in water and immerse them for a few moments in iodine solution. What is the result? Does the iodine reveal the presence of starch? If you can find some striped grasses, treat them in the same way. Do all parts of the grasses show starch?

Conditions upon which leaves depend. - The results indicate that the leaves contain starch, one of the carbohydrates manufactured by the chloroplasts. It is necessary now to determine by experiment the conditions upon which leaves depend in doing this work. The following is a simple and convenient way.

Exercise. - Place some rapidly growing plant like the bean or geranium in the dark for two or three days. When you remove it from the dark, observe the pale sickly appearance of the leaves. Much of the green pigment has disappeared. Cut off one or two leaves and test them as you did the others. Before restoring the plant to the sunlight cover a portion of the upper surface of a large wellexposed leaf with tin-foil or black paper. Some design may be cut out of the tin-foil to expose a part of the leaf. Now place the plant

${ }^{1}$ To prepare iodine solution dissolve five grams of potassium iodide in three or four ounces of water. Then add one gram of iodine and let the mixture stand for ten or twelve hours. An occasional shaking will aid in dissolving the iodine. Add enough water to make one quart. 
where it will have the sunlight for several hours. At the end of the time remove the covered leaf and another that has been well exposed to the sunlight. Treat them with boiling water and alcohol as previously described, and test with iodine solution. What does the test reveal? In what parts of the leaf is the presence of starch indicaterl by the color? In what is a little or no starch shown? What do you find as to the extent of the starch in the uncovered leaf? What do these facts signify?

The uncovered leaf and the exposed parts of the other leaf show by their color that they contain starch. The protected parts indicate by their reaction the absence of starch. The facts seem to mean that leaves manufacture starch when they are exposed to light. In leaves kept in darkness not only is there no starch produced but that which was formed during the day is removed. If this evidence is not sufficient, test and compare leaves cut off such plants as the bean, clover, or geranium early in the morning with those removed late in the afternoon. Observe plants which are kept in shady places, or in greenhouses where the light is poor, as during a long spell of cloudy weather.

Photosynthesis. - The chloroplasts or manufacturing centers of the leaf produce sugars and starch only in sunlight. In the dark or at night the starch is removed from the leaf to other parts of the plant. This process of fixing carbon or combining it with water to form carbohydrates (page 129) by means of the energy derived from the sun is sometimes called photosynthesis, meaning "putting together by means of light." The term emphasizes the dependence upon the light of green plants in manufacturing food. An idea of the tremendous work done in breaking up carbon dioxid by the chloroplasts with the aid of the sun's radiant energy may be obtained from the fact that, outside the leaf, carbon dioxid can be decomposed by heat energy of a temperature of about $2372^{\circ} \mathrm{F}$.

Proteins. - Sugar, starch, and other carbohydrates are not the only foods manufactured by plants. There is a different group of foods, in the most important of which is found nitrogen 
combined with carbon, oxygen, hydrogen, and mineral substances, such as sulfur and phosphorus. These nitrogen compounds are the proteins. They are less in quantity than the carbohydrates, but not in importance, for they are indispensable to plant life and growth. The substance composing the cells, which is called protoplasm, is very similar in composition to the complex proteins. Proteins are manufactured in the leaves, by a process somewhat like photosynthesis, also in the roots, and possibly in other parts of plants. They are made by plants in the absence of light, but are produced in larger amounts in light. They are present in all the live centers of the plant, in the protoplasm of the cells, some dissolved in cell sap and others contained in seeds, such as cereals, beans, peas, and in roots, tubers, and bulbs. Proteins resemble the albumen or white of an egg, and, like this, coagulate when heated. They become yellow when stained with iodine solution.

251. Digestion of starch. - In the process of photosynthesis a sugar much like cane sugar is first of all produced in the leaves; but when the amount exceeds that needed for immediate consumption by the plant in its growth and work, the excess is converted by the chloroplasts into starch grains. In darkness or at night plants cease to manufacture sugar and starch, but growth continues. Growth requires food and so makes use of the starch stored by day. But starch grains are insoluble in pure water, as you may demonstrate by trial. They are moreover too large to be carried through the very minute sap canals to distant parts of the plant. They must therefore be made soluble for circulation, that is, digested. How then do plants digest starch or other solid food substances?

Diastase. - The cell sap contained in the chloroplasts is not like pure water, but is a very complex solution, It contains among other things certain substances which render soluble such solid and insoluble foods as starch, and certain proteins. These substances are called digestive ferments or enzymes. Starch, which is the most abundant of the solid foods stored up 
by the plant during its day's work, is made soluble by a particular kind of digestive ferment or enzyme known as diastase. Diastase dissolves the starch grains produced in the leaves, converting them into sugar, which being soluble is taken into the circulation and distributed among the various centers of growth. This enzyme, generally inactive or even absent in the bright sunlight, becomes especially abundant in the dark, so that by the return of the daylight the starch has been digested and removed from the leaves. Thus the leaves are made ready for another day's labor in producing food.

Diastase is not confined merely to the leaves, but is found in new stems, buds, and other actively growing parts of the plant. Here it does the same work of changing starch into sugar. It is found also in potato tubers and especially in the neighborhood of " eyes" where growth occurs. It is plentiful in the seeds of cereals and grasses. One of the best examples of the working of diastase is furnished in grains of barley. One finds by tasting barley grains that the endosperm is not sweet. Barley grains soaked in water have the same starchy nature. But taste the endosperm of grains which have just begun to sprout (sprout them between sheets of damp blotting paper); this is found to be sweet, in fact is a kind of sugar which has been produced by diastase acting upon the starch of the grain.

Experiment. - The conversion of starch into sugar very much in the manner of its digestion in the plant may be illustrated by a simple experiment. Make a thin starch paste in a beaker. Add five drops of hydrochloric acid and boil slowly for twenty minutes. With a glass rod put a drop of it on blue litmus paper. If the solution is acid, it will turn the paper red. Dissolve a level teaspoonful of bicarbonate of soda in about ten times its volume of water. Add the soda solution very gradually to the starch paste, testing this from time to time with litmus paper until it fails to give the acid reaction. The acid of the solution should be just neutralized. The solution is found to be sweet to the taste, due to a kind of sugar called glucose, which is soluble in water and similar to the sugar made by diastase acting upon starch in the plant. 
252. The plant's use of foods. - What is done with the sugars and starch, the proteins, fats, and oils which plants manufacture? Leaves, as you have demonstrated, do not, when left in the dark, retain the food they create by means of the sunlight. What disposition is made of the food? Part of it is immediately consumed in the work of respiration and manufacture of food. Part of it is digested and distributed as nutrition to the mature plant cells which are actively at work in different parts of the plant, and for the making of cellulose to thicken cell walls. Wood is largely cellulose; cotton is practically pure cellulose. Part of the food is utilized in producing new cells and cell walls wherever growth is taking place, as in buds, stems, and roots. A part is used in making various compounds which give odor and color to flowers, and flavors to fruit and seed. Or it may remain in the sap as sugar. What plants do you know that have sweet sap? What use do we make of such sap?

253. Where plants store food. - As plants mature or approach the end of their annual growth they manufacture more food than they can make use of in the ways just stated. The excess food is transferred and deposited in reserve food centers for future use. Such reserve centers ore seeds, buds, stems, roots and root stalks, tubers, and bulbs. Many plants, as corn, wheat, oats, barley, beans, and peas, store this in grains or seeds to serve as nutriment for their offspring in early growth. Where do potatoes lay up this reserve? Where do such plants as carrots, beets, sweet potatoes, parsnips, and turnips accumulate their food supply? For what purpose? Where do the onion, lily, and similar plants deposit it? Trees not only provide their young in seeds with nutriment, but also lay up a sufficient supply in special parts for beginning their own growth the succeeding season.

Exercise: Tests of food storage. - As starch is the most common of the foods deposited in solid form, it is worth while to make a series of tests on different parts and products of plants to determine its 
presence and relative abundance. You have already found it in the potato. Prepare in the same way and apply the starch test to such food plants as the carrot, turnip, onion, and others available. Corn, wheat, barley, rice, and other grains may readily be examined in this way. Cut a grain of wheat transversely (Fig. 159), scrape very delicately a little of the endosperm and spread it out in a little water on a microscope slide. Cover with a thin glass and examine with a low-power microscope. Put a drop of iodine solution at the edge of the cover glass and note its effect upon the particles. Does the color produced indicate the presence of starch? Stems, last year's buds, and roots may be examined. Cut exceedingly thin cross sections, immerse them for a moment in iodine solution, and then mount in water on a

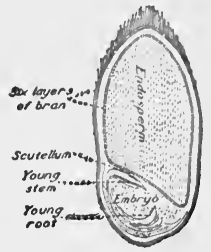

Fig. 159. - Lengthwise section of the wheat grain. Flour is made chiefly of the endosperm. microscope slide. Examine closely and observe in what parts of the tissue the largest amount of starch is indicated.

\section{SUMMARY}

Plants manufacture their own food; differing in this from animals. The materials used are soil water and carbon dioxid from the air.

Sugar is manufactured in the chloroplasts of the green leaves by the aid of the energy of sunlight. The process is called photosynthesis. The sugar may be converted into starch in the leaf. The presence of starch is shown by the iodine test.

Plants manufacture proteins also, which contain nitrogen and sometimes sulfur and phosphorus.

Proteins are abundant in seeds.

Starch is insoluble. It is changed in the leaf into soluble sugar by a digestive ferment, or enzyme, known as diastase.

The sugar produced in the leaf is carried in the sap to various parts of the plant and may remain as sugar or be converted into cellulose and other substances, from which new growth is made or new plants started.

Plants store up food either for their new growth or for their offspring, in various centers, such as seeds, fruits, buds, stems, roots and root stalks, bulbs, and tubers. Many of such stores are appropriated for food by man and other animals. 


\section{REVIEW QUESTIONS}

1. What is one of the important differences between plants and animals? 2. What are the sources of the materials used by plants in food manufacture? 3. By what organs do plants take carbon dioxid from the air? 4. To what is the color of the leaves due? 5. What is the function of chloroplasts? 6. What is the first food produced in the leaf? The second?

7. How can you prove the presence of starch? 8. (a) What is the relation of sunlight to starch production? (b) How can you prove your answers? 9. (a) Mention some carbohydrates. . (b) Of what are they composed? 10. (a) What is the meaning of photosynthesis? (b) Where does it take place?

(c) By what agencies?

11. (a) Of what simple substances are proteins composed? (b) Where are they found in a plant? (c) How may their presence be indicated? 12. What chemical change takes place in barley when it sprouts? By what is this change effected? 13. (a) Why does the starch disappear from the leaves at night? (b) Is starch soluble in pure water? (c) What does cell sap contain which makes starch soluble? (d) In what conditions is the diastase especially active? (e) What is the product of the action of diastase on starch?

14. (a) How may starch be converted into sugar in the laboratory? (b) How is the acid finally neutralized? 15. What uses do plants make of the food they manufacture? 16. Mention several ways in which plants store food. 17. How can you prove the presence of starch in the stores of food which the plants make? 


\section{CHAPTER XII}

\section{INSECTS AND HUMAN HEALTH}

\section{A Typical Germ Disease - Malaria}

Malaria is the most nearly universal of all diseases of man. Not only its immediate effects, but also its after effects have long been considered serious and difficult to control. To-day, however, the course and character of the disease, as well as its cause and the agent by which it is transmitted, are well known and largely under control. This result has been brought about by the research of several men, extending over many years. The story of the investigation is a striking. example of the way in which scientific work is carried on by many individuals to a common end. For this reason malaria may be taken as a type study of the diseases which have been conquered by the use of scientific knowledge.

254. History of the disease. - The downfall of Egypt, which is still mosquito plagued, of Mesopotamia and Athens, and of Rome in a later age, was probably hastened by mosquitodistributed diseases. The people of these countries made use of mosquito netting, brushes, and fans, but they apparently did not connect the mosquito with the diseases which were decimating the population. Early in the eighteenth century gnats or mosquitoes were supposed to have some share in the distribution of "marsh diseases," by carrying the "miasmic or malarial" poison and injecting it through their sharp beaks into human beings and animals. The poisonous matter was imagined by some to be animalculæ, or microörganisms as we should call them to-day. This idea received no support in science until nearly the end of the nineteenth century. 


\section{Transmitted
by bite of
mosquito.}

Red
Corpuscle
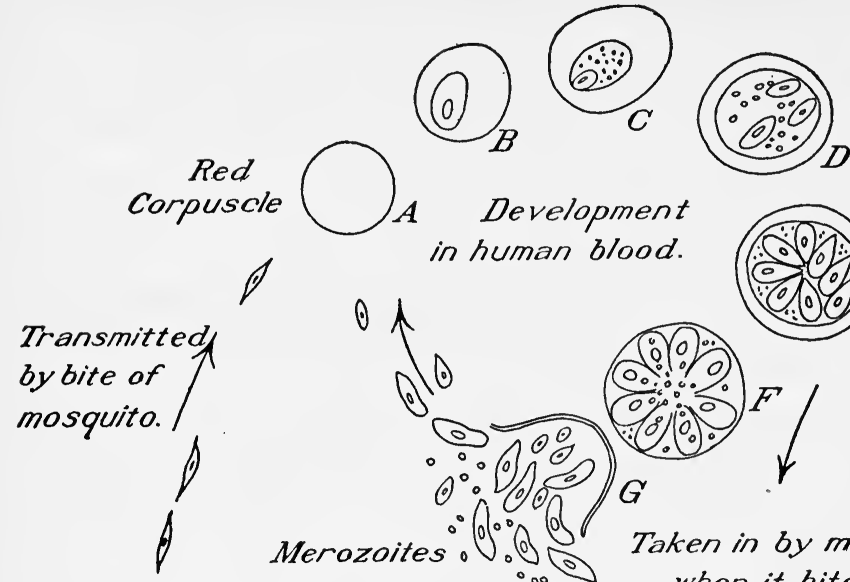

1)

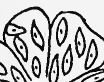

In salivary gland

5 (2) of mosquito.

Merozoites $: 0600$ Taken in by mósquito

Development in human blood.

$0 \uparrow$
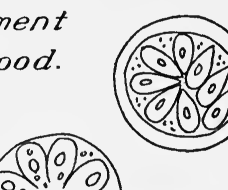

(a)

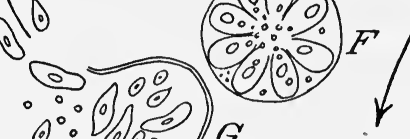

$\therefore 000$

- 100 $\therefore$ when it bites.

;0 $a$ O

Sexual forms 60 in mosquito's stomach.

$\theta$

00 sporozoites in

$\theta$

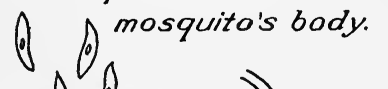
400

Fertilized cell.
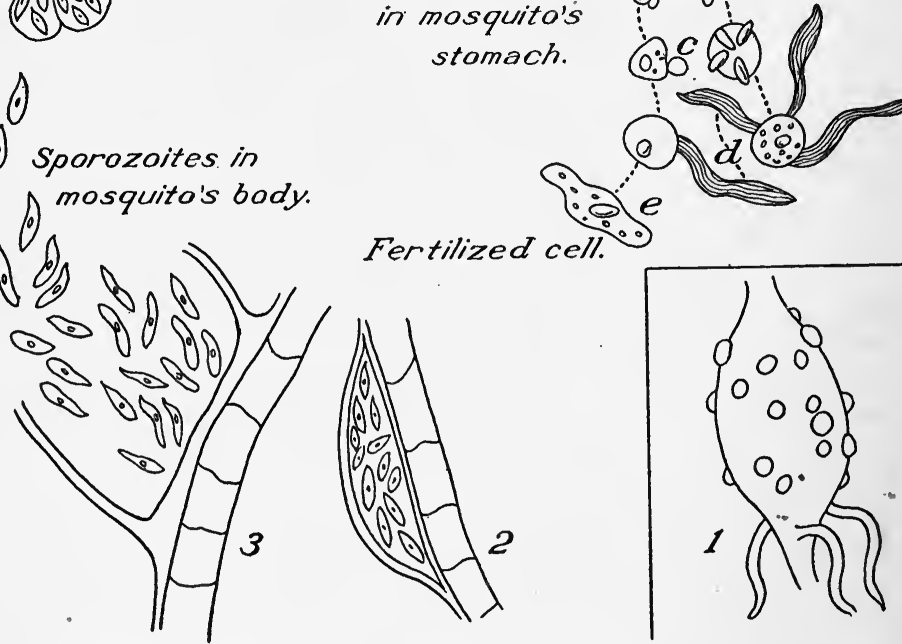

Frg. 160. - Life history of the malarial parasite in man and mosquito. $1,2,3$, parasites on the outer wall of mosquito's stomach. 
white cells of the blood, called the phagocytes (page 601). The parasite feeds upon the substance of the cell, growing and multiplying by the simple division of its own substance into a large number of minute spores called merozoites. In its digestion the parasite throws off several waste products, among them the black granules which are always present in malarial infection. At last when all the cell's substance is consumed, as at the end of three days in the tertian fever, or of four days in the quartan fever, the wall of the red cell bursts, liberating in the blood of the infected person a horde of merozoites with their waste products. As nearly all cells burst at about the same time, the waste products which are poisonous or toxic cause the attack of fever and chills, or ague, as it is popularly called.

The number of parasites in the blood is thus increased rapidly with ever greater destruction of the red corpuscles and more severe attacks of fever and chills. Each merozoite repeats the cycle of development just described. Most of them reproduce by simple division; but there are a few which reproduce sexually, not in the human blood, but only in the body of the Anopheles mosquito. This is one of the most important facts discovered by Ross.

In the mosquito. - When an Anopheles sucks the blood of a malarial patient, it ingests with the blood many parasites. All of these except the sexual forms (Fig. 160, $a, b$ ), which are crescent in shape, are digested and perish. The sexual parasites continue the development, each form throwing out polar branches that unite with their opposites and produce in this way a fertilized cell. This fertilized body, resembling a sharply pointed rod, pierces and escapes to the outer wall of the mosquito's stomach (Fig. 160, e, 1, 2, 3), attached to which it grows to several times its original size and multiplies by simple division into a number of bodies called sporoblasts. These multiply and develop in a similar way, producing large numbers of minute hair-like organisms, known as sporozoites, which find their 
way to the salivary glands of the mosquito or to other parts of the body.

The complete development of the parasite in the mosquito requires from ten to fourteen days. Only after the development is complete does the mosquito become infective. Then when it bites an individual, it pours into the wound a quantity of saliva containing a number of the sporozoites, which at once begin their life cycle unless they are destroyed by the protective phagocytes of the blood plasm.

A mosquito does not expel all of the sporozoites from the salivary glands at once, but may infect several individuals over a period of many days or weeks. All the parasites do not escape through the salivary glands, some working their way into the ovaries and entering the eggs developing there. The young mosquitoes hatched from such eggs may introduce the malarial parasites into human circulation just as effectively as if they had been directly infected. This fact is important in understanding an outbreak of malarial fever that cannot be explained otherwise.

If the adult parasites are not taken from the human system by a mosquito, they perish, except in a few cases where the female forms survive in man for years. Then with no apparent means of being fertilized, they begin to multiply by division: Thus it happens that one who has recovered from malaria may have after several years a recurrence of the disease, and without a new inoculation by a mosquito.

257. Practical tests of the theory. - The popular mind is usually slow to accept the results of science, and even medical practitioners may reject the conclusions of scientific investigators in their own field. So it was with the work of Ross, and with that of others, as Pasteur, Koch, and Finlay. To secure the popular recognition of truths practical tests and demonstrations must be made. Even to-day, in spite of the work of science, the superstitious idea persists, as it did after the results of Ross's work had been announced, that malaria is caused by marsh or swamp air (mal aria meaning bad air). 
In Italy. - With the opposition of medical practice to the new theory advanced by Ross and others, a series of practical tests was made to demonstrate its truth. A test undertaken by an Italian scientist, Grassi, was to prove that the bite of the Anopheles is the only way of transmission of the disease, and also to formulate a set of rules which should banish malaria from Italy. A group of railroad workers, about four hundred men, women, and children, lived in a malarial district near Salerno. Their cottages were equipped with screens, and the people remained within doors from sunset until dawn, pursuing their regular work during the day. The result was that all individuals escaped infection, although three experienced a recurrence of the disease.

Another experiment made by four medical authorities of London was carried out in a notorious malarial district, in the Roman Campagna. In a house which they had built and made mosquito-proof they remained from shortly before six o'clock in the evening until broad daylight in the morning. A careful observation showed that the female Anopheles appeared regularly at sunset, and never during the day. By day they visited the villages of the neighborhood, examining many cases of malaria, and were exposed to bad weather. They kept the windows of their house open day and night so as to admit the miasmic air of the marsh ; but they developed not the slightest trace of malaria. They exposed themselves to all possible ways of infection except the bite of the mosquito, and took no quinine or other drugs as preventives. This demonstration carried great weight with many medical men and others; but another test was made to establish the theory beyond any doubt.

In England. - Dr. Manson, a son of Sir Patrick Manson who had advanced the hypothesis that malaria is transmitted by blood-sucking insects, and Dr. Warren, both London physicians, undertook the crucial test. They allowed themselves to be bitten by Anopheles mosquitoes which had 
been fed on the blood of a malarial patient and had become infective. After eighteen days both developed typical cases of malarial fever of the double tertian or three-day character. Microscopic examination of the blood revealed the presence of the malarial parasites. As neither of the men had had this disease previously, the result established the truth of the theory.

258. An example of scientific method. - This is an excellent example of the way in which scientific men deal with a difficulty or a problem. It illustrates the spirit of those who do the scientific work of the world, and may serve as a guide to every one in striving to understand the difficulties and problems of daily experience. Some of the chief steps involved in the whole course of investigation may be stated briefly in order to emphasize the method of scientific procedure.

1. The first aim is to locate exactly and form a clear idea of the difficulty experienced. This means that facts must be compiled, and a definite statement made of the problem in order to make clear what is known and what is unknown.

2. This statement of the problem and its difficulties suggests possible explanations or solutions.

3. The explanations or solutions suggested are carefully considered before applying experimental or practical tests in order to avoid waste of time and energy.

4. The promising explanations are tested in various ways. Of a thousand ideas advanced it may often happen that only one is worthy of thorough test. It was so with the problem of malaria and its transmission. The idea selected for thorough test was that malaria and possibly other diseases may be transmitted by blood-sucking insects. It had to be proved by the discovery of new facts and then tested by these facts.

5. Practical tests may be necessary, as with malaria. When it had been proved that the bite of the Anopheles mosquito, which had become infective, inoculated healthy persons with the malarial parasite, the case seemed to be closed. But it also 
had to be proved that in no other way could the malarial germ be transmitted.

6. The next step after the practical test is the acceptance of the theory, for it now stands uncontradicted by all known facts. In the case of malaria this acceptance leads to control of the disease and the agency of its transmission.

259. Control of malaria. - Knowledge of the cause of malaria and the way of its transmission opened up another field for scientific investigation. It became necessary, since the Anopheles mosquito had been proven guilty, to learn its life history and habits in order that effective measures might be employed to exterminate it. It was necessary also to discover how the parasite in the human system may be controlled or destroyed. A study of the mosquito is outlined in the following section. We here consider briefly the control of the disease and the prevention of its spread by animal agencies.

The parasite in the human system is destroyed by quinine, the only known specific for the disease; but since quinine is not equally safe for all persons, it should be taken only when prescribed. The patient should be isolated so that no mosquitoes can feed upon the blood, and thus becoming infective agents, spread the disease. This is the only way of preventing its transmission to healthy individuals.

260. Malaria, a typical disease caused by protozoa. Malaria is typical of all diseases known to be caused by animal parasites and transmitted by animal agencies. Its wide geographical distribution has made it of primary interest to those who labor to conserve as well as to restore the health of mankind. Its successful mastery justifies at least the following practical suggestions.

First. There is sufficient reason to believe that the means will be discovered of destroying all pathogenic protozoa and bacteria or of neutralizing their poisonous products or toxins in the human system. This is now done in several diseases. 
Vaccination is actively preventive of smallpox, and immunity to typhoid fever is acquired by a similar method. Diphtheria is treated successfully by inoculation with diphtheria serum (see page 606); and within recent years certain drugs have been discovered which are specific for several common and dangerous infectious diseases.

Second. Simple but adequate preventive measures can be employed by everyone to block or destroy the animal agencies or other media of transmission of pathogenic germs. Following the discoveries by Ross, scientific investigation has proven that several dangerous diseases are caused by animal or plant parasites (pages $588 \mathrm{ff}$.) which are distributed by insects. Other varieties of the mosquito are known to spread yellow fever and dengue or break-bone fever; the common or domestic fly is a carrier of the typhoid fever germ ; the tsetse fly spreads sleeping sickness; while fleas, bedbugs, ticks, and lice are carriers of germs causing cholera, bubonic plague, spotted fever, and typhus fever. Although many diseases have not been thoroughly investigated and their causes discovered, it is certainly a wise method of procedure to employ adequate measures to prevent the transmission of any disease by insects, other animals, utensils, clothing, food, water, or other possible agencies.

261. Yellow fever. - In the year 1881 Dr. Carlos Finlay suggested that yellow fever may be transmitted from an infected person to a healthy one by a mosquito. He failed to prove this by his experiments, because he did not know that a mosquito after feeding on the blood of a patient does not become infective under a period of twelve or more days. This fact was discovered by others, and Dr. Finlay's hypothesis was proven to be true by a series of practical tests.

The triumph of scientific knowledge over the cause and agency of this disease has been witnessed in many localities. In the southern states, in Havana, and in the Panama Canal Zone, where yellow fever had held full sway, there' is now scarcely a trace of the disease. This is due to the extermination or control 
of the Stegomyia mosquito, the sole agency of transfer of the yellow fever parasite (see pages $542-3$ ). ${ }^{1}$

262. Infantile paralysis. - The disease known popularly as infantile paralysis and to medical science as acute anterior poliomyelitis is a current problem of great interest and difficulty. During recent years there have been several widespread epidemics with great mortality among young children. Nearly two thirds of those who do not die are permanently crippled in some way.

Careful investigation has not so far been able to discover the cause of the disease, the agencies of its transmission, or the means of controlling it in the human system. It is supposed that the cause is a microörganism, whether a bacterium or a protozoan is uncertain. It is ultra-microscopic, that is, so small that it cannot be discovered by the aid of the microscope. Its presence is assumed from the effects observed in the course of the disease.

Search for the agencies of transmission has not met with positive results. Investigations have been made of the house fly, stable fly, bedbug, mosquitoes, and lice as possible carriers, but sufficient evidence has not been found to warrant a final conclusion as to their agency in transmitting it. It has also been suggested that the disease may be communicated by contact with the infected individual, and that it may be transmitted by food, milk, water, clothing, or dust, or in fact by any medium which has been contaminated by contact with the patient.

In the treatment of the disease, various means have been employed, but the evidence at present is far from conclusive as to the merits of any particular remedy or method tried.

The problem is of so great interest to human welfare as to command the efforts of scientific men; and, like malaria and yellow fever, its cause and ways of transmission will be found and brought under control through the research of scientists.

1 An excellent account of the conquest of yellow fever and the Stegomyia is presented in the book "Walter Reed and Yellow Fever," by Dr. H. A. Kelly. 


\section{SUMMARY}

Malaria is typical of the diseases that have been brought under control by scientific knowledge.

Malaria is supposed to have been the cause of the decline of some ancient peoples.

The conquest of a disease depends upon the knowledge of its cause, transmission, and control.

In the study of malaria its cause was discovered to be an animal parasite in the human blood.

Because one stage of its life history was missing in the human blood, search for it was made in blood-sucking insects, particularly the mosquito.

Traces of the parasite were found in the Anopheles mosquito. This discovery led to the working out of the complete life history of the parasite.

The theory of the transmission of malaria by the mosquito was put to practical tests by showing: (1) that persons could live in "malarial swamps" without contracting the disease if they were protected from mosquitoes; (2) that persons not in malarial regions contracted malaria when bitten by an infective mosquito.

The conquest of malaria illustrates the way in which men who are scientific in spirit and method attack and solve problems concerning human welfare.

The control of infectious diseases depends first upon the prevention of the transmission of the germs to uninfected persons, and second, upon the destruction of the germs or the neutralization of their toxins in infected persons.

Yellow fever was conquered by preventing the transmission of the germs, by means of the destruction of the Stegomyia mosquito.

The cause and means of transmission of infantile paralysis are problems under investigation.

\section{REVIEW QUESTIONS}

1. Why is the study of malaria important? 2. Why was this name given to the disease? 3. When did the discussion of living disease germs begin? State the theories then advanced. 4. What was Laveran's contribution to the knowledge of malaria? 5. Give an account of the discoveries of Golgi. 6. Describe the chief types of malaria. 7. What was Manson's theory of its transmission? 8. State the problem as presented to Ronald Ross. 9. Give an account of the 
efforts of Ross, and their results. 10. Where did he find the missing stage in the life history of the mosquito? 11. What did this discovery mean in the conquest of the disease? 12. How did the knowledge of the cause of malaria and its transmission influence the investigation of yellow fever and other diseases? 13. What other insects are disease carriers?

14. Describe the life history of the malarial parasite in man, accounting for its introduction, its source of food, the fate of the red corpuscles of the blood, and the effect of the parasites' waste products upon the patient. 15. Give an account of its removal from man; its course of development in the mosquito. 16. In what ways may the parasites be distributed by the mosquito? 17. Explain how an outbreak of malaria may occur without a new inoculation by mosquitoes.

18. (a) Describe the practical tests of Ross's theory of malarial transmission, made in the malarial district in Italy. (b) What did they prove? 19. Describe the practical test of the theory made in England. What was proven? 20. How do men of scientific habits of work deal with a problem? 21. What is the value of practical tests of a proved theory? 22. How may the malarial parasite be controlled or destroyed in man? 23. What knowledge is necessary for the prevention of the transmission of malaria? 24. What lessons does the conquest of malaria teach as to the control of infectious diseases and the prevention of their transmission?

25. Give an account of the conquest of yellow fever. 26. Why is infantile paralysis an important problem to-day? 27. What is known of the cause of the disease? 28. What is known of its ways of transmission?

\section{The Mosquito}

263. Relation to human health. - As already stated in the study of malaria, the Anopheles mosquito is widely distributed in the United States as well as in other countries. In the past it has wrought great disaster to human health and life; but to-day in many localities, where malaria was formerly prevalent, rarcely a case can be found because of the relentless war of crtermination waged against this mosquito. The Panama Canal Zone, where in the past yellow fever and malaria made human occupation almost impossible, is now become as safe as any place on earth, through intelligent use of preventive measures 
in exterminating the Anopheles, which transmits malaria, and the Stegomyia, which transmits yellow fever.

264. Life history and habits of the mosquito. - As is the case with all winged insects, the several varieties of the mosquito have a life story which is the same in all general features. The study of the common Culex or domestic variety of the mosquito, which is selected for convenience, will provide, therefore, a sufficient foundation for the understanding of the slight variations in the Anopheles and the Stegomyia. The experiments outlined will afford opportunity to verify and supplement the brief account here presented of its life history and habits.

265. The eggs. - All mosquitoes lay their eggs in water, usually in that which is stagnant or still and rich in food

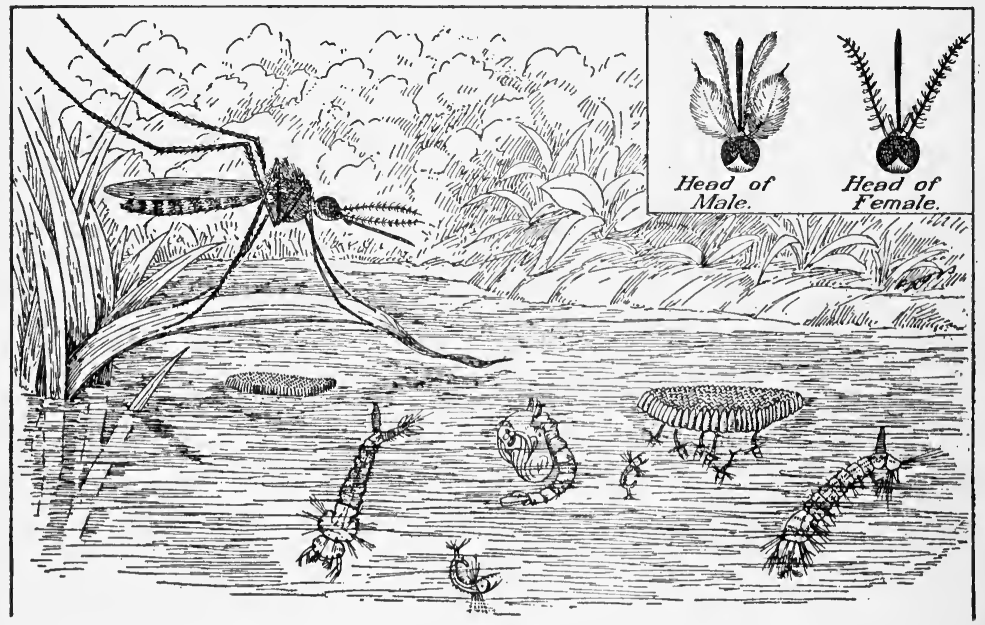

FIG. 161. - Life story of the Culex mosquito. Compare each stage of the. Culex with the corresponding stage of the Anopheles.

materials for the young. The eggs of the Culex cohere in rafts or masses (Fig. 161). The color of the upper surface of the eggs is gray to brown or sooty in appearance, and of the under surface silvery to water-white. Each mass may consist of from 
one hundred to four hundred eggs. It floats on the surface of the water like cork. The eggs of the Stegomyia are only slightly less massed. The Anopheles oviposits her eggs singly (Fig. 162). Each egg, the shape of which is regular and elliptical at both

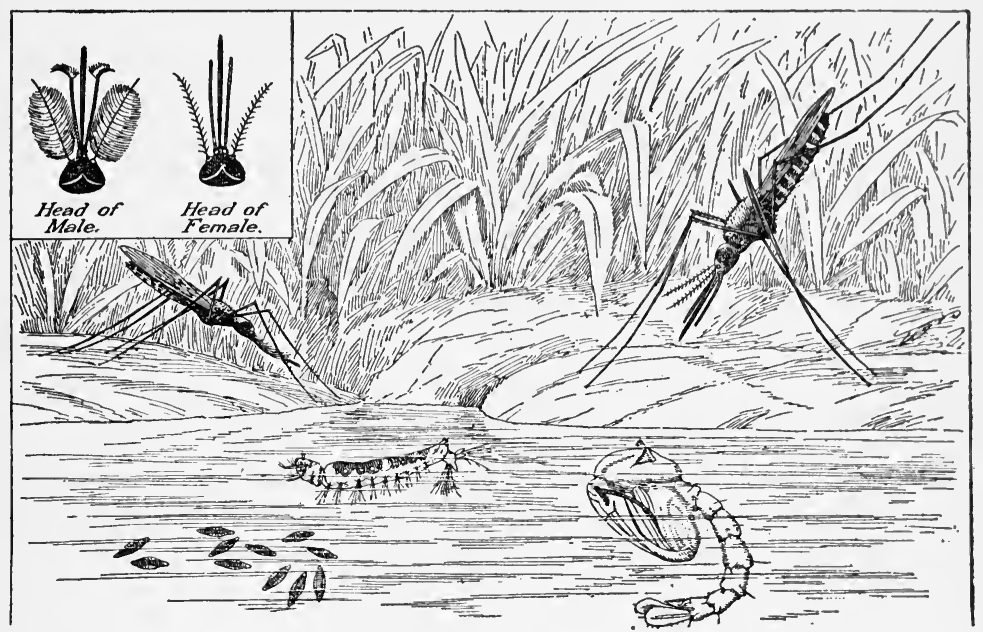

Frg. 162. - Life story of the Anopheles mosquito, the carrier of the malaria plasmodium. Characteristic positions of the adult mosquito, the pupa and the larva. Compare with the Culex mosquito, Fig. 161.

ends, lies on its side and floats on the surface of the water. They usually remain close together. The Anopheles lays from fifty to a hundred eggs at a time.

Exercise: Life history of the mosquito. - Collect mosquito eggs. Get some larvæ or wigglers from a pond, and a quart of the water in which the larvæ are found. Mosquito eggs may be secured by setting a pail of not too clean water with a few small bits of wood floating on the surface, in places frequented by mosquitoes. Examine in the morning and when you find eggs, transfer them to the breeding jars. If you have a good supply of eggs and larvæ, take out at stated intervals one or two to preserve and arrange them to show stages of development. A record of dates, temperatures, and condition of the water should be kept, and the important data summarized on the labels pasted on the jars. 
Glass jars, of pint or quart capacity, make good containers. Equip one with a pasteboard box having two sides covered with screen; cover the other jars with netting or wire screen. Label the jars as No. 1, No. 2, and so on. Large labels on which important data may be recorded are serviceable.

In jar No. 1, containing about a pint of water obtained with the wigglers, put a dozen of the larvæ and a small bit of an egg-raft.

In No. 2, with the same amount of water, put some larvæ and a bit of an egg-raft. Keep this jar in a dark place as cool as possible.

In No. 3 put about one half pint of distilled water and some larvæ.

Jar No. 4 is to be half filled with pond water and supplied with eggs and some larvæ. Place this where the temperature may be $85^{\circ}$ F. to $90^{\circ} \mathrm{F}$., as upon a steam radiator or a warm water bath.

Keep complete and exact records of your observations, arranged under separate headings for each culture. Follow progress of development as fully as possible. Note behavior of larvæ in the different media and at different temperatures and explain. Observe the larvæ in the distilled water. What is the source of their food? What do the facts show as to the life habits of the mosquito in the larval stage?

Follow closely the specimens in jar No. 1, their behavior as larvæ, as pupæ, and their transformation into the adult mosquitoes.

Feed adult mosquitoes small pieces of banana or other juicy fruit. Make outline sketches showing chief features of larvæ, pupæ, and adult mosquito; outline sketches are even more valuable for the purpose than finished drawings. When adult mosquitoes appear in No. 4, transfer them to screen box on No. 1. How do the adults behave toward the pupæ or the latest born mosquitoes?

Into a jar containing clear water put some larvæ. As soon as they have grown familiar with their habitat, and breathe as larvæ do, cover the surface of the water with a small amount of kerosene or coal oil. How do the larvæ behave? Explain the result. Other points may be taken up in connection with the study in the text, and tested experimentally with the captive mosquitoes.

266. Larva. - In warm weather, as in summer, the eggs hatch in from sixteen to forty-eight hours. : In colder weather they are slower and may be weeks in hatching. Each egg produces a mosquito larva, commonly known as a "wiggler." While all mosquitoes in the early stages are aquatic, they are also direct air-breathers. They are equipped for getting oxygen directly from the air, and quickly perish when prevented 
from breathing. The breathing tubes, which issue from the eighth segment of the abdomen, are of varying lengths in different varieties. In the Culex the tube is longer than in either Anopheles or Stegomyia. The Culex in breathing or feeding is usually head down and tail up. When it breathes, the end of the tube projects slightly above the surface of the water. It feeds near the bottom or sides of the pool. The Anopheles is normally parallel to the surface in breathing and feeding.

The food of the larvæ is made up largely of microscopic forms of plant and animal life, as spores of algæ, diatoms, protozoa, and decaying organic matter; and if other supplies fail, they feed upon the eggs and larvæ of their own kind. The larvæ in the distilled or filtered water will probably show you this fact.

The wigglers or larvce are quite active. Their manner of locomotion is very interesting. When threatened by a hand or other moving object, they wiggle quite rapidly to the bottom of the pool. Although they are not as luscious morsels as the pupæ for fish or other predatory foe, they have their enemies and need power to escape. The chief natural foes of larvæ and pupæ are fish, especially goldfish and the top-minnow, the water beetle known as the back swimmer or water boatman, the larvæ of the dragon fly, and occasionally larvæ of other species of mosquitoes.

The larve, at first small, grow rapidly. Within two or three days the first skin becomes too tight and inelastic. There is formed a new skin and the old one is molted. Molting occurs four times during the larval stage of development. . Each time the color of the skin is darker. The intervals between molting vary from two days to several.

After eight days, if the weather and food conditions are favorable, the larva enters the pupal stage. The larva may develop much more slowly, and in winter may continue in the hibernating state. If you can find a pitcher plant with its water 
content frozen hard, you may secure a supply of hibernating larvæ. These, if the ice be very slowly melted and the water gradually warmed, will revive and begin their life routine.

267. The pupa. - The pupal stage lasts from one day to as many as conditions determine. The pupa bobs about near the surface of the water, eating nothing, but still wonderfully active if necessary. A tempting bite for fish and the water boatman, it must be ready to dart like a flash to safety. Try to catch them in water.

In the pupal state one may discern, delicately outlined within the thin covering, the head, wings, legs; and antennæ of the adult mosquito. The usual position of the pupa near the surface of the water shows a new way of getting air. The old tube is replaced by a pair of ear-like tubes issuing from the back of the body or thorax. In the Anopheles pupa (Fig. 162), these are long and sharp at the ends. In the Culex (Fig. 161), they are conical, like gently tapering funnels.

268. The adult. - The adult mosquito issues after twentyfour hours or more. For half an hour its wings are unfit for use, and it remains floating on the pupa skin at the mercy of wind or wave or any enemy. Slight rippling of the water upsets it and causes it to drown. Food during this stage is obtained from the water or any substances into which it happens to thrust its beak. When its wings are strong, it finds food in the vegetation near its birthplace, the juices of plants and fruits being its main supply.

The ratio of males to females is probably seven to one. The male remains near the place of birth, feeding and associating with females for a few days, when he dies ; or he may complete his life span, which is very short, without feeding. The female soon makes longer journeys in search of food. She usually derives sustenance from the juices of plants and fruits, but apparently prefers a diet of the blood of animals and men. After a period of two weeks or more, she usually seeks her birthplace, or, if not that, any suitable place where she may 
deposit her eggs. This she does three or four times during her life. If the female is born late in summer, she may hibernate; in the succeeding season of warm weather she will revive and proceed to lay eggs.

Males and females are readily distinguished by certain structural features. The males have very long palpi, and a proboscis which is not fitted for piercing. The antennæ are covered with fine hair, giving them a feathery appearance. Only the females are able to bite, the proboscis being equipped with half a dozen very fine, sharp, needle-like instruments that can pierce the toughest skin. When these needles are thrust into the skin and reach a blood supply, a quantity of saliva is injected and then the blood is sucked up and ingested.

The natural food of mosquitoes is not in any large measure the blood of men or other animals. There are extensive regions where mosquitoes ábound and warm-blooded animals seldom wander. It seems certain that only a small number of mosquitoes have the opportunity of banqueting on the blood of human beings. Those that do succeed are likely to produce more eggs and oftener, because of their superior diet.

Because plant and fruit juices are the natural food of mosquitoes, it is supposed that the salivary secretion poured by the insect into the food before being swallowed renders it easier to suck and also prepares it for digestion. The plant juices may coagulate and harden unless mixed with the saliva. When mosquitoes feed on men and animals, they inject a quantity of saliva into the wound, whether necessary or not. Some of it is undoubtedly taken up again with the food ; but part is slowly spread by the circulation of the blood and causes the irritation and swelling experienced by many persons.

Distribution. - The Culex mosquito is very widely distributed geographically; but it is always domestic, that is, closely associated with human habitation. Its habitual breeding places are usually provided by human carelessness, and are such as old cans, pans, bottles, barrels, neglected watering troughs, 
gutters, rain and drain pipes, clogged-up eaves or exposed tanks in attic or basement, cesspools, imperfectly covered wells, and in short any vessel containing a supply of dirty water in which a mosquito may deposit her eggs.

The Culex differs in habits from the forest or marsh mosquito, the Anopheles, which lays her eggs in pools of water, shallow ponds, marshes, stagnant or sluggish streams. Small lakes in public parks of large towns or cities, irrigation ditches in which water may stand for a considerable period or flow very slowly, may account for the occasional presence of the forest mosquito with the common variety. The two varieties are easily distinguished by the fact that both tend to remain near their breeding places. For this reason the common Culex will always outnumber the marsh or forest kind in or near any community. The converse is true in the vicinity of woods or marshes.

The color and markings of the Culex are plain and unobtrusive. It is usually gray with a tendency toward brown, but sometimes black. When it rests on a wall or ceiling, its position is characteristic (Fig. 161). It stands with its body nearly parallel to the surface and is apparently "humpbacked." The palpi are quite short in comparison with the proboscis.

The Anopheles is generally easily recognized. Its spotted wings are often taken as its chief characteristic and from this it has the name Anopheles maculipennis (spotted wing). But this feature varies among the several varieties. There are also groups of other species that have spotted wings. This feature, if taken alone, is therefore unreliable in identifying the insect. The plainest and most certain features are the feathery palpi of the mouth, which are quite as long as the proboscis. A characteristic of the Anopheles which cannot be mistaken is its standing or resting position. It always holds its body in a straight line and at a wide angle or nearly perpendicular to the surface upon which it is standing (Fig. 162).

Stegomyia. - Another species of the order to which the Culex and Anopheles belong, is the Stegomyia calopus, sometimes called 
the tiger mosquito. This is the carrier of the yellow fever virus. It is therefore important to be able to recognize it. Its habitat is usually in the tropical and subtropical regions; but it is often transported into the colder climates of the temperate zones. It is remarkably domestic in its habits and instincts. Its breeding places are much the same as those of the common Culex.

- The color and markings of the Stegomyia (Fig. 163) are characteristic. The body, abdomen, wings, and legs are brilliantly marked with black and white stripes. It is thus conspicuous among its more somber-colored relatives. Although it feeds by night, it has acquired a reputation for unusual activity by day. For this reason it is often called the day mosquito. In the daytime, however, it seeks the darker places in houses and dark-colored clothing. Otherwise its habits and life story are the same as those of the Culex and the Anopheles.

Their rôle in the spread of disease.The investigations have shown clearly that the Anopheles mosquito is the sole agency in the transmission of malaria

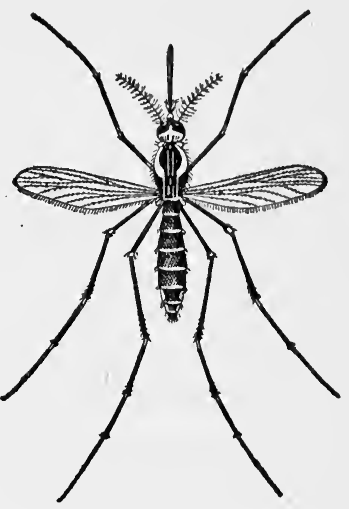

FIG. 163. - Stegomyia (female) mosquito. This transmits the germ causing yellow fever. and that the Stegomyia plays a similar rôle in the spread of yellow fever. The common Culex of this country is not proven to be a disease carrier, although it is seriously suspected and is under investigation. But a variety of the Culex called the fatigans, which is widely distributed in the tropical region and especially in the Philippines, transmits the germ of dengue fever.

269. Preventive measures. - Because of their agency in the transmission of disease germs and their annoying character even when they are not infective, mosquitoes are grouped with the enemies of human welfare, against which a war of extermina- 
tion is being waged. The various methods employed in bringing about their extermination are based upon exact knowledge of their life history and habits. This knowledge makes it possible to devise simple and effective measures.

The fact that all mosquito larvæ are aquatic and direct air-breathers points the way clearly to their destruction. Any means that prevents their getting to the air insures their death. A thin skin of kerosene is used for this purpose. The oilcovered water is destructive not only of the larvæ, but also of mosquitoes alighting on it. If mosquitoes find no water in which they can deposit their eggs, they lay them in grass or leaves where destruction is almost inevitable.

Drainage of marshes, stocking ponds with fish that prey upon mosquitoes in any stage of development, covering small pools, ponds, and the like with some viscous substance - as kerosene, - have all proven to be effective in destroying the mosquito. Among the more immediate measures which every one should take is to remove or destroy all breeding places due to carelessness. Old cans, bottles, troughs, and any receptacle that can hold dirty water should not be allowed to accumulate in corners or alleys, nor should garbage piles be permitted; for in all of these mosquitoes may deposit their eggs. Water tanks, barrels, and drain pipes need constant care for sanitary reasons, and they should be screened also to prevent mosquitoes and other insects from entering them.

When mosquitoes are prevalent in spite of such preventive measures, they should be kept out of houses and other buildings by screens. Certain preparations are useful, moreover, for those exposed in outdoor duties to the attack of the pests. Among such oil of eucalyptus either by itself or in mixture with other substances may be applied to the face, hands, and ankles. Oil of citronella is commonly used and is less disagreeable than some other applications. A more effective application is a mixture of two parts of oil of citronella, two parts of spirits of camphor, and one part of oil of cedar. 
When mosquitoes infest a room, they may be killed by means of chlorine gas. The room cannot be occupied for many hours afterward because of the noxious fumes. The gas may be prepared by putting into a large glass or crockery dish half a teacupful of chloride of lime; pour upon this about one fluid ounce of dilute sulfuric acid. Leave the room immediately and close the door, stopping up larger cracks to prevent the escape of the gas. Care should be taken not to inhale the gas when opening the room later.

\section{SUMMARY}

A knowledge of the life history and habits of mosquitoes is necessary to the control of certain diseases, such as malaria, transmitted by the Anopheles mosquito, and yellow fever, by the Stegomyia. The Culex, or domestic mosquito, is annoying, but not known to be dangerous.

The food of the adult mosquito consists mainly of the juices of plants. Only the females suck blood.

The life history of the mosquito, like that of all winged insects, includes four stages: the egg, larval, pupal, and adult.

Knowledge of the life history and habits of mosquitoes makes easy their destruction: (1) by draining swamps, (2) by destroying eggs, larvæ, and pupæ by means of a film of oil.

Additional protection may be had by the use of screens and certain oils.

\section{REVIEW QUESTIONS}

1. Why is knowledge of the mosquito important? 2. Where is the Anopheles mosquito found? 3. Give reasons for the selection of the common mosquito for study. 4. Describe the eggs of the Culex, as to appearance, places deposited, number, and time of hatching in usual conditions; also those of the Anopheles.

5. Describe the larvæ of the Culex as to appearance, locomotion, food, breathing, enemies, and growth; describe the larvæ of the Anopheles in the same way. 6. What stage follows the larval stage? Describe. 7. Describe the way in which the adult mosquito emerges from the pupa skin. 8. Give an account of the life of the adult mosquito, its food and habits. 9. Why do mosquito bites cause swelling? 10. Describe the external features of $(a)$ the Culex; $(b)$ the Anopheles; (c) the Stegomyia. 11. Of what use is the knowledge of their life history and habits? 12. What measures are employed to prevent the breeding of mosquitoes? 13. How may the adults be kept from attacking us? 14. How may mosquitoes in a room be killed? 


\section{CHAPTER XIII}

\section{MICROÖRGANISMS IN RELATION TO MAN}

\section{The Dust of the Atmosphere}

270. Composition of the air. - The atmosphere of the earth is a mixture of yarious substances. From the time of Lavoisier, chemists have known of its chief constituents, nitrogen, oxygen, and carbon dioxid. Recently, however, they have discovered in it several relatively unimportant inert gases, such as argon, helium, neon, and krypton. Traces of ammonia, hydrogen, and hydrogen peroxid are also found in the air.

The volumes of the four chief gases found in a liter, or 1000 cubic centimeters, of dry air are as follows :

$\begin{array}{lllllllll}\text { Nitrogen } & . & . & . & . & . & . & . & 784 \text { cubic centimeters } \\ \text { Oxygen } & . & . & . & . & . & . & . & 209.4 \text { cubic centimeters } \\ \text { Argon } & . & . & . & . & . & . & . & 6.3 \text { cubic centimeters } \\ \text { Carbon dioxid } & . & . & . & . & . & . & \cdot & 0.3 \text { cubic centimeter } \\ & & & & & & & 999.9 \text { cubic centimeters }\end{array}$

271. Dust in the air. - It is a matter of common experience that the atmosphere we breathe contains besides these substances a quantity of water vapor and of more or less minute dust particles. Water vapor in condensation is supposed to collect about dust particles in forming cloud, mist, fog, or rain. That dust is abundant in the air may be observed in a ray of sunshine passing through a darkened room; it is seen to form a veritable cloud. The particles observed are comparatively coarse, the vastly greater bulk of suspended dust being invisible to the unaided eye.

The air we breathe is obviously not always clean or pure, 
but more or less filled with microscopic dust particles. The fine, almost invisible particles often fill eyes, nostrils, mouth, and throat, and occasion at least temporary discomfort and pain. Dust accumulation in homes, offices, school buildings, and factories requires a great deal of human ingenuity and labor to effect its removal. It adheres to walls and floors, rugs and carpets, furniture and clothing, and accumulates with astonishing rapidity. It seems to be present always and everywhere, and to demand unceasing efforts to prevent its insidious conquest of human comfort and even health and life.

Means of removing dust. - The methods devised to remove dust even temporarily from buildings suggest the seriousness and magnitude of the problem. The common broom, the agelong weapon of attack upon dust and dirt, the feather duster, brush and dust cloth, carpet sweeper, dustless duster, chemical preparations for collecting and holding dust instead of allowing it to be scattered in the air, and vacuum cleaners of varying efficiency are some of the means employed. This does not complete the list, for water, the most abundant and universal cleanser in nature, must be included; and with water a score or more of chemical substances used to increase its cleansing properties. One should note some of the ways in which water is utilized to conquer dust. Floors, streets, and highways are often sprinkled, unless the use of oil has made it unnecessary. Water is used for washing ail durable parts of the house, for washing clothes, utensils, and so on indefinitely. It may be used cold or hot, or in the form of steam.

Hard and soft water. - Many difficulties may be presented in the use of water as a cleanser. Water varies greatly in quality because of the different kinds of rock materials through which it may have passed (see page 196), and which it in some measure dissolves away and retains in solution. It may, as is commonly stated, be hard or soft in varying degrees. Hard water is unsatisfactory for cleansing purposes at least, and useless with ordinary soaps. Did you ever try to wash anything 
with sea water and soap? A great deal of well water is almost as hard as that of the ocean. In some sections of the country, where the well water is hard, cisterns are often used to collect and conserve rain water for household uses. Hard water, however, can be softened by heat or by the addition of such chemical substances as carbonate of soda (sal soda), caustic soda, borax, and ammonia. Many washing powders are mixtures of soap and soda or caustic soda, and such mixtures both soften the water and combine with grease and dirt, facilitating their removal. From a survey of the numerous washing compounds on the market and of what they are advertised to accomplish, it would appear that the warfare against dust is of vital concern to every one. That dust, moreover, is somehow dangerous to health and life may be inferred from the large number of articles on the market for cleansing our bodies and for the removal of dust from the more delicate membranes of nose, mouth, and throat.

Materials in dust. - It is astonishing. to note the various kinds of materials contained in dust. If one should select a limited area of the street and list the materials in process of becoming dust, one would discover enough to disturb the usual complacent attitude toward dust and dirt. There are fragments of hair, cells from skins and bodies of animals and men, soot, bits of cloth, wood, paper, stone, steel and other metals, tobacco, filth of various sorts, sputum, - all being mixed and ground into a more or less impalpable powder to be diffused through the air with every breeze. This should be supplemented with the fact that many a cigar fragment and the sputum and droplets of moisture in speaking, coughing, and sneezing may come from persons who are victims of infectious disease.

272. What dust may carry. - It is a well-established fact that microbes of diphtheria, measles, whooping cough, influenza, pneumonia, tuberculosis, and epidemic meningitis are expelled in just this manner. These may float about in 
the air for hours, and finally become attached to dust particles with which they are distributed. They invade nose, mouth, throat, and perhaps reach the lungs. Experiments have proved that tubercle germs expelled by a consumptive are sometimes thrown two yards distant and remain in the air several hours. Disease germs such as these when attached to dust particles may live and be virulent for months. These facts suggest the need of knowing what invisible living things may be mingled and distributed with dust, and what may be their significance in relation to man.

\section{Molds}

273. How to discover what dust carries. - It is comparatively simple to devise means for detecting some of the living things that are distributed broadcast with the dust in the air. The following experiments are to be considered primarily as suggestive; others may be devised by the student either to supplement them or to be used as substitutes.

Exercise: Growing molds. - Boil until thoroughly cooked several medium-sized potatoes. Sterilize several glass tumblers and saucers by dry heat in an oven with a temperature of approximately $150^{\circ} \mathrm{C}$. for an hour, or in water brought to boil and kept boiling for several minutes. When the potatoes are cool, eut into two equal sections with a knife sterilized in a flame or in boiling water. Place one section on a saucer and cover immediately with a sterilized glass. Expose the other sections to the air for ten to fifteen minutes, and then seal under the sterilized glasses. Label the former No. 1, and the latter No. 2. Take some dust from the sweepings of a room and put upon the cut surface of another section, and label this No. 3. Expose a fourth section to the air when a class is moving, or in a crowded assembly and label this No. 4. See that glasses fit closely on saucers and prevent evaporation of moisture from their contents.

Place the "cultures," as these are called, in a dark part of the room. The temperature should be kept at about $75^{\circ} \mathrm{F}$. to $80^{\circ} \mathrm{F}$., to insure quick results. Perhaps another part of the building may offer better advantages than the room in which experiments are made. When the cultures have been properly put where they are to remain, a small quantity of lukewarm water that has been boiled may be poured into each saucer. 
Make full and accurate record of preparations of each culture, the kind and duration of exposure or manner of treatment. No. 1, which was not exposed, is to serve as a "control," and will remain practically unchanged unless it has been carelessly exposed to infection. Observe your cultures at intervals of a day or two. When they present changes in appearance, as size, color, or other features, make sketches and descriptive records of such.

In a similar way other materials may be prepared for mold cultures, such as small pieces of lemon, apple, cake, bread, cheese, banana, etc., and each kept warm and moist under a glass tumbler.

274. Molds. - In a few days you will find several small masses of whitish thread-like or fibrous material growing on the

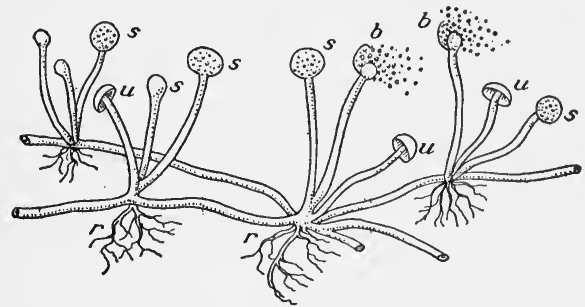

Fig. 164. - Method of growth and reproduction of black mold; $r$, root-like absorbing branches; $s$, sporangia; $b$, sporangia bursting and scattering spores; $u$, umbrella shape of dried sporangia after seed dispersal. of the colors by touching each gently with a sterilized toothpick and by drawing this over a freshly cut surface of boiled potato. Label each specimen, naming color transplanted, and treat all as you did the first cultures. When they grow and show colors, compare with the originals.

Propagation of molds. - The color is the sign of fruition of the plants. Spores (Fig. 165) are being produced, which are a kind of seed and secure reproduction. With the aid of a magnifying glass, examine the thread-like stems bearing spores and the spore-capsules called sporangia, or spore clusters. Examine spores when being dispersed or coming from the burst sporangium. Observe how light the spores are, flying in a 
cloud-like dust when disturbed. Disturb the mold in one of your cultures in a single ray of sunlight admitted into a darkened room. The number of spores thus scattered is practically countless. The spores float easily in gently moving air and are widely distributed. As seeds they grow wherever they find suitable conditions, and produce more spores.

In specimen No. 1, you will probably find no sign of growing life or mold. This may lead to the conclusion that where spores have not been planted, mold will not appear. If your control is in good condition, expose it to the air near the floor of a room when people are moving about, or inoculate it with the spores from another culture. If the latter, note the color of the spores. Observe as directed for the others.

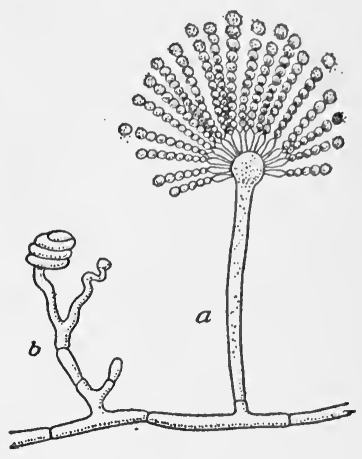

Fig. 165. - Development of spores in brown and graygreen molds; $a$, ripe branch ready to scatter spores; $b, a$ young branch.

Molds are small thread-like plants easily visible to the unaided eye. They are included in a larger group of plants, called fungi. The fungi include several other groups of plants, such as mushrooms, toadstools, the various smuts and rusts, and the groups of much smaller plants called yeasts. The fungi as a class or group are included in the large general group Plants. Closely related to the fungi or spore-producing plants is a group of plants called bacteria. Bacteria are microscopic in size, reproducing generally by simple division or fission. The two groups, fungi and bacteria, are not, however, sharply distinguished, and the terms are often used interchangeably in discussion. The border species between the two groups are not clearly differentiated and may easily be assigned to one class or the other.

275. Varieties of fungi. - There are many varieties of fungi, which are generally distinguished by differences in size and color. Some may be as large as the puffball or giant toadstool, 
and others may be so minute that they can be seen only with the aid of a microscope. In color they vary widely. They may be blue, black or brown, pink, red, yellow or green, though the green is not like that of ordinary green plants.

Classification on the basis of food source. - All fungi are alike in respect to the source of the food material. They require the same chemical substances for food that ordinary green plants use and derive directly from the air and the soil. But the fungi can utilize only such materials as are already organized. The fungi are thus dependent upon organic matter for their food supply. They have therefore very few of the features of green plants and differ from them fundamentally in their products. Green plants in their nutrition and growth are peculiarly constructive or creative of organic compounds such as starch, sugar, and proteins. But the fungi in general and the molds in particular are destructive, bringing about decomposition and decay.

Saprophytes. - The fungi derive the materials they need for food either from organic substances that were once alive and are now decaying, or from living plants and animals. Those that derive their food from dead or decaying organic matter are called saprophytes. Common examples of such are abundant, as the slime molds on damp rotting branches, trunks, and leaves of trees, the toadstools, and the molds often found on bread, fruit, and vegetables, and on milk and cheese.

Some of the common saprophytic molds are important just because of the work they do in initiating the decay and destruction of dead organic materials, as trees, leaves, vegetation, and animal matter. They aid in restoring to the soil substances which are necessary to the growth of living things, and at the same time in ridding the earth of vast amounts of useless matter.

Certain saprophytic molds are utilized in the production of certain foods and drinks. Some species convert sugar properly aërated and warmed into citric acid. Other molds together with bacteria are extensively employed in the manufacture of 
cheese. Stilton cheese, which is an English product, is ripened by the action of molds, and the characteristic flavor of Roquefort is due to the activities of molds. Camembert cheese depends for its ripening and flavor upon at least two kinds of molds; the one species reducing the acidity and softening the curd, and the other developing the characteristic flavor of the cheese. One of these molds is the Oidium lactis (Fig. 166), which is found generally in milk and cheese, where it lives on the lactic acid produced by bacteria, thus making it possible for

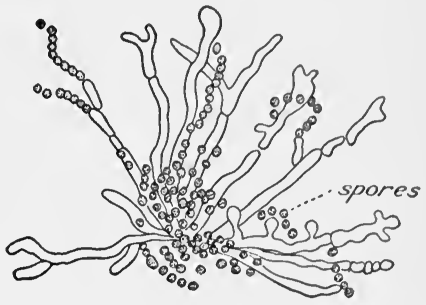

Fig. 166. - The mold Oidium lactis consumes the lactic acid formed in souring milk, in checse, and in other milk products. This figure shows how the mold branches and produces spores by which it is propagated. these and other useful bacteria to continue their growth and work (pages 584-5). The other mold is the Penicillium (Fig. 167), of which there are many widely distributed varieties. Each

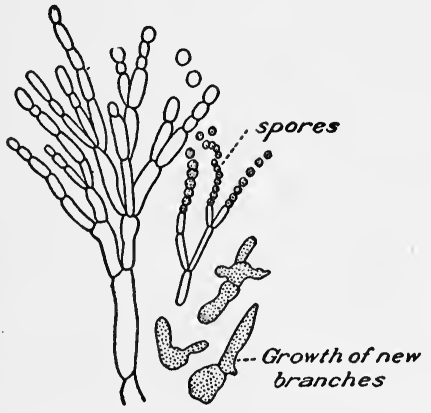

Fig. 167. - The Penicillium camembertii, the mold that produces the characteristic flavor of Camembert cheese; a common species of the Penicillium genus. species produces its characteristic result; one gives to Roquefort cheese its bitter flavor, and others give different results in different media, as in Brie, Gorgonzola, Limburger, and other kinds of soft cheese. In ripening cheese the molds grow on the surface, but their products, which are the digestive fluids or enzymes (page 518), penetrate and gradually convert the hard curd into a soft smooth mass, and develop its peculiar flavor. A cheese is ripe when the enzymes have changed the entire mass. Unripened cheese is almost indigestible and flavorless. Ripe or mature cheese is a very digestible food and 
richly flavored in contrast with the usual hard cheddar or socalled American cheese.

Molds of certain kinds modify food materials in several disagreeable ways, as in appearance, taste, odor, and in the beginnings of disintegration, which is a forerunner of decay. It is not difficult to find in any place where foods are kept molds that are responsible for these results.

Parasites. - The fungi or molds that get their food from living organisms, either plant or animal, are parasites. The organism upon which they live is called the host. The fungi may be the active cause of disease, or they may enter the body after a disease has begun, and aggravate it to a dangerous degree.

The disease-causing molds or fungi that infect man and other animals are usually restricted to limited areas or organs, such as the ear, nose, mouth and throat passages, the lungs, and the skin. A common disease known as ringworm, or in a more severe form sometimes called barber's itch, is caused by a particular species of mold. This mold attacks men and many domestic animals, such as the horse, dog, and cat, which spread the infection. It is restricted to the harder, drier parts of the skin, upon which it may form scabs and from which the hair falls out. The disease, while not serious or affecting the general health, is irritating and difficult to heal. It is easily communicated through contact or by the use of articles that have been handled by an infected person. A fungus that is indirectly of some use to man is called the fly fungus, which in the latter part of summer causes an epidemic among flies.

Molds that cause diseases of plants and plant products can be mentioned here only in the briefest way. Molds in general initiate the decay of all fruit and vegetables. They also start the wet-rot and dry-rot of potatoes and cause potato scab. The black rot, brown rot, and bitter rot of fruits, the smuts and rusts of grains, such as oats, wheat, and corn, the mildews on grapes and other plants, are all of them due to parasitic 
molds of different varieties. A certain species infests the roots of many vegetables, such as cabbage and turnip, causing the familiar disease known as clubroot, in which the root is distorted and swollen in a peculiar way.

276. Distribution. - Molds are adapted through their spores to universal distribution by moving air, dust, water, or any object on earth. They make any place their habitat, such as factory, store, and home, where they cause trouble and perhaps loss for all concerned. Hardly any place in the home is exempt. Evidence of their presence is sometimes found in basement and attic, closets, pantries, and refrigerators. One may easily discover whether or not mold spores are present in any place by exposing for a while pieces of moistened bread, fruit, meat, or cheese. Even damp cloth, leather, and wood furnish nutriment for molds. Mildew is a mold which grows on any of these materials that are sufficiently moist and warm.

277. Their vitality. - Molds and spores are also capable of resisting successfully many extreme conditions in nature. The spores are very dry, and remain uninjured by prolonged drought, or excessive moisture. They are not impaired when subjected to such extremes of temperature as occur in nature. Indeed, spores withstand a temperature that approaches closely the boiling point of water. It is usually supposed that they are destroyed by being boiled in water. Spores have been cooled to extremely low temperatures, just a few degrees above absolute zero $\left(-459^{\circ} \mathrm{F}\right.$., $-273^{\circ} \mathrm{C}$.), and suffered no injury. Thus molds through their spores are adapted to survive and retain their vitality indefinitely in practically every combination of conditions in the natural environment.

278. Their rate of growth. - Molds vary in their rate of growth and fruition. Some grow very rapidly, completing their life cycle within twenty-four hours. Though common experience is often a good witness to the speed with which mold spores germinate and secure strong foothold in food materials, you may verify this by experiment and observation. 
richly flavored in contrast with the usual hard cheddar or socalled American cheese.

Molds of certain kinds modify food materials in several disagreeable ways, as in appearance, taste, odor, and in the beginnings of disintegration, which is a forerunner of decay. It is not difficult to find in any place where foods are kept molds that are responsible for these results.

Parasites. - The fungi or molds that get their food from living organisms, either plant or animal, are parasites. The organism upon which they live is called the host. The fungi may be the active cause of disease, or they may enter the body after a disease has begun, and aggravate it to a dangerous degree.

The disease-causing molds or fungi that infect man and other animals are usually restricted to limited areas or organs, such as the ear, nose, mouth and throat passages, the lungs, and the skin. A common disease known as ringworm, or in a more severe form sometimes called barber's itch, is caused by a particular species of mold. This mold attacks men and many domestic animals, such as the horse, dog, and cat, which spread the infection. It is restricted to the harder, drier parts of the skin, upon which it may form scabs and from which the hair falls out. The disease, while not serious or affecting the general health, is irritating and difficult to heal. It is easily communicated through contact or by the use of articles that have been handled by an infected person. A fungus that is indirectly of some use to man is called the fly fungus, which in the latter part of summer causes an epidemic among flies.

Molds that cause diseases of plants and plant products can be mentioned here only in the briefest way. Molds in general initiate the decay of all fruit and vegetables. They also start the wet-rot and dry-rot of potatoes and cause potato scab. The black rot, brown rot, and bitter rot of fruits, the smuts and rusts of grains, such as oats, wheat, and corn, the mildews on grapes and other plants, are all of them due to parasitic 
molds of different varieties. A certain species infests the roots of many vegetables, such as cabbage and turnip, causing the familiar disease known as clubroot, in which the root is distorted and swollen in a peculiar way.

276. Distribution. - Molds are adapted through their spores to universal distribution by moving air, dust, water, or any object on earth. They make any place their habitat, such as factory, store, and home, where they cause trouble and perhaps loss for all concerned. Hardly any place in the home is exempt. Evidence of their presence is sometimes found in basement and attic, closets, pantries, and refrigerators. One may easily discover whether or not mold spores are present in any place by exposing for a while pieces of moistened bread, fruit, meat, or cheese. Even damp cloth, leather, and wood furnish nutriment for molds. Mildew is a mold which grows on any of these materials that are sufficiently moist and warm.

277. Their vitality. - Molds and spores are also capable of resisting successfully many extreme conditions in nature. The spores are very dry, and remain uninjured by prolonged drought, or excessive moisture. They are not impaired when subjected to such extremes of temperature as occur in nature. Indeed, spores withstand a temperature that approaches closely the boiling point of water. It is usually supposed that they are destroyed by being boiled in water. Spores have been cooled to extremely low temperatures, just a few degrees above absolute zero $\left(-459^{\circ} \mathrm{F}\right.$., $-273^{\circ} \mathrm{C}$.), and suffered no injury. Thus molds through their spores are adapted to survive and retain their vitality indefinitely in practically every combination of conditions in the natural environment.

278. Their rate of growth. - Molds vary in their rate of growth and fruition. Some grow very rapidly, completing their life cycle within twenty-four hours. Though common experience is often a good witness to the speed with which mold spores germinate and secure strong foothold in food materials, you may verify this by experiment and observation. 
Every housekeeper recognizes the need of unceasing vigilance in guarding against molds in foods and food containers, as pantry, bread boxes, and refrigerator, and is well aware of the promptness with which molds begin to work their undesirable results.

Favorable conditions. - Prevention of molds depends upon knowledge and control of the essential external conditions in which they thrive. These conditions have been suggested in the directions for experiments for mold cultures, but may be summarized briefly.

The first and most important of the external conditions favorable to the growth of molds and spores when nutritive materials are available is moisture. This must be sufficient not only to dissolve the food materials in a suitable concentrated solution, but also and especially to keep the cells of the plants full, firm, and turgid. Molds may rest when the moisture is inadequate to growth.

The second condition is temperature, in adaptation to which molds show remarkable variation. Some species of mold grow slowly, but fully, at a temperature as low as $35^{\circ} \mathrm{F}$. Such molds may sometimes be found in refrigerators. Other undesirable molds flourish best at a temperature ranging from $70^{\circ} \mathrm{F}$. to $100^{\circ} \mathrm{F}$.

The third condition is light, which is vitally important to chlorophyll-bearing plants, and is also important, but in different degree, to molds and fungi generally. Many species of mold can live and grow in bright sunlight, but most do better in darkness, or where the light is subdued. The more common and troublesome species require darkness, partly because this may allow a very slow loss of moisture through evaporation, but chiefly because light is injurious to them and restrictive of their growth. A few species of mold exhibit a remarkable habit of developing all parts of their structure in the darkness, except the reproductive parts, which are formed only in light. But sunlight is generally destructive of molds. 
Air is the fourth necessary condition. Molds require air, as may be seen by their growth only where air is available. But moving air is unfavorable, not only disturbing the molds, but hastening the evaporation of moisture. Air that is stagnant or static, as in food containers, such as closed dishes, is by far the most favorable.

\section{SUMMARY}

Nitrogen and oxygen are the chief constituents of the atmosphere. Small amounts of other gases are found, also water vapor and dust.

The constant accumulation of dust necessitates unceasing vigilance to prevent its becoming a source of discomfort and possible menace to health.

Many devices are employed in the warfare against dust, but water is the universal and most effective cleanser.

Hard water, which is not as useful as soft, may be softened by heat or by the addition of chemicals, such as washing soda.

Street dust is made up of many different materials and may carry disease germs which endanger health.

Molds belong to the group of fungi, plants which derive their food materials from other plants or animals.

Fungi and molds differ from the green plants in being destructive, promoting decomposition and decay.

Saprophytes are plants that get their food from organic matter that is dead or decaying.

Parasites are such plants (or animals) as derive their food from living plants or animals.

Some of the saprophytic molds are useful in promoting the decay of organic matter.

Many molds are industrially useful, as in the production of certain foods and beverages.

Certain parasitic molds cause diseases of plants, men, and other animals.

Molds and their spores resist extremely high and low temperatures, as well as moisture and drought.

Conditions favorable to growth of molds are moisture, warmth, dim light, and still air.

\section{REVIEW QUESTIONS}

1. What are the four principal constituents of the air? 2. What other substances are present in small amounts? 3. How does the dust of the atmosphere affect our health? Our homes? Our clothing? 
4. What measures are taken to get rid of dust? 5. What are the advantages and disadvantages of oiled floors? 6. How does water vary in usefulness? 7. How can hard water be softened? 8. Of what materials is street dust made up? 9. How may disease germs be carried by dust?

10. What is a "culture"? 11. Describe the preparation of cultures. 12. What is the object of boiling the potatoes used for cultures and sterilizing the containers? 13. What conditions are necessary for the growth of molds? 14. What is meant by a control culture? 15. What colors may molds have? What does the color indicate?

16. Describe how molds reproduce. 17. Will molds start to grow where no spores are planted? 18. To what group of plants do molds belong? 19. To what other plants are they related?

20. Compare fungi and green plants as to the source of their food materials; the character of their work. 21. What are saprophytes? 22. How are saprophytic molds useful in nature? 23. How are they useful in industries? 24. Explain the changes in cheese effected by molds. 25. What undesirable effects do molds produce in foods? 26. How are parasitic molds distinguished from saprophytes? 27. What is a host? 28. What is the relation of certain fungi to human health? To the health of the housefly? To the health of plants? 29. Account for the universal distribution of molds. 30. What natural conditions are molds fitted to survive? 31. Of what use is the knowledge of their rate of growth? 32. Summarize the conditions favoring the growth of molds. 33. How may knowledge of these conditions be used in preventing their growth or destroying the spores?

\section{YeAstS}

279. Other plants distributed in the air. - Molds comprise only a small part of the known list of invisible plants that are present in and distributed by the atmosphere. The conditions that are especially favorable to molds, like those secured in your experiments, are not equally favorable to all kinds of in-" visible plants or their spores, or at least do not make their products visible. Consequently we must provide different and suitable conditions just as a gardener provides different kinds of soil for various plants in the garden. One of the simplest ways of cultivating certain other kinds of air-dis- 
tributed plants and of making their products visible is described in the following experiment, which is just the familiar way of making "salt-rising" bread. This is an old practice in different countries, and from it have been developed various methods of making leavened or raised bread.

Exercise. - Heat about half a cup of water to a temperature of $90^{\circ} \mathrm{F}$. In this dissolve one teaspoonful of salt and stir in enough flour, which has been warmed to about the same temperature, to make a thick, smooth batter. (The salt merely prevents the mixture from becoming sour.) Cover the mixture with a piece of glass or other close-fitting covering, and place the vessel containing the batter in a dish of warm water, which should be kept at the same temperature for several hours. Observe it at intervals, but do not let it be chilled by cold air. Note change in volume. When the batter is very light, examine under a magnifying glass a small lump which has been carefully removed. If a high-power microseope is available, mix a small portion of the light dough with some lukewarm water in a test tube by shaking until the mixture is smooth. Examine a drop on a glass under the microscope. Make a record, with sketches, of what you see.

280. Making bread. - If bread making is done at home, carefully observe the preparation for the next baking. Make a record of the quantities of materials used, method of mixing them, and care of the mixture overnight. You may find that the sponge is a success only if certain conditions are provided, such as a temperature not below $75^{\circ} \mathrm{F}$. and sufficient moisture.

The next morning or several hours after its preparation, the sponge is several times larger than its original volume, is full of pores, and has a peculiar odor. The pores and what they signify will be considered a little later. Examine a small portion of the sponge with a magnifying glass. How does it compare with the result of your examination of the "salt-rising" sponge? Make a microscopic examination of this as you did of the salt-rising.

The remaining steps in making bread are interesting. The sponge is stiffened with flour added gradually until the whole mass may be poured upon the kneading board. It is then 
kneaded with firm pressure for about fifteen minutes, in order to mix thoroughly the ingredients, until it becomes elastic and blisters of gas form near the surface. The dough is put into a covered bowl and kept at a constant temperature not lower than $75^{\circ} \mathrm{F}$., until it rises to two and one half times its original volume. It is again kneaded lightly, with just enough flour for handling, in order to break up the large bubbles of gas and make the dough of fine texture. It is shaped into loaves, which are allowed to rise to twice their volume before being placed in the oven. The baking, which requires about one hour at a moderate temperature, stops further change in the dough. The heat changes the starch on the surface of the loaf to a brown sweetish substance, called dextrine, which forms the crust.

The rate at which sponge and dough rise depends upon the amount and kind of yeast, the temperature, and the moisture. These conditions are carefully controlled in the modern bakery. The bakery methods generally are such as to bring about a rapid rate of rising, whiteness and fineness of texture of bread with less frequent kneading. All these results are obtained by using a large amount of relatively pure yeast, with sugar and the right degree of temperature and moisture.

Bakery bread is usually whiter and finer in texture than the homemade product, but it dries out quickly and has at no time the flavor and keeping qualities of bread made in the slower way. Sometimes certain materials are used to whiten and refine the bakery bread; these do not as a rule add to the food value and may decrease it, and are therefore undesirable.

In many parts of this country and in Europe it has long been a practice to keep a small lump of dough from one baking for leavening the next sponge. The bread produced is slightly. different from that made with the usual yeast cake. It has a richer flavor and other desirable qualities. But the dough develops an excessive amount of acid because of a certain kind of fermentation which occurs.

Why dough rises. - We have now to inquire into the causes 
of the rising of the sponge and dough. In the experiment you found that a mere mixture of flour and water, when kept at a fairly constant temperature, acted just as the sponge to which yeast was added which you observed at home. What eauses the batter to rise? Or what causes the sponge, to which a small cake of yeast is added, to rise? Is it just a physical or chemical change brought about by the combination of the different substances, such as the milk, potato, flour, moisture, and salt, and by keeping the mixture at a certain temperature? Or do these conditions make possible a different kind of change, which is not merely physical or chemical, but a living process also? In order to observe just what takes place and what causes the changes, it will be necessary to make use of experiments such as the following.

Exercise. - Prepare a simple yeast mixture by dissolving a small cake of compressed or dried yeast in a pint of warm water and adding one ounce of molasses or an equivalent amount of sugar. Mix these thoroughly in a large, wide-mouth bottle and place it where the temperature will not fall below $75^{\circ} \mathrm{F}$. In the course of an hour or two bubbles will be formed. Taste the mixture at the beginning and then at intervals of an hour for several hours. When it is working well, close the bottle with a stopper through which you have inserted a glass tube. Attach to the tube a piece of rubber tubing and run it into a test tube half full of limewater. What does the change in the limewater indicate as to the kind of gas produced by the yeast? (See page 124.)

At the same time you prepare the yeast mixture, arrange the following for later observation and use. Prepare three test tubes by thoroughly cleansing and sterilizing them. Sterilize also one rubber stopper. Label the tubes. Fill one about two thirds full of fresh cider or grape juice, and close loosely with a clean cotton stopper. Fill the others with about the same quantity and heat directly over a flame or indirectly in boiling water to a temperature of $176^{\circ} \mathrm{F}$. to $180^{\circ}$ F. Close one at once with the sterilized stopper. Let the other remain open. Put them all where they may be observed easily. Keep a record of the procedure and of the facts observed in the behavior of each, until other tests are made. What gas is being produced, as shown by the bubbles rising from the bottom? 
After the yeast mixture has stood for two days, you may test it by tasting and smelling it. Record the results and compare them with the results of similar tests made at the beginning. For the next step you need a flask equipped as described on page 305. Pour a quantity of the yeast mixture into the flask, and heat, collecting and condensing the vapor in a clean bottle. Keep delivery tube cool with wet cloth. When you have three or four ounces of condensed vapor, redistill it until you have about one eighth of the first quantity of the distilled liquor. This latter distillation should be done slowly and very carefully. Examine the product by tasting and smelling. Try burning a small quantity of it in a spoon. Does it produce a flame? What flame do you know which this resembles?

The observed facts indicate several changes in the mixture. The sweetened substance produced after a short time a gas which is shown by the test to be carbon dioxid. The process continued until the sugary substance became an alcoholic mixture from which alcohol is distilled, as is shown by the tests. The process is known as alcoholic fermentation. It might be continued longer with different results, as later experiments will show.

In making bread the process of alcoholic fermentation is started promptly by adding some sugar or molasses to the yeast mixture. The result is that carbon dioxid and alcohol are quickly produced and the dough is made porous and light. The fermentation is stopped by baking, which also causes the gas to expand and be driven out with the alcohol, producing a bread that is light and digestible.

It is now evident that the sponge or dough rises because of the carbon dioxid gas which is produced in the fermentation. As the dough imprisons the gas, it is filled with small cavities full of gas and thus made to increase in volume, or rise. There is, however, another question which demands an änswer. What is it that produces the carbon dioxid and alcohol? Are these products the result of merely chemical changes in the dough or of changes of a different nature? We have carried the problem along to a new difficulty which must now be at- 
tacked. Possibly the solutions in the test tubes may help in solving the problem.

Exercise. - Examine the test tubes, tasting and smelling the contents of each and recording the results. Is the preparation which was not heated, sour or acid? Let it remain after testing, as directed, to see whether or not it will eventually become vinegar. When bubbles are rising freely, take a few drops with a medicine dropper from near the bottom of the tube, and put them on a glass slide for examination with the microscope. Examine them carefully, record, and make sketches of what you observe.

281. The yeast plant. - Examine in the same way a few drops taken from the yeast mixture and compare the two.

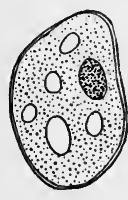

A

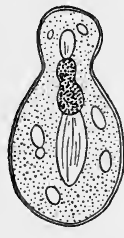

$B$

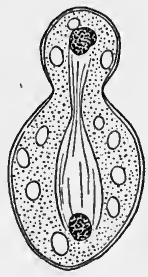

C
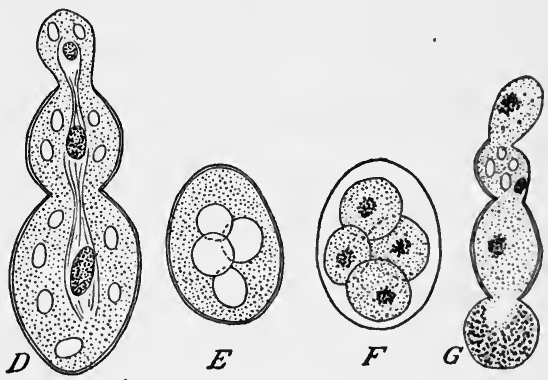

Fig. 168. - Yeast Cell. $A$, a typical yeast cell with nucleus and several vacuoles which are filled with cell sap. $B$ and $C$, stages in the process of budding, the nucleus dividing in the formation of the new cell. $D$, a bud still attached to parent plant, producing a new bud. $E$ and $F$, formation of spores, the nucleus divided into four, each new nucleus acquiring a cell wall and becoming a spore, the parent cell wall serving as a sac. $G$, a spore developing into a yeast cell which buds and forms a colony.

What do the results indicate as to the changes which are taking place in each? Do you think the same process is occurring in both? If the microscope is high power enough, it will make clearly visible the minute bodies which produce the changes in the liquids and the yeast mixture. The small bodies which the microscope reveals are egg-shaped or oval, almost transparent, and they may be separate or joined in chains or treelike forms. They may be like the forms shown in Fig. 168. 
These small bodies are yeast plants, microscopic organisms that live and thrive in such conditions as have been provided.

Growth and reproduction. - The living, growing yeast plant is a single-cell organism, with a nucleus or "knot" near one end, and the whole enveloped in a cell wall of cellulose. As the cell grows, it develops within it one or more small drops of an oily substance, or "cell sap"; these drops are called vacuoles. The cells also begin to multiply by a process known as budding. The first appearance of a new cell is as a bud on the side of the parent plant, where it increases until it is fully grown. It may remain thus attached in a time of rapid growth or it may separate. Either the parent cell or the new cell may produce a new bud, and this process continues with the plants appearing in oddly formed groups as long as the nutritive conditions are favorable. If the conditions become unfavorable, the plants separate and become inactive. When favorable conditions are restored, the plants resume active growth and multiplication. The inactivity of the yeast may be due to a lack of nutritive material or to toxic (poisonous) waste products, which the plants themselves have thrown off. But an interesting fact is that just when this condition is brought about, another variety of plant begins to develop and produce fermentation of a different character. The dead yeast plants may be distinguished easily from the active and resting plants by their opaqueness.

Size of yeast plants. - Yeast plants are much smaller than molds, so minute, in fact, that a hundred or more placed side by side would hardly equal the thickness of the paper upon which this is printed. The size varies in different conditions, such as the nature of the food solution and the temperature. But the size is not an important item in identifying yeasts, molds, or bacteria. A compressed yeast cake is made up of yeast plants, perhaps several million, and may have a small percentage of starch to dry and preserve it. The yeast is alive, but inactive, as it cannot grow under such conditions. 
Exercise. - Examine now the liquid that was heated and kept unexposed to the air. Do you find similar bodies in it? The boiling would destroy any living organisms in the fresh fruit juice, and the stopper would prevent others from entering.

What do you find in the other liquid which was heated and left exposed to the air? Are the yeast plants evident in any way? From what source can they come? Fermentation in this tube indicates that the yeast plants entered it from the air. The simple mixture of flour, salt, and water developed fermentation which seems to have been due to the work of yeast plants. In this case the yeast plants may have come from the air or may have been in the flour and water.

Distribution of yeast. - How difficult it is to keep the microscopic plants out of fruit and other preparations may be inferred from a few results of investigation. Samples of apple ciders made in the usual way showed from 50,000 to $30,000,000$ yeast plants or spores per cubic centimeter. A cubic centimeter of apple butter contained nearly $10,000,000$, and an equal quantity of currant jam had the moderate number of $40,000,000$. Fortunately most varieties of the yeast plant are harmless; but a few cause disease or serious intestinal troubles.

The facts indicate that yeast is widely and abundantly distributed. How is this done? Do the yeast plants themselves get carried about or do they, like molds, produce "seed" or spores which are transported by the moving air? If it were practicable to carry on a series of experiments with the yeast plants, putting them into different kinds of conditions, it would be found that yeast plants in certain unfavorable conditions, as when deprived of food, do produce spores. The little plants divide into two or more minute bodies which eventually break through the cell wall of the yeast and escape as spores. Spores are exceedingly light and small, and are carried like impalpable dust far and wide in a gently moving air. The spores successfully resist unusual extremes of heat, cold, and dryness. They are found practically everywhere, as every case of fermentation of any sort indicates. They are in the air, in water, in the soil, and are attached to all kinds of 
plants and especially their fruits and grains. Whenever they find suitable food, moisture, and temperature, they begin immediately to develop, bud, and produce fermentation.

Wild and cultivated yeasts. - It is customary to classify yeasts of the harmless and beneficial kinds as wild and cultivated. The wild yeasts are such as were captured and cultivated in your flour and water mixture and in the fruit juices. The cultivated yeasts are represented by those in the commercial yeast cake, either the dry or the compressed, and by others mentioned later as the top and the bottom yeasts.

Industrial uses of yeast. - Because of their economic importance yeasts have been thoroughly studied and are utilized in various ways. Bread making is practically world wide and supports a great industry of producing and marketing yeast in different forms, such as the compressed cake, the dried cake, or the liquid form. Such industries as manufacturing vinegar, as brewing and distilling make use of different kinds of yeasts and bacteria which are associated with them; and they have through scientific study acquired control of the different kinds of yeasts and their products. In brewing, for example, there are two kinds of yeast used, one being called top yeast, and the other bottom yeast. The top yeast grows in fermentation on the surface of the brew, while the bottom yeast develops at the bottom of the brew. In their action upon sugary solutions each of these evolves very different and characteristic products. The yeasts employed in the production of distilled liquors are of another kind, producing great fermentation with the evolution of large amounts of alcohol. Yeasts differ greatly in different regions, as may be especially noted in connection with the grape-growing and wine-making districts. In Europe. and in America special local varieties of yeasts are cultivated and employed in making wine, and these give the distinctive bouquet and qualities of famous vintages. In this country several varieties of yeast are now available which are utilized in developing distinctive and delicate flavors and qualities in cider. 
Still different varieties are used in the fermentation of milk. One which was originally cultivated by a Russian tribe dwelling by the river Kuma has come into wide use in preparing the fermented milk known as Kumiss. Another variety produces the well-known Bulgarian milk beverage, called Matzoon, which has become popular largely because of the theory of Metchnikoff as to its value in prolonging life. These are only a few of the many varieties discovered by modern scientific investigation and made useful to man.

282. What occurs in rising bread. - We may now resume the study of the process which takes place in rising bread or sponge. This is already recognized as complex, involving several different varieties of yeast and bacterial action. The yeast plants begin to develop immediately in the right conditions, utilizing the available sugar which they decompose. Flour contains a small amount of sugar besides the large amount of starch. The sugar, which is a carbohydrate composed of carbon, hydrogen, and oxygen (pages 129,515 ), is converted by the yeast plants into carbon dioxid and alcohol. Alcohol also is composed of carbon, hydrogen, and oxygen. The carbon dioxid is imprisoned by the dough which is made to swell or rise by the multiplying and expanding bubbles of gas. The alcohol combines with the water in the dough and is evaporated in the baking. While the alcoholic fermentation is going on, another kind of ferment called diastase (page 518), which is contained in the flour, becomes active and changes part of the starch into a form of sugar. If the process of rising is of the slower kind and made with a relatively impure yeast, as in the homemade bread, it allows certain bacteria to develop which act upon the nitrogenous substances, such as gluten, and produce in the bread a peculiarly fine flavor.

If a dough is allowed to stand too long or is kept at too high a temperature, it becomes more or less sour or acid. This change is effected by other kinds of bacteria. These begin to grow and multiply rapidly in such conditions and to produce 
acid of one kind or another. The acidity of the bread may be due to lactic or to acetic acid or to a combination of the two. You may identify the lactic acid by means of the acid in sour milk, for milk is made sour by bacteria which produce lactic acid. Acetic acid is that which is found in vinegar. It is produced by yeast and a species of bacteria called acetic bacteria. Yeasts cause fermentation, in which are evolved carbon dioxid and alcohol. Then the acetic bacteria, which can act only with great difficulty upon sugar, begin to work rapidly on the alcohol, which they oxidize to form acetic acid.

It is comparatively easy to prevent the dough from becoming acid when one knows the causes which produce it. A poor grade of flour usually contains more kinds and more bacteria than a high grade of flour. With a strictly high-grade flour and a good, perfectly fresh yeast, and all materials mixed and kneaded in clean utensils with clean hands, the primary conditions for a sweet bread are provided. The dough should be kept at a temperature of not lower than $75^{\circ} \mathrm{F}$. in warm weather and between $85^{\circ}$ and $90^{\circ} \mathrm{F}$. in cold weather. This will ordinarily insure complete fermentation within six to eight hours. Detrimental bacterial action does not pass beyond control or affect the flavor of bread which is prepared within this period of time.

\section{SUMMARY}

Leavened bread is made, (1) by the "salt rising" method, (2) by the use of dough from a former baking, (3) by prepared yeast.

In each case yeast plants grow and multiply in the dough, producing carbon dioxid and alcohol.

If the sponge is kept warm, yeast develops more rapidly than the bacteria which make the bread sour.

The universal distribution of yeasts is indicated by their appearance in " salt rising" bread and in fruit juices.

Yeast plants reproduce rapidly by budding, and in unfavorable conditions produce spores. In many food products enormous numbers may develop.

Most varieties of yeast are useful and harmless. 
Domestic varieties of yeast are cultivated for specific purposes as bread making and brewing.

Yeast develops best in a dilute sugar or molasses solution. The starch of flour is partly converted into sugar by diastase and so rendered available for the yeast plants.

Starch, sugar, and alcohol contain the same elements (carbon, hydrogen, and oxygen) but in different proportions.

\section{REVIEW QUESTIONS}

1. Describe the experiment with "salt-rising" dough. 2. How is bread made at home? What materials are used, in what quantities, and how mixed? 3. Why is bread kneaded? 4. What does the baking do to yeast action? 5. What change does heat cause in the surface of the loaf? 6. By what conditions can the rising of dough be controlled? 7. What conditions hasten the bread-making process? 8. What qualities are gained by the slower process? 9. What are the sources of yeast for bread making?

10. Describe the experiment with yeast and molasses, and state what products were found. How are they identified? 11. Describe the experiments with fruit juices, and state the results. Give the reasons for the special treatment of each sample.

12. What substances are produced in the rising sponge and dough? What becomes of them? 13. Why does dough rise? 14. Describe the yeast plant and its methods of reproduction. 15. Compare yeast plants and molds as to size and methods of reproduction. 16. How do yeast plants get into food? 17. Give instances of the number of yeast plants in food products. 18. Under what conditions are yeast spores produced? 19. Distinguish between wild and cultivated yeast.

20. Of what industrial importance are yeasts? 21. What yeasts are used in brewing? 22. How is wine made? 23. What are Kumiss and Matzoon? 24. What is the first action of yeast in dough? 25. Upon what does yeast live? 26. What are the products of alcoholic fermentation? 27. What is diastase? What change in flour is made by it? 28. What changes produce the fine flavors of bread made by the slow process? 29. If dough is allowed to stand too long what change occurs? 30 . How and by what is lactic acid produced? 31. How and by what is acetic acid produced? 32. What condition is necessary to produce vinegar? 33. How can souring of bread be prevented? 


\section{BACTERIA}

283. Nature and distribution. - Frequent reference has been made to the action of the microscopic plants, called bacteria, in connection with the putrefaction of sewage, with their growth upon the roots of leguminous plants, with their work in making bread dough sour, and in converting alcoholic solutions into acetic acid or vinegar. These are only a few instances to suggest the extent and abundance of bacterial plant life. Like molds and yeasts, bacteria are found to be universal, for they begin growth wherever conditions are favorable. They are found in the air, soil, water, on fruit and vegetables, and in the bodies; tissues, and circulatory fluids of animals and men. That they are abundant may be inferred from the countless number found in a very small amount of decaying plant or animal matter, and from the fact that putrefaction and decay, which are the work of bacteria, are practically everywhere and at all times. Every household furnishes evidence of their presence, and every housekeeper, while perhaps not aware of the nature of the enemies against which war is waged, devotes thought and time to the preservation of food from their action. To defeat or destroy bacteria is the purpose of most of the labor involved in the refrigerating, cooking, canning, smoking, drying, and salting of food materials.

284. Bacteria and disease. - We are familiar with the statement that microbes, germs, or bacteria cause certain diseases. The disease-causing bacteria have been the special subject of scientific study, with the result that it is now well established that many infectious diseases are due to bacterial activities and products. The species of bacteria which cause disease, and are classed therefore as pathogenic, are comparatively few ; and they are included among the parasites, of which certain fungi or molds have been mentioned (page 554). Of many infectious diseases due to parasitic bacteria the most common are tuberculosis, pneumonia, diphtheria, typhoid fever, 
influenza, grippe, cholera, lockjaw or tetanus, anthrax, whooping cough, and one variety of dysentery, generally known as summer complaint or diarrhœa of infants.

This list of diseases is sufficient to emphasize the necessity of knowing the life history and habits of pathogenic bacteria. This knowledge is necessary in order to secure such control of them as will prevent or cure the diseases caused by them. The need of this knowledge is emphasized also by the wellknown bacterial infection of plants and the lower animals, to which is due an annual loss of several million dollars. What we need to know is briefly: (1) in what conditions bacteria best develop ; (2) how they multiply and at what rate; (3) what are their products and the effects of these products upon the organism in which they are evolved; (4) and how bacteria are transferred from one place to another. The knowledge of these items leads directly to the discovery of the ways and means of making conditions unfavorable for their growth and multiplication, of destroying or neutralizing their products in the human body, and of preventing their transfer from an infected to a healthy individual.

As stated above, only a few species of bacteria are pathogenic, or cause diseases in plants and animals. Very many kinds are included in the group of bacteria causing decay and putrefaction ; but there are many others which are entirely harmless, and still others which are indispensable in various ways. The human body is literally alive with many kinds of bacteria, the skin and hair, the mucous membranes of eyes, nostrils, mouth, throat, and the alimentary canal, having each its peculiar kind of bacteria. In normally healthy individuals pathogenic forms may be found, but these seem for the time powerless to cause disease; only when bodily conditions are favorable do they begin their undesirable activities.

285. Useful bacteria. - The useful varieties of bacteria are many. They are indispensable in agriculture, in brewing, in the manufacture of vinegar, wines, and other liquors, and of 
the finest grades of butter and cheese ; in tanning leather, curing tobacco, making hemp and linen. Under the guidance of scientific knowledge, cultures of pure bacteria are now prepared for the market. These are adapted to several of the purposes above indicated; for example, in agriculture to inoculate such legumes as alfalfa, clover, soy beans, vetches, and the like.

The nitrogen cycle. - The importance of bacteria which are mainly concerned in putrefaction and decay, in taking nitrogen

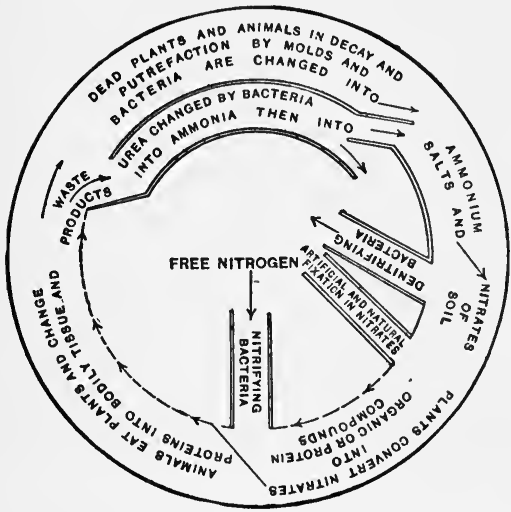

Fig. 169. - The nitrogen cycle. Trace the complete cycle of nitrogen from the air through plant and animal life to the air. Bacterial action is indicated by double lines, plant and animal action by broken lines.

from the air and fixing it in compounds available for green plants, and in other ways converting substances into suitable plant food materials, may be seen in the accompanying diagram (Fig. 169). Nitrogen is one of the chief materials of plant food, but must be furnished in compounds which plants can absorb and assimilate. Such compounds are nitrates. Nitrogen exists in abundance in organic matter, such as wood, straw, manure, and animal bodies; but green plants cannot make use of it in this form. Molds begin the decomposition of organic matter, and bacteria carry on the process, utilizing the organic materials as food and throwing off new compounds, which are directly available as food material of green plants. For example, ammonia is a nitrogen compound, but in this form is not useful for plants. It is taken as a food by a certain species of bacteria, which in using it combine it with oxygen or oxidize it, forming a nitrate. Again the free nitrogen of the air is 
absorbed by another species of bacteria growing in the roots, the nodules, of leguminous plants (Fig. 170), and is "fixed" or combined with other elements in compounds that can be assimilated directly by the plants.

Nitrogen compounds, produced by bacteria (Fig. 169) are absorbed by chlorophyll-bearing plants and changed into organic compounds of which nitrogen is ${ }^{\circ}$ the principal constituent. These compounds are called proteins (page 517). Animals and men live on plants and plant products. The edible parts are changed in the course of nutrition into tissue for the growth and repair of the body, and partly into waste products that are excreted. Of the waste products urea, for example, is converted by a species of bacteria into ammonia, which in turn is oxidized by another species and made into a nitrate. The dead remains of plants and animals are subject to decay, the work of saprophytic molds and bacteria, in which process nitrogen, with other elements, is restored to the soil and started upon a new cycle. A curious fact to be

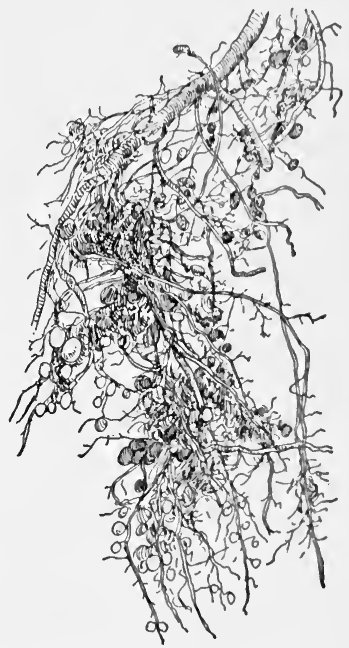

Fig. 170. - A legume root showing nodules formed by nitrogen-fixing baeteria. noted is that nitrogen compounds are in certain conditions attacked and broken up by denitrifying bacteria (page 472). But, on the whole, the cycle of nitrogen in its different combinations indicates the complete interdependence of molds, bacteria, green plants, animals, and men.

286. Classification. - On the basis of the sources of the nutritive materials upon which they depend, bacteria may be roughly grouped into three classes:

(1) The saprophytes, the bacteria which derive their food materials from dead and decaying organic matter; 
(2) The parasites, those that depend upon living organisms, both plant and animal;

(3) The independent bacteria, those that get their food materials from inorganic substances.

To the third class belong the nitrifying bacteria, some of which, attached to leguminous plants, take nitrogen and perhaps carbon dioxid directly from the air; the iron bacteria, one species of which probably produces the deposits of free iron ochre; and the sulfur bacteria, which abound in water containing sulfur.

Bacteria may also be classified as to their independence upon oxygen, as the aërobic and the anaërobic (page 216).

287. Study of bacteria through their effects. - The study of bacteria by direct observation is beset with many difficulties because of their extremely small size. Microscopes of high power are necessary, and stains must often be employed to bring out detailed structures. But a rough general idea may be formed from examination of some of the effects of bacteria in different substances. These give an acquaintance with the principal forms, the rapidity of their multiplication in suitable conditions, and important results of their growth.

Exercise. - The potato cultures prepared for molds, if these have been preserved, may be observed first. You will probably find growths upon the cut surface that are different in outline and color from the molds. Many such growths are white, but there are other sorts of bacteria that produce green, yellow, red, and blue. The outline forms indicate the extent of the colony produced from a single bacterium. Examine them with a magnifying glass. What features do you find generally distinguishing them from molds? How can their presence be accounted for? Why are they so late in development compared with the molds? What does the difference in areas covered by the colonies indicate?

Exercise: A culture medium. - Potato medium as previously prepared will do, but other culture media may be necessary. A simple medium may be made with beef bouillon (such as may be bought in a can) to which is added one per cent of peptone and ten per cent of gelatine to stiffen it. Heat the mixture gradually until the gelatine 
is melted and then boil for several minutes. Test for acidity with litmus paper, and add enough baking soda to neutralize and give an alkaline reaction. Filter while hot through absorbent cotton in a glass funnel two thirds full, into which the mixture is slowly poured ; fill sterilized test tubes about one third full, and close with a cotton plug. The tubes with their contents should be steamed for half an hour or more to insure sterilization. This should be repeated two or three times at intervals of a day.

Exercise: Cultures. - The medium thus prepared may be inoculated in the tubes or melted and poured into watch glasses or Petri dishes for plate cultures. Plate cultures have several advantages, such as separating the individual bacteria and showing to the naked eye the separate colonies when developed. To inoculate, melt the mixture in several tubes and add to each any of the following materials : a cubic centimeter of faucet water, a cubic centimeter of boiled water in which a small piece of putrefying meat has been shaken, a drop of saliva, a hair from the head, a bit of substance gathered on a toothpick from the teeth, or some dust from the floor. Mix the contents of each tube thoroughly and pour into the watch glass; cover immediately. Label each and make a record of the preparations. Fill three more dishes with the mixture, covering them at once. When cool, touch the surface of one with the finger; expose another to the air of the room when the class is moving about; expose another when all is quiet. Place all the dishes where the light is dim and the temperature is likely to remain about $70^{\circ} \mathrm{F}$. (as in the furnace room). Observe at intervals and make a record of growth, number of colonies, color, and general appearance. Put on a glass slide and cover with a thin glass a drop of some stagnant water in which a crumb of bread or cake has been left for several days. Examine it with a high-power microscope. Examine also a drop of some water in which a bit of decaying meat has been shaken.

Forms of bacteria. - The form of the microörganisms which may be observed in these preparations is spherical. This is the simplest type of bacteria, and is exceedingly small. The name given to this group is coccus (cocci, plural), (Fig. 1'71 a). The cocci may be congregated in groups varying in number, size, and shape. The grouping may be due to the manner of reproduction by division, as determined by external conditions of warmth, moisture, and nutritive materials. If the division occurs in one plane, forming a pattern like a string of beads, 
the group is called streptococcus (Fig. $171 b$ ). If reproduction occurs in two planes with such a mass as $(c)$, the group is micrococcus. Other forms of groups as in $(d)$ are produced, depending especially upon the nutritive conditions.

Bacteria of still different forms may be discovered in the microscopic field. Many types assume a cylindrical, or rodlike form (e), which may be either straight or bent. The bacteria found in sour milk are rod-shaped of different lengths. But

0 $\infty$

a

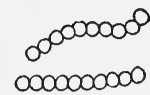

b

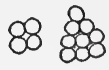

c

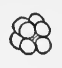

d
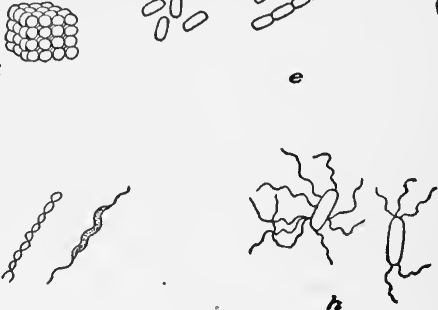
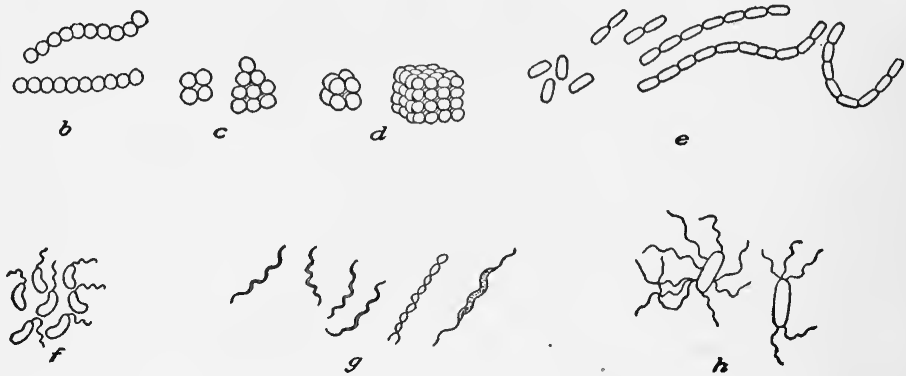

Fig. 171. - Typical forms of bacteria: $a, b, c$, and $d$, typical groups of cocci ; $e$, straight rod-shaped bacteria and ways of grouping; $f$ and $g$, spiral forms, $f$, the vibrio of cholera; $h$, motile forms, of ten called bacilli, equipped with flagella as means of locomotion. Compare these forms of bacteria with the protozoa, Fig. 172.

the extremely short ones are single bacteria, and the longer ones are short rods joined together end to end. One may find a short rod dividing itself into two sections. This is the usual manner of reproduction. Continued observation will reveal the full growth and reproduction of the minute plants, for this may easily occur within an hour.

The rod-shaped bacteria are divided into two classes, the motionless and the motile. The motile has organs of locomotion called flagella, exceedingly fine, hair-like extensions which are curved in various directions. It is impossible to detect the flagella without staining and a very high-power microscope. It is customary to call all motile bacteria bacilli (bacillus, singular). An example is the typhoid bacillus (Fig. $171 h$ ). 
Bacilli move at different rates of speed, some very slowly and others very rapidly. The swiftly moving forms may travel at a rate equivalent to that of an automobile making thirty miles an hour. It should also be noted that a bacterium has about as much free moving space in a drop of water as a fish in a goodsized lake. The manner of locomotion varies with different species, some traveling in straight courses, others following tortuous routes.

The bent rod-shaped bacteria are generally called spirilla, for they assume a more or less spiral form. A very slightly bent form is the vibrio of cholera (Fig. $171 \mathrm{f}$ ), and a very pronounced spiral form $(g)$ is found in the mucus or tartar of the teeth. Both of the cylindrical forms appear separately or grouped in pairs joined end to end, or in long bead-like strings.

Size of bacteria. - Bacteria are the smallest of plants, but probably not the smallest living organisms. Most bacteria are microscopic in size, but a few varieties are ultra-microscopic, revealed only through their effects or products. Bacteria range in size from $\frac{1}{10000}$ of an inch in diameter to $\frac{1}{50000}$ inch.

The usual practice is to speak of their size in terms of the metric system, as $\frac{1}{1000}$ of a millimeter (called one micron or $1 \mu)$. This is equivalent to about $\frac{1}{25000}$ of an inch. The size may be appreciated by a concrete comparison. To equal the thickness of ordinary book paper, it would require three to four thousand bacteria of medium diameter, placed side by side. Some bacteria are much smaller, as the influenza bacillus, which is about $\frac{1}{125000}$ of an inch (.2 micron) and has a lengthwise diameter of $\frac{1}{5000}$ inch (.5 micron).

Reproduction. - The rate and manner of reproduction of bacteria are of practical importance. The usual manner of reproduction is vegetative, or by simple division. Some types also reproduce by spores, as may be determined by the external conditions. Bacteria on the average reach maturity in favorable conditions in about half an hour. As soon as mature, each begins to divide itself into two sections and quickly there are 
two cells, which carry on the same process indefinitely. Thus a single bacterium may produce many millions within twenty-four hours. The weight of the mass which might be produced from one ancestor in seventy-two hours, if uninterrupted, has been estimated at just a little less than 5000 tons. Is it difficult to understand how fruit, vegetables, and meat pass so quickly from a sound to a decaying or putrefactive condition? Or how a slight wound may, if neglected, quickly become painful and dangerous? The bacterium causing lockjaw, when introduced through a wound in foot or hand, multiplies in the favorable conditions provided in the human body with wonderful rapidity and in a week or ten days may defy curative treatment.

Habitat. - Mention has been made of the extent and abundance of bacteria. They are universal and thrive wherever nutritive and other conditions are favorable. They are found in air, water, milk, and meat, and so on throughout the whole list of things which concern human life. Their abundance in the air varies with conditions. Bright sunlight, or the atmosphere of the desert, high mountains, and mid-ocean are decidedly unfavorable and cause reduction of numbers. Rain washes them from the air; and prolonged low temperature, as in winter, seems to kill many kinds which do not happen to be protected. Wind scatters them widely. In the country there are comparatively few species and of these a small number; cities show by tests not only greater numbers but more varieties.

Rooms and workshops occupied by many persons reveal a superabundance of bacteria, which becomes excessive when the air is still or gently moving. Any estimate of the number of bacteria in a given place can be only approximate. In a cubic meter of outdoor air in a city have been found one hundred fifty bacteria. In an equal volume of air in a room where several people are working, the number varies from fifteen to twenty thousand. In a sleeping room with the usual ventilation, occupied by three persons, an equal volume of air con- 
tained more than five thousand. Bacteria have strong domestic tendencies, abounding in close association with mankind.

In all surface water, as in springs, wells, brooks, rivers, lakes, and oceans, bacteria of certain kinds are present. Stagnant water is very favorable to them. Water into which sewage is emptied contains not only the usual species and numbers, but many forms which come from infected persons and are therefore dangerous to human life and health. When shellfish, such as oysters, clams, and others, occupy such contaminated waters and are used for food, they are a source of danger.

All kinds of food materials may be infested with bacteria. Milk, even when the strictest sanitary precautions are taken in milking and handling, is alive with them. They are collected from the air, the body of the cow, the milk vessels, and the persons handling the milk. The varieties collected are many, some useful and others useless, and possibly others harmful or disease-causing, as those introduced by a typhoid fever carrier in handling milk. Blue or otherwise colored milk, sour, curdling, "slimy" milk are caused by the activities of different kinds of bacteria. How such changes may be retarded and dangerous bacteria destroyed will be taken up later. Other food materials may have a large allotment. Eggs just beyond the point of freshness, meats that have become tender with storage, vegetables and fruits of all sorts, these have their freight of bacteria. Moldy bread and cake are alive with them. But it is not necessary that foods should be tainted or discolored to prove the presence of the microörganisms. Food products purchased in the market and sold as edible show astonishingly large numbers of bacteria. One sample of catsup showed by count over 5,000,000 bacteria to the cubic centimeter. Another revealed 400,000,000 bacteria and 7,500,000 spores per cubic centimeter. A plum jam had half a million bacteria and as many yeasts per cubic centimeter. The number of bacteria and other organisms in any food is definitely related to the cleanliness of the place where it is prepared. 
288. External conditions affecting bacteria. - The conditions upon which bacteria depend for life and multiplication are physical, chemical, and biological. The physical conditions are generally those considered for molds and yeasts, namely, moisture, temperature, light, nutritive materials, and possibly electricity. These need only summary statement. While moisture is necessary to growth, bacteria are very resistant to drought, and their spores remain alive for years in extreme dryness. Two facts make this clear: first, the spores contain practically no moisture, and second, the protective membrane of spores and bacteria is almost impermeable to water.

Temperature varies for different kinds and for different individuals of a kind. Some are rendered inactive by freezing, as those in milk; hence by keeping a liquid frozen the number may be diminished. But those surviving resume growth as soon as sufficient warmth is provided. Many kinds resist extremely low temperatures. Some investigators have kept certain species in a temperature of $-252^{\circ} \mathrm{C}$. for twenty hours with no injury to the organisms.

High temperatures have a deterrent influence upon certain kinds. Pasteurizing milk, or keeping it from fifteen to thirty minutes at a temperature of $140^{\circ}$ to $160^{\circ} \mathrm{F}$., checks the growth of bacteria and kills any of the disease-causing variety, as those causing typhoid fever. But bacteria of the "heat-loving" variety thrive only at a high temperature. Some of these grow rapidly in a temperature of $160^{\circ} \mathrm{F}$. and become inactive when the temperature falls much below $125^{\circ} \mathrm{F}$. Such bacteria are found in compost, in vegetable matter in silos, in hay, and tobacco, all of which develop in fermentation a high temperature. The spontaneous heat of moist cotton waste is due to bacteria of the heat-loving variety. Many kinds, including those causing diseases in animals and men, are adapted to the temperature of the bodies in which they are found.

Destruction of bacteria by heat is possible, but not always practicable. In food materials cooking usually destroys them, 
although it may not affect the spores. Some spores have been boiled in water for ten or more hours without injury; indeed few spores are destroyed by the ordinary boiling temperature unless continued for more than an hour. This is a practical point in preserving food materials, as in canning fruits and vegetables. Where canning is done in factories, temperatures higher than that of the ordinary boiling point are secured under pressure, in order to sterilize the food completely. Fruits are generally free from spores and do not therefore require more than a few minutes of boiling. Vegetables, such as corn, beans, and peas, contain heat-resisting spores which cannot be killed under two or more hours of boiling under pressure. Where heating under pressure is impracticable, the method of repeating the boiling on at least three successive days is employed. Substances are boiled for a time to destroy bacteria, and as soon as sufficiently cooled the spores begin to develop. Boiling ten or fifteen minutes destroys germinated spores, and a third boiling does away with possible survivors. Such fruit juices as cider, or such vegetables as tomatoes, are easily sterilized with one or two short periods of heating. Milk is exceedingly difficult to sterilize, as boiling changes its character, and it often contains spores that are very resistant to high temperatures.

Of the remaining physical conditions, light is important. Molds, yeasts, and bacteria, in their natural habitats, are not wholly exposed to direct sunlight for any considerable period. Practically all kinds are impaired, if not destroyed, by bright sunlight in the course of a few hours. Other conditions figure in this, such as moisture in the atmosphere. This suggests the possibility that light is not so directly influential in killing bacteria as in changing the conditions of the nutritive substances on which they exist. It evaporates the moisture rapidly and may produce other changes which make nutrition impossible and death or a resting state inevitable.

Certain kinds of bacteria are light-loving, as the purple bacteria, which live largely on hydrogen sulfid and absorb 
carbon dioxid directly in sunlight as do the chlorophyll-bearing plants. But most kinds like the darkness in some degree. The pathogenic variety of bacteria may be killed by exposure to direct sunlight, - a fact of value in housekeeping, and in the treatment of certain skin diseases due to fungi or bacteria. Concentrated light from an electric are has been employed beneficially in the treatment of such diseases. What influence the Roentgen ray and radium ray may have is not definitely known, though their use has been claimed as helpful in certain kinds of skin diseases.

Certain chemical conditions affect the growth and multiplication of bacteria. A few varieties thrive well in acid solutions, but most flourish in alkaline conditions. Lime, a common alkali, has a destructive influence upon some varieties but favors others. This accounts in part for the hygienic character of fresh whitewash, mortar, and lime plaster (page 418). Lime is used in agriculture to correct an acid condition of the soil, which favors useless bacteria and throws the useful bacteria into an inactive state. Acids are detrimental to many kinds. The acid secretions of the stomach, for example, are germicidal for many kinds of bacteria, and serve in this way to protect the body from these invading enemies. Almost all fruits are acid enough to prevent the action of bacteria; their decay is initiated by molds that flourish on acid solutions.

Chemical conditions which either destroy or prevent the growth of bacteria are of practical value. Although very little is known at present of the way in which poisonous substances used as germicides act upon the bacteria, that limited knowledge has been utilized in obtaining suitable preservatives for many kinds of foods. Experience has long since hit upon many * non-poisonous preservatives which now have scientific sanction. Such preservatives are not only aseptic, checking bacterial growth, but also free from danger to human health. The most common is salt, a strong solution of which has long been used as an aseptic and preservative. Others are acetic acid, or vine- 
gar, and sugar ; and many spices, as pepper, cinnamon, cloves, allspice, sage, and nutmeg.

Preservative compounds are sometimes used which contain certain mildly poisonous substances and are therefore germicidal. In these compounds may be found such substances as formalin, borax, boric acid, benzoic acid or benzoate of soda, saltpeter, and salicylic acid. These, even in small quantities, may be injurious to human health if their use is long continued. Because of the danger from cumulative poisoning, the use of such preservatives is inadvisable. Their use in foods for the public market is prohibited by law except in one or two cases, in which the amount and kind of substance used must be kept within certain limits and published on the labels. It has been a common practice to preserve butter from becoming rancid by the addition of a small amount of borax or formalin. One twelfth of an ounce of pure borax in one quart of milk prevents the growth of bacteria, and a very small amount of formalin has a similar effect. But either practice is objectionable. Formalin in milk and other foods has a chemical effect upon the protein substances, rendering them difficult of digestion.

Most food substances are safely and satisfactorily preserved by thorough drying or cooking, by sugar, salt or vinegar solutions, or by refrigeration. With clean handling and sterilized containers these methods have all the advantages and none of the dangers involved in the use of chemical preservatives.

Only a few of the substances valuable as germicides or as aseptics need be mentioned. Carbolic acid or phenol in dilute solution is often used, but it is effective for only a few kinds of bacteria and acts slowly except when very strong. Chlorine in the form of chlorid of lime is a valuable germicide and disinfectant for many purposes, such as waste water pipes and sinks, but its general use is objectionable because of its pungent odor. Formalin is becoming the leader of all disinfectants and germicides, because of its convenience and effectiveness (page 598). Perhaps one of the best and most useful aseptics is 
hydrogen peroxid. This in ordinary strength acts as an oxidizing agent, and in a sufficiently concentrated form is a good disinfectant. Cuts and abrasions of the skin are advantageously bathed with hydrogen peroxid. In dilute solutions it is an excellent gargle.

Tooth powders and mouth washes are sometimes supposed or claimed to be germicidal. These usually contain some of the essential oils, such as peppermint, eucalyptus, thyme, and some contain hydrogen peroxid. The solutions are necessarily weak and possibly only slightly disturbing to bacteria. The mechanical action of the toothbrush with a plentiful supply of water performs the really effective work of prophylaxis.

All soaps are prophylactic, that is, preventive of bacterial growth. They are so because they promote cleanliness, and cleanliness is prophylactic. But soaps are not disinfectant unless they are kept for a long time in contact with the body. The chief value of soap is detergent, or to remove dirt, which property is associated with the mechanical action involved in its use. Other hygienic values claimed for a soap are usually fictitious or, if present, are at the expense of its cleansing qualities. In general a good vegetable oil soap, vigorously applied, is, next to a strong salt solution, one of the most convenient and cleansing aseptics.

The biological conditions of bacterial life and growth are many and complex. Some varieties are interdependent or coöperative, and some in conflict with others. Just what may determine such relations is not known. Bacteria have been studied mostly in the laboratory, in pure cultures, while in nature varieties are ordinarily mixed, except in some diseases of plants and animals.

A few examples of relations between different groüs of bacteria are taken from familiar substances. In milk one species of bacteria produces lactic acid. If no interference occurs, the acid soon becomes excessive for the acid-producing bacteria, which cease to develop in the medium they created. 
But lactic acid is exactly the food material for the mold, oidium lactis, which begins to develop and by consuming the acid maintains the condition in which the acid-producing bacteria thrive. Another instance is found in the relations of certain soil bac- teria. Protein substances in decaying organic matter are changed by bacteria into ammonia. A different species of bacteria attacks and uses the ammonia, oxidizing it to form compounds which are called nitrites. As soon as nitrites are present in the soil, another species of bacteria begins to develop, and using the nitrites as food material, combines them with oxygen and produces nitrates. These nitrogen compounds are at once available to green plants. Microörganisms of this species are called the nitrate bacteria. This helps in understanding the production of the great beds of sodium nitrate or saltpeter in Chile and Peru from the accumulations of seaweeds in inland seas. The seaweeds were gradually decayed and changed into ammonia, then into sodium nitrite, and finally into sodium nitrate.

These examples indicate ways in which many microscopic plants are interdependent or coöperative among themselves. They may further suggest how chlorophyll-bearing plants are dependent upon the work of microörganisms, and how we who depend upon the green plants for food and clothing are also dependent upon the invisible multitudes of bacteria which are growing everywhere.

\section{SUMMARY}

Bacteria like molds and yeasts are dependent plants having no chlorophyll.

Many kinds of bacteria are useful; a few are pathogenic.

Pathogenic bacteria cause such diseases as tuberculosis, pneumonia, diphtheria, typhoid fever, cholera, and tetanus.

Some bacteria produce putrefaction and decay, others prepare the nitrates necessary for plant growth.

Bacteria may be classified on the basis of their source of food into three groups: (1) saprophytes, (2) parasites, (3) independent bacteria.

Bacteria are also classified on the basis of their form and appear- 
ance into the spherical, the rod-shaped, and the spiral. To the rodshaped belong the bacilli causing typhoid fever.

Bacteria are the smallest plants, ranging from $\frac{1}{10,000}$ to $\frac{1}{50,000}$ of an inch in diameter. They reproduce by division and by spores, and they live wherever the nutritive and other conditions are favorable.

Many bacteria endure freezing. Pathogenic bacteria are killed by boiling. Most kinds of bacteria, except in the spore stage, are weakened or killed by sunlight.

Food preservatives are germicidal or disinfectant (killing bacteria), or aseptic (preventing their growth). They may be harmless to human beings, as are spices and salt; or probably harmful, as benzoate of soda; or poisonous, as formalin.

Other aseptics and disinfectants are carbolic acid, which is dangerous when strong, and hydrogen peroxid, which is safe.

There is often a mutual dependence among groups of bacteria.

\section{REVIEW QUESTIONS}

1. Mention five instances in which the work of bacteria is useful to human life. 2. Where are bacteria found? How abundant are they? 3. What is a common evidence of the presence of bacteria?

4. To what group do the pathogenic bacteria belong? 5. What are some diseases caused by bacteria? 6. Why is the study of bacteria important? 7. What four facts do we need to know? 8. To what does this knowledge lead? 9. Into what groups may bacteria be classified in accordance with their relation to human welfare? 10. Where are bacteria found in the human body? Are they always harmful?

11. Name several industries which are dependent upon the work of bacteria. 12. What groups of bacteria are especially important for plants? 13. What important element of plant food do bacteria contribute? 14. In what form does it exist abundantly? 15. How do bacteria enable green plants to get their food? 16. How is the free nitrogen of the air made available for certain plants? 17 . What use is made of nitrogen by green plants? 18. What use do animals and men make of plant products? 19. What becomes of the waste products of animal life? 20. How is nitrogen again restored to the soil? 21. What do the denitrifying bacteria do? 22. What does the complete cycle of nitrogen show?

23. What are the three main classes of bacteria? 24. What is the characteristic of $(a)$ the saprophytes, $(b)$ the parasites, $(c)$ the independent bacteria? 25. (a) What are some of the independent bacteria?

(b) What are the sources of their food?

(c) What are 
their products? 26. How may bacteria be classified on the basis of their relation to oxygen?

27. Why is the study of bacteria difficult? 28. What methods are used to make bacteria visible? 29. How are bacteria grown for study? 30. Why is it necessary to sterilize materials and containers? 31. From what sources may cultures be inoculated to obtain growths of bacteria? 32. What conditions favor their growth?

33. What is the simplest form of bacteria? 34. What different forms do bacteria take in different conditions? 35. What are the classes of rod-shaped bacteria? 36. Give an example of motile bacteria. 37. How do they move? 38. Illustrate their rate of motion. 39. Give examples of bent rod-shaped bacteria. 40. Give an example of a pronounced spiral form. Where may it be found? 41. How may the size of bacteria be estimated? 42. How many of the medium size would equal the thickness of ordinary paper? 43. Compare with these the size of the influenza bacteria.

44. How do bacteria usually reproduce? 45. In what other manner do they sometimes reproduce? 46. How rapidly do they multiply? 47. What conditions are unfavorable to bacteria? 48. Where are the greatest numbers found? 49. What forms are found in water under ordinary conditions? 50. When polluted by sewage? 51. What common foods are especially liable to be infected with bacteria?

52. How are bacteria affected by drought? 53. What is the effect of freezing? 54. What is the effect of heat? 55. What temperature kills the harmful bacteria in milk? 56. What bacteria thrive in high temperatures? 57. Where are they useful? 58. What is the effect of cooking on bacteria? 59. How may the spores be killed? 60. Why are certain vegetables, as corn, beans, and peas, difficult to can? 61. By what method can they be sterilized in the home? 62. What is the effect of light on bacteria? 63. What kind thrives in light? 64. What use is made of light in medicine?

65. What is the effect of whitewash on some bacteria? 66. What condition of the soil hinders the beneficial bacteria? 67. How is this corrected? 68. What protection has the stomach against eertain bacteria? Why? 69. How is the decay of fruit begun?

70. Mention the most familiar and safe substances to preserve food. 71. What dangerous preservatives are sometimes used? 72. What germicides are useful in household sanitation? 73. Name some useful in the body. 74. What is the value of so-called germicidal mouthwashes, tooth pastes, and soaps? 75. Give instances in which bacteria are helpful to each other. 76 . In what ways is the work of microorganisms necessary to other forms of life? 


\section{CHAPTER XIV}

\section{THE PROTECTION OF HEALTH}

\section{INFECTION AND ImMUNITY}

THE application of recently acquired knowledge in biological research to the control and prevention of disease forms one of the most interesting chapters in the history of the struggle for human health. The results indicate clearly that the control of disease depends upon the following facts :

289. Classes of disease. - Diseases are of two general classes, the infectious and the non-infectious. Infectious diseases are always caused by living microörganisms. These

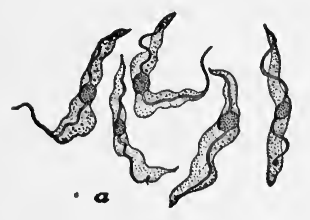

Fig. 172. - Two types of disease-causing protozoa: $a$, trypanosome, the cause of sleeping sickness; $b$, spirichæta, the cause of relapsing fever, showing manner of reproduction by lengthwise division.

\section{as molds, yeasts, and bacteria. Pro}

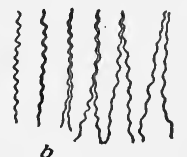
microörganisms are usually described as pathogenic to distinguish them from the harmless and beneficial varieties.

Infectious diseases. - The microörganisms are of two kinds : animal parasites, or protozoa, and plant parasites, Protozoa (Fig. 172) are known to be the cause of such diseases as malaria, yellow fever, rabies or hydrophobia, amœbic or chronic dysentery, and possibly of smallpox and scarlet fever. Bacteria (Fig. 173) are the causative organisms in such diseases as typhoid fever, tuberculosis, diphtheria, pneumonia, influenza or grippe, colds, whooping cough, cholera, tetanus (lockjaw), leprosy, rheumatism, bubonic plague, certain forms of diarrhea and infantile paralysis. 
Non-infectious diseases. - The non-infectious diseases are not caused by microörganisms. They may be due to faults
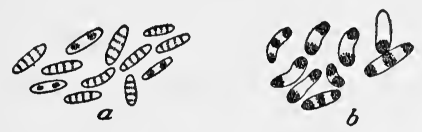

6
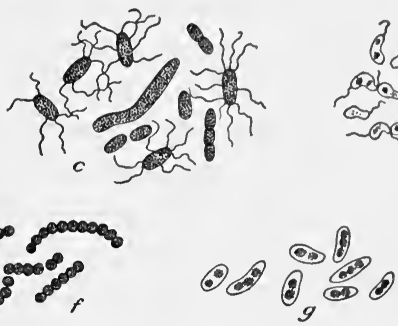

Fig. 173. - Types of pathogenic bacteria: $a$, tuberculosis; $b$, diphtheria; $c$, typhoid fever; $d$, vibrio of cholera $e$, anthrax; $f$, erysipelas; $g$, pneumonia.

in diet, in work, or in rest. They include such diseases as diabetes, some forms of indigestion, nervous disorders, and certain kinds of heart trouble.

290. Transfer of disease germs. - The infective organism, or that which causes a disease, must be transferred by some agent from a patient or a disease carrier to another person who, if susceptible, becomes a victim of the disease. The agencies and ways by which pathogenic germs are transferred are now definitely known in the case of several common diseases. The spread of infectious disease can be controlled by blocking the ways or by destroying the agencies of transmission. The essential facts may be presented in the following way:

I. Infective germs of many diseases are thrown off by way of the mucus-lined openings of the body, such as the nose and mouth. They gain entrance into the body of a healthy individual through such openings. The germs which cause tuberculosis, pneumonia, typhoid fever, diphtheria, influenza or grippe, whooping cough, colds, measles, scarlet fever, cholera, and diarrheal disorders are usually received through the nose or mouth. The germs causing smallpox, chicken pox, and leprosy escape through sores and attack another individual through cuts, sores, or the mucus-lined openings, as the nose and mouth. 
II. The microörganisms which cause rabies, anthrax, and tetanus gain entrance into the body of man or animal through cuts and abrasions of the skin. Tetanus bacteria are abundant in the soil of certain localities and may infect wounds in the feet or hands when these are contaminated with dirt. A dirty nail or splinter may be infected with the tetanus germ, and, if accidentally forced through the skin, leaves the germ to infect the individual. Dust particles lodging upon the skin may carry pathogenic germs and be driven deep into the flesh by cuts. It is necessary merely to mention the cases of those infected with tetanus through wounds made by percussion caps, firecrackers, and so on. Wounds made by any unclean object should be bathed thoroughly with a disinfectant.

III. There are certain diseases, the germs of which may be received through the eyes and through sores or breaks in the skin or in the mucous membrane, such as chapped lips. The germs of these diseases may be conveyed by the hands to the face and mouth, by such public utilities as the telephone, drinking cups, and towels.

IV. The animal parasites which cause malaria, dengue fever, and yellow fever are introduced into the body by the bite of mosquitoes, and that causing sleeping sickness by the bite of the tsetse fly. Infantile paralysis may be transmitted by the stable fly or by fleas, though this is far from being established. Bubonic plague is usually transmitted by fleas which attack both human beings and rodents, such as the rat and the ground squirrel. Rocky Mountain spotted fever, a disease now found in all of the Rocky Mountain and Pacific coast states, is transmitted by the tick. Typhus fever, often epidemic in military camps, is transmitted by vermin, such as the body louse. Its. prevention is a matter of cleanliness and destruction of vermin.

The hands are active in transmitting disease germs, though this may not at first be suspected. It requires but a little observation to become convinced of the unusual opportunity of hands to transfer infective germs. Note how frequently and 
automatically the hands touch the face and lips and eyes, and how easily minute germ-infected particles of dust and moisture may be transferred from hands to face. A healthy person may touch some infected article or person and carry the infective germs eventually to the face, eyes, or lips, or to some abrasion in the skin. Or they may transfer the germs to some common utility, as a towel or dish, and thus make possible their transfer to another person. Food, such as milk, fruit, bread, cakes, and candies, may be infected by handling with unwashed hands.

291. Resistance to disease. - The constitution of the individual who receives the infective organisms is very important in respect to the growth of the germs and the development of the disease. An individual may be immune, that is, resist successfully the invading germs and the disease. Immunity varies in degree from partial to total. It may be due generally to the following causes.

I. It may be natural, that is, due to a constitution inherited from stalwart, disease-resisting family and race. This is aided by maintaining a general condition of health and vigor through proper diet and hygiene.

II. (1) It may be acquired in recovery from a former attack of certain diseases, such as chicken pox, smallpox, measles, scarlet fever, typhus fever, mumps, plague, cholera, and typhoid fever.

(2) It may be acquired artificially by specific treatment, as by vaccination, or inoculation of specific serum containing bacteria or bacterial products, or both.

Immunity when acquired either in the recovery from a given disease or artificially through vaccination is always specific. One who has recovered from an attack of the mumps is specifically immune against mumps, but not against any other disease. Vaccination against smallpox or typhoid fever develops specific immunity against smallpox or typhoid fever.

Degrees of resistance. - An individual may on the contrary 
be susceptible in varying degrees to one kind of infection or another. Susceptibility means lack of resistance to disease microbes and their poisonous products. The conditions determining susceptibility correspond to those eited for immunity, namely, racial, family, and individual. One race may be extremely susceptible to tuberculosis or smallpox, but quite resistant to yellow fever or scarlatina. Differences in family susceptibility are commonly assumed, as when it is said that scarlet fever or tuberculosis seems to run in this family or that. Individual members of a family vary remarkably, however, as one member, though exposed in all possible ways, may remain immune, while all others of the family are afflicted with the disease. Diet, personal hygiene, and previous illness are important factors in determining susceptibility, as well as immunity.

Conditions favoring resistance. - Certain familiar, but often neglected conditions will, if faithfully maintained, contribute. greatly to natural resistance to pathogenic microbes and their toxins. The chief of these conditions is to keep the body and mind strong and vigorous through a proper distribution of work, play, exercise, rest, and sleep, through a sane diet, and habitual cleanliness. Health gained and sustained through the care and use of all the bodily and mental powers is a prophylactic difficult to equal by any artificial means.

Why an invading germ may not cause disease. - The infective organism is very much like a seed which depends for its growth upon the condition of the soil upon which it falls. If the soil is favorable, having sufficient food materials, moisture, a suitable temperature, air, and so on, the seed will develop, mature, and bear fruit. So it is with the infective germ. If the body into which it is introduced is susceptible, that is, affords suitable conditions, as adequate moisture, temperature, mucus, and circulatory fluids, the germ will develop, multiply, and overwhelm the body with its products. There are, however, three possibilities of failure for the invading germ. First, it may find 
utterly adverse conditions in the organism to which it is transferred and it will be unable therefore to grow. Second, it may grow for a time, but be unable to produce or throw off the poisonous substances or toxins, which constitute the principal danger to the body. Third, the germ may grow, multiplying and evolving the toxins which endanger health, but these may be neutralized by natural antitoxins and thus fail to cause the disease. The individual thus infected may experience no interruption of function of an organ or lowering of the general health. The mere introduction into the human system of the infective microbe does not, therefore, necessarily mean disease. The microbe must develop, multiplying and evolving toxins, and these must act injuriously upon the body or cause the disease characteristic of the infective organism.

292. Prevention of infection. - The following measures and precautions are suggested for the control and prevention of the transmission of disease-causing microbes.

I. Artificial immunity. - The most effective preventive of the spread of disease would be to render immune all individuals, or at least all who are likely to be exposed to infection. This would block completely and promptly the transmission of disease by creating conditions making it impossible. Modern science in investigating the conditions upon which health and disease depend has discovered so far ways and means of immunizing against only a few diseases.

1. Vaccines. - Artificial immunity is produced by vaccination or inoculation by hypodermic injection. Vaccination gives active artificial immunity. It is usually the introduction into the blood through a cut or abrasion of the skin of a modified or weakened virus that has been developed in the blood of another person or an animal infected with the disease. The first result of vaccination is the development of a very mild type of the disease, in which are produced by the bodily cells such substances as render the individual actively immune for a considerable period of time. Vaccination is extensively practiced 
for such diseases as smallpox and typhoid fever, and may on occasion be extended to cholera and bubonic plague for men, and anthrax in the case of animals.

2. Antitoxins. - The injection of antitoxins and of bacterial substances in the form of serum has become quite general in late years. Antitoxins are employed especially in diphtheria and tetanus. The antitoxin when injected is diffused through the circulatory system where it neutralizes toxins that may be produced by the bacteria. It does not stimulate the bodily cells to produce antitoxins or increase the natural defenses, and can give, therefore, only temporary protection. It acts primarily as a curative or therapeutic agent, and incidentally gives a temporary and passive immunity to the specific disease.

II. Control of disease germs in the body. - Natural immunity is greatly limited, and artificial immunity can be acquired through vaccines and antitoxins for only a few infective diseases. This makes it necessary to find other ways for preventing the spread of infection to the healthy. A fact of fundamental importance is that pathogenic germs multiply and produce their toxins in the living body of a patient. They must, therefore, be destroyed, either at their source of generation or after they leave the patient. Unfortunately, it is not as a general rule possible to destroy by specific treatment the disease germs in the patient except by such means as would destroy the patient. In only a few diseases, such as malaria, is destruction of the parasites in the patient possible. The primary aim of medical practice is, therefore, to assist the natural energies to overcome the disease and to restore health. It aims only incidentally to destroy the pathogenic organisms.

In the case of certain diseases, as typhoid fever and diphtheria, the pathogenic germs continue to flourish in the body of the recovered patient, some being expelled from time to time in such ways as may expose others to infection. An individual who may be such an incubator and carrier of disease germs suffers no ill effects, because the toxins produced are neutralized. 
Such a disease carrier, because he is unsuspecting and unsuspected, may be a great menace to public health. A recovered typhoid fever patient may be a carrier, or indeed one who has never had the disease and is immune to it may be a typhoid carrier. Several epidemics have been caused by carriers, who, though never victims of the disease, spread the bacteria broadcast. Where conditions point to such a possibility, a prompt and thorough examination of the blood of the suspected individual should be made, and adequate measures should be taken to protect the public.

III. 1. Control of germs after leaving the body. - A third method of control is to destroy the germs after they leave the body of the patient, or the "incubator" individual. This demands unremitting care on the part of the patient, of those in charge of the patient, of the "carrier," and of any who may be exposed to infection. The germs must be destroyed by disinfecting or destroying all discharges and materials used by the patient.

The success of this method depends upon the habits and ideals of the persons concerned. It is the habits formed in health, that are directly involved in controlling disease. Careless habits, disregard for the health and comfort of others, as shown by speaking, coughing, or sneezing into their faces, using common drinking cups and towels, and the like, and allowing them to be used by others, make the problem exceedingly difficult.

This has a wider application than may be recognized at first glance. Careful investigation has made it quite certain that more than half the total number of persons afflicted with communicable diseases are not confined to bed or to the house. The vast majority of the infected class may go about freely everywhere. Of course there is also the constant presence in public places of those who are in the incipient or the convalescent stage of disease, and are dangerous to the public. Little effort or thought seems to be given to protecting the healthy from infection. But the time is probably near at hand when 
either self-control or control by others will be compulsory. Control should begin with and by the infective persons, for they can prevent their discharges from reaching others. But where such control is not exercised, control by others for the sake of the general welfare is inevitable.

2. Practical preventive measures. - There are several rather simple precautions which every person should take, but which are stated chiefly with reference to persons suffering from infectious disease.

a. Discharges through nose and mouth should be carefully confined to cloths that may be disinfected or burned. The same methods of disinfection should be used with all discharges from the patient or with all materials and utensils used and handled by him. Since it is not easy to know whether or not one is a carrier of infection, it should be the habitual practice of every person to take similar precautions.

$b$. Persons known to be infective should not be permitted to work in stores, markets, dairies, or restaurants, where food, water, milk, or public utilities are handled. They should not make use of public drinking or eating utensils, towels, books, telephones, and so on. This may seem at first a personal hardship, but it is certainly not justifiable to expose others to disease.

c. Eventually it will be recognized as necessary to adopt more vigorous measures in behalf of the public health. Strict supervision of health should become commonplace. Probably all infective individuals should be isolated for curative purposes, as is now done in the case of such diseases as leprosy, smallpox, typhus fever, and scarlet fever. This will insure the control of all bodily discharges, with disinfection, and destruction of disease germs. In such diseases as tuberculosis, pneumonia, influenza, grippe, and colds, the danger of which is not now sufficiently recognized, isolation will become the rule. By thorough isolation of the patient, of nurses, and of all exposed persons until danger has passed, infection can be definitely 
checked. It should be considered a crime to endanger, knowingly or not, the public health.

$d$. No material media of communication between a patient and the public should be allowed. This is usually restricted to letters, books, common utensils, and the like, but it should include insects, as flies, fleas, mosquitoes, and animals, as mice, cats, and dogs, all of which may be carriers of infectious diseases.

How to keep well. - So much may be said of the infective individual and his share of the responsibility. Those who enjoy health have a duty to fulfill in order to retain health. The natural defensive organs and elements of the body play a large rôle in the maintenance of health, but they need assistance to operate effectively. The skin, which requires frequent and thorough cleansing, is, in normal condition, a protective organ that no germ unaided can penetrate. The mucous secretion of mouth and nose passages and the gastric secretion have a limited germicidal power; the phagocytes and chemical substances in the blood and tissues are relentless in defending the organism against disease germs and their products; but favorable conditions are necessary to enable these to perform their work.

Some of these conditions are to keep clean all the parts or organs of one's body, mouth and teeth, throat, nose, and skin, and especially the hands, through the daily routine. Hands may be contaminated by anything, as door knobs, hangers in street cars, money, books, papers, telephones, and public utilities of all sorts. After handling, one may well be careful about touching the hands to the face or mouth before they are thoroughly cleansed. Mouth and teeth must be kept clean by frequent washing. Ordinary germicides are not usually strong enough to be useful without injuring the delicate mucous linings of nose and throat. Perfect cleanliness is the safest means of defense.

Disinfection of parts of the house and surroundings where conditions become unsanitary is sometimes necessary. It is 
usually directed by public health officials after a case of infectious disease. But it is now generally recognized that the usual practice is not thorough and is worse than no disinfection because it does not destroy the disease germs, but merely quiets the suspicion and consequently lessens the precautions of those who occupy the room or use articles exposed to infection. Concurrent disinfection or that done throughout the course of the disease is the most efficient sort. When this is thoroughly done, it may render disinfection after the recovery of the patient unnecessary.

For some kinds of disease, as, for example, tuberculosis and anthrax, subsequent disinfection is indispensable, because the germs adhere to walls, furniture, and clothing, and remain alive and virulent for months. Disinfection by fumigation may be made fairly effective. But it should not prevent thorough cleaning of ceiling, walls, and floor of the room, the removal of the old wall paper, if such is used, and fresh decoration of walls and woodwork. Hot water and soap may, when vigorously used, be germicidal as well as cleansing.

Several kinds of disinfectants have come into common use. Sulfur is often burned in a closed room; it destroys bacteria and insects imprisoned there, but it also destroys the colors of textiles by bleaching them. Formalin is preferable, though it fails often to kill certain kinds of pestiferous insects that may inhabit a room. There are many methods of using formalin. It may be evaporated on hot radiators, or in other ways. Sometimes formalin lamps are burned, though these can hardly be considered satisfactory.

A convenient method of fumigation is as follows: The quantities of materials necessary for a room $10^{\prime}$ by $10^{\prime}$ by $10^{\prime}$ are given; these may serve as a unit in computing for larger rooms. All windows and doorways, except one, should be tightly sealed with strips of paper. Articles in the room should be opened so that the disinfectant may have ready access to all parts. A large galvanized metal pail or tub of about six- 


\section{The Protection of Health}

teen quarts' capacity is to be placed in the room upon bricks or other non-combustible material. Into this is put twelve ounces of permanganate of potash, in crystals, and upon it is poured about one quart of formalin. The one doing this should leave the room immediately and seal the door with paper strips. The temperature in the room should be kept above $70^{\circ}$ Fahrenheit, and the room remain closed from eight to ten hours. A thorough airing and cleaning should follow.

\section{Modern Theories of Immunity}

In current explanations of immunity or resistance to disease, the general assumption is that microbes may be checked in their multiplication and development, and their toxins neutralized by the natural energies of the body. The natural energies that are chiefly concerned in the subjection of the microbes, or the neutralization of their toxins, are supposed to be of two kinds, chemical and biological.

293. Ehrlich's chemical theory. - The chemical energies concerned in immunity have been explained by the famous German bio-chemist, Ehrlich, in what is often called the chemical side-chain theory. According to this theory, the cells of the organism are composed of chemical substances, which have also side-chains or associates especially adapted to unite with other chemical substances. The side chains are normally concerned in absorbing and transmitting to the cells the food products taken into the blood in the process of nutrition. But in abnormal conditions, as when the organism is invaded by disease bacteria, the chemical side chains or associates exhibit a peculiar capacity of absorbing and neutralizing poisonous or toxic substances produced by the bacteria. The cells, moreover, directly they are affected through their side chains by the bacterial toxin, are incited to extraordinary activity, producing more of the side-chain substances which eventually split off from the cells and circulate independently in the system. The split off side chains or chemical substances form the antitoxin. 
An antitoxin possesses far greater capacity for uniting with bacterial toxin than do the side chains that remain attached to the cells. The attached side chains retain largely their nutritive function, and only incidentally combine with toxic substances. Thus the antitoxin acts as a prophylactic, preventing the free poisons from reaching and uniting with the cells. It does this as long as it is sufficient in amount and potency, and free toxin is produced in the body. But in certain kinds of infectious disease the toxins unite with cells before these can produce the necessary antitoxins. This union of toxin and cell varies in stability. In tetanus, for example, the poisons unite most readily and firmly with the cells, and especially with nerve cells, which form very little if any of the antitoxin. This union is exceedingly difficult, if not impossible, for an antitoxin to break down. Tetanus antitoxin prepared artificially and injected unites freely with and neutralizes the free toxin in the system. If it is injected directly after infection and in sufficient amounts, it acts as a prophylactic, preventing the development of the disease. But when the disease is well developed, that is, when the toxin has united with a great deal of the cellular tissue, the antitoxin may only rarely effect a cure, for it fails to break down the chemical union of cell and toxin.

How an antitoxin may bring about a cure is made quite clear by the chemical side chain theory. In a disease like diphtheria, where the union of cell and toxin is more easily broken down than in the case of tetanus, an antitoxin may be curative as well as prophylactic. In diphtheria the bacterial poison rapidly combines with the cells before sufficient antitoxin can be formed to guard them. But as soon as the antitoxin is formed in sufficient amounts by the cells responding to toxic stimulation, or as soon as it is injected, it begins not only to absorb and neutralize the free toxins in the system, but also to break down the union of cell and toxin. Thus antitoxin in diphtheria performs a twofold service; it is prophylactic in neutralizing 
the free toxin, and curative in breaking down the chemical union of cells and toxin.

294. Metchnikoff's biological theory. - The biological energies that work to the preservation of health or confer immunity have been explained most clearly by Metchnikoff and his school, in what is known as the phagocytic theory. Certain white cells of the blood plasma, and perhaps certain other cells of the body, are phagocytes, devouring or absorbing not only the toxins produced by bacteria and animal parasites, but the bacteria and animal parasites as well. They may be considered the scavengers of the organism. Reference has been made to this function of the phagocytic cells in connection with the malarial parasite. The number and absorptive capacity of these cells will vary with the general health of the individual. The phagocytes may be sufficient to confer on the individual natural immunity, by neutralizing the toxins, or by attacking and destroying the bacteria or animal parasites. Immunity acquired through infection is explained by the fact that the protective cells have increased sufficiently in capacity and in number to destroy the invading germs and to neutralize their poisonous products.

Both biological and chemical factors seem to be necessary in understanding the conditions of immunity and of recovering from disease with the aid of antitoxin. The phagocytic cells, multiplying under stress of infection, attack and digest some species of invading microörganisms. The chemical substances, formed by the cells as side chains to receive food materials, split off in abnormal conditions and act as antitoxins. These combine freely with the respective toxins to form new chemical compounds that are inert and not injurious to the body cells or tissue. Thus the antitoxins are protective energies in the service of the cells. Apparently immunity is dependent upon the biological phagocytes and the chemical substances which form antitoxins.

Practical value of the chemical theory. - The knowledge which 
has been gained of the chemical nature of antitoxins produced by the bodily cells in resisting infective germs, of the toxins formed by the bacteria, and of the new compounds resulting from the union of these two substances is of great practical importance. It throws light upon the natural processes and elements that wage war against invading microörganisms and are active during recovery from disease. It makes possible the artificial preparation and efficient use of antitoxins both for prophylactic and for curative purposes. This constitutes one of the most noteworthy achievements of modern medicine. To-day artificial antitoxins are prepared and used for all bacteria that produce soluble poisons, - poisons, that is, which spread through and affect all surrounding substance, gland, tissue, or blood. They have proved to be especially successful in curing diphtheria and in preventing tetanus; and promise to become equally valuable in treatment of epidemic dysentery, rabies, and other diseases. There is another point strongly emphasized by this knowledge of the processes and the substances involved in recovery from disease. It is that the body should have such hygienic care and medical treatment as will enable it to marshal all its natural energies of defense against the invading organisms, their toxic products, and the resulting diseases.

\section{SUMMARY}

Infectious diseases are due to microörganisms; non-infectious to various causes, as faults in diet, and in amounts of exercise and rest.

Pathogenic germs are transferred directly or indirectly from persons or animals. They may enter the body through cuts or abrasions in the skin, or insect bites, or through the mucous membrane.

Immunity to any disease may be natural, as an inherited quality. It may also be acquired either from having recovered from the disease, or from having been vaccinated against it.

The germ, or infective organism, to produce the disease, must not only find lodgment in an individual, but develop, multiply, and evolve toxins that are not neutralized by the body.

Vaccination, as against_smallpox and typhoid fever, is the inoculation 
of an individual with germs of a mild type or of a kindred disease. It gives active immunity.

Inoculation with antitoxin is curative or therapeutic and gives passive immunity by neutralizing the toxin produced by the pathogenic organisms.

The three methods of preventing the spread of infectious diseases are :

1. Immunizing by vaccination or inoculation.

2. Destruction of disease germs in the patient.

3. Blocking all the ways of transmission and destruction or disinfection of all infective material that may serve as a carrier of the germs.

Immunity is due to chemical substances, which are produced by the living cells of the body and are capable of neutralizing toxins, and to the phagocytes, which, like scavengers, destroy invading germs.

\section{REVIEW QUESTIONS}

1. Upon what facts does the control of disease depend? 2. Name the two general classes of disease. 3. By what are infectious diseases always caused? 4. (a) What are the two kinds of pathogenic germs? (b) What is a parasite? 5. (a) Mention several diseases caused by protozoa. (b) Several caused by bacteria. 6. (a) Mention some non-infectious diseases. (b) To what may such diseases be due?

7. How are disease germs thrown off by a patient? Give instances. 8. How may they be received by other persons? 9. (a) How may tetanus bacteria be received? (b) How should suspected wounds be treated? 10. Why should public utilities, such as the telephone, and utensils, such as drinking cups, be considered dangerous to health? 11. Mention diseases transferred by insects, and how transfer may be prevented. 12. Describe the possible rôle of the hands in disease transmission. 13. How may food and drink be contaminated?

14. What is immunity? 15. What is natural immunity? 16. In what ways may immunity be acquired? 17 . What is meant by specific immunity? 18. What does susceptibility to disease mean? 19. Mention important conditions determining susceptibility. 20. What conditions favor natural resistance to disease? 21. What does prophylactic mean? 22. Why may invading germs fail to cause disease? 23. What conditions are necessary for the germ to cause disease? 24. What would be the most effective measure to prevent the spread of disease? 25. How is active artificial immunity produced? 26. Toward what diseases is such immunity effective? 
27. What use is made of antitoxins? 28. What is an antitoxin? 29. What sort of protection does it give?

30. In what ways may disease germs be controlled or destroyed? 31. (a) What is a disease carrier? (b) Why is such an unsuspected carrier more dangerous than a patient suffering with the disease? 32. What precautions should those afflicted with a disease take to protect others? 33. What conditions necessitate public control of disease? 34. What means should one in good health take to protect oneself from disease? 35. Mention precautions which should be taken in the market, in dairies and restaurants, and in the home? 36. What are the advantages and disadvantages of isolation of the patient and persons exposed to infection? 37. Mention material means by which disease may be communicated. 38. In what ways does cleanliness help us to keep well? 39. When is disinfection necessary? 40. (a) What is concurrent disinfection? (b) What are its advantages? 41. (a) When is subsequent disinfection indispensable? (b) What are the most effective means? 42. Mention several disinfectants, their advantages, and disadvantages.

43. State Ehrlich's theory of immunity, explaining the function of the chemical side chains in normal and abnormal conditions; the formation of antitoxins; the action of the antitoxin. 44. Explain why tetanus is difficult to cure. 45. Explain how the antitoxin for diphtheria acts, according to the chemical theory.

46. What is the theory of Metchnikoff? 47. How does this theory apply in the case of malaria? 48. How may immunity be acquired in accordance with Metchnikoff's theory? 49. Show that both theories may be true and necessary. 50. What use is made of the knowledge of the chemical nature of antitoxins produced in the body when it is resisting disease? 51. For what kind of bacterial diseases are artificial antitoxins prepared and used?

\section{Vaccines AND Antitoxins}

295. Vaccines. - In the eighteenth century Lady Mary Montagu introduced into western Europe the practice of inoculation for smallpox, which had long been common in Turkey and many sections of the Orient. As generally practiced, the healthy person was inoculated through an open cut in the skin with the virus taken from the smallpox sore of a patient with a mild form of the disease. Although this was 
dangerous, producing a genuine but generally mild type of smallpox, it was preferable to loss of eyesight or life through a severe attack. By 1796 Edward Jenner had successfully proved by experiments that vaccination with the virus from cattle afflicted with cowpox causes a local and mild development of the disease, in the course of which defensive substances which give active immunity to smallpox for a number of years are produced in the blood. This method eliminated the risks incurred by the older practice, and at the same time greatly reduced the ravages of smallpox. Vaccination, if made universal and compulsory, would eliminate entirely this loathsome disease.

The discoveries of Jenner and later those of Pasteur have brought about a revolution in preventive medical practice. The use of vaccines, antitoxins, and similar biological products is increasing constantly. Vast quantities of such products are annually prepared under the most exacting and hygienic requirements and are distributed through public and private dispensaries. Inasmuch as all products must be pure, specific, and have a definite potency, their production and distribution were put by Congressional act under Federal control and assigned to the Treasury Department which acts under the Public Health and Marine Hospital Service. Constant supervision of manufacture and thorough testing at practically every point in their distribution insure a dependable and safe product.

Vaccines result in active immunity and are used most commonly for smallpox and typhoid fever for man, and anthrax for animals. During the year 1914 more than 100,000 persons and in 1917 nearly ten times this number were immunized by typhoid vaccination under the direction of the United States Public Health Service. Typhoid vaccination is as simple in performance as smallpox vaccination and produces no ill results. Vaccination is sometimes used against cholera and bubonic plague. New vaccines are being tried and may eventually come into common use. 
296. Antitoxins. - Antitoxins are curative and also give passive immunity for a relatively short. period. The best known and perhaps the most important are those for diphtheria and tetanus, the tuberculins and many others being still in the experimental stage. Antitoxins are employed also in immunizing animals against certain kinds of infection, such as hogcholera and influenza in horses.

Because of the importance of the antitoxins, a brief account of the manufacture of the diphtheria antitoxin, which is typical, is presented to show the exact care and precautions exercised in its preparation.

An antitoxin serum, as it is seen in the market, is in either dried or liquid form. It is administered in solution hypodermically, and as it is absorbed, begins immediately to neutralize the free toxins in the body and to break down any chemical unions of toxin and cells. It is a specific, attacking only that toxin for which it is the antitoxin.

The preparation of diphtheria antitoxin is begun with a culture taken from a patient, and this culture is developed in a special medium kept at a suitable temperature. The resulting culture must be freed from any microbes of different kinds. This is done by inoculating with it tested guinea pigs, in which the bacteria of diphtheria may be isolated and secured in perfect purity. Purity of bacteria is necessary in order to get a specific antitoxin and to avoid danger to life or health by the introduction of harmful microbes. Tests to determine purity are employed at every step of the process.

The bacteria of diphtheria obtained from the guinea pigs are now put into a beef broth medium and incubated at a temperature of $37.5^{\circ} \mathrm{C}$. for seven to ten days. They develop rapidly and produce their toxic substances. The prodùct is examined with the aid of a microscope to detect any possible contamination. The pure culture is treated with a preservative, such as carbolic acid, and filtered to remove all the bacteria from the toxic fluid or serum. The filtered serum is bottled 
and kept at a definite low temperature in a refrigerator. This is done to allow the poison to "season" or mature. At first it deteriorates rapidly, but is finally found, by tests which are made at intervals of a month, to be relatively constant or stable. It is then possible to determine the amount of the dose. This is the diphtheria toxin.

Horses to be used in preparing the antitoxin for diphtheria must be absolutely sound. The antitoxin stable, scrupulously sanitary in all its appointments and conditions, never receives an animal which does not meet satisfactorily every known test. The selected horses are inoculated with the diphtheria toxin the dose of which, beginning with a very small fraction of a cubic centimeter, is gradually increased to the maximum, perhaps 600 cubic centimeters. Inoculations are made at intervals of three to five days, the frequency together with the amount of serum to be injected being determined by the reaction of the animal to previous injections, as shown in fever, and by its complete recovery. If injections can be given at short intervals, the resulting antitoxin serum will have the greatest potency. The treatment is continued usually for a period of from forty to sixty days. When antitoxin is produced freely, as after twenty-eight to thirty days, just as much blood is drawn from the jugular vein of the animal as it can spare without injury.

The blood serum is stored for two days or more in tall glass cylinders, to allow the blood to coagulate. The clotted blood is then removed, leaving a clear, amber-like fluid, which is the diphtheria antitoxin serum. This is treated with a preservative, filtered, examined, and tested for potency, purity, and safety. It is stored in small containers for distribution and use. After the product is safely packed, samples are taken at random and examined independently by experts for possible contamination. Only after this final examination and approval is the product distributed.

When a serum is placed on the market samples are purchased and tested by Federal experts. If Federal examination reveals 
a potency or purity below the standard, or the wrong kind and amount of preservative, the manufacturer must at once withdraw that lot from the market. Thus a standard product is insured which can be used with safety and give dependable results.

\section{SUMMARY}

Edward Jenner, in 1796, improved the method of vaccination against smallpox by using the virus or pus from cattle afflicted with cowpox.

The discoveries of Pasteur revolutionized preventive medical practice and initiated the use of inoculation in the treatment of rabies and anthrax.

Vaccines are used to immunize man against smallpox, typhoid fever, cholera, and bubonic plague.

Antitoxins are used to cure and give passive immunity to diphtheria and tetanus.

The production of antitoxins, as for diphtheria, is a process requiring extreme care to insure a pure serum of known potency.

\section{REVIEW QUESTIONS}

1. (a) What was the common method of preventing smallpox before 1796? (b) What change in the method was made by Edward Jenner? 2. What is the difference between vaccines and antitoxins? 3 . How are the purity, potency, and safety of vaccines and antitoxins insured? 4. Describe the preparation of diphtheria antitoxin. 5. What department of the Federal government supervises and tests the products?

\section{SUGGESTIVE QUESTIONS}

1. Show that the sun is the source of your energy. 2. It has been said that the total weight of microörganisms is greater than the total weight of the animals of the earth. Show that the estimate is a fair one. 3. Would you destroy, if you could, all bacteria for the sake of getting rid of the pathogenic kinds? Would you destroy all fungi if you could? Would you destroy the insects of the order Diptera? Give reasons for your answers. 4. Which do you think are more dangerous, insects or snakes? 5. In most cities ordinances provide for fines and imprisonment for spitting in public places. Should this ordinance be enforced? Does its enforcement interfere with personal liberty?

6. The average length of life of man in civilized countries is increas- 
ing. Why? 7. In what ways do modern ideas and practices accord with the old adage " An ounce of prevention is worth a pound of cure"? 8. It is reported that every member of an Aretic expedition was free from colds except on two oceasions; one when the cabin carpet was taken up and cleaned, the other when new underclothing was unpacked. Explain. 9. What is the dust of a "puff ball"? 10. Man has been instrumental in the extermination of the wild pigeon, and possibly of the cavebear and the mastodon. It is not known that he has exterminated a single species of bacteria or protozoa. Explain. 11. Which has been the more important to human welfare, the microscope or the telescope? Why? 12. What new uses have recently been found for waxed or paraffined paper? Why? 13. Did bacteria exist before man? Give reasons for your answer.

14. Compare the city and the country as to advantages and disadvantages in respect to health. 15. Some people keep the window shades drawn to keep out the sunlight. What reasons are there for and against this practice? 16. Primitive people used open, smoky fires in their tepees or huts. How was this hygienic? 17. Are the natural defenses or the special measures against disease the more important? 18. What scientific reasons are there for the use of rugs rather than carpets? For the use of the vacuum cleaner rather than the broom? 19. What are some of the reasons why smallpox is no longer epidemic in the United States?

20. By what means does man strengthen his natural defenses against disease? What practices do you think tend to weaken the natural defenses? 21. Why should you be interested in maintaining good health? Why should you be interested in the health of others? 22 . Why is the fungus disease of the fly more likely to be epidemic among flies in the fall than in midsummer? 23. What are some of the differences between seeds and spores? 24. In what respect do fungi and bacteria resemble animals? green plants? 25. Do you know of any kind of plant or animal that is not useful to some other kind? 26. What would you presume to be the business of a company from its name "Farmogerm Company"? 27. What other living things besides the crops and his stock should the farmer consider in his business? 28. Are all kinds of plants and animals that you know useful to man? Support your answer with specific references. 


\section{INDEX}

Numbers refer to pages. The principal references are indicated by black-face type, e.g. 172.

Absolute humidity, 45, 54

pressure (steam), 154, $311 \mathrm{ff}$.

temperature, 88, 311

zero, 90, 311, 392

Acetic acid, $128 \mathrm{ff} ., 568,582$

Acetylene, oxy-acetylene flame, 82 gas light, 171

Acid, acetic, $128 \mathrm{ff}$.

citric, 129

hydrochloric, 120

lactic, 553, 568, 584

sulfuric, 119

Adaptation, a condition of natural selection, 478, 480, 489 ff., 492,512

Aëration, agencies of soil, 470

Aërobic bacteria, 216, 574

Aëroplane, see Airplane

Ague, 527

Air, composition of, 76, 131, 546

compressibility of, 231

expansion of, $43,64 \mathrm{ff} ., 97,229,231$

humidity of, see Atmosphere

in soil, 469

saturation of, $43 \mathrm{ff}$.

weight of, $11 \mathrm{ff}$., 42,64

Airplane, 389, 394 ff., 398 ff., 401

Alcohol, boiling point, 313

distillation of, 562

freezing point of, 90

production of, $129,562,566,568 \mathrm{ff}$. wood, 128

Algæ, 220, 224

Alluvial soil, 456, 474

Alternating current, 320, 327, 328, 334

Altitude and air pressure, $10 \mathrm{ff}$., 391, 392, 393

Aluminum, $167 \mathrm{ff}$.
Ammonia, occurrence of, 155, 163, $546,572 \mathrm{ff}$.

use in refrigeration, $151 \mathrm{ff}$., $156 \mathrm{ff}$., 164

Ammonia water, 164

Ammonium sulfate, $163 \mathrm{ff}$.

Anaërobic bacteria, 216, 574

Anemometer, 23

Aneroid barometer, 89

Aniline, 164

Animals and soil aëration, 471

Anopheles mosquito, 525 ff., 542

Anther of flower, $484 \mathrm{ff}$., 494

Anthracene, 164

Anthracite, 144, 414

Anthrax, microörganism causing, 589 cure and prevention of, 594, 598, 605

Anticyclone, 21, $31 \mathrm{ff}$.

Antiseptic, see Germicide

Antitoxins, artificial, 600, 602, 606, 608 natural, $593 \mathrm{ff}$., $599,600 \mathrm{ff}$.

public control of, 605

Arc, electric, 174 ff., 248

Arch bridge, 287, 289

Archimedes, principle of buoyancy, $340 \mathrm{ff}$., 368

and the lever, 258

Argon, 546

Arid soil, 457, 465

Armature, 322, 324 ff.

Arrowrock dam, 202 ff.

Artesian wells, 194 ff.

Artificial gas, 119, 145, $161 \mathrm{ff}$.

Artificial selection, 479 ff., 496

Aseptics, 122, 582, 586, 599

Ash, 125, 138, 144, 515

Ashokan reservoir, 191 
Atmosphere, $1 \mathrm{ff}$., 546 and altitude, 391

density of, 11, 42, 63, 392, 393

depth of, 12, 104, $391 \mathrm{ff}$.

electrification of, 38

exploration of, 391, 401

humidity of, $43 \mathrm{ff} ., 103,306$

pressure of, $10,11,15,43,390$, 391, $392 \mathrm{ff}$.

temperature of, $5,10,18,84,390 \mathrm{ff}$.

Babbitt metal, $260 \mathrm{ff}$.

Bacillus, 217, 222, 576

Bacteria, aërobic, 216, 222, 574

acid-producing, 568, 576, 585

anaërobic, 216, 222, 574

and decay, 213 ff., 221, 572, 578, 585

and disease, $217 \mathrm{ff} ., 221 \mathrm{ff} ., 571$

classification of, $216,573 \mathrm{ff}$.

control of, $580 \mathrm{ff}$., 609

cultivated, 572

denitrifying, 473,573

distribution of, $\mathbf{5 7 0}, 578$

forms of, 575 ff., 586

in food, 579

in water, $219,221,570,579$

independent, 574

industrial uses of, $568,571,572$

iron, 574

nitrifying, $75,471,475,572 \mathrm{ff} ., 585$

pathogenic, 217, 221 ff., 570,582 , $585 \mathrm{ff} ., 602$

putrefying, $215 \mathrm{ff}$., 570

reproduction of, $576 \mathrm{ff}$.

saprophytic, $\mathbf{5 7 3}$

size of, $551,577,586$

soil, $75,470 \mathrm{ff}$.

sulfur, 574

useful, 75, 567, $571 \mathrm{ff}$.

Balloon, 389 ff., 401

Barium salts, 176

Barograph, 16, 17

Barometer, aneroid, 89

construction of, 13

correction for altitude, 15

kinds of, $15 \mathrm{ff}$.

water, 15

reading, 14,15

Battery, 332 ff., 335

Bearings, friction-reducing, $260 \mathrm{ff}$.

Beaufort wind scale, 23
Benzene, 164

Benzine, 129, 314

Bessemer converter, 442, 443, 451

Bicycle, 261, 285

Big Creek hydro-electric plant, 236, $240,247,249$

Biplane, 389

Blast furnace, $437 \mathrm{ff} ., 451$

Blizzard, 31

Body temperature, 87, 89

Boiling, 303 ff., 306

and pressure, 155, $299 \mathrm{ff}$.

temperature, 155, $299 \mathrm{ff}$.

Bread, $559 \mathrm{ff}$.

Brick, $419 \mathrm{ff}$.

Bridges, $280 \mathrm{ff}$.

British thermal unit (B. t. u.), 126, 142, 307

Bubonic plague, 532

Buds and budding, 498, 501, 503

Building materials, $403 \mathrm{ff}$.

Buoyancy, 338 ff., 345, 392

Burbank, Luther, $481 \mathrm{ff}$.

Burning point, see Kindling temperature

Burning, see Combustion and Oxidation

Butter, 572

Calcium carbonates, $135,139,417$

Calms, belt of, 31

Calorie, $142 \mathrm{ff}$.

gram, 142

Calyx, 484, 494

Camembert cheese, 553

Canals, $382 \mathrm{ff}$.

in United States, $\mathbf{3 8 5}, 388$

Canning of foods, $580 \mathrm{ff}$.

Cantilever bridge, $283 \mathrm{ff}$.

Capillarity, 467, 474

Carbohydrates, 129, 139, 515, 516

Carbolic acid, 164, 583

Carbon, 81, 124, 128, 137, 168 ff., 175, 443,515

cycle, 136

dioxid, 81, 124 ff., 156, 177, 411, $515,546,562 \mathrm{ff}$.

monoxid, 126, 138, 163

Carbonated water, 134, 411

Carbonates, 135, 139, 411 ff., 433

Carborundum, 426 ff.

Carburetor, $\mathbf{3 3 1}$ 
Cast iron, 441, 451

Cavendish, 117

Cellulose, $520 \mathrm{ff}$.

Cement, 419, $426 \mathrm{ff}$.

Center, of buoyancy, 345, 351

of gravity, 344 ff., 349, 399

Centigrade scale, $87 \mathrm{ff}$.

Cerium, 160

Cesspool, 214

Charcoal, 81, 129, 138, 145

Cheese, molds in, 553, 554

Chemical change, 59 ff., 109, 131, 139 energy, 109

Chemistry and industry, $418 \mathrm{ff}$., 438

Chicago water supply, 192

Chlorid of lime, 583

Chlorine, 545, 583

Chlorophyll, 515

Chloroplasts, 515, 516, 521

Cholera, 208, 217, 577

Chronometer, $376 \mathrm{ff}$.

Cirro-stratus clouds, 28

Cisterns, 197, 548

Citric acid, 129

Clay, 407, 414, 420 ff., 427, 456

Climate, 1, 2 ff.

Clinical thermometer, $\mathbf{8 8}$

Close-pollination, $488 \mathrm{ff}$., 495

Cloud, 28 ff., 44, 50, 55

Cloudburst, $36 \mathrm{ff}$.

Coal, 129, 132, 136 ff. anthracite, 414, 415

bituminous, 138, 144, 163, $414 \mathrm{ff} .$, 445

distillation of, 163

economy of, 127, $235 \mathrm{ff} ., 271,295$, 303

gas, 163, 445

heat value of, $142 \mathrm{ff}$., 144

supply of, 234

tar, 164 ff., 406

Coke, 128, 138, 144, 163

Cold storage, see Refrigeration

Color and eye-hygiene, 177, 182

of flowers, 491, 495

of objects, 179

Combustion, 59, 69 ff., 80, 113 ff., 125, 138

Commerce of Great Lakes, $385 \mathrm{ff}$.

Commutator, 322, 325

Compass, $370 \mathrm{ff}$.

Composition of forces, $360 \mathrm{ff}$.
Compound engines, $310 \mathrm{ff}$.

substance, 113, 130, 139

Compressor pump, 151, 154

Concrete, 419, 427

Condensation, 306

effect on climate of, 35,306

Condenser, steam, $295 \mathrm{ff}$.

Conduction of heat, $93 \mathrm{ff} ., 104,111$

Conductivity of substances, electricity, $167 \mathrm{ff} ., 449$

heat, $95,111,167$

Conductor, heat, 94, 104, 111

Convection, 96 ff., 111

currents, 65, 102, 111

Cooper-Hewitt lamp, 178 ff.

Copper, 168 ff., 176

Corn, 487 ff.

Corolla, 483, 494

Cross-pollination, 488 ff., 495, 503

Crucible steel, 446, 451

Crushing force, 282 strength, 282, 431

Crystals, formation in rock, $408 \mathrm{ff}$.

Culex mosquito, 524 ff., 536 ff., 545

Cultivation of soil, necessity for, $\mathbf{4 7 5}$

Cumulus clouds, 28

Curves, in roads, 274, 278

Cuttings of plants, $499 \mathrm{ff}$., 503

Cyclone, 20 ff., 33, 50

Dams, 200

Decay, 213, 552

Declination of magnetic needle, $372 \mathrm{ff}$.

Dengue fever, 532,543

Density, 342 ff., 351, 392 ff.

Desert land, reclamation of, $203 \mathrm{ff}$.

Dew, 48, $52 \mathrm{ff}$. point, $48 \mathrm{ff} ., 55$

Dextrine, $\mathbf{5 6 0}$

Diamond, 128

Diastase, 518 ff., 521, 567, 569

Diescl engine, 368

Digestion of starch by plants, 518, 519, 521

Digestive ferments, $518 \mathrm{ff}$., 521,553

Diphtheria, 532, 594, 602, 606 ff.

Dirigible balloon, $393 \mathrm{ff}$.

Disease, control of, 523, $531 \mathrm{ff} ., 588$. 591,603

causing bacteria, 217, 548, 570, 592

of plants, $\mathbf{5 5 4}$ 
Disease, prevention of, 592 ff., 605, 608 transmission of, 187, 207, 523, $532 \mathrm{ff} ., 548,589$

resistance to, $591 \mathrm{ff}$.

Disinfectants, 583, 586

Disinfection, 597, 598 ff.

Distillation, $313 \mathrm{ff}$.

destructive, 155, 157, 163

fractional, 314

Division, in propagation of plants,

Doldrums, 31 $499,503,551,563,577$

Dominant characters, 507 ff., 513

Draft, $65 \mathrm{ff}$.

Dry cell, electric, $\mathbf{3 3 2}$

Dust in air, 197, $546 \mathrm{ff}$.

Dysentery, 217, 571

Eccentric, 294

Efficiency of machines, $243 \mathrm{ff} ., 249$, 272,363

Ehrlich's theory of immunity, 599

Electric, arc light, 171, 174

current, alternating, 320 ff., 327 ff., 334

direct, 321, 327, 334

uses of, 165, 202, 248 ff., 316, $320,368,446$

energy, distribution of, $38 \mathrm{ff}$. measuring, 145,173

production of, $234 \mathrm{ff}$., 246, 320, $325 \mathrm{ff} ., 332$

transmission of, 246, $247 \mathrm{ff}$.

furnace, $427,446,451$

generator, 319, $324 \mathrm{ff} ., 334 \mathrm{ff}$.

lighting, cost of, 159, 161

methods of, $\mathbf{1 6 5}$

locomotive, 318

motor, $318,321 \mathrm{ff}$.

Electricity, as energy, 109, $246 \mathrm{ff}$. source of heat, 145

Electrification of the air, $38 \mathrm{ff}$.

Electrode, 178, 184

Electrolysis of water, 130,506

Electromagnet, 323 ff., 335

Element or simple substance, 119, 130, 139

Endosperm, 519, 521

Energy, electric, transmission of, 246, 247, 249

kinds of, 109, 308, 319

measurement of, $243,245,249,354$
Energy, radiant, $106 \mathrm{ff}$.

sources of, $233,245,308$

sun's, $66,106,517$

transmission of, $104 \mathrm{ff}$., $245 \mathrm{ff}$., 258

transformations of, $108 \mathrm{ff}$., $245 \mathrm{ff}$., $308 \mathrm{ff} ., 319,324,334$

Engines, simple and compound, $310 \mathrm{ff}$., 315

Enzyme, 518 ff., 521, 553

Epidemic, 207

Equilibrium, 344, 348, 395

Ether, 108

Ethyl chlorid, 156

Eugenics, 505

Evaporation, 43, 305 ff., 315

effect on climate, 47,306

Expansion, an effect of heat, 64, 91, 108, 109

Fahrenheit scale, 86 ff.

Felspar, 408 ff., 415, 421, 425

Fermentation, 133, 213, 562, 566, 580

Fertilization of flower, 485, 494, 497

Fertilizer, 443

Filtration of water, $208 \mathrm{ff}$., 220

Fire, conditions of, $69 \mathrm{ff}$.

kindling a, 60

uses of, 58

Floral envelope, 483, 494

Flower, parts of, $483 \mathrm{ff} ., 494$

Fly fungus, 554

Fly, as disease carrier, 532 ff.

Food of plants, $459 \mathrm{ff} ., 464,515,520 \mathrm{ff}$.

Foot-pounds, $243 \mathrm{ff}$., 269

Force pump, $230 \mathrm{ff}$.

Forces, graphic representation of, 354 ff., $360 \mathrm{ff}$.

Formalin, 583, 586, $598 \mathrm{ff}$.

Fractional distillation, $314 \mathrm{ff}$.

Friction, 258 ff., 278, 398

Frost, 53

prediction of, $\mathbf{5 4}$

Fuel, heat values of, $142 \mathrm{ff}$.

kinds of, 59, 66, 144

Fungi, 551 ff., 557

Galvanometer, $\mathbf{3 2 7}$

Ganges River, water of, 193

Gas, artificial, 145, $161 \mathrm{ff}$.

lighting, history of, $158 \mathrm{ff}$.

mantle, Welsbach, 160 ff., 171

natural, 129, 136 
Gases, expansion of, 64, 85, 153

Gasoline, 129, 139, 143 ff., 314 motor, 330 ff., 400

Generator, electric, 325, 327

Germicide, 582 ff., 597

Gilbert's theory of magnetism, $373 \mathrm{ff}$. Girder, principle of, $280 \mathrm{ff}$.

Glacial moraine, 457, 474

Glass, 168, $421 \mathrm{ff}$.

Glucose, 519

Gluten, 567

Gneiss, 414

Grades, 268 ff., 318

Gradient, of roads, $272 \mathrm{ff} ., 318$ of winds, 25

Grafting, 502, 503

Granite, 408 ff., 415

Graphite, 128, 414, 415

Gyro-compass, $375 \mathrm{ff}$.

Gyroscope, 375 ff., 399

Hail, 36, 52, 55

Harbors, 379 ff., 387

Heat, absorption of, 109, $152 \mathrm{ff}$. capacity of substances, $100 \mathrm{ff} ., 111$, 142,307

of water, 101, 142, 304

. energy, $107 \mathrm{ff} ., 295 \mathrm{ff}$., $306 \mathrm{ff} ., 31.1$ measuring, $141 \mathrm{ff}$.

of the liquid, 304

of melting, or fusion, 149, 156

of steam, $303 \mathrm{ff} ., 305,309,312,315$

measuring, 306

sources of, $59 \mathrm{ff} ., 93,108$

transfer of, $93 \mathrm{ff}$.

units of measure of, $141 \mathrm{ff}$., 306

values of fuels, $142 \mathrm{ff}$., 144

Helium, 90, 546

Hematite, 434, 540

Heredity, in plants, 480, 493, 503, 514

Mendel's law of, $505 \mathrm{ff}$., 508, $511 \mathrm{ff}$.

Hero's steam engine, $364 \mathrm{ff}$.

Hookworm, 217

Horsepower, 243 ff., 249

and efficiency of machines, $243 \mathrm{ff}$. 248

Humid soil, 457

Humidity of atmosphere, 43 ff., 103, $305 \mathrm{ff}$.

Humus, 455 ff., 471,474

Hurricanes, 22 ff., 30 ff., 51

Hybridization, 497 ff., 503
Hybrids, 497 ff., 503, 506 ff., 514

Hydraulic press, 230

Hydrocarbons, 129, 139, 144, 162 ff., 413

Hydrochloric acid, 120, 519

Hydrogen, 117 ff., 120 ff., 138, 144, $391 \mathrm{ff} ., 515$

peroxid, $122,546,584$

Hygienic lighting, 182

Hygrometer, 46 ff., 54

Ice, manufacture of, $151 \mathrm{ff}$.

Ice cream freezer, 150

Igneous rock, 410, $413 \mathrm{ff}$.

Illuminants, $159 \mathrm{ff}$.

Illumination, standards of, $176 \mathrm{ff}$.

Immunity, artificial, 591, 593, 603, 605

biological or phagocytic theory of, $601 \mathrm{ff}$.

chemical theory of, $599 \mathrm{ff}$., $601 \mathrm{ff}$. how acquired, 532, 591, $602 \mathrm{ff}$.

natural, 591, 602

Improvement of plants, $481,496 \mathrm{ff}$., $503,511 \mathrm{ff}$.

Incandescent lamp, electric, 160, 165 ff., 183

Inclined plane, $268 \mathrm{ff}$.

Inertia, $275 \mathrm{ff}$.

law of, 276

Infantile paralysis, 533

Infectious diseascs, 217, 588, 589, 603

Inflammable air, 117

Inland waterways, $380 \mathrm{ff}$.

Inoculation, 532, $591 \mathrm{ff} ., 603 \mathrm{ff}$.

Insects and cross-pollination, $490 \mathrm{ff}$., 494

and human health, 523, 532, 534, 590

Insulators, 96, $167 \mathrm{ff}$.

Iron, production of, 430, 431, $434 \mathrm{ff}$., 540

properties of, 168, $431 \mathrm{ff} ., 491 \mathrm{ff}$. uses of, $429 \mathrm{ff}$.

Iron ore, distribution of, $386,432, \mathbf{4 3 3}$, $450 \mathrm{ff}$.

kinds of, 433, 450

mining, 435, 436, 451

reduction of, $438 \mathrm{ff}$., 451

Irrigation, 201, 203 ff., 465

Isobars, $9 \mathrm{ff}$.

and altitude, 10 
Isotherms, $5 \mathrm{ff}$., 10 and altitude, 10

Keokuk power plant, 243, 249

Kerosene, 129, 139, 143, 159, 313, 544

Kilowatt hour, 145

Kindling temperature, $61 \mathrm{ff}$.

Kite, why it flies, $396 \mathrm{ff}$.

Koch and filtration of water, 208, 528

Krypton, 546

Lactic acid, 553, 568, 584

Lacustrian soil, $\mathbf{4 5 7}, 474$

Larva of mosquito, 538, 539

Latent heat, $305 \mathrm{ff} ., 315$

Latitude, determination of, $377 \mathrm{ff}$.

Lava, 409, $414 \mathrm{ff}$.

Lavoisier and chemistry, $70 \mathrm{ff}$., 74, 77, 118

Layering, in propagation of plants, $500 \mathrm{ff}$.

Lead, 70, 81, 168, 169

Leaves, $465 \mathrm{ff}$.

function of, $515 \mathrm{ff}$.

Legumes, 471, 475, 573

Lever, 254 ff., 262, 349

Lift pump, $15 \mathrm{ff}$., 230

Light as energy, $107 \mathrm{ff}$.

Lighting, artificial, $158 \mathrm{ff}$.

Lightning, $39 \mathrm{ff}$.

Lignite, 129, $414 \mathrm{ff}$.

Lime, 417, 426, 439, 443, 582

Limerock, 410 ff., 439,451

Limestone, 410 ff., 438

Limewater, 124, 132, 138, 418

Litharge, 81

Loam, 456, 474

Local storms, $34 \mathrm{ff}$. winds, 22

Locks, canal, operation of, 357,387

Locomotives, $289 \mathrm{ff}$.

Lodestone, 371

Log, of ship, $377 \mathrm{ff}$.

London water supply, 193

Los Angeles water supply, 189, 226

Lows, see Cyclone.

Lubricating oil and friction, 260

Lumbering, 405

Machines, 151, 243, 245 ff., 249, 254 ff., $258,308 \mathrm{ff} ., 330 \mathrm{ff} ., 349$

Magnet, poles of, $372,374 \mathrm{ff}$.
Magnetic needle, $371 \mathrm{ff}$.

variation of, 372

Magnetic property of iron, 324, 370, $432,449 \mathrm{ff}$.

Magnetite, 176, 434, 450

Magneto, 326, 335

Malaria, 523 ff., 594

Malarial parasite, $524 \mathrm{ff}$.

Marble, $413 \mathrm{ff}$.

Marseilles-Rhone canal, 385

Matches, 61

Measurement of work, 244, $268 \mathrm{ff}$.

Mendel, 497, $505 \mathrm{ff}$.

Mendel's law, $508 \mathrm{ff}$.

Mercury, boiling point of, 89

freezing point of, 90

resistance to electric current, 168,169

uses of, $14,85,86$

vapor lamp, $178 \mathrm{ff}$.

Merozoites, 527

Metals, relative abundance, $\mathbf{4 3 3}$

Metamorphic rock, $413 \mathrm{ff}$.

Metchnikoff's theory of immunity, $601 \mathrm{ff}$.

Meter, electric, 173

Method, scientific, 530

Mica, 408, 409, 415

Microörganisms, $546 \mathrm{ff}$.

Mildew, $\mathbf{5 5 5}$

Mississippi River, control of, 381

Molds, $550 \mathrm{ff} ., 557,594 \mathrm{ff}$. and decay, 554, 557, 572

and disease, $554 \mathrm{ff}$.

classification of, $552 \mathrm{ff}$.

control of, $556 \mathrm{ff}$.

distribution of, $\mathbf{5 5 5}$

spores of, 550

useful, $552 \mathrm{ff}$., 557

Molecular theory, $107 \mathrm{ff} ., 375$

Molecules, 107, 305

Moraine, 474, 547

Mosquito, $523 \mathrm{ff}$., $536 \mathrm{ff}$. control of, $544 \mathrm{ff}$.

Motor, electric, $320 \mathrm{ff}$. gasoline, $330 \mathrm{ff}$.

Mulch, 468

Naphtha, 129

Natural selection, $477 \mathrm{ff}$. conditions of, $479 \mathrm{ff}$.

Navigation, instruments of, $370 \mathrm{ff}$. 
Nectar of plants, $492 \mathrm{ff}$.

Neon, 546

Nernst lamp, 168

New York City water supply, $190 \mathrm{ff}$., 226

Newton's law of inertia, 276 motion, $350 \mathrm{ff}$.

Niagara Falls, electric plant, 243, 247,249

estimated power of, 385

Nimbus clouds, 30

Nitrates, 75, 475, 572 ff., 585

Nitrifying bacteria, 75, 475, $572 \mathrm{ff}$., 585

Nitrogen, 74 ff., 144, 517 ff., 521, 546, $572 \mathrm{ff}$.

as plant food, $471 \mathrm{ff}$., $572 \mathrm{ff}$.

cycle, 572

Nodules on legume roots, 471, 573

Norther, 31

Odor of flowers, $491 \mathrm{ff} ., 495$

Oïdium lactis, 553, 585

Oil lamp, 158

Ooze, 412, 414

Open-hearth process, $443 \mathrm{ff} ., 451$

Osmosis, 461 ff., 474

Ovary of flower, $484 \mathrm{ff} ., 494$

Overshot wheel, 238

Oxidation, 80, 123 ff., 137

Oxids, 81, 123 ff.

Oxygen, 76 ff., 123, 138 ff., 314, 515, 546

Panama canal, 357, 382 ff., 532

Pantagraph, 320

Paraffin, 129, 131, 139, 314

Parallelogram of forces, $360 \mathrm{ff}$.

Parasites, animal, 524 ff., 531, 557, 588, 590

plant, 532, 554, 557, 570, 574, 585

Pascal's principle, 229, 233

Pasteur's discoveries, 213, 528, 605, 608

Pathogenic bacteria, 217, 570 ff., 588

Peat, 129, 455

Pelton wheel, 238 ff., 248

Penicillium, 553

Petals, 483, 494

Petroleum, 129, 145, 314

Phagocytes, 527, 597, 601, 603

Phagocytic theory of immunity, 601, 603

Phenol, see Carbolic acid

Phosphorus, 61, 79, 443, 518, 521
Photosynthesis, 517, 518, 521

Physical change, 60, 131, 139

Pig iron, 439 ff., 451

Pistil, 484 ff., 494

Pistillate flower, 487, 494

Planetary winds, 64, 102

Plant food, 459 ff., 464, 471

Plants, and climate, 2

light, 466, 478, 479, 517 ff., 581

soil, $454 \mathrm{ff}$.

soil air, $469 \mathrm{ff} ., 472,474$

moisture, $457 \mathrm{ff} ., 474$

temperature, $472 \mathrm{ff} ., 475$

storage of food, $\mathbf{5 2 0}$

Plasmodium, 524

Platinum, 166, 168

Pollen, 219, $484 \mathrm{ff}$.

Pollination, $483 \mathrm{ff}$., 494

and variation, 493,513

control of, $496 \mathrm{ff}$.

Poliomyelitis, $\mathbf{5 3 3}$

Porcelain, 421, 427

Porphyritic rock, 408, 409.

Power, importance of, 233 sources of, $233 \mathrm{ff}$., 246

Pratt truss, 283

Preservation of wood, $405 \mathrm{ff}$.

Preservatives, 582, 586

Pressure, of air, $11 \mathrm{ff}$., $24 \mathrm{ff}$., 43, $391 \mathrm{ff}$. water, $225 \mathrm{ff} ., 299,309,341$

Priestley, 70, 77

Prism, analysis and synthesis of light by, $180 \mathrm{ff}$.

Propagation of plants, 498, 499, 503

Proteins, 517 ff., 573

Protoplasm, 460, 518

Protozoa, 524, 531, 588, 590

Public health, $208 \mathrm{ff} ., 210,212,595 \mathrm{ff}$.

Puddling furnace, $449 \mathrm{ff}$.

Pump, centrifugal, $231 \mathrm{ff}$.

force, $230 \mathrm{ff}$.

lift or suction, $15 \mathrm{ff}$., 230

Pupa of mosquito, $\mathbf{5 4 0}$

Purification of water, domestic, 197, 219

municipal, $218 \mathrm{ff}$.

Putrefaction, $213 \mathrm{ff}$., 570, $578 \mathrm{ff}$.

Pyrometer, 89

Quartz, 408, 415, $424 \mathrm{ff}$.

Quicklime, 417, 426

Quinine, 529, 531 
Rabies, 590

Radiant energy, 106 ff., 111

Radiation, 103, 106 ff., 110

Railroads, 253, 266 ff., 276, 316 ff.

Rain, 50 ff., 197

Rainfall, distribution of, 51, 196 and "lows," 28

Recessive characters, 507 ff., 514

Reciprocating engine, 289 ff., 309 ff., $365 \mathrm{ff}$.

Refrigeration, $147 \mathrm{ff}$. substances used in, 156 temperatures of, 152

Refrigerator, $147 \mathrm{ff}$.

Regenerative braking, 319

Relative density, 97 ff., 342 ff., 351 densities, table of, 343 humidity, 46 ff., 54, 103

Reproductive organs of flower, $484 \mathrm{ff}$., 494

Reservoirs, $218 \mathrm{ff}$.

Resolution of forces, $353 \mathrm{ff}$., $359 \mathrm{ff}$.

Respiration, 133, 520

Rigidity, 432, 450

Ringworm, 554

Roads, 264 ff., 278

Roquefort cheese, 553

Root hairs, $462 \mathrm{ff}$.

Roots, 461, 470, 515, 520

Sand filter, 208, $220 \mathrm{ff}$.

Saccharin, 164

Saltpeter, 74 ff., 585

Sand, 407, 423, 424 ff., 456

Sandrock, 406 ff., 411, 415

Saprophytes, 552, 557, 573

Saturation of air, 43 ff., $48 \mathrm{ff}$., $305 \mathrm{ff}$. Scarlet fever, $588 \mathrm{ff}$.

Scheele, 70, 74, 77

Scientific method, steps in, $530 \mathrm{ff}$.

Screw propeller, $363 \mathrm{ff}$.

Secondary cell, electric, $333 \mathrm{ff}$.

Sedimentary rocks, 407, 410, 413

Sedimentation, 219, 457

Seed, selection of, 493, 502

Seedlings, 498, 502

Selection, artificial, 479, 480 natural, $477 \mathrm{ff}$.

Sepals, 483 ff., 494

Septic tank, 214 ff., 218

Serum, 532, 591, $606 \mathrm{ff}$.

Sewage, as disease carrier, 210
Sewage, bacteria in, $215 \mathrm{ff}$. disposal, municipal, 211, 212, 218 rural, 213

Shale, 408, $413 \mathrm{ff}$., $419 \mathrm{ff}$.

Silica, 424 ff., 439, 442. 444

Silicon, $424 \mathrm{ff} ., 433,441,442 \mathrm{ff}$

Silt, 456, 474

Silver, 168

Simoom, 34, 41

Slag, 439, 443 ff., 449, 451

Slaked lime, $417 \mathrm{ff} ., 426$

Slate, $407 \mathrm{ff} ., 411,415$

Smallpox, 532, 591, $604 \mathrm{ff}$.

Smoke, 125 ff., 138

Snow, 52, 55

Soil, aëration, agents of, $470 \mathrm{ff}$., 475 air, $469 \mathrm{ff}$., 475

and plants, 454 ff., 414

classification of, $456 \mathrm{ff}$.

composition of, $455 \mathrm{ff}$.

formation of, $454 \mathrm{ff}$.

humus in, 455, 459, 474

temperature, $\mathbf{4 7 2} \mathrm{ff}$.

water, amount of, $458 \mathrm{ff} ., 465,474$

character of, $459 \mathrm{ff}$., 474

movement of, $466 \mathrm{ff}$., 474

Solar motor, $\mathbf{6 7}$

Soo canal, 385 ff., 388

Soot, 125 ff., 138

Spectrum, 180 ff., 184

Spirilla, 577

Spontaneous combustion, 80

Sporangia, 550

Spores, 550, 555, 557, 563, 565, 568, $580 \mathrm{ff}$.

Sporoblasts, 527

Sporozoites, $527 \mathrm{ff}$.

Sports, 496 ff., 503

Springs, 196, 198

Stability, 344, 346, 351, 398, 401

Stalactites, 411

Stalagmites, 411

Stamens, 484, 494

Staminate flower, 487, 494

Standpipe, 229

Starch, 516 ff., 520 ff., 567, 569

Steam, $297 \mathrm{ff.,} 305 \mathrm{ff}$., 315, $364 \mathrm{ff}$.

engine, operation of, $293 \mathrm{ff} ., 310$

ff., $364 \mathrm{ff}$.

generation of, $306 \mathrm{ff}$.

heating, 101, 306

turbine, 312, 364, 369 
Steamship, $362 \mathrm{ff}$.

Steel, and iron, importance of, $429 \mathrm{ff}$. kinds of, $446 \mathrm{ff}$., 451

manufacture of, $442 \mathrm{ff}$., 451 tempering, $447 \mathrm{ff}$.

Stegomyia mosquito, 533, 536 ff., 542 ff., 545

Sterilization of food, $580 \mathrm{ff}$.

Stone, $406 \mathrm{ff}$.

Storage, cell, electric, $333 \mathrm{ff}$. of water, $218 \mathrm{ff}$.

Storm warnings, 5, 31

Storms, local, $34 \mathrm{ff}$.

Stratified rock, 407, 410, $414 \mathrm{ff}$.

Strontium salts, 176

Submarine, 368

Sugar, $129,515,517 \mathrm{ff} ., 521,566,569$

Sulfur, $61 \mathrm{ff} ., 81,144,433,443,518$, 521,598

Sulfur dioxid, $81,156 \mathrm{ff}$.

Sulfuric acid, 119,545

Sunlight, analysis of, 180 and bacteria, $581 \mathrm{ff}$.

and molds, $556 \mathrm{ff}$. and plants, $517 \mathrm{ff}$.

Sun's energy, 67 ff., 104 ff., 517 temperature, 89

Superheated steam, $309 \mathrm{ff} ., 312$

Suspension bridge, 280, $285 \mathrm{ff}$.

Tangential force, $274 \mathrm{ff} ., 277$

Tartaric acid, 129

Temperature, $84 \mathrm{ff} ., 89 \mathrm{ff}$., 96

and altitude, 10, $392 \mathrm{ff}$.

and microörganisms, 556, 568, 580

extreme, 89 ff., 150, 392

feeling of, 84

kindling, 61

measurement of, $85,89 \mathrm{ff}$.

of soil, $472 \mathrm{ff}$.

Tempering steel, $447 \mathrm{ff}$.

Tensile force, 282

strength, 282, 431

Tetanus antitoxin, 600, 602

Thermograph, 89

Thermometer, $84 \mathrm{ff}$.

Thermostat, 103

Thorium, $160 \mathrm{ff}$.

Thunderstorm, $34 \mathrm{ff}$.

Tin, 169

Tornado, $34 \mathrm{ff} ., 38,41$

Toxic products, 527, 531, 564
Toxins, 527, 531, 593 ff., 599, 600 ff., 607

Tractive effort, 272, 292, 318, 320

Transformer, electric, 247, $328 \mathrm{ff}$.

Transmission of electric energy, 246 ff., 249,328

Transpiration, $463 \mathrm{ff}$.

Transplanting, 463

Transportation, importance of, 251 , 368

in air, $389 \mathrm{ff}$.

motive force and, 253, $265 \mathrm{ff}$., $270 \mathrm{ff} ., 289 \mathrm{ff}$., $318 \mathrm{ff}$., $320 \mathrm{ff}$., $330,349,353,363,399$ ff.

on land, $251 \mathrm{ff}$.

on water, $337 \mathrm{ff}$.

roads and, $264 \mathrm{ff}$.

- vehicles of, $253 \mathrm{ff}$., $339 \mathrm{ff}$., $362 \mathrm{ff}$., $389 \mathrm{ff}$.

waterways and, $337 \mathrm{ff}$., $379 \mathrm{ff}$.

Truss bridge, $281 \mathrm{ff}$.

Tsetse fly, 532, 590

Tubular frame bridge, $284 \mathrm{ff}$.

Tungsten, $166 \mathrm{ff}$.

Tunnels, 268

Turbine, steam, 310, 364 ff., 369

water, $241 \mathrm{ff} ., 249$

Typhoid carriers, 217, 594

fever, 207, $208 \mathrm{ff} ., 576,586,591$, 594

bacillus, 589

Typhoons, 27, 30, 33

Typhus fever, 532, $590 \mathrm{ff}$.

Undershot water wheel, 238

Unit character in inheritance, 506, 513

Vaccination, 532, 591, 604, 605

Vaccines, 593, 604

Vapor lamps, electric, $177 \mathrm{ff}$.

Vaporization, 152, 155, $305 \mathrm{ff}$.

Variation, in plants, $480 \mathrm{ff}$., 493, $496 \mathrm{ff}$., · $503,507,513$

of compass, $372 \mathrm{ff}$.

Ventilation, $102 \mathrm{ff}$.

Vitiated air, $\mathbf{7 4}$

Voltage, electric, 329

Volume of steam, 308, 315

Walschaert's gear, 294

Water, carbonated, 134, 411

composition of, $114 \mathrm{ff.}, 13 \mathrm{~s}$ 
Water, decomposition of, $115 \mathrm{ff.,} 118$ Weather Bureau, map reading, 10 energy of falling, $233 \mathrm{ff}$., $243 \mathrm{ff}$., Wells, deep, 194 $246 \mathrm{ff} ., 249$

hard, 196, $223 \mathrm{ff} ., 547,548,557$

heat capacity, 101

level of ground, $194 \mathrm{ff}$.

plant's use of, $463 \mathrm{ff}$.

power, $233 \mathrm{ff}$., 248

cost of, 235

neglect of, 235,271

pressure, 201, $225 \mathrm{ff} ., 341$

transmission of, 229,341

solvent power of, 135, 196, $411 \mathrm{ff}$., 415,459

supply, $188 \mathrm{ff}$.

and disease, 187, 198, $206 \mathrm{ff} .$, 210,225

and sewage disposal, $210 \mathrm{ff}$., 225

control of, 187, 223

transportation on, $337 \mathrm{ff}$.

trap, 213

underground, 196, $466 \mathrm{ff}$.

uses of, $98 \mathrm{ff}$., 100, $186 \mathrm{ff}$., 230 , $234 \mathrm{ff}$., $248,305 \mathrm{ff}$.

wheels, $238 \mathrm{ff}$.

Water-gas, 119, 162

Waterspout, $36 \mathrm{ff}$., 41

Weather, 1 ff.

Weather Bureau, the United States, 4, 5 forecast, $\mathbf{2}, 54$ shallow, $198 \mathrm{ff}$.

Welsbach mantle, $160 \mathrm{ff}$.

Wheat, improvement of, 512 grain, constituents of, 521

Winds, $19 \mathrm{ff}$., 31, 64, 111 direction of, $3,19 \mathrm{ff}$. local, 22 planetary, 102 velocity of, $3,22 \mathrm{ff} ., 31$

Wiring a house, 172

Wood, composition and heat value of, 144

distillation of, 128

properties and use, $342 \mathrm{ff}$., $404 \mathrm{ff}$.

Work, measurement of, $244 \mathrm{ff}$., $268 \mathrm{ff}$. of steam, $311 \mathrm{ff}$.

Wright brothers and the airplane, 395, 401

Wrought iron, 449

Yeasts, 551, $558 \mathrm{ff} ., 563 \mathrm{ff} ., 568 \mathrm{ff}$. distribution of, $565 \mathrm{ff}$., 568 uses of, $560,565 \mathrm{ff} ., 569$

Yellow fever, 532 ff., 543

Zero, 86, 87 absolute, $88,90,311,392$

Zinc, 119 
THE following pages contain advertisements
of other Macmillan educational publications 



\section{PRACTICAL PHYSICS}

By N. HENRY BLACK

Science Master in the Roxbury Latin School, Boston

AND

\section{Professor HARVEY N. DAVIS \\ Of Harvard University}

Cloth, I I mo, Illustrated, ix and 487 pages, $\$ 1.25$

The remarkable record that this text has made in the short period it has been on the market is sufficient testimony as to its adaptability to high school needs. And it is not difficult to sum up the features of the book that give it preëminence in its field.

It is written by experts in the field who are, moreover, thoroughly acquainted with the needs and limitations of the high school course.

It is practical in fact as well as in name. It connects the fundamental principles of physics with the everyday affairs of life, by introducing each subject through some familiar experience, by using the appliances of modern industrial and commercial life as illustrations of the principles studied, and by suggesting research questions at ends of chapters that send pupils afield for information.

The rate of progress is adjusted to the ability of the average class to proceed. The pace is slow in the early part of the book, more rapid later. Topics under any one subject are arranged in what experience has shown to be the most teachable order.

The manner of presentation shows exceptional skill in the actual work of teaching. The method is inductive and pedagogically sound - first, the familiar facts, then the underlying principles, then the application to less familiar facts. New subjects are introduced by illustrations from daily life - not by definitions. Principles are introduced by illustrative experiments or by appeal to familiar experience - not stated first and illustrated afterwards.

\section{THE MACMILLAN COMPANY}

\section{4-66 FIFTH AVENUE}




\title{
New Physical Geography
}

\author{
BY RALPH S. TARR
}

Late Professor of Physical Geography in Cornell University

Cloth, r2mo, fully illustrated, pp. 457. \$1.00 net

Some points of interest in connection with this book are :

I. The author has an international reputation as an authority in the field of geology and physical geography.

2. In both content and method of presentation the book makes a strong appeal to the human interest of the pupil. It is well written.

3. It gives particular attention to the geography of the United States.

4. The illustrations are numerous, superior in quality, and significant, and they are used systematically throughout the book.

5. Topic summaries, topical outlines, and review questions following each chapter make its use easy for both pupil and teacher.

6. Experience shows that it possesses to an unusual degree those qualities that make a book a success in the classroom. It is widely used with great satisfaction.

\section{THE MACMILLAN COMPANY}

Publishers

64-66 Fifth Avenue
New York 


\section{Elementary Biology \\ BY JAMES E. PEABODY}

Head of Department of Biology in the Morris High School

New York City, and

\section{ARTHUR E. HUNT}

Head of Department of Biology in Manual Training High School Brooklyn

Cloth, 12 mo, illustrated, 593 pages

\section{List Price, \$1.25}

\section{Elementary Plant Biology}

Elementary Human Biology . . . . . . . . . . .65

Elementary Biology - Animal and Human . . . . . 1.12

This book, a product of many years of successful teaching experience in secondary schools, emphasizes throughout the relation of the science of biology to human welfare. Both matter and method are chosen with reference to this end. Structure and classification are not neglected, but they are made subordinate to function, because the latter is more significant for boys and girls of high school age. The activities of plants and animals and their use by man are shown to be closely related to human welfare, for example, the use of plants for food and clothing; the relation of mosquitoes to malaria and yellow fever and of flies to typhoid fever; the work of bees and silkworms; the importance of birds as destroyers of insects; the work of bacteria as the friends or as the foes of man, etc.

The significant character of the book is further emphasized by simplicity of style, abundant half-tone illustrations, chapter summaries, and interesting laboratory exercises. Text and laboratory manual are com. bined in one. In the appendix are found valuable suggestions for teachers and pupils concerning laboratory equipment, special topics, notebooks, and reference books.

\section{THE MACMILLAN COMPANY}

Boston

Chicago
64-66 Fifth Avenue, New York City

Atlanta
Dallas

San Francisco 


\section{Chemistry}

By WILliaM CONGER MORGaN, Ph.D. (Yale)

Assistant Professor of Chemistry in the University of California, and

JAMES A. LYMAN, Рн.D. (Јohns Hopkins)

Professor of Chemistry in Pomona College

Cloth $12 m 0 \quad 429$ pages $\$ r .25$

\section{A Laboratory Manual in Chemistry}

By the SAME AUTHORS

Cloth I2mo 142 pages $\$ .40$

\section{Chemistry and Laboratory Manual}

$\$ 1.40$

The characteristic feature of these new books is the success with which the facts of everyday life have been utilized and made an essential part of chemical science. This feature has been most enthusiastically commended by teachers who have used the books in the classroom.

With the practical application of principles is combined scientific accuracy and completeness. The treatment has been made interesting without any sacrifice of thoroughness.

The half-tone illustrations are both attractive and significant.

The questions following each chapter serve at once to provoke thought and to relate the science of chemistry to the experiences of common life.

The laboratory exercises are so chosen and worked out as to combine with the study of the fundamental principles of the science, illustrations of its bearing upon human welfare. Chemical science is humanized throughout.

\section{THE MACMILLAN COMPANY}

\section{Publishers 64-66 Fifth Avenue New York}







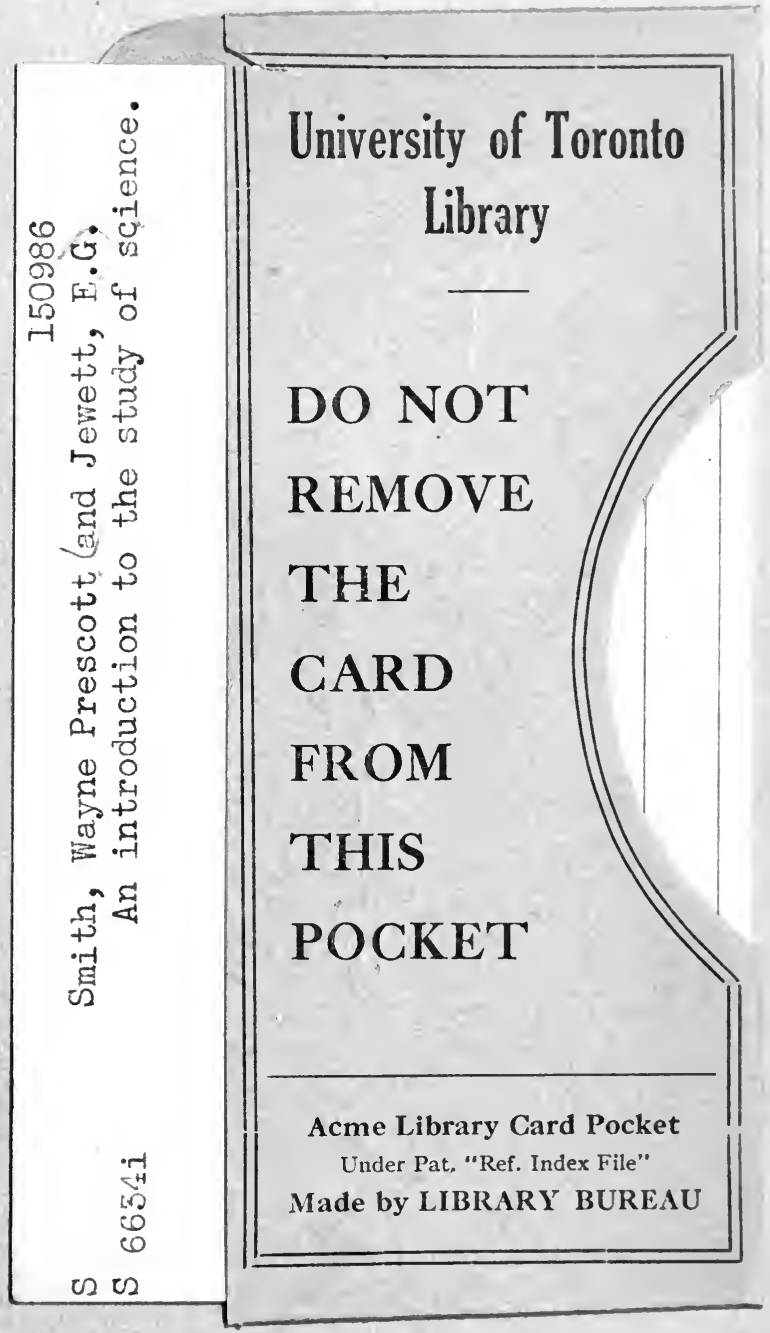



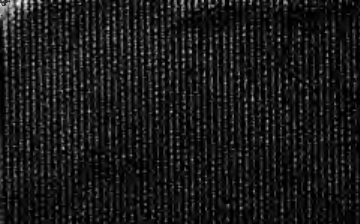

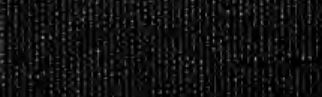

(1) 